\title{
Data Summary Report for Hanford Site Coal Ash Characterization
}

\section{February 2012}

For Public Release 


\section{TRADEMARK DISCLAIMER}

Reference herein to any specific commercial product, process, or service by trade name, trademark, manufacturer, or otherwise, does not necessarily constitute or imply its endorsement,

recommendation, or favoring by the United States Government or any agency thereof or its contractors or subcontractors.

This report has been reproduced from the best available copy.

Printed in the United States of America 


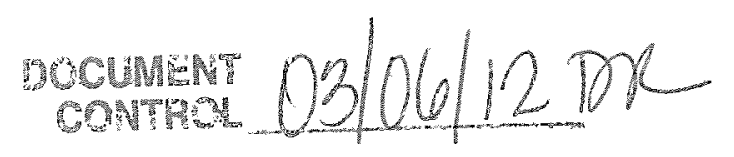

WCH-506

Rev. 0

\section{STANDARD APPROVAL PAGE}

Title: Data Summary Report for Hanford Site Coal Ash Characterization

Author Name: H. M. Sulloway, Environmental Sciences

Approval: $\quad$ J. A. Lerch, Washington Closure Hanford, Mission Completion Manager

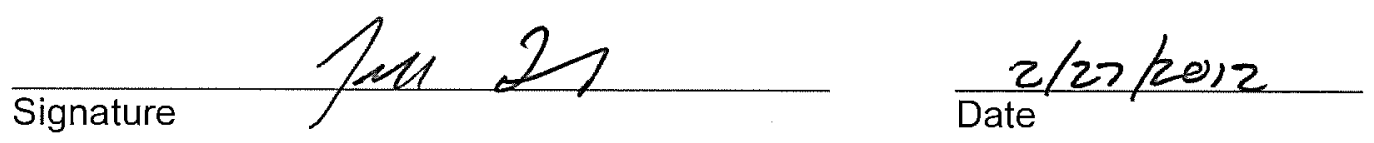

The approval signature on this page indicates that this document has been authorized for information release to the public through appropriate channels. No other forms or signatures are required to document this information release. 

WCH-506

Rev. 0

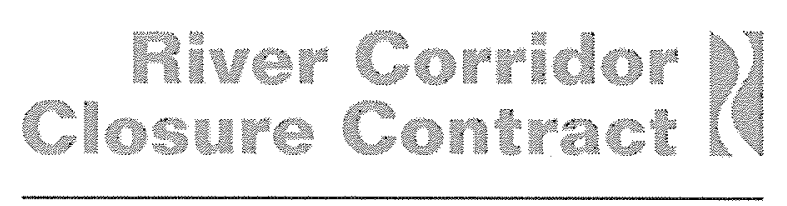

\title{
Data Summary Report for Hanford Site Coal Ash Characterization
}

\author{
February 2012
}

Author:

\author{
H. M. Sulloway
}

For Public Release 



\section{TABLE OF CONTENTS}

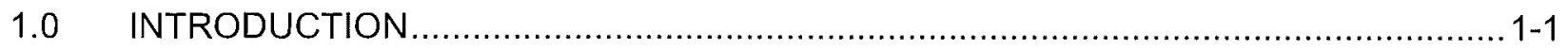

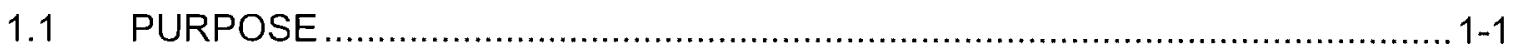

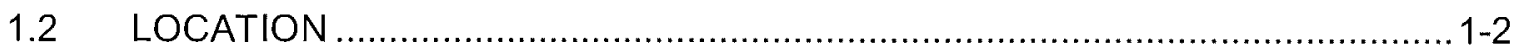

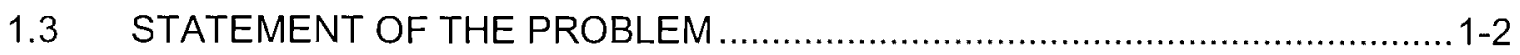

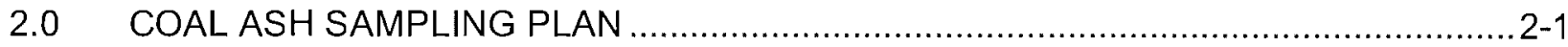

$2.1 \quad$ OVERVIEW OF SAMPLE DESIGN ..........................................................

2.2 LEACHING AND METALS ANALYSIS ..........................................................

2.3 CONTAMINANTS OF POTENTIAL CONCERN …...........................................2-2

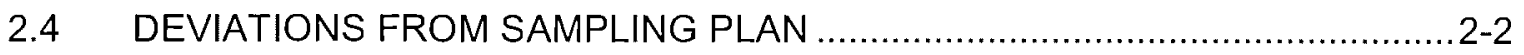

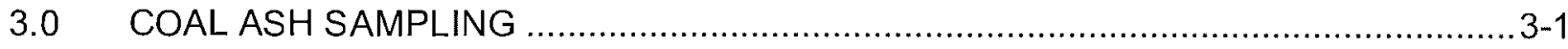

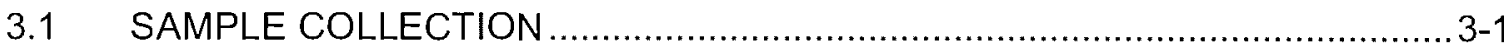

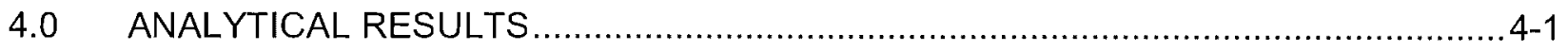

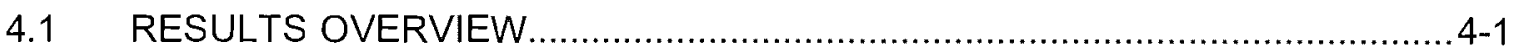

4.2 METALS ANALYSIS FOR SURFACE SAMPLES ...................................... $4-1$

4.3 METALS ANALYSIS FOR DEPTH SAMPLES ........................................... 4-5

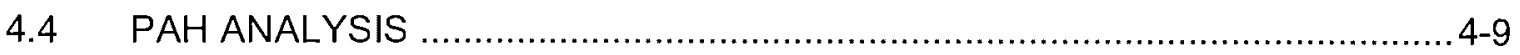

$4.5 \quad$ BATCH LEACH TEST FOR DEPTH SAMPLES ….................................... $4-9$

4.6 ADDITIONAL BATCH LEACH TESTS WITH NON-PH-ADJUSTED WATER.. 4-14

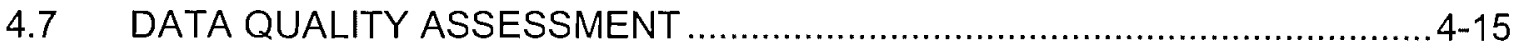

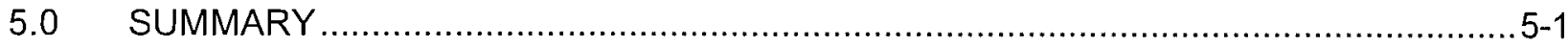

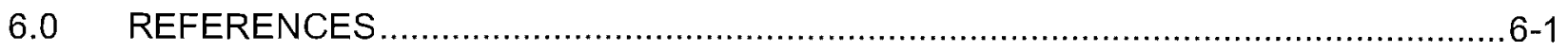




\section{APPENDICES}

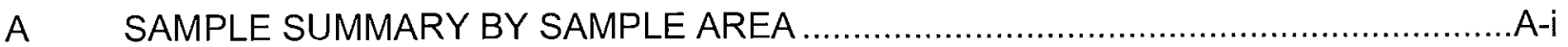

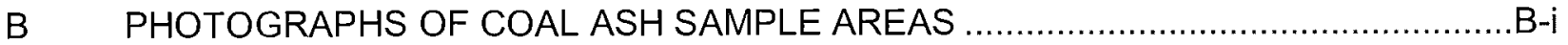

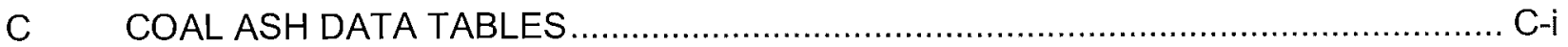

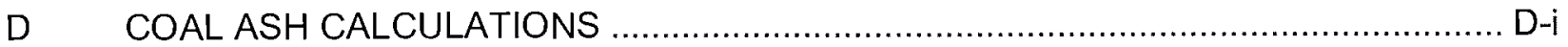

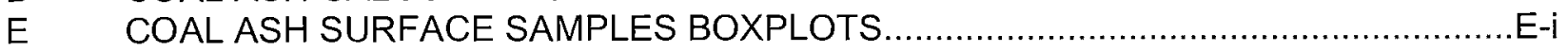

F COAL ASH SURFACE AND DEPTH SAMPLE BOXPLOTS $\ldots \ldots \ldots \ldots \ldots \ldots \ldots \ldots \ldots \ldots . . . . \ldots \ldots$

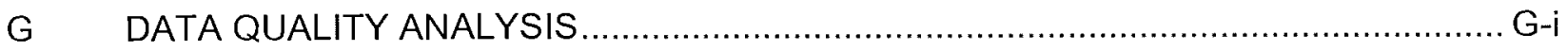

\section{FIGURES}

3-1. Coal Ash at the 126-B-1 Sampling Site.....................................................

3-2. Coal Ash at the 300 Area Sampling Site ..........................................................

\section{TABLES}

2-1. Coal Ash Sample and Analysis Summary..........................................................

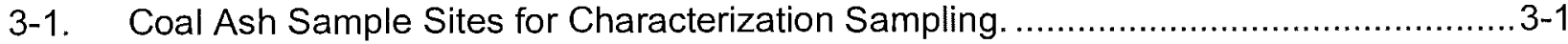

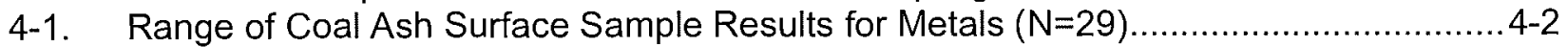

4-2. $\quad 90^{\text {th }}$ Percentile Values of Coal Ash Surface Samples from

Five Sample Sites $(\mathrm{N}=29)$

4-3. $\quad 90^{\text {th }}$ Percentile Upper Tolerance Limit Values of Coal Ash Surface Samples from

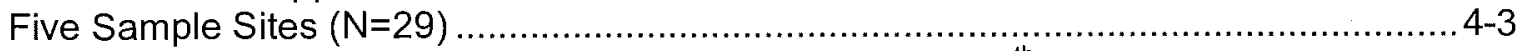

4-4. Coal Ash Sample Sites with Highest Median Values and $90^{\text {th }}$ Percentile Values .........4-4

4-5. Range of Coal Ash Sample Results for Metals for Three Surface/Depth Sampling

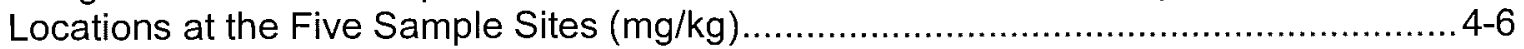

4-6. Mean Analyte Concentrations and Confidence Limits of Combined Surface and Depth Samples from Five Sample Sites........................................................... 4-8

4-7. Range of Coal Ash Sample Results for Detected Polycyclic Aromatic Hydrocarbons for Three Surface/Depth Sampling Locations at the Five Sample Sites ....................4-10

4-8. $\quad$ Leachate Results from Coal Ash Depth Samples .............................................. 4-11

4-9. Identification of Coal Ash Surface/Depth Samples with Highest Arsenic Concentrations.

4-10. Arsenic Leachate Results from Coal Ash Surface/Depth Samples with Highest Arsenic Concentrations. 


\section{ACRONYMS}

$\begin{array}{ll}\text { bgs } & \text { below ground surface } \\ \text { DQA } & \text { data quality assessment } \\ \text { Ecology } & \text { Washington State Department of Ecology } \\ \text { EPA } & \text { U.S. Environmental Protection Agency } \\ \text { ICP-MS } & \text { inductively coupled plasma-mass spectrometry } \\ \text { IQR } & \text { interquartile range } \\ \text { KPA } & \text { kinetic phosphorescence analysis } \\ \text { LCL } & \text { lower confidence limit } \\ \text { PAH } & \text { polycyclic aromatic hydrocarbons } \\ \text { PQL } & \text { practical quantitation limit } \\ \text { QC } & \text { quality control } \\ \text { RCRA } & \text { Resource Conservation and Recovery Act } \\ \text { SAP } & \text { sampling and analysis plan } \\ \text { UCL } & \text { upper confidence limit } \\ \text { UTL } & \text { upper tolerance limit } \\ \text { WIDS } & \text { Waste Information Database System }\end{array}$


WCH-506

Rev. 0 


\section{METRIC CONVERSION CHART}

\begin{tabular}{|c|c|c|c|c|c|}
\hline \multicolumn{3}{|c|}{ Into Metric Units } & \multicolumn{3}{|c|}{ Out of Metric Units } \\
\hline If You Know & Multiply By & To Get & If You Know & Multiply By & To Get \\
\hline Length & & & Length & & \\
\hline inches & 25.4 & millimeters & millimeters & 0.039 & inches \\
\hline inches & 2.54 & centimeters & centimeters & 0.394 & inches \\
\hline feet & 0.305 & meters & meters & 3.281 & feet \\
\hline yards & 0.914 & meters & meters & 1.094 & yards \\
\hline miles & 1.609 & kilometers & kilometers & 0.621 & miles \\
\hline Area & & & Area & & \\
\hline sq. inches & 6.452 & sq. centimeters & sq. centimeters & 0.155 & sq. inches \\
\hline sq. feet & 0.093 & sq. meters & sq. meters & 10.76 & sq. feet \\
\hline sq. yards & 0.836 & sq. meters & sq. meters & 1.196 & sq. yards \\
\hline sq. miles & 2.6 & sq. kilometers & sq. kilometers & 0.4 & sq. miles \\
\hline acres & 0.405 & hectares & hectares & 2.47 & acres \\
\hline Mass (weight) & & & Mass (weight) & & \\
\hline ounces & 28.35 & grams & grams & 0.035 & ounces \\
\hline pounds & 0.454 & kilograms & kilograms & 2.205 & pounds \\
\hline ton & 0.907 & metric ton & metric ton & 1.102 & ton \\
\hline Volume & & & Volume & & \\
\hline teaspoons & 5 & milliliters & milliliters & 0.033 & $\begin{array}{l}\text { fluid } \\
\text { ounces }\end{array}$ \\
\hline tablespoons & 15 & milliliters & liters & 2.1 & pints \\
\hline fluid ounces & 30 & milliliters & liters & 1.057 & quarts \\
\hline cups & 0.24 & liters & liters & 0.264 & gallons \\
\hline pints & 0.47 & liters & cubic meters & 35.315 & cubic feet \\
\hline quarts & 0.95 & liters & cubic meters & 1.308 & $\begin{array}{l}\text { cubic } \\
\text { yards }\end{array}$ \\
\hline gallons & 3.8 & liters & & & \\
\hline cubic feet & 0.028 & cubic meters & & & \\
\hline cubic yards & 0.765 & cubic meters & & & \\
\hline Temperature & & & Temperature & & \\
\hline Fahrenheit & $\begin{array}{l}\text { subtract } 32 \text {, } \\
\text { then multiply } \\
\text { by } 5 / 9\end{array}$ & Celsius & Celsius & $\begin{array}{l}\text { multiply by } 9 / 5 \text {, } \\
\text { then add } 32\end{array}$ & Fahrenheit \\
\hline Radioactivity & & & Radioactivity & & \\
\hline picocuries & 37 & millibecquerel & millibecquerels & 0.027 & picocuries \\
\hline
\end{tabular}


WCH-506

Rev. 0 


\subsection{INTRODUCTION}

There are significant deposits of coal ash at various locations in the river corridor operable units from previous coal-fired power plant operations. Although coal ash from Hanford boiler facilities was previously determined to be nondangerous and nonregulated from a Resource Conservation and Recovery Act (RCRA) hazardous waste perspective, a more complete study of coal ash at primary disposal locations was undertaken to further characterize the concentrations and leachability of coal ash metals in various Hanford Site locations.

This study is detailed in the DOE/RL-2010-113, Sampling and Analysis Plan for Characterization of Hanford Site Coal Ash Components (SAP) (DOE-RL 2011a). Coal ash sampling was carried out in 2011 and included surface sampling, depth sampling, and batch leach studies from selected river corridor coal ash sites. Surface samples of coal ash from five Hanford Site operable units were analyzed for metals including mercury. A subset of these sample locations

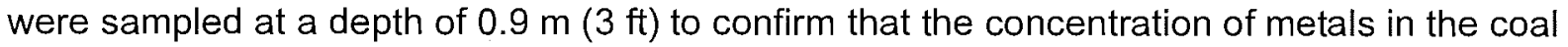
ash is consistent between the surface and depth. A subset of the samples was analyzed for polycyclic aromatic hydrocarbons (PAHs) and total uranium (metal). In addition to directly measuring the concentration of metals in the ash, the samples obtained at depth underwent a serial batch leaching procedure to quantify potential environmental availability.

\section{$1.1 \quad$ PURPOSE}

The purpose of this report is to present data and findings from sampling and analysis of five distinct areas of coal ash within the Hanford Site River Corridor. The sampling objectives include the following:

- Quantify the concentration of metals in coal ash samples collected at 29 statistically determined surface locations and 3 depth locations at each of 5 separate coal ash disposal areas.

- Determine the $90^{\text {th }}$ percentile values and corresponding $95 \%$ upper confidence limits (UCL) on the $90^{\text {th }}$ percentile values (upper tolerance limit [UTL]) for metals from coal ash surface samples at each of five sampling areas. Compare values as appropriate to examine the relative homogeneity of metal concentrations between the coal ash areas. Prepare additional statistical tests as appropriate.

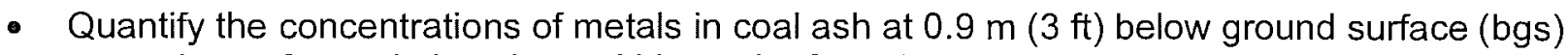
at a subset of sample locations within each of the five sample areas. Compare with data from the corresponding surface samples to determine if the metal concentrations within the ash are relatively homogeneous.

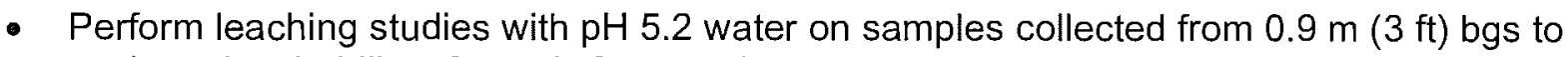
evaluate leachability of metals from coal ash. Perform follow-on leaching studies with non-pH-adjusted water on the samples with the highest arsenic concentrations.

- Quantify PAHs and uranium (total) at a subset of the sample locations at each of the five sample areas. 


\subsection{LOCATION}

In the Hanford Site River Corridor area, coal-fired boilers were used to generate steam in the 100-B/C, 100-D, 100-F, 100-H, and 300 Areas. Multiple coal-fired boiler houses were also constructed in Hanford Camps to support Hanford construction activities. The coal ash from Hanford Site powerhouses in reactor areas was transported to nearby unlined disposal basins via subsurface pipelines. The coal ash was mixed with river water for sluicing through these pipelines. During operations, coal ash from the disposal basins was periodically removed and deposited at various locations in the vicinity of the disposal basins during necessary cleanout operations. Coal ash remains in the various disposal basins and surrounding areas in varying quantities. Excess coal ash was also used as backfill material at unrelated sites, such as decommissioned septic tanks, as well as in Hanford Site infrastructure, such as road bedding.

As described in the SAP, coal ash from five of the River Corridor operable units was sampled. Four of the sample locations (126-B-1, 126-D-1, 126- $\mathrm{H}-1$, and 600-207) are waste sites previously reclassified as Rejected in the Waste Information Database System (WIDS). The fifth sample location is a coal ash disposal area in the 300 Area that is outside of the primary ash disposal basin. The 300 Area Ash sampling location is not an identified waste site.

\subsection{STATEMENT OF THE PROBLEM}

While there are no current sources of coal ash production at the Hanford Site, there are multiple locations within the River Corridor where coal ash from previous production was deposited. Coal ash from Hanford boiler facilities was previously determined to be nondangerous and nonregulated from a RCRA dangerous waste perspective. In light of the U.S. Environmental Protection Agency's (EPA) proposal to regulate coal ash from operating electric utilities and independent power producers (EPA 2010a), along with EPA's risk assessment for coal combustion wastes (EPA 2010b), a study was undertaken to characterize the metals (including mercury) present in coal ash at various Hanford Site locations. 


\subsection{COAL ASH SAMPLING PLAN}

\subsection{OVERVIEW OF SAMPLE DESIGN}

Hanford Site coal ash was characterized using the sampling and analytical strategies detailed in the SAP (DOE-RL 2011a). Five coal ash areas, each in a different Hanford Site operable unit, were identified for sampling. A statistical sampling design was used to locate 29 surface samples at each of the 5 sample areas. A subset of the surface sample locations (three

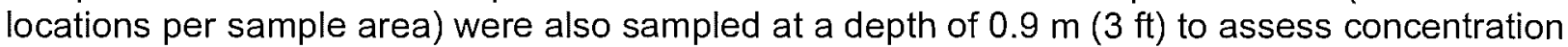
variability with depth. In addition to directly measuring the concentration of metals in the ash, batch leach testing was performed on the coal ash samples collected at depth to quantify potential environmental availability. Further analysis for PAHs and total uranium was conducted on coal ash samples collected at depth, as well as the corresponding surface sample. A summary of the analyses conducted on samples from each sample area is presented in Table 2-1.

Table 2-1. Coal Ash Sample and Analysis Summary.

\begin{tabular}{|c|c|c|c|}
\hline \multirow[b]{2}{*}{ Analyte } & \multicolumn{3}{|c|}{ Sample Location } \\
\hline & $\begin{array}{c}\text { Surface Only } \\
\text { (26 Locations/Areas) }\end{array}$ & $\begin{array}{c}\text { Surface with } \\
\text { Corresponding Depth } \\
\text { ( } 3 \text { Locations/Areas) }\end{array}$ & $\begin{array}{c}\text { Depth } \\
\text { (3 Locations/Areas) }\end{array}$ \\
\hline Metals and mercury & $\mathrm{X}$ & $x$ & $\mathrm{X}$ \\
\hline $\mathrm{PAH}$ & - & $\mathrm{X}$ & $\mathrm{X}$ \\
\hline Uranium (metal) & - & $\bar{X}$ & $\mathrm{X}$ \\
\hline $\begin{array}{l}\text { Batch leach test with } \\
\text { leachate analysis for } \\
\text { metals, mercury, } \\
\text { and uranium (metal) }\end{array}$ & - & - & $x$ \\
\hline
\end{tabular}

- $\quad$ = analysis not performed

$\mathrm{PAH}=$ polycyclic aromatic hydrocarbons

$X \quad=$ analysis performed

The statistical sampling design for this study was based on obtaining sufficient samples ( 29 per sample area) such that $90^{\text {th }}$ percentile values for the coal ash analytes could be computed. The $90^{\text {th }}$ percentile values are used by the Washington State Department of Ecology (Ecology) to determine background concentrations (Ecology 1994). While the subject of this sampling is not to quantify background concentrations, determining $90^{\text {th }}$ percentile values and the corresponding $95 \%$ UCL on the $90^{\text {th }}$ percentile (UTL) for metals in coal ash provides a measure of metals concentrations existing in coal ash from different operable units. Additional statistical analysis of the data is reported in Section 4.0.

\subsection{LEACHING AND METALS ANALYSIS}

As described in the SAP, standardized batch leach tests were performed on samples collected at $0.9 \mathrm{~m}$ (3 ft) bgs using a leach procedure based on ASTM D3987, Standard Test Method for Shake Extraction of Solid Waste with Water (ASTM 2004). The procedure recommends using material screened through $0.95-\mathrm{cm}$ (3/8-in.) mesh. Demineralized water ( $\mathrm{pH}$ adjusted to 5.2) 
was used as the leaching liquid. Three coal ash samples from each of the five study areas were leached at coal ash to water weight ratios of 1 to 1,1 to 2.5 , and 1 to 5 (total number of leach tests $=45$ ) with at least one test in each series duplicated and one duplicate leach test for each coal ash sample (total number of duplicate leach tests $=15$ ). The coal ash-water mixtures were placed in clean, water-tight sample containers (extraction vessels) and rotated end over end through the vessel centerline at a rate of about 30 rotations per minute for 18 hours. Following 18 hours of mixing, the coal ash-water slurry was filtered using a $0.45 \mu \mathrm{m}$ filter.

The leachate was analyzed to determine the concentration of leachable metals including uranium (total) and mercury. The final $\mathrm{pH}$ of each leachate was also reported. Quadruplicate analyses for metals and mercury of the coal ash sample from which the batch leach test material was obtained were also performed.

To investigate any potential effect of leach fluid $\mathrm{pH}$ on the final concentration of leachable arsenic, a second set of batch leach tests was performed on a subset of samples. Additional coal ash had been collected during sampling for the depth samples along with the corresponding surface samples such that a second leach test could be performed. After the arsenic concentration of all surface-depth sample pairs was completed, the three samples with the highest arsenic concentrations were selected to undergo the second batch leach test. The second batch leach test was conducted identically to the first test, except the leach fluid was non-pH-adjusted demineralized water. Analytes for the leachate for the second set of batch leach tests included antimony, arsenic, barium, beryllium, boron, cadmium, chromium (total), cobalt, copper, lead, manganese, molybdenum, nickel, silver, selenium, thallium, vanadium, and zinc.

\subsection{CONTAMINANTS OF POTENTIAL CONCERN}

The contaminants of potential concern for all coal ash samples are metals, including mercury. The metals analyzed by inductively coupled plasma-mass spectrometry (ICP-MS) using EPA method 6010 include antimony, arsenic, barium, beryllium, boron, cadmium, chromium (total), cobalt, copper, lead, manganese, molybdenum, nickel, selenium, silver, thallium, vanadium, and zinc. Selected samples were also analyzed for total uranium by kinetic phosphorescence analysis (KPA) and PAHs using EPA method 8270.

\subsection{DEVIATIONS FROM SAMPLING PLAN}

Two approved change notices are associated with the SAP. TPA-CN-431 (DOE-RL 2011b) added analysis of uranium (total metal) to a subset of the samples. Prior to coal ash sampling, the contract laboratories tested their ability to quantify uranium metal from coal ash using ICP-MS and KPA. Due to elevated practical quantitation limits (PQLs) for uranium analysis using ICP-MS in preliminary analyses with coal ash, analysis for uranium metal was performed using KPA.

As documented in TPA-CN-451 (DOE-RL 2011c), adjustment to the original 126-D-1 sampling plan was required due to the excavation activities from the 100-D-31:7 pipeline remediation. The excavation had covered a significant portion of the coal ash sample area (nine sample locations) with up to $0.3 \mathrm{~m}(1 \mathrm{ft})$ of sand and soil with cobble. Due to the requirement that the coal ash samples be composed of no more than $5 \%$ noncoal ash material (e.g., soil, plant roots, rocks), a new sample area boundary that excluded this disturbed area was created and a new 
statistical sampling design of 29 sample locations was prepared and approved by the regulators.

Due to conditions in the field, the planned locations for several samples were adjusted to a nearby location with sufficient coal ash to obtain a sample. Coordinates for the final sample locations were recorded and are presented with the sample information in Appendix $A$. 
WCH-506

Rev. 0 


\subsection{COAL ASH SAMPLING}

\subsection{SAMPLE COLLECTION}

Coal ash characterization samples were collected as described in the SAP (DOE-RL 2011a). Sampling occurred at five disposal sites located in separate operable units (Table 3-1). Four of the sampling sites are rejected WIDS sites while the 300 Area Ash site is an area of coal ash deposition located in the northeast portion of the 300 Area and next to the Columbia River. Photographs of the five sample sites are presented in Appendix B.

Table 3-1. Coal Ash Sample Sites for Characterization Sampling.

\begin{tabular}{|l|c|c|c|}
\hline Sample Site & Operable Unit & WIDS Site & WIDS Status \\
\hline $126-\mathrm{B}-1$ & $100-\mathrm{BC}-1$ & $126-\mathrm{B}-1$ & Rejected \\
\hline $126-\mathrm{D}-1$ & $100-\mathrm{DR}-1$ & $126-\mathrm{D}-1$ & Rejected \\
\hline $126-\mathrm{H}-1$ & $100-\mathrm{HR}-2$ & $126-\mathrm{H}-1$ & Rejected \\
\hline 300 Area Ash & NA & NA & NA \\
\hline $600-207$ & $100-1 \mathrm{U}-6$ & $600-207$ & Rejected \\
\hline
\end{tabular}

NA $=$ not applicable

WIDS = Waste Information Database System

Coal ash sampling commenced in April 2011 at the 100-B sample site (WIDS site 126-B-1). A total of 23 of the 29 sample locations were sampled in April. Sample work was suspended to address potential worker protection measures. The final six sample locations at 126-B-1 were sampled in August 2011. The remaining four coal ash sample sites were sampled in August/September 2011. Field sampling work was recorded in Logbook EL-1658, Characterization of Hanford Coal Ash Components (WCH 2011).

Sample coordinates from the SAP were located and staked prior to sample collection. Per the SAP instruction, coal ash samples were to contain no more than $5 \%$ soil or other non-coal ash material (such as rocks or plant roots). In cases where material from the prescribed sample locations could not meet these requirements, the sample location was moved to a nearby area of coal ash. Final coordinates for each sample location are presented in Appendix A. Photographs of representative coal ash sample locations are presented in Figures 3-1 and 3-2.

As prescribed in the SAP, three sample locations at each sample site were sampled at both the surface and at a depth of $0.9 \mathrm{~m}(3 \mathrm{ft})$. The locations for depth sampling were chosen at the sampler's discretion and were dependant on the availability of coal ash at depth. Identification of the three sample locations per sample site from which depth samples were obtained is presented in Appendix A tables. 
Figure 3-1. Coal Ash at the 126-B-1 Sampling Site.

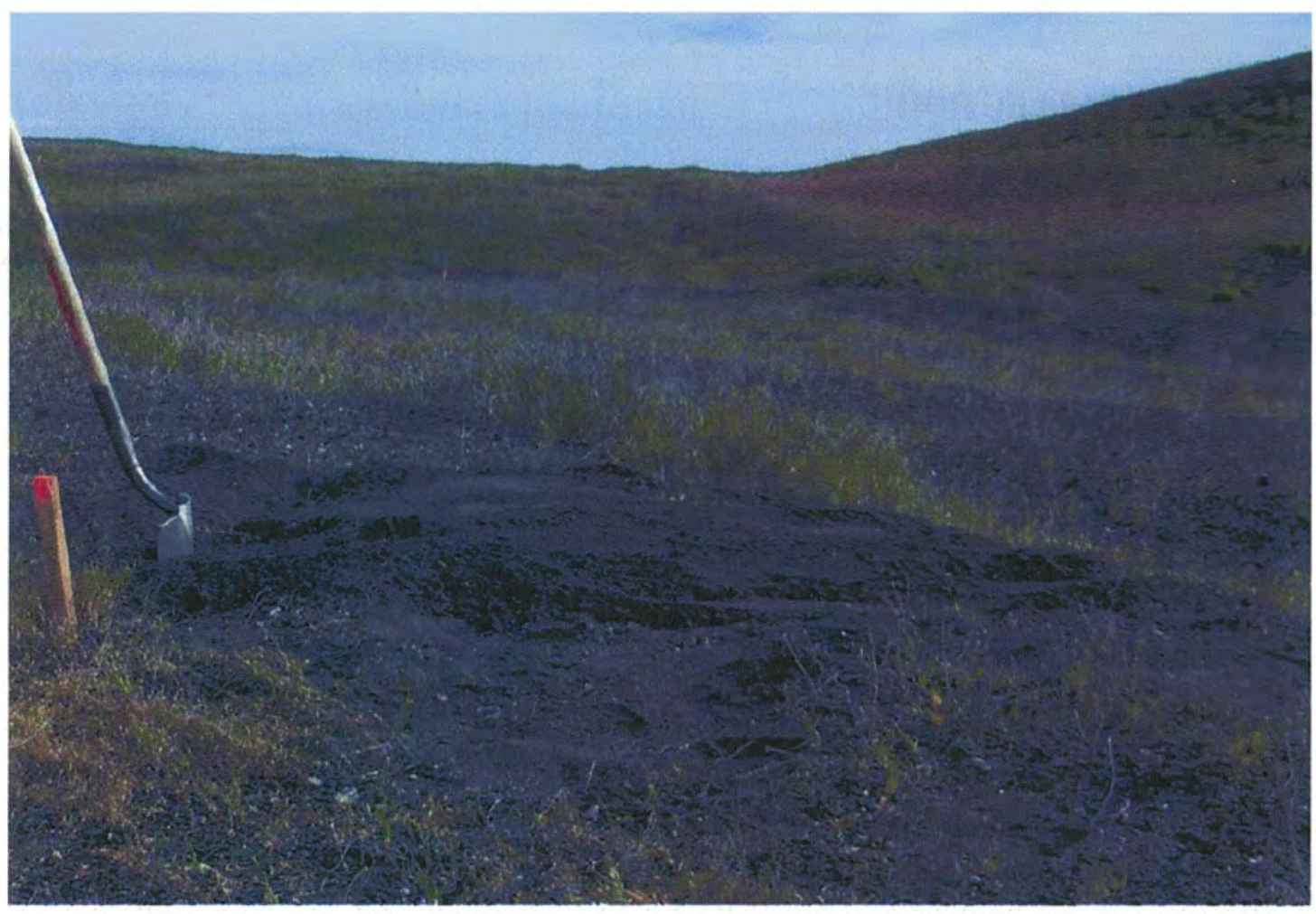

Figure 3-2. Coal Ash at the 300 Area Sampling Site.

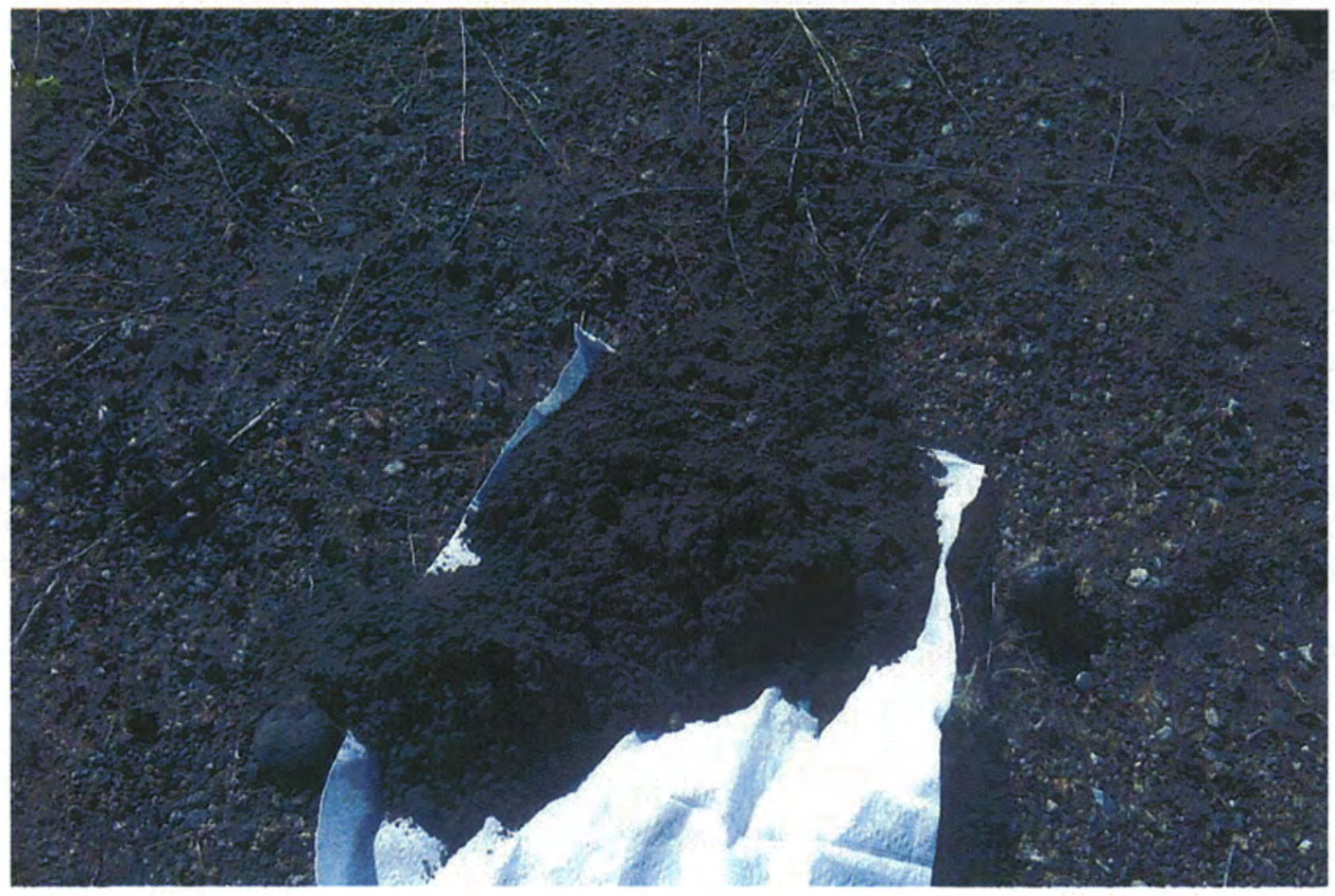




\subsection{ANALYTICAL RESULTS}

\subsection{RESULTS OVERVIEW}

Analytical results from the coal ash samples collected under the SAP and subsequent batch leaching results are reported in Appendix C. Tabulated data is presented to facilitate comparison between the sample areas. For the purposes of calculations, all nondetect data was set to one-half the PQL, and the results of primary-duplicate sample pairs were averaged to obtain one value to represent the sample location.

\subsection{METALS ANALYSIS FOR SURFACE SAMPLES}

At each of the five sample sites, coal ash surface samples were collected at 29 locations. The range of sample results for each analyte is presented in Table 4-1.

Sample data from each of the five coal ash sample sites were used to compute $90^{\text {th }}$ percentile values for metals with at least one detection per sample site using ProUCL 4.1 (EPA 2010c). The $90^{\text {th }}$ percentile represented the value separating the lower $90 \%$ of the data set from the upper $10 \%$ of the data. The $90^{\text {th }}$ percentile values are used by Ecology to determine background concentrations (Ecology 1994).

As discussed in the SAP, a statistical sampling design with 29 samples was required to further calculate a $95 \%$ UCL on the $90^{\text {th }}$ percentile. This calculation is also referred to as an UTL: a one-sided upper confidence limit on the specified percentile. Data from each analyte with at least one detection per sample area were used to produce $90^{\text {th }}$ percentile values using ProUCL 4.1 (EPA 2010c). For nondetect results within these data sets, one-half the PQL was used as the sample result in the calculation. In cases where a duplicate sample was obtained for quality control (QC) purposes, the results from the primary and duplicate sample were averaged to obtain a single value for the sample. Results from the $90^{\text {th }}$ percentile calculation are presented in Table 4-2 and results from the $90^{\text {th }}$ percentile UTL are presented in Table 4-3. Calculations of these values are provided in Appendix D. Antimony, silver, and thallium were not detected in any samples from one or more of the sampling sites. The $90^{\text {th }}$ percentile UTL calculations were not performed for sample sites with no detections of these metals.

Additional statistical analysis of the surface sample data sets from the five sample sites was performed using the nonparametric Kruskal-Wallis one-way analysis of variance test using ProUCL 4.1 (EPA 2010c). The null hypothesis for the Kruskal-Wallis test is that the medians of the data sets (sample sites) are equal. The Kruskal-Wallis tests were conducted at a $5 \%$ significance level. The $p$-value calculated by the analysis determines if the null hypothesis is accepted or rejected. If the $p$-value is greater than 0.05 the null hypothesis is accepted (medians are equal), but if the $p$-value is less than or equal to 0.05 the null hypothesis is rejected (medians are not equal).

Calculation of the Kruskal-Wallis median equality test using ProUCL 4.1 (EPA 2010c) is documented in Appendix $\mathrm{D}$. Of the analytes that met the threshold for analysis (detections in at least three sample sites), the null hypothesis is rejected for all except vanadium. Therefore, vanadium is the only analyte for which the Kruskal-Wallis test found the medians to be statistically equal. 
Table 4-1. Range of Coal Ash Surface Sample Results for Metals (N=29). ${ }^{\text {a }}$

\begin{tabular}{|c|c|c|c|c|c|c|c|c|c|c|}
\hline \multirow{2}{*}{ Analyte } & \multicolumn{2}{|c|}{$126-B-1(\mathrm{mg} / \mathrm{kg})$} & \multicolumn{2}{|c|}{$126-\mathrm{D}-1(\mathrm{mg} / \mathrm{kg})$} & \multicolumn{2}{|c|}{$126-\mathrm{H}-1$ (mg/kg) } & \multicolumn{2}{|c|}{$\begin{array}{l}300 \text { Area Ash } \\
(\mathrm{mg} / \mathrm{kg})\end{array}$} & \multicolumn{2}{|c|}{$600-207(\mathrm{mg} / \mathrm{kg})$} \\
\hline & Low & High & Low & High & Low & High & Low & High & Low & High \\
\hline Antimony & $\mathrm{NA}$ & 1.91 & $\mathrm{NA}$ & 0.965 & NA & 0.300 & $\mathrm{NA}$ & NA & $\mathrm{NA}$ & NA \\
\hline Arsenic & 1.33 & 9.59 & 2.15 & 16.7 & 2.05 & 4.72 & 1.60 & 3.91 & 3.39 & 10.6 \\
\hline Barium & 184 & 2,340 & 407 & 1,770 & 313 & 1,330 & 177 & 782 & 379 & 1,250 \\
\hline Beryllium & 0.632 & 3.22 & 0.588 & 2.61 & 0.429 & 1.70 & 0.544 & 1.95 & 0.46 & 1.46 \\
\hline Boron & 66.3 & 328 & 33.6 & 489 & 20.2 & 455 & 65.4 & 476 & 31.2 & 162 \\
\hline Cadmium & 0.096 & 0.914 & 0.127 & 0.445 & 0.14 & 0.577 & 0.094 & 0.366 & 0.122 & 0.232 \\
\hline Chromium, total & 4.76 & 25.5 & 5.70 & 12.9 & 6.38 & 16.5 & 7.15 & 15.8 & 6.81 & 16.7 \\
\hline Cobalt & 1.00 & 15.1 & 2.31 & 13.7 & 2.14 & 9.94 & 1.9 & 5.12 & 3.21 & 7.14 \\
\hline Copper & 8.6 & 98.3 & 9.66 & 59.4 & 16.7 & 46.8 & 14.7 & 172 & 16.9 & 46.8 \\
\hline Lead & 2.85 & 35.8 & 1.9 & 16.8 & 1.64 & 8.64 & 3.33 & 17.2 & 4.23 & 14.5 \\
\hline Manganese & 13.1 & 521 & 108 & 890 & 67.7 & 300 & 35.5 & 213 & 205 & 537 \\
\hline Mercury & 0.022 & 0.861 & 0.012 & 0.189 & NA & 0.177 & 0.034 & 1.02 & 0.009 & 0.046 \\
\hline Molybdenum & 0.314 & 34.7 & 0.786 & 4.42 & 0.493 & 3.52 & 0.472 & 2.12 & 1.01 & 3.13 \\
\hline Nickel & 3.02 & 31.5 & 4.63 & 20.5 & 5.88 & 22.4 & 5.73 & 14.9 & 7.95 & 19.7 \\
\hline Selenium & $\mathrm{NA}$ & 2.00 & $\mathrm{NA}$ & 1.14 & NA & 2.06 & 0.491 & 2.65 & 0.898 & 2.27 \\
\hline Silver & NA & $\mathrm{NA}$ & NA & NA & $\mathrm{NA}$ & NA & NA & NA & NA & $\mathrm{NA}$ \\
\hline Thallium & NA & $\mathrm{NA}$ & NA & 0.242 & NA & NA & $\mathrm{NA}$ & NA & NA & $\mathrm{NA}$ \\
\hline Vanadium & 9.73 & 113 & 20.9 & 77.5 & 22.8 & 60.0 & 21.3 & 46.2 & 25.4 & 102 \\
\hline Zinc & 9.43 & 104 & 11.8 & 75.7 & 11.2 & 76.9 & 12.6 & 53.0 & 15.8 & 28.5 \\
\hline
\end{tabular}

${ }^{a}$ For each sample site, 29 surface samples with 2 duplicates were collected. Primary-duplicate sample pairs were averaged to obtain one value for their respective sample location.

NA $=$ not applicable, all sample results were nondetects 
Table 4-2. $90^{\text {th }}$ Percentile Values of Coal Ash Surface Samples from Five Sample Sites $(N=29)$.

\begin{tabular}{|l|c|c|c|c|c|}
\hline \multirow{2}{*}{ Analyte } & \multicolumn{6}{|c|}{$\mathbf{9 0}^{\text {th }}$ Percentile for Coal Ash Surface Samples (mg/kg) } \\
\cline { 2 - 6 } & $\mathbf{1 0 0 - B}$ & $\mathbf{1 0 0 - D}$ & $\mathbf{1 0 0 - H}$ & $\mathbf{3 0 0}$ Area & $\mathbf{6 0 0 - 2 0 7}$ \\
\hline Antimony & 0.844 & 0.633 & 0.290 & NA & NA \\
\hline Arsenic & 7.46 & 7.78 & 3.80 & 3.64 & 6.69 \\
\hline Barium & 1,988 & 1,480 & 1,024 & 715 & 989 \\
\hline Beryllium & 1.94 & 1.88 & 1.31 & 1.42 & 1.12 \\
\hline Boron & 271 & 318 & 300 & 258 & 87.8 \\
\hline Cadmium & 0.508 & 0.383 & 0.353 & 0.313 & 0.205 \\
\hline Chromium, total & 13.5 & 12.2 & 13.6 & 14.9 & 12.0 \\
\hline Cobalt & 7.02 & 9.19 & 8.53 & 4.26 & 5.89 \\
\hline Copper & 39.3 & 31.6 & 41.1 & 26.7 & 30.0 \\
\hline Lead & 16.4 & 11.0 & 6.31 & 14.1 & 7.12 \\
\hline Manganese & 350 & 458 & 246 & 188 & 415 \\
\hline Mercury & 0.246 & 0.115 & 0.132 & 0.482 & 0.030 \\
\hline Molybdenum & 2.58 & 2.86 & 2.06 & 1.39 & 2.34 \\
\hline Nickel & 15.0 & 15.3 & 19.5 & 12.6 & 16.7 \\
\hline Selenium & 1.32 & 0.927 & 1.51 & 1.74 & 1.93 \\
\hline Silver & $\mathrm{NA}$ & $\mathrm{NA}$ & $\mathrm{NA}$ & $\mathrm{NA}$ & $\mathrm{NA}$ \\
\hline Thallium & $\mathrm{NA}$ & 0.243 & $\mathrm{NA}$ & $\mathrm{NA}$ & $\mathrm{NA}$ \\
\hline Vanadium & 49.8 & 53.8 & 53.0 & 43.1 & 45.5 \\
\hline Zinc & 60.5 & 56.6 & 48.9 & 37.4 & 25.4 \\
\hline The & & & & \\
\hline
\end{tabular}

a Total of 29 surface samples with 2 duplicates collected per sample area. Each primary-duplicate sample pair was averaged to obtain one value for the sample location. Nondetect data set to one-half the practical quantitation limit.

$\mathrm{NA}=$ not applicable, no detections in data set

Table 4-3. $90^{\text {th }}$ Percentile Upper Tolerance Limit Values of Coal Ash Surface Samples from Five Sample Sites $(N=29)$. (2 Pages)

\begin{tabular}{|l|c|c|c|c|c|}
\hline \multirow{2}{*}{ Analyte } & \multicolumn{5}{|c|}{$\mathbf{9 0}^{\text {th }}$ Percentile Upper Tolerance Limit Values for } \\
& \multicolumn{5}{|c|}{ Coal Ash Surface Samples (mg/kg) } \\
\cline { 2 - 6 } & $\mathbf{1 0 0 - B}$ & $\mathbf{1 0 0 - D}$ & $\mathbf{1 0 0 - H}$ & $\mathbf{3 0 0}$ Area & $\mathbf{6 0 0 - 2 0 7}$ \\
\hline Antimony & 1.26 & 0.894 & 0.295 & NA & NA \\
\hline Arsenic & 8.45 & 13.6 & 4.34 & 3.87 & 8.54 \\
\hline Barium & 2,250 & 1,750 & 1,250 & 754 & 1,060 \\
\hline Beryllium & 2.39 & 2.51 & 1.44 & 1.66 & 1.19 \\
\hline Boron & 327 & 410 & 367 & 369 & 137 \\
\hline Cadmium & 0.899 & 0.443 & 0.388 & 0.338 & 0.211 \\
\hline Chromium, total & 16.2 & 12.5 & 14.4 & 15.6 & 12.9 \\
\hline Cobalt & 8.22 & 11.6 & 9.81 & 4.89 & 6.07 \\
\hline Copper & 94.3 & 34.8 & 43.5 & 28.8 & 37.0 \\
\hline Lead & 19.0 & 15.1 & 7.43 & 15.2 & 9.30 \\
\hline Manganese & 396 & 689 & 283 & 210 & 486 \\
\hline Mercury & 0.338 & 0.139 & 0.176 & 0.713 & 0.042 \\
\hline Molybdenum & 3.85 & 3.54 & 2.44 & 1.50 & 2.58 \\
\hline Nickel & 15.8 & 17.5 & 20.4 & 13.2 & 16.7 \\
\hline Selenium & 1.44 & 0.955 & 1.63 & 2.46 & 2.00 \\
\hline Silver & $\mathrm{NA}$ & $\mathrm{NA}$ & $\mathrm{NA}$ & $\mathrm{NA}$ & NA \\
\hline Thallium & $\mathrm{NA}$ & 0.245 & $\mathrm{NA}$ & $\mathrm{NA}$ & $\mathrm{NA}$ \\
\hline Vanadium & 64.0 & 59.7 & 58.4 & 45.7 & 50.1 \\
\hline
\end{tabular}


Table 4-3. $90^{\text {th }}$ Percentile Upper Tolerance Limit Values of Coal Ash Surface Samples from Five Sample Sites $(\mathrm{N}=29)$. (2 Pages)

\begin{tabular}{|l|c|c|c|c|c|}
\hline \multirow{3}{*}{ Analyte } & \multicolumn{5}{|c|}{$90^{\text {th }} \begin{array}{c}\text { Percentile Upper Tolerance Limit Values for } \\
\text { Coal Ash Surface Samples (mg/kg) }\end{array}$} \\
\cline { 2 - 6 } & $100-\mathrm{B}$ & $100-\mathrm{D}$ & $100-\mathrm{H}$ & 300 Area & $600-207$ \\
\hline Zinc & 94.8 & 69.7 & 53.6 & 43.3 & 26.9 \\
\hline
\end{tabular}

a Total of 29 surface samples with 2 duplicates collected per sample area. Each primary-duplicate sample pair was averaged to obtain one value for the sample location. Nondetect data set to one-half the practical quantitation limit.

$\mathrm{NA}=$ not applicable, no detections in data set

Further characterization of the surface sample data was prepared with box plots using ProUCL 4.1 (EPA 2010c). Box plots of analyte data sets with at least one detection per sample area are presented in Appendix E. The box plots provide a visual representation of the respective data set. The vertical width of the box in the box plot is the differences in the data percentiles or interquartile range (IQR) $\left(75^{\text {th }}\right.$ percentile [Q3] minus the $25^{\text {th }}$ percentile [Q1]). The horizontal bars, also known as whiskers or fences, represent Q1- (1.5*IQR) (lower whisker) and $\mathrm{Q} 3+\left(1.5^{*} \mathrm{IQR}\right)$ (upper whisker). The band within the box represents the median. Data points outside of the whiskers are considered potential outliers.

As seen in the Appendix $\mathrm{E}$ box plots, the medians of most analytes differ between the sample sites. The box plots present a visual representation of the medians for each analyte, the relative homogeneity of the data set, and data that are above the Q3. Sample sites with the maximum analyte medians relative to $90^{\text {th }}$ percentile values are identified in Table 4-4. As shown in Appendix $E$ and Table 4-4, metals concentrations are not consistently higher or lower at any one site relative to the others. While the medians of the data are not statistically equal, this is most likely a function of considerable variability in metals concentrations within coal ash, and there is limited evidence to suggest that coal ash deposits in different areas of the River Corridor are appreciably different.

Table 4-4. Coal Ash Sample Sites with Highest Median Values and $90^{\text {th }}$ Percentile Values. (2 Pages)

\begin{tabular}{|c|c|c|}
\hline Analyte & $\begin{array}{c}\text { Site with Highest } \\
\text { Median }\end{array}$ & $\begin{array}{l}\text { Site with Highest } \\
90^{\text {th }} \text { Percentile }\end{array}$ \\
\hline Antimony & $126-\mathrm{B}-1$ & $126-\mathrm{B}-1$ \\
\hline Arsenic & $600-207$ & $126-D-1$ \\
\hline Barium & 126-D-1 & 126-B-1 \\
\hline Beryllium & 126-B-1/126-D-1 & 126-B-1 \\
\hline Boron & 300 Area Ash & 126-D-1 \\
\hline Cadmium & 126-B-1 & $126-B-1$ \\
\hline Chromium (total) & 300 Area Ash & 300 Area Ash \\
\hline Cobalt & $126-\mathrm{H}-1$ & 126-D-1 \\
\hline Copper & $126-\mathrm{H}-1$ & 126-H-1 \\
\hline Lead & 300 Area Ash & 126-B-1 \\
\hline Manganese & $600-207$ & 126-D-1 \\
\hline Mercury & 300 Area Ash & 300 Area Ash \\
\hline Molybdenum & $600-207$ & $126-\mathrm{D}-1$ \\
\hline Nickel & 126-H-1 & $126-\mathrm{H}-1$ \\
\hline Selenium & $600-207$ & $600-207$ \\
\hline
\end{tabular}


Table 4-4. Coal Ash Sample Sites with Highest Median Values and $90^{\text {th }}$ Percentile Values. (2 Pages)

\begin{tabular}{|l|c|c|}
\hline \multicolumn{1}{|c|}{ Analyte } & $\begin{array}{c}\text { Site with Highest } \\
\text { Median }\end{array}$ & $\begin{array}{c}\text { Site with Highest } \\
\mathbf{9 0}^{\text {th }} \text { Percentile }\end{array}$ \\
\hline Vanadium & $126-\mathrm{H}-1$ & $126-\mathrm{D}-1$ \\
\hline Zinc & $126-\mathrm{B}-1$ & $126-\mathrm{B}-1$ \\
\hline
\end{tabular}

\subsection{METALS ANALYSIS FOR DEPTH SAMPLES}

Analysis of the results was conducted from the three sets of samples collected at both surface

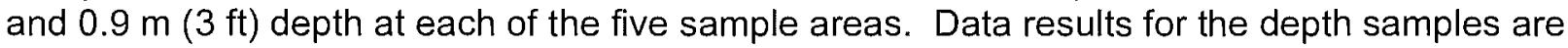
presented in Appendix C. Table 4-5 compares the range of data for the three depth samples with the range of data for the corresponding three surface samples at each of the five sample sites separately. For each analyte, Table 4-6 presents the average and standard deviation as well as the lower confidence limit (LCL) and upper confidence limit (UCL) for a two-sided $95 \%$ confidence interval for the collective means of all 15 surface sample results relative to all 15 depth sample results. Box plots of the three surface and three depth results by analyte are presented in Appendix $F$ for each analyte at each site with at least one detection in the respective data set. Given each data set contains only three samples per box plot, whiskers were not generated for these box plots.

As seen in Table 4-6, the mean analyte concentration from all 15 surface samples are slightly greater than, or essentially equal to, that of the mean concentrations for all 15 depth samples, with the exception of boron, lead, and mercury. In the case of boron, the confidence limits of the sample sets overlap, indicating that the means are not significantly different. However, for lead and mercury, the standard deviations for the depth results are up to two times the mean which skews the LCL to less than 0 . This indicates that the underlying populations for these analytes are quite skewed and the two-sided $95 \%$ confidence intervals are not applicable in these cases.

Further evaluation of surface and depth sample data for lead and mercury data at each of the five sites using box plots (Appendix F) illustrates the source of the variability. As discussed, the mean concentration of lead from all depth samples is greater than that from all surface samples (Table 4-6). The box plot for lead results from the three depth samples at $126-\mathrm{H}-1$ is skewed to the high end (Figure F-10) which is due to a single, elevated result (132 mg/kg). However, all three lead depth sample results at $126-\mathrm{H}-1$ are greater than those from the three corresponding surface samples (Table 4-5), as well as all other 126- $\mathrm{H}-1$ surface samples (range of 1.64 to $8.64 \mathrm{mg} / \mathrm{kg}$ ) (Table 4-1). The highest lead result in the depth samples was $132 \mathrm{mg} / \mathrm{kg}$ which is over 10 times higher than the corresponding surface sample $(5.21 \mathrm{mg} / \mathrm{kg})$ and the $90^{\text {th }}$ percentile value for lead at $126-\mathrm{H}-1(6.31 \mathrm{mg} / \mathrm{kg})$. 
Table 4-5. Range of Coal Ash Sample Results for Metals for Three Surface/Depth Sampling Locations at the Five Sample Sites (mg/kg). ${ }^{a}$ (2 Pages)

\begin{tabular}{|c|c|c|c|c|c|c|c|c|c|c|}
\hline \multirow{2}{*}{ Analyte } & \multicolumn{2}{|c|}{ 126-B-1 } & \multicolumn{2}{|c|}{ 126-D-1 } & \multicolumn{2}{|c|}{$126-\mathrm{H}-1$} & \multicolumn{2}{|c|}{300 Area Ash } & \multicolumn{2}{|c|}{$600-207$} \\
\hline & Min & Max & Min & Max & Min & Max & Min & Max & Min & Max \\
\hline Antimony (S) & NA & 1.26 & 0.470 & 0.605 & NA & NA & NA & NA & NA & NA \\
\hline Antimony (D) & NA & 0.956 & NA & NA & NA & NA & NA & $\mathrm{NA}$ & NA & 0.520 \\
\hline Arsenic (S) & 2.50 & 8.09 & 2.52 & 6.00 & 2.51 & 2.94 & 2.25 & 2.45 & 4.29 & 4.92 \\
\hline Arsenic (D) & 2.50 & 7.88 & 2.32 & 4.61 & 2.24 & 11.7 & 2.70 & 3.33 & 6.46 & 10.6 \\
\hline Barium (S) & 556 & 1,164 & 743 & 1,470 & 527 & 1,017 & 306 & 590 & 915 & 1,230 \\
\hline Barium (D) & 681 & 1,130 & 871 & 1,470 & 487 & 1,000 & 526 & 629 & 653 & 711 \\
\hline Beryllium (S) & 1.03 & 1.35 & 1.21 & 1.31 & 0.833 & 1.31 & 0.544 & 1.09 & 0.903 & 1.43 \\
\hline Beryllium (D) & 1.14 & 1.68 & 1.10 & 1.62 & 0.691 & 1.28 & 0.818 & 1.03 & 0.658 & 1.21 \\
\hline Boron (S) & 96.3 & 269 & 142 & 317 & 145 & 455 & 65.4 & 198 & 103 & 146 \\
\hline Boron (D) & 35.6 & 279 & 169 & 419 & 199 & 404 & 256 & 360 & 147 & 168 \\
\hline Cadmium (S) & 0.117 & 0.332 & 0.129 & 0.176 & 0.148 & 0.200 & 0.201 & 0.240 & 0.122 & 0.223 \\
\hline Cadmium (D) & 0.135 & 0.239 & 0.074 & 0.208 & 0.184 & 0.252 & 0.207 & 0.267 & 0.111 & 0.186 \\
\hline Chromium, total $(\mathrm{S})$ & 8.75 & 10.6 & 8.03 & 12.6 & 8.57 & 16.5 & 12.0 & 12.5 & 11.5 & 16.1 \\
\hline Chromium, total (D) & 7.34 & 13.3 & 7.64 & 8.73 & 8.52 & 11.2 & 9.87 & 11.6 & 7.50 & 8.99 \\
\hline Cobalt (S) & 2.51 & 4.42 & 3.46 & 9.87 & 2.14 & 3.77 & 2.83 & 4.23 & 5.74 & 7.11 \\
\hline Cobalt (D) & 2.64 & 5.12 & 4.21 & 6.82 & 1.72 & 3.52 & 2.16 & 2.94 & 3.56 & 4.42 \\
\hline Copper (S) & 21.9 & 28.8 & 20.8 & 31.0 & 16.7 & 33.8 & 14.7 & 19.6 & 28.2 & 46.8 \\
\hline Copper (D) & 19.6 & 26.2 & 13.6 & 31.8 & 18.1 & 25.3 & 15.5 & 19.7 & 21.3 & 21.9 \\
\hline Lead (S) & 5.59 & 11.7 & 1.90 & 16.8 & 4.25 & 5.12 & 7.13 & 9.66 & 5.28 & 9.30 \\
\hline Lead (D) & 4.33 & 11.8 & 3.24 & 3.50 & 18.3 & 132 & 8.18 & 14.5 & 3.30 & 5.61 \\
\hline Manganese (S) & 57.5 & 297 & 121 & 244 & 67.7 & 138 & 99.4 & 213 & 410 & 486 \\
\hline Manganese (D) & 38.5 & 187 & 91.4 & 255 & 82.1 & 148 & 66.3 & 95.9 & 250 & 327 \\
\hline Mercury (S) & 0.049 & 0.118 & 0.055 & 0.119 & 0.018 & 0.049 & 0.060 & 0.713 & NA & 0.027 \\
\hline Mercury (D) & 0.026 & 0.080 & 0.036 & 0.052 & 0.035 & 0.863 & 0.291 & 3.32 & NA & NA \\
\hline Molybdenum (S) & 1.06 & 1.17 & 1.03 & 2.10 & 0.708 & 2.44 & 0.472 & 0.993 & 2.02 & 2.34 \\
\hline Molybdenum (D) & 1.31 & 1.59 & 1.47 & 1.67 & 0.916 & 1.96 & 0.690 & 0.923 & 1.78 & 2.16 \\
\hline Nickel (S) & 7.02 & 10.1 & 8.04 & 14.9 & 5.88 & 10.9 & 8.37 & 9.21 & 16.2 & 19.3 \\
\hline Nickel (D) & 7.29 & 11.2 & 10.6 & 11.5 & 4.79 & 10.6 & 5.84 & 6.53 & 9.42 & 11.2 \\
\hline Selenium (S) & 0.331 & 1.31 & NA & 0.995 & 0.341 & 0.762 & 0.877 & 1.21 & 1.22 & 2.00 \\
\hline Selenium (D) & NA & 0.367 & 0.686 & 0.837 & NA & 1.18 & 1.19 & 1.77 & 1.29 & 1.85 \\
\hline Thallium (S) & $\mathrm{NA}$ & NA & NA & 0.198 & $\mathrm{NA}$ & NA & $\mathrm{NA}$ & NA & $\mathrm{NA}$ & $\mathrm{NA}$ \\
\hline Thallium (D) & NA & NA & NA & NA & NA & NA & NA & NA & NA & NA \\
\hline Uranium (metal) (S) & 2.67 & 5.39 & 3.66 & 4.07 & 2.24 & 4.01 & 3.28 & 4.22 & 2.56 & 4.68 \\
\hline Uranium (metal) (D) & 3.56 & 6.75 & 3.56 & 4.46 & 2.78 & 4.25 & 2.61 & 3.14 & 1.76 & 3.28 \\
\hline
\end{tabular}


Table 4-5. Range of Coal Ash Sample Results for Metals for Three Surface/Depth Sampling Locations at the Five Sample Sites (mg/kg). ${ }^{a}$ (2 Pages)

\begin{tabular}{|c|c|c|c|c|c|c|c|c|c|c|}
\hline \multirow{2}{*}{ Analyte } & \multicolumn{2}{|c|}{$126-B-1$} & \multicolumn{2}{|c|}{$126-D-1$} & \multicolumn{2}{|c|}{$126-\mathrm{H}-1$} & \multicolumn{2}{|c|}{300 Area Ash } & \multicolumn{2}{|c|}{$600-207$} \\
\hline & Min & Max & Min & Max & Min & Max & Min & Max & Min & Max \\
\hline Vanadium (S) & 27.3 & 39.3 & 27.6 & 38.5 & 23.8 & 42.2 & 32.8 & 44.4 & 43.7 & 78.7 \\
\hline Vanadium (D) & 28.7 & 33.5 & 29.7 & 41.8 & 19.6 & 28.9 & 24.3 & 28.3 & 32.1 & 36.8 \\
\hline $\operatorname{Zinc}(\mathrm{S})$ & 13.6 & 35.8 & 12.1 & 21.2 & 14.4 & 24.2 & 27.8 & 35.8 & 22.1 & 25.3 \\
\hline Zinc (D) & 15.1 & 29.7 & 10.3 & 33.6 & 21.0 & 31.3 & 26.7 & 27.8 & 16.0 & 18.1 \\
\hline
\end{tabular}

a Values determined from the three depth samples and the three corresponding surface samples for each of five sample sites. Each primary-duplicate sample pair was averaged to obtain one value for the sample location.
$\mathrm{D} \quad=$ depth
$\operatorname{Max}=$ maximum
Min $=$ minimum
NA = not applicable, sample results are nondetects
$\mathrm{S}=$ surface 
Table 4-6. Mean Analyte Concentrations and Confidence Limits of Combined Surface and Depth Samples from Five Sample Sites. ${ }^{a}$

\begin{tabular}{|c|c|c|c|c|c|c|c|c|c|}
\hline \multirow[b]{2}{*}{ Analyte } & \multicolumn{4}{|c|}{ Surface Concentration $(\mathrm{N}=15)(\mathrm{mg} / \mathrm{kg})$} & \multicolumn{4}{|c|}{ Depth Concentration $(\mathrm{N}=15)(\mathrm{mg} / \mathrm{kg})$} & \multirow{2}{*}{$\begin{array}{l}\text { Do Confidence } \\
\text { Limits Overlap? }\end{array}$} \\
\hline & Mean & $\begin{array}{l}\text { Standard } \\
\text { Deviation }\end{array}$ & LCL & UCL & Mean & $\begin{array}{l}\text { Standard } \\
\text { Deviation }\end{array}$ & LCL & UCL & \\
\hline Antimony & 0.372 & 0.267 & 0.224 & 0.519 & 0.296 & 0.142 & 0.218 & 0.375 & Yes \\
\hline Arsenic & 4.31 & 2.37 & 3.00 & 5.62 & 4.20 & 2.67 & 2.72 & 5.68 & Yes \\
\hline Barium & 833 & 322 & 654 & 1011 & 803 & 271 & 653 & 953 & Yes \\
\hline Beryllium & 1.10 & 0.254 & 0.956 & 1.24 & 1.11 & 0.310 & 0.943 & 1.29 & Yes \\
\hline Boron & 190 & 99.2 & 135 & 245 & 239 & 103 & 182 & 296 & Yes \\
\hline Cadmium & 0.189 & 0.056 & 0.157 & 0.220 & 0.187 & 0.053 & 0.158 & 0.216 & Yes \\
\hline Chromium & 11.8 & 2.44 & 10.4 & 13.1 & 9.43 & 1.76 & 8.46 & 10.4 & Yes \\
\hline Cobalt & 4.58 & 1.98 & 3.49 & 5.67 & 3.65 & 1.42 & 2.86 & 4.44 & Yes \\
\hline Copper & 26.2 & 8.46 & 21.5 & 30.9 & 21.2 & 4.56 & 18.7 & 23.8 & Yes \\
\hline Lead & 7.82 & 4.08 & 5.57 & 10.1 & 17.0 & 32.5 & -1.06 & 35.0 & NA \\
\hline Manganese & 212 & 149 & 129 & 294 & 157 & 91.9 & 106 & 207 & Yes \\
\hline Mercury & 0.116 & 0.185 & 0.013 & 0.219 & 0.359 & 0.853 & -0.113 & 0.832 & NA \\
\hline Molybdenum & 1.41 & 0.650 & 1.05 & 1.77 & 1.43 & 0.439 & 1.19 & 1.68 & Yes \\
\hline Nickel & 11.0 & 3.91 & 8.88 & 13.2 & 8.78 & 2.33 & 7.48 & 10.1 & Yes \\
\hline Selenium & 0.904 & 0.493 & 0.631 & 1.18 & 0.938 & 0.632 & 0.588 & 1.29 & Yes \\
\hline Uranium & 3.62 & 0.832 & 3.16 & 4.08 & 3.60 & 1.16 & 2.96 & 4.24 & Yes \\
\hline Vanadium & 39.3 & 12.8 & 32.2 & 46.4 & 29.9 & 5.29 & 27.0 & 32.9 & Yes \\
\hline Zinc & 24.1 & 7.98 & 19.7 & 28.5 & 23.6 & 6.92 & 19.8 & 27.5 & Yes \\
\hline
\end{tabular}

${ }^{a}$ Values determined from 15 depth samples and the 15 corresponding surface samples for each of 5 sample sites. Each primary-duplicate sample pair was averaged to obtain one value for the sample location. Nondetect data set to one-half the practical quantitation limit.

$\mathrm{LCL}=$ lower confidence limit

$\mathrm{NA}=$ not applicable

$\mathrm{UCL}=$ upper confidence limit 
As with lead, the mean concentration of mercury from all depth samples is greater than that from all surface samples (Table 4-6). As shown in the box plots (Figure F-12), the medians for the three surface and three depth sample results for mercury in the 300 Area Ash were relatively similar, however, the depth data was skewed higher than the surface sample data. Individual mercury results from depth samples at 300 Area Ash are all higher than those from the corresponding surface samples, but 2 of the 3 sample results $(0.291$ and $0.501 \mathrm{mg} / \mathrm{kg})$ were within the range of all 29 surface sample results $(0.034$ to $1.02 \mathrm{mg} / \mathrm{kg})($ Table $4-5)$. The single highest mercury result from 300 Area Ash $(3.02 \mathrm{mg} / \mathrm{kg}$ ) was from the third depth sample result. This trend of higher mercury at 300 Area Ash is also observed in the surface sample box plots (Figure E-12), which shows a similar elevated skewness to the box plot with two results greater than the Q3.

Comparison of results from the depth samples with the corresponding surface samples at each of the five sample sites indicates that the mean concentrations for all analytes, with the exception of lead and mercury, are not statistically different due to overlap of the LCL and UCL. For both lead and mercury, depth sample results from one of the five sites are greater than all other results. The potential exists that there is a significant difference in the lead concentrations between the coal ash at surface and at depth in 126- $\mathrm{H}-1$ and in the mercury concentrations at 300 Area Ash

\subsection{PAH ANALYSIS}

Analyses for PAHs were conducted on the three coal ash samples collected at both surface and

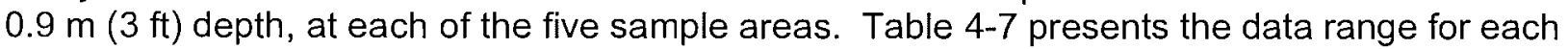
of the three depth samples, along with the data range for the three corresponding surface samples, at each of the five sample sites. Due to the significant number of nondetects amongst the data sets, box plots were not prepared. In cases where a duplicate sample was obtained for QC purposes, the results from the primary and duplicate sample were averaged to obtain a single value for the sample. If the primary or duplicate sample was a nondetect result, one-half the PQL was used as the sample result prior to averaging.

Acenaphthene, acenaphthylene, benzo(a)pyrene, dibenz(a,h)anthracene, fluorene, and indeno(1,2,3-cd)pyrene were not detected in any samples. Maximum values of detected PAHs were relatively low with maximum detected values measured primarily in the surface samples.

\subsection{BATCH LEACH TEST FOR DEPTH SAMPLES}

To quantify the potential environmental availability of coal ash metals, all coal ash samples

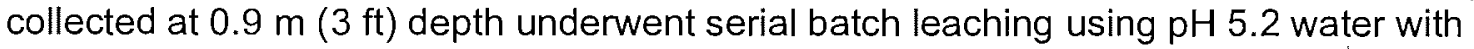
subsequent analysis for metals including mercury. Coal ash samples were leached with three coal ash:water ratios of 1:1, 1:2.5, and 1:5, with one of each ratio duplicated for each sample. These ratios result in a much lower dilution than that assumed when establishing cleanup levels, where default soil-to-groundwater dilution ratios of 1:100 (WAC 173-340-740 [Ecology 1996]) or 1:20 (WAC 173-340-747 [Ecology 2007]) are used. Data results from the solid and leachate samples associated with the batch leaching tests are presented in Appendix C. A summary of the leachate results, with no adjustment for coal ash:leachate ratio, is presented in Table 4-8. 
Table 4-7. Range of Coal Ash Sample Results for Detected Polycyclic Aromatic Hydrocarbons for Three Surface/Depth Sampling Locations at the Five Sample Sites.

\begin{tabular}{|c|c|c|c|c|c|c|c|c|c|c|}
\hline \multirow{2}{*}{ Analyte } & \multicolumn{2}{|c|}{$126-\mathrm{B}-1(\mathrm{mg} / \mathrm{kg})$} & \multicolumn{2}{|c|}{$126-\mathrm{D}-1$ (mg/kg) } & \multicolumn{2}{|c|}{$126-\mathrm{H}-1(\mathrm{mg} / \mathrm{kg})$} & \multicolumn{2}{|c|}{$\begin{array}{c}300 \text { Area Ash } \\
(\mathrm{mg} / \mathrm{kg})\end{array}$} & \multicolumn{2}{|c|}{$600-207(\mathrm{mg} / \mathrm{kg})$} \\
\hline & Min & Max & Min & Max & Min & Max & Min & $\operatorname{Max}$ & Min & Max \\
\hline Anthracene (S) & NA & 0.0502 & NA & NA & NA & NA & NA & $\mathrm{NA}$ & NA & NA \\
\hline Anthracene (D) & $\mathrm{NA}$ & NA & NA & $\mathrm{NA}$ & NA & NA & $\mathrm{NA}$ & NA & NA & NA \\
\hline Benzo(a)anthracene (S) & $\mathrm{NA}$ & 0.117 & NA & NA & NA & NA & NA & $\mathrm{NA}$ & $\mathrm{NA}$ & NA \\
\hline Benzo(a)anthracene (D) & $\mathrm{NA}$ & NA & $\mathrm{NA}$ & NA & $\mathrm{NA}$ & NA & NA & NA & NA & NA \\
\hline Benzo(b)fluoranthene (S) & NA & $\mathrm{NA}$ & $\mathrm{NA}$ & $\mathrm{NA}$ & NA & NA & $\mathrm{NA}$ & NA & $\mathrm{NA}$ & 0.071 \\
\hline Benzo(b)fluoranthene (D) & NA & NA & NA & NA & $\mathrm{NA}$ & NA & $\mathrm{NA}$ & NA & NA & NA \\
\hline Benzo(ghi)perylene (S) & NA & NA & NA & NA & NA & NA & $\mathrm{NA}$ & NA & NA & $\mathrm{NA}$ \\
\hline Benzo(ghi)perylene (D) & NA & $\mathrm{NA}$ & NA & NA & $\mathrm{NA}$ & 0.585 & NA & $\mathrm{NA}$ & NA & NA \\
\hline Benzo(k)fluoranthene (S) & $\mathrm{NA}$ & 0.0839 & NA & NA & NA & NA & NA & $\mathrm{NA}$ & NA & 0.065 \\
\hline Benzo(k)fluoranthene (D) & NA & NA & NA & $\mathrm{NA}$ & $\mathrm{NA}$ & NA & NA & NA & NA & NA \\
\hline Chrysene (S) & 0.0637 & 0.122 & NA & $\mathrm{NA}$ & $\mathrm{NA}$ & NA & NA & NA & NA & 0.100 \\
\hline Chrysene (D) & NA & NA & $\mathrm{NA}$ & NA & NA & NA & NA & NA & NA & NA \\
\hline Fluoranthene (S) & 0.0651 & 0.0780 & $\mathrm{NA}$ & NA & $\mathrm{NA}$ & NA & $\mathrm{NA}$ & NA & 0.057 & 0.259 \\
\hline Fluoranthene (D) & NA & NA & NA & NA & $\mathrm{NA}$ & NA & $\mathrm{NA}$ & $\mathrm{NA}$ & NA & 0.195 \\
\hline Naphthalene (S) & 0.257 & 0.964 & NA & 0.619 & 0.316 & 0.691 & NA & NA & 0.287 & 0.429 \\
\hline Naphthalene (D) & NA & 0.234 & NA & 0.253 & 0.137 & 0.181 & NA & 0.124 & NA & 0.451 \\
\hline Phenanthrene (S) & 0.164 & 0.281 & NA & 0.259 & NA & 0.250 & NA & NA & 0.095 & 0.316 \\
\hline Phenanthrene (D) & 0.079 & 0.119 & NA & NA & NA & 0.111 & NA & $\mathrm{NA}$ & NA & 0.302 \\
\hline Pyrene (S) & 0.0649 & 0.131 & $\mathrm{NA}$ & $\mathrm{NA}$ & $\mathrm{NA}$ & NA & NA & NA & NA & 0.115 \\
\hline Pyrene (D) & NA & NA & NA & NA & $\mathrm{NA}$ & 0.104 & NA & NA & NA & NA \\
\hline
\end{tabular}

${ }^{a}$ Values determined from the three depth samples and the three corresponding surface samples for each of five sample sites. Primary-duplicate sample pairs were averaged to obtain one value for the sample location, as appropriate.

$\mathrm{D} \quad=$ depth

Max = maximum

Min $=$ minimum

NA = not applicable, sample results are nondetects

$\mathrm{S} \quad=$ surface 
Table 4-8. Leachate Results from Coal Ash Depth Samples. (3 Pages)

\begin{tabular}{|c|c|c|c|c|c|c|c|c|c|c|c|c|c|c|c|c|}
\hline \multirow{2}{*}{$\begin{array}{l}\text { Ratio, Coal } \\
\text { Ash:Water }\end{array}$} & \multicolumn{4}{|c|}{ 126-B-1 (mg/L) } & \multicolumn{3}{|c|}{ 126-D-1 (mg/L) } & \multicolumn{3}{|c|}{$126-\mathrm{H}-1(\mathrm{mg} / \mathrm{L})$} & \multicolumn{3}{|c|}{300 Area Ash $(\mathrm{mg} / \mathrm{L})$} & \multicolumn{3}{|c|}{$600-207(\mathrm{mg} / \mathrm{L})$} \\
\hline & J1HHW3 & J1HJ08 & J1HJ09 & $\mathrm{J} 1 \mathrm{HJ} 10^{\mathrm{a}}$ & $\mathrm{J} 1 \mathrm{~J} 442$ & $\mathrm{~J} 1 \mathrm{~J} 443$ & $\mathrm{~J} 1 \mathrm{~J} 444$ & J1HJ80 & J1HJ81 & J1HJ82 & J1HJV1 & J1HJV2 & J1HJV0 & J1HHT8 & J1HHT9 & J1HHV0 \\
\hline & \multicolumn{16}{|c|}{ Arsenic } \\
\hline $1: 1$ & $\mathrm{U}$ & $\mathrm{U}$ & $\mathrm{U}$ & $\mathrm{U}$ & $\mathrm{U}$ & $\mathrm{U}$ & $\mathrm{U}$ & $\mathrm{U}$ & $\mathrm{U}$ & $\mathrm{U}$ & $\mathrm{U}$ & $\mathrm{U}$ & $\mathrm{U}$ & $\mathrm{U}$ & U & $\mathrm{U}$ \\
\hline $1: 1$ & $\cdots$ & -- & -- & $\mathrm{U}$ & $\mathrm{U}$ & -- & - & $\cdots$ & $\cdots$ & $\mathrm{U}$ & - & - & U & U & - & - \\
\hline $1: 2.5$ & 0.011 & $\mathrm{U}$ & 0.003 & 0.006 & $\mathrm{U}$ & $\mathrm{U}$ & $\mathrm{U}$ & $\mathrm{U}$ & $\mathrm{U}$ & $U$ & $U$ & $\mathrm{U}$ & $\mathrm{U}$ & 0.002 & 0.003 & $U$ \\
\hline $1: 2.5$ & 0.010 & $\mathrm{U}$ & -- & -- & -- & $\mathrm{U}$ & -- & $\mathrm{U}$ & -- & - & $\mathrm{U}$ & -- & -- & -- & 0.004 & -- \\
\hline $1: 5$ & 0.007 & $\mathrm{U}$ & 0.003 & 0.005 & $\mathrm{U}$ & $\mathrm{U}$ & $\mathrm{U}$ & $\mathrm{U}$ & $\mathrm{U}$ & $\mathrm{U}$ & $\mathrm{U}$ & $\mathrm{U}$ & $\mathrm{U}$ & 0.003 & U & 0.002 \\
\hline \multirow[t]{2}{*}{$1: 5$} & -- & -- & 0.003 & -- & -- & $\cdots$ & U & -- & $\mathrm{U}$ & - & - & $\mathrm{U}$ & - & -- & - & U \\
\hline & \multicolumn{16}{|c|}{ Barium } \\
\hline $1: 1$ & 0.031 & 0.035 & 0.035 & 0.056 & 0.048 & 0.046 & 0.032 & 0.076 & 0.066 & 0.039 & 0.045 & 0.048 & 0.042 & 0.039 & 0.027 & 0.037 \\
\hline $1: 1$ & -- & -- & -- & 0.035 & 0.190 & -- & -- & -- & -- & 0.041 & - & -- & 0.037 & 0.041 & - & -- \\
\hline $1: 2.5$ & 0.020 & 0.034 & 0.037 & 0.035 & 0.041 & 0.032 & 0.029 & 0.075 & 0.069 & 0.042 & 0.036 & 0.037 & 0.034 & 0.036 & 0.026 & 0.033 \\
\hline $1: 2.5$ & 0.019 & 0.033 & -- & -- & -- & 0.033 & -- & 0.077 & -- & - & 0.037 & -- & -- & -- & 0.029 & -- \\
\hline $1: 5$ & 0.013 & 0.039 & 0.026 & 0.031 & 0.037 & 0.031 & 0.024 & 0.077 & 0.081 & 0.039 & 0.036 & 0.034 & 0.033 & 0.039 & 0.029 & 0.035 \\
\hline \multirow[t]{2}{*}{$1: 5$} & $-\cdot$ & -- & 0.027 & -- & -- & $\cdots$ & 0.023 & -- & 0.079 & - & - & 0.034 & -- & $\cdots$ & - & 0.034 \\
\hline & \multicolumn{16}{|c|}{ Boron } \\
\hline $1: 1$ & 3.31 & 2.55 & 5.66 & 8.57 & 13.5 & 20.2 & 49.9 & 13.4 & 4.95 & 10.2 & 43.4 & 58.4 & 77.8 & 24.8 & 11.7 & 29.0 \\
\hline $1: 1$ & -- & -- & -- & 8.62 & 14.7 & -- & -- & -- & $-\cdots$ & 11.2 & - & -- & 70.0 & 26.2 & - & -- \\
\hline $1: 2.5$ & 1.92 & 1.55 & 3.93 & 4.36 & 8.56 & 12.7 & 47.5 & 7.18 & 2.82 & 6.66 & 22.9 & 33.7 & 40.4 & 15.1 & 7.66 & 19.0 \\
\hline $1: 2.5$ & 1.75 & 1.48 & -- & -- & -- & 12.6 & -- & 6.79 & -- & - & 21.6 & -- & -- & -- & 8.71 & -- \\
\hline $1: 5$ & 0.98 & 1.06 & 2.36 & 2.31 & 4.54 & 6.74 & 28.1 & 4.04 & 1.75 & 4.11 & 13.0 & 19.3 & 23.1 & 10.4 & 5.48 & 12.5 \\
\hline \multirow[t]{2}{*}{$1: 5$} & -- & -- & 2.23 & -- & -- & $\cdots$ & 30.3 & -- & 1.75 & - & - & 20.2 & -- & -- & - & 11.3 \\
\hline & \multicolumn{16}{|c|}{ Cadmium } \\
\hline $1: 1$ & $\mathrm{U}$ & $U$ & $U$ & $\mathrm{U}$ & $\mathrm{U}$ & U & $\mathrm{U}$ & $U$ & $\mathrm{U}$ & U & U & $U$ & $\mathrm{U}$ & 0.0020 & 0.0020 & 0.0020 \\
\hline $1: 1$ & -- & -- & -- & U & $\mathrm{U}$ & -- & -- & $\cdots$ & - & $U$ & - & -- & $U$ & 0.0020 & - & -- \\
\hline $1: 2.5$ & $\mathrm{U}$ & $U$ & $U$ & $U$ & $U$ & $U$ & $U$ & $\mathrm{U}$ & $\mathrm{U}$ & $\mathrm{U}$ & $\mathrm{U}$ & $U$ & $U$ & 0.00047 & 0.0010 & 0.00051 \\
\hline $1: 2.5$ & $\mathrm{U}$ & $U$ & -- & - & - & $\mathrm{U}$ & -- & $\mathrm{U}$ & -- & - & $\mathrm{U}$ & -- & $-\cdots$ & -- & 0.0010 & -- \\
\hline $1: 5$ & $\mathrm{U}$ & $\mathrm{U}$ & $\mathrm{U}$ & $U$ & $U$ & $\mathrm{U}$ & $\mathrm{U}$ & $\mathrm{U}$ & $\mathrm{U}$ & $\mathrm{U}$ & $\mathrm{U}$ & $\mathrm{U}$ & $\mathrm{U}$ & 0.00049 & 0.0010 & 0.0010 \\
\hline \multirow[t]{2}{*}{$1: 5$} & -- & - & $U$ & - & -- & -- & U & - & U & - & - & U & $\cdots$ & $-\cdots$ & - & 0.0010 \\
\hline & \multicolumn{16}{|c|}{ Chromium (total) } \\
\hline $1: 1$ & $\mathrm{U}$ & U & $\mathrm{U}$ & U & 0.015 & 0.003 & 0.010 & 0.005 & 0.008 & 0.003 & 0.003 & 0.003 & 0.003 & $\mathrm{U}$ & 0.004 & 0.004 \\
\hline $1: 1$ & -- & -- & -- & $\mathrm{U}$ & 0.007 & -- & $\ldots$ & $\cdots$ & -- & $\mathrm{U}$ & - & -- & $\mathrm{U}$ & 0.003 & - & $-\cdots$ \\
\hline $1: 2.5$ & 0.002 & $\mathrm{U}$ & 0.003 & 0.002 & $\mathrm{U}$ & $\mathrm{U}$ & U & 0.003 & U & U & $\mathrm{U}$ & $\mathrm{U}$ & $\mathrm{U}$ & 0.001 & 0.001 & 0.001 \\
\hline $1: 2.5$ & 0.001 & $U$ & $-\cdots$ & -- & -- & $\mathrm{U}$ & -- & 0.003 & -- & - & 0.003 & -- & -- & -- & 0.002 & \\
\hline $1: 5$ & 0.001 & U & 0.002 & 0.001 & $\mathrm{U}$ & $\mathrm{U}$ & U & $\mathrm{U}$ & U & $U$ & $\mathrm{U}$ & $\mathrm{U}$ & U & 0.001 & 0.002 & 0.001 \\
\hline \multirow[t]{2}{*}{$1: 5$} & -- & - & 0.002 & $-\cdots$ & - & -- & $\mathrm{U}$ & - & U & - & - & $\mathrm{U}$ & -- & -- & - & $\mathrm{U}$ \\
\hline & \multicolumn{16}{|c|}{ Cobalt } \\
\hline $1: 1$ & $\mathrm{U}$ & U & U & $\mathrm{U}$ & $\mathrm{U}$ & $\mathrm{U}$ & $\mathrm{U}$ & $\mathrm{U}$ & $U$ & $u$ & $\mathrm{U}$ & U & $\mathrm{U}$ & $\mathrm{U}$ & $\mathrm{U}$ & U \\
\hline $1: 1$ & -- & -- & -- & $U$ & $\mathrm{U}$ & $\cdots$ & -- & -- & -- & $\mathrm{U}$ & - & -- & U & U & - & -- \\
\hline $1: 2.5$ & $\mathrm{U}$ & $\mathrm{U}$ & $U$ & U & $\mathrm{U}$ & $\mathrm{U}$ & $\mathrm{U}$ & U & $\mathrm{U}$ & u & U & U & $\mathrm{U}$ & $\mathrm{U}$ & U & $\mathrm{U}$ \\
\hline $1: 2.5$ & $\mathrm{U}$ & $\mathrm{U}$ & -- & -- & -- & $\mathrm{U}$ & - & U & - & - & $\mathrm{U}$ & -- & -- & -- & 0.001 & -- \\
\hline $1: 5$ & $U$ & $U$ & $U$ & $U$ & U & $\mathrm{U}$ & $U$ & U & U & $U$ & $U$ & $U$ & $U$ & $U$ & $\mathrm{U}$ & $U$ \\
\hline $1: 5$ & - & - & $\mathrm{U}$ & - & $=$ & - & $\mathrm{U}$ & - & $\mathrm{U}$ & - & - & U & -- & -- & - & U \\
\hline
\end{tabular}


Table 4-8. Leachate Results from Coal Ash Depth Samples. (3 Pages)

\begin{tabular}{|c|c|c|c|c|c|c|c|c|c|c|c|c|c|c|c|c|}
\hline \multirow{2}{*}{$\begin{array}{l}\text { Ratio, Coal } \\
\text { Ash:Water }\end{array}$} & \multicolumn{4}{|c|}{ 126-B-1 (mg/L) } & \multicolumn{3}{|c|}{ 126-D-1 (mg/L) } & \multicolumn{3}{|c|}{$126-\mathrm{H}-1(\mathrm{mg} / \mathrm{L})$} & \multicolumn{3}{|c|}{300 Area Ash $(\mathrm{mg} / \mathrm{L})$} & \multicolumn{3}{|c|}{$600-207(\mathrm{mg} / \mathrm{L})$} \\
\hline & J1HHW3 & J1HJ08 & J1HJ09 & J1HJ10 & J1J442 & $\mathrm{J} 1 \mathrm{~J} 443$ & $\mathrm{~J} 1 \mathrm{~J} 444$ & J1HJ80 & J1HJ81 & \begin{tabular}{|l} 
J1HJ82 \\
\end{tabular} & J1HJV1 & J1HJV2 & J1HJV0 & J1HHT8 & J1HHT9 & J1HHV0 \\
\hline & \multicolumn{16}{|c|}{ Copper } \\
\hline $1: 1$ & $\mathrm{U}$ & U & U & $\bar{U}$ & $\mathrm{U}$ & $U$ & $U$ & $\mathrm{U}$ & U & $\mathrm{U}$ & $\bar{U}$ & $\mathrm{U}$ & $\mathrm{U}$ & $\mathrm{U}$ & $\bar{U}$ & $U$ \\
\hline $1: 1$ & -- & -- & -- & U & $\mathrm{U}$ & -- & - & -- & -- & U & - & -- & $U$ & $U$ & - & -- \\
\hline $1: 2.5$ & 0.003 & $U$ & $U$ & $U$ & $\bar{U}$ & $\bar{U}$ & $\mathrm{U}$ & $\mathrm{U}$ & U & $\mathrm{U}$ & $\mathrm{U}$ & U & $\mathrm{U}$ & $\mathrm{U}$ & $\mathrm{U}$ & $\mathrm{U}$ \\
\hline $1: 2.5$ & 0.003 & U & - & $-\cdots$ & -- & $\mathrm{U}$ & -- & $\mathrm{U}$ & -- & - & $\mathrm{U}$ & -- & -- & -- & U & - \\
\hline $1: 5$ & 0.003 & U & $\mathrm{U}$ & $\mathrm{U}$ & U & $\mathrm{U}$ & $\bar{U}$ & U & $\bar{U}$ & U & U & $\mathrm{U}$ & $\mathrm{U}$ & 0.003 & $\mathrm{U}$ & $\mathrm{U}$ \\
\hline \multirow[t]{2}{*}{$1: 5$} & -- & -- & $U$ & -- & -- & -- & $\mathrm{U}$ & -- & $\mathrm{U}$ & - & - & $\mathrm{U}$ & - & -- & - & $\mathrm{U}$ \\
\hline & \multicolumn{16}{|c|}{ Lead } \\
\hline $1: 1$ & $U$ & $U$ & $U$ & $\mathrm{U}$ & $\mathrm{U}$ & $\mathrm{U}$ & $U$ & $\mathrm{U}$ & U & U & $\mathrm{U}$ & $\mathrm{U}$ & U & 0.022 & $\mathrm{U}$ & 0.022 \\
\hline $1: 1$ & $\cdots$ & -- & -- & U & $\mathrm{U}$ & -- & -- & -- & - & $U$ & - & -- & $\mathrm{U}$ & 0.023 & - & -- \\
\hline $1: 2.5$ & $\mathrm{U}$ & U & $\mathrm{U}$ & U & $\mathrm{U}$ & $\mathrm{U}$ & U & U & $U$ & $U$ & $U$ & $\mathrm{U}$ & $\mathrm{U}$ & 0.014 & 0.003 & 0.018 \\
\hline $1: 2.5$ & U & $U$ & -- & -- & -- & $\mathrm{U}$ & - & 0.011 & -- & - & $U$ & -- & -- & -- & 0.004 & -- \\
\hline $1: 5$ & $\mathrm{U}$ & U & $\mathrm{U}$ & $\bar{U}$ & $\mathrm{U}$ & $\bar{U}$ & $\mathrm{U}$ & $\mathrm{U}$ & $U$ & U & $U$ & $U$ & $\mathrm{U}$ & 0.009 & 0.003 & 0.012 \\
\hline \multirow[t]{2}{*}{$1: 5$} & -- & $\cdots$ & $\mathrm{U}$ & -- & -- & -- & $U$ & -- & U & - & - & $\mathrm{U}$ & -- & -- & - & 0.010 \\
\hline & \multicolumn{16}{|c|}{ Manganese } \\
\hline $1: 1$ & 0.013 & 0.081 & $\mathrm{U}$ & 0.014 & $U$ & $\mathrm{U}$ & 0.288 & $U$ & $\mathrm{U}$ & $\mathrm{U}$ & $\mathrm{U}$ & $\mathrm{U}$ & $\mathrm{U}$ & 0.424 & 0.099 & 0.165 \\
\hline $1: 1$ & -- & -- & - & 0.011 & $U$ & -- & -- & -- & -- & U & - & -- & $U$ & 0.443 & - & -- \\
\hline $1: 2.5$ & 0.001 & 0.038 & 0.001 & 0.001 & $\mathrm{U}$ & $\mathrm{U}$ & 0.349 & U & $\mathrm{U}$ & $\mathrm{U}$ & U & U & $\mathrm{U}$ & 0.341 & 0.066 & 0.140 \\
\hline $1: 2.5$ & 0.001 & 0.036 & $\cdots$ & - & -- & $\mathrm{U}$ & -- & $\mathrm{U}$ & -- & - & $U$ & -- & $-\cdots$ & -- & 0.072 & -- \\
\hline $1: 5$ & 0.001 & 0.023 & 0.001 & 0.001 & $\mathrm{U}$ & $\mathrm{U}$ & 0.187 & $U$ & $U$ & $U$ & $\mathrm{U}$ & $\mathrm{U}$ & $\mathrm{U}$ & 0.303 & 0.048 & 0.122 \\
\hline \multirow[t]{2}{*}{$1: 5$} & -- & -- & 0.001 & -- & -- & -- & 0.201 & -- & $\mathrm{U}$ & - & - & $U$ & -- & -- & - & 0.120 \\
\hline & \multicolumn{16}{|c|}{ Mercury } \\
\hline $1: 1$ & U & $\mathrm{U}$ & $\mathrm{U}$ & $\mathrm{U}$ & $\mathrm{U}$ & $\mathrm{U}$ & $\mathrm{U}$ & 0.000186 & 0.000339 & 0.000366 & 0.00023 & 0.00040 & 0.00023 & $\mathrm{U}$ & $\mathrm{U}$ & $\mathrm{U}$ \\
\hline $1: 1$ & - & -- & -- & U & $\mathrm{U}$ & -- & -- & -- & $\ldots$ & 0.000202 & - & -- & 0.00024 & $\mathrm{U}$ & - & $-\cdot$ \\
\hline $1: 2.5$ & U & $U$ & $\mathrm{U}$ & U & U & $U$ & U & 0.000103 & 0.000115 & 0.000115 & 0.00013 & 0.00013 & 0.00013 & $U$ & $U$ & U \\
\hline $1: 2.5$ & U & U & -- & -- & - & $\mathrm{U}$ & - & 0.000111 & -- & - & 0.00011 & -- & -- & -- & $U$ & $\cdots$ \\
\hline $1: 5$ & $\mathrm{U}$ & $U$ & $\mathrm{U}$ & U & $\mathrm{U}$ & $U$ & U & 0.000100 & 0.000117 & 0.000102 & 0.00013 & 0.00015 & 0.00012 & $U$ & $\mathrm{U}$ & $\mathrm{U}$ \\
\hline \multirow[t]{2}{*}{$1: 5$} & -- & -- & $U$ & -- & -- & -- & $U$ & -- & 0.000110 & - & - & 0.00013 & -- & -- & - & U \\
\hline & \multicolumn{16}{|c|}{ Molybdenum } \\
\hline $1: 1$ & 0.011 & 0.012 & 0.035 & 0.015 & 0.111 & 0.054 & 0.003 & 0.017 & 0.010 & 0.018 & 0.060 & 0.069 & 0.133 & 0.003 & 0.197 & 0.008 \\
\hline $1: 1$ & -- & -- & $-\cdots$ & 0.015 & 0.110 & -- & & -- & -- & 0.019 & - & -- & 0.120 & 0.003 & & -- \\
\hline $1: 2.5$ & 0.003 & 0.008 & 0.011 & 0.007 & 0.059 & 0.035 & 0.005 & 0.008 & 0.003 & 0.008 & 0.032 & 0.035 & 0.062 & 0.001 & 0.103 & 0.004 \\
\hline $1: 2.5$ & 0.003 & 0.008 & -- & $-\cdots$ & -- & 0.034 & -- & 0.009 & -- & - & 0.028 & & $\cdots$ & -- & 0.112 & -- \\
\hline $1: 5$ & 0.002 & 0.008 & 0.006 & 0.007 & 0.035 & 0.018 & 0.004 & 0.007 & $\mathrm{U}$ & 0.005 & 0.016 & 0.019 & 0.035 & 0.001 & 0.057 & 0.003 \\
\hline $1: 5$ & -- & -- & 0.006 & -- & -- & -- & 0.003 & -- & $\mathrm{U}$ & - & - & 0.019 & -- & -- & - & 0.003 \\
\hline
\end{tabular}


Table 4-8. Leachate Results from Coal Ash Depth Samples. (3 Pages)

\begin{tabular}{|c|c|c|c|c|c|c|c|c|c|c|c|c|c|c|c|c|}
\hline \multirow{2}{*}{$\begin{array}{l}\text { Ratio, Coal } \\
\text { Ash:Water }\end{array}$} & \multicolumn{4}{|c|}{$126-\mathrm{B}=1(\mathrm{mg} / \mathrm{L})$} & \multicolumn{3}{|c|}{$126-\mathrm{D}-1$ (mg/L) } & \multicolumn{3}{|c|}{$126-\mathrm{H}-1(\mathrm{mg} / \mathrm{L})$} & \multicolumn{3}{|c|}{300 Area Ash (mg/L) } & \multicolumn{3}{|c|}{$600-207(\mathrm{mg} / \mathrm{L})$} \\
\hline & J1HHW3 & J1HJ08 & J1HJ09 & J1HJ10 & J1J442 & J1J443 & $\mathrm{J} 1 \mathrm{~J} 444$ & J1HJ80 & $\mathrm{J} 1 \mathrm{HJ} 81$ & J1HJ82 & J1HJV1 & J1HJV2 & J1HJV0 & J1HHT8 & J1HHT9 & J1HHV0 \\
\hline & \multicolumn{16}{|c|}{ Nickel } \\
\hline $1: 1$ & 0.005 & $U$ & $\mathrm{U}$ & $\bar{U}$ & 0.006 & U & $U$ & $U$ & $U$ & $U$ & $\mathrm{U}$ & $U$ & $U$ & $\bar{U}$ & $\bar{U}$ & 0.004 \\
\hline $1: 1$ & -- & -- & -- & $U$ & U & -- & -- & -- & -- & $U$ & - & -. & $U$ & $U$ & - & -- \\
\hline $1: 2.5$ & 0.001 & 0.001 & $U$ & 0.001 & $U$ & $U$ & 0.003 & $\mathrm{U}$ & $U$ & $\bar{U}$ & $U$ & $U$ & $U$ & 0.001 & $\bar{U}$ & 0.001 \\
\hline $1: 2.5$ & 0.001 & $\mathrm{U}$ & -- & -- & -- & $U$ & -- & 0.003 & -. & - & $\mathrm{U}$ & -- & -- & -- & $u$ & -- \\
\hline $1: 5$ & 0.001 & 0.001 & 0.001 & 0.001 & $U$ & $U$ & $\mathrm{U}$ & $\mathrm{U}$ & $U$ & $\bar{U}$ & $\bar{U}$ & $\bar{U}$ & $U$ & 0.001 & $U$ & 0.001 \\
\hline \multirow[t]{2}{*}{$1: 5$} & -- & -- & 0.001 & -- & - & -- & $U$ & -- & $U$ & - & - & $U$ & - & -- & - & 0.001 \\
\hline & \multicolumn{16}{|c|}{ Selenium } \\
\hline $1: 1$ & $U$ & $U$ & $\bar{U}$ & $\bar{U}$ & 0.089 & $\bar{U}$ & $\bar{U}$ & $\mathrm{U}$ & $U$ & 0.019 & 0.062 & 0.064 & 0.175 & $\mathrm{U}$ & 0.416 & $\bar{U}$ \\
\hline $1: 1$ & $\ldots$ & -- & -- & $U$ & 0.092 & -- & - & - & -. & 0.018 & - & -- & 0.151 & $U$ & - & -- \\
\hline $1: 2.5$ & $u$ & $U$ & 0.003 & 0.003 & 0.049 & $u$ & $\mathrm{U}$ & $\mathrm{U}$ & $u$ & $\mathrm{U}$ & 0.027 & 0.042 & 0.079 & $U$ & 0.210 & 0.003 \\
\hline $1: 2.5$ & $\mathrm{U}$ & 0.003 & -- & -- & -- & $\mathrm{U}$ & -- & $U$ & -- & - & 0.024 & -- & -- & -- & 0.239 & -- \\
\hline $1: 5$ & $\bar{U}$ & U & $\bar{U}$ & $U$ & 0.018 & $\mathrm{U}$ & $\bar{U}$ & $U$ & $\bar{U}$ & $\bar{U}$ & $\mathrm{U}$ & 0.019 & 0.041 & $\bar{U}$ & 0.112 & $\bar{U}$ \\
\hline \multirow[t]{2}{*}{$1: 5$} & -- & - & U & $-\cdots$ & -- & -- & $U$ & - & $\mathrm{U}$ & - & - & 0.019 & -- & -- & - & $\mathrm{U}$ \\
\hline & \multicolumn{16}{|c|}{ Uranium (metal) } \\
\hline $1: 1$ & 0.00222 & 0.00006 & 0.00642 & 0.00516 & 0.0125 & 0.00366 & 0 & 0.00177 & 0.00109 & 0.00594 & 0.0186 & 0.0289 & 0.0273 & 0.000030 & 0.000897 & $\bar{U}$ \\
\hline $1: 1$ & -- & -- & -- & 0.00519 & 0.0110 & -- & $-\cdots$ & -- & -- & 0.00596 & -- & -- & 0.0270 & $\mathrm{U}$ & - & - \\
\hline $1: 2.5$ & 0.00127 & 0.00005 & 0.00335 & 0.00268 & 0.00615 & 0.00235 & 0.000032 & 0.000897 & 0.000677 & 0.00334 & 0.00831 & 0.0158 & 0.0130 & $U$ & 0.000520 & $U$ \\
\hline $1: 2.5$ & 0.00130 & 0.00005 & -- & -- & -- & 0.00231 & -- & 0.000842 & -- & - & 0.00825 & -- & -- & - & 0.000491 & - \\
\hline $1: 5$ & \begin{tabular}{|l|}
0.00109 \\
\end{tabular} & 0.00010 & 0.00227 & 0.00211 & 0.00301 & 0.00153 & 0 & $\mathrm{U}$ & 0.000322 & 0.00228 & 0.00527 & 0.0106 & 0.00902 & $\mathrm{U}$ & 0.000354 & U \\
\hline \multirow[t]{2}{*}{$1: 5$} & \begin{tabular}{|l|}
-- \\
\end{tabular} & \begin{tabular}{|c|}
-- \\
\end{tabular} & 0.00244 & -- & -- & \begin{tabular}{|l|}
-- \\
\end{tabular} & 0 & 0.000551 & 0.000407 & - & - & 0.0109 & -- & -- & - & $U$ \\
\hline & \multicolumn{16}{|c|}{ Vanadium } \\
\hline $1: 1$ & 0.067 & 0.039 & 0.035 & 0.057 & 0.116 & 0.088 & 0.106 & 0.046 & 0.032 & 0.033 & 0.077 & 0.073 & 0.123 & 0.090 & 0.073 & 0.092 \\
\hline $1: 1$ & -- & -- & -- & 0.056 & 0.123 & -- & -- & -- & -- & 0.037 & - & $-\cdots$ & 0.118 & 0.089 & - & -- \\
\hline $1: 2.5$ & 0.061 & 0.017 & 0.037 & 0.047 & 0.094 & 0.066 & 0.114 & 0.034 & 0.028 & 0.025 & 0.049 & 0.049 & 0.083 & 0.015 & $U$ & 0.007 \\
\hline $1: 2.5$ & 0.058 & 0.017 & -- & -- & -- & 0.069 & -- & 0.032 & -- & - & 0.048 & -- & -- & -- & $\mathrm{U}$ & -- \\
\hline $1: 5$ & 0.046 & 0.015 & 0.032 & 0.036 & 0.072 & 0.051 & 0.084 & 0.027 & 0.021 & 0.019 & 0.033 & 0.033 & 0.057 & 0.018 & 0.013 & 0.012 \\
\hline \multirow[t]{2}{*}{$1: 5$} & - & - & 0.030 & -- & -- & -- & 0.085 & -- & 0.020 & - & - & 0.033 & -- & -- & - & 0.014 \\
\hline & \multicolumn{16}{|c|}{ Zinc } \\
\hline $1: 1$ & $U$ & $U$ & $U$ & $\bar{U}$ & U & $U$ & $U$ & $U$ & $U$ & $U$ & $\bar{U}$ & $U$ & $U$ & $U$ & $U$ & $U$ \\
\hline $1: 1$ & -- & -- & -- & $\mathrm{U}$ & $U$ & -. & -- & -- & -- & $U$ & - & - & $U$ & $\mathrm{U}$ & - & -- \\
\hline $1: 2.5$ & $\mathrm{U}$ & $U$ & $U$ & 0.012 & $U$ & $U$ & $U$ & $U$ & $U$ & $U$ & $U$ & $U$ & $U$ & $U$ & $U$ & $U$ \\
\hline $1: 2.5$ & $\mathrm{U}$ & $U$ & -- & -- & -- & $U$ & - & $U$ & -- & - & $U$ & -- & -- & - & U & $\cdots$ \\
\hline $1: 5$ & U & $U$ & $U$ & $u$ & $U$ & $U$ & $U$ & $U$ & $U$ & $U$ & $U$ & $U$ & $U$ & $U$ & $U$ & $U$ \\
\hline \multirow[t]{2}{*}{$1: 5$} & -- & -- & $U$ & -- & -- & -- & $\mathrm{U}$ & - & U & - & - & $U$ & -- & -- & - & $U$ \\
\hline & \multicolumn{16}{|c|}{ Final $\mathrm{pH}$} \\
\hline $1: 1$ & 8.79 & 7.89 & 9.18 & 8.58 & 8.39 & 8.24 & 7.45 & 8.41 & 9.01 & 8.46 & 8.41 & 8.21 & 8.17 & 7.68 & 7.44 & 7.61 \\
\hline $1: 1$ & -- & -- & -- & 8.91 & 8.61 & - & -- & -- & -- & 8.43 & - & - & 8.26 & 6.99 & - & -- \\
\hline $1: 2.5$ & 9.01 & 7.72 & 9.22 & 9.02 & 8.89 & 8.36 & 6.88 & 9.05 & 9.17 & 8.56 & 8.58 & 8.42 & 8.78 & 7.16 & 8.02 & 7.63 \\
\hline $1: 2.5$ & 9.13 & 7.58 & -- & -- & -- & 8.38 & -- & 9.12 & -- & - & 8.59 & -- & -- & -- & 8.23 & -- \\
\hline $1: 5$ & 9.28 & 7.57 & 9.52 & 8.57 & 9.12 & 8.48 & 6.78 & 9.31 & 9.35 & 8.64 & 8.80 & 8.61 & 8.7 & 7.09 & 8.41 & 7.58 \\
\hline $1: 5$ & $-\cdots$ & -- & 9.55 & -- & -- & -- & 6.76 & -- & 9.31 & - & - & 8.63 & -- & -- & - & 7.49 \\
\hline
\end{tabular}

${ }^{\text {a }}$ Sample J1HHW3 was inadvertently processed through leach testing. J1HHW3 is the surface sample associated with depth sample $\mathrm{J} 1 \mathrm{HJ} 10$.

$-=$ no leach test performed

$\mathrm{U}=$ undetected 
Antimony, beryllium, silver, and thallium were not quantified in any leachate samples. Barium, boron, uranium, and vanadium were measured in nearly all samples. Total chromium was measured in one or more leachate samples from each area with a maximum concentration of $0.015 \mathrm{mg} / \mathrm{L}$ at a $1: 1$ ratio at $126-\mathrm{D}-1$. Lead was measured in a single leachate sample from $126-\mathrm{H}-1$ at $0.011 \mathrm{mg} / \mathrm{L}$ at a $1: 2.5 \mathrm{coal}$ ash:water ratio. Final $\mathrm{pH}$ values in leachate ranged from 6.99 to 9.55 .

\subsection{ADDITIONAL BATCH LEACH TESTS WITH NON-PH-ADJUSTED WATER}

To investigate any potential effect of leach fluid $\mathrm{pH}$ on the final concentration of leachable arsenic, a second set of batch leach tests was performed on a subset of samples. After analysis for metals had been completed on all the surface/depth samples, the arsenic results were evaluated to choose the samples for this second leach test. Table 4-9 shows the samples with the highest arsenic results that were chosen for subsequent leach tests with non-pH-adjusted water. Two of the samples with the highest arsenic concentrations were surface samples and had not undergone batch leach testing. Therefore, these two surface samples underwent batch leach testing with both $\mathrm{pH} 5.2$ and non-pH-adjusted water. A summary of the leachate results for arsenic with no adjustment for coal ash:leachate ratio is presented in Table 4-10.

Table 4-9. Identification of Coal Ash Surface/Depth Samples with Highest Arsenic Concentrations.

\begin{tabular}{|c|c|c|c|c|c|c|}
\hline \multirow{2}{*}{$\begin{array}{l}\text { Sample } \\
\text { Location }\end{array}$} & \multirow{2}{*}{$\begin{array}{l}\text { Sample } \\
\text { Number }\end{array}$} & \multicolumn{3}{|c|}{ Arsenic } & \multirow{2}{*}{$\begin{array}{l}\text { Leached with } \\
\text { Non-pH-Adjusted Water }\end{array}$} & \multirow{2}{*}{$\begin{array}{l}\text { Leached with } \\
\text { pH 5.2 Water }\end{array}$} \\
\hline & & $\mathrm{mg} / \mathrm{kg}$ & $\mathbf{Q}$ & PQL & & \\
\hline $\begin{array}{l}100-\mathrm{B}-25 \\
\text { (surface) }\end{array}$ & J1HHX8 & 10.3 & & 2.83 & $x$ & $x$ \\
\hline $\begin{array}{l}\text { 100-B-25 } \\
\text { (depth) }\end{array}$ & J1HJ08 & 8.09 & & 2.68 & $x$ & $\begin{array}{l}\text { Yes, depth } \\
\text { sample }\end{array}$ \\
\hline $\begin{array}{c}100-\mathrm{H}-25 \\
\text { (depth) }\end{array}$ & J1HJ80 & 11.7 & & 0.86 & $x$ & $\begin{array}{l}\text { Yes, depth } \\
\text { sample }\end{array}$ \\
\hline $\begin{array}{c}600-15 \\
\text { (surface) }\end{array}$ & J1HHN7 & 10.6 & & 0.96 & $x$ & $x$ \\
\hline
\end{tabular}

$\mathrm{PQL}=$ practical quantitation limit

$\mathrm{Q}=$ qualifier

$X$ = additional leach test performed

Table 4-10. Arsenic Leachate Results from Coal Ash Surface/Depth Samples with Highest Arsenic Concentrations. (2 Pages)

\begin{tabular}{|c|c|c|c|c|c|c|c|c|}
\hline \multirow[b]{2}{*}{$\begin{array}{l}\text { Coal Ash: } \\
\text { Water Ratio }\end{array}$} & \multicolumn{8}{|c|}{ Arsenic (mg/L) } \\
\hline & $\begin{array}{l}\text { J1HHX8 } \\
\text { (pH 5.2) }\end{array}$ & $\begin{array}{l}\text { J1HHX8 } \\
(\mathrm{pH} \mathrm{6.4)}\end{array}$ & $\begin{array}{l}\text { J1HJ08 } \\
\text { (pH 5.2) }\end{array}$ & $\begin{array}{l}\text { J1HJ08 } \\
\text { (pH 6.4) }\end{array}$ & $\begin{array}{l}\text { J1HJ80 } \\
\text { (pH 5.2) }\end{array}$ & $\begin{array}{l}\text { J1HJ80 } \\
\text { (pH 6.4) }\end{array}$ & $\begin{array}{l}\text { J1HHN7 } \\
\text { (pH 5.2) }\end{array}$ & $\begin{array}{l}\text { J1HHN7 } \\
\text { (pH 6.4) }\end{array}$ \\
\hline $1: 1$ & 0.015 & $\mathrm{U}$ & $\mathrm{U}$ & $\mathrm{U}$ & U & $U$ & $\mathrm{U}$ & U \\
\hline $1: 1$ & -- & -- & -- & $\mathrm{U}$ & -- & -- & -- & - \\
\hline $1: 2.5$ & 0.008 & 0.007 & $\mathrm{U}$ & 0.002 & $\mathrm{U}$ & 0.006 & 0.003 & 0.002 \\
\hline $1: 2.5$ & -- & 0.006 & $\mathrm{U}$ & $-\cdots$ & $\mathrm{U}$ & 0.006 & 0.005 & - \\
\hline $1: 5$ & 0.008 & 0.006 & $\mathrm{U}$ & $\mathrm{U}$ & $\mathrm{U}$ & 0.005 & 0.003 & $\mathrm{U}$ \\
\hline
\end{tabular}


Table 4-10. Arsenic Leachate Results from Coal Ash Surface/Depth Samples with Highest Arsenic Concentrations. (2 Pages)

\begin{tabular}{|c|c|c|c|c|c|c|c|c|}
\hline \multirow[b]{2}{*}{$\begin{array}{c}\text { Coal Ash: } \\
\text { Water Ratio }\end{array}$} & \multicolumn{8}{|c|}{ Arsenic (mg/L) } \\
\hline & $\begin{array}{l}\text { J1HHX8 } \\
\text { (pH 5.2) }\end{array}$ & $\begin{array}{l}\text { J1HHX8 } \\
\text { (pH 6.4) }\end{array}$ & $\begin{array}{l}\mathrm{J} 1 \mathrm{HJ} 08 \\
(\mathrm{pH} \mathrm{5.2)}\end{array}$ & $\begin{array}{l}\text { J1HJ08 } \\
\text { (pH 6.4) }\end{array}$ & $\begin{array}{l}\mathrm{J} 1 \mathrm{HJ} 80 \\
(\mathrm{pH} 5.2)\end{array}$ & $\begin{array}{l}\text { J1HJ80 } \\
(\mathrm{pH} 6.4)\end{array}$ & $\begin{array}{l}\text { J1HHN7 } \\
\text { (pH 5.2) }\end{array}$ & $\begin{array}{l}\text { J1HHN7 } \\
(\mathrm{pH} 6.4)\end{array}$ \\
\hline $1: 5$ & 0.007 & -- & - & -- & -- & -. & -- & 0.002 \\
\hline
\end{tabular}

- $=$ no leach test performed

$\mathrm{U}=$ undetected

In some samples ( $\mathrm{J} 1 \mathrm{HJ} 08$ and $\mathrm{J} 1 \mathrm{HJ} 80$ ), low-level detections of arsenic in leachate were observed with unadjusted leachate $(\mathrm{pH} 6.4)$ relative to $\mathrm{pH} 5.2$ leachate. However, the concentrations are consistent with other $\mathrm{pH} 5.2$ leach test results and were below the PQL (approximately $0.015 \mathrm{mg} / \mathrm{L}$ ). Analytical results from the solid and leachate samples associated with the batch leaching tests, including other analytes, are presented in Appendix C.

\subsection{DATA QUALITY ASSESSMENT}

A data quality assessment (DQA) was performed to compare the sampling approach and analytical data with the sampling and data requirements specified in the SAP (DOE-RL 2011a) and associated change notices (DOE-RL 2011b, 2011c). The DQA for the coal ash characterization sampling established that the data are of the right type, quality, and quantity to support site verification decisions within specified error tolerances. The data set was found to be acceptable for decision-making purposes. The analytical sample data are stored in the environmental-restoration project-specific database for data evaluation prior to its archival in Hanford Environmental Information System and are summarized in Appendix C. The detailed DQA is presented in Appendix $G$. 
WCH-506

Rev. 0 


\subsection{SUMMARY}

Coal ash samples from five sites in separate River Corridor operable units were collected and analyzed per the SAP with subsequent approved change notices (DOE-RL 2011a, 2011b, and 2011c). Statistical surface sample results $(N=29)$ from each site were used to prepare $90^{\text {th }}$ percentile values and $90^{\text {th }}$ percentile UTL values to characterize metal concentrations. These values for metals in coal ash provide a means to compare concentrations between coal ash from different operable units. Additional analysis of the surface sample data sets was performed using the Kruskal-Wallis median equality test. Test results indicate that vanadium is the only analyte for which the medians of the data from all five sample sites are statistically equal. Surface sample data was further characterized through box plots. Variability in the $90^{\text {th }}$ percentile values is observed between the sample sites, the box plots provide a visual representation of the overall variability in the data.

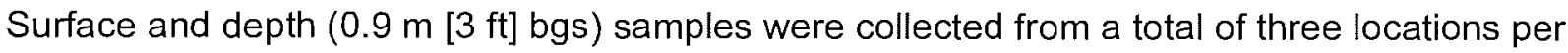
site to determine if the metals concentration varied with depth. Box plots were generated to depict the surface and depth sampling results by analyte at each of the sites. Determination of the means for each analyte including all surface and depth results was performed. Analytes showing significant variability are lead at $126-\mathrm{H}-1$ and mercury at 300 Area Ash. For both lead and mercury, depth sample results from the abovementioned sites are greater than all other results due to a single elevated result. A limited number of low concentration PAHs were detected in the surface and depth samples.

Batch leach tests conducted on depth samples indicate leachable barium, boron, uranium, and vanadium are present at each sample site. Additional leach tests conducted on a sample subset with the most elevated arsenic concentrations indicate that use of non-pH-adjusted leachate solution does not affect the concentration of leachable arsenic from coal ash.

Collectively, the results of this coal ash characterization effort provide data that can be used for further evaluation and consideration of coal ash throughout the river corridor. 
WCH-506

Rev. 0 


\subsection{REFERENCES}

ASTM, 2004, Standard Test Method for Shake Extraction of Solid Waste with Water, ASTM D3987-06, American Society for Testing and Materials, West Conshohocken, Pennsylvania.

DOE-RL, 2011a, Sampling and Analysis Plan for Characterization of Hanford Site Coal Ash Components, DOE/RL-2010-113, Rev. 0, U.S. Department of Energy, Richland Operations Office, Richland, Washington.

DOE-RL, 2011b, Tri-Party Agreement Change Notice, TPA-CN-431, Modify Sampling and Analysis Plan for Characterization of Hanford Site Coal Ash Components to add Additional Analysis for Total Uranium on a Subset of Coal Ash Samples, (DOE/RL-2010-113, Rev. 0), U.S. Department of Energy, Richland Operations Office, Richland, Washington.

DOE-RL, 2011c, Tri-Party Agreement Change Notice, TPA-CN-451, Modify Sampling and Analysis Plan for Characterization of Hanford Site Coal Ash Components to 1) Update Sample Area of 126-D-1 to Exclude an Area Containing Minimal Coal Ash and 2) Identify Waste Management Approach for Waste Related to Coal Ash Sampling Activities (DOE/RL-2010-113, Rev. 0), U.S. Department of Energy, Richland Operations Office, Richland, Washington.

Ecology, 1994, Natural Background Soil Metal Concentrations in Washington State, Publication No. 94-115, Washington State Department of Ecology, Olympia, Washington.

Ecology, 1996, Washington Administrative Code, Title 173, Chapter 340, "Model Toxics Control Act-Cleanup," Part VII, "Cleanup Standards," WAC 173-340-747, "Deriving Soil Concentrations for Groundwater Protection," Washington State Department of Ecology, Olympia, Washington.

Ecology, 2007, Washington Administrative Code, Title 173, Chapter 340, "Model Toxics Control Act-Cleanup," Part VII, "Cleanup Standards," WAC 173-340-740, "Unrestricted Land Use Soil Cleanup Standards," Washington State Department of Ecology, Olympia, Washington.

EPA, 2010a, Coal Combustion Residuals, U.S. Environmental Protection Agency, Washington, D.C. < www.epa.gov/epawaste/nonhaz/industrial/special/fossil/ccrrule/index.htm $>$.

EPA, 2010b, Draft: Human and Ecological Risk Assessment of Coal Combustion Wastes, EPA-HQ-RCRA-2009-0640-0002, U.S. Environmental Protection Agency, Washington, D.C. < www.regulations.gov/4t!documentDetail; $D=E P A-H Q-R C R A-2009-$ 0640-0002 >.

EPA, 2010c, ProUCL, Version 4.1, U.S. Environmental Protection Agency, Washington, D.C. $<$ http://www.epa.gov/esd/tsc/install.htm>. 
WCH-506

Rev. 0

Resource Conservation and Recovery Act of 1976, 42 U.S.C. 6901, et seq., as amended.

WCH, 2011, Characterization of Hanford Coal Ash Components, Logbook EL-1658, Washington Closure Hanford, Richland, Washington. 


\section{APPENDIX A}

\section{SAMPLE SUMMARY BY SAMPLE SITE}


WCH-506

Rev. 0 
Table A-1. 126-B-1 Sample Summary.

\begin{tabular}{|c|c|c|c|c|}
\hline \multirow{2}{*}{$\begin{array}{l}\text { Sample } \\
\text { Location }\end{array}$} & \multirow{2}{*}{$\begin{array}{c}\text { HEIS } \\
\text { Number }\end{array}$} & \multirow{2}{*}{$\begin{array}{c}\text { Sample } \\
\text { Date }\end{array}$} & \multicolumn{2}{|c|}{ Washington State Plane } \\
\hline & & & Easting (m) & Northing (m) \\
\hline 100-B-01 & J1HHV4 & $4 / 27 / 11$ & 564953.23 & 145012.09 \\
\hline $100-B-02$ & J1HHV5 & $4 / 27 / 11$ & 564972.68 & 145007.32 \\
\hline $100-B-03$ & J1HHV6 & $4 / 27 / 11$ & 564992.12 & 145001.56 \\
\hline $100-B-04$ & J1HHV7 & $4 / 27 / 11$ & 564968.81 & 145025.55 \\
\hline 100-B-05 & J1HHV8 & $4 / 27 / 11$ & 564988.26 & 145018.78 \\
\hline $100-B-06$ & J1HHV9 & $4 / 27 / 11$ & 565007.70 & 145012.02 \\
\hline 100-B-07 & J1HHW0 & $4 / 27 / 11$ & 565027.15 & 145005.25 \\
\hline 100-B-08 & J1HHW1 & $4 / 27 / 11$ & 565046.60 & 144998.49 \\
\hline $100-B-09$ & J1HHW2 & $8 / 23 / 11$ & 564964.94 & 145045.77 \\
\hline $100-B-10$ & J1HHW3 & $4 / 27 / 11$ & 564984.39 & 145039.01 \\
\hline $\begin{array}{c}\text { 100-B-10 } \\
\text { (depth) }\end{array}$ & J1HJ10 & $4 / 27 / 11$ & 564984.39 & 145039.01 \\
\hline $100-\mathrm{B}-11$ & J1HHW4 & $4 / 27 / 11$ & 565003.84 & 145032.24 \\
\hline $100-B-12$ & J1HHW5 & $4 / 27 / 11$ & 565023.29 & 145025.48 \\
\hline $100-\mathrm{B}-13$ & J1HHW6 & $4 / 27 / 11$ & 565039.73 & 145018.71 \\
\hline $100-B-14$ & J1HHW7 & $8 / 23 / 11$ & 564961.08 & 145065.99 \\
\hline 100-B-15 & J1HHW8 & $4 / 27 / 11$ & 564980.53 & 145059.23 \\
\hline $\begin{array}{c}\text { Duplicate of } \\
\text { J1HHW8 }\end{array}$ & J1HJ04 & $4 / 27 / 11$ & 564980.53 & 145059.23 \\
\hline $100-\mathrm{B}-16$ & J1HHW9 & $4 / 26 / 11$ & 564999.97 & 145052.47 \\
\hline 100-B-17 & $\mathrm{J} 1 \mathrm{HHX0}$ & 4/27/11 & 565019.42 & 145045.70 \\
\hline $\begin{array}{c}100-\mathrm{B}-17 \\
\text { (depth) }\end{array}$ & J1HJ09 & $4 / 27 / 11$ & 565019.42 & 145045.70 \\
\hline $100-B-18$ & J1HHX1 & $4 / 27 / 11$ & 565039.87 & 145038.94 \\
\hline 100-B-19 & $\mathrm{J} 1 \mathrm{HHX} 2$ & $8 / 23 / 11$ & 564957.21 & 145086.22 \\
\hline $100-B-20$ & $\mathrm{~J} 1 \mathrm{HH} \times 3$ & $8 / 23 / 11$ & 564976.66 & 145079.45 \\
\hline $100-B-21$ & J1HHX4 & $4 / 25 / 11$ & 564996.11 & 145077.26 \\
\hline $100-B-22$ & J1HHX5 & $4 / 26 / 11$ & 565015.55 & 145065.92 \\
\hline $100-B-23$ & $\mathrm{~J} 1 \mathrm{HH} \times 6$ & $4 / 26 / 11$ & 565035.00 & 145059.16 \\
\hline $100-B-24$ & $\mathrm{~J} 1 \mathrm{HHX7}$ & $4 / 26 / 11$ & 564972.79 & 145099.68 \\
\hline $100-B-25$ & J1HHX8 & $4 / 25 / 11$ & 564992.24 & 145089.91 \\
\hline $\begin{array}{c}\text { Duplicate of } \\
\text { J1HHX8 }\end{array}$ & J1HJ03 & $4 / 25 / 11$ & 564992.24 & 145089.91 \\
\hline $\begin{array}{c}\text { 100-B-25 } \\
\text { (depth) }\end{array}$ & J1HJ08 & $4 / 26 / 11$ & 564992.24 & 145089.91 \\
\hline $100-B-26$ & J1HHX9 & $8 / 23 / 11$ & 565011.69 & 145086.15 \\
\hline 100-B-27 & J1HJ00 & $4 / 27 / 11$ & 565031.14 & 145079.38 \\
\hline $100-B-28$ & J1HJ01 & $8 / 23 / 11$ & 565027.27 & 145099.61 \\
\hline $100-\mathrm{B}-29$ & J1HJ02 & $8 / 23 / 11$ & 565046.72 & 145092.84 \\
\hline Equipment blank & J1HJ05 & $4 / 25 / 11$ & NA & NA \\
\hline
\end{tabular}

$\mathrm{HEIS}=$ Hanford Environmental Information Service $\mathrm{NA}=$ not applicable 
Table A-2. 126-D-1 Sample Summary.

\begin{tabular}{|c|c|c|c|c|}
\hline \multirow{2}{*}{$\begin{array}{l}\text { Sample } \\
\text { Location }\end{array}$} & \multirow{2}{*}{$\begin{array}{c}\text { HEIS } \\
\text { Number }\end{array}$} & \multirow{2}{*}{$\begin{array}{c}\text { Sample } \\
\text { Date }\end{array}$} & \multicolumn{2}{|c|}{ Washington State Plane } \\
\hline & & & Easting (m) & Northing (m) \\
\hline 100-D-01 & J1J3W3 & $8 / 9 / 11$ & 573360.49 & 152130.02 \\
\hline $100-D-02$ & J1J3W4 & $8 / 22 / 11$ & 573358.68 & 152107.74 \\
\hline 100-D-03 & J1J3W5 & $8 / 22 / 11$ & 573352.88 & 152085.46 \\
\hline 100-D-04 & J1J3W6 & $8 / 23 / 11$ & 573381.68 & 152137.87 \\
\hline $100-\mathrm{D}-05$ & J1J3W7 & $8 / 23 / 11$ & 573377.88 & 152115.59 \\
\hline 100-D-06 & J1J3W8 & $8 / 22 / 11$ & 573374.07 & 152093.31 \\
\hline 100-D-07 & J1J3W9 & $8 / 22 / 11$ & 573370.27 & 152071.03 \\
\hline 100-D-08 & $\mathrm{J} 1 \mathrm{~J} 3 \times 0$ & $8 / 11 / 11$ & 573366.47 & 152048.75 \\
\hline 100-D-08 (depth) & $\mathrm{J} 1 \mathrm{~J} 444$ & $8 / 11 / 11$ & 573366.47 & 152071.03 \\
\hline 100-D-09 & $\mathrm{J} 1 \mathrm{~J} 3 \mathrm{X} 1$ & $8 / 11 / 11$ & 573362.66 & 152026.46 \\
\hline $100-\mathrm{D}-10$ & $\mathrm{~J} 1 \mathrm{~J} 3 \times 2$ & $8 / 11 / 11$ & 573358.86 & 152004.18 \\
\hline $100-D-11$ & $\mathrm{~J} 1 \mathrm{~J} 3 \times 3$ & $8 / 23 / 11$ & 573402.88 & 152145.71 \\
\hline $100-D-12$ & $\mathrm{~J} 1 \mathrm{~J} 3 \mathrm{X} 4$ & $8 / 9 / 11$ & 573399.08 & 152123.43 \\
\hline $100-\mathrm{D}-13$ & J1J3X5 & $8 / 22 / 11$ & 573391.16 & 152100.95 \\
\hline $100-\mathrm{D}-14$ & J1J3X6 & $8 / 22 / 11$ & 573391.47 & 152078.87 \\
\hline $100-\mathrm{D}-15$ & $\mathrm{~J} 1 \mathrm{~J} 3 \mathrm{X} 7$ & $8 / 11 / 11$ & 573387.66 & 152056.59 \\
\hline $100-D-16$ & J1J3X8 & $8 / 22 / 11$ & 573383.86 & 152034.31 \\
\hline $100-D-17$ & $\mathrm{~J} 1 \mathrm{~J} 3 \times 9$ & $8 / 22 / 11$ & 573380.06 & 152012.03 \\
\hline $\begin{array}{c}\text { Duplicate of } \\
\mathrm{J} 1 \mathrm{~J} 3 \times 9\end{array}$ & J1J413 & $8 / 22 / 11$ & 573380.06 & 152012.03 \\
\hline $100-D-18$ & $\mathrm{~J} 1 \mathrm{~J} 400$ & $8 / 9 / 11$ & 573424.08 & 152153.56 \\
\hline $100-D-19$ & $\mathrm{~J} 1 \mathrm{~J} 401$ & $8 / 11 / 11$ & 573420.27 & 152131.28 \\
\hline 100-D-19 (depth) & $\mathrm{J} 1 \mathrm{~J} 443$ & $8 / 11 / 11$ & 573420.27 & 152131.28 \\
\hline $100-\mathrm{D}-20$ & $\mathrm{~J} 1 \mathrm{~J} 402$ & $8 / 23 / 11$ & 573416.47 & 152116.00 \\
\hline $100-\mathrm{D}-21$ & $\mathrm{~J} 1 \mathrm{~J} 403$ & $8 / 23 / 11$ & 573444.28 & 152161.41 \\
\hline $100-D-22$ & $\mathrm{~J} 1 \mathrm{~J} 404$ & $8 / 23 / 11$ & 573441.47 & 152139.13 \\
\hline $100-D-23$ & $\mathrm{~J} 1 \mathrm{~J} 405$ & $8 / 23 / 11$ & 573437.67 & 152118.85 \\
\hline $100-D-24$ & $\mathrm{~J} 1 \mathrm{~J} 406$ & $8 / 23 / 11$ & 573466.47 & 152169.25 \\
\hline $100-D-25$ & $\mathrm{~J} 1 \mathrm{~J} 407$ & $8 / 23 / 11$ & 573462.67 & 152146.97 \\
\hline $100-D-26$ & $\mathrm{~J} 1 \mathrm{~J} 408$ & $8 / 11 / 11$ & 573458.87 & 152124.69 \\
\hline $\begin{array}{c}\text { Duplicate of } \\
\mathrm{J} 1 \mathrm{~J} 408\end{array}$ & $\mathrm{~J} 1 \mathrm{~J} 412$ & $8 / 11 / 11$ & 573458.87 & 152124.69 \\
\hline 100-D-26 (depth) & $\mathrm{J} 1 \mathrm{~J} 442$ & $8 / 11 / 11$ & 573458.87 & 152124.69 \\
\hline $100-\mathrm{D}-27$ & $\mathrm{~J} 1 \mathrm{~J} 409$ & $8 / 23 / 11$ & 573481.71 & 152154.62 \\
\hline 100-D-28 & $\mathrm{J} 1 \mathrm{~J} 410$ & $8 / 23 / 11$ & 573480.06 & 152132.54 \\
\hline 100-D-29 & $\mathrm{J} 1 \mathrm{~J} 411$ & $8 / 11 / 11$ & 573476.26 & 152110.26 \\
\hline Equipment blank & $\mathrm{J} 1 \mathrm{~J} 414$ & $8 / 11 / 11$ & NA & NA \\
\hline
\end{tabular}

HEIS = Hanford Environmental Information Service $\mathrm{NA}=$ not applicable 
Table A-3. 126-H-1 Sample Summary.

\begin{tabular}{|c|c|c|c|c|}
\hline \multirow{2}{*}{$\begin{array}{l}\text { Sample } \\
\text { Location }\end{array}$} & \multirow{2}{*}{$\begin{array}{c}\text { HEIS } \\
\text { Number }\end{array}$} & \multirow{2}{*}{$\begin{array}{c}\text { Sample } \\
\text { Date }\end{array}$} & \multicolumn{2}{|c|}{ Washington State Plane } \\
\hline & & & Easting (m) & Northing (m) \\
\hline $100-\mathrm{H}-01$ & $\mathrm{~J} 1 \mathrm{HJ} 48$ & $8 / 27 / 11$ & 577278.93 & 152906.74 \\
\hline $100-\mathrm{H}-02$ & $\mathrm{~J} 1 \mathrm{HJ} 49$ & $8 / 27 / 11$ & 577280.28 & 152927.94 \\
\hline $100-\mathrm{H}-03$ & $\mathrm{~J} 1 \mathrm{HJ} 50$ & $8 / 28 / 11$ & 577297.96 & 152916.17 \\
\hline $100-\mathrm{H}-04$ & J1HJ51 & $8 / 28 / 11$ & 577315.63 & 152904.40 \\
\hline $100-\mathrm{H}-05$ & J1HJ52 & $8 / 27 / 11$ & 577279.63 & 152949.13 \\
\hline $100-\mathrm{H}-06$ & J1HJ53 & $8 / 28 / 11$ & 577299.31 & 152937.36 \\
\hline $100-\mathrm{H}-07$ & J1HJ54 & $8 / 28 / 11$ & 577316.99 & 152925.59 \\
\hline $100-\mathrm{H}-08$ & J1HJ55 & $8 / 28 / 11$ & 577334.67 & 152913.82 \\
\hline $100-\mathrm{H}-09$ & J1HJ56 & $8 / 28 / 11$ & 577352.34 & 152902.05 \\
\hline $100-\mathrm{H}-10$ & J1HJ57 & $8 / 27 / 11$ & 577282.99 & 152970.32 \\
\hline $100-\mathrm{H}-11$ & J1HJ58 & $8 / 27 / 11$ & 577300.67 & 152958.55 \\
\hline $100-\mathrm{H}-12$ & J1HJ59 & $8 / 28 / 11$ & 577318.34 & 152946.78 \\
\hline $100-\mathrm{H}-13$ & J1HJ60 & $8 / 28 / 11$ & 577336.02 & 152935.01 \\
\hline $100-\mathrm{H}-14$ & J1HJ61 & $8 / 28 / 11$ & 577353.70 & 152923.24 \\
\hline $100-\mathrm{H}-15$ & J1HJ62 & $8 / 28 / 11$ & 577371.37 & 152911.47 \\
\hline $100-\mathrm{H}-16$ & $\mathrm{~J} 1 \mathrm{HJ} 63$ & $8 / 27 / 11$ & 577284.34 & 152991.52 \\
\hline $\begin{array}{c}\text { Duplicate of } \\
\mathrm{J} 1 \mathrm{HJ} 63\end{array}$ & J1HJ78 & $8 / 27 / 11$ & 577284.34 & 152991.52 \\
\hline $100-\mathrm{H}-17$ & J1HJ64 & $8 / 27 / 11$ & 577303.02 & 152972.75 \\
\hline $100-\mathrm{H}-17$ (depth) & J1HJ82 & $8 / 27 / 11$ & 577303.02 & 152972.75 \\
\hline $100-\mathrm{H}-18$ & J1HJ65 & $8 / 27 / 11$ & 577319.70 & 152967.98 \\
\hline $100-\mathrm{H}-19$ & J1HJ66 & $8 / 27 / 11$ & 577337.37 & 152956.21 \\
\hline $100-\mathrm{H}-20$ & J1HJ67 & $8 / 24 / 11$ & 577355.05 & 152944.44 \\
\hline $100-\mathrm{H}-20$ (depth) & J1HJ81 & $8 / 24 / 11$ & 577355.05 & 152944.44 \\
\hline $100-\mathrm{H}-21$ & J1HJ68 & $8 / 28 / 11$ & 577372.73 & 152932.67 \\
\hline $100-\mathrm{H}-22$ & J1HJ69 & $8 / 27 / 11$ & 577303.38 & 153000.94 \\
\hline $100-\mathrm{H}-23$ & J1HJ70 & $8 / 27 / 11$ & 577321.05 & 152989.17 \\
\hline $100-\mathrm{H}-24$ & J1HJ71 & $8 / 27 / 11$ & 577338.73 & 152977.40 \\
\hline $100-\mathrm{H}-25$ & $\mathrm{~J} 1 \mathrm{HJ} 72$ & $8 / 24 / 11$ & 577356.41 & 152965.63 \\
\hline $\begin{array}{c}\text { Duplicate of } \\
\mathrm{J} 1 \mathrm{HJ} 72\end{array}$ & J1HJ77 & $8 / 24 / 11$ & 577356.41 & 152965.63 \\
\hline 100-H-25 (depth) & J1HJ80 & $8 / 24 / 11$ & 577356.41 & 152965.63 \\
\hline $100-\mathrm{H}-26$ & J1HJ73 & $8 / 28 / 11$ & 577374.08 & 152953.86 \\
\hline $100-\mathrm{H}-27$ & J1HJ74 & $8 / 27 / 11$ & 577340.08 & 152998.59 \\
\hline $100-\mathrm{H}-28$ & J1HJ75 & $8 / 27 / 11$ & 577357.76 & 152986.82 \\
\hline $100-\mathrm{H}-29$ & J1HJ76 & $8 / 28 / 11$ & 577375.44 & 152977.05 \\
\hline Equipment blank & J1HJ79 & $8 / 24 / 11$ & NA & NA \\
\hline
\end{tabular}

HEIS = Hanford Environmental Information Service $\mathrm{NA}=$ not applicable 
Table A-4. 300 Area Sample Summary.

\begin{tabular}{|c|c|c|c|c|}
\hline \multirow{2}{*}{$\begin{array}{c}\text { Sample } \\
\text { Location }\end{array}$} & \multirow{2}{*}{$\begin{array}{c}\text { HEIS } \\
\text { Number }\end{array}$} & \multirow{2}{*}{$\begin{array}{c}\text { Sample } \\
\text { Date }\end{array}$} & \multicolumn{2}{|c|}{ Washington State Plane } \\
\hline & & & Easting (m) & Northing (m) \\
\hline $300-01$ & J1HJN8 & $8 / 26 / 11$ & 594486.57 & 116009.32 \\
\hline $300-02$ & J1HJN7 & $8 / 25 / 11$ & 594495.38 & 116006.38 \\
\hline 300-02 (depth) & J1HJV1 & $8 / 25 / 11$ & 594495.38 & 116006.38 \\
\hline $300-03$ & J1HJN6 & $8 / 25 / 11$ & 594484.71 & 116018.41 \\
\hline $300-03$ (depth) & $\mathrm{J} 1 \mathrm{HJV} 2$ & $8 / 25 / 11$ & 594484.71 & 116018.41 \\
\hline $300-04$ & J1HJN9 & $8 / 25 / 11$ & 594493.52 & 116015.48 \\
\hline $\begin{array}{c}\text { Duplicate of } \\
\text { J1HJN9 }\end{array}$ & J1HJT5 & $8 / 25 / 11$ & 594493.52 & 116015.48 \\
\hline 300-04 (depth) & J1HJV0 & $8 / 25 / 11$ & 594493.52 & 116015.48 \\
\hline $300-05$ & J1HJP0 & $8 / 26 / 11$ & 594456.42 & 116036.32 \\
\hline $300-06$ & J1HJP1 & $8 / 26 / 11$ & 594482.85 & 116027.51 \\
\hline $\begin{array}{c}\text { Duplicate of } \\
\text { J1HJP1 }\end{array}$ & J1HJT6 & $8 / 26 / 11$ & 594482.85 & 116027.51 \\
\hline $300-07$ & J1HJP2 & $8 / 26 / 11$ & 594491.66 & 116024.57 \\
\hline $300-08$ & J1HJP3 & $8 / 26 / 11$ & 594454.56 & 116045.42 \\
\hline $300-09$ & J1HJP4 & $8 / 26 / 11$ & 594463.37 & 116042.48 \\
\hline $300-10$ & J1HJP5 & $8 / 26 / 11$ & 594472.18 & 116039.54 \\
\hline $300-11$ & J1HJP6 & $8 / 26 / 11$ & 594480.99 & 116036.61 \\
\hline $300-12$ & J1HJP7 & $8 / 26 / 11$ & 594489.80 & 116033.67 \\
\hline $300-13$ & J1HJP8 & $8 / 26 / 11$ & 594461.51 & 116051.58 \\
\hline $300-14$ & J1HJP9 & $8 / 26 / 11$ & 594470.32 & 116048.64 \\
\hline $300-15$ & J1HJR0 & $8 / 26 / 11$ & 594479.13 & 116045.70 \\
\hline $300-16$ & J1HJR1 & $8 / 26 / 11$ & 594487.94 & 116042.77 \\
\hline $300-17$ & J1HJR2 & $8 / 26 / 11$ & 594459.65 & 116060.67 \\
\hline $300-18$ & J1HJR3 & $8 / 26 / 11$ & 594468.46 & 116057.74 \\
\hline $300-19$ & J1HJR4 & $8 / 26 / 11$ & 594477.27 & 116054.80 \\
\hline $300-20$ & J1HJR5 & $8 / 26 / 11$ & 594486.07 & 116051.86 \\
\hline $300-21$ & J1HJR6 & $8 / 26 / 11$ & 594466.60 & 116066.83 \\
\hline $300-22$ & J1HJR7 & $8 / 26 / 11$ & 594475.40 & 116063.90 \\
\hline $300-23$ & J1HJR8 & $8 / 26 / 11$ & 594484.21 & 116060.96 \\
\hline $300-24$ & J1HJR9 & $8 / 26 / 11$ & 594464.73 & 116075.93 \\
\hline $300-25$ & J1HJT0 & $8 / 26 / 11$ & 594473.54 & 116073.00 \\
\hline $300-26$ & J1HJT1 & $8 / 26 / 11$ & 594482.35 & 116070.06 \\
\hline $300-27$ & J1HJT2 & $8 / 26 / 11$ & 594471.68 & 116082.09 \\
\hline $300-28$ & J1HJT3 & $8 / 26 / 11$ & 594480.49 & 116079.16 \\
\hline $300-29$ & J1HJT4 & $8 / 26 / 11$ & 594469.82 & 116091.19 \\
\hline Equipment blank & J1HJT7 & $8 / 25 / 11$ & NA & NA \\
\hline
\end{tabular}

HEIS = Hanford Environmental Information Service $\mathrm{NA}=$ not applicable 
Table A-5. 600-207 Area Sample Summary.

\begin{tabular}{|c|c|c|c|c|}
\hline \multirow{2}{*}{$\begin{array}{c}\text { Sample } \\
\text { Location }\end{array}$} & \multirow{2}{*}{$\begin{array}{c}\text { HEIS } \\
\text { Number }\end{array}$} & \multirow{2}{*}{$\begin{array}{c}\text { Sample } \\
\text { Date }\end{array}$} & \multicolumn{2}{|c|}{ Washington State Plane } \\
\hline & & & Easting $(\mathrm{m})$ & Northing (m) \\
\hline $600-01$ & J1HHM3 & $9 / 28 / 11$ & 588354.10 & 136806.92 \\
\hline $600-02$ & J1HHM4 & $9 / 28 / 11$ & 588373.44 & 136806.57 \\
\hline $600-03$ & J1HHM5 & $9 / 28 / 11$ & 588392.78 & 136806.21 \\
\hline $600-04$ & J1HHM6 & $9 / 28 / 11$ & 588412.12 & 136805.86 \\
\hline $600-05$ & J1HHM7 & $9 / 28 / 11$ & 588344.74 & 136823.85 \\
\hline $600-06$ & J1HHM8 & $9 / 28 / 11$ & 588364.08 & 136823.49 \\
\hline $600-07$ & J1HHM9 & $9 / 27 / 11$ & 588383.42 & 136823.14 \\
\hline $600-07$ (depth) & J1HHT0 & $9 / 27 / 11$ & 588383.42 & 136823.14 \\
\hline $600-08$ & J1HHN0 & 9/28/11 & 588402.76 & 136822.78 \\
\hline $600-09$ & J1HHN1 & 9/28/11 & 588422.10 & 136822.43 \\
\hline $600-10$ & J1HHN2 & 9/28/11 & 588354.72 & 136840.42 \\
\hline $600-11$ & J1HHN3 & 9/28/11 & 588374.06 & 136840.06 \\
\hline $600-12$ & J1HHN4 & 9/28/11 & 588393.40 & 136839.71 \\
\hline $600-13$ & J1HHN5 & 9/28/11 & 588412.74 & 136839.36 \\
\hline $600-14$ & J1HHN6 & 9/28/11 & 588345.36 & 136857.35 \\
\hline $600-15$ & J1HHN7 & $9 / 27 / 11$ & 588364.70 & 136856.99 \\
\hline $\begin{array}{c}\text { Duplicate of } \\
\text { J1HHN7 }\end{array}$ & J1HHR2 & $9 / 27 / 11$ & 588364.70 & 136856.99 \\
\hline $600-15$ (depth) & J1HHT9 & 9/27/11 & 588364.70 & 136856.99 \\
\hline $600-16$ & J1HHN8 & $9 / 28 / 11$ & 588384.04 & 136856.64 \\
\hline $600-17$ & J1HHN9 & $9 / 28 / 11$ & 588403.37 & 136856.28 \\
\hline $600-18$ & J1HHP0 & 9/28/11 & 588422.71 & 136855.93 \\
\hline $600-19$ & J1HHP1 & $9 / 28 / 11$ & 588355.33 & 136873.92 \\
\hline $600-20$ & J1HHP2 & $9 / 28 / 11$ & 588374.67 & 136873.56 \\
\hline $600-21$ & J1HHP3 & $9 / 28 / 11$ & 588394.01 & 136873.21 \\
\hline $600-22$ & J1HHP4 & $9 / 27 / 11$ & 588345.97 & 136890.84 \\
\hline $600-23$ & J1HHP5 & 9/27/11 & 588365.31 & 136890.49 \\
\hline $600-23$ (depth) & J1HHV0 & 9/27/11 & 588365.31 & 136890.49 \\
\hline $600-24$ & J1HHP6 & $9 / 28 / 11$ & 588384.65 & 136890.13 \\
\hline $\begin{array}{l}\text { Duplicate of } \\
\text { J1HHP6 }\end{array}$ & J1HHR3 & $9 / 28 / 11$ & 588384.65 & 136890.13 \\
\hline $600-25$ & J1HHP7 & $9 / 27 / 11$ & 588355.95 & 136907.41 \\
\hline $600-26$ & J1HHP8 & $9 / 28 / 11$ & 588375.29 & 136907.06 \\
\hline $600-27$ & J1HHP9 & $9 / 27 / 11$ & 588346.59 & 136924.34 \\
\hline $600-28$ & J1HHR0 & $9 / 27 / 11$ & 588365.93 & 136923.98 \\
\hline $600-29$ & J1HHR1 & $9 / 28 / 11$ & 588355.00 & 136932.00 \\
\hline Equipment blank & J1HHT7 & 9/27/11 & NA & NA \\
\hline
\end{tabular}

HEIS = Hanford Environmental Information Service $\mathrm{NA}=$ not applicable 
WCH-506

Rev. 0 


\section{APPENDIX B}

\section{PHOTOGRAPHS OF COAL ASH SAMPLE AREAS}


WCH-506

Rev. 0 
Figure B-1. 126-B-1 Coal Ash Sample Site and Surrounding Coal Ash Area.

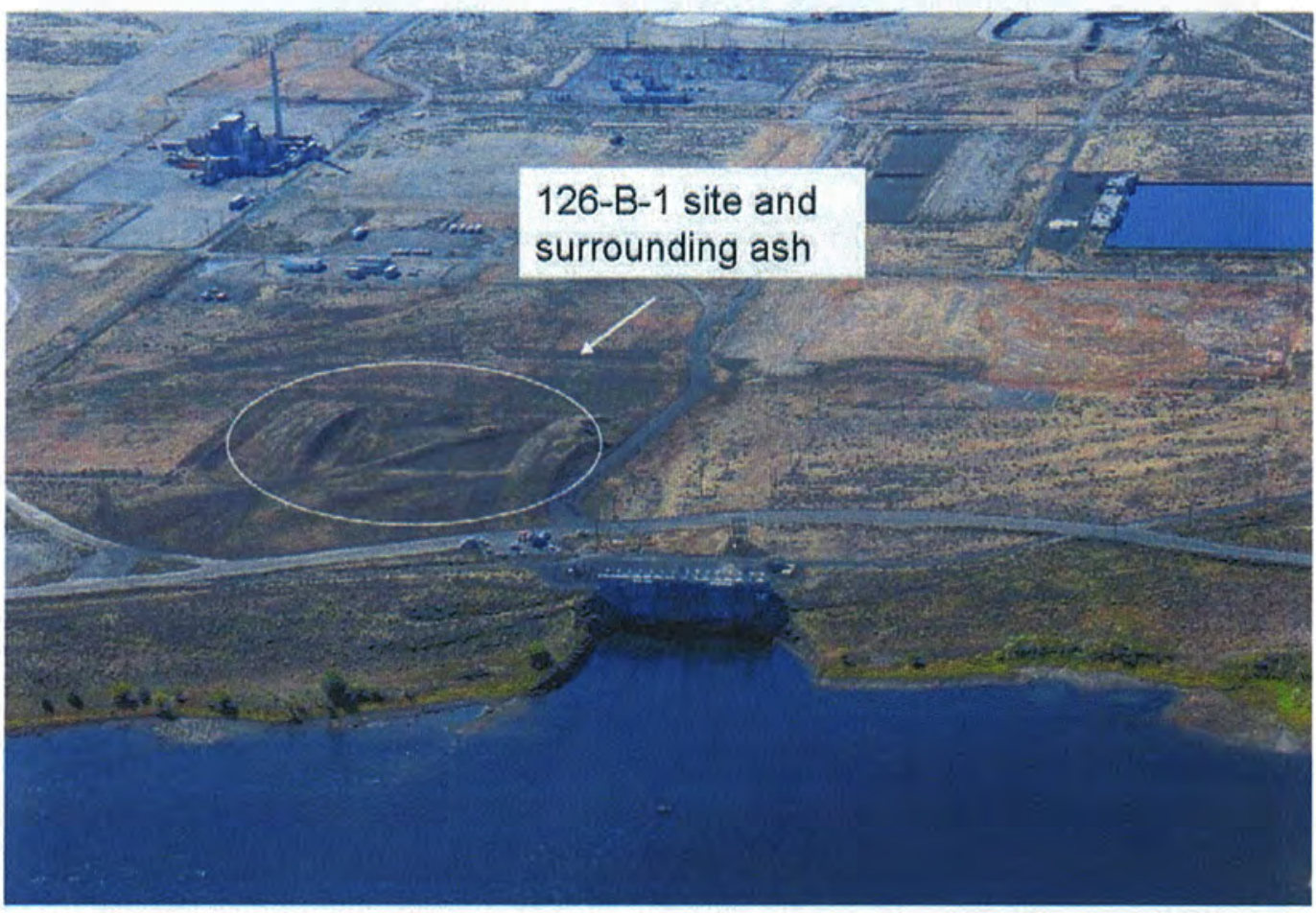

Figure B-2. View of Side Ash Berm from Within 126-B-1 Site.

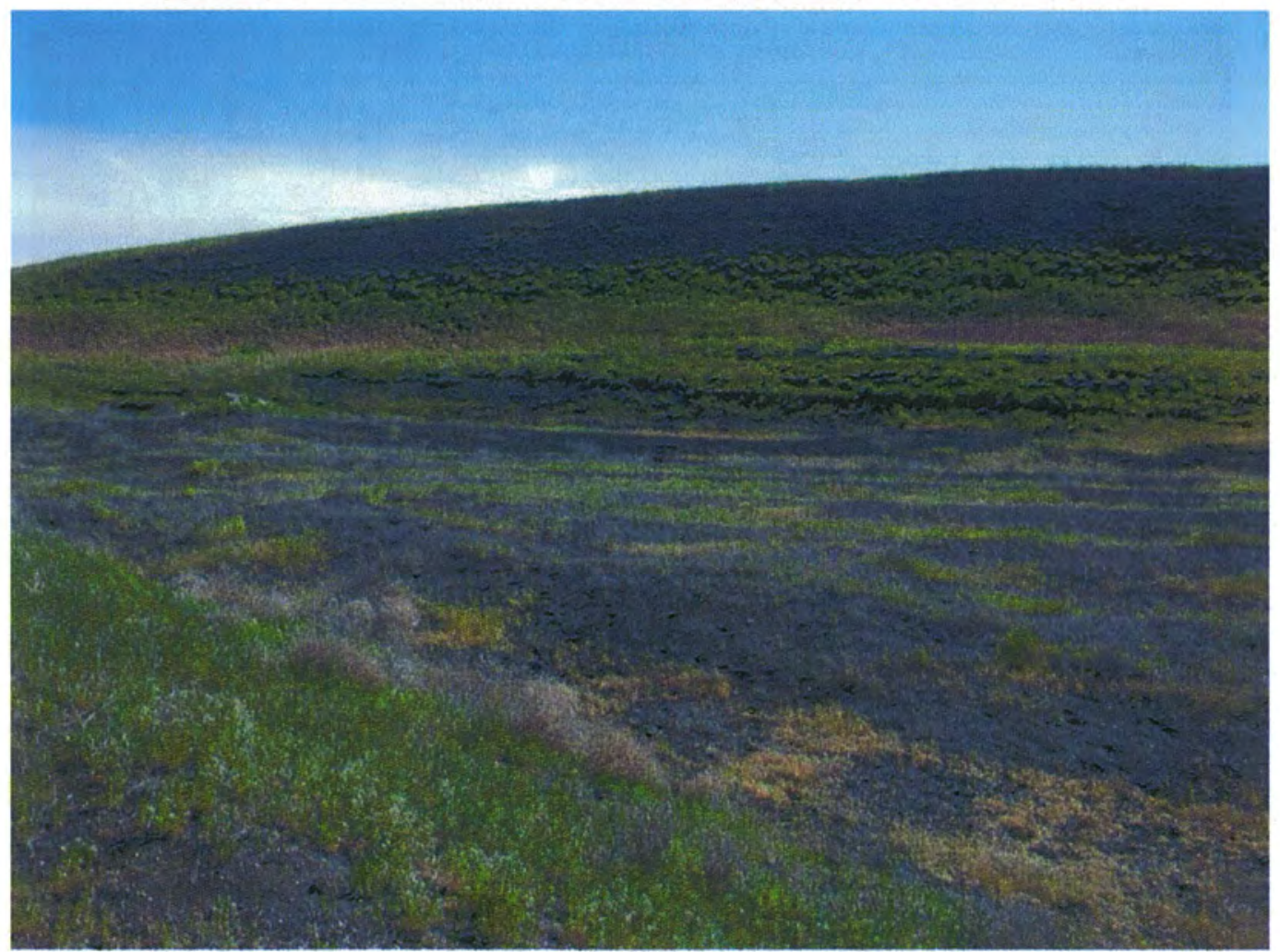


Figure B-3. View of Midline Berm Within 126-B-1 Site.

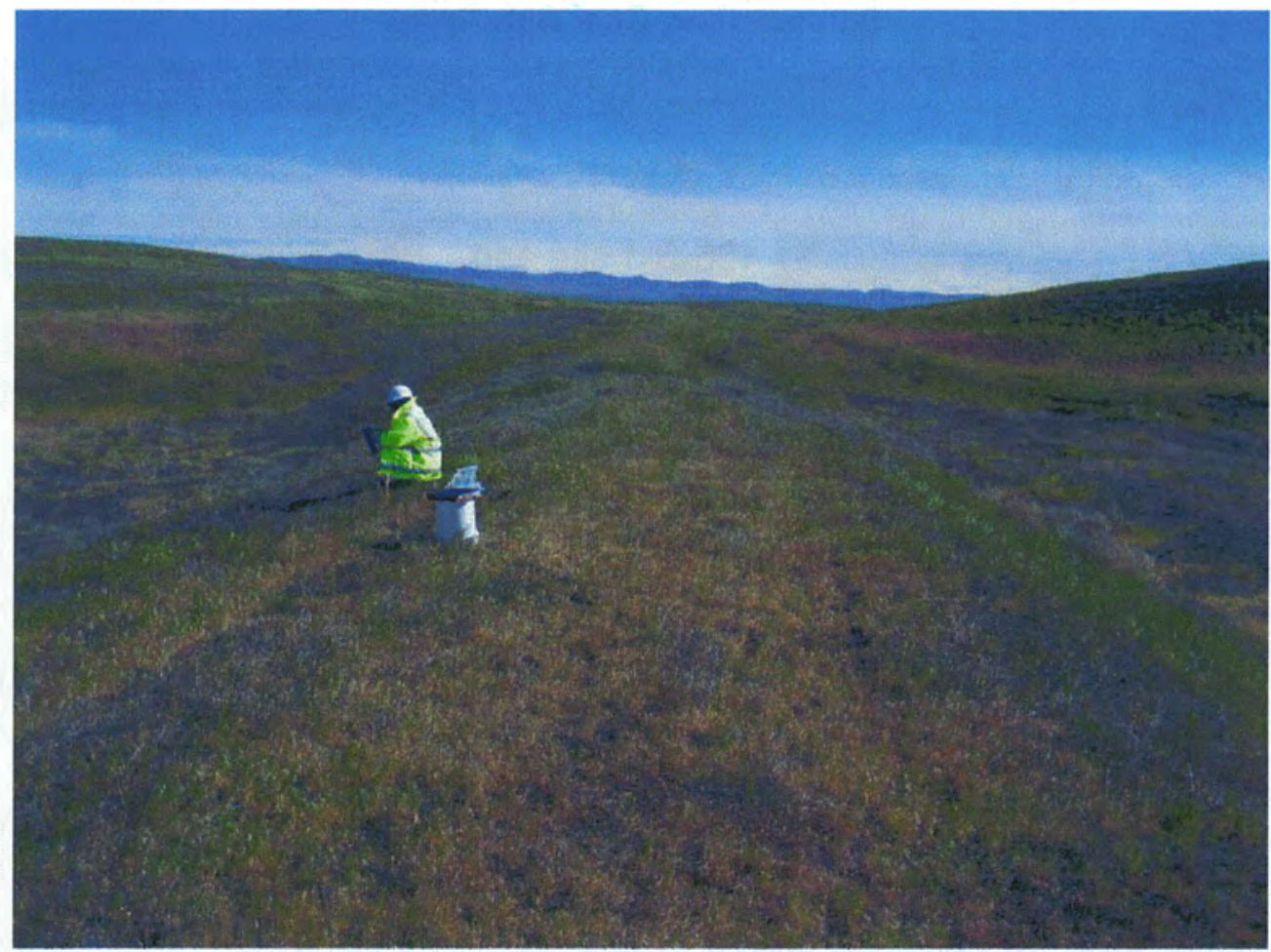

Figure B-4. 126-D-1 Coal Ash Sample Site and Surrounding Coal Ash Area (View to North).

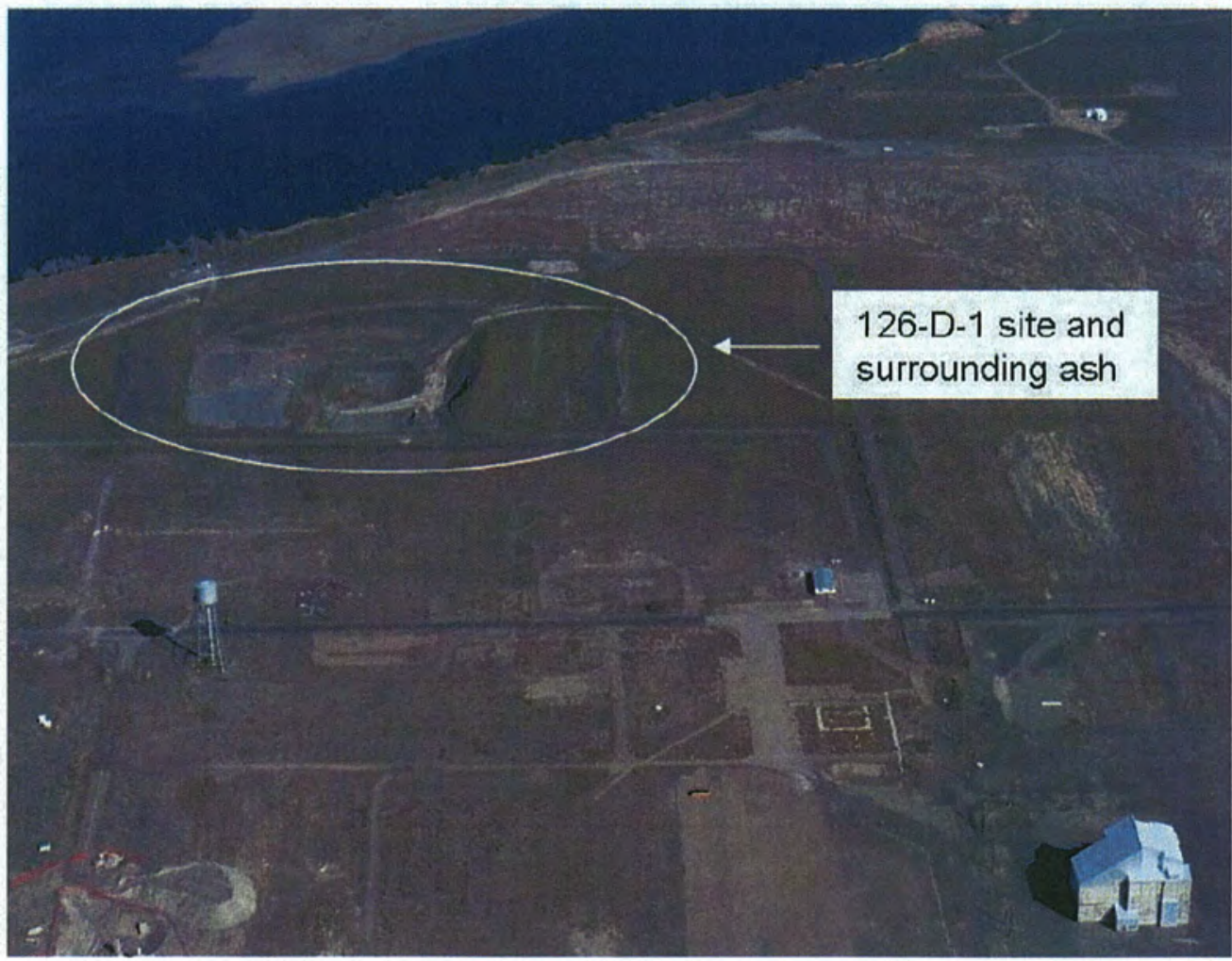


Figure B-5. 126-D-1 Coal Ash Sample Site and Surrounding Coal Ash Berms After 100-D-31:7 Pipeline Backfill (View to South).

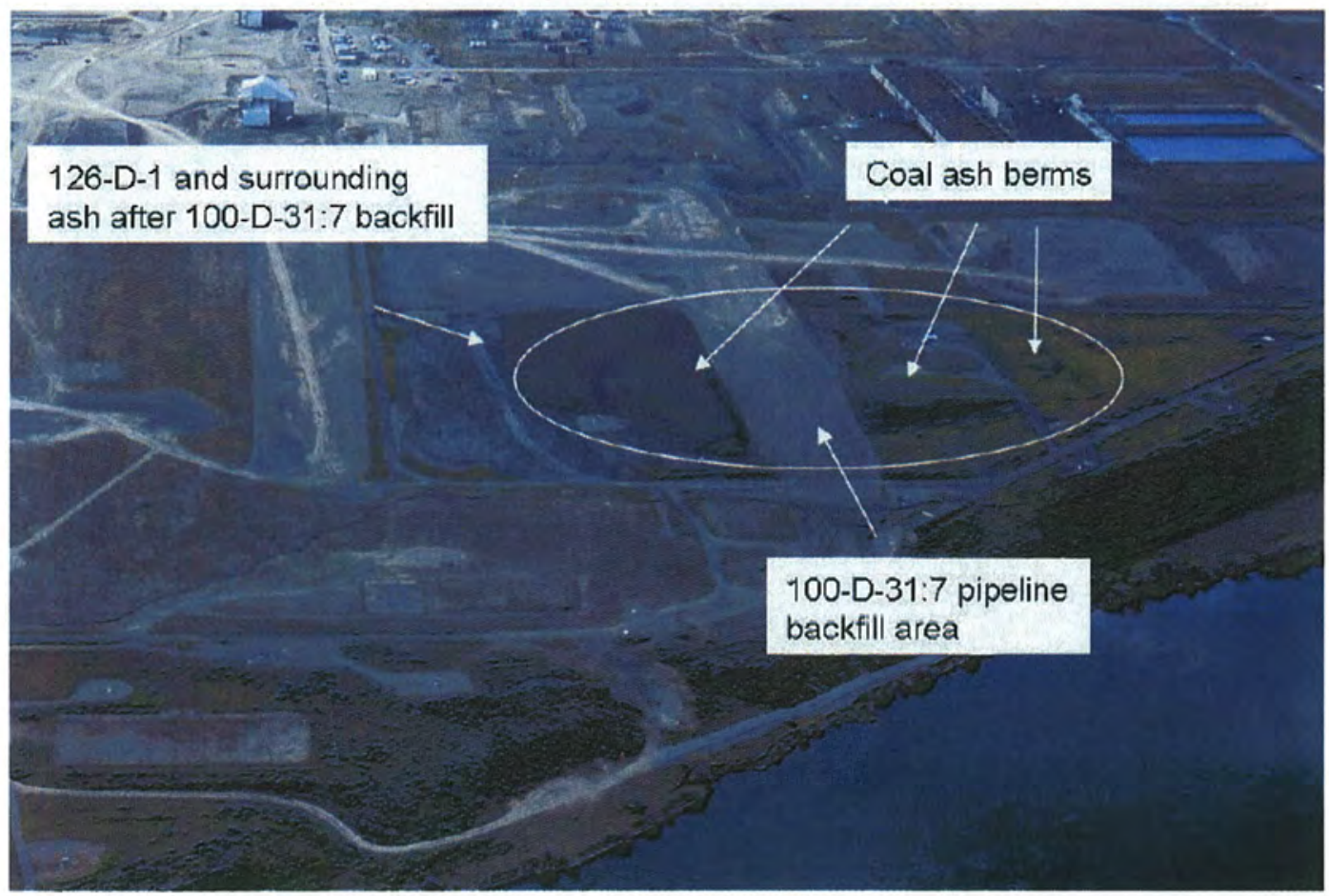

Figure B-6. View of West Coal Ash Berm from the North Coal Ash Berm at 126-D-1 Coal Ash Sample Site. 
Figure B-7. View of North Coal Ash Berm from the West Coal Ash Berm at 126-D-1

Coal Ash Sample Site.

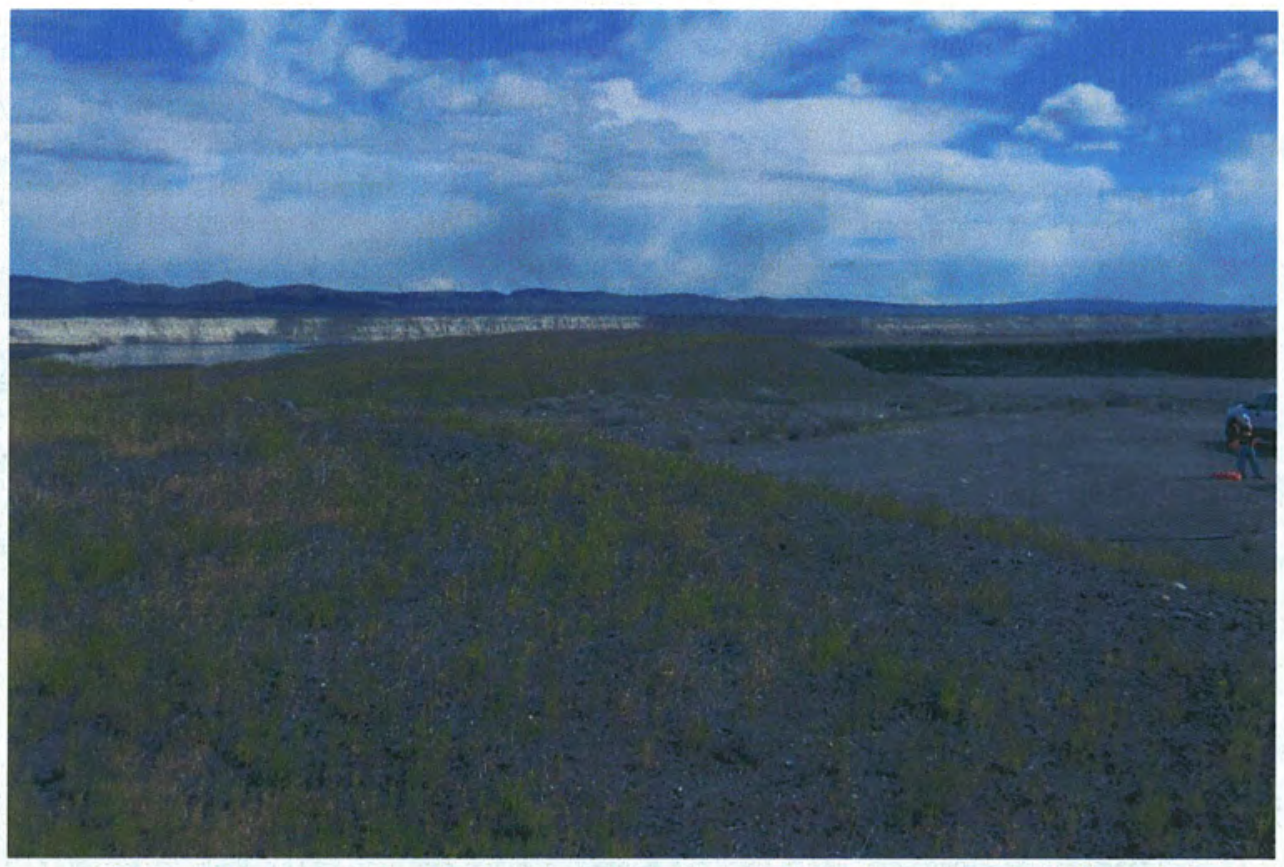

Figure B-8. Overland View of 126-H-1 Coal Ash Sample Site with Neighboring Ash Berm.

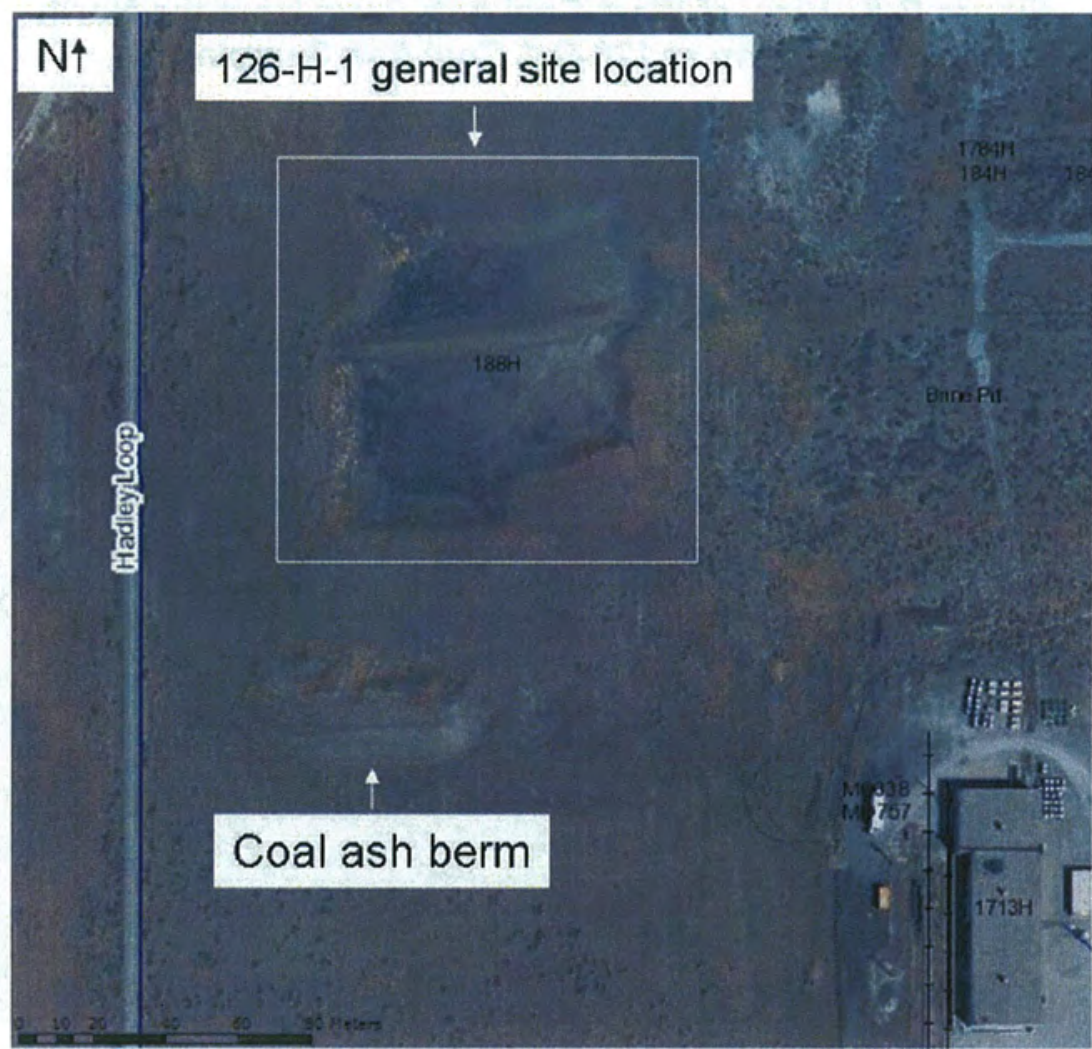


Figure B-9. View to North of Midline Berm at 126-H-1 Site.

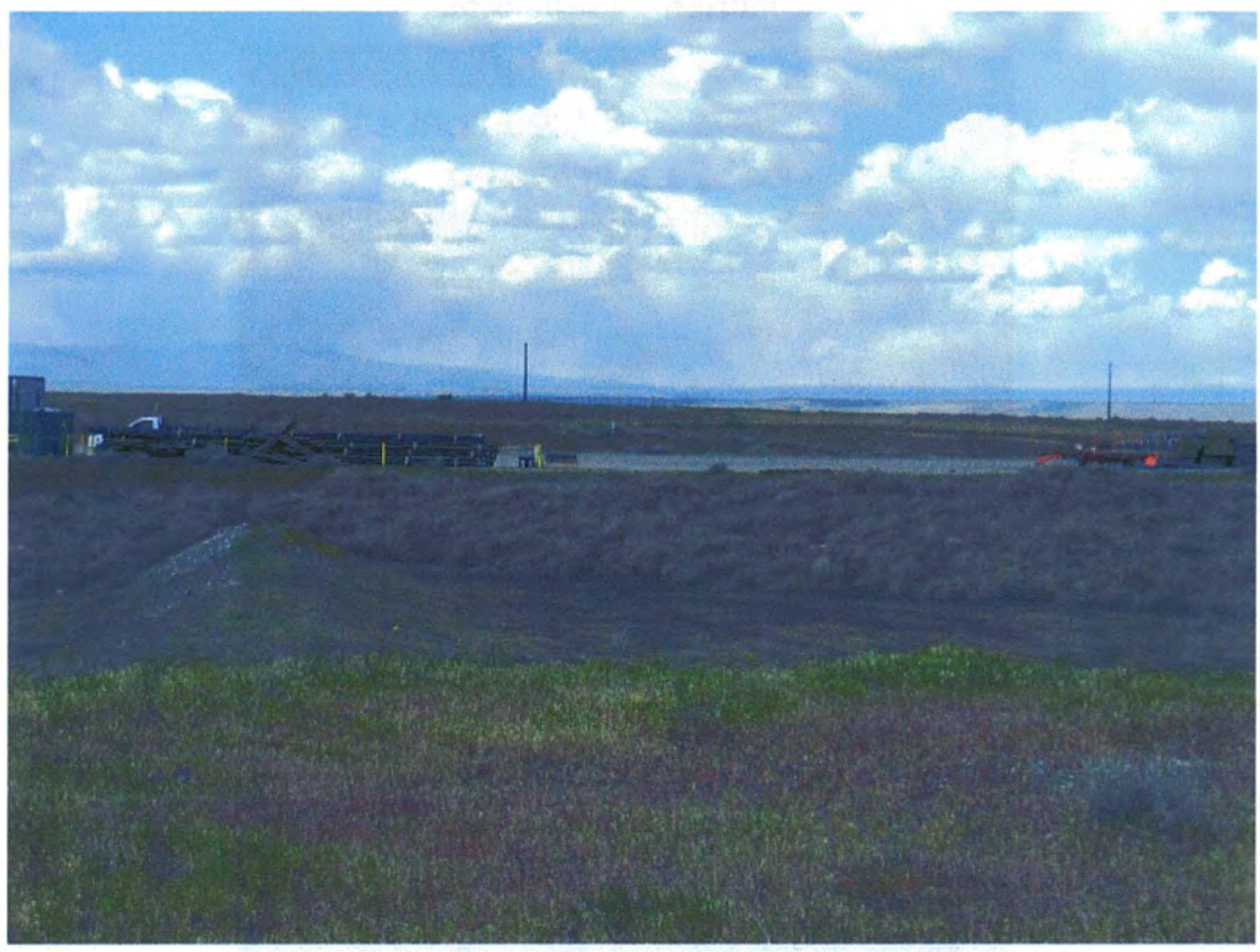

Figure B-10. View to North of Midline Berm at 126-H-1 Site.

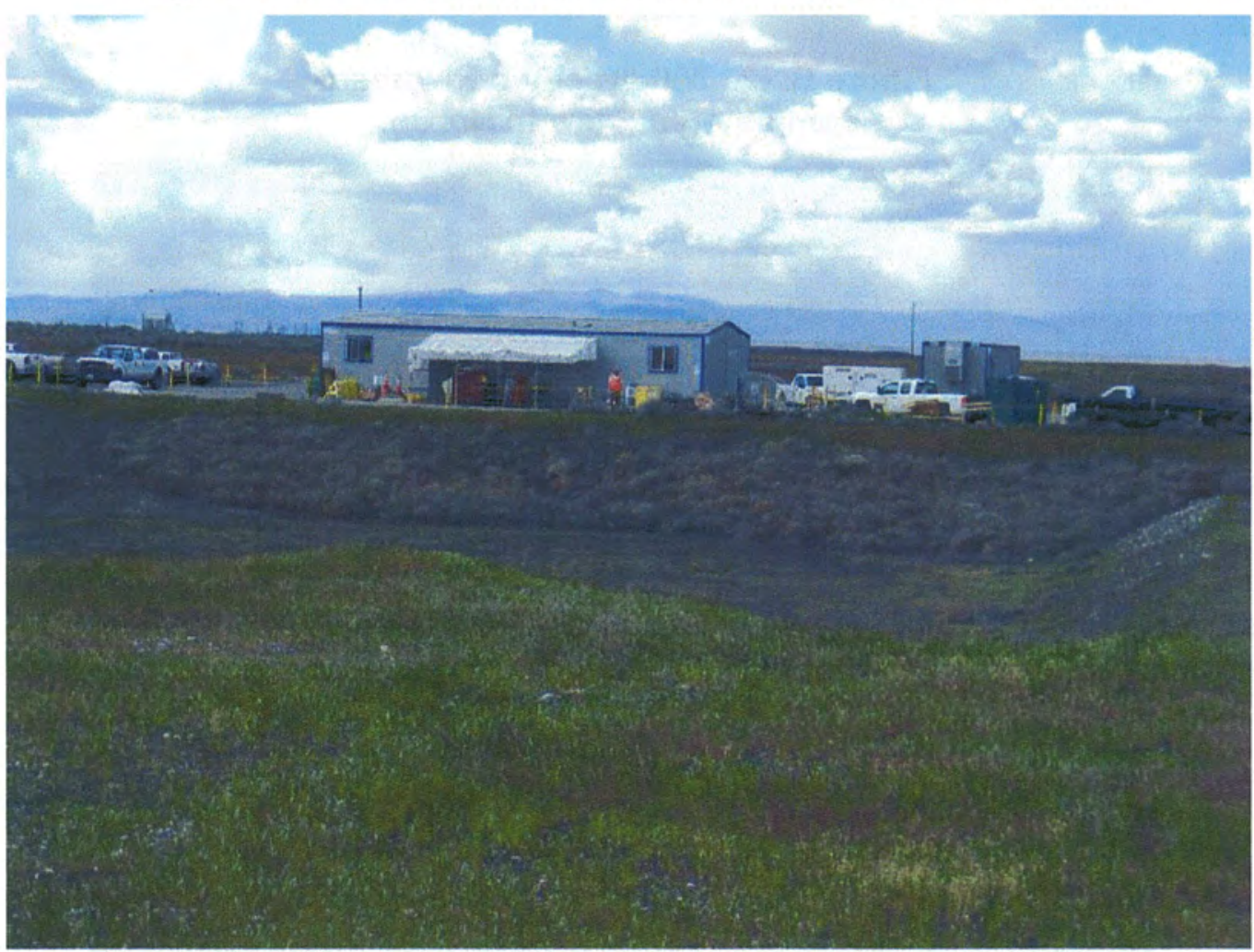


Figure B-11. Overland View of 300 Area

Coal Ash Sample Site.

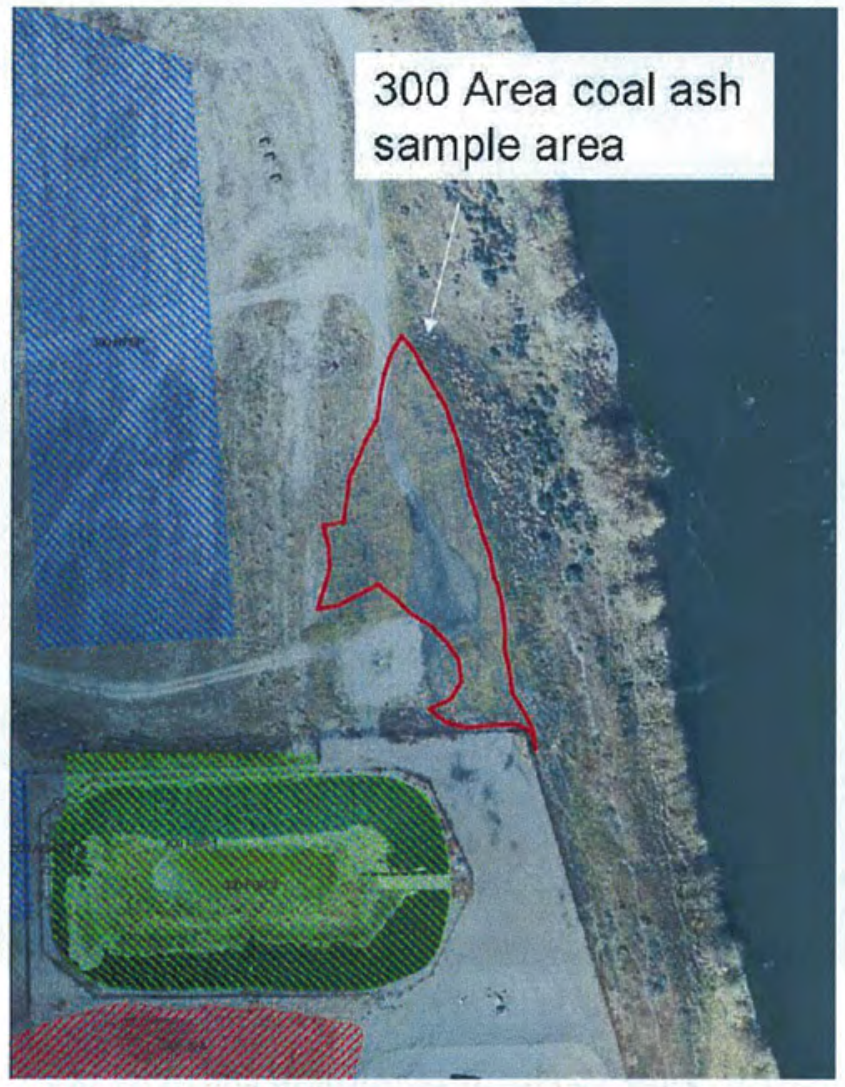

Figure B-12. 300 Area Ash Riverbank Sample Area (View to North).

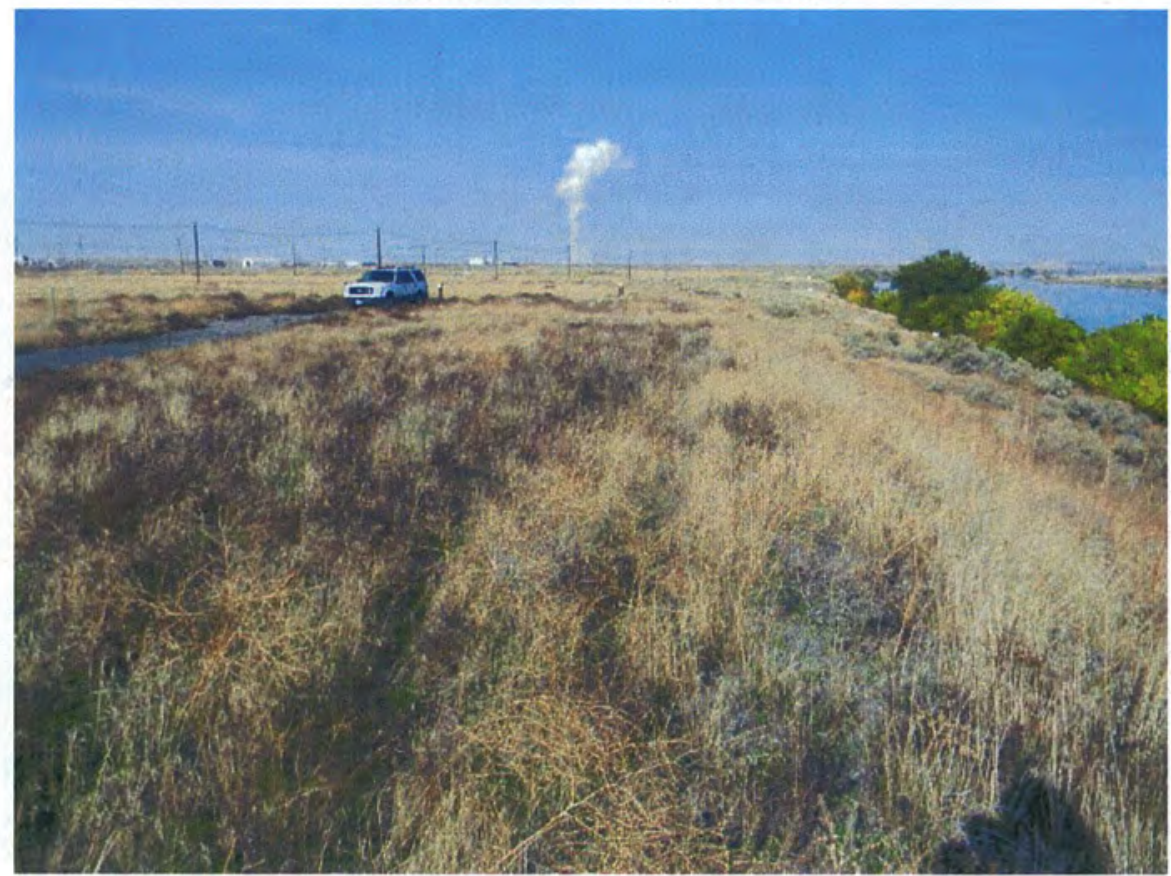


Figure B-13. 300 Area Ash Sample Area (View to South).

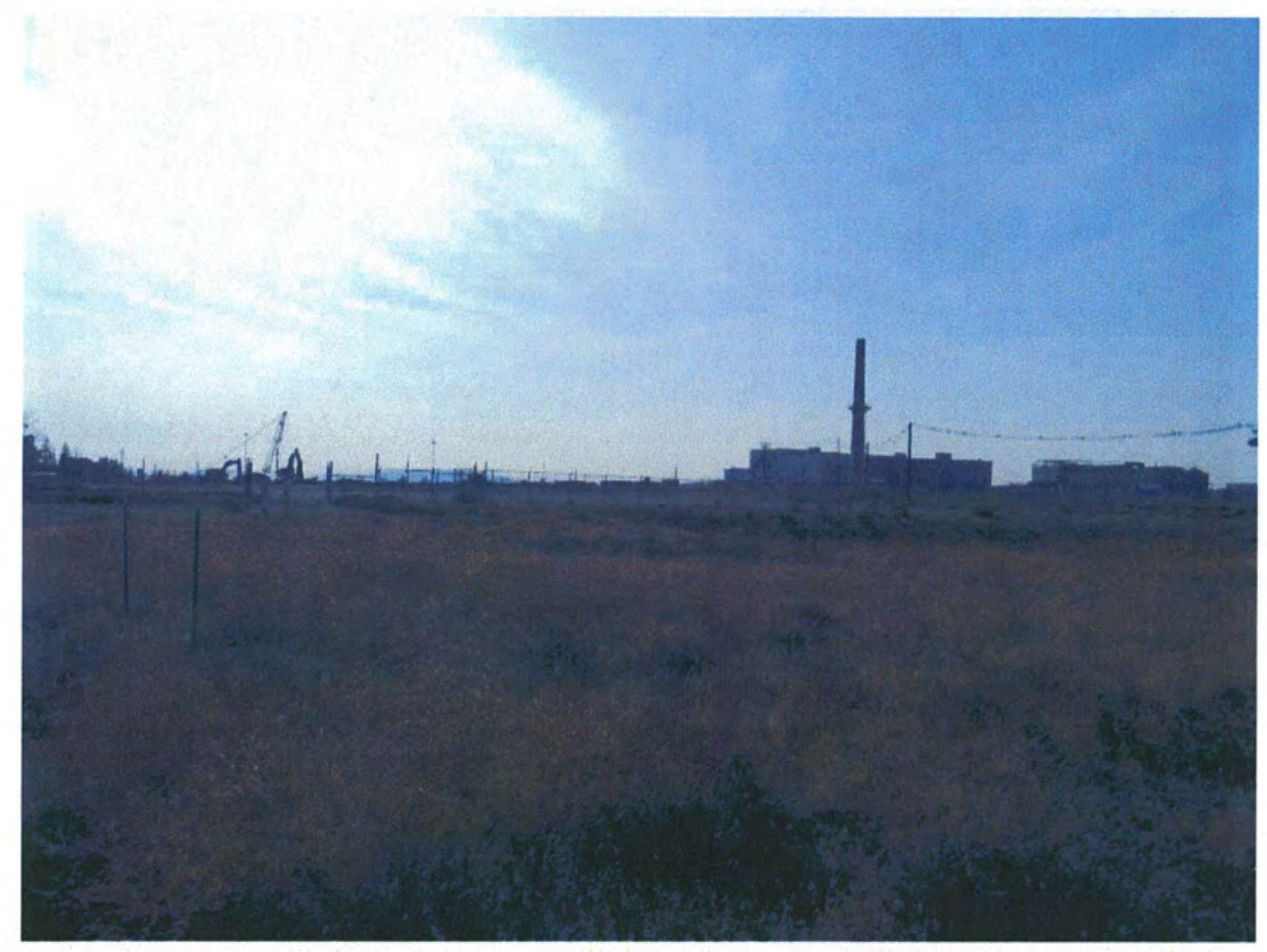

Figure B-14. Overland View of 600-207 Coal Ash Site.

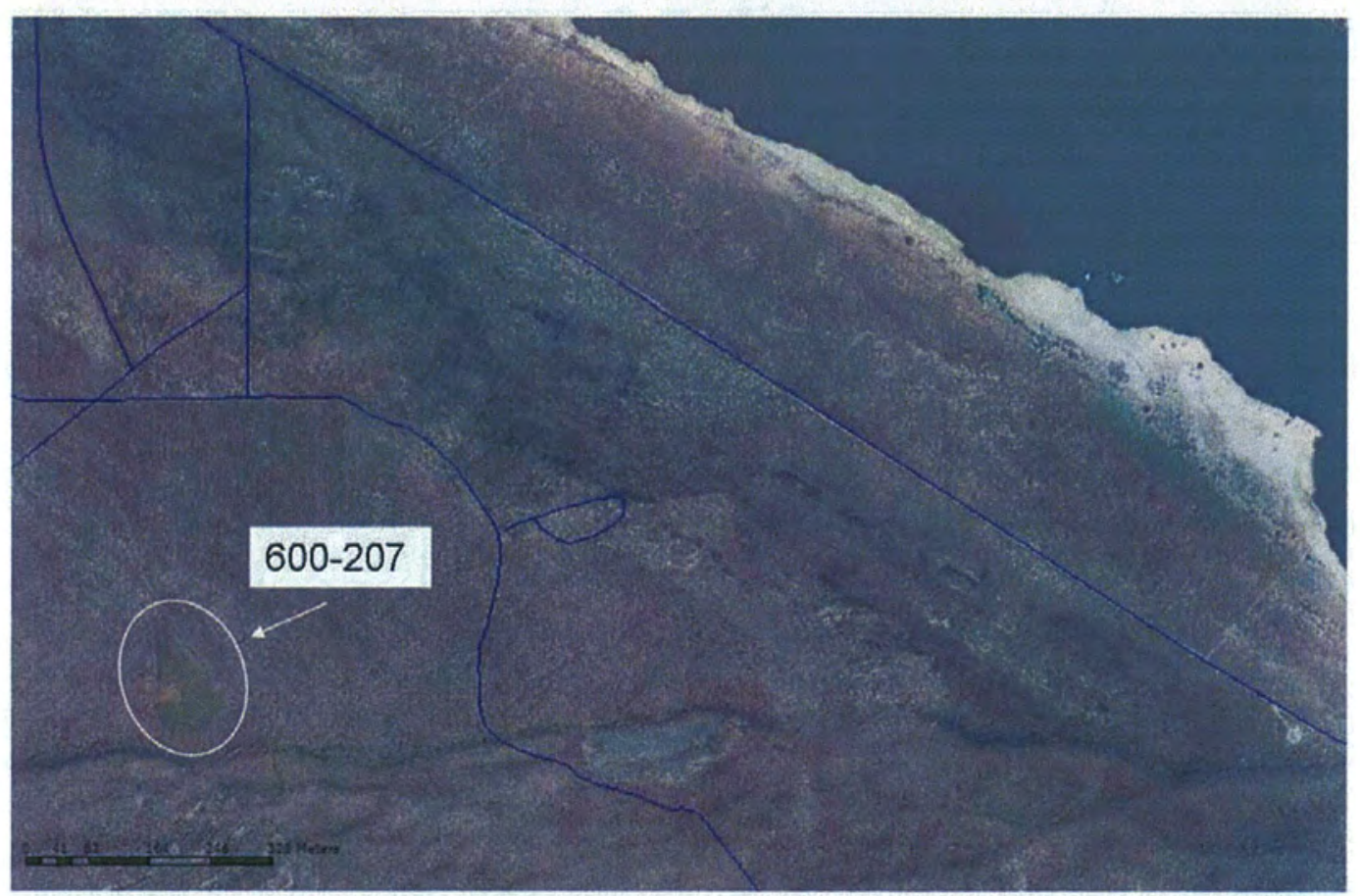


Figure B-15. 600-207 Coal Ash Site (View to South).

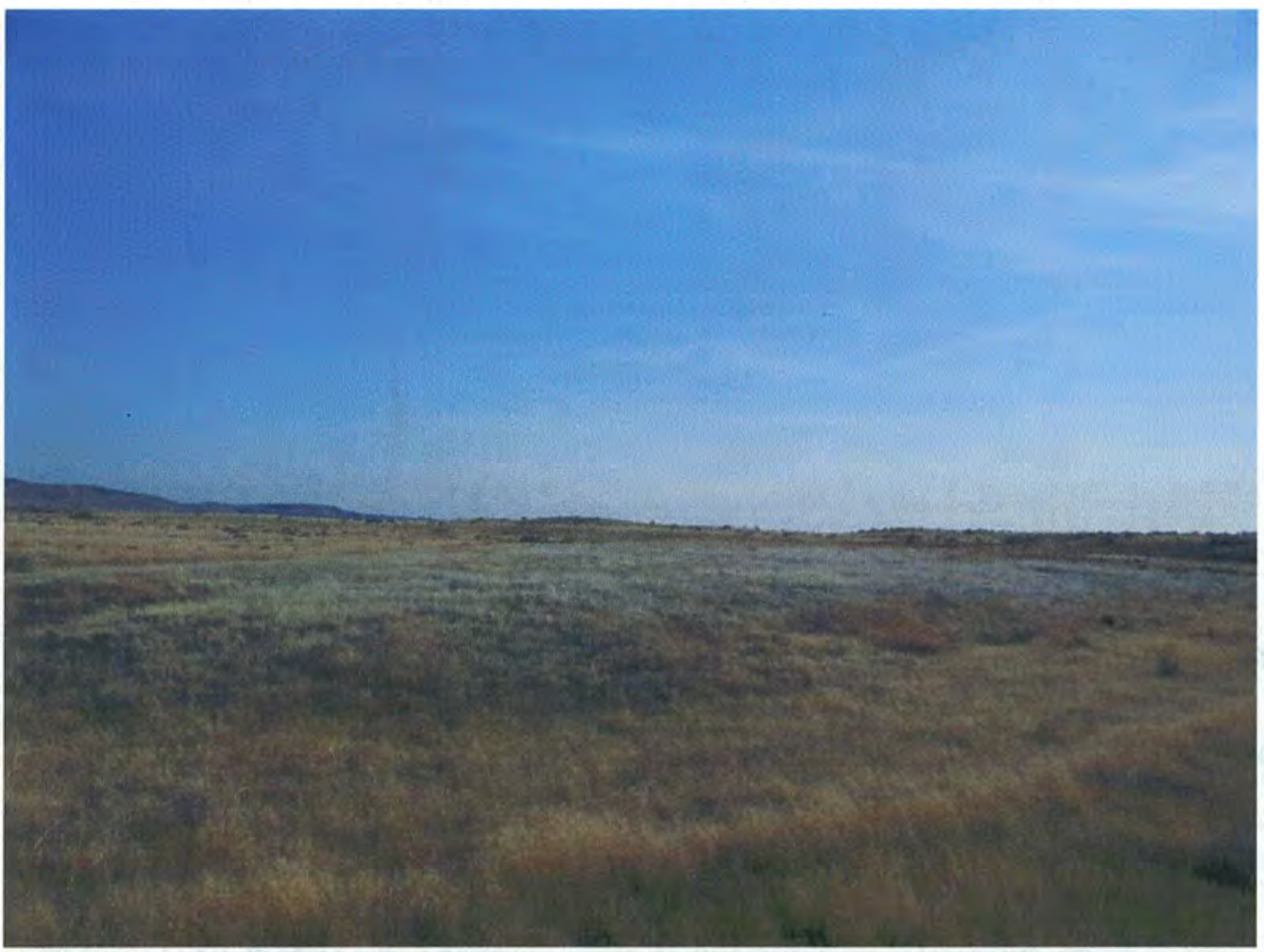

Figure B-16. Sample Location Within 600-207 Coal Ash Site.

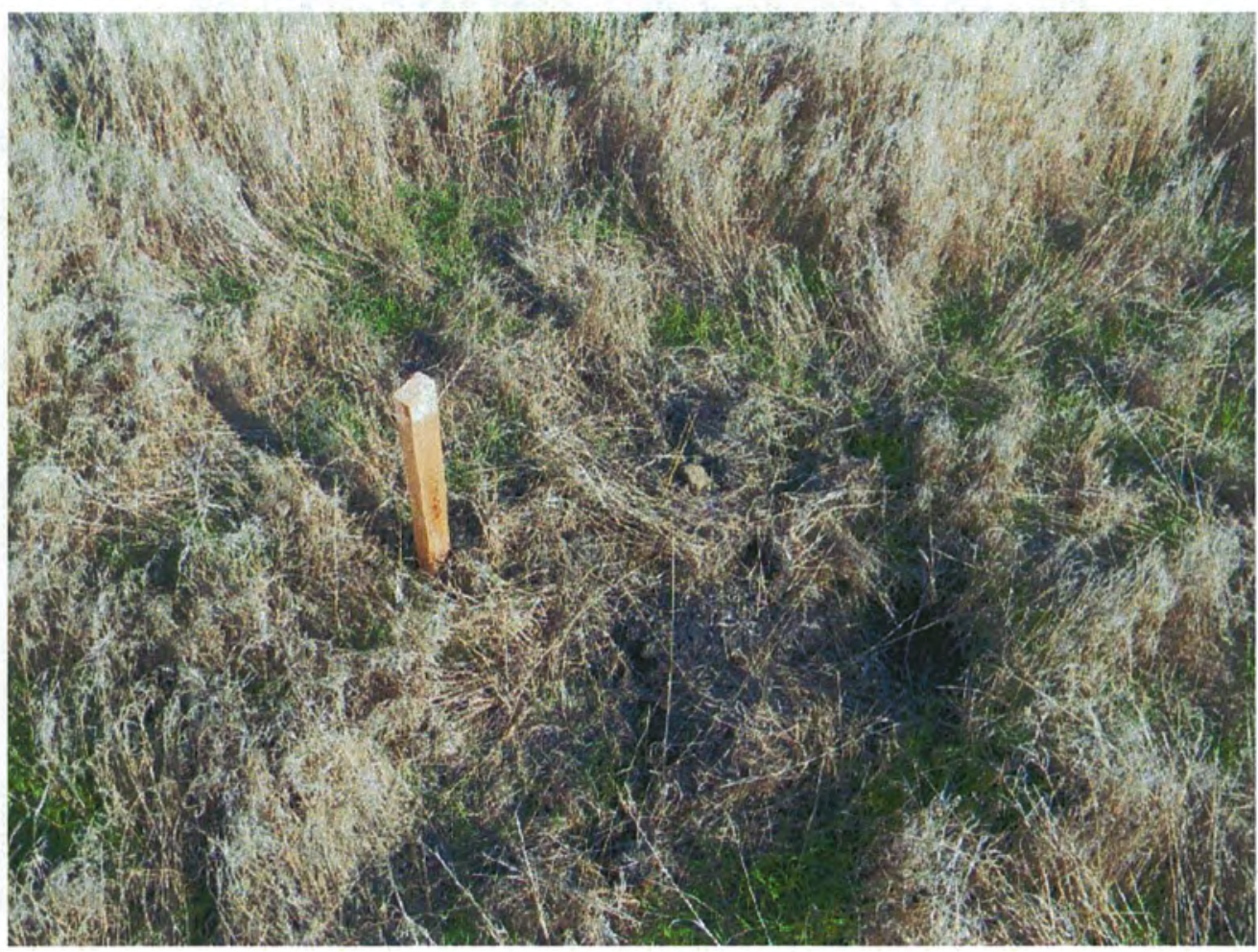




\section{APPENDIX C}

COAL ASH DATA TABLES 
WCH-506

Rev. 0 
Table C-1. 126-B-1 Coal Ash Sample Results. (5 Pages)

\begin{tabular}{|c|c|c|c|c|c|c|c|c|c|c|c|c|c|c|c|c|c|}
\hline \multirow{2}{*}{ Sample Location } & \multirow{2}{*}{$\begin{array}{c}\text { HEIS } \\
\text { Number }\end{array}$} & \multirow{2}{*}{$\begin{array}{c}\text { Sample } \\
\text { Date }\end{array}$} & \multicolumn{3}{|c|}{ Antimony } & \multicolumn{3}{|c|}{ Arsenic } & \multicolumn{3}{|c|}{ Barium } & \multicolumn{3}{|c|}{ Beryllium } & \multicolumn{3}{|c|}{ Boron } \\
\hline & & & $\mathrm{mg} / \mathrm{kg}$ & $\mathbf{Q}$ & PQL & $\mathrm{mg} / \mathrm{kg}$ & Q & PQL & $\mathrm{mg} / \mathrm{kg}$ & Q & $\mathrm{PQL}$ & $\mathrm{mg} / \mathrm{kg}$ & Q & PQL & $\mathrm{mg} / \mathrm{kg}$ & Q & PQL \\
\hline $100-\mathrm{B}-01$ & J1HHV4 & $4 / 27 / 11$ & 0.588 & $\mathrm{U}$ & 0.59 & 5.85 & & 0.98 & 1970 & & 0.49 & 1.93 & & 0.20 & 109 & & 1.96 \\
\hline 100-B-02 & J1HHV5 & $4 / 27 / 11$ & 0.265 & B & 0.52 & 4.21 & & 0.86 & 1660 & & 0.43 & 1.44 & & 0.17 & 88.4 & & 1.72 \\
\hline $100-\mathrm{B}-03$ & J1HHV6 & $4 / 27 / 11$ & 0.566 & $\mathrm{U}$ & 0.57 & 4.62 & & 0.94 & 1620 & & 0.47 & 1.21 & & 0.19 & 130 & & 1.89 \\
\hline 100-B-04 & J1HHV7 & $4 / 27 / 11$ & 0.588 & $\mathrm{U}$ & 0.59 & 2.41 & & 0.98 & 793 & & 0.49 & 1.15 & & 0.20 & 161 & & 1.96 \\
\hline 100-B-05 & J1HHV8 & $4 / 27 / 11$ & 0.304 & B & 0.57 & 4.46 & & 0.94 & 824 & & 0.47 & 1.23 & & 0.19 & 328 & & 1.89 \\
\hline $100-\mathrm{B}-06$ & J1HHV9 & $4 / 27 / 11$ & 0.566 & $\mathrm{U}$ & 0.57 & 5.37 & & 0.94 & 1450 & & 0.47 & 1.32 & & 0.19 & 108 & & 1.89 \\
\hline 100-B-07 & J1HHW0 & $4 / 27 / 11$ & 0.536 & U & 0.54 & 3.43 & & 0.89 & 1160 & & 0.45 & 1.16 & & 0.18 & 76.5 & & 1.79 \\
\hline 100-B-08 & J1HHW1 & $4 / 27 / 11$ & 0.517 & U & 0.52 & 4.76 & & 0.86 & 2060 & & 0.43 & 1.86 & & 0.17 & 277 & & 1.72 \\
\hline 100-B-09 & J1HHW2 & $8 / 23 / 11$ & 0.469 & $\mathrm{U}$ & 0.47 & 4.28 & & 0.78 & 1200 & & 0.39 & 1.48 & & 0.16 & 250 & & 1.56 \\
\hline $100-\mathrm{B}-10$ & J1HHW3 & $4 / 27 / 11$ & 0.566 & $\mathrm{U}$ & 0.57 & 2.98 & & 0.94 & 597 & & 0.47 & 1.03 & & 0.19 & 169 & & 1.89 \\
\hline 100-B-10 (Depth) & $\mathrm{J} 1 \mathrm{HJ} 10$ & $4 / 27 / 11$ & 0.556 & $\mathrm{U}$ & 0.56 & 2.87 & & 0.93 & 916 & & 0.46 & 1.18 & & 0.19 & 279 & & 1.85 \\
\hline $100-\mathrm{B}-11$ & J1HHW4 & $4 / 27 / 11$ & 0.577 & $\mathrm{U}$ & 0.58 & 3.20 & & 0.96 & 958 & & 0.48 & 1.56 & & 0.19 & 265 & & 1.92 \\
\hline 100-B-12 & J1HHW5 & $4 / 27 / 11$ & 0.577 & $\mathrm{U}$ & 0.58 & 2.63 & & 0.96 & 696 & & 0.48 & 1.54 & & 0.19 & 256 & & 1.92 \\
\hline 100-B-13 & J1HHW6 & $4 / 27 / 11$ & 0.505 & $B$ & 0.54 & 7.36 & & 0.89 & 2340 & & 0.45 & 2.39 & & 0.18 & 93.8 & & 1.79 \\
\hline $100-B-14$ & J1HHW7 & $8 / 23 / 11$ & 0.600 & $\mathrm{U}$ & 0.60 & 8.45 & & 1.00 & 1170 & & 0.50 & 1.24 & & 0.20 & 169 & & 2.00 \\
\hline 100-B-15 & J1HHW8 & $4 / 27 / 11$ & 0.545 & $\mathrm{U}$ & 0.55 & 3.01 & & 0.91 & 792 & & 0.46 & 1.17 & & 0.18 & 211 & & 1.82 \\
\hline $\begin{array}{c}\text { Duplicate of } \\
\text { J1HHW8 }\end{array}$ & J1HJ04 & $4 / 27 / 11$ & 1.76 & $U$ & 1.76 & 3.41 & & 2.94 & 753 & & 1.47 & 1.19 & & 0.59 & 240 & & 5.88 \\
\hline $100-B-16$ & J1HHW9 & $4 / 26 / 11$ & 0.376 & UJB & 0.46 & 4.07 & & 0.76 & 1360 & & 0.38 & 1.50 & & 0.15 & 129 & & 1.52 \\
\hline $100-B-17$ & $\mathrm{~J} 1 \mathrm{HHX0}$ & $4 / 27 / 11$ & 0.484 & UJ & 0.48 & 2.50 & & 0.81 & 556 & & 0.40 & 1.31 & & 0.16 & 269 & & 1.61 \\
\hline 100-B-17 (Depth) & J1HJ09 & $4 / 27 / 11$ & 0.469 & UJ & 0.47 & 2.50 & & 0.78 & 681 & & 0.39 & 1.68 & & 0.16 & 262 & & 1.56 \\
\hline 100-B-18 & $\mathrm{J} 1 \mathrm{HHX} 1$ & $4 / 27 / 11$ & 0.818 & UJB & 1.58 & 5.69 & & 2.63 & 599 & & 1.32 & 1.36 & & 0.53 & 156 & & 5.26 \\
\hline 100-B-19 & $\mathrm{J} 1 \mathrm{HHX} 2$ & $8 / 23 / 11$ & 0.469 & $\mathrm{U}$ & 0.47 & 2.74 & & 0.78 & 783 & & 0.39 & 1.16 & & 0.16 & 205 & & 1.56 \\
\hline $100-B-20$ & $\mathrm{~J} 1 \mathrm{HH} \times 3$ & $8 / 23 / 11$ & 0.588 & $\mathrm{U}$ & 0.59 & 3.4 & & 0.98 & 866 & & 0.49 & 1.22 & & 0.20 & 194 & & 1.96 \\
\hline 100-B-21 & $\mathrm{J} 1 \mathrm{HHX} 4$ & $4 / 25 / 11$ & 0.918 & UJB & 1.55 & 4.76 & & 2.59 & 406 & & 1.29 & 1.12 & & 0.52 & 145 & & 5.17 \\
\hline $100-\mathrm{B}-22$ & J1HHX5 & $4 / 26 / 11$ & 0.416 & UJB & 0.57 & 1.33 & & 0.94 & 184 & & 0.47 & 0.632 & & 0.19 & 66.3 & & 1.89 \\
\hline $100-B-23$ & $\mathrm{~J} 1 \mathrm{HHX6}$ & $4 / 26 / 11$ & 1.91 & $\mathrm{~J}$ & 1.70 & 9.59 & & 2.83 & 2250 & & 1.42 & 3.22 & & 0.57 & 327 & & 5.66 \\
\hline $100-B-24$ & $\mathrm{~J} 1 \mathrm{HHX7}$ & $4 / 26 / 11$ & 1.00 & UJB & 1.76 & 5.42 & & 2.94 & 1310 & & 1.47 & 1.60 & & 0.59 & 192 & & 5.88 \\
\hline $100-\mathrm{B}-25$ & $\mathrm{~J} 1 \mathrm{HHX} 8$ & $4 / 25 / 11$ & 1.69 & $\mathrm{BJ}$ & 1.70 & 10.3 & & 2.83 & 1420 & & 1.42 & 1.65 & & 0.57 & 107 & & 5.66 \\
\hline $\begin{array}{c}\text { Duplicate of } \\
\text { J1HHX8 }\end{array}$ & J1HJ03 & $4 / 25 / 11$ & 1.64 & $\mathrm{U}$ & 1.64 & 5.46 & & 2.73 & 907 & & 1.36 & 1.04 & & 0.55 & 85.5 & & 5.45 \\
\hline 100-B-25 (Depth) & $\mathrm{J1HJ08}$ & $4 / 26 / 11$ & 0.956 & UJB & 1.61 & 8.09 & & 2.68 & 1130 & & 1.34 & 1.14 & & 0.54 & 35.6 & & 5.36 \\
\hline $100-\mathrm{B}-26$ & J1HHX9 & $8 / 23 / 11$ & 0.441 & $\mathrm{U}$ & 0.44 & 3.14 & & 0.74 & 1000 & & 0.37 & 1.06 & & 0.15 & 174 & & 1.47 \\
\hline $100-B-27$ & $\mathrm{~J} 1 \mathrm{HJ} 00$ & $4 / 27 / 11$ & 1.67 & $\mathrm{U}$ & 1.67 & 3.19 & & 2.78 & 497 & & 1.39 & 0.894 & & 0.56 & 92.6 & & 5.56 \\
\hline $100-B-28$ & $\mathrm{~J} 1 \mathrm{HJ} 01$ & $8 / 23 / 11$ & 0.259 & B & 0.48 & 4.16 & & 0.79 & 969 & & 0.40 & 1.18 & & 0.16 & 267 & & 1.59 \\
\hline $100-B-29$ & $\mathrm{~J} 1 \mathrm{HJ} 02$ & $8 / 23 / 11$ & 0.550 & & 0.44 & 5.94 & & 0.73 & 1360 & & 0.36 & 1.98 & & 0.15 & 68.1 & & 1.45 \\
\hline Equipment Blank & J1HJ05 & 4/25/11 & 0.429 & $U$ & 0.43 & 0.302 & $B$ & 0.71 & 1.94 & & 0.36 & 0.054 & B & 0.14 & 1.43 & $U$ & 1.43 \\
\hline
\end{tabular}


Table C-1. 126-B-1 Coal Ash Sample Results. (5 Pages)

\begin{tabular}{|c|c|c|c|c|c|c|c|c|c|c|c|c|c|c|c|c|c|}
\hline \multirow{2}{*}{ Sample Location } & \multirow{2}{*}{$\begin{array}{c}\text { HEIS } \\
\text { Number }\end{array}$} & \multirow{2}{*}{$\begin{array}{c}\text { Sample } \\
\text { Date }\end{array}$} & \multicolumn{3}{|c|}{ Cadmium } & \multicolumn{3}{|c|}{ Chromium } & \multicolumn{3}{|c|}{ Cobalt } & \multicolumn{3}{|c|}{$\begin{array}{l}\text { Copper } \\
\end{array}$} & \multicolumn{3}{|c|}{ Lead } \\
\hline & & & $\mathrm{mg} / \mathrm{kg}$ & $\mathbf{Q}$ & $\mathrm{PQL}$ & $\mathrm{mg} / \mathrm{kg}$ & Q & $\mathrm{PQL}$ & $\mathrm{mg} / \mathrm{kg}$ & $\mathbf{Q}$ & $\mathrm{PQL}$ & $\mathrm{mg} / \mathrm{kg}$ & $\mathbf{Q}$ & $\mathrm{PQL}$ & $\mathrm{mg} / \mathrm{kg}$ & $\mathbf{Q}$ & PQL \\
\hline 100-B-01 & J1HHV4 & $4 / 27 / 11$ & 0.198 & & 0.20 & 8.33 & & 0.20 & 8.22 & & 1.96 & 39.2 & & 0.98 & 2.89 & & 0.49 \\
\hline $100-B-02$ & J1HHV5 & $4 / 27 / 11$ & 0.311 & & 0.17 & 8.03 & & 0.17 & 4.49 & & 1.72 & 22.7 & & 0.86 & 4.19 & & 0.43 \\
\hline 100-B-03 & J1HHV6 & 4/27/11 & 0.293 & & 0.19 & 9.87 & & 0.19 & 6.47 & & 1.9 & 32.0 & & 0.9 & 4.86 & & 0.47 \\
\hline $100-B-04$ & J1HHV7 & $4 / 27 / 11$ & 0.175 & $B$ & 0.20 & 8.46 & & 0.20 & 4.68 & & 2.0 & 35.4 & & 1.0 & 35.8 & & 0.49 \\
\hline 100-B-05 & J1HHV8 & $4 / 27 / 11$ & 0.506 & & 0.19 & 16.2 & & 0.19 & 4.12 & & 1.9 & 98.3 & & 0.9 & 13.5 & & 0.47 \\
\hline 100-B-06 & J1HHV9 & 4/27/11 & 0.243 & & 0.19 & 9.83 & & 0.19 & 7.07 & & 1.9 & 28.6 & & 0.9 & 3.84 & & 0.47 \\
\hline 100-B-07 & J1HHW0 & $4 / 27 / 11$ & 0.224 & & 0.18 & 8.47 & & 0.18 & 5.31 & & 1.8 & 24.6 & & 0.9 & 5.55 & & 0.45 \\
\hline $100-B-08$ & J1HHW1 & $4 / 27 / 11$ & 0.215 & & 0.17 & 8.17 & & 0.17 & 7.01 & & 1.72 & 39.5 & & 0.86 & 2.85 & & 0.43 \\
\hline 100-B-09 & J1HHW2 & $8 / 23 / 11$ & 0.205 & & 0.16 & 12.5 & & 0.16 & 5.41 & & 1.56 & 32.4 & & 0.78 & 6.08 & & 0.39 \\
\hline $100-\mathrm{B}-10$ & J1HHW3 & $4 / 27 / 11$ & 0.117 & $\mathrm{~B}$ & 0.19 & 8.75 & & 0.19 & 4.42 & & 1.89 & 28.8 & & 0.94 & 5.59 & & 0.47 \\
\hline 100-B-10 (Depth) & $\mathrm{J} 1 \mathrm{HJ} 10$ & $4 / 27 / 11$ & 0.239 & & 0.19 & 7.34 & & 0.19 & 3.44 & & 1.85 & 21.0 & & 0.93 & 11.8 & & 0.46 \\
\hline $100-B-11$ & J1HHW4 & 4/27/11 & 0.221 & & 0.19 & 12.9 & & 0.19 & 3.10 & & 1.92 & 23.7 & & 0.96 & 6.05 & & 0.48 \\
\hline $100-B-12$ & J1HHW5 & $4 / 27 / 11$ & 0.167 & B & 0.19 & 12.7 & & 0.19 & 2.98 & & 1.92 & 22.2 & & 0.96 & 5.80 & & 0.48 \\
\hline $100-B-13$ & J1HHW6 & $4 / 27 / 11$ & 0.518 & & 0.18 & 8.91 & & 0.18 & 4.87 & & 1.79 & 25.4 & & 0.89 & 3.72 & & 0.45 \\
\hline $100-B-14$ & J1HHW7 & $8 / 23 / 11$ & 0.360 & & 0.20 & 11.5 & & 0.20 & 4.59 & & 2.00 & 27.3 & & 1.00 & 7.72 & & 0.50 \\
\hline 100-B-15 & J1HHW8 & $4 / 27 / 11$ & 0.182 & $B$ & 0.18 & 10.3 & & 0.18 & 4.88 & & 1.82 & 27.4 & & 0.91 & 6.73 & & 0.46 \\
\hline $\begin{array}{c}\text { Duplicate of } \\
\text { J1HHW8 }\end{array}$ & $\mathrm{J} 1 \mathrm{HJO4}$ & $4 / 27 / 11$ & 0.240 & B & 0.59 & 11.3 & & 0.59 & 4.56 & $B$ & 5.88 & 26.8 & & 2.94 & 7.10 & & 1.47 \\
\hline $100-B-16$ & J1HHW9 & 4/26/11 & 0.182 & & 0.15 & 6.74 & & 0.15 & 3.55 & & 1.52 & 19.1 & & 0.76 & 3.18 & & 0.38 \\
\hline $100-\mathrm{B}-17$ & $\mathrm{~J} 1 \mathrm{HHX0}$ & $4 / 27 / 11$ & 0.221 & & 0.16 & 10.6 & & 0.16 & 2.51 & & 1.61 & 21.9 & & 0.81 & 6.28 & & 0.40 \\
\hline 100-B-17 (Depth) & J1HJ09 & $4 / 27 / 11$ & 0.135 & B & 0.16 & 13.3 & & 0.16 & 2.64 & & 1.56 & 19.6 & & 0.78 & 4.33 & & 0.39 \\
\hline $100-\mathrm{B}-18$ & $\mathrm{~J} 1 \mathrm{HHX} 1$ & $4 / 27 / 11$ & 0.337 & $\mathrm{~B}$ & 0.53 & 10.5 & & 0.53 & 3.97 & $\mathrm{~B}$ & 5.26 & 25.6 & & 2.63 & 6.58 & & 1.32 \\
\hline 100-B-19 & J1HHX2 & $8 / 23 / 11$ & 0.265 & & 0.16 & 9.66 & & 0.16 & 2.79 & & 1.56 & 20.9 & & 0.78 & 10.7 & & 0.39 \\
\hline $100-B-20$ & $\mathrm{J1HHX3}$ & $8 / 23 / 11$ & 0.187 & B & 0.20 & 11.1 & & 0.20 & 4.88 & & 1.96 & 29.1 & & 0.98 & 5.77 & & 0.49 \\
\hline $100-B-21$ & $\mathrm{J1HHX4}$ & $4 / 25 / 11$ & 0.914 & & 0.52 & 14.4 & & 0.52 & 3.06 & B & 5.17 & 25.0 & & 2.59 & 18.4 & & 1.29 \\
\hline $100-B-22$ & $\mathrm{~J} 1 \mathrm{HHX5}$ & $4 / 26 / 11$ & 0.096 & B & 0.19 & 4.76 & & 0.19 & 1.00 & $\mathrm{~B}$ & 1.89 & 8.6 & & 0.94 & 6.50 & & 0.47 \\
\hline $100-B-23$ & $\mathrm{J1HHX6}$ & $4 / 26 / 11$ & 0.899 & & 0.57 & 25.5 & & 0.57 & 15.1 & & 5.66 & 94.3 & & 2.83 & 19.0 & & 1.42 \\
\hline $100-B-24$ & $\mathrm{~J} 1 \mathrm{HHX7}$ & $4 / 26 / 11$ & 0.219 & B & 0.59 & 13.3 & & 0.59 & 5.84 & $\mathrm{~B}$ & 5.88 & 36.7 & & 2.94 & 10.6 & & 1.47 \\
\hline 100-B-25 & $\mathrm{J} 1 \mathrm{HHX8}$ & $4 / 25 / 11$ & 0.385 & $\mathrm{~B}$ & 0.57 & 11.2 & & 0.57 & 4.66 & $\mathrm{~B}$ & 5.66 & 28.4 & & 2.83 & 13.4 & & 1.42 \\
\hline $\begin{array}{c}\text { Duplicate of } \\
\text { J1HHX8 }\end{array}$ & J1HJ03 & $4 / 25 / 11$ & 0.278 & B & 0.55 & 8.34 & & 0.55 & 4.12 & B & 5.45 & 20.2 & & 2.73 & 9.95 & & 1.36 \\
\hline 100-B-25 (Depth) & J1HJ08 & $4 / 26 / 11$ & 0.202 & B & 0.54 & 10.6 & & 0.54 & 5.12 & B & 5.36 & 26.2 & & 2.68 & 4.91 & & 1.34 \\
\hline $100-\mathrm{B}-26$ & J1HHX9 & $8 / 23 / 11$ & 0.354 & & 0.15 & 12.3 & & 0.15 & 2.18 & & 1.47 & 17.7 & & 0.74 & 12.2 & & 0.37 \\
\hline 100-B-27 & $\mathrm{J} 1 \mathrm{HJO0}$ & 4/27/11 & 0.397 & $B$ & 0.56 & 10.4 & & 0.56 & 5.56 & $\mathrm{U}$ & 5.56 & 22.0 & & 2.78 & 9.69 & & 1.39 \\
\hline $100-B-28$ & $\mathrm{~J} 1 \mathrm{HJO1}$ & $8 / 23 / 11$ & 0.373 & & 0.16 & 13.2 & & 0.16 & 2.69 & & 1.59 & 26.2 & & 0.79 & 15.9 & & 0.40 \\
\hline $100-\mathrm{B}-29$ & $\mathrm{~J} 1 \mathrm{HJO2}$ & $8 / 23 / 11$ & 0.251 & & 0.15 & 7.66 & & 0.15 & 4.02 & & 1.45 & 22.8 & & 0.73 & 3.87 & & 0.36 \\
\hline Equipment Blank & $\mathrm{J} 1 \mathrm{HJ} 05$ & $4 / 25 / 11$ & 0.143 & U & 0.14 & 0.206 & & 0.14 & 1.43 & U & 1.43 & 0.714 & U & 0.71 & 0.405 & & 0.36 \\
\hline
\end{tabular}


Table C-1. 126-B-1 Coal Ash Sample Results. (5 Pages)

\begin{tabular}{|c|c|c|c|c|c|c|c|c|c|c|c|c|c|c|c|c|c|}
\hline \multirow{2}{*}{ Sample Location } & \multirow{2}{*}{$\begin{array}{c}\text { HEIS } \\
\text { Number }\end{array}$} & \multirow{2}{*}{$\begin{array}{c}\text { Sample } \\
\text { Date }\end{array}$} & \multicolumn{3}{|c|}{ Manganese } & \multicolumn{3}{|c|}{ Mercury } & \multicolumn{3}{|c|}{ Molybdenum } & \multicolumn{3}{|c|}{ Nickel } & \multicolumn{3}{|c|}{ Selenium } \\
\hline & & & $\mathrm{mg} / \mathrm{kg}$ & Q & PQL & $\mathrm{mg} / \mathrm{kg}$ & $\mathbf{Q}$ & PQL & $\mathrm{mg} / \mathrm{kg}$ & $\mathbf{Q}$ & PQL & $\mathrm{mg} / \mathrm{kg}$ & $\mathbf{Q}$ & PQL & $\mathrm{mg} / \mathrm{kg}$ & $\mathbf{Q}$ & PQL \\
\hline 100-B-01 & J1HHV4 & $4 / 27 / 11$ & 335 & & 4.90 & 0.072 & & 0.03 & 2.40 & & 1.96 & 11.8 & & 3.92 & 0.294 & $\bar{U}$ & 0.29 \\
\hline $100-\mathrm{B}-02$ & J1HHV5 & $4 / 27 / 11$ & 296 & & 4.31 & 0.057 & & 0.02 & 1.72 & B & 1.72 & 9.09 & & 3.45 & 0.264 & & 0.26 \\
\hline 100-B-03 & J1HHV6 & $4 / 27 / 11$ & 396 & & 4.72 & 0.060 & & 0.03 & 1.47 & $\mathrm{~B}$ & 1.89 & 15.1 & & 3.77 & 0.283 & $\mathrm{U}$ & 0.28 \\
\hline 100-B-04 & J1HHV7 & $4 / 27 / 11$ & 94.3 & & 4.90 & 0.062 & & 0.03 & 1.22 & B & 1.96 & 9.93 & & 3.92 & 0.332 & & 0.29 \\
\hline $100-B-05$ & J1HHV8 & $4 / 27 / 11$ & 199 & & 4.72 & 0.861 & & 0.02 & 2.88 & & 1.89 & 15.0 & & 3.77 & 0.479 & & 0.28 \\
\hline $100-\mathrm{B}-06$ & J1HHV9 & $4 / 27 / 11$ & 348 & & 4.72 & 0.064 & & 0.03 & 34.7 & & 1.89 & 12.3 & & 3.77 & 0.497 & & 0.28 \\
\hline $100-\mathrm{B}-07$ & J1HHW0 & 4/27/11 & 218 & & 4.46 & 0.065 & & 0.03 & 1.16 & B & 1.79 & 10.5 & & 3.57 & 0.268 & $U$ & 0.27 \\
\hline $100-B-08$ & J1HHW1 & $4 / 27 / 11$ & 213 & & 4.31 & 0.046 & & 0.03 & 2.51 & & 1.72 & 15.8 & & 3.45 & 0.329 & & 0.26 \\
\hline 100-B-09 & J1HHW2 & $8 / 23 / 11$ & 157 & & 3.91 & 0.292 & & 0.02 & 2.26 & & 1.56 & 13.0 & & 3.12 & 0.495 & & 0.23 \\
\hline $100-\mathrm{B}-10$ & J1HHW3 & $4 / 27 / 11$ & 104 & & 4.72 & 0.049 & & 0.03 & 1.08 & $\mathrm{~B}$ & 1.89 & 9.93 & & 3.77 & 0.618 & & 0.28 \\
\hline 100-B-10 (Depth) & $\mathrm{J1HJ10}$ & $4 / 27 / 11$ & 110 & & 4.63 & 0.042 & & 0.03 & 1.40 & $B$ & 1.85 & 7.29 & & 3.70 & 0.278 & $\mathrm{U}$ & 0.28 \\
\hline $100-\mathrm{B}-11$ & J1HHW4 & $4 / 27 / 11$ & 68.8 & & 4.81 & 0.059 & & 0.02 & 1.42 & B & 1.92 & 8.27 & & 3.85 & 0.323 & & 0.29 \\
\hline $100-B-12$ & J1HHW5 & $4 / 27 / 11$ & 60.9 & & 4.81 & 0.062 & & 0.03 & 1.34 & B & 1.92 & 8.38 & & 3.85 & 0.409 & & 0.29 \\
\hline $100-B-13$ & J1HHW6 & $4 / 27 / 11$ & 521 & & 4.46 & 0.075 & & 0.03 & 2.21 & & 1.79 & 9.05 & & 3.57 & 0.414 & & 0.27 \\
\hline $100-B-14$ & J1HHW7 & $8 / 23 / 11$ & 182 & & 5.00 & 0.196 & & 0.03 & 1.63 & $B$ & 2.00 & 10.6 & & 4.00 & 1.26 & & 0.30 \\
\hline 100-B-15 & J1HHW8 & $4 / 27 / 11$ & 94.8 & & 4.55 & 0.060 & & 0.03 & 1.59 & $\mathrm{~B}$ & 1.82 & 10.3 & & 3.64 & 0.443 & & 0.27 \\
\hline $\begin{array}{c}\text { Duplicate of } \\
\text { J1HHW8 }\end{array}$ & $\mathrm{J} 1 \mathrm{HJO4}$ & $4 / 27 / 11$ & 107 & & 14.7 & 0.077 & & 0.03 & 1.42 & B & 5.88 & 10.3 & B & 11.8 & 0.882 & $\mathrm{U}$ & 0.88 \\
\hline 100-B-16 & J1HHW9 & $4 / 26 / 11$ & 283 & & 3.79 & 0.022 & B & 0.03 & 1.33 & B & 1.52 & 7.12 & & 3.03 & 0.361 & & 0.23 \\
\hline $100-B-17$ & $\mathrm{~J} 1 \mathrm{HHX0}$ & $4 / 27 / 11$ & 57.5 & & 4.03 & 0.053 & & 0.03 & 1.17 & B & 1.61 & 7.02 & & 3.23 & 0.331 & & 0.24 \\
\hline 100-B-17 (Depth) & J1HJ09 & $4 / 27 / 11$ & 38.5 & & 3.91 & 0.080 & & 0.03 & 1.31 & $\mathrm{~B}$ & 1.56 & 7.67 & & 3.12 & 0.367 & & 0.23 \\
\hline 100-B-18 & $\mathrm{J} 1 \mathrm{HHX1}$ & $4 / 27 / 11$ & 145 & & 13.2 & 0.037 & & 0.03 & 1.47 & B & 5.26 & 9.39 & $\mathrm{~B}$ & 10.5 & 0.789 & $\mathrm{U}$ & 0.79 \\
\hline 100-B-19 & $\mathrm{J} 1 \mathrm{HHX} 2$ & $8 / 23 / 11$ & 109 & & 3.9 & 0.080 & & 0.02 & 1.20 & B & 1.56 & 7.20 & & 3.12 & 0.798 & & 0.23 \\
\hline $100-B-20$ & $\mathrm{~J} 1 \mathrm{HH} \times 3$ & $8 / 23 / 11$ & 102 & & 4.9 & 0.200 & & 0.03 & 1.85 & B & 1.96 & 11.2 & & 3.92 & 0.648 & & 0.29 \\
\hline 100-B-21 & $\mathrm{J} 1 \mathrm{HHX} 4$ & $4 / 25 / 11$ & 116 & & 12.9 & 0.338 & & 0.03 & 0.752 & B & 5.17 & 9.09 & $B$ & 10.3 & 2.00 & & 0.78 \\
\hline 100-B-22 & $\mathrm{J} 1 \mathrm{HH} \times 5$ & $4 / 26 / 11$ & 13.1 & & 4.72 & 0.043 & & 0.03 & 0.314 & B & 1.89 & 3.02 & $B$ & 3.77 & 0.664 & & 0.28 \\
\hline 100-B-23 & $\mathrm{J} 1 \mathrm{HH} \times 6$ & $4 / 26 / 11$ & 344 & & 14.2 & 0.146 & & 0.03 & 3.85 & B & 5.66 & 31.5 & & 11.3 & 1.36 & & 0.85 \\
\hline 100-B-24 & $\mathrm{J} 1 \mathrm{HHX7}$ & $4 / 26 / 11$ & 159 & & 14.7 & 0.087 & & 0.03 & 1.67 & B & 5.88 & 13.8 & & 11.8 & 0.882 & $U$ & 0.88 \\
\hline $100-\mathrm{B}-25$ & $\mathrm{~J} 1 \mathrm{HHX} 8$ & $4 / 25 / 11$ & 391 & & 14.2 & 0.115 & & 0.03 & 1.14 & $B$ & 5.66 & 10.5 & $B$ & 11.3 & 1.58 & & 0.85 \\
\hline $\begin{array}{c}\text { Duplicate of } \\
\text { J1HHX8 }\end{array}$ & J1HJ03 & $4 / 25 / 11$ & 202 & & 13.6 & 0.120 & & 0.03 & 0.972 & B & 5.45 & 9.70 & B & 10.9 & 1.03 & & 0.82 \\
\hline 100-B-25 (Depth) & $\mathrm{J1HJ08}$ & $4 / 26 / 11$ & 187 & & 13.4 & 0.026 & $B$ & 0.03 & 1.59 & $B$ & 5.36 & 11.2 & & 10.7 & 0.804 & $U$ & 0.80 \\
\hline $100-\mathrm{B}-26$ & J1HHX9 & $8 / 23 / 11$ & 71.2 & & 3.7 & 0.092 & & 0.03 & 0.734 & B & 1.47 & 6.63 & & 2.94 & 1.22 & & 0.22 \\
\hline 100-B-27 & $\mathrm{J1HJ00}$ & $4 / 27 / 11$ & 85.0 & & 13.9 & 0.235 & & 0.03 & 0.654 & B & 5.56 & 6.92 & B & 11.1 & 0.876 & & 0.83 \\
\hline $100-\mathrm{B}-28$ & $\mathrm{J1HJ01}$ & $8 / 23 / 11$ & 83.5 & & 4.0 & 0.195 & & 0.03 & 1.10 & $\mathrm{~B}$ & 1.59 & 8.14 & & 3.17 & 1.44 & & 0.24 \\
\hline $100-B-29$ & $\mathrm{~J} 1 \mathrm{HJ} 02$ & $8 / 23 / 11$ & 358 & & 3.6 & 0.052 & & 0.03 & 1.69 & & 1.45 & 7.70 & & 2.90 & 0.476 & & 0.22 \\
\hline Equipment Blank & $\mathrm{J1HJ05}$ & $4 / 25 / 11$ & 5.24 & & 3.57 & 0.025 & $\mathrm{U}$ & 0.03 & 1.43 & U & 1.43 & 2.86 & $\mathrm{U}$ & 2.86 & 0.214 & $U$ & 0.21 \\
\hline
\end{tabular}


Table C-1. 126-B-1 Coal Ash Sample Results. (5 Pages)

\begin{tabular}{|c|c|c|c|c|c|c|c|c|c|c|c|c|c|c|c|c|c|}
\hline \multirow{2}{*}{ Sample Location } & \multirow{2}{*}{$\begin{array}{c}\text { HEIS } \\
\text { Number }\end{array}$} & \multirow{2}{*}{$\begin{array}{c}\text { Sample } \\
\text { Date }\end{array}$} & \multicolumn{3}{|c|}{ Silver } & \multicolumn{3}{|c|}{ Thallium } & \multicolumn{3}{|c|}{ Uranium (KPA) } & \multicolumn{3}{|c|}{ Vanadium } & \multicolumn{3}{|c|}{ Zinc } \\
\hline & & & $\mathrm{mg} / \mathrm{kg}$ & $\mathbf{Q}$ & PQL & $\mathrm{mg} / \mathrm{kg}$ & $\mathbf{Q}$ & PQL & ug/g & $\mathbf{Q}$ & MDA & $\mathrm{mg} / \mathrm{kg}$ & $\mathbf{Q}$ & PQL & $\mathrm{mg} / \mathrm{kg}$ & $\mathbf{Q}$ & PQL \\
\hline $100-\mathrm{B}-01$ & J1HHV4 & $4 / 27 / 11$ & 0.196 & $\mathrm{U}$ & 0.20 & 0.490 & $\mathrm{U}$ & 0.490 & & & & 64.0 & & 2.45 & 20.4 & & 9.80 \\
\hline $100-B-02$ & J1HHV5 & $4 / 27 / 11$ & 0.172 & $\mathrm{U}$ & 0.17 & 0.431 & $U$ & 0.431 & & & & 30.6 & & 2.16 & 41.8 & & 8.62 \\
\hline $100-\mathrm{B}-03$ & J1HHV6 & $4 / 27 / 11$ & 0.189 & $\mathrm{U}$ & 0.19 & 0.472 & $\mathrm{U}$ & 0.472 & & & & 48.0 & & 2.36 & 55.5 & & 9.43 \\
\hline 100-B-04 & J1HHV7 & $4 / 27 / 11$ & 0.196 & $\mathrm{U}$ & 0.20 & 0.490 & $U$ & 0.490 & & & & 37.7 & & 2.45 & 38.9 & & 9.80 \\
\hline 100-B-05 & J1HHV8 & $4 / 27 / 11$ & 0.189 & $\mathrm{U}$ & 0.19 & 0.472 & $\mathrm{U}$ & 0.472 & & & & 33.4 & & 2.36 & 80.7 & & 9.43 \\
\hline $100-B-06$ & J1HHV9 & $4 / 27 / 11$ & 0.189 & $\mathrm{U}$ & 0.19 & 0.472 & $\mathrm{U}$ & 0.472 & & & & 48.7 & & 2.36 & 36.0 & & 9.43 \\
\hline 100-B-07 & J1HHW0 & $4 / 27 / 11$ & 0.179 & $\mathrm{U}$ & 0.18 & 0.446 & $\mathrm{U}$ & 0.446 & & & & 38.4 & & 2.23 & 34.1 & & 8.93 \\
\hline 100-B-08 & J1HHW1 & $4 / 27 / 11$ & 0.172 & $\mathrm{U}$ & 0.17 & 0.431 & $\mathrm{U}$ & 0.431 & & & & 54.1 & & 2.16 & 23.7 & & 8.62 \\
\hline $100-B-9$ & J1HHW2 & $8 / 23 / 11$ & 0.156 & $U$ & 0.16 & 0.391 & $U$ & 0.390 & & & & 43.7 & & 1.95 & 42.5 & & 7.81 \\
\hline 100-B-10 & J1HHW3 & $4 / 27 / 11$ & 0.189 & $\mathrm{U}$ & 0.19 & 0.472 & $\mathrm{U}$ & 0.472 & 2.67 & & 0.123 & 39.3 & & 2.36 & 13.6 & & 9.43 \\
\hline 100-B-10 (Depth) & $\mathrm{J} 1 \mathrm{HJ} 10$ & $4 / 27 / 11$ & 0.185 & $U$ & 0.19 & 0.463 & $\mathrm{U}$ & 0.463 & 4.28 & & 0.123 & 28.7 & & 2.31 & 29.7 & & 9.26 \\
\hline $100-B-11$ & J1HHW4 & $4 / 27 / 11$ & 0.192 & $\mathrm{U}$ & 0.19 & 0.481 & $U$ & 0.481 & & & & 32.1 & & 2.40 & 26.8 & & 9.62 \\
\hline $100-B-12$ & J1HHW5 & $4 / 27 / 11$ & 0.192 & $\mathrm{U}$ & 0.19 & 0.481 & $U$ & 0.481 & & & & 30.7 & & 2.40 & 29.8 & & 9.62 \\
\hline $100-B-13$ & J1HHW6 & $4 / 27 / 11$ & 0.179 & $\mathrm{U}$ & 0.18 & 0.446 & $\mathrm{U}$ & 0.446 & & & & 32.5 & & 2.23 & 54.5 & & 8.93 \\
\hline $100-B-14$ & J1HHW7 & $8 / 23 / 11$ & 0.200 & $\mathrm{U}$ & 0.20 & 0.500 & $\mathrm{U}$ & 0.500 & & & & 37.0 & & 2.50 & 47.6 & & 10.00 \\
\hline $100-B-15$ & J1HHW8 & $4 / 27 / 11$ & 0.182 & $\mathrm{U}$ & 0.18 & 0.455 & $\mathrm{U}$ & 0.455 & & & & 35.7 & & 2.27 & 26.9 & & 9.09 \\
\hline $\begin{array}{c}\text { Duplicate of } \\
\text { J1HHW8 }\end{array}$ & J1HJ04 & $4 / 27 / 11$ & 0.588 & $U$ & 0.59 & 1.47 & $U$ & 1.47 & & & & 37.4 & & 7.35 & 48.6 & & 29.4 \\
\hline $100-B-16$ & J1HHW9 & $4 / 26 / 11$ & 0.152 & $U$ & 0.15 & 0.379 & $\mathrm{U}$ & 0.379 & & & & 24.6 & & 1.89 & 24.6 & & 7.58 \\
\hline $100-\mathrm{B}-17$ & $\mathrm{~J} 1 \mathrm{HHX0}$ & $4 / 27 / 11$ & 0.161 & $\mathrm{U}$ & 0.16 & 0.403 & $\mathrm{U}$ & 0.403 & 5.39 & & 0.128 & 27.3 & & 2.02 & 32.4 & & 8.06 \\
\hline 100-B-17 (Depth) & J1HJ09 & $4 / 27 / 11$ & 0.156 & $\mathrm{U}$ & 0.16 & 0.391 & $U$ & 0.391 & 6.75 & & 0.128 & 29.2 & & 1.95 & 15.1 & & 7.81 \\
\hline $100-B-18$ & J1HHX1 & $4 / 27 / 11$ & 0.526 & $U$ & 0.53 & 1.32 & $U$ & 1.32 & & & & 31.4 & & 6.58 & 37.3 & & 26.3 \\
\hline $100-B-19$ & $\mathrm{~J} 1 \mathrm{HHX} 2$ & $8 / 23 / 11$ & 0.156 & $\mathrm{U}$ & 0.16 & 0.391 & $U$ & 0.390 & & & & 28.3 & & 1.95 & 36.3 & & 7.8 \\
\hline $100-B-20$ & $\mathrm{~J} 1 \mathrm{HHX} 3$ & $8 / 23 / 11$ & 0.196 & $\mathrm{U}$ & 0.20 & 0.490 & $\mathrm{U}$ & 0.490 & & & & 38.2 & & 2.45 & 21.8 & & 9.8 \\
\hline $100-B-21$ & $\mathrm{~J} 1 \mathrm{HHX} 4$ & $4 / 25 / 11$ & 0.517 & $\mathrm{U}$ & 0.52 & 1.29 & $\mathrm{U}$ & 1.29 & & & & 29.3 & & 6.47 & 104 & & 25.9 \\
\hline $100-B-22$ & $\mathrm{J1HHX5}$ & $4 / 26 / 11$ & 0.189 & $\mathrm{U}$ & 0.19 & 0.472 & $\mathrm{U}$ & 0.472 & & & & 9.73 & & 2.36 & 9.43 & $B$ & 9.43 \\
\hline $100-B-23$ & $\mathrm{J1HHX6}$ & $4 / 26 / 11$ & 0.566 & $\mathrm{U}$ & 0.57 & 1.42 & $\mathrm{U}$ & 1.42 & & & & 113 & & 7.08 & 94.8 & & 28.3 \\
\hline $100-B-24$ & $\mathrm{~J} 1 \mathrm{HHX7}$ & $4 / 26 / 11$ & 0.588 & $\mathrm{U}$ & 0.59 & 1.47 & $\mathrm{U}$ & 1.47 & & & & 46.3 & & 7.35 & 28.0 & $\mathrm{~B}$ & 29.4 \\
\hline 100-B-25 & $\mathrm{J1HHX8}$ & $4 / 25 / 11$ & 0.566 & $\mathrm{U}$ & 0.57 & 1.42 & $\mathrm{U}$ & 1.42 & 4.13 & & 0.128 & 36.2 & & 7.08 & 38.0 & & 28.3 \\
\hline $\begin{array}{c}\text { Duplicate of } \\
\mathrm{J} 1 \mathrm{HHX8}\end{array}$ & $\mathrm{J} 1 \mathrm{HJ} 03$ & $4 / 25 / 11$ & 0.545 & $U$ & 0.55 & 1.36 & $U$ & 1.36 & 3.25 & & 0.123 & 27.6 & & 6.82 & 33.5 & & 27.3 \\
\hline 100-B-25 (Depth) & $\mathrm{J} 1 \mathrm{HJ} 08$ & $4 / 26 / 11$ & 0.536 & $\mathrm{U}$ & 0.54 & 1.34 & $\mathrm{U}$ & 1.34 & 3.56 & & 0.128 & 33.5 & & 6.70 & 23.8 & $B$ & 26.8 \\
\hline $100-B-26$ & $\mathrm{~J} 1 \mathrm{HHX9}$ & $8 / 23 / 11$ & 0.147 & $\mathrm{U}$ & 0.15 & 0.368 & $\mathrm{U}$ & 0.37 & & & & 25.9 & & 1.84 & 41.8 & & 7.4 \\
\hline $100-B-27$ & $\mathrm{~J} 1 \mathrm{HJO0}$ & $4 / 27 / 11$ & 0.556 & $\mathrm{U}$ & 0.56 & 1.39 & $\mathrm{U}$ & 1.39 & & & & 28.9 & & 6.94 & 43.4 & & 27.8 \\
\hline $100-B-28$ & $\mathrm{J1HJ01}$ & $8 / 23 / 11$ & 0.159 & $\mathrm{U}$ & 0.16 & 0.397 & $\mathrm{U}$ & 0.40 & & & & 29.1 & & 1.98 & 46.6 & & 7.9 \\
\hline $100-B-29$ & $\mathrm{~J} 1 \mathrm{HJO2}$ & $8 / 23 / 11$ & 0.145 & $\mathrm{U}$ & 0.15 & 0.362 & $\mathrm{U}$ & 0.36 & & & & 30.6 & & 1.81 & 29.8 & & 7.3 \\
\hline Equipment Blank & $\mathrm{J} 1 \mathrm{HJ} 05$ & $4 / 25 / 11$ & 0.143 & $\mathrm{U}$ & 0.14 & 0.357 & $\mathrm{U}$ & 0.357 & & & & 0.701 & B & 1.79 & 2.37 & B & 7.14 \\
\hline
\end{tabular}


Table C-1. 126-B-1 Coal Ash Sample Results. (5 Pages)

\begin{tabular}{|c|c|c|c|c|c|c|c|c|c|c|c|c|c|c|c|c|c|c|c|c|}
\hline \multirow{2}{*}{$\begin{array}{c}\text { Sample } \\
\text { Location }\end{array}$} & \multirow{2}{*}{$\begin{array}{c}\text { HEIS } \\
\text { Number }\end{array}$} & \multirow{2}{*}{$\begin{array}{c}\text { Sample } \\
\text { Date }\end{array}$} & \multicolumn{3}{|c|}{ Acenaphthene } & \multicolumn{3}{|c|}{ Acenaphthylene } & \multicolumn{3}{|c|}{ Anthracene } & \multicolumn{3}{|c|}{ Benzo(a)anthracene } & \multicolumn{3}{|c|}{ Benzo(a)pyrene } & \multicolumn{3}{|c|}{ Benzo(b)fluoranthene } \\
\hline & & & $u g / k g$ & Q & $\mathrm{PQL}$ & $u g / k g$ & $\mathbf{Q}$ & $\mathrm{PQL}$ & $u g / k g$ & Q & $\mathrm{PQL}$ & ug/kg & Q & PQL & ug/kg & Q & PQL & ug/kg & $\mathbf{Q}$ & PQL \\
\hline $100-B-10$ & J1HHW3 & $4 / 27 / 11$ & 328 & 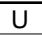 & 328 & 328 & 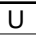 & 328 & 50.2 & $\mathrm{~J}$ & 328 & 328 & $U$ & 328 & 328 & $\bar{U}$ & 328 & 328 & $\bar{U}$ & 328 \\
\hline $\begin{array}{c}\text { 100-B-10 } \\
\text { (Depth) }\end{array}$ & $\mathrm{J} 1 \mathrm{HJ} 10$ & $4 / 27 / 11$ & 322 & $\mathrm{u}$ & 322 & 322 & $u$ & 322 & 322 & $\mathrm{U}$ & 322 & 322 & $\mathrm{u}$ & 322 & 322 & $\mathrm{u}$ & 322 & 322 & $\mathrm{u}$ & 322 \\
\hline $100-\mathrm{B}-17$ & $\mathrm{~J} 1 \mathrm{HHX0}$ & $4 / 27 / 11$ & 324 & $\mathrm{U}$ & 324 & 324 & $\mathrm{U}$ & 324 & 324 & $\mathrm{U}$ & 324 & 324 & $\mathrm{U}$ & 324 & 324 & $\mathrm{U}$ & 324 & 324 & $\mathrm{U}$ & 324 \\
\hline $\begin{array}{c}\text { 100-B-17 } \\
\text { (Depth) }\end{array}$ & J1HJ09 & $4 / 27 / 11$ & 327 & $u$ & 327 & 327 & $\mathrm{u}$ & 327 & 327 & $\mathrm{U}$ & 327 & 327 & $u$ & 327 & 327 & u & 327 & 327 & U & 327 \\
\hline $100-\mathrm{B}-25$ & J1HHX8 & $4 / 25 / 11$ & 328 & $\mathrm{U}$ & 328 & 328 & $\mathrm{U}$ & 328 & 328 & $\mathrm{U}$ & 328 & 73.6 & $\mathrm{~J}$ & 328 & 328 & $\mathrm{U}$ & 328 & 328 & $\mathrm{U}$ & 328 \\
\hline $\begin{array}{c}\text { Duplicate of } \\
\mathrm{J} 1 \mathrm{HHX8}\end{array}$ & $\mathrm{J} 1 \mathrm{HJ} 03$ & $4 / 25 / 11$ & 322 & $\mathrm{u}$ & 322 & 322 & u & 322 & 322 & U & 322 & 322 & U & 322 & 322 & U & 322 & 322 & U & 322 \\
\hline $\begin{array}{c}100-\mathrm{B}-25 \\
\text { (Depth) }\end{array}$ & $\mathrm{J} 1 \mathrm{HJ} 08$ & 4/26/11 & 328 & u & 328 & 328 & U & 328 & 328 & u & 328 & 328 & U & 328 & 328 & U & 328 & 328 & U & 328 \\
\hline $\begin{array}{c}\text { Equipment } \\
\text { Blank }\end{array}$ & $\mathrm{J} 1 \mathrm{HJ} 05$ & 4/25/11 & 316 & U & 316 & 316 & U & 316 & 316 & U & 316 & 316 & U & 316 & 316 & U & 316 & 316 & u & 316 \\
\hline
\end{tabular}

\begin{tabular}{|c|c|c|c|c|c|c|c|c|c|c|c|c|c|c|c|c|c|c|c|c|}
\hline \multirow{2}{*}{$\begin{array}{c}\text { Sample } \\
\text { Location }\end{array}$} & \multirow{2}{*}{$\begin{array}{c}\text { HEIS } \\
\text { Number }\end{array}$} & \multirow{2}{*}{$\begin{array}{c}\text { Sample } \\
\text { Date }\end{array}$} & \multicolumn{3}{|c|}{ Benzo(ghi)perylene } & \multicolumn{3}{|c|}{ Benzo(k)fluoranthene } & \multicolumn{3}{|c|}{ Chrysene } & \multicolumn{3}{|c|}{ Dibenz(a,h)anthracene } & \multicolumn{3}{|c|}{ Fluoranthene } & \multicolumn{3}{|c|}{ Fluorene } \\
\hline & & & $u g / k g$ & Q & PQL & ug/kg & $\mathbf{Q}$ & $\mathrm{PQL}$ & $u g / k g$ & Q & PQL & ug/kg & Q & $\mathrm{PQL}$ & ug/kg & $\mathbf{Q}$ & $\mathrm{PQL}$ & ug/kg & $\mathbf{Q}$ & PQL \\
\hline $100-B-10$ & J1HHW3 & $4 / 27 / 11$ & 328 & $\mathrm{U}$ & 328 & 328 & $\mathrm{U}$ & 328 & 63.7 & $\mathrm{~J}$ & 328 & 328 & $\mathrm{U}$ & 328 & 71.3 & $\mathrm{~J}$ & 328 & 328 & $\mathrm{U}$ & 328 \\
\hline $\begin{array}{c}\text { 100-B-10 } \\
\text { (Depth) }\end{array}$ & $\mathrm{J} 1 \mathrm{HJ} 10$ & $4 / 27 / 11$ & 322 & $u$ & 322 & 322 & $\mathrm{u}$ & 322 & 322 & $u$ & 322 & 322 & $u$ & 322 & 322 & $u$ & 322 & 322 & $u$ & 322 \\
\hline $100-\mathrm{B}-17$ & J1HHXO & $4 / 27 / 11$ & 324 & $\mathrm{U}$ & 324 & 324 & $\mathrm{U}$ & 324 & 65.0 & $\mathrm{~J}$ & 324 & 324 & $\mathrm{U}$ & 324 & 78.0 & $\mathrm{~J}$ & 324 & 324 & $\mathrm{U}$ & 324 \\
\hline $\begin{array}{c}\text { 100-B-17 } \\
\text { (Depth) }\end{array}$ & J1HJ09 & $4 / 27 / 11$ & 327 & $u$ & 327 & 327 & $u$ & 327 & 327 & $u$ & 327 & 327 & $u$ & 327 & 327 & $U$ & 327 & 327 & $u$ & 327 \\
\hline $100-\mathrm{B}-25$ & J1HHX8 & $4 / 25 / 11$ & 328 & $\mathrm{U}$ & 328 & 83.9 & $\mathrm{~J}$ & 328 & 83.4 & $\mathrm{~J}$ & 328 & 328 & $u$ & 328 & 74.9 & $\mathrm{~J}$ & 328 & 328 & $U$ & 328 \\
\hline $\begin{array}{c}\text { Duplicate of } \\
\mathrm{J} 1 \mathrm{HHX} 8\end{array}$ & $\mathrm{~J} 1 \mathrm{HJ} 03$ & $4 / 25 / 11$ & 322 & $u$ & 322 & 322 & u & 322 & 322 & U & 322 & 322 & U & 322 & 55.2 & $\mathrm{~J}$ & 322 & 322 & U & 322 \\
\hline $\begin{array}{c}100-\mathrm{B}-25 \\
\text { (Depth) }\end{array}$ & $\mathrm{J} 1 \mathrm{HJ} 08$ & $4 / 26 / 11$ & 328 & u & 328 & 328 & u & 328 & 328 & U & 328 & 328 & U & 328 & 328 & U & 328 & 328 & u & 328 \\
\hline $\begin{array}{l}\text { Equipment } \\
\text { Blank }\end{array}$ & $\mathrm{J} 1 \mathrm{HJ} 05$ & $4 / 25 / 11$ & 316 & U & 316 & 316 & U & 316 & 316 & U & 316 & 316 & U & 316 & 316 & U & 316 & 316 & U & 316 \\
\hline
\end{tabular}

\begin{tabular}{|l|l|l|l|l|l|l}
\hline Sample & HEIS & Sample & Indeno(1,2,3-cd)pyrene & Naphthalene & Phenanthrene & Pyrene
\end{tabular}

\begin{tabular}{|c|c|c|c|c|c|c|c|c|c|c|c|c|c|c|}
\hline \multirow{2}{*}{$\begin{array}{l}\text { Sample } \\
\text { Location }\end{array}$} & \multirow{2}{*}{$\begin{array}{c}\text { HEIS } \\
\text { Number }\end{array}$} & \multirow{2}{*}{$\begin{array}{c}\text { Sample } \\
\text { Date }\end{array}$} & \multicolumn{3}{|c|}{ Indeno(1,2,3-cd)pyrene } & \multicolumn{3}{|c|}{ Naphthalene } & \multicolumn{3}{|c|}{ Phenanthrene } & \multicolumn{3}{|c|}{ Pyrene } \\
\hline & & & $u g / k g$ & $\mathbf{Q}$ & $\mathrm{PQL}$ & ug/kg & $\mathbf{Q}$ & PQL & ug/kg & Q & PQL & ug/kg & $\mathbf{Q}$ & PQL \\
\hline $100-B-10$ & J1HHW3 & 4/27/11 & 328 & $\bar{U}$ & 328 & 257 & $\mathrm{~J}$ & 328 & 240 & $\mathrm{~J}$ & 328 & 64.9 & $\mathrm{~J}$ & 328 \\
\hline $\begin{array}{c}\begin{array}{c}100-\mathrm{B}-10 \\
\text { (Depth) }\end{array} \\
\end{array}$ & $\mathrm{J} 1 \mathrm{HJ} 10$ & $4 / 27 / 11$ & 322 & $\mathrm{u}$ & 322 & 125 & $\mathrm{~J}$ & 322 & 79.0 & $\mathrm{~J}$ & 322 & 322 & $u$ & 322 \\
\hline $100-\mathrm{B}-17$ & $\mathrm{~J} 1 \mathrm{HHXO}$ & 4/27/11 & 324 & $U$ & 324 & 852 & & 324 & 164 & $\mathrm{~J}$ & 324 & 69.4 & $\mathrm{~J}$ & 324 \\
\hline $\begin{array}{c}\text { 100-B-17 } \\
\text { (Depth) }\end{array}$ & J1HJ09 & $4 / 27 / 11$ & 327 & $\mathrm{u}$ & 327 & 234 & $\mathrm{~J}$ & 327 & 90.3 & $\mathrm{~J}$ & 327 & 327 & U & 327 \\
\hline $100-B-25$ & J1HHX8 & 4/25/11 & 328 & $\mathrm{U}$ & 328 & 1730 & & 328 & 393 & & 328 & 100 & $\mathrm{~J}$ & 328 \\
\hline $\begin{array}{c}\text { Duplicate of } \\
\mathrm{J} 1 \mathrm{HHX8}\end{array}$ & $\mathrm{J} 1 \mathrm{HJ} 03$ & $4 / 25 / 11$ & 322 & $\mathrm{u}$ & 322 & 198 & $\mathrm{~J}$ & 322 & 168 & $\mathrm{~J}$ & 322 & 322 & $u$ & 322 \\
\hline $\begin{array}{c}\text { 100-B-25 } \\
\text { (Depth) }\end{array}$ & $\mathrm{J} 1 \mathrm{HJ} 08$ & $4 / 26 / 11$ & 328 & $\mathrm{u}$ & 328 & 328 & $u$ & 328 & 119 & $\mathrm{~J}$ & 328 & 328 & $u$ & 328 \\
\hline $\begin{array}{l}\text { Equipment } \\
\text { Blank }\end{array}$ & J1HJ05 & $4 / 25 / 11$ & 316 & $u$ & 316 & 316 & $u$ & 316 & 316 & $\mathrm{U}$ & 316 & 316 & $u$ & 316 \\
\hline
\end{tabular}


Table C-2. 126-B-1 Coal Ash Pre-Leaching Quadruplicate Sample Results. (2 Pages)

\begin{tabular}{|c|c|c|c|c|c|c|c|c|c|c|c|c|c|c|c|c|c|}
\hline \multirow{2}{*}{ Sample Location } & \multirow{2}{*}{$\begin{array}{c}\text { HEIS } \\
\text { Number }\end{array}$} & \multirow{2}{*}{$\begin{array}{c}\text { Sample } \\
\text { Date }\end{array}$} & \multicolumn{3}{|c|}{ Antimony } & \multicolumn{3}{|c|}{ Arsenic } & \multicolumn{3}{|c|}{ Barium } & \multicolumn{3}{|c|}{ Beryllium } & \multicolumn{3}{|c|}{ Boron } \\
\hline & & & $\mathrm{mg} / \mathrm{kg}$ & $\mathbf{Q}$ & PQL & $\mathrm{mg} / \mathrm{kg}$ & $\mathbf{Q}$ & PQL & $\mathrm{mg} / \mathrm{kg}$ & $\mathbf{Q}$ & PQL & $\mathbf{m g} / \mathbf{k g}$ & $\mathbf{Q}$ & PQL & $\mathrm{mg} / \mathrm{kg}$ & $\mathbf{Q}$ & PQL \\
\hline $100-B-10$ & J1HHW3-1 & $4 / 27 / 11$ & 0.556 & $\mathrm{U}$ & 0.56 & 3.10 & & 0.93 & 701 & & 0.46 & 1.12 & & 0.19 & 217 & & 1.85 \\
\hline 100-B-10 & J1HHW3-2 & $4 / 27 / 11$ & 0.545 & $\mathrm{U}$ & 0.55 & 3.14 & & 0.91 & 665 & & 0.46 & 1.13 & & 0.18 & 217 & & 1.82 \\
\hline $100-B-10$ & J1HHW3-3 & $4 / 27 / 11$ & 0.566 & $\mathrm{U}$ & 0.57 & 2.80 & & 0.94 & 609 & & 0.47 & 1.09 & & 0.19 & 208 & & 1.89 \\
\hline 100-B-10 & J1HHW3-4 & $4 / 27 / 11$ & 0.545 & $\mathrm{U}$ & 0.55 & 3.04 & & 0.91 & 675 & & 0.46 & 1.07 & & 0.18 & 210 & & 1.82 \\
\hline 100-B-25 (Depth) & $\mathrm{J} 1 \mathrm{HJ} 08-1$ & $4 / 26 / 11$ & 0.657 & $\mathrm{U}$ & 0.66 & 11.1 & & 1.10 & 1790 & & 0.55 & 1.65 & & 0.22 & 69.1 & & 2.19 \\
\hline 100-B-25 (Depth) & $\mathrm{J} 1 \mathrm{HJ} 08-2$ & $4 / 26 / 11$ & 0.635 & $\mathrm{U}$ & 0.64 & 9.25 & & 1.06 & 2030 & & 0.53 & 1.82 & & 0.21 & 166 & & 2.12 \\
\hline 100-B-25 (Depth) & $\mathrm{J} 1 \mathrm{HJ} 08-3$ & $4 / 26 / 11$ & 0.434 & B & 0.64 & 8.86 & & 1.06 & 1500 & & 0.53 & 1.43 & & 0.21 & 56.8 & & 2.12 \\
\hline 100-B-25 (Depth) & $\mathrm{J} 1 \mathrm{HJ} 08-4$ & $4 / 26 / 11$ & 0.397 & B & 0.72 & 11.1 & & 1.20 & 1720 & & 0.60 & 1.52 & & 0.24 & 57.2 & & 2.40 \\
\hline 100-B-17 (Depth) & $\mathrm{J} 1 \mathrm{HJ} 09-1$ & $4 / 27 / 11$ & 0.636 & $\mathrm{U}$ & 0.64 & 2.66 & & 1.06 & 910 & & 0.53 & 1.98 & & 0.21 & 352 & & 2.12 \\
\hline 100-B-17 (Depth) & $\mathrm{J} 1 \mathrm{HJ} 09-2$ & $4 / 27 / 11$ & 0.614 & $\mathrm{U}$ & 0.61 & 2.57 & & 1.02 & 866 & & 0.51 & 1.88 & & 0.21 & 317 & & 2.05 \\
\hline 100-B-17 (Depth) & $\mathrm{J} 1 \mathrm{HJ} 09-3$ & $4 / 27 / 11$ & 0.614 & $\mathrm{U}$ & 0.61 & 2.41 & & 1.02 & 766 & & 0.51 & 1.76 & & 0.21 & 300 & & 2.05 \\
\hline 100-B-17 (Depth) & J1HJ09-4 & $4 / 27 / 11$ & 0.584 & $\mathrm{U}$ & 0.58 & 2.14 & & 0.97 & 732 & & 0.49 & 1.65 & & 0.20 & 275 & & 1.95 \\
\hline 100-B-10 (Depth) & $\mathrm{J} 1 \mathrm{HJ} 10-1$ & $4 / 27 / 11$ & 0.517 & $\mathrm{U}$ & 0.52 & 3.30 & & 0.86 & 896 & & 0.43 & 1.17 & & 0.17 & 247 & & 1.72 \\
\hline 100-B-10 (Depth) & $\mathrm{J} 1 \mathrm{HJ} 10-2$ & $4 / 27 / 11$ & 0.517 & $\mathrm{U}$ & 0.52 & 2.94 & & 0.86 & 922 & & 0.43 & 1.29 & & 0.17 & 240 & & 1.72 \\
\hline 100-B-10 (Depth) & $\mathrm{J} 1 \mathrm{HJ} 10-3$ & $4 / 27 / 11$ & 0.353 & B & 0.52 & 3.03 & & 0.86 & 1120 & & 0.43 & 1.13 & & 0.17 & 229 & & 1.72 \\
\hline 100-B-10 (Depth) & J1HJ10-4 & $4 / 27 / 11$ & 0.55 & $\mathrm{U}$ & 0.55 & 2.94 & & 0.91 & 938 & & 0.46 & 1.19 & & 0.18 & 292 & & 1.82 \\
\hline
\end{tabular}

\begin{tabular}{|c|c|c|c|c|c|c|c|c|c|c|c|c|c|c|c|c|c|}
\hline \multirow{2}{*}{ Sample Location } & \multirow{2}{*}{$\begin{array}{c}\text { HEIS } \\
\text { Number }\end{array}$} & \multirow{2}{*}{$\begin{array}{c}\text { Sample } \\
\text { Date }\end{array}$} & \multicolumn{3}{|c|}{ Cadmium } & \multicolumn{3}{|c|}{ Chromium } & \multicolumn{3}{|c|}{ Cobalt } & \multicolumn{3}{|c|}{ Copper } & \multicolumn{3}{|c|}{ Lead } \\
\hline & & & $\mathrm{mg} / \mathrm{kg}$ & Q & PQL & $\mathrm{mg} / \mathrm{kg}$ & Q & $\mathrm{PQL}$ & $\mathrm{mg} / \mathrm{kg}$ & $\mathbf{Q}$ & $\mathrm{PQL}$ & $\mathrm{mg} / \mathrm{kg}$ & $\mathbf{Q}$ & PQL & $\mathrm{mg} / \mathrm{kg}$ & $\mathbf{Q}$ & $\mathrm{PQL}$ \\
\hline $100-B-10$ & J1HHW3-1 & $4 / 27 / 11$ & 0.133 & B & 0.19 & 9.10 & & 0.19 & 4.51 & & 1.85 & 29.6 & & 0.93 & 9.63 & & 0.46 \\
\hline $100-B-10$ & J1HHW3-2 & $4 / 27 / 11$ & 0.119 & B & 0.18 & 9.44 & & 0.18 & 4.53 & & 1.82 & 30.0 & & 0.91 & 7.95 & & 0.46 \\
\hline $100-B-10$ & J1HHW3-3 & $4 / 27 / 11$ & 0.144 & $\mathrm{~B}$ & 0.19 & 8.31 & & 0.19 & 4.13 & & 1.89 & 24.4 & & 0.94 & 7.04 & & 0.47 \\
\hline $100-B-10$ & J1HHW3-4 & $4 / 27 / 11$ & 0.159 & $B$ & 0.18 & 8.23 & & 0.18 & 4.37 & & 1.82 & 30.1 & & 0.91 & 13.7 & & 0.46 \\
\hline 100-B-25 (Depth) & J1HJ08-1 & $4 / 26 / 11$ & 0.268 & & 0.22 & 14.1 & & 0.22 & 6.74 & & 2.19 & 38.5 & & 1.10 & 8.17 & & 0.55 \\
\hline 100-B-25 (Depth) & J1HJ08-2 & $4 / 26 / 11$ & 0.232 & & 0.21 & 10.3 & & 0.21 & 6.43 & & 2.12 & 31.5 & & 1.06 & 5.38 & & 0.53 \\
\hline 100-B-25 (Depth) & J1HJ08-3 & $4 / 26 / 11$ & 0.244 & & 0.21 & 11 & & 0.21 & 5.44 & & 2.12 & 30.6 & & 1.06 & 6.73 & & 0.53 \\
\hline 100-B-25 (Depth) & J1HJ08-4 & $4 / 26 / 11$ & 0.282 & & 0.24 & 13.7 & & 0.24 & 6.49 & & 2.40 & 34.3 & & 1.20 & 7.09 & & 0.60 \\
\hline 100-B-17 (Depth) & J1HJ09-1 & $4 / 27 / 11$ & 0.163 & $B$ & 0.21 & 14.9 & & 0.21 & 3.31 & & 2.12 & 25.7 & & 1.06 & 6.94 & & 0.53 \\
\hline 100-B-17 (Depth) & J1HJ09-2 & $4 / 27 / 11$ & 0.188 & $B$ & 0.21 & 15.1 & & 0.21 & 3.13 & & 2.05 & 26.6 & & 1.02 & 7.00 & & 0.51 \\
\hline 100-B-17 (Depth) & J1HJ09-3 & $4 / 27 / 11$ & 0.138 & B & 0.21 & 13.3 & & 0.21 & 2.88 & & 2.05 & 23.3 & & 1.02 & 7.08 & & 0.51 \\
\hline 100-B-17 (Depth) & J1HJ09-4 & $4 / 27 / 11$ & 0.128 & $B$ & 0.20 & 11.4 & & 0.20 & 2.75 & & 1.95 & 21.3 & & 0.97 & 5.04 & & 0.49 \\
\hline 100-B-10 (Depth) & $\mathrm{J} 1 \mathrm{HJ} 10-1$ & $4 / 27 / 11$ & 0.213 & & 0.17 & 10.3 & & 0.17 & 3.56 & & 1.72 & 26.2 & & 0.86 & 38.7 & & 0.43 \\
\hline 100-B-10 (Depth) & $\mathrm{J} 1 \mathrm{HJ} 10-2$ & $4 / 27 / 11$ & 0.227 & & 0.17 & 9.20 & & 0.17 & 4.14 & & 1.72 & 36.8 & & 0.86 & 14.9 & & 0.43 \\
\hline 100-B-10 (Depth) & $\mathrm{J} 1 \mathrm{HJ} 10-3$ & $4 / 27 / 11$ & 0.29 & & 0.17 & 7.31 & & 0.17 & 3.61 & & 1.72 & 27.0 & & 0.86 & 25.7 & & 0.43 \\
\hline 100-B-10 (Depth) & J1HJ10-4 & $4 / 27 / 11$ & 0.247 & & 0.18 & 8.72 & & 0.18 & 3.49 & & 1.82 & 25.5 & & 0.91 & 12.1 & & 0.46 \\
\hline
\end{tabular}


Table C-2. 126-B-1 Coal Ash Pre-Leaching Quadruplicate Sample Results. (2 Pages)

\begin{tabular}{|c|c|c|c|c|c|c|c|c|c|c|c|c|c|c|c|c|c|}
\hline \multirow{2}{*}{ Sample Location } & \multirow{2}{*}{$\begin{array}{c}\text { HEIS } \\
\text { Number }\end{array}$} & \multirow{2}{*}{$\begin{array}{c}\text { Sample } \\
\text { Date }\end{array}$} & \multicolumn{3}{|c|}{ Manganese } & \multicolumn{3}{|c|}{ Mercury } & \multicolumn{3}{|c|}{ Molybdenum } & \multicolumn{3}{|c|}{ Nickel } & \multicolumn{3}{|c|}{ Selenium } \\
\hline & & & $\mathrm{mg} / \mathrm{kg}$ & Q & PQL & $\mathrm{mg} / \mathrm{kg}$ & Q & PQL & $\mathrm{mg} / \mathrm{kg}$ & Q & PQL & $\mathrm{mg} / \mathrm{kg}$ & $\mathbf{Q}$ & PQL & $\mathrm{mg} / \mathrm{kg}$ & $\mathbf{Q}$ & PQL \\
\hline $100-B-10$ & J1HHW3-1 & $4 / 27 / 11$ & 94.9 & & 4.63 & 0.038 & & 0.03 & 1.18 & $B$ & 1.85 & 10.4 & & 3.70 & 0.278 & $\mathrm{U}$ & 0.28 \\
\hline $100-B-10$ & J1HHW3-2 & $4 / 27 / 11$ & 96.1 & & 4.55 & 0.042 & & 0.03 & 1.22 & $B$ & 1.82 & 10.1 & & 3.64 & 0.367 & & 0.27 \\
\hline 100-B-10 & J1HHW3-3 & $4 / 27 / 11$ & 84.9 & & 4.72 & 0.044 & & 0.03 & 1.12 & $B$ & 1.89 & 9.04 & & 3.77 & 0.464 & & 0.28 \\
\hline $100-\mathrm{B}-10$ & J1HHW3-4 & $4 / 27 / 11$ & 89.6 & & 4.55 & 0.045 & & 0.03 & 1.26 & $B$ & 1.82 & 9.38 & & 3.64 & 0.421 & & 0.27 \\
\hline 100-B-25 (Depth) & J1HJ08-1 & $4 / 26 / 11$ & 269 & & 5.48 & 0.038 & & 0.03 & 2.90 & & 2.19 & 15.7 & & 4.38 & 1.32 & & 0.33 \\
\hline 100-B-25 (Depth) & $\mathrm{J} 1 \mathrm{HJ} 08-2$ & $4 / 26 / 11$ & 175 & & 5.29 & 0.046 & & 0.03 & 2.43 & & 2.12 & 13.8 & & 4.23 & 0.823 & & 0.32 \\
\hline 100-B-25 (Depth) & J1HJ08-3 & $4 / 26 / 11$ & 228 & & 5.29 & 0.037 & & 0.03 & 2.27 & & 2.12 & 13.1 & & 4.23 & 1.39 & & 0.32 \\
\hline 100-B-25 (Depth) & $\mathrm{J} 1 \mathrm{HJ} 08-4$ & $4 / 26 / 11$ & 254 & & 6.00 & 0.037 & & 0.03 & 3.32 & & 2.40 & 15.2 & & 4.80 & 1.47 & & 0.36 \\
\hline 100-B-17 (Depth) & J1HJ09-1 & $4 / 27 / 11$ & 52.0 & & 5.30 & 0.033 & & 0.03 & 1.60 & $B$ & 2.12 & 9.21 & & 4.24 & 0.343 & & 0.32 \\
\hline 100-B-17 (Depth) & J1HJ09-2 & $4 / 27 / 11$ & 46.8 & & 5.12 & 0.035 & & 0.03 & 1.52 & $\mathrm{~B}$ & 2.05 & 8.90 & & 4.09 & 0.307 & $\mathrm{U}$ & 0.31 \\
\hline 100-B-17 (Depth) & J1HJ09-3 & $4 / 27 / 11$ & 37.8 & & 5.12 & 0.037 & & 0.03 & 1.31 & $\mathrm{~B}$ & 2.05 & 8.22 & & 4.09 & 0.339 & & 0.31 \\
\hline 100-B-17 (Depth) & J1HJ09-4 & $4 / 27 / 11$ & 43.5 & & 4.87 & 0.042 & & 0.03 & 1.28 & B & 1.95 & 7.52 & & 3.89 & 0.292 & $\mathrm{U}$ & 0.29 \\
\hline 100-B-10 (Depth) & $\mathrm{J} 1 \mathrm{HJ} 10-1$ & $4 / 27 / 11$ & 136 & & 4.31 & 0.033 & & 0.03 & 1.23 & B & 1.72 & 8.91 & & 3.45 & 0.301 & & 0.26 \\
\hline 100-B-10 (Depth) & $\mathrm{J} 1 \mathrm{HJ} 10-2$ & $4 / 27 / 11$ & 159 & & 4.31 & 0.039 & & 0.02 & 1.56 & B & 1.72 & 10.5 & & 3.45 & 0.259 & $\mathrm{U}$ & 0.26 \\
\hline 100-B-10 (Depth) & $\mathrm{J} 1 \mathrm{HJ} 10-3$ & $4 / 27 / 11$ & 140 & & 4.31 & 0.029 & & 0.02 & 1.28 & $B$ & 1.72 & 8.04 & & 3.45 & 0.412 & & 0.26 \\
\hline 100-B-10 (Depth) & J1HJ10-4 & $4 / 27 / 11$ & 141 & & 4.55 & 0.040 & & 0.02 & 1.46 & B & 1.82 & 8.20 & & 3.64 & 0.273 & $U$ & 0.27 \\
\hline
\end{tabular}

\begin{tabular}{|c|c|c|c|c|c|c|c|c|c|c|c|c|c|c|}
\hline Sample Location & $\begin{array}{c}\text { HEIS } \\
\text { Number }\end{array}$ & $\begin{array}{c}\text { Sample } \\
\text { Date }\end{array}$ & \multicolumn{3}{|c|}{ Silver } & \multicolumn{3}{|c|}{ Thallium } & \multicolumn{3}{|c|}{ Vanadium } & \multicolumn{3}{|c|}{ Zinc } \\
\hline $100-B-10$ & J1HHW3-2 & $4 / 27 / 11$ & 0.182 & $U$ & 0.18 & 0.455 & $U$ & 0.46 & 40.6 & & 2.27 & 15.8 & & 9.09 \\
\hline $100-B-10$ & J1HHW3-3 & 4/27/11 & 0.189 & $U$ & 0.19 & 0.472 & $U$ & 0.47 & 35.1 & & 2.36 & 14.6 & & 9.43 \\
\hline 100-B-25 (Depth) & J1HJ08-1 & $4 / 26 / 11$ & 0.219 & $U$ & 0.22 & 0.548 & $U$ & 0.55 & 52.0 & & 2.74 & 31.1 & & 11.0 \\
\hline 100-B-25 (Depth) & J1HJ08-2 & $4 / 26 / 11$ & 0.212 & $U$ & 0.21 & 0.529 & $U$ & 0.53 & 49.5 & & 2.65 & 20.7 & & 10.6 \\
\hline 100-B-25 (Depth) & J1HJ08-3 & $4 / 26 / 11$ & 0.212 & $U$ & 0.21 & 0.529 & $U$ & 0.53 & 41.4 & & 2.65 & 27.8 & & 10.6 \\
\hline 100-B-17 (Depth) & J1HJ09-2 & 4/27/11 & 0.205 & $U$ & 0.21 & 0.512 & $U$ & 0.51 & 34.3 & & 2.56 & 22.2 & & 10.2 \\
\hline 100-B-17 (Depth) & J1HJ09-3 & $4 / 27 / 11$ & 0.205 & $U$ & 0.21 & 0.512 & $U$ & 0.51 & 32.2 & & 2.56 & 22.5 & & 10.2 \\
\hline 100-B-17 (Depth) & J1HJ09-4 & $4 / 27 / 11$ & 0.195 & $\mathrm{U}$ & 0.20 & 0.487 & $U$ & 0.49 & 29.2 & & 2.43 & 15.8 & & 9.73 \\
\hline 100-B-10 (Depth) & $\mathrm{J} 1 \mathrm{HJ} 10-1$ & 4/27/11 & 0.172 & $U$ & 0.17 & 0.431 & $U$ & 0.43 & 28.8 & & 2.16 & 31.1 & & 8.62 \\
\hline 100-B-10 (Depth) & $\mathrm{J} 1 \mathrm{HJ} 10-2$ & $4 / 27 / 11$ & 0.172 & $U$ & 0.17 & 0.431 & $U$ & 0.43 & 30.5 & & 2.16 & 38.3 & & 8.62 \\
\hline 100-B-10 (Depth) & $\mathrm{J} 1 \mathrm{HJ} 10-3$ & $4 / 27 / 11$ & 0.172 & $U$ & 0.17 & 0.431 & $U$ & 0.43 & 27.4 & & 2.16 & 44.5 & & 8.62 \\
\hline
\end{tabular}


Table C-3. 126-B-1 Coal Ash Leaching Sample Results. (2 Pages)

\begin{tabular}{|c|c|c|c|c|c|c|c|c|c|c|c|c|c|c|c|c|c|c|}
\hline \multirow{2}{*}{ Sample Location } & \multirow{2}{*}{$\begin{array}{c}\text { Coal Ash:Water } \\
\text { Ratio }\end{array}$} & \multirow{2}{*}{$\begin{array}{c}\text { HEIS } \\
\text { Number }\end{array}$} & \multirow{2}{*}{$\begin{array}{l}\text { Sample } \\
\text { Date }\end{array}$} & \multicolumn{3}{|c|}{ Antimony } & \multicolumn{3}{|c|}{$\begin{array}{c}\text { Arsenic } \\
\end{array}$} & \multicolumn{3}{|c|}{ Barium } & \multicolumn{3}{|c|}{ Beryllium } & \multicolumn{3}{|c|}{ Boron } \\
\hline & & & & $\mathrm{mg} / \mathrm{L}$ & Q & $\mathrm{PQL}$ & $\mathrm{mg} / \mathrm{L}$ & Q & $\mathrm{PQL}$ & $\mathrm{mg} / \mathrm{L}$ & $\mathbf{Q}$ & $\mathrm{PQL}$ & $\mathrm{mg} / \mathrm{L}$ & $\mathbf{Q}$ & PQL & $\mathrm{mg} / \mathrm{L}$ & Q & PQL \\
\hline $100-B-10$ & $1: 1$ & \begin{tabular}{|l|} 
J1HHW 3-A1 \\
\end{tabular} & $4 / 27 / 11$ & 0.200 & $u$ & 0.20 & 0.150 & $u$ & 0.15 & 0.031 & & 0.010 & 0.010 & $U$ & 0.010 & 3.31 & & 0.15 \\
\hline $100-B-10$ & $1: 2.5$ & \begin{tabular}{|l|}
$\mathrm{J} 1 \mathrm{HHW} 3-\mathrm{B} 1$ \\
\end{tabular} & $4 / 27 / 11$ & 0.020 & $U$ & 0.02 & 0.011 & $\mathrm{~B}$ & 0.02 & 0.020 & & 0.003 & 0.001 & $U$ & 0.001 & 1.92 & & 0.02 \\
\hline $100-\mathrm{B}-10$ & $1: 2.5$ & \begin{tabular}{|l|} 
J1HHW 3-B2 \\
\end{tabular} & $4 / 27 / 11$ & 0.020 & $U$ & 0.02 & 0.010 & $B$ & 0.02 & 0.019 & & 0.002 & 0.001 & $U$ & 0.001 & 1.75 & & 0.02 \\
\hline $100-B-10$ & $1: 5$ & \begin{tabular}{|l|}
$\mathrm{J} 1 \mathrm{HHW} 3-\mathrm{C} 1$ \\
\end{tabular} & $4 / 27 / 11$ & 0.020 & $U$ & 0.02 & 0.007 & $\mathrm{~B}$ & 0.02 & 0.013 & & 0.002 & 0.001 & $U$ & 0.001 & 0.98 & & 0.02 \\
\hline 100-B-25 (Depth) & $1: 1$ & \begin{tabular}{|l|} 
J1HJ08-A1 \\
\end{tabular} & $4 / 26 / 11$ & 0.200 & 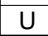 & 0.20 & 0.150 & 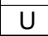 & 0.15 & 0.035 & & 0.010 & 0.010 & $U$ & 0.010 & 2.55 & & 0.15 \\
\hline 100-B-25 (Depth) & $1: 2.5$ & J1HJ08-B1 & $4 / 26 / 11$ & 0.020 & $U$ & 0.02 & 0.015 & $u$ & 0.02 & 0.034 & & 0.001 & 0.001 & $U$ & 0.001 & 1.55 & & 0.02 \\
\hline 100-B-25 (Depth) & $1: 2.5$ & $\mathrm{~J} 1 \mathrm{HJ} 08-\mathrm{B} 2$ & $4 / 26 / 11$ & 0.020 & $U$ & 0.02 & 0.015 & $U$ & 0.02 & 0.033 & & 0.001 & 0.001 & $U$ & 0.001 & 1.48 & & 0.02 \\
\hline 100-B-25 (Depth) & $1: 5$ & J1HJ08-C1 & $4 / 26 / 11$ & 0.020 & $U$ & 0.02 & 0.015 & $U$ & 0.02 & 0.039 & & 0.001 & 0.001 & $U$ & 0.001 & 1.06 & & 0.02 \\
\hline 100-B-17 (Depth) & $1: 1$ & J1HJ09-A1 & $4 / 27 / 11$ & 0.200 & $U$ & 0.20 & 0.150 & $U$ & 0.15 & 0.035 & & 0.010 & 0.010 & $\mathrm{U}$ & 0.010 & 5.66 & & 0.15 \\
\hline 100-B-17 (Depth) & $1: 2.5$ & J1HJ09-B1 & $4 / 27 / 11$ & 0.020 & $U$ & 0.02 & 0.003 & $B$ & 0.02 & 0.037 & & 0.001 & 0.001 & $\mathrm{U}$ & 0.001 & 3.93 & & 0.02 \\
\hline 100-B-17 (Depth) & $1: 5$ & J1HJ09-C1 & $4 / 27 / 11$ & 0.020 & $U$ & 0.02 & 0.003 & B & 0.02 & 0.026 & & 0.001 & 0.001 & 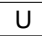 & 0.001 & 2.36 & & 0.02 \\
\hline 100-B-17 (Depth) & $1: 5$ & J1HJ09-C2 & $4 / 27 / 11$ & 0.020 & $U$ & 0.02 & 0.003 & B & 0.02 & 0.027 & & 0.001 & 0.001 & $U$ & 0.001 & 2.23 & & 0.02 \\
\hline 100-B-10 (Depth) & $1: 1$ & J1HJ10-A1 & $4 / 27 / 11$ & 0.200 & $U$ & 0.20 & 0.150 & $U$ & 0.15 & 0.056 & & 0.010 & 0.010 & $U$ & 0.010 & 8.57 & & 0.15 \\
\hline 100-B-10 (Depth) & $1: 1$ & $\mathrm{~J} 1 \mathrm{HJ} 10-\mathrm{A} 2$ & $4 / 27 / 11$ & 0.200 & $U$ & 0.20 & 0.150 & $U$ & 0.15 & 0.035 & & 0.010 & 0.010 & $U$ & 0.010 & 8.62 & & 0.15 \\
\hline 100-B-10 (Depth) & $1: 2.5$ & $\mathrm{~J} 1 \mathrm{HJ} 10-\mathrm{B} 1$ & $4 / 27 / 11$ & 0.020 & $U$ & 0.02 & 0.006 & $\mathrm{~B}$ & 0.02 & 0.035 & & 0.003 & 0.001 & $\mathrm{U}$ & 0.001 & 4.36 & & 0.02 \\
\hline 100-B-10 (Depth) & $1: 5$ & J1HJ10-C1 & $4 / 27 / 11$ & 0.020 & $U$ & 0.02 & 0.005 & $\mathrm{~B}$ & 0.02 & 0.031 & & 0.003 & 0.001 & $U$ & 0.001 & 2.31 & & 0.02 \\
\hline
\end{tabular}

\begin{tabular}{|c|c|c|c|c|c|c|c|c|c|c|c|c|c|c|c|c|c|c|}
\hline \multirow{2}{*}{ Sample Location } & \multirow{2}{*}{\begin{tabular}{|c} 
Coal Ash:Water \\
Ratio
\end{tabular}} & \multirow{2}{*}{$\begin{array}{c}\text { HEIS } \\
\text { Number }\end{array}$} & \multirow{2}{*}{$\begin{array}{c}\text { Sample } \\
\text { Date }\end{array}$} & \multicolumn{3}{|c|}{ Cadmium } & \multicolumn{3}{|c|}{ Chromium } & \multicolumn{3}{|c|}{ Cobalt } & \multicolumn{3}{|c|}{ Copper } & \multicolumn{3}{|c|}{ Lead } \\
\hline & & & & $\mathrm{mg} / \mathrm{L}$ & Q & $\mathrm{PQL}$ & $\mathrm{mg} / \mathrm{L}$ & Q & $\mathrm{PQL}$ & $\mathrm{mg} / \mathrm{L}$ & $\mathbf{Q}$ & $\mathrm{PQL}$ & $\mathrm{mg} / \mathrm{L}$ & $\mathbf{Q}$ & $\mathrm{PQL}$ & $\mathrm{mg} / \mathrm{L}$ & Q & $\mathrm{PQL}$ \\
\hline 100-B-10 & 1:1 & J1HHW3-A1 & $4 / 27 / 11$ & 0.030 & $u$ & 0.030 & 0.050 & U & 0.050 & 0.020 & $u$ & 0.020 & 0.200 & U & 0.20 & 0.100 & U & 0.100 \\
\hline $100-B-10$ & $1: 2.5$ & J1HHW3-B1 & $4 / 27 / 11$ & 0.003 & $U$ & 0.003 & 0.002 & $B$ & 0.010 & 0.002 & $U$ & 0.002 & 0.003 & $B$ & 0.02 & 0.010 & $U$ & 0.010 \\
\hline $100-B-10$ & $1: 2.5$ & \begin{tabular}{|l|}
$\mathrm{J} 1 \mathrm{HHW} 3-\mathrm{B} 2$ \\
\end{tabular} & $4 / 27 / 11$ & 0.003 & $U$ & 0.003 & 0.001 & $\mathrm{~B}$ & 0.010 & 0.002 & $U$ & 0.002 & 0.003 & $\mathrm{~B}$ & 0.02 & 0.010 & $U$ & 0.010 \\
\hline 100-B-10 & $1: 5$ & J1HHW3-C1 & $4 / 27 / 11$ & 0.003 & $u$ & 0.001 & 0.001 & $B$ & 0.010 & 0.002 & $u$ & 0.002 & 0.003 & B & 0.02 & 0.010 & $u$ & 0.010 \\
\hline 100-B-25 (Depth) & $1: 1$ & \begin{tabular}{|l|} 
J1HJ08-A1 \\
\end{tabular} & $4 / 26 / 11$ & 0.030 & $U$ & 0.030 & 0.050 & $\mathrm{U}$ & 0.050 & 0.020 & $U$ & 0.020 & 0.200 & $U$ & 0.20 & 0.100 & $U$ & 0.100 \\
\hline 100-B-25 (Depth) & $1: 2.5$ & J1HJ08-B1 & $4 / 26 / 11$ & 0.003 & $U$ & 0.003 & 0.005 & $\mathrm{u}$ & 0.010 & 0.002 & $U$ & 0.002 & 0.020 & $U$ & 0.02 & 0.010 & $U$ & 0.010 \\
\hline 100-B-25 (Depth) & $1: 2.5$ & J1HJ08-B2 & $4 / 26 / 11$ & 0.003 & $U$ & 0.003 & 0.005 & $\mathrm{U}$ & 0.010 & 0.002 & $U$ & 0.002 & 0.020 & $U$ & 0.02 & 0.010 & $U$ & 0.010 \\
\hline 100-B-25 (Depth) & $1: 5$ & $\mathrm{~J} 1 \mathrm{HJ} 08-\mathrm{C} 1$ & $4 / 26 / 11$ & 0.003 & $U$ & 0.003 & 0.005 & $\mathrm{U}$ & 0.010 & 0.002 & $U$ & 0.002 & 0.020 & $U$ & 0.02 & 0.010 & $U$ & 0.010 \\
\hline 100-B-17 (Depth) & $1: 1$ & J1HJ09-A1 & $4 / 27 / 11$ & 0.030 & $U$ & 0.030 & 0.050 & $\mathrm{U}$ & 0.050 & 0.020 & $U$ & 0.020 & 0.200 & $U$ & 0.20 & 0.100 & $U$ & 0.100 \\
\hline 100-B-17 (Depth) & $1: 2.5$ & J1HJ09-B1 & $4 / 27 / 11$ & 0.003 & $U$ & 0.003 & 0.003 & $\mathrm{~B}$ & 0.010 & 0.002 & $U$ & 0.002 & 0.020 & $U$ & 0.02 & 0.010 & $U$ & 0.010 \\
\hline 100-B-17 (Depth) & $1: 5$ & J1HJ09-C1 & $4 / 27 / 11$ & 0.003 & $U$ & 0.003 & 0.002 & $B$ & 0.010 & 0.002 & $u$ & 0.002 & 0.020 & $U$ & 0.02 & 0.010 & $U$ & 0.010 \\
\hline 100-B-17 (Depth) & $1: 5$ & J1HJ09-C2 & $4 / 27 / 11$ & 0.003 & $U$ & 0.003 & 0.002 & $\mathrm{~B}$ & 0.010 & 0.002 & $U$ & 0.002 & 0.020 & $U$ & 0.02 & 0.010 & $U$ & 0.010 \\
\hline 100-B-10 (Depth) & $1: 1$ & J1HJ10-A1 & $4 / 27 / 11$ & 0.030 & $U$ & 0.030 & 0.050 & $\mathrm{u}$ & 0.050 & 0.020 & $U$ & 0.020 & 0.200 & $U$ & 0.20 & 0.100 & $U$ & 0.100 \\
\hline 100-B-10 (Depth) & $1: 1$ & J1HJ10-A2 & $4 / 27 / 11$ & 0.030 & $\mathrm{U}$ & 0.030 & 0.050 & $\mathrm{U}$ & 0.050 & 0.020 & $U$ & 0.020 & 0.200 & $U$ & 0.20 & 0.100 & $U$ & 0.100 \\
\hline 100-B-10 (Depth) & $1: 2.5$ & J1HJ10-B1 & $4 / 27 / 11$ & 0.003 & $\mathrm{U}$ & 0.003 & 0.002 & $\mathrm{~B}$ & 0.010 & 0.002 & $U$ & 0.002 & 0.020 & $U$ & 0.02 & 0.010 & $U$ & 0.010 \\
\hline 100-B-10 (Depth) & $1: 5$ & J1HJ10-C1 & $4 / 27 / 11$ & 0.003 & $U$ & 0.001 & 0.001 & $\mathrm{~B}$ & 0.010 & 0.002 & $U$ & 0.002 & 0.020 & $U$ & 0.02 & 0.010 & $U$ & 0.010 \\
\hline
\end{tabular}




\begin{tabular}{|c|c|c|c|c|c|c|c|c|c|c|c|c|c|c|c|c|c|c|c|}
\hline \multirow{3}{*}{ 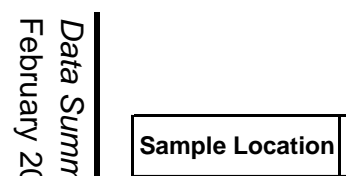 } & \multicolumn{18}{|c|}{ Table C-3. 126-B-1 Coal Ash Leaching Sample Results. (2 Pages) } & \\
\hline & Coal Ash:Water & HEIS & Sample & \multicolumn{3}{|c|}{ Manganese } & \multicolumn{3}{|c|}{ Mercury } & \multicolumn{3}{|c|}{ Molybdenum } & \multicolumn{3}{|c|}{ Nickel } & \multicolumn{3}{|c|}{ Selenium } & \\
\hline & Ratio & Number & Date & $\mathrm{mg} / \mathrm{L}$ & Q & PQL & $\mathrm{mg} / \mathrm{L}$ & 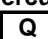 & PQL & $\mathrm{mg} / \mathrm{L}$ & Q & PQL & $\mathrm{mg} / \mathrm{L}$ & $\mathrm{Q}$ & PQL & $\mathrm{mg} / \mathrm{L}$ & $\mathrm{Q}$ & PQL & \\
\hline 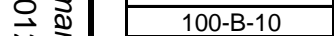 & $1: 1$ & J1HHW3-A1 & $4 / 27 / 11$ & 0.013 & $\mathrm{~B}$ & 0.050 & 0.002 & $\mathrm{U}$ & 0.0020 & 0.011 & $\mathrm{~B}$ & 0.030 & 0.005 & $\mathrm{~B}$ & 0.200 & 0.200 & $U$ & 0.200 & \\
\hline $100-B-10$ & $1: 2.5$ & J1HHW3-B1 & $4 / 27 / 11$ & 0.001 & $\mathrm{~B}$ & 0.010 & 0.0002 & $\mathrm{U}$ & 0.0004 & 0.003 & $\mathrm{~B}$ & 0.002 & 0.001 & $\mathrm{~B}$ & 0.020 & 0.020 & U & 0.020 & \\
\hline $100-B-10$ & $1: 2.5$ & J1HHW3-B2 & $4 / 27 / 11$ & 0.001 & $\mathrm{~B}$ & 0.010 & 0.0002 & $\mathrm{u}$ & 0.0002 & 0.003 & $\mathrm{~B}$ & 0.002 & 0.001 & $\mathrm{~B}$ & 0.020 & 0.020 & $\mathrm{u}$ & 0.020 & \\
\hline $100-B-10$ & $1: 5$ & J1HHW3-C1 & $4 / 27 / 11$ & 0.001 & $B$ & 0.010 & 0.0002 & $U$ & 0.0002 & 0.002 & $B$ & 0.001 & 0.001 & $B$ & 0.020 & 0.020 & $u$ & 0.020 & \\
\hline \begin{tabular}{|l|} 
100-B-25 (Depth) \\
\end{tabular} & $1: 1$ & \begin{tabular}{|l|} 
J1HJ08-A1 \\
\end{tabular} & $4 / 26 / 11$ & 0.081 & & 0.050 & 0.002 & $\mathrm{U}$ & 0.0020 & 0.012 & $\mathrm{~B}$ & 0.030 & 0.200 & $\mathrm{U}$ & 0.200 & 0.200 & $U$ & 0.200 & \\
\hline \begin{tabular}{|l|}
$100-B-25$ (Depth) \\
\end{tabular} & $1: 2.5$ & J1HJ08-B1 & $4 / 26 / 11$ & 0.038 & & 0.010 & 0.0002 & $\mathrm{U}$ & 0.0002 & 0.008 & & 0.003 & 0.001 & $\mathrm{~B}$ & 0.020 & 0.020 & U & 0.020 & \\
\hline \begin{tabular}{|l|}
$100-B-25$ (Depth) \\
\end{tabular} & $1: 2.5$ & J1HJ08-B2 & $4 / 26 / 11$ & 0.036 & & 0.010 & 0.0002 & $\mathrm{U}$ & 0.0002 & 0.008 & & 0.003 & 0.020 & $U$ & 0.020 & 0.003 & B & 0.020 & \\
\hline 100-B-25 (Depth) & $1: 5$ & J1HJ08-C1 & $4 / 26 / 11$ & 0.023 & & 0.010 & 0.0002 & $\mathrm{U}$ & 0.0002 & 0.008 & & 0.003 & 0.001 & $B$ & 0.020 & 0.020 & $U$ & 0.020 & \\
\hline \begin{tabular}{|l|} 
100-B-17 (Depth) \\
\end{tabular} & $1: 1$ & J1HJ09-A1 & $4 / 27 / 11$ & 0.050 & $\mathrm{U}$ & 0.050 & 0.002 & $\mathrm{U}$ & 0.0020 & 0.035 & & 0.030 & 0.200 & $\mathrm{U}$ & 0.200 & 0.200 & $U$ & 0.200 & \\
\hline 100-B-17 (Depth) & $1: 2.5$ & J1HJ09-B1 & $4 / 27 / 11$ & 0.001 & $B$ & 0.010 & 0.0002 & $\mathrm{U}$ & 0.0002 & 0.011 & & 0.003 & 0.020 & $U$ & 0.020 & 0.003 & B & 0.020 & \\
\hline \begin{tabular}{|l|}
$100-B-17$ (Depth) \\
\end{tabular} & $1: 5$ & $\mathrm{~J} 1 \mathrm{HJ} 09-\mathrm{C} 1$ & $4 / 27 / 11$ & 0.001 & $\mathrm{~B}$ & 0.010 & 0.0002 & $\mathrm{U}$ & 0.0002 & 0.006 & & 0.003 & 0.001 & $\mathrm{~B}$ & 0.020 & 0.020 & $u$ & 0.020 & \\
\hline 100-B-17 (Depth) & $1: 5$ & J1HJ09-C2 & 4/27/11 & 0.001 & $B$ & 0.010 & 0.0002 & $U$ & 0.0002 & 0.006 & & 0.003 & 0.001 & $B$ & 0.020 & 0.020 & $u$ & 0.020 & \\
\hline \begin{tabular}{|l|}
$100-B-10$ (Depth) \\
\end{tabular} & $1: 1$ & J1HJ10-A1 & $4 / 27 / 11$ & 0.014 & $\mathrm{~B}$ & 0.050 & 0.002 & $\mathrm{U}$ & 0.0020 & 0.015 & B & 0.030 & 0.200 & $U$ & 0.200 & 0.200 & $U$ & 0.200 & \\
\hline \begin{tabular}{|l|} 
100-B-10 (Depth) \\
\end{tabular} & $1: 1$ & J1HJ10-A2 & $4 / 27 / 11$ & 0.011 & B & 0.050 & 0.002 & $\mathrm{U}$ & 0.0020 & 0.015 & B & 0.030 & 0.200 & $U$ & 0.200 & 0.200 & $u$ & 0.200 & \\
\hline \begin{tabular}{|l|}
$100-B-10$ (Depth) \\
\end{tabular} & $1: 2.5$ & $\mathrm{~J} 1 \mathrm{HJ} 10-\mathrm{B} 1$ & $4 / 27 / 11$ & 0.001 & $\mathrm{~B}$ & 0.010 & 0.0002 & $\mathrm{U}$ & 0.0002 & 0.007 & & 0.007 & 0.001 & $\mathrm{~B}$ & 0.020 & 0.003 & $\mathrm{~B}$ & 0.020 & \\
\hline 100-B-10 (Depth) & $1: 5$ & $\mathrm{~J} 1 \mathrm{HJ} 10-\mathrm{C} 1$ & $4 / 27 / 11$ & 0.001 & $\mathrm{~B}$ & 0.010 & 0.0002 & $\mathrm{U}$ & 0.0002 & 0.007 & & 0.007 & 0.001 & $\mathrm{~B}$ & 0.020 & 0.020 & $\mathrm{U}$ & 0.020 & \\
\hline \multirow{2}{*}{ Sample Location } & \multirow{2}{*}{$\begin{array}{c}\text { Coal Ash:Water } \\
\text { Ratio }\end{array}$} & \multirow{2}{*}{$\begin{array}{c}\text { HEIS } \\
\text { Number }\end{array}$} & \multirow{2}{*}{$\begin{array}{c}\text { Sample } \\
\text { Date }\end{array}$} & \multicolumn{3}{|c|}{$\begin{array}{l}\text { Silver } \\
\end{array}$} & \multicolumn{3}{|c|}{ Thallium } & \multicolumn{3}{|c|}{ Uranium (KPA) } & \multicolumn{3}{|c|}{ Vanadium } & \multicolumn{3}{|c|}{ Zinc } & \\
\hline & & & & $\mathrm{mg} / \mathrm{L}$ & 0 & PQL & $\mathrm{mg} / \mathrm{L}$ & 0 & $\mathrm{PQL}$ & ug/L & 0 & MDA & $\mathrm{mg} / \mathrm{L}$ & 0 & PQL & $\mathrm{mg} / \mathrm{L}$ & 0 & PQL & $\mathrm{pH}$ \\
\hline $100-B-10$ & $1: 1$ & J1HHW3-A1 & $4 / 27 / 11$ & 0.060 & $\mathrm{U}$ & 0.06 & 0.150 & $\mathrm{U}$ & 0.15 & 2.22 & & 0.02 & 0.067 & & 0.05 & 0.500 & $\mathrm{U}$ & 0.50 & 8.79 \\
\hline $100-B-10$ & $1: 2.5$ & J1HHW3-B1 & $4 / 27 / 11$ & 0.006 & $\mathrm{U}$ & 0.01 & 0.015 & $\mathrm{U}$ & 0.02 & 1.27 & & 0.02 & 0.061 & & 0.01 & 0.050 & $U$ & 0.05 & 9.01 \\
\hline $100-B-10$ & $1: 2.5$ & J1HHW3-B2 & $4 / 27 / 11$ & 0.006 & $U$ & 0.01 & 0.015 & $U$ & 0.02 & 1.30 & & 0.02 & 0.058 & & 0.01 & 0.050 & u & 0.05 & 9.13 \\
\hline $100-B-10$ & $1: 5$ & J1HHW3-C1 & $4 / 27 / 11$ & 0.006 & $\mathrm{U}$ & 0.01 & 0.015 & $\mathrm{U}$ & 0.02 & 1.09 & & 0.02 & 0.046 & & 0.01 & 0.050 & $\mathrm{U}$ & 0.05 & 9.28 \\
\hline 100-B-25 (Depth) & $1: 1$ & \begin{tabular}{|l|}
$\mathrm{J} 1 \mathrm{H} J 08-\mathrm{A} 1$ \\
\end{tabular} & $4 / 26 / 11$ & 0.060 & $U$ & 0.06 & 0.150 & $U$ & 0.15 & 0.056 & & 0.04 & 0.039 & $B$ & 0.05 & 0.500 & $U$ & 0.50 & 7.89 \\
\hline 100-B-25 (Depth) & $1: 2.5$ & $\mathrm{~J} 1 \mathrm{HJ} 08-\mathrm{B} 1$ & $4 / 26 / 11$ & 0.006 & $\mathrm{U}$ & 0.01 & 0.015 & $\mathrm{U}$ & 0.02 & 0.050 & & 0.02 & 0.017 & & 0.01 & 0.050 & $U$ & 0.05 & 7.72 \\
\hline \begin{tabular}{|l|} 
100-B-25 (Depth) \\
\end{tabular} & $1: 2.5$ & J1HJ08-B2 & $4 / 26 / 11$ & 0.006 & $\mathrm{U}$ & 0.01 & 0.015 & $\mathrm{U}$ & 0.02 & 0.046 & & 0.02 & 0.017 & & 0.01 & 0.050 & 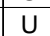 & 0.05 & 7.58 \\
\hline \begin{tabular}{|l|}
$100-B-25$ (Depth) \\
\end{tabular} & $1: 5$ & J1HJ08-C1 & $4 / 26 / 11$ & 0.006 & $\mathrm{U}$ & 0.01 & 0.015 & $\mathrm{U}$ & 0.02 & 0.098 & & 0.02 & 0.015 & & 0.01 & 0.050 & $U$ & 0.05 & 7.57 \\
\hline \begin{tabular}{|l|}
$100-B-17$ (Depth) \\
\end{tabular} & $1: 1$ & J1HJ09-A1 & $4 / 27 / 11$ & 0.060 & $U$ & 0.06 & 0.150 & $U$ & 0.15 & 6.42 & & 0.13 & 0.035 & $\mathrm{~B}$ & 0.05 & 0.500 & U & 0.50 & 9.18 \\
\hline \begin{tabular}{|l|}
$100-B-17$ (Depth) \\
\end{tabular} & $1: 2.5$ & J1HJ09-B1 & $4 / 27 / 11$ & 0.006 & $\mathrm{U}$ & 0.01 & 0.015 & $U$ & 0.02 & 3.35 & & 0.02 & 0.037 & & 0.01 & 0.050 & U & 0.05 & 9.22 \\
\hline \begin{tabular}{|l|}
$100-B-17$ (Depth) \\
\end{tabular} & $1: 5$ & J1HJ09-C1 & $4 / 27 / 11$ & 0.006 & $U$ & 0.01 & 0.015 & $\mathrm{U}$ & 0.02 & 2.27 & & 0.02 & 0.032 & & 0.01 & 0.050 & $u$ & 0.05 & 9.52 \\
\hline \begin{tabular}{|l|} 
100-B-17 (Depth) \\
\end{tabular} & $1: 5$ & J1HJ09-C2 & $4 / 27 / 11$ & 0.006 & $U$ & 0.01 & 0.015 & $\mathrm{U}$ & 0.02 & 2.44 & & 0.02 & 0.030 & & 0.01 & 0.050 & $U$ & 0.05 & 9.55 \\
\hline \begin{tabular}{|l|}
$100-B-10$ (Depth) \\
\end{tabular} & $1: 1$ & J1HJ10-A1 & $4 / 27 / 11$ & 0.060 & $U$ & 0.06 & 0.150 & $U$ & 0.15 & 5.16 & & 0.02 & 0.057 & & 0.05 & 0.500 & $U$ & 0.50 & 8.58 \\
\hline \begin{tabular}{|l|}
$100-B-10$ (Depth) \\
\end{tabular} & $1: 1$ & $\mathrm{~J} 1 \mathrm{HJ} 10-\mathrm{A} 2$ & $4 / 27 / 11$ & 0.060 & $\mathrm{U}$ & 0.06 & 0.150 & $\mathrm{U}$ & 0.15 & 5.19 & & 0.02 & 0.056 & & 0.05 & 0.500 & 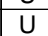 & 0.50 & 8.91 \\
\hline \begin{tabular}{|l|}
$100-B-10$ (Depth) \\
\end{tabular} & $1: 2.5$ & $\mathrm{~J} 1 \mathrm{HJ} 10-\mathrm{B} 1$ & $4 / 27 / 11$ & 0.006 & $\mathrm{U}$ & 0.01 & 0.015 & $\mathrm{U}$ & 0.02 & 2.68 & & 0.02 & 0.047 & & 0.01 & 0.012 & $\mathrm{~B}$ & 0.05 & 9.02 \\
\hline \begin{tabular}{|l|}
$100-B-10$ (Depth) \\
\end{tabular} & $1: 5$ & $\mathrm{~J} 1 \mathrm{HJ} 10-\mathrm{C} 1$ & $4 / 27 / 11$ & 0.006 & $\mathrm{U}$ & 0.01 & 0.015 & $\mathrm{U}$ & 0.02 & 2.11 & & 0.02 & 0.036 & & 0.01 & 0.050 & $u$ & 0.05 & 8.57 \\
\hline
\end{tabular}


Table C-4. 126-D-1 Coal Ash Sample Results. (5 Pages)

\begin{tabular}{|c|c|c|c|c|c|c|c|c|c|c|c|c|c|c|c|c|c|}
\hline \multirow{2}{*}{ Sample Location } & \multirow{2}{*}{$\begin{array}{c}\text { HEIS } \\
\text { Number }\end{array}$} & \multirow{2}{*}{$\begin{array}{c}\text { Sample } \\
\text { Date }\end{array}$} & \multicolumn{3}{|c|}{ Antimony } & \multicolumn{3}{|c|}{ Arsenic } & \multicolumn{3}{|c|}{ Barium } & \multicolumn{3}{|c|}{ Beryllium } & \multicolumn{3}{|c|}{ Boron } \\
\hline & & & $\mathrm{mg} / \mathrm{kg}$ & $\mathbf{Q}$ & $\mathrm{PQL}$ & $\mathrm{mg} / \mathrm{kg}$ & $\mathbf{Q}$ & $\mathrm{PQL}$ & $\mathrm{mg} / \mathrm{kg}$ & Q & $\mathrm{PQL}$ & $\mathrm{mg} / \mathrm{kg}$ & $\mathbf{Q}$ & $\mathrm{PQL}$ & $\mathrm{mg} / \mathrm{kg}$ & $\mathbf{Q}$ & $\mathrm{PQL}$ \\
\hline 100-D-01 & J1J3W3 & $8 / 9 / 11$ & 0.965 & $\mathrm{~J}$ & 0.49 & 10.2 & & 0.82 & 1770 & & 0.41 & 2.50 & & 0.16 & 50.1 & $\mathrm{~J}$ & 1.64 \\
\hline 100-D-02 & J1J3W4 & $8 / 22 / 11$ & 0.536 & $\mathrm{U}$ & 0.54 & 3.87 & & 0.89 & 1030 & & 0.45 & 1.36 & & 0.18 & 197 & & 1.79 \\
\hline 100-D-03 & J1J3W5 & $8 / 22 / 11$ & 0.626 & & 0.48 & 13.6 & & 0.79 & 1470 & & 0.40 & 2.51 & & 0.16 & 33.6 & & 1.59 \\
\hline 100-D-04 & J1J3W6 & $8 / 23 / 11$ & 0.448 & $U$ & 0.45 & 2.70 & & 0.75 & 949 & & 0.37 & 1.04 & & 0.15 & 182 & & 1.49 \\
\hline 100-D-05 & J1J3W7 & $8 / 23 / 11$ & 0.497 & & 0.48 & 16.7 & & 0.79 & 1750 & & 0.40 & 2.61 & & 0.16 & 71.3 & & 1.59 \\
\hline 100-D-06 & J1J3W8 & $8 / 22 / 11$ & 0.441 & $U$ & 0.44 & 2.41 & & 0.74 & 1080 & & 0.37 & 1.53 & & 0.15 & 489 & & 1.47 \\
\hline $100-D-07$ & J1J3W9 & $8 / 22 / 11$ & 0.517 & $\mathrm{U}$ & 0.52 & 5.42 & & 0.86 & 1310 & & 0.43 & 1.38 & & 0.17 & 324 & & 1.72 \\
\hline 100-D-08 & $\mathrm{J1J3 \times 0}$ & $8 / 11 / 11$ & 0.470 & $\mathrm{BJ}$ & 0.58 & 2.32 & & 0.96 & 1470 & & 0.48 & 1.31 & & 0.19 & 317 & $\mathrm{~J}$ & 1.92 \\
\hline 100-D-08 (Depth) & $\mathrm{J} 1 \mathrm{~J} 444$ & $8 / 11 / 11$ & 0.476 & UJ & 0.48 & 2.52 & & 0.79 & 1060 & & 0.40 & 1.45 & & 0.16 & 419 & $\mathrm{~J}$ & 1.59 \\
\hline 100-D-09 & $\mathrm{J1J3X1}$ & $8 / 11 / 11$ & 0.419 & BJ & 0.53 & 2.38 & & 0.88 & 781 & & 0.44 & 1.70 & & 0.18 & 155 & $\mathrm{~J}$ & 1.75 \\
\hline $100-D-10$ & $\mathrm{J1J3X2}$ & $8 / 11 / 11$ & 0.894 & $\mathrm{~J}$ & 0.44 & 3.42 & & 0.74 & 526 & & 0.37 & 0.595 & & 0.15 & 38.4 & $\mathrm{~J}$ & 1.47 \\
\hline $100-D-11$ & $\mathrm{J1} 1 \mathrm{~J} 3 \times 3$ & $8 / 23 / 11$ & 0.268 & B & 0.46 & 7.17 & & 0.77 & 1240 & & 0.39 & 1.43 & & 0.15 & 115 & & 1.54 \\
\hline 100-D-12 & $\mathrm{J1J3X4}$ & $8 / 9 / 11$ & 0.510 & BJ & 0.53 & 4.37 & & 0.88 & 907 & & 0.44 & 1.41 & & 0.18 & 269 & $\mathrm{~J}$ & 1.75 \\
\hline 100-D-13 & $\mathrm{J1J3 \times 5}$ & $8 / 22 / 11$ & 0.566 & $\mathrm{U}$ & 0.57 & 3.26 & & 0.94 & 972 & & 0.47 & 1.06 & & 0.19 & 118 & & 1.89 \\
\hline $100-D-14$ & $\mathrm{~J} 1 \mathrm{~J} 3 \times 6$ & $8 / 22 / 11$ & 0.588 & $\mathrm{U}$ & 0.59 & 5.23 & & 0.98 & 1210 & & 0.49 & 1.30 & & 0.20 & 94.1 & & 1.96 \\
\hline 100-D-15 & $\mathrm{J1J3 \times 7}$ & $8 / 11 / 11$ & 0.532 & $\mathrm{BJ}$ & 0.60 & 4.02 & & 1.00 & 1520 & & 0.50 & 1.43 & & 0.20 & 410 & $\mathrm{~J}$ & 2.00 \\
\hline 100-D-16 & $\mathrm{J1J3X8}$ & $8 / 22 / 11$ & 0.500 & $\mathrm{U}$ & 0.50 & 2.27 & & 0.83 & 703 & & 0.42 & 0.763 & & 0.17 & 141 & & 1.67 \\
\hline $100-\mathrm{D}-17$ & $\mathrm{J1J3 \times 9}$ & $8 / 22 / 11$ & 0.517 & $\mathrm{U}$ & 0.52 & 3.68 & & 0.86 & 1110 & & 0.43 & 1.20 & & 0.17 & 170 & & 1.72 \\
\hline Duplicate of J1J3X9 & $\mathrm{J} 1 \mathrm{~J} 413$ & $8 / 22 / 11$ & 0.566 & $\mathrm{U}$ & 0.57 & 3.30 & & 0.94 & 1410 & & 0.47 & 1.28 & & 0.19 & 231 & & 1.89 \\
\hline $100-\mathrm{D}-18$ & $\mathrm{J1J} 400$ & $8 / 9 / 11$ & 0.659 & $\mathrm{~J}$ & 0.53 & 5.51 & & 0.88 & 1380 & & 0.44 & 1.43 & & 0.18 & 129 & $\mathrm{~J}$ & 1.75 \\
\hline $100-\mathrm{D}-19$ & J1J401 & $8 / 11 / 11$ & 0.605 & $\mathrm{~J}$ & 0.48 & 4.61 & & 0.79 & 743 & & 0.40 & 1.21 & & 0.16 & 142 & $\mathrm{~J}$ & 1.59 \\
\hline 100-D-19 (Depth) & J1J443 & $8 / 11 / 11$ & 0.545 & UJ & 0.55 & 6.00 & & 0.91 & 1470 & & 0.46 & 1.62 & & 0.18 & 195 & $\mathrm{~J}$ & 1.82 \\
\hline 100-D-20 & $\mathrm{J} 1 \mathrm{~J} 402$ & $8 / 23 / 11$ & 0.500 & $\mathrm{U}$ & 0.50 & 3.00 & & 0.83 & 733 & & 0.42 & 1.09 & & 0.17 & 147 & & 1.67 \\
\hline 100-D-21 & $\mathrm{J} 1 \mathrm{~J} 403$ & $8 / 23 / 11$ & 0.566 & $\mathrm{U}$ & 0.57 & 3.38 & & 0.94 & 843 & & 0.47 & 1.31 & & 0.19 & 227 & & 1.89 \\
\hline $100-D-22$ & J1J404 & $8 / 23 / 11$ & 0.508 & $\mathrm{U}$ & 0.51 & 3.19 & & 0.85 & 951 & & 0.42 & 1.24 & & 0.17 & 249 & & 1.69 \\
\hline $100-D-23$ & $\mathrm{~J} 1 \mathrm{~J} 405$ & $8 / 23 / 11$ & 0.536 & $\mathrm{U}$ & 0.54 & 2.99 & & 0.89 & 734 & & 0.45 & 1.03 & & 0.18 & 91.1 & & 1.79 \\
\hline 100-D-24 & $\mathrm{J} 1 \mathrm{~J} 406$ & $8 / 23 / 11$ & 0.500 & $\mathrm{U}$ & 0.50 & 2.15 & & 0.83 & 407 & & 0.42 & 0.588 & & 0.17 & 86.1 & & 1.67 \\
\hline $100-D-25$ & J1J407 & $8 / 23 / 11$ & 0.517 & $U$ & 0.52 & 3.13 & & 0.86 & 941 & & 0.43 & 1.37 & & 0.17 & 312 & & 1.72 \\
\hline $100-D-26$ & J1J408 & $8 / 11 / 11$ & 0.556 & $\mathrm{~J}$ & 0.51 & 3.91 & & 0.85 & 879 & & 0.42 & 1.17 & & 0.17 & 181 & $\mathrm{~J}$ & 1.69 \\
\hline Duplicate of J1J408 & $\mathrm{J} 1 \mathrm{~J} 412$ & $8 / 11 / 11$ & 0.545 & UJ & 0.55 & 4.34 & & 0.91 & 1140 & & 0.46 & 1.42 & & 0.18 & 218 & $\mathrm{~J}$ & 1.82 \\
\hline 100-D-26 (Depth) & $\mathrm{J} 1 \mathrm{~J} 442$ & $8 / 11 / 11$ & 0.435 & UJ & 0.44 & 3.16 & & 0.73 & 871 & & 0.36 & 1.10 & & 0.15 & 169 & $\mathrm{~J}$ & 1.45 \\
\hline $100-\mathrm{D}-27$ & $\mathrm{~J} 1 \mathrm{~J} 409$ & $8 / 23 / 11$ & 0.341 & $\mathrm{~B}$ & 0.58 & 5.75 & & 0.96 & 1110 & & 0.48 & 1.72 & & 0.19 & 234 & & 1.92 \\
\hline 100-D-28 & $\mathrm{J} 1 \mathrm{~J} 410$ & $8 / 23 / 11$ & 0.536 & $\mathrm{U}$ & 0.54 & 4.42 & & 0.89 & 1330 & & 0.45 & 1.35 & & 0.18 & 118 & & 1.79 \\
\hline 100-D-29 & J1J411 & $8 / 11 / 11$ & 0.515 & BJ & 0.59 & 5.11 & & 0.98 & 1320 & & 0.49 & 1.25 & & 0.20 & 238 & $\mathrm{~J}$ & 1.96 \\
\hline Equip blank & J1J414 & $8 / 11 / 11$ & 0.508 & UJ & 0.51 & 0.847 & $U$ & 0.85 & 7.38 & & 0.42 & 0.054 & $B$ & 0.17 & 1.69 & UJ & 1.69 \\
\hline
\end{tabular}


Table C-4. 126-D-1 Coal Ash Sample Results. (5 Pages)

\begin{tabular}{|c|c|c|c|c|c|c|c|c|c|c|c|c|c|c|c|c|c|}
\hline \multirow{2}{*}{ Sample Location } & \multirow{2}{*}{$\begin{array}{c}\text { HEIS } \\
\text { Number }\end{array}$} & \multirow{2}{*}{$\begin{array}{c}\text { Sample } \\
\text { Date }\end{array}$} & \multicolumn{3}{|c|}{ Cadmium } & \multicolumn{3}{|c|}{ Chromium } & \multicolumn{3}{|c|}{ Cobalt } & \multicolumn{3}{|c|}{ Copper } & \multicolumn{3}{|c|}{ Lead } \\
\hline & & & $\mathrm{mg} / \mathrm{kg}$ & $\mathbf{Q}$ & $\mathrm{PQL}$ & $\mathrm{mg} / \mathrm{kg}$ & Q & PQL & $\mathrm{mg} / \mathrm{kg}$ & $\mathbf{Q}$ & $\mathrm{PQL}$ & $\mathrm{mg} / \mathrm{kg}$ & Q & PQL & $\mathrm{mg} / \mathrm{kg}$ & $\mathbf{Q}$ & $\mathrm{PQL}$ \\
\hline $100-D-01$ & J1J3W3 & $8 / 9 / 11$ & 0.430 & & 0.16 & 7.31 & & 0.16 & 3.86 & & 1.64 & 23.2 & & 0.82 & 3.91 & $\mathrm{~J}$ & 0.41 \\
\hline $100-D-02$ & J1J3W4 & $8 / 22 / 11$ & 0.227 & & 0.18 & 7.31 & & 0.18 & 6.04 & & 1.79 & 19.6 & & 0.89 & 3.02 & & 0.45 \\
\hline 100-D-03 & J1J3W5 & $8 / 22 / 11$ & 0.371 & & 0.16 & 6.61 & & 0.16 & 2.31 & & 1.59 & 19.9 & & 0.8 & 3.71 & & 0.40 \\
\hline $100-D-04$ & J1J3W6 & $8 / 23 / 11$ & 0.208 & & 0.15 & 11.1 & & 0.15 & 5.04 & & 1.49 & 29.1 & & 0.8 & 6.19 & & 0.37 \\
\hline 100-D-05 & J1J3W7 & $8 / 23 / 11$ & 0.311 & & 0.16 & 8.18 & & 0.16 & 3.63 & & 1.59 & 20.4 & & 0.8 & 4.05 & & 0.40 \\
\hline 100-D-06 & J1J3W8 & $8 / 22 / 11$ & 0.186 & & 0.15 & 8.72 & & 0.15 & 11.6 & & 1.47 & 12.7 & & 0.7 & 1.91 & & 0.37 \\
\hline $100-D-07$ & J1J3W9 & $8 / 22 / 11$ & 0.254 & & 0.17 & 12.1 & & 0.17 & 8.48 & & 1.72 & 15.0 & & 0.9 & 3.61 & & 0.43 \\
\hline $100-\mathrm{D}-08$ & $\mathrm{~J} 1 \mathrm{~J} 3 \times 0$ & $8 / 11 / 11$ & 0.129 & B & 0.19 & 8.03 & & 0.19 & 9.87 & & 1.92 & 20.8 & & 0.96 & 1.90 & $\mathrm{~J}$ & 0.48 \\
\hline 100-D-08 (Depth) & J1J444 & $8 / 11 / 11$ & 0.178 & & 0.16 & 7.64 & & 0.16 & 6.82 & & 1.59 & 13.6 & & 0.79 & 3.50 & $\mathrm{~J}$ & 0.40 \\
\hline 100-D-09 & J1J3X1 & $8 / 11 / 11$ & 0.127 & $\mathrm{~B}$ & 0.18 & 5.70 & & 0.18 & 4.88 & & 1.75 & 9.66 & & 0.88 & 2.57 & $\mathrm{~J}$ & 0.44 \\
\hline $100-D-10$ & $\mathrm{~J} 1 \mathrm{~J} 3 \mathrm{X} 2$ & $8 / 11 / 11$ & 0.267 & & 0.15 & 8.53 & & 0.15 & 7.19 & & 1.47 & 23.1 & & 0.74 & 9.50 & $\mathrm{~J}$ & 0.37 \\
\hline $100-D-11$ & J1J3X3 & $8 / 23 / 11$ & 0.445 & & 0.15 & 8.72 & & 0.15 & 4.41 & & 1.54 & 24.2 & & 0.77 & 4.09 & & 0.39 \\
\hline $100-D-12$ & J1J3X4 & $8 / 9 / 11$ & 0.165 & B & 0.18 & 12.5 & & 0.18 & 4.12 & & 1.75 & 28.3 & & 0.88 & 15.1 & $\mathrm{~J}$ & 0.44 \\
\hline 100-D-13 & J1J3X5 & $8 / 22 / 11$ & 0.228 & & 0.19 & 6.95 & & 0.19 & 6.2 & & 1.89 & 34.1 & & 0.94 & 2.42 & & 0.47 \\
\hline 100-D-14 & J1J3X6 & $8 / 22 / 11$ & 0.293 & & 0.20 & 11.6 & & 0.20 & 9.02 & & 1.96 & 59.4 & & 0.98 & 3.59 & & 0.49 \\
\hline 100-D-15 & $\mathrm{J} 1 \mathrm{~J} 3 \mathrm{X} 7$ & $8 / 11 / 11$ & 0.138 & B & 0.20 & 11.2 & & 0.20 & 13.7 & & 2.00 & 13.4 & & 1.00 & 3.15 & $\mathrm{~J}$ & 0.50 \\
\hline $100-D-16$ & J1J3X8 & $8 / 22 / 11$ & 0.256 & & 0.17 & 6.36 & & 0.17 & 6.3 & & 1.67 & 12.8 & & 0.83 & 2.70 & & 0.42 \\
\hline $100-D-17$ & J1J3X9 & $8 / 22 / 11$ & 0.447 & & 0.17 & 9.89 & & 0.17 & 5.18 & & 1.72 & 20.9 & & 0.86 & 10.4 & & 0.43 \\
\hline Duplicate of J1J3X9 & $\mathrm{J} 1 \mathrm{~J} 413$ & $8 / 22 / 11$ & 0.438 & & 0.19 & 10.3 & & 0.19 & 4.59 & & 1.89 & 17.7 & & 0.94 & 8.56 & & 0.47 \\
\hline $100-\mathrm{D}-18$ & $\mathrm{~J} 1 \mathrm{~J} 400$ & $8 / 9 / 11$ & 0.311 & & 0.18 & 8.57 & & 0.18 & 4.48 & & 1.75 & 21.7 & & 0.88 & 4.27 & $\mathrm{~J}$ & 0.44 \\
\hline 100-D-19 & $\mathrm{J} 1 \mathrm{~J} 401$ & $8 / 11 / 11$ & 0.176 & & 0.16 & 12.6 & & 0.16 & 4.59 & & 1.59 & 31 & & 0.79 & 16.8 & $\mathrm{~J}$ & 0.40 \\
\hline 100-D-19 (Depth) & $\mathrm{J} 1 \mathrm{~J} 443$ & $8 / 11 / 11$ & 0.208 & & 0.18 & 7.76 & & 0.18 & 5.63 & & 1.82 & 23.7 & & 0.91 & 3.24 & $\mathrm{~J}$ & 0.46 \\
\hline 100-D-20 & $\mathrm{J} 1 \mathrm{~J} 402$ & $8 / 23 / 11$ & 0.211 & & 0.17 & 10.6 & & 0.17 & 5.24 & & 1.67 & 30.9 & & 0.83 & 8.68 & & 0.42 \\
\hline $100-D-21$ & $\mathrm{~J} 1 \mathrm{~J} 403$ & $8 / 23 / 11$ & 0.267 & & 0.19 & 8.21 & & 0.19 & 5.25 & & 1.89 & 24.8 & & 0.94 & 3.65 & & 0.47 \\
\hline $100-D-22$ & J1J404 & $8 / 23 / 11$ & 0.212 & & 0.17 & 10.2 & & 0.17 & 3.25 & & 1.69 & 23.6 & & 0.85 & 10.1 & & 0.42 \\
\hline $100-D-23$ & $\mathrm{~J} 1 \mathrm{~J} 405$ & $8 / 23 / 11$ & 0.203 & & 0.18 & 9.26 & & 0.18 & 4.49 & & 1.79 & 25.5 & & 0.89 & 6.44 & & 0.45 \\
\hline $100-D-24$ & $\mathrm{~J} 1 \mathrm{~J} 406$ & $8 / 23 / 11$ & 0.270 & & 0.17 & 7.29 & & 0.17 & 4.46 & & 1.67 & 18.2 & & 0.83 & 5.01 & & 0.42 \\
\hline $100-D-25$ & $\mathrm{~J} 1 \mathrm{~J} 407$ & $8 / 23 / 11$ & 0.216 & & 0.17 & 10.8 & & 0.17 & 2.81 & & 1.72 & 21.9 & & 0.86 & 9.61 & & 0.43 \\
\hline $100-\mathrm{D}-26$ & $\mathrm{~J} 1 \mathrm{~J} 408$ & $8 / 11 / 11$ & 0.156 & B & 0.17 & 11.4 & & 0.17 & 3.44 & & 1.69 & 23.8 & & 0.85 & 13.2 & $\mathrm{~J}$ & 0.42 \\
\hline Duplicate of J1J408 & $\mathrm{J} 1 \mathrm{~J} 412$ & $8 / 11 / 11$ & 0.190 & & 0.18 & 12.9 & & 0.18 & 3.48 & & 1.82 & 28.0 & & 0.91 & 16.4 & $\mathrm{~J}$ & 0.46 \\
\hline 100-D-26 (Depth) & $\mathrm{J} 1 \mathrm{~J} 442$ & 8/11/11 & 0.074 & $B$ & 0.15 & 8.73 & & 0.15 & 4.21 & & 1.45 & 31.8 & & 0.73 & 3.35 & $\mathrm{~J}$ & 0.36 \\
\hline $100-\mathrm{D}-27$ & $\mathrm{~J} 1 \mathrm{~J} 409$ & $8 / 23 / 11$ & 0.179 & $\mathrm{~B}$ & 0.19 & 7.72 & & 0.19 & 3.97 & & 1.92 & 16.5 & & 0.96 & 2.18 & & 0.48 \\
\hline $100-D-28$ & $\mathrm{~J} 1 \mathrm{~J} 410$ & $8 / 23 / 11$ & 0.192 & & 0.18 & 8.30 & & 0.18 & 8.56 & & 1.79 & 34.8 & & 0.89 & 2.34 & & 0.45 \\
\hline $100-D-29$ & $\mathrm{~J} 1 \mathrm{~J} 411$ & $8 / 11 / 11$ & 0.230 & & 0.20 & 12.5 & & 0.20 & 4.61 & & 1.96 & 28.5 & & 0.98 & 7.97 & $\mathrm{~J}$ & 0.49 \\
\hline Equip blank & J1J414 & $8 / 11 / 11$ & 0.169 & $\mathrm{U}$ & 0.17 & 0.169 & U & 0.17 & 1.69 & U & 1.69 & 0.847 & $\mathrm{U}$ & 0.85 & 0.512 & $\mathrm{~J}$ & 0.42 \\
\hline
\end{tabular}




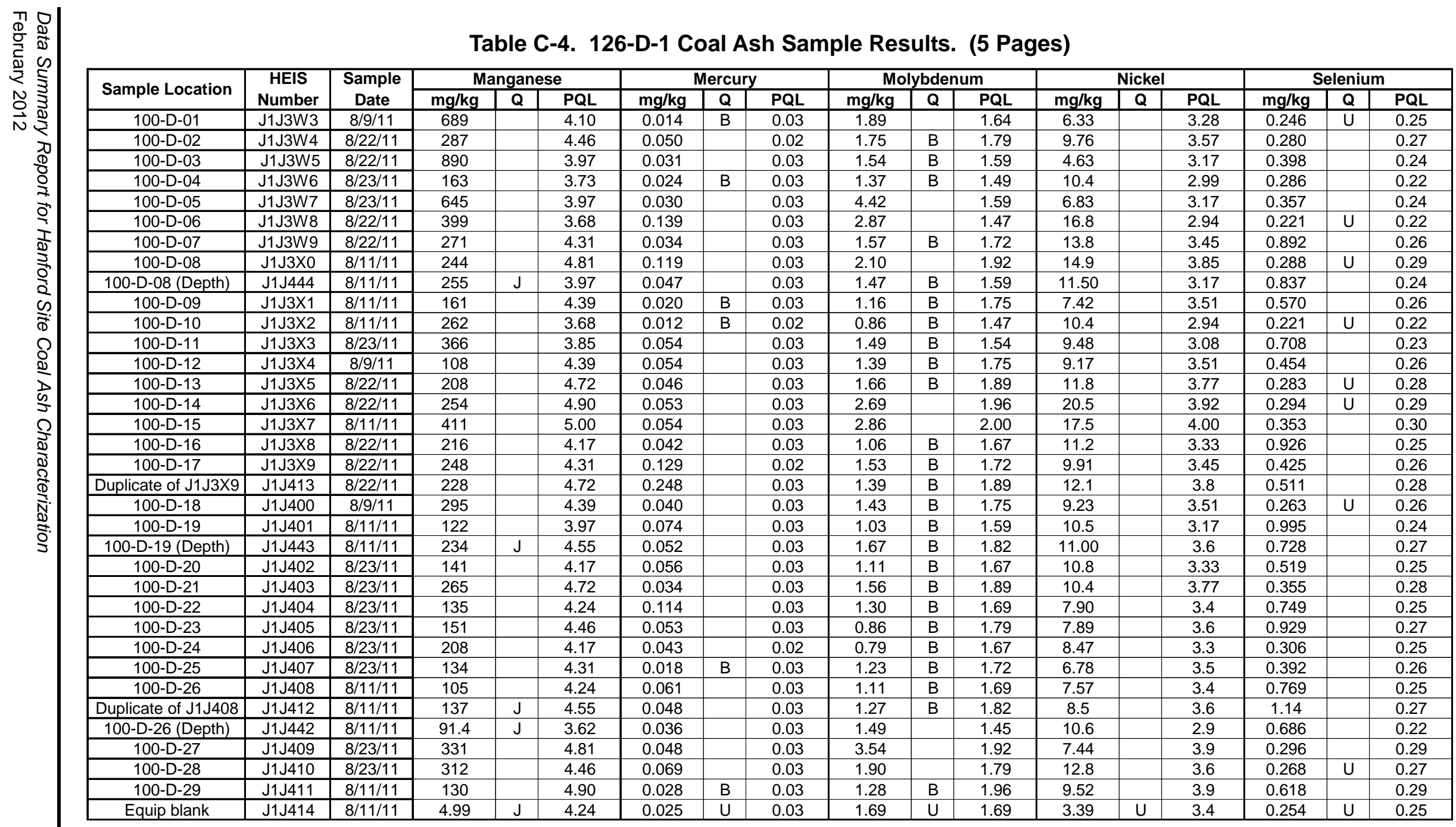


Table C-4. 126-D-1 Coal Ash Sample Results. (5 Pages)

\begin{tabular}{|c|c|c|c|c|c|c|c|c|c|c|c|c|c|c|c|c|c|}
\hline \multirow{2}{*}{ Sample Location } & \multirow{2}{*}{$\begin{array}{c}\text { HEIS } \\
\text { Number }\end{array}$} & \multirow{2}{*}{$\begin{array}{c}\text { Sample } \\
\text { Date }\end{array}$} & \multicolumn{3}{|c|}{ Silver } & \multicolumn{3}{|c|}{ Thallium } & \multicolumn{3}{|c|}{ Uranium (KPA) } & \multicolumn{3}{|c|}{ Vanadium } & \multicolumn{3}{|c|}{ Zinc } \\
\hline & & & $\mathrm{mg} / \mathrm{kg}$ & $\mathrm{Q}$ & PQL & $\mathrm{mg} / \mathrm{kg}$ & Q & PQL & $u g / g$ & $\mathrm{Q}$ & MDA & $\mathrm{mg} / \mathrm{kg}$ & Q & PQL & $\mathrm{mg} / \mathrm{kg}$ & Q & $\begin{array}{l}\mathrm{PQL} \\
\end{array}$ \\
\hline $100-\mathrm{D}-01$ & J1J3W3 & $8 / 9 / 11$ & 0.164 & $\mathrm{U}$ & 0.16 & 0.410 & UJ & 0.41 & & & & 27.9 & & 2.05 & 75.7 & $\mathrm{~J}$ & 8.20 \\
\hline 100-D-02 & J1J3W4 & $8 / 22 / 11$ & 0.179 & $U$ & 0.18 & 0.446 & $\mathrm{U}$ & 0.45 & & & & 33.4 & & 2.23 & 24.4 & & 8.93 \\
\hline 100-D-03 & J1J3W5 & $8 / 22 / 11$ & 0.159 & $U$ & 0.16 & 0.397 & $U$ & 0.40 & & & & 22.2 & & 1.98 & 31.4 & & 7.94 \\
\hline 100-D-04 & J1J3W6 & $8 / 23 / 11$ & 0.149 & $\mathrm{U}$ & 0.15 & 0.373 & $U$ & 0.37 & & & & 47.6 & & 1.87 & 33.9 & & 7.46 \\
\hline $100-D-05$ & J1J3W7 & $8 / 23 / 11$ & 0.159 & $\mathrm{U}$ & 0.16 & 0.397 & $\mathrm{U}$ & 0.40 & & & & 26.4 & & 1.98 & 43.8 & & 7.94 \\
\hline $100-\mathrm{D}-06$ & J1J3W8 & $8 / 22 / 11$ & 0.147 & $U$ & 0.15 & 0.368 & $U$ & 0.37 & & & & 25.7 & & 1.84 & 16.5 & & 7.35 \\
\hline $100-D-07$ & J1J3W9 & $8 / 22 / 11$ & 0.172 & $U$ & 0.17 & 0.431 & $\mathrm{U}$ & 0.43 & & & & 31.8 & & 2.16 & 19.0 & & 8.62 \\
\hline 100-D-08 & $\mathrm{J} 1 \mathrm{~J} 3 \times 0$ & $8 / 11 / 11$ & 0.192 & $U$ & 0.19 & 0.481 & UJ & 0.48 & 3.66 & & 0.143 & 27.6 & & 2.40 & 12.1 & $\mathrm{~J}$ & 9.62 \\
\hline 100-D-08 (Depth) & $\mathrm{J} 1 \mathrm{~J} 444$ & 8/11/11 & 0.159 & $U$ & 0.16 & 0.397 & UJ & 0.40 & 3.56 & & 0.143 & 31.2 & & 1.98 & 30.8 & $\mathrm{~J}$ & 7.94 \\
\hline 100-D-09 & J1J3X1 & $8 / 11 / 11$ & 0.175 & $\mathrm{U}$ & 0.18 & 0.439 & UJ & 0.44 & & & & 20.9 & & 2.19 & 15.0 & $\mathrm{~J}$ & 8.77 \\
\hline $100-D-10$ & $\mathrm{~J} 1 \mathrm{~J} 3 \times 2$ & 8/11/11 & 0.147 & $\mathrm{U}$ & 0.15 & 0.242 & BJ & 0.37 & & & & 52.5 & & 1.84 & 69.7 & $\mathrm{~J}$ & 7.35 \\
\hline 100-D-11 & $\mathrm{J} 1 \mathrm{~J} 3 \times 3$ & $8 / 23 / 11$ & 0.154 & $\mathrm{U}$ & 0.15 & 0.385 & $\mathrm{U}$ & 0.39 & & & & 36.4 & & 1.92 & 50.5 & & 7.69 \\
\hline $100-D-12$ & J1J3X4 & $8 / 9 / 11$ & 0.175 & $\mathrm{U}$ & 0.18 & 0.439 & UJ & 0.44 & & & & 38.9 & & 2.19 & 23.4 & $\mathrm{~J}$ & 8.77 \\
\hline $100-\mathrm{D}-13$ & J1J3X5 & $8 / 22 / 11$ & 0.189 & $U$ & 0.19 & 0.472 & $\mathrm{U}$ & 0.47 & & & & 59.7 & & 2.36 & 26.4 & & 9.43 \\
\hline $100-D-14$ & J1J3X6 & $8 / 22 / 11$ & 0.196 & $\mathrm{U}$ & 0.20 & 0.490 & $\mathrm{U}$ & 0.49 & & & & 77.5 & & 2.45 & 27.4 & & 9.80 \\
\hline $100-D-15$ & $\mathrm{J1J3X7}$ & 8/11/11 & 0.200 & $\mathrm{U}$ & 0.20 & 0.500 & UJ & 0.50 & & & & 26.6 & & 2.50 & 11.8 & $\mathrm{~J}$ & 10.0 \\
\hline $100-D-16$ & J1J3X8 & $8 / 22 / 11$ & 0.167 & $U$ & 0.17 & 0.417 & $\mathrm{U}$ & 0.42 & & & & 27.7 & & 2.08 & 31.6 & & 8.33 \\
\hline 100-D-17 & J1J3X9 & $8 / 22 / 11$ & 0.172 & $\bar{U}$ & 0.17 & 0.431 & $\mathrm{U}$ & 0.43 & & & & 34.9 & & 2.16 & 65.2 & & 8.62 \\
\hline Duplicate of $\mathrm{J1J3 \times 9}$ & J1J413 & $8 / 22 / 11$ & 0.189 & $U$ & 0.19 & 0.472 & $\mathrm{U}$ & 0.47 & & & & 38.5 & & 2.36 & 56.6 & & 9.43 \\
\hline 100-D-18 & $\mathrm{J} 1 \mathrm{~J} 400$ & $8 / 9 / 11$ & 0.175 & $\mathrm{U}$ & 0.18 & 0.439 & UJ & 0.44 & & & & 31.8 & & 2.19 & 55.5 & $\mathrm{~J}$ & 8.77 \\
\hline 100-D-19 & $\mathrm{J} 1 \mathrm{~J} 401$ & $8 / 11 / 11$ & 0.159 & $\mathrm{U}$ & 0.16 & 0.198 & BJ & 0.40 & 3.95 & & 0.143 & 38.5 & & 1.98 & 21.2 & $\mathrm{~J}$ & 7.94 \\
\hline 100-D-19 (Depth) & $\mathrm{J} 1 \mathrm{~J} 443$ & $8 / 11 / 11$ & 0.182 & $\bar{U}$ & 0.18 & 0.455 & UJ & 0.46 & 4.46 & & 0.143 & 29.7 & & 2.27 & 33.6 & $\mathrm{~J}$ & 9.09 \\
\hline $100-\mathrm{D}-20$ & J1J402 & $8 / 23 / 11$ & 0.167 & $U$ & 0.17 & 0.417 & $\mathrm{U}$ & 0.42 & & & & 45.1 & & 2.08 & 28.4 & & 8.33 \\
\hline $100-D-21$ & J1J403 & 8/23/11 & 0.189 & $\mathrm{U}$ & 0.19 & 0.472 & $\mathrm{U}$ & 0.47 & & & & 38.3 & & 2.36 & 39.5 & & 9.43 \\
\hline $100-D-22$ & $\mathrm{~J} 1 \mathrm{~J} 404$ & $8 / 23 / 11$ & 0.169 & $\mathrm{U}$ & 0.17 & 0.424 & $\mathrm{U}$ & 0.42 & & & & 33.6 & & 2.12 & 22.1 & & 8.47 \\
\hline $100-\mathrm{D}-23$ & J1J405 & $8 / 23 / 11$ & 0.179 & $\mathrm{U}$ & 0.18 & 0.446 & $\mathrm{U}$ & 0.45 & & & & 47.3 & & 2.23 & 24.9 & & 8.93 \\
\hline $100-\mathrm{D}-24$ & J1J406 & $8 / 23 / 11$ & 0.167 & $\mathrm{U}$ & 0.17 & 0.417 & $\mathrm{U}$ & 0.42 & & & & 35.5 & & 2.08 & 36.6 & & 8.33 \\
\hline $100-D-25$ & $\mathrm{~J} 1 \mathrm{~J} 407$ & $8 / 23 / 11$ & 0.172 & $U$ & 0.17 & 0.431 & $\mathrm{U}$ & 0.43 & & & & 33.3 & & 2.16 & 21.8 & & 8.62 \\
\hline $100-D-26$ & $\mathrm{~J} 1 \mathrm{~J} 408$ & $8 / 11 / 11$ & 0.169 & $U$ & 0.17 & 0.424 & UJ & 0.42 & 4.22 & & 0.143 & 33.2 & & 2.12 & 15.2 & $\mathrm{~J}$ & 8.47 \\
\hline Duplicate of J1J408 & $\mathrm{J} 1 \mathrm{~J} 412$ & $8 / 11 / 11$ & 0.182 & $U$ & 0.18 & 0.455 & UJ & 0.46 & 3.92 & & 0.143 & 41.3 & & 2.27 & 22.9 & $\mathrm{~J}$ & 9.09 \\
\hline 100-D-26 (Depth) & $\mathrm{J} 1 \mathrm{~J} 442$ & $8 / 11 / 11$ & 0.145 & $\mathrm{U}$ & 0.15 & 0.362 & UJ & 0.36 & 3.85 & & 0.143 & 41.8 & & 1.81 & 10.3 & $\mathrm{~J}$ & 7.25 \\
\hline $100-\mathrm{D}-27$ & J1J409 & $8 / 23 / 11$ & 0.192 & $U$ & 0.19 & 0.481 & $\mathrm{U}$ & 0.48 & & & & 22.5 & & 2.40 & 16.7 & & 9.62 \\
\hline $100-\mathrm{D}-28$ & J1J410 & $8 / 23 / 11$ & 0.179 & $U$ & 0.18 & 0.446 & $U$ & 0.45 & & & & 59.1 & & 2.23 & 22.5 & & 8.93 \\
\hline $100-\mathrm{D}-29$ & J1J411 & $8 / 11 / 11$ & 0.196 & $U$ & 0.20 & 0.49 & UJ & 0.49 & & & & 37.6 & & 2.45 & 37.0 & $\mathrm{~J}$ & 9.80 \\
\hline Equip blank & J1J414 & $8 / 11 / 11$ & 0.169 & $U$ & 0.17 & 0.424 & UJ & 0.42 & & & & 0.308 & $\mathrm{~B}$ & 2.12 & 1.08 & $\mathrm{~J}$ & 8.47 \\
\hline
\end{tabular}


Table C-4. 126-D-1 Coal Ash Sample Results. (5 Pages)

\begin{tabular}{|c|c|c|c|c|c|c|c|c|c|c|c|c|c|c|c|c|c|c|c|c|}
\hline \multirow{2}{*}{ Sample Location } & \multirow{2}{*}{$\begin{array}{c}\text { HEIS } \\
\text { Number }\end{array}$} & \multirow{2}{*}{$\begin{array}{c}\text { Sample } \\
\text { Date }\end{array}$} & \multicolumn{3}{|c|}{ Acenaphthene } & \multicolumn{3}{|c|}{ Acenaphthylene } & \multicolumn{3}{|c|}{ Anthracene } & \multicolumn{3}{|c|}{ Benzo(a)anthracene } & \multicolumn{3}{|c|}{ Benzo(a)pyrene } & \multicolumn{3}{|c|}{ Benzo(b)fluoranthene } \\
\hline & & & ug/kg & Q & PQL & $u g / \mathrm{kg}$ & $\mathrm{Q}$ & $\mathrm{PQL}$ & ug/kg & Q & $\mathrm{PQL}$ & ug/kg & Q & PQL & $u g / \mathrm{kg}$ & $\mathrm{Q}$ & $\mathrm{PQL}$ & ug/kg & Q & PQL \\
\hline $100-D-08$ & $\mathrm{~J} 1 \mathrm{~J} 3 \mathrm{X} \times 0$ & $8 / 11 / 11$ & 338 & UJ & 338 & 338 & UJ & 338 & 338 & $\frac{U}{U J}$ & 338 & 338 & UJ & 338 & 338 & UJ & 338 & 338 & UJ & 338 \\
\hline 100-D-08 (Depth) & $\mathrm{J1JJ444}$ & $8 / 11 / 11$ & 330 & UJ & 330 & 330 & UJ & 330 & 330 & UJ & 330 & 330 & UJ & 330 & 330 & UJ & 330 & 330 & UJ & 330 \\
\hline $100-\mathrm{D}-19$ & $\mathrm{~J} 1 \mathrm{~J} 401$ & $8 / 11 / 11$ & 337 & UDJ & 337 & 337 & UDJ & 337 & 337 & UDJ & 337 & 337 & UDJ & 337 & 337 & UDJ & 337 & 337 & UDJ & 337 \\
\hline 100-D-19 (Depth) & $\mathrm{J1J} 443$ & $8 / 11 / 11$ & 328 & UDJ & 328 & 328 & UDJ & 328 & 328 & UDJ & 328 & 328 & UDJ & 328 & 328 & UDJ & 328 & 328 & UDJ & 328 \\
\hline $100-\mathrm{D}-26$ & $\mathrm{~J} 1 \mathrm{~J} 408$ & $8 / 11 / 11$ & 339 & UDJ & 339 & 339 & UDJ & 339 & 339 & UDJ & 339 & 339 & UDJ & 339 & 339 & UDJ & 339 & 339 & UDJ & 339 \\
\hline Duplicate of J1J408 & $\mathrm{J} 1 \mathrm{~J} 412$ & $8 / 11 / 11$ & 336 & UDJ & 336 & 336 & UDJ & 336 & 336 & UDJ & 336 & 336 & UDJ & 336 & 336 & UDJ & 336 & 336 & UDJ & 336 \\
\hline 100-D-26 (Depth) & $\mathrm{J} 1 \mathrm{~J} 442$ & $8 / 11 / 11$ & 336 & UJ & 336 & 336 & UJ & 336 & 336 & UJ & 336 & 336 & UJ & 336 & 336 & UJ & 336 & 336 & UJ & 336 \\
\hline Equip blank & J1J414 & $8 / 11 / 11$ & 326 & UJ & 326 & 326 & UJ & 326 & 326 & UJ & 326 & 326 & UJ & 326 & 326 & UJ & 326 & 326 & UJ & 326 \\
\hline \multirow{2}{*}{ Sample Location } & \multirow{2}{*}{$\begin{array}{c}\text { HEIS } \\
\text { Number }\end{array}$} & \multirow{2}{*}{$\begin{array}{c}\text { Sample } \\
\text { Date }\end{array}$} & \multicolumn{3}{|c|}{ Benzo(ghi)perylene } & \multicolumn{3}{|c|}{ Benzo(k)fluoranthene } & \multicolumn{3}{|c|}{$\begin{array}{c}\text { Chrysene } \\
\end{array}$} & \multicolumn{3}{|c|}{ Dibenz(a,h)anthracene } & \multicolumn{3}{|c|}{ Fluoranthene } & \multicolumn{3}{|c|}{ Fluorene } \\
\hline & & & ug $/ \mathrm{kg}$ & $\mathrm{Q}$ & $\mathrm{PQL}$ & $u g / k g$ & $\mathrm{Q}$ & $\mathrm{PQL}$ & ug/kg & $\mathrm{Q}$ & $\mathrm{PQL}$ & ug/kg & $Q$ & $\mathrm{PQL}$ & $u g / \mathbf{k g}$ & $\mathrm{Q}$ & $\mathrm{PQL}$ & $u g / \mathrm{kg}$ & $Q$ & PQL \\
\hline $100-\mathrm{D}-08$ & $\mathrm{~J} 1 \mathrm{~J} 3 \mathrm{X} 0$ & $8 / 11 / 11$ & 338 & UJ & 338 & 338 & UJ & 338 & 338 & UJ & 338 & 338 & UJ & 338 & 338 & UJ & 338 & 338 & UJ & 338 \\
\hline 100-D-08 (Depth) & $\mathrm{J} 1 \mathrm{~J} 444$ & $8 / 11 / 11$ & 330 & UJ & 330 & 330 & UJ & 330 & 330 & UJ & 330 & 330 & UJ & 330 & 330 & UJ & 330 & 330 & UJ & 330 \\
\hline $100-\mathrm{D}-19$ & $\mathrm{~J} 1 \mathrm{~J} 401$ & $8 / 11 / 11$ & 337 & UDJ & 337 & 337 & UDJ & 337 & 337 & UDJ & 337 & 337 & UDJ & 337 & 337 & UDJ & 337 & 337 & UDJ & 337 \\
\hline 100-D-19 (Depth) & J1J443 & $8 / 11 / 11$ & 328 & UDJ & 328 & 328 & UDJ & 328 & 328 & UDJ & 328 & 328 & UDJ & 328 & 328 & UDJ & 328 & 328 & UDJ & 328 \\
\hline $100-D-26$ & J1J408 & $8 / 11 / 11$ & 339 & UDJ & 339 & 339 & UDJ & 339 & 339 & UDJ & 339 & 339 & UDJ & 339 & 339 & UDJ & 339 & 339 & UDJ & 339 \\
\hline Duplicate of J1J408 & $\mathrm{J} 1 \mathrm{~J} 412$ & $8 / 11 / 11$ & 336 & UDJ & 336 & 336 & UDJ & 336 & 336 & UDJ & 336 & 336 & UDJ & 336 & 336 & UDJ & 336 & 336 & UDJ & 336 \\
\hline 100-D-26 (Depth) & $\mathrm{J} 1 \mathrm{~J} 442$ & $8 / 11 / 11$ & 336 & UJ & 336 & 336 & UJ & 336 & 336 & UJ & 336 & 336 & UJ & 336 & 336 & UJ & 336 & 336 & UJ & 336 \\
\hline Equip blank & J1J414 & $8 / 11 / 11$ & 326 & UJ & 326 & 326 & UJ & 326 & 326 & UJ & 326 & 326 & UJ & 326 & 326 & UJ & 326 & 326 & UJ & 326 \\
\hline
\end{tabular}

\begin{tabular}{|c|c|c|c|c|c|c|c|c|c|c|c|c|c|c|}
\hline \multirow{2}{*}{ Sample Location } & \multirow{2}{*}{$\begin{array}{c}\text { HEIS } \\
\text { Number }\end{array}$} & \multirow{2}{*}{$\begin{array}{c}\text { Sample } \\
\text { Date }\end{array}$} & \multicolumn{3}{|c|}{ Indeno(1,2,3-cd)pyrene } & \multicolumn{3}{|c|}{ Naphthalene } & \multicolumn{3}{|c|}{ Phenanthrene } & \multicolumn{3}{|c|}{ Pyrene } \\
\hline & & & $u g / k g$ & $\mathbf{Q}$ & $\mathrm{PQL}$ & $u g / \mathrm{kg}$ & Q & $\mathrm{PQL}$ & $u g / k g$ & Q & $\mathrm{PQL}$ & $u g / \mathrm{kg}$ & Q & $\mathrm{PQL}$ \\
\hline $100-D-08$ & $\mathrm{J1} 1 \mathrm{~J} 3 \times 0$ & $8 / 11 / 11$ & 338 & UJ & 338 & 338 & UJ & 338 & 338 & UJ & 338 & 338 & UJ & 338 \\
\hline 100-D-08 (Depth) & $\mathrm{J} 1 \mathrm{~J} 444$ & $8 / 11 / 11$ & 330 & UJ & 330 & 330 & UJ & 330 & 330 & UJ & 330 & 330 & UJ & 330 \\
\hline $100-D-19$ & $\mathrm{~J} 1 \mathrm{~J} 401$ & $8 / 11 / 11$ & 337 & UDJ & 337 & 369 & DJ & 1310 & 259 & $\mathrm{JD}$ & 1310 & 337 & UDJ & 337 \\
\hline 100-D-19 (Depth) & J1J443 & $8 / 11 / 11$ & 328 & UDJ & 328 & 253 & DJ & 1270 & 328 & UDJ & 328 & 328 & UDJ & 328 \\
\hline $100-\mathrm{D}-26$ & $\mathrm{J1J} 408$ & $8 / 11 / 11$ & 339 & UDJ & 339 & 1070 & DJ & 1320 & 281 & DJ & 1320 & 339 & UDJ & 339 \\
\hline Duplicate of J1J408 & $\mathrm{J1J412}$ & $8 / 11 / 11$ & 336 & UDJ & 336 & 336 & UDJ & 336 & 336 & UDJ & 336 & 336 & UDJ & 336 \\
\hline 100-D-26 (Depth) & $\mathrm{J} 1 \mathrm{~J} 442$ & $8 / 11 / 11$ & 336 & UJ & 336 & 143 & $\mathrm{~J}$ & 616 & 336 & UJ & 336 & 336 & UJ & 336 \\
\hline Equip blank & $\mathrm{J} 1 \mathrm{~J} 414$ & $8 / 11 / 11$ & 326 & UJ & 326 & 326 & UJ & 326 & 326 & UJ & 326 & 326 & UJ & 326 \\
\hline
\end{tabular}


Table C-5. 126-D-1 Coal Ash Pre-Leaching Quadruplicate Sample Results. (2 Pages)

\begin{tabular}{|c|c|c|c|c|c|c|c|c|c|c|c|c|c|c|c|c|c|}
\hline \multirow{2}{*}{ Sample Location } & \multirow{2}{*}{$\begin{array}{c}\text { HEIS } \\
\text { Number }\end{array}$} & \multirow{2}{*}{$\begin{array}{c}\text { Sample } \\
\text { Date }\end{array}$} & \multicolumn{3}{|c|}{ Antimony } & \multicolumn{3}{|c|}{ Arsenic } & \multicolumn{3}{|c|}{ Barium } & \multicolumn{3}{|c|}{ Beryllium } & \multicolumn{3}{|c|}{ Boron } \\
\hline & & & $\mathrm{mg} / \mathrm{kg}$ & $\mathbf{Q}$ & PQL & $\mathrm{mg} / \mathrm{kg}$ & $\mathbf{Q}$ & PQL & $\mathrm{mg} / \mathrm{kg}$ & $\mathbf{Q}$ & PQL & $\mathrm{mg} / \mathrm{kg}$ & Q & $\mathrm{PQL}$ & $\mathrm{mg} / \mathrm{kg}$ & $\mathbf{Q}$ & PQL \\
\hline 100-D-26 (Depth) & J1J442-1 & $8 / 11 / 11$ & 0.492 & $\mathrm{U}$ & 0.49 & 3.51 & & 0.82 & 1010 & & 0.41 & 1.16 & & 0.16 & 173 & & 1.64 \\
\hline 100-D-26 (Depth) & $\mathrm{J} 1 \mathrm{~J} 442-2$ & $8 / 11 / 11$ & 0.448 & $\mathrm{U}$ & 0.45 & 3.34 & & 0.75 & 990 & & 0.37 & 1.11 & & 0.15 & 180 & & 1.49 \\
\hline 100-D-26 (Depth) & J1J442-3 & $8 / 11 / 11$ & 0.588 & $\mathrm{U}$ & 0.59 & 3.31 & & 0.98 & 1140 & & 0.49 & 1.25 & & 0.20 & 207 & & 1.96 \\
\hline 100-D-26 (Depth) & $\mathrm{J} 1 \mathrm{~J} 442-4$ & $8 / 11 / 11$ & 0.469 & U & 0.47 & 2.83 & & 0.78 & 871 & & 0.39 & 1.09 & & 0.16 & 155 & & 1.56 \\
\hline 100-D-19 (Depth) & $\mathrm{J} 1 \mathrm{~J} 443-1$ & $8 / 11 / 11$ & 0.526 & U & 0.53 & 8.66 & & 0.88 & 1150 & & 0.44 & 1.48 & & 0.18 & 230 & & 1.75 \\
\hline 100-D-19 (Depth) & $\mathrm{J} 1 \mathrm{~J} 443-2$ & $8 / 11 / 11$ & 0.435 & $\mathrm{U}$ & 0.44 & 9.28 & & 0.73 & 1140 & & 0.36 & 1.29 & & 0.15 & 187 & & 1.45 \\
\hline 100-D-19 (Depth) & J1J443-3 & $8 / 11 / 11$ & 0.556 & $\mathrm{U}$ & 0.56 & 8.04 & & 0.93 & 1180 & & 0.46 & 1.44 & & 0.19 & 210 & & 1.85 \\
\hline 100-D-19 (Depth) & J1J443-4 & $8 / 11 / 11$ & 0.536 & $\mathrm{U}$ & 0.54 & 7.68 & & 0.89 & 1240 & & 0.45 & 1.48 & & 0.18 & 238 & & 1.79 \\
\hline 100-D-08 (Depth) & J1J444-1 & $8 / 11 / 11$ & 0.545 & $\mathrm{U}$ & 0.55 & 2.33 & & 0.91 & 1030 & & 0.46 & 1.57 & & 0.18 & 445 & & 1.82 \\
\hline 100-D-08 (Depth) & J1J444-2 & $8 / 11 / 11$ & 0.476 & $\mathrm{U}$ & 0.48 & 2.35 & & 0.79 & 1140 & & 0.40 & 1.41 & & 0.16 & 463 & & 1.59 \\
\hline 100-D-08 (Depth) & J1J444-3 & $8 / 11 / 11$ & 0.545 & $\mathrm{U}$ & 0.55 & 2.55 & & 0.91 & 1100 & & 0.46 & 1.35 & & 0.18 & 408 & & 1.82 \\
\hline 100-D-08 (Depth) & $\mathrm{J} 1 \mathrm{~J} 444-4$ & $8 / 11 / 11$ & 0.600 & $\mathrm{U}$ & 0.60 & 2.64 & & 1.00 & 1150 & & 0.50 & 1.56 & & 0.20 & 468 & & 2.00 \\
\hline \multirow{2}{*}{ Sample Location } & HEIS & Sample & \multicolumn{3}{|c|}{ Cadmium } & \multicolumn{3}{|c|}{ Chromium } & \multicolumn{3}{|c|}{ Cobalt } & \multicolumn{3}{|c|}{ Copper } & \multicolumn{3}{|c|}{ Lead } \\
\hline & Number & Date & $\mathrm{mg} / \mathrm{kg}$ & $\mathbf{Q}$ & PQL & $\mathrm{mg} / \mathrm{kg}$ & $\mathbf{Q}$ & PQL & $\mathrm{mg} / \mathrm{kg}$ & Q & PQL & $\mathrm{mg} / \mathrm{kg}$ & Q & $\mathrm{PQL}$ & $\mathrm{mg} / \mathrm{kg}$ & $\mathbf{Q}$ & PQL \\
\hline 100-D-26 (Depth) & J1J442-1 & $8 / 11 / 11$ & 0.078 & $\mathrm{~B}$ & 0.16 & 9.67 & & 0.16 & 6.11 & & 1.64 & 35.2 & & 0.82 & 3.40 & & 0.41 \\
\hline 100-D-26 (Depth) & $\mathrm{J} 1 \mathrm{~J} 442-2$ & $8 / 11 / 11$ & 0.091 & $B$ & 0.15 & 10.3 & & 0.15 & 5.05 & & 1.49 & 36.1 & & 0.75 & 3.99 & & 0.37 \\
\hline 100-D-26 (Depth) & $\mathrm{J} 1 \mathrm{~J} 442-3$ & $8 / 11 / 11$ & 0.090 & $\mathrm{~B}$ & 0.20 & 10.5 & & 0.20 & 5.48 & & 1.96 & 39.2 & & 0.98 & 3.37 & & 0.49 \\
\hline 100-D-26 (Depth) & $\mathrm{J1J} 442-4$ & $8 / 11 / 11$ & 0.079 & $\mathrm{~B}$ & 0.16 & 8.91 & & 0.16 & 5.11 & & 1.56 & 32.0 & & 0.78 & 3.52 & & 0.39 \\
\hline 100-D-19 (Depth) & J1J443-1 & $8 / 11 / 11$ & 0.243 & & 0.18 & 9.35 & & 0.18 & 5.35 & & 1.75 & 29.1 & & 0.88 & 7.60 & & 0.44 \\
\hline 100-D-19 (Depth) & J1J443-2 & $8 / 11 / 11$ & 0.217 & & 0.15 & 8.72 & & 0.15 & 5.45 & & 1.45 & 25.9 & & 0.73 & 4.08 & & 0.36 \\
\hline 100-D-19 (Depth) & J1J443-3 & $8 / 11 / 11$ & 0.219 & & 0.19 & 8.36 & & 0.19 & 4.97 & & 1.85 & 26.5 & & 0.93 & 3.76 & & 0.46 \\
\hline 100-D-19 (Depth) & J1J443-4 & $8 / 11 / 11$ & 0.272 & & 0.18 & 9.46 & & 0.18 & 4.84 & & 1.79 & 27.6 & & 0.89 & 4.48 & & 0.45 \\
\hline 100-D-08 (Depth) & J1J444-1 & $8 / 11 / 11$ & 0.171 & B & 0.18 & 7.04 & & 0.18 & 6.81 & & 1.82 & 11.7 & & 0.91 & 3.66 & & 0.46 \\
\hline 100-D-08 (Depth) & $\mathrm{J1J444-2}$ & $8 / 11 / 11$ & 0.180 & & 0.16 & 7.30 & & 0.16 & 7.24 & & 1.59 & 12.5 & & 0.79 & 3.29 & & 0.40 \\
\hline 100-D-08 (Depth) & J1J444-3 & $8 / 11 / 11$ & 0.171 & $B$ & 0.18 & 7.14 & & 0.18 & 7.02 & & 1.82 & 15.7 & & 0.91 & 3.48 & & 0.46 \\
\hline 100-D-08 (Depth) & J1J444-4 & $8 / 11 / 11$ & 0.183 & B & 0.20 & 7.94 & & 0.20 & 7.77 & & 2.00 & 13.9 & & 1.00 & 3.36 & & 0.50 \\
\hline
\end{tabular}


Table C-5. 126-D-1 Coal Ash Pre-Leaching Quadruplicate Sample Results. (2 Pages)

\begin{tabular}{|c|c|c|c|c|c|c|c|c|c|c|c|c|c|c|c|c|c|}
\hline \multirow{2}{*}{ Sample Location } & \multirow{2}{*}{$\begin{array}{c}\text { HEIS } \\
\text { Number }\end{array}$} & \multirow{2}{*}{$\begin{array}{c}\text { Sample } \\
\text { Date }\end{array}$} & \multicolumn{3}{|c|}{ Manganese } & \multicolumn{3}{|c|}{ Mercury } & \multicolumn{3}{|c|}{ Molybdenum } & \multicolumn{3}{|c|}{ Nickel } & \multicolumn{3}{|c|}{ Selenium } \\
\hline & & & $\mathrm{mg} / \mathrm{kg}$ & Q & PQL & $\mathrm{mg} / \mathrm{kg}$ & $\mathbf{Q}$ & PQL & $\mathrm{mg} / \mathrm{kg}$ & Q & PQL & $\mathrm{mg} / \mathrm{kg}$ & $\mathbf{Q}$ & PQL & mg/kg & $\mathbf{Q}$ & PQL \\
\hline 100-D-26 (Depth) & J1J442-1 & $8 / 11 / 11$ & 110 & & 4.10 & 0.027 & & 0.03 & 1.60 & B & 1.64 & 12.9 & & 3.28 & 0.465 & & 0.25 \\
\hline 100-D-26 (Depth) & J1J442-2 & $8 / 11 / 11$ & 122 & & 3.73 & 0.028 & & 0.03 & 1.58 & & 1.49 & 12.2 & & 2.99 & 0.528 & & 0.22 \\
\hline 100-D-26 (Depth) & $\mathrm{J} 1 \mathrm{~J} 442-3$ & $8 / 11 / 11$ & 111 & & 4.90 & 0.023 & B & 0.03 & 1.82 & $B$ & 1.96 & 12.3 & & 3.92 & 0.469 & & 0.29 \\
\hline 100-D-26 (Depth) & J1J442-4 & $8 / 11 / 11$ & 115 & & 3.91 & 0.042 & & 0.02 & 1.42 & B & 1.56 & 11.6 & & 3.12 & 0.586 & & 0.23 \\
\hline 100-D-19 (Depth) & J1J443-1 & $8 / 11 / 11$ & 211 & & 4.39 & 0.043 & & 0.03 & 1.77 & & 1.75 & 11.6 & & 3.51 & 0.931 & & 0.26 \\
\hline 100-D-19 (Depth) & $\mathrm{J} 1 \mathrm{~J} 443-2$ & $8 / 11 / 11$ & 182 & & 3.62 & 0.048 & & 0.02 & 1.55 & & 1.45 & 11.6 & & 2.90 & 0.709 & & 0.22 \\
\hline 100-D-19 (Depth) & J1J443-3 & $8 / 11 / 11$ & 208 & & 4.63 & 0.057 & & 0.02 & 1.81 & B & 1.85 & 10.9 & & 3.70 & 1.06 & & 0.28 \\
\hline 100-D-19 (Depth) & J1J443-4 & $8 / 11 / 11$ & 190 & & 4.46 & 0.053 & & 0.02 & 2.03 & & 1.79 & 10.9 & & 3.57 & 0.789 & & 0.27 \\
\hline 100-D-08 (Depth) & J1J444-1 & $8 / 11 / 11$ & 264 & & 4.55 & 0.049 & & 0.03 & 1.59 & B & 1.82 & 11.1 & & 3.64 & 0.789 & & 0.27 \\
\hline 100-D-08 (Depth) & J1J444-2 & $8 / 11 / 11$ & 283 & & 3.97 & 0.055 & & 0.03 & 1.47 & $B$ & 1.59 & 11.5 & & 3.17 & 0.702 & & 0.24 \\
\hline 100-D-08 (Depth) & J1J444-3 & $8 / 11 / 11$ & 275 & & 4.55 & 0.027 & & 0.03 & 1.56 & B & 1.82 & 11.8 & & 3.64 & 0.919 & & 0.27 \\
\hline 100-D-08 (Depth) & J1J444-4 & $8 / 11 / 11$ & 276 & & 5.00 & 0.034 & & 0.03 & 1.87 & $B$ & 2.00 & 12.5 & & 4.00 & 0.832 & & 0.30 \\
\hline
\end{tabular}

\begin{tabular}{|c|c|c|c|c|c|c|c|c|c|c|c|c|c|c|}
\hline \multirow{2}{*}{ Sample Location } & \multirow{2}{*}{$\begin{array}{c}\text { HEIS } \\
\text { Number }\end{array}$} & \multirow{2}{*}{$\begin{array}{c}\text { Sample } \\
\text { Date }\end{array}$} & \multicolumn{3}{|c|}{ Silver } & \multicolumn{3}{|c|}{ Thallium } & \multicolumn{3}{|c|}{ Vanadium } & \multicolumn{3}{|c|}{ Zinc } \\
\hline & & & $\mathrm{mg} / \mathrm{kg}$ & $\mathbf{Q}$ & PQL & $\mathrm{mg} / \mathrm{kg}$ & $\mathbf{Q}$ & PQL & $\mathrm{mg} / \mathrm{kg}$ & $\mathbf{Q}$ & PQL & $\mathrm{mg} / \mathrm{kg}$ & Q & PQL \\
\hline 100-D-26 (Depth) & J1J442-1 & $8 / 11 / 11$ & 0.164 & $\mathrm{U}$ & 0.16 & 0.410 & $\dot{U}$ & 0.41 & 49.7 & & 2.05 & 11.4 & & 8.20 \\
\hline 100-D-26 (Depth) & $\mathrm{J} 1 \mathrm{~J} 442-2$ & $8 / 11 / 11$ & 0.149 & $\mathrm{U}$ & 0.15 & 0.373 & $U$ & 0.37 & 47.8 & & 1.87 & 13.4 & & 7.46 \\
\hline 100-D-26 (Depth) & $\mathrm{J} 1 \mathrm{~J} 442-3$ & $8 / 11 / 11$ & 0.196 & $\mathrm{U}$ & 0.20 & 0.490 & $\mathrm{U}$ & 0.49 & 51.1 & & 2.45 & 14.2 & & 9.80 \\
\hline 100-D-26 (Depth) & J1J442-4 & $8 / 11 / 11$ & 0.156 & $U$ & 0.16 & 0.391 & $U$ & 0.39 & 42.6 & & 1.95 & 23.7 & & 7.81 \\
\hline 100-D-19 (Depth) & J1J443-1 & $8 / 11 / 11$ & 0.175 & $\mathrm{U}$ & 0.18 & 0.439 & $U$ & 0.44 & 35.1 & & 2.19 & 40.3 & & 8.77 \\
\hline 100-D-19 (Depth) & J1J443-2 & $8 / 11 / 11$ & 0.145 & $\mathrm{U}$ & 0.15 & 0.362 & $U$ & 0.36 & 32.0 & & 1.81 & 36.4 & & 7.25 \\
\hline 100-D-19 (Depth) & J1J443-3 & $8 / 11 / 11$ & 0.185 & $U$ & 0.19 & 0.463 & $U$ & 0.46 & 32.3 & & 2.31 & 37.5 & & 9.26 \\
\hline 100-D-19 (Depth) & J1J443-4 & $8 / 11 / 11$ & 0.179 & $\mathrm{U}$ & 0.18 & 0.446 & $U$ & 0.45 & 34.9 & & 2.23 & 43.0 & & 8.93 \\
\hline 100-D-08 (Depth) & J1J444-1 & 8/11/11 & 0.182 & $U$ & 0.18 & 0.455 & $U$ & 0.46 & 25.2 & & 2.27 & 28.9 & & 9.09 \\
\hline 100-D-08 (Depth) & J1J444-2 & $8 / 11 / 11$ & 0.159 & $U$ & 0.16 & 0.397 & $U$ & 0.40 & 27.1 & & 1.98 & 34.9 & & 7.94 \\
\hline 100-D-08 (Depth) & J1J444-3 & $8 / 11 / 11$ & 0.182 & $\mathrm{U}$ & 0.18 & 0.455 & $U$ & 0.46 & 27.4 & & 2.27 & 30.6 & & 9.09 \\
\hline 100-D-08 (Depth) & J1J444-4 & $8 / 11 / 11$ & 0.200 & $U$ & 0.20 & 0.500 & $U$ & 0.50 & 29.0 & & 2.50 & 29.8 & & 10.0 \\
\hline
\end{tabular}


Table C-6. 126-D-1 Coal Ash Leaching Sample Results. (2 Pages)

\begin{tabular}{|c|c|c|c|c|c|c|c|c|c|c|c|c|c|c|c|c|c|c|}
\hline \multirow{2}{*}{ Sample Location } & \multirow{2}{*}{$\begin{array}{c}\text { Coal Ash:Water } \\
\text { Ratio }\end{array}$} & \multirow{2}{*}{$\begin{array}{c}\text { HEIS } \\
\text { Number }\end{array}$} & \multirow{2}{*}{$\begin{array}{c}\text { Sample } \\
\text { Date }\end{array}$} & \multicolumn{3}{|c|}{ Antimony } & \multicolumn{3}{|c|}{ Arsenic } & \multicolumn{3}{|c|}{ Barium } & \multicolumn{3}{|c|}{ Beryllium } & \multicolumn{3}{|c|}{ Boron } \\
\hline & & & & $\mathrm{mg} / \mathrm{L}$ & $\mathbf{Q}$ & PQL & $\mathrm{mg} / \mathrm{L}$ & $\mathbf{Q}$ & PQL & $\mathrm{mg} / \mathrm{L}$ & $\mathbf{Q}$ & $\mathrm{PQL}$ & $\mathrm{mg} / \mathrm{L}$ & $\mathrm{Q}$ & PQL & $\mathrm{mg} / \mathrm{L}$ & $\mathbf{Q}$ & $\mathrm{PQL}$ \\
\hline 100-D-26 (Depth) & $1: 1$ & J1J442-A1 & $8 / 11 / 11$ & 0.100 & $\mathrm{U}$ & 0.10 & 0.075 & $\mathrm{U}$ & 0.08 & 0.048 & & 0.01 & 0.005 & $\mathrm{U}$ & 0.01 & 13.5 & & 0.08 \\
\hline 100-D-26 (Depth) & $1: 1$ & J1J442-A2 & $8 / 11 / 11$ & 0.100 & $\mathrm{U}$ & 0.10 & 0.075 & $\mathrm{U}$ & 0.08 & 0.19 & & 0.01 & 0.005 & $U$ & 0.01 & 14.7 & & 0.08 \\
\hline 100-D-26 (Depth) & $1: 2.5$ & J1J442-B1 & $8 / 11 / 11$ & 0.100 & $U$ & 0.10 & 0.075 & $\mathrm{U}$ & 0.08 & 0.041 & & 0.01 & 0.005 & $U$ & 0.01 & 8.56 & & 0.08 \\
\hline 100-D-26 (Depth) & $1: 5$ & J1J442-C1 & $8 / 11 / 11$ & 0.100 & $\mathrm{U}$ & 0.10 & 0.075 & $\mathrm{U}$ & 0.08 & 0.037 & & 0.01 & 0.005 & $U$ & 0.01 & 4.54 & & 0.08 \\
\hline 100-D-19 (Depth) & $1: 1$ & J1J443-A1 & $8 / 11 / 11$ & 0.100 & $\mathrm{U}$ & 0.10 & 0.075 & $\mathrm{U}$ & 0.08 & 0.046 & & 0.01 & 0.005 & $U$ & 0.01 & 20.2 & & 0.08 \\
\hline 100-D-19 (Depth) & $1: 2.5$ & J1J443-B1 & $8 / 11 / 11$ & 0.100 & $U$ & 0.10 & 0.075 & $\mathrm{U}$ & 0.08 & 0.032 & & 0.01 & 0.005 & $U$ & 0.01 & 12.7 & & 0.08 \\
\hline 100-D-19 (Depth) & $1: 2.5$ & J1J443-B2 & $8 / 11 / 11$ & 0.100 & $\mathrm{U}$ & 0.10 & 0.075 & $\mathrm{U}$ & 0.08 & 0.033 & & 0.01 & 0.005 & $U$ & 0.01 & 12.6 & & 0.08 \\
\hline 100-D-19 (Depth) & $1: 5$ & J1J443-C1 & $8 / 11 / 11$ & 0.100 & $\mathrm{U}$ & 0.10 & 0.075 & $\mathrm{U}$ & 0.08 & 0.031 & & 0.01 & 0.005 & $U$ & 0.01 & 6.74 & & 0.08 \\
\hline 100-D-08 (Depth) & $1: 1$ & J1J444-A1 & $8 / 11 / 11$ & 0.100 & $\mathrm{U}$ & 0.10 & 0.075 & $\mathrm{U}$ & 0.08 & 0.032 & & 0.01 & 0.005 & $U$ & 0.01 & 49.9 & & 0.08 \\
\hline 100-D-08 (Depth) & $1: 2.5$ & J1J444-B1 & $8 / 11 / 11$ & 0.100 & $U$ & 0.10 & 0.075 & $\mathrm{U}$ & 0.08 & 0.029 & & 0.01 & 0.005 & $U$ & 0.01 & 47.5 & & 0.08 \\
\hline 100-D-08 (Depth) & $1: 5$ & J1J444-C1 & $8 / 11 / 11$ & 0.100 & $\mathrm{U}$ & 0.10 & 0.075 & $\mathrm{U}$ & 0.08 & 0.024 & & 0.01 & 0.005 & $\mathrm{U}$ & 0.01 & 28.1 & & 0.08 \\
\hline 100-D-08 (Depth) & $1: 5$ & J1J444-C2 & $8 / 11 / 11$ & 0.100 & $\mathrm{U}$ & 0.10 & 0.075 & $\mathrm{U}$ & 0.08 & 0.023 & & 0.01 & 0.005 & $U$ & 0.01 & 30.3 & & 0.08 \\
\hline
\end{tabular}

\begin{tabular}{|c|c|c|c|c|c|c|c|c|c|c|c|c|c|c|c|c|c|c|}
\hline \multirow{2}{*}{ Sample Location } & \multirow{2}{*}{$\begin{array}{c}\text { Coal Ash:Water } \\
\text { Ratio }\end{array}$} & \multirow{2}{*}{$\begin{array}{c}\text { HEIS } \\
\text { Number }\end{array}$} & \multirow{2}{*}{$\begin{array}{c}\text { Sample } \\
\text { Date }\end{array}$} & \multicolumn{3}{|c|}{ Cadmium } & \multicolumn{3}{|c|}{ Chromium } & \multicolumn{3}{|c|}{ Cobalt } & \multicolumn{3}{|c|}{ Copper } & \multicolumn{3}{|c|}{ Lead } \\
\hline & & & & $\mathrm{mg} / \mathrm{L}$ & $\mathbf{Q}$ & PQL & $\mathrm{mg} / \mathrm{L}$ & $\mathbf{Q}$ & $\mathrm{PQL}$ & $\mathrm{mg} / \mathrm{L}$ & $\mathrm{Q}$ & PQL & $\mathrm{mg} / \mathrm{L}$ & $\mathrm{Q}$ & $\mathrm{PQL}$ & $\mathrm{mg} / \mathrm{L}$ & $\mathrm{Q}$ & PQL \\
\hline 100-D-26 (Depth) & $1: 1$ & J1J442-A1 & $8 / 11 / 11$ & 0.015 & $U$ & 0.02 & 0.015 & $B$ & 0.03 & 0.010 & $\mathrm{U}$ & 0.01 & 0.100 & $\mathrm{U}$ & 0.10 & 0.05 & $U$ & 0.05 \\
\hline 100-D-26 (Depth) & $1: 1$ & $\mathrm{~J} 1 \mathrm{~J} 442-\mathrm{A} 2$ & $8 / 11 / 11$ & 0.015 & $U$ & 0.02 & 0.007 & B & 0.03 & 0.010 & $\mathrm{U}$ & 0.01 & 0.100 & $U$ & 0.10 & 0.05 & $U$ & 0.05 \\
\hline 100-D-26 (Depth) & $1: 2.5$ & J1J442-B1 & 8/11/11 & 0.015 & $U$ & 0.02 & 0.025 & $U$ & 0.03 & 0.010 & $\mathrm{U}$ & 0.01 & 0.100 & $U$ & 0.10 & 0.05 & $U$ & 0.05 \\
\hline 100-D-26 (Depth) & $1: 5$ & J1J442-C1 & $8 / 11 / 11$ & 0.015 & $U$ & 0.02 & 0.025 & $\mathrm{U}$ & 0.03 & 0.010 & $\mathrm{U}$ & 0.01 & 0.100 & $U$ & 0.10 & 0.05 & $U$ & 0.05 \\
\hline 100-D-19 (Depth) & $1: 1$ & J1J443-A1 & $8 / 11 / 11$ & 0.015 & $U$ & 0.02 & 0.003 & B & 0.03 & 0.010 & $\mathrm{U}$ & 0.01 & 0.100 & $U$ & 0.10 & 0.05 & $U$ & 0.05 \\
\hline 100-D-19 (Depth) & $1: 2.5$ & J1J443-B1 & $8 / 11 / 11$ & 0.015 & $U$ & 0.02 & 0.025 & $\mathrm{U}$ & 0.03 & 0.010 & $\mathrm{U}$ & 0.01 & 0.100 & $U$ & 0.10 & 0.05 & $\mathrm{U}$ & 0.05 \\
\hline 100-D-19 (Depth) & $1: 2.5$ & J1J443-B2 & $8 / 11 / 11$ & 0.015 & $\mathrm{U}$ & 0.02 & 0.025 & $\mathrm{U}$ & 0.03 & 0.010 & $\mathrm{U}$ & 0.01 & 0.100 & $U$ & 0.10 & 0.05 & $\mathrm{U}$ & 0.05 \\
\hline 100-D-19 (Depth) & $1: 5$ & J1J443-C1 & $8 / 11 / 11$ & 0.015 & $U$ & 0.02 & 0.025 & $U$ & 0.03 & 0.010 & $\mathrm{U}$ & 0.01 & 0.100 & $U$ & 0.10 & 0.05 & $U$ & 0.05 \\
\hline 100-D-08 (Depth) & $1: 1$ & J1J444-A1 & $8 / 11 / 11$ & 0.015 & $U$ & 0.02 & 0.010 & B & 0.03 & 0.010 & $\mathrm{U}$ & 0.01 & 0.100 & $U$ & 0.10 & 0.05 & $U$ & 0.05 \\
\hline 100-D-08 (Depth) & $1: 2.5$ & J1J444-B1 & $8 / 11 / 11$ & 0.015 & $U$ & 0.02 & 0.025 & $U$ & 0.03 & 0.010 & $\mathrm{U}$ & 0.01 & 0.100 & $U$ & 0.10 & 0.05 & $\mathrm{U}$ & 0.05 \\
\hline 100-D-08 (Depth) & $1: 5$ & \begin{tabular}{|l|}
$\mathrm{J} 1 \mathrm{~J} 444-\mathrm{C} 1$ \\
\end{tabular} & $8 / 11 / 11$ & 0.015 & $U$ & 0.02 & 0.025 & $U$ & 0.03 & 0.010 & $U$ & 0.01 & 0.100 & $U$ & 0.10 & 0.05 & $U$ & 0.05 \\
\hline 100-D-08 (Depth) & $1: 5$ & \begin{tabular}{|l|}
$\mathrm{J} 1 \mathrm{~J} 444-\mathrm{C} 2$ \\
\end{tabular} & $8 / 11 / 11$ & 0.015 & $U$ & 0.02 & 0.025 & $U$ & 0.03 & 0.010 & $\mathrm{U}$ & 0.01 & 0.100 & $U$ & 0.10 & 0.05 & $U$ & 0.05 \\
\hline
\end{tabular}


Table C-6. 126-D-1 Coal Ash Leaching Sample Results. (2 Pages)

\begin{tabular}{|c|c|c|c|c|c|c|c|c|c|c|c|c|c|c|c|c|c|c|}
\hline \multirow{2}{*}{ Sample Location } & \multirow{2}{*}{$\begin{array}{l}\text { Coal Ash:Water } \\
\text { Ratio }\end{array}$} & \multirow{2}{*}{$\begin{array}{c}\text { HEIS } \\
\text { Number }\end{array}$} & \multirow{2}{*}{$\begin{array}{l}\text { Sample } \\
\text { Date }\end{array}$} & \multicolumn{3}{|c|}{ Manganese } & \multicolumn{3}{|c|}{ Mercury } & \multicolumn{3}{|c|}{ Molybdenum } & \multicolumn{3}{|c|}{ Nickel } & \multicolumn{3}{|c|}{ Selenium } \\
\hline & & & & $\mathrm{mg} / \mathrm{L}$ & Q & PQL & $\mathrm{mg} / \mathrm{L}$ & Q & $\mathrm{PQL}$ & $\mathrm{mg} / \mathrm{L}$ & Q & PQL & $\mathrm{mg} / \mathrm{L}$ & $\mathrm{Q}$ & $\mathrm{PQL}$ & $\mathrm{mg} / \mathrm{L}$ & $\mathrm{Q}$ & PQL \\
\hline 100-D-26 (Depth) & $1: 1$ & J1J442-A1 & $8 / 11 / 11$ & 0.025 & U & 0.03 & 0.001 & $\bar{U}$ & 0.0010 & 0.111 & & 0.02 & 0.006 & $\mathrm{~B}$ & 0.10 & 0.089 & $\mathrm{~B}$ & 0.10 \\
\hline 100-D-26 (Depth) & $1: 1$ & J1J442-A2 & 8/11/11 & 0.025 & $\mathrm{U}$ & 0.03 & 0.001 & $\mathrm{U}$ & 0.0010 & 0.110 & & 0.02 & 0.100 & $U$ & 0.10 & 0.092 & B & 0.10 \\
\hline 100-D-26 (Depth) & $1: 2.5$ & \begin{tabular}{|l|}
$\mathrm{J} 1 \mathrm{~J} 442-\mathrm{B} 1$ \\
\end{tabular} & $11 / 11$ & 0.025 & $U$ & 0.03 & 0.0002 & $\mathrm{U}$ & 0.0002 & 0.059 & & 0.02 & 0.100 & $U$ & 0.10 & 0.049 & B & 0.10 \\
\hline 100-D-26 (Depth) & $1: 5$ & \begin{tabular}{|l|}
$\mathrm{J} 1 \mathrm{~J} 442-\mathrm{C} 1$ \\
\end{tabular} & $8 / 11 / 11$ & 0.025 & $U$ & 0.03 & 0.0002 & $U$ & 0.0002 & 0.035 & & 0.02 & 0.100 & $U$ & 0.10 & 0.018 & $\mathrm{~B}$ & 0.10 \\
\hline 100-D-19 (Depth) & $1: 1$ & \begin{tabular}{|l|}
$\mathrm{J} 1 \mathrm{~J} 443-\mathrm{A} 1$ \\
\end{tabular} & $8 / 11 / 11$ & 0.025 & $U$ & 0.03 & 0.001 & $U$ & 0.0010 & 0.054 & & 0.02 & 0.100 & $U$ & 0.10 & 0.100 & $U$ & 0.10 \\
\hline & $1: 2.5$ & \begin{tabular}{|l|}
$\mathrm{J} 1 \mathrm{~J} 443-\mathrm{B} 1$ \\
\end{tabular} & $8 / 1$ & 0.025 & $\bar{U}$ & 0.03 & 0.0002 & $U$ & 0.0002 & 0.035 & & 0.02 & 0.100 & $U$ & 0.10 & 0.100 & $\bar{U}$ & 0.10 \\
\hline 100-D-19 (Depth) & $1: 2.5$ & \begin{tabular}{|l|}
$\mathrm{J} 1 \mathrm{~J} 443-\mathrm{B} 2$ \\
\end{tabular} & $8 / 11 / 11$ & 0.025 & $U$ & 0.03 & 0.0002 & $U$ & 0.0002 & 0.034 & & 0.02 & 0.100 & $U$ & 0.10 & 0.100 & $\mathrm{U}$ & 0.10 \\
\hline 100-D-19 (Depth) & $1: 5$ & \begin{tabular}{|l|}
$\mathrm{J} 1 \mathrm{~J} 443-\mathrm{C} 1$ \\
\end{tabular} & $8 / 11 / 11$ & 0.025 & $\mathrm{U}$ & 0.03 & 0.0002 & $U$ & 0.0002 & 0.018 & & 0.02 & 0.100 & $U$ & 0.10 & 0.100 & $U$ & 0.10 \\
\hline 100-D-08 (Depth) & $1: 1$ & \begin{tabular}{|l|}
$\mathrm{J} 1 \mathrm{~J} 444-\mathrm{A} 1$ \\
\end{tabular} & $8 / 11 / 11$ & 0.288 & & 0.03 & 0.001 & $U$ & 0.0010 & 0.003 & B & 0.02 & 0.100 & $U$ & 0.10 & 0.100 & $U$ & 0.10 \\
\hline 100-D-08 (Depth) & $1: 5$ & \begin{tabular}{|l|}
$\mathrm{J} 1 \mathrm{~J} 444-\mathrm{C} 1$ \\
\end{tabular} & $8 / 11 / 11$ & 0.187 & & 0.03 & 0.0002 & $U$ & 0.0002 & 0.004 & $B$ & 0.02 & 0.100 & $U$ & 0.10 & 0.100 & $\mathrm{U}$ & 0.10 \\
\hline 100-D-08 (Depth) & $1: 5$ & J1J444-C2 & $8 / 11 / 11$ & 0.201 & & 0.03 & 0.0002 & $U$ & 0.0002 & 0.003 & $B$ & 0.02 & 0.100 & $U$ & 0.10 & 0.100 & $\mathrm{U}$ & 0.10 \\
\hline
\end{tabular}

\begin{tabular}{|c|c|c|c|c|c|c|c|c|c|c|c|c|c|c|c|c|c|c|c|}
\hline \multirow{2}{*}{ Sample Location } & \multirow{2}{*}{\begin{tabular}{|c|}
$\begin{array}{c}\text { Coal Ash:Water } \\
\text { Ratio }\end{array}$ \\
\end{tabular}} & \multirow{2}{*}{$\begin{array}{c}\text { HEIS } \\
\text { Number }\end{array}$} & \multirow{2}{*}{$\begin{array}{c}\text { Sample } \\
\text { Date }\end{array}$} & \multicolumn{3}{|c|}{ Silver } & \multicolumn{3}{|c|}{ Thallium } & \multicolumn{3}{|c|}{ Uranium (KPA) } & \multicolumn{3}{|c|}{ Vanadium } & \multicolumn{3}{|c|}{ Zinc } & \multirow[b]{2}{*}{$\mathrm{pH}$} \\
\hline & & & & $\mathrm{mg} / \mathrm{L}$ & Q & PQL & $\mathrm{mg} / \mathrm{L}$ & Q & PQL & ug/L & Q & MDA & $\mathrm{mg} / \mathrm{L}$ & Q & $\mathrm{PQL}$ & $\mathrm{mg} / \mathrm{L}$ & $\mathrm{Q}$ & PQL & \\
\hline 100-D-26 (Depth) & $1: 1$ & J1J442-A1 & $8 / 11 / 11$ & 0.030 & $\mathrm{U}$ & 0.03 & 0.075 & 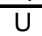 & 0.08 & 12.5 & & 0.034 & 0.116 & & 0.03 & 0.250 & U & 0.25 & 8.39 \\
\hline 100-D-26 (Depth) & $1: 1$ & J1J442-A2 & $8 / 11 / 11$ & 0.030 & $\mathrm{U}$ & 0.03 & 0.075 & $U$ & 0.08 & 11.0 & & 0.034 & 0.123 & & 0.03 & 0.250 & $U$ & 0.25 & 8.61 \\
\hline 100-D-26 (Depth) & $1: 2.5$ & J1J442-B1 & $8 / 11 / 11$ & 0.030 & $\mathrm{U}$ & 0.03 & 0.075 & $\mathrm{U}$ & 0.08 & 6.15 & & 0.034 & 0.094 & & 0.03 & 0.250 & $U$ & 0.25 & 8.89 \\
\hline 100-D-26 (Depth) & $1: 5$ & J1J442-C1 & $8 / 11 / 11$ & 0.030 & $\mathrm{U}$ & 0.03 & 0.075 & $U$ & 0.08 & 3.01 & & 0.034 & 0.072 & & 0.03 & 0.250 & $U$ & 0.25 & 9.12 \\
\hline 100-D-19 (Depth) & $1: 1$ & J1J443-A1 & $8 / 11 / 11$ & 0.030 & $U$ & 0.03 & 0.075 & $U$ & 0.08 & 3.66 & & 0.034 & 0.088 & & 0.03 & 0.250 & $U$ & 0.25 & 8.24 \\
\hline 100-D-19 (Depth) & $1: 2.5$ & \begin{tabular}{|l|} 
J1J443-B1 \\
\end{tabular} & $8 / 11 / 11$ & 0.030 & $\mathrm{U}$ & 0.03 & 0.075 & $\mathrm{U}$ & 0.08 & 2.35 & & 0.034 & 0.066 & & 0.03 & 0.250 & $u$ & 0.25 & 8.36 \\
\hline 100-D-19 (Depth) & $1: 2.5$ & J1J443-B2 & $8 / 11 / 11$ & 0.030 & $U$ & 0.03 & 0.075 & U & 0.08 & 2.31 & & 0.034 & 0.069 & & 0.03 & 0.250 & $U$ & 0.25 & 8.38 \\
\hline 100-D-19 (Depth) & $1: 5$ & J1J443-C1 & $8 / 11 / 11$ & 0.030 & $U$ & 0.03 & 0.075 & $U$ & 0.08 & 1.53 & & 0.064 & 0.051 & & 0.03 & 0.250 & $U$ & 0.25 & 8.48 \\
\hline 100-D-08 (Depth) & $1: 1$ & J1J444-A1 & $8 / 11 / 11$ & 0.030 & $U$ & 0.03 & 0.075 & $U$ & 0.08 & 0 & $\mathrm{U}$ & 0.034 & 0.106 & & 0.03 & 0.250 & $U$ & 0.25 & 7.45 \\
\hline 100-D-08 (Depth) & $1: 2.5$ & \begin{tabular}{|l|} 
J1J444-B1 \\
\end{tabular} & $8 / 11 / 11$ & 0.030 & $\mathrm{U}$ & 0.03 & 0.075 & $U$ & 0.08 & 0.032 & $\mathrm{U}$ & 0.034 & 0.114 & & 0.03 & 0.250 & $U$ & 0.25 & 6.88 \\
\hline 100-D-08 (Depth) & $1: 5$ & J1J444-C1 & $8 / 11 / 11$ & 0.030 & $U$ & 0.03 & 0.075 & $U$ & 0.08 & 0 & $U$ & 0.034 & 0.084 & & 0.03 & 0.250 & $U$ & 0.25 & 6.78 \\
\hline \begin{tabular}{|l|}
$100-D-08$ (Depth) \\
\end{tabular} & $1: 5$ & J1J444-C2 & $8 / 11 / 11$ & 0.030 & $\mathrm{U}$ & 0.03 & 0.075 & $\mathrm{U}$ & 0.08 & 0 & $\mathrm{U}$ & 0.034 & 0.085 & & 0.03 & 0.250 & $U$ & 0.25 & 6.76 \\
\hline
\end{tabular}




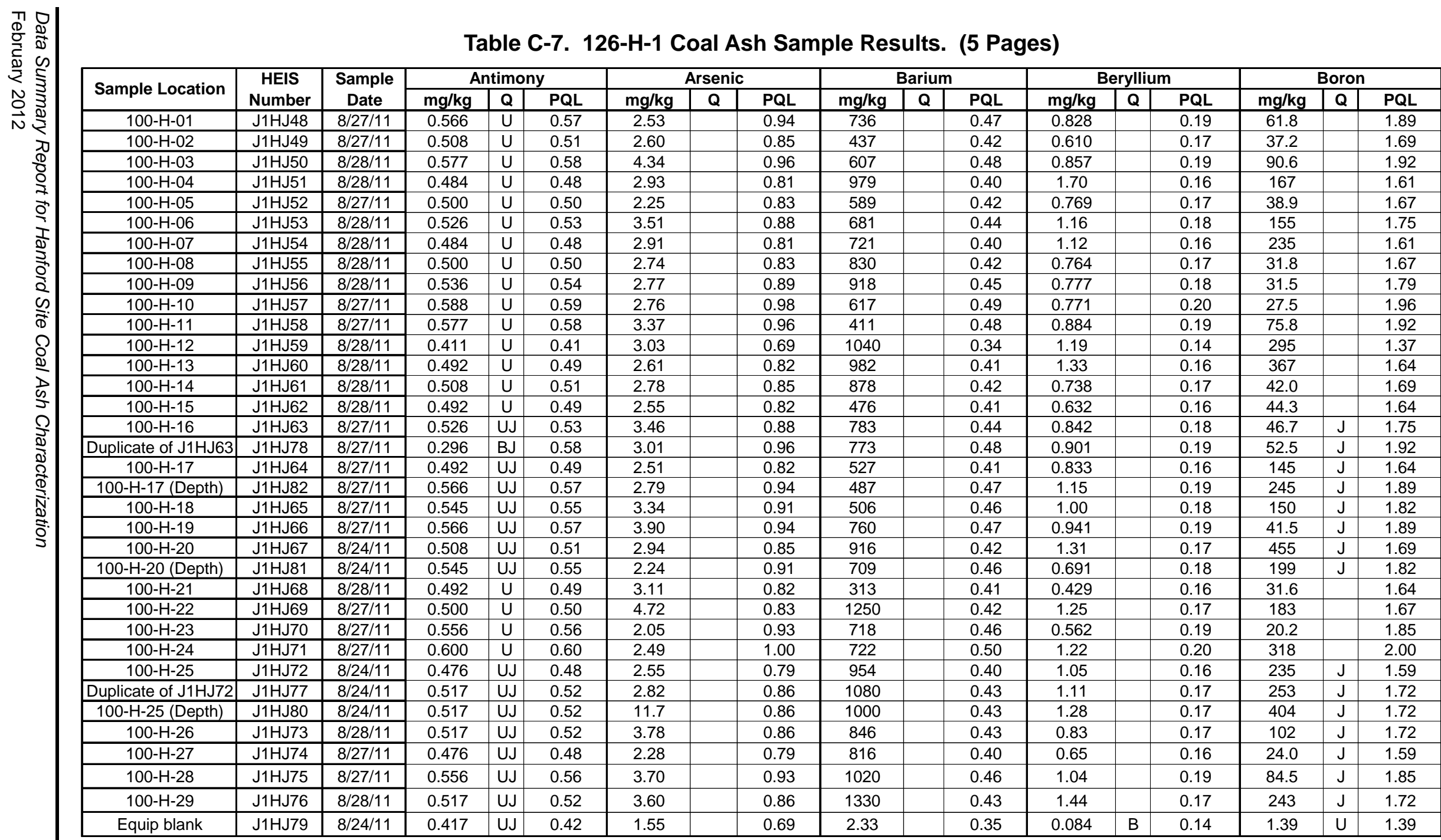




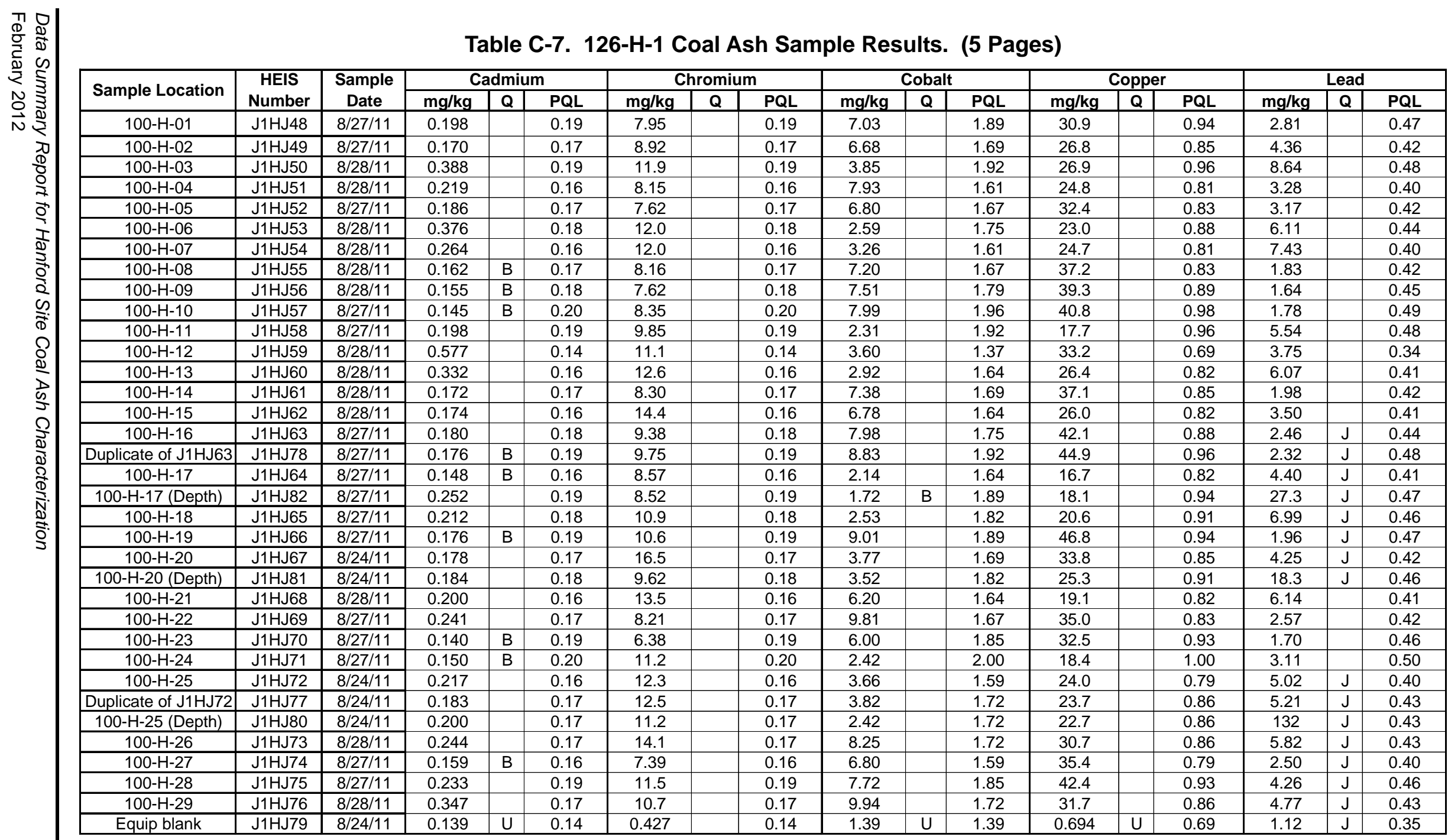


Table C-7. 126-H-1 Coal Ash Sample Results. (5 Pages)

\begin{tabular}{|c|c|c|c|c|c|c|c|c|c|c|c|c|c|c|c|c|c|}
\hline \multirow{2}{*}{ Sample Location } & \multirow{2}{*}{$\begin{array}{c}\text { HEIS } \\
\text { Number }\end{array}$} & \multirow{2}{*}{$\begin{array}{c}\text { Sample } \\
\text { Date }\end{array}$} & \multicolumn{3}{|c|}{ Manganese } & \multicolumn{3}{|c|}{ Mercury } & \multicolumn{3}{|c|}{ Molybdenum } & \multicolumn{3}{|c|}{ Nickel } & \multicolumn{3}{|c|}{ Selenium } \\
\hline & & & $\mathrm{mg} / \mathrm{kg}$ & $\mathbf{Q}$ & PQL & $\mathrm{mg} / \mathrm{kg}$ & $\mathbf{Q}$ & PQL & $\mathrm{mg} / \mathrm{kg}$ & Q & PQL & $\mathrm{mg} / \mathrm{kg}$ & Q & $\mathrm{PQL}$ & $\mathrm{mg} / \mathrm{kg}$ & Q & $\mathrm{PQL}$ \\
\hline 100-H-01 & J1HJ48 & $8 / 27 / 11$ & 169 & & 4.72 & 0.022 & $B$ & 0.03 & 1.30 & $B$ & 1.89 & 14.4 & & 3.77 & 0.283 & $\mathrm{U}$ & 0.28 \\
\hline $100-\mathrm{H}-02$ & J1HJ49 & $8 / 27 / 11$ & 172 & & 4.24 & 0.035 & & 0.03 & 0.997 & B & 1.69 & 13.6 & & 3.39 & 0.349 & & 0.25 \\
\hline $100-\mathrm{H}-03$ & $\mathrm{~J} 1 \mathrm{HJ} 50$ & $8 / 28 / 11$ & 99.7 & & 4.81 & 0.176 & & 0.03 & 0.607 & $B$ & 1.92 & 10.2 & & 3.85 & 1.58 & & 0.29 \\
\hline $100-\mathrm{H}-04$ & J1HJ51 & $8 / 28 / 11$ & 146 & & 4.03 & 0.100 & & 0.03 & 1.52 & $\mathrm{~B}$ & 1.61 & 16.4 & & 3.23 & 0.540 & & 0.24 \\
\hline $100-\mathrm{H}-05$ & $\mathrm{~J} 1 \mathrm{HJ} 52$ & $8 / 27 / 11$ & 223 & & 4.17 & 0.023 & $\mathrm{~B}$ & 0.03 & 1.46 & $\mathrm{~B}$ & 1.67 & 14.8 & & 3.33 & 0.319 & & 0.25 \\
\hline $100-\mathrm{H}-06$ & $\mathrm{~J} 1 \mathrm{HJ} 53$ & $8 / 28 / 11$ & 84.7 & & 4.39 & 0.146 & & 0.03 & 0.710 & $\mathrm{~B}$ & 1.75 & 8.08 & & 3.51 & 1.63 & & 0.26 \\
\hline $100-\mathrm{H}-07$ & $\mathrm{~J} 1 \mathrm{HJ} 54$ & $8 / 28 / 11$ & 82.7 & & 4.03 & 0.053 & & 0.03 & 1.23 & $\mathrm{~B}$ & 1.61 & 8.93 & & 3.23 & 0.981 & & 0.24 \\
\hline $100-\mathrm{H}-08$ & J1HJ55 & $8 / 28 / 11$ & 97.8 & & 4.17 & 0.015 & $B$ & 0.03 & 1.41 & $B$ & 1.67 & 19.3 & & 3.33 & 0.250 & $\mathrm{U}$ & 0.25 \\
\hline $100-\mathrm{H}-09$ & $\mathrm{~J} 1 \mathrm{HJ} 56$ & $8 / 28 / 11$ & 80.0 & & 4.46 & 0.112 & & 0.03 & 1.55 & B & 1.79 & 20.4 & & 3.57 & 0.277 & & 0.27 \\
\hline $100-\mathrm{H}-10$ & $\mathrm{~J} 1 \mathrm{HJ} 57$ & $8 / 27 / 11$ & 128 & & 4.90 & 0.012 & B & 0.02 & 1.62 & B & 1.96 & 18.5 & & 3.92 & 0.294 & $\mathrm{U}$ & 0.29 \\
\hline $100-\mathrm{H}-11$ & $\mathrm{~J} 1 \mathrm{HJ} 58$ & $8 / 27 / 11$ & 76.0 & & 4.81 & 0.088 & & 0.03 & 0.493 & $\mathrm{~B}$ & 1.92 & 6.72 & & 3.85 & 2.06 & & 0.29 \\
\hline $100-\mathrm{H}-12$ & J1HJ59 & $8 / 28 / 11$ & 96.4 & & 3.42 & 0.177 & & 0.03 & 2.00 & & 1.37 & 9.71 & & 2.74 & 0.508 & & 0.21 \\
\hline $100-\mathrm{H}-13$ & J1HJ60 & $8 / 28 / 11$ & 77.7 & & 4.10 & 0.042 & & 0.03 & 1.86 & & 1.64 & 9.15 & & 3.28 & 0.591 & & 0.25 \\
\hline $100-\mathrm{H}-14$ & J1HJ61 & $8 / 28 / 11$ & 120 & & 4.24 & 0.011 & $B$ & 0.03 & 1.46 & B & 1.69 & 18.5 & & 3.39 & 0.337 & & 0.25 \\
\hline $100-\mathrm{H}-15$ & J1HJ62 & $8 / 28 / 11$ & 225 & & 4.10 & 0.034 & & 0.02 & 0.861 & B & 1.64 & 14.4 & & 3.28 & 0.246 & $\mathrm{U}$ & 0.25 \\
\hline $100-\mathrm{H}-16$ & J1HJ63 & $8 / 27 / 11$ & 134 & $\mathrm{~J}$ & 4.39 & 0.015 & $\mathrm{~B}$ & 0.03 & 1.51 & $\mathrm{~B}$ & 1.75 & 20.4 & $\mathrm{~J}$ & 3.51 & 0.263 & $\mathrm{U}$ & 0.26 \\
\hline Duplicate of $\mathrm{J} 1 \mathrm{HJ} 63$ & J1HJ78 & $8 / 27 / 11$ & 135 & $\mathrm{~J}$ & 4.81 & 0.029 & & 0.03 & 1.78 & B & 1.92 & 20.4 & $\mathrm{~J}$ & 3.85 & 0.288 & $\mathrm{U}$ & 0.29 \\
\hline $100-\mathrm{H}-17$ & $\mathrm{~J} 1 \mathrm{HJ} 64$ & $8 / 27 / 11$ & 67.7 & $\mathrm{~J}$ & 4.10 & 0.049 & & 0.03 & 0.708 & $\mathrm{~B}$ & 1.64 & 5.88 & $\mathrm{~J}$ & 3.28 & 0.762 & & 0.25 \\
\hline 100-H-17 (Depth) & $\mathrm{J} 1 \mathrm{HJ} 82$ & $8 / 27 / 11$ & 82.1 & $\mathrm{~J}$ & 4.72 & 0.053 & & 0.03 & 0.916 & $B$ & 1.89 & 4.79 & $\mathrm{~J}$ & 3.77 & 1.18 & & 0.28 \\
\hline $100-\mathrm{H}-18$ & $\mathrm{~J} 1 \mathrm{HJ} 65$ & $8 / 27 / 11$ & 81.8 & $\mathrm{~J}$ & 4.55 & 0.129 & & 0.03 & 0.786 & B & 1.82 & 7.05 & $\mathrm{~J}$ & 3.64 & 1.49 & & 0.27 \\
\hline $100-\mathrm{H}-19$ & J1HJ66 & $8 / 27 / 11$ & 154 & $\mathrm{~J}$ & 4.72 & 0.025 & $U$ & 0.03 & 2.11 & & 1.89 & 22.4 & $\mathrm{~J}$ & 3.77 & 0.283 & $\mathrm{U}$ & 0.28 \\
\hline $100-\mathrm{H}-20$ & J1HJ67 & $8 / 24 / 11$ & 122 & $\mathrm{~J}$ & 4.24 & 0.018 & B & 0.03 & 2.44 & & 1.69 & 10.9 & $\mathrm{~J}$ & 3.39 & 0.341 & & 0.25 \\
\hline 100-H-20 (Depth) & $\mathrm{J1HJ81}$ & $8 / 24 / 11$ & 148 & $\mathrm{~J}$ & 4.55 & 0.863 & & 0.02 & 1.96 & & 1.82 & 10.6 & $\mathrm{~J}$ & 3.64 & 0.273 & $\mathrm{U}$ & 0.27 \\
\hline $100-\mathrm{H}-21$ & $\mathrm{~J} 1 \mathrm{HJ} 68$ & $8 / 28 / 11$ & 268 & & 4.10 & 0.069 & & 0.03 & 0.628 & B & 1.64 & 12.4 & & 3.28 & 0.348 & & 0.25 \\
\hline $100-\mathrm{H}-22$ & $\mathrm{~J} 1 \mathrm{HJ} 69$ & $8 / 27 / 11$ & 240 & & 4.17 & 0.012 & $\mathrm{~B}$ & 0.02 & 3.52 & & 1.67 & 16.3 & & 3.33 & 0.250 & $\mathrm{U}$ & 0.25 \\
\hline $100-\mathrm{H}-23$ & $\mathrm{~J} 1 \mathrm{HJ} 70$ & $8 / 27 / 11$ & 70.2 & & 4.63 & 0.025 & $\mathrm{U}$ & 0.03 & 1.22 & $\mathrm{~B}$ & 1.85 & 15.9 & & 3.70 & 0.278 & $\mathrm{U}$ & 0.28 \\
\hline $100-\mathrm{H}-24$ & J1HJ71 & $8 / 27 / 11$ & 72.2 & & 5.00 & 0.023 & $\mathrm{U}$ & 0.02 & 1.26 & B & 2.00 & 6.94 & & 4.00 & 0.571 & & 0.30 \\
\hline $100-\mathrm{H}-25$ & $\mathrm{~J} 1 \mathrm{HJ} 72$ & $8 / 24 / 11$ & 153 & $\mathrm{~J}$ & 3.97 & 0.027 & & 0.03 & 1.44 & $B$ & 1.59 & 10.1 & $\mathrm{~J}$ & 3.17 & 0.338 & & 0.24 \\
\hline Duplicate of $\mathrm{J} 1 \mathrm{HJ} 72$ & $\mathrm{~J} 1 \mathrm{HJ} 77$ & $8 / 24 / 11$ & 122 & $\mathrm{~J}$ & 4.31 & 0.019 & $B$ & 0.03 & 1.49 & $\mathrm{~B}$ & 1.72 & 10.2 & $\mathrm{~J}$ & 3.45 & 0.504 & & 0.26 \\
\hline 100-H-25 (Depth) & $\mathrm{J} 1 \mathrm{HJ} 80$ & $8 / 24 / 11$ & 90.9 & $\mathrm{~J}$ & 4.31 & 0.035 & & 0.03 & 1.41 & $\mathrm{~B}$ & 1.72 & 6.96 & $\mathrm{~J}$ & 3.45 & 0.259 & $\mathrm{U}$ & 0.26 \\
\hline $100-\mathrm{H}-26$ & J1HJ73 & $8 / 28 / 11$ & 283 & $\mathrm{~J}$ & 4.31 & 0.072 & & 0.02 & 1.25 & $\mathrm{~B}$ & 1.72 & 15.6 & $\overline{\mathrm{J}}$ & 3.45 & 0.259 & $\mathrm{U}$ & 0.26 \\
\hline $100-\mathrm{H}-27$ & J1HJ74 & $8 / 27 / 11$ & 78.8 & $\mathrm{~J}$ & 3.97 & 0.011 & B & 0.03 & 1.40 & B & 1.59 & 17.8 & $\mathrm{~J}$ & 3.17 & 0.238 & $\mathrm{U}$ & 0.24 \\
\hline $100-\mathrm{H}-28$ & J1HJ75 & $8 / 27 / 11$ & 141 & $\mathrm{~J}$ & 4.63 & 0.051 & & 0.03 & 1.81 & B & 1.85 & 19.2 & $\mathrm{~J}$ & 3.70 & 0.510 & & 0.28 \\
\hline $100-\mathrm{H}-29$ & J1HJ76 & $8 / 28 / 11$ & 300 & $\mathrm{~J}$ & 4.31 & 0.075 & & 0.03 & 2.05 & & 1.72 & 17.8 & $\mathrm{~J}$ & 3.45 & 0.349 & & 0.26 \\
\hline Equip blank & J1HJ79 & $8 / 24 / 11$ & 7.47 & $\mathrm{~J}$ & 3.47 & 0.024 & $\mathrm{U}$ & 0.02 & 1.39 & $\mathrm{U}$ & 1.39 & 2.78 & UJ & 2.78 & 0.208 & $\mathrm{U}$ & 0.21 \\
\hline
\end{tabular}




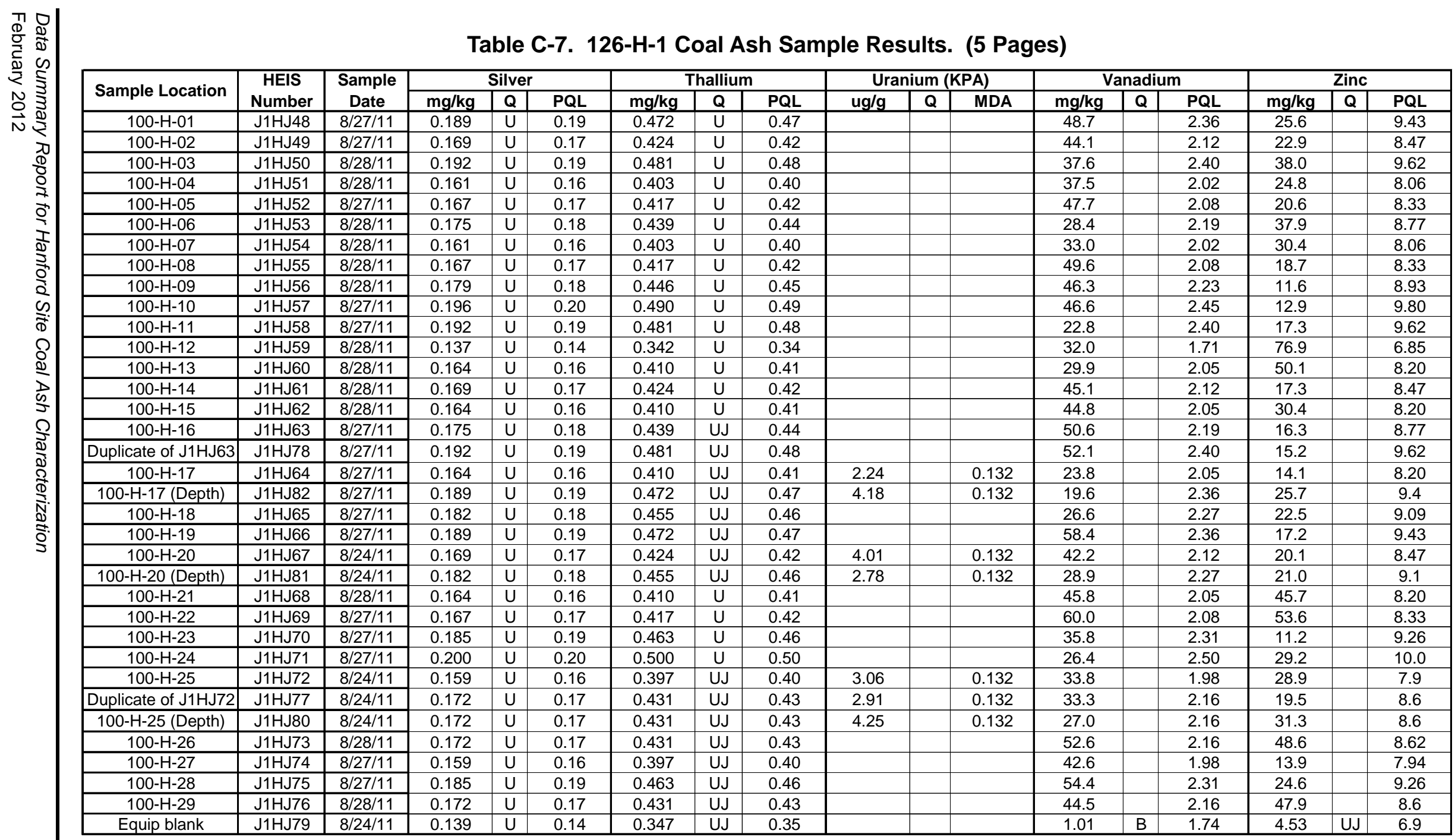


Table C-7. 126-H-1 Coal Ash Sample Results. (5 Pages)

\begin{tabular}{|c|c|c|c|c|c|c|c|c|c|c|c|c|c|c|c|c|c|c|c|c|}
\hline \multirow{2}{*}{ Sample Location } & \multirow{2}{*}{$\begin{array}{c}\text { HEIS } \\
\text { Number }\end{array}$} & \multirow{2}{*}{$\begin{array}{c}\text { Sample } \\
\text { Date }\end{array}$} & \multicolumn{3}{|c|}{ Acenaphthene } & \multicolumn{3}{|c|}{ Acenaphthylene } & \multicolumn{3}{|c|}{ Anthracene } & \multicolumn{3}{|c|}{ Benzo(a)anthracene } & \multicolumn{3}{|c|}{ Benzo(a)pyrene } & \multicolumn{3}{|c|}{ Benzo(b)fluoranthene } \\
\hline & & & ug/kg & $Q$ & $\mathrm{PQL}$ & ug/kg & Q & PQL & ug/kg & $\mathrm{Q}$ & $\mathrm{PQL}$ & ug/kg & Q & $\mathrm{PQL}$ & $u g / k g$ & Q & $\mathrm{PQL}$ & ug/kg & Q & PQL \\
\hline $100-\mathrm{H}-17$ & J1HJ64 & $8 / 27 / 11$ & 336 & $\dot{U}$ & 336 & 336 & $\mathrm{U}$ & 336 & 336 & $\dot{U}$ & 336 & 336 & $\mathrm{U}$ & 336 & 336 & $\dot{U}$ & 336 & 336 & $\dot{U}$ & 336 \\
\hline $100-\mathrm{H}-20$ & $\mathrm{~J} 1 \mathrm{HJ} 67$ & $8 / 24 / 11$ & 336 & UD & 336 & 336 & UD & 336 & 336 & UD & 336 & 336 & UD & 336 & 336 & UD & 336 & 336 & UD & 336 \\
\hline $100-\mathrm{H}-25$ & $\mathrm{~J} 1 \mathrm{HJ} 72$ & $8 / 24 / 11$ & 332 & UD & 332 & 332 & UD & 332 & 332 & UD & 332 & 332 & UD & 332 & 332 & UD & 332 & 332 & UD & 332 \\
\hline Duplicate of $\mathrm{J} 1 \mathrm{HJ} 72$ & $\mathrm{~J} 1 \mathrm{HJ} 77$ & $8 / 24 / 11$ & 335 & $\mathrm{U}$ & 335 & 335 & $\mathrm{U}$ & 335 & 335 & $\mathrm{U}$ & 335 & 335 & $\mathrm{U}$ & 335 & 335 & $\mathrm{U}$ & 335 & 335 & $\mathrm{U}$ & 335 \\
\hline 100-H-17 (Depth) & J1HJ82 & $8 / 27 / 11$ & 335 & $U$ & 335 & 335 & $U$ & 335 & 335 & $U$ & 335 & 335 & $U$ & 335 & 335 & $U$ & 335 & 335 & $U$ & 335 \\
\hline $100-\mathrm{H}-20$ (Depth) & J1HJ81 & $8 / 24 / 11$ & 339 & $U$ & 339 & 339 & $U$ & 339 & 339 & $U$ & 339 & 339 & $U$ & 339 & 339 & $U$ & 339 & 339 & $U$ & 339 \\
\hline $100-\mathrm{H}-25$ (Depth) & $\mathrm{J} 1 \mathrm{HJ} 80$ & $8 / 24 / 11$ & 338 & $U$ & 338 & 338 & $U$ & 338 & 338 & $U$ & 338 & 338 & $U$ & 338 & 338 & $U$ & 338 & 338 & $U$ & 338 \\
\hline Equip blank & J1HJ79 & $8 / 24 / 11$ & 332 & $\mathrm{U}$ & 332 & 332 & U & 332 & 332 & $\mathrm{U}$ & 332 & 332 & U & 332 & 332 & U & 332 & 332 & $U$ & 332 \\
\hline
\end{tabular}

\begin{tabular}{|c|c|c|c|c|c|c|c|c|c|c|c|c|c|c|c|c|c|c|c|c|}
\hline \multirow{2}{*}{ Sample Location } & \multirow{2}{*}{$\begin{array}{c}\text { HEIS } \\
\text { Number }\end{array}$} & \multirow{2}{*}{$\begin{array}{c}\text { Sample } \\
\text { Date }\end{array}$} & \multicolumn{3}{|c|}{ Benzo(ghi)perylene } & \multicolumn{3}{|c|}{ Benzo(k)fluoranthene } & \multicolumn{3}{|c|}{ Chrysene } & \multicolumn{3}{|c|}{ Dibenz(a,h)anthracene } & \multicolumn{3}{|c|}{$\begin{array}{l}\text { Fluoranthene } \\
\end{array}$} & \multicolumn{3}{|c|}{$\begin{array}{l}\text { Fluorene } \\
\end{array}$} \\
\hline & & & $\mathrm{ug} / \mathrm{kg}$ & $Q$ & PQL & $\mathrm{ug} / \mathrm{kg}$ & $Q$ & $\mathrm{PQL}$ & ug/kg & $Q$ & $\mathrm{PQL}$ & $\mathrm{ug} / \mathrm{kg}$ & Q & $\mathrm{PQL}$ & $\mathrm{ug} / \mathrm{kg}$ & Q & $\mathrm{PQL}$ & ug/kg & Q & $\mathrm{PQL}$ \\
\hline $100-\mathrm{H}-17$ & $\mathrm{~J} 1 \mathrm{HJ} 64$ & $8 / 27 / 11$ & 336 & U & 336 & 336 & $U$ & 336 & 336 & $U$ & 336 & 336 & U & 336 & 336 & $\mathrm{U}$ & 336 & 336 & U & 336 \\
\hline $100-\mathrm{H}-20$ & $\mathrm{~J} 1 \mathrm{HJ} 67$ & $8 / 24 / 11$ & 336 & UD & 336 & 336 & UD & 336 & 336 & UD & 336 & 336 & UD & 336 & 336 & UD & 336 & 336 & UD & 336 \\
\hline $100-\mathrm{H}-25$ & $\mathrm{~J} 1 \mathrm{HJ} 72$ & $8 / 24 / 11$ & 332 & UD & 332 & 332 & UD & 332 & 332 & UD & 332 & 332 & UD & 332 & 332 & UD & 332 & 332 & UD & 332 \\
\hline Duplicate of J1HJ72 & $\mathrm{J} 1 \mathrm{HJ} 77$ & $8 / 24 / 11$ & 335 & $\mathrm{U}$ & 335 & 335 & $\mathrm{U}$ & 335 & 335 & $\mathrm{U}$ & 335 & 335 & $\mathrm{U}$ & 335 & 335 & U & 335 & 335 & $\mathrm{U}$ & 335 \\
\hline 100-H-17 (Depth) & $\mathrm{J} 1 \mathrm{HJ} 82$ & $8 / 27 / 11$ & 335 & $\mathrm{U}$ & 335 & 335 & $\mathrm{U}$ & 335 & 335 & $\mathrm{U}$ & 335 & 335 & $\mathrm{U}$ & 335 & 335 & $\mathrm{U}$ & 335 & 335 & $\mathrm{U}$ & 335 \\
\hline 100-H-20 (Depth) & $\mathrm{J} 1 \mathrm{HJ} 81$ & $8 / 24 / 11$ & 339 & U & 339 & 339 & $u$ & 339 & 339 & 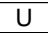 & 339 & 339 & $u$ & 339 & 339 & $U$ & 339 & 339 & 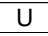 & 339 \\
\hline 100-H-25 (Depth) & $\mathrm{J} 1 \mathrm{HJ} 80$ & $8 / 24 / 11$ & 585 & $\mathrm{~J}$ & 656 & 338 & $U$ & 338 & 338 & $U$ & 338 & 338 & $U$ & 338 & 338 & $U$ & 338 & 338 & $U$ & 338 \\
\hline Equip blank & J1HJ79 & $8 / 24 / 11$ & 332 & $U$ & 332 & 332 & $\mathrm{U}$ & 332 & 332 & $U$ & 332 & 332 & $U$ & 332 & 332 & $U$ & 332 & 332 & $U$ & 332 \\
\hline
\end{tabular}

\begin{tabular}{|c|c|c|c|c|c|c|c|c|c|c|c|c|c|c|}
\hline \multirow{2}{*}{ Sample Location } & \multirow{2}{*}{$\begin{array}{c}\text { HEIS } \\
\text { Number }\end{array}$} & \multirow{2}{*}{$\begin{array}{c}\text { Sample } \\
\text { Date }\end{array}$} & \multicolumn{3}{|c|}{ Indeno(1,2,3-cd)pyrene } & \multicolumn{3}{|c|}{ Naphthalene } & \multicolumn{3}{|c|}{ Phenanthrene } & \multicolumn{3}{|c|}{ Pyrene } \\
\hline & & & ug/kg & $\mathrm{Q}$ & $\mathrm{PQL}$ & ug/kg & Q & PQL & ug/kg & $\mathbf{Q}$ & PQL & $u g / k g$ & Q & PQL \\
\hline $100-\mathrm{H}-17$ & J1HJ64 & $8 / 27 / 11$ & 336 & $U^{2}$ & 336 & 357 & $\mathrm{~J}$ & 633 & 240 & $\mathrm{~J}$ & 633 & 336 & 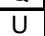 & 336 \\
\hline $100-\mathrm{H}-20$ & $\mathrm{~J} 1 \mathrm{HJ} 67$ & $8 / 24 / 11$ & 336 & UD & 336 & 691 & $\mathrm{JD}$ & 1270 & 250 & $\mathrm{JD}$ & 1270 & 336 & UD & 336 \\
\hline $100-\mathrm{H}-25$ & $\mathrm{~J} 1 \mathrm{HJ} 72$ & $8 / 24 / 11$ & 332 & UD & 332 & 464 & $\mathrm{JD}$ & 1260 & 332 & UD & 332 & 332 & UD & 332 \\
\hline Duplicate of J1HJ72 & $\mathrm{J} 1 \mathrm{HJ} 77$ & $8 / 24 / 11$ & 335 & $U$ & 335 & 335 & $\mathrm{U}$ & 335 & 335 & $\mathrm{U}$ & 335 & 335 & $\mathrm{U}$ & 335 \\
\hline 100-H-17 (Depth) & J1HJ82 & $8 / 27 / 11$ & 335 & $U$ & 335 & 174 & $\mathrm{~J}$ & 633 & 110 & $\mathrm{~J}$ & 633 & 104 & $\mathrm{~J}$ & 633 \\
\hline $100-\mathrm{H}-20$ (Depth) & $\mathrm{J1HJ81}$ & $8 / 24 / 11$ & 339 & $\mathrm{U}$ & 339 & 181 & $\mathrm{~J}$ & 657 & 111 & $\mathrm{~J}$ & 657 & 339 & $\mathrm{U}$ & 339 \\
\hline $100-\mathrm{H}-25$ (Depth) & $\mathrm{J} 1 \mathrm{HJ} 80$ & $8 / 24 / 11$ & 338 & $U$ & 338 & 137 & $\mathrm{~J}$ & 656 & 338 & $\mathrm{U}$ & 338 & 338 & $U$ & 338 \\
\hline Equip blank & J1HJ79 & $8 / 24 / 11$ & 332 & U & 332 & 332 & U & 332 & 332 & $U$ & 332 & 332 & $U$ & 332 \\
\hline
\end{tabular}


Table C-8. 126-H-1 Coal Ash Pre-Leaching Quadruplicate Sample Results. (2 Pages)

\begin{tabular}{|c|c|c|c|c|c|c|c|c|c|c|c|c|c|c|c|c|c|}
\hline \multirow{2}{*}{ Sample Location } & \multirow{2}{*}{$\begin{array}{c}\text { HEIS } \\
\text { Number }\end{array}$} & \multirow{2}{*}{$\begin{array}{c}\text { Sample } \\
\text { Date }\end{array}$} & \multicolumn{3}{|c|}{ Antimony } & \multicolumn{3}{|c|}{ Arsenic } & \multicolumn{3}{|c|}{ Barium } & \multicolumn{3}{|c|}{ Beryllium } & \multicolumn{3}{|c|}{ Boron } \\
\hline & & & $\mathrm{mg} / \mathrm{kg}$ & $\mathbf{Q}$ & PQL & $\mathrm{mg} / \mathrm{kg}$ & Q & $\mathrm{PQL}$ & $\mathrm{mg} / \mathrm{kg}$ & $\mathbf{Q}$ & $\mathrm{PQL}$ & $\mathrm{mg} / \mathrm{kg}$ & Q & PQL & $\mathrm{mg} / \mathrm{kg}$ & $\mathbf{Q}$ & $\mathrm{PQL}$ \\
\hline 100-H-25 (Depth) & J1HJ80-1 & $8 / 24 / 11$ & 0.484 & $\bar{U}$ & 0.48 & 2.22 & & 0.81 & 943 & & 0.40 & 1.49 & & 0.16 & 392 & & 1.61 \\
\hline 100-H-25 (Depth) & $\mathrm{J} 1 \mathrm{HJ} 80-2$ & $8 / 24 / 11$ & 0.441 & $\mathrm{U}$ & 0.44 & 2.42 & & 0.74 & 964 & & 0.37 & 1.39 & & 0.15 & 347 & & 1.47 \\
\hline 100-H-25 (Depth) & J1HJ80-3 & $8 / 24 / 11$ & 0.588 & $U$ & 0.59 & 2.27 & & 0.98 & 1130 & & 0.49 & 1.30 & & 0.20 & 377 & & 1.96 \\
\hline 100-H-25 (Depth) & J1HJ80-4 & $8 / 24 / 11$ & 0.577 & $U$ & 0.58 & 2.41 & & 0.96 & 941 & & 0.48 & 1.20 & & 0.19 & 370 & & 1.92 \\
\hline 100-H-20 (Depth) & J1HJ81-1 & 8/24/11 & 0.455 & $\mathrm{U}$ & 0.46 & 1.70 & & 0.76 & 468 & & 0.38 & 0.525 & & 0.15 & 146 & & 1.52 \\
\hline 100-H-20 (Depth) & J1HJ81-2 & $8 / 24 / 11$ & 0.500 & $U$ & 0.50 & 3.41 & & 0.83 & 844 & & 0.42 & 0.978 & & 0.17 & 223 & & 1.67 \\
\hline 100-H-20 (Depth) & J1HJ81-3 & $8 / 24 / 11$ & 0.515 & $B$ & 0.60 & 3.20 & & 1.00 & 871 & & 0.50 & 0.896 & & 0.20 & 276 & & 2.00 \\
\hline 100-H-20 (Depth) & J1HJ81-4 & $8 / 24 / 11$ & 0.508 & $\mathrm{U}$ & 0.51 & 2.31 & & 0.85 & 907 & & 0.42 & 0.871 & & 0.17 & 265 & & 1.69 \\
\hline 100-H-17 (Depth) & J1HJ82-1 & $8 / 27 / 11$ & 0.588 & $U$ & 0.59 & 2.74 & & 0.98 & 559 & & 0.49 & 1.20 & & 0.20 & 263 & & 1.96 \\
\hline 100-H-17 (Depth) & J1HJ82-2 & $8 / 27 / 11$ & 0.577 & $U$ & 0.58 & 2.53 & & 0.96 & 485 & & 0.48 & 1.07 & & 0.19 & 223 & & 1.92 \\
\hline 100-H-17 (Depth) & J1HJ82-3 & 8/27/11 & 0.484 & $U$ & 0.48 & 2.78 & & 0.81 & 603 & & 0.40 & 1.24 & & 0.16 & 263 & & 1.61 \\
\hline 100-H-17 (Depth) & J1HJ82-4 & $8 / 27 / 11$ & 0.577 & $U$ & 0.58 & 2.83 & & 0.96 & 563 & & 0.48 & 1.24 & & 0.19 & 262 & & 1.92 \\
\hline
\end{tabular}

\begin{tabular}{|c|c|c|c|c|c|c|c|c|c|c|c|c|c|c|c|c|c|}
\hline \multirow{2}{*}{ Sample Location } & \multirow{2}{*}{$\begin{array}{c}\text { HEIS } \\
\text { Number }\end{array}$} & \multirow{2}{*}{$\begin{array}{c}\text { Sample } \\
\text { Date }\end{array}$} & \multicolumn{3}{|c|}{ Cadmium } & \multicolumn{3}{|c|}{ Chromium } & \multicolumn{3}{|c|}{ Cobalt } & \multicolumn{3}{|c|}{ Copper } & \multicolumn{3}{|c|}{ Lead } \\
\hline & & & $\mathrm{mg} / \mathrm{kg}$ & $\mathbf{Q}$ & PQL & $\mathrm{mg} / \mathrm{kg}$ & Q & PQL & $\mathrm{mg} / \mathrm{kg}$ & Q & PQL & $\mathrm{mg} / \mathrm{kg}$ & Q & PQL & $\mathrm{mg} / \mathbf{k g}$ & $\bar{Q}$ & PQL \\
\hline 100-H-25 (Depth) & $\mathrm{J} 1 \mathrm{HJ} 80-1$ & $8 / 24 / 11$ & 0.172 & & 0.16 & 10.7 & & 0.16 & 2.26 & & 1.61 & 28.2 & & 0.81 & 11.9 & & 0.40 \\
\hline 100-H-25 (Depth) & $\mathrm{J} 1 \mathrm{HJ} 80-2$ & $8 / 24 / 11$ & 0.167 & & 0.15 & 12.1 & & 0.15 & 2.52 & & 1.47 & 80.9 & & 0.74 & 16.7 & & 0.37 \\
\hline 100-H-25 (Depth) & J1HJ80-3 & $8 / 24 / 11$ & 0.152 & $B$ & 0.20 & 10.4 & & 0.20 & 2.48 & & 1.96 & 27.2 & & 0.98 & 7.79 & & 0.49 \\
\hline 100-H-25 (Depth) & J1HJ80-4 & $8 / 24 / 11$ & 0.142 & B & 0.19 & 10.8 & & 0.19 & 2.22 & & 1.92 & 22.4 & & 0.96 & 17.1 & & 0.48 \\
\hline 100-H-20 (Depth) & J1HJ81-1 & $8 / 24 / 11$ & 0.156 & & 0.15 & 16.1 & & 0.15 & 3.08 & & 1.52 & 22.8 & & 0.76 & 170 & & 0.38 \\
\hline 100-H-20 (Depth) & J1HJ81-2 & $8 / 24 / 11$ & 0.147 & $B$ & 0.17 & 12.4 & & 0.17 & 5.31 & & 1.67 & 36.1 & & 0.83 & 23.0 & & 0.42 \\
\hline 100-H-20 (Depth) & J1HJ81-3 & $8 / 24 / 11$ & 0.180 & $\mathrm{~B}$ & 0.20 & 13.7 & & 0.20 & 4.48 & & 2.00 & 31.7 & & 1.00 & 40.0 & & 0.50 \\
\hline 100-H-20 (Depth) & $\mathrm{J} 1 \mathrm{HJ} 81-4$ & 8/24/11 & 0.181 & & 0.17 & 10.8 & & 0.17 & 4.14 & & 1.69 & 29.0 & & 0.85 & 19.6 & & 0.42 \\
\hline 100-H-17 (Depth) & J1HJ82-1 & $8 / 27 / 11$ & 0.195 & $B$ & 0.20 & 8.38 & & 0.20 & 1.61 & $B$ & 1.96 & 18.6 & & 0.98 & 25.3 & & 0.49 \\
\hline 100-H-17 (Depth) & J1HJ82-2 & $8 / 27 / 11$ & 0.168 & B & 0.19 & 7.52 & & 0.19 & 1.47 & B & 1.92 & 16.9 & & 0.96 & 22.2 & & 0.48 \\
\hline 100-H-17 (Depth) & $\mathrm{J} 1 \mathrm{HJ} 82-3$ & $8 / 27 / 11$ & 0.212 & & 0.16 & 8.45 & & 0.16 & 1.72 & & 1.61 & 19.7 & & 0.81 & 27.0 & & 0.40 \\
\hline 100-H-17 (Depth) & J1HJ82-4 & $8 / 27 / 11$ & 0.209 & & 0.19 & 8.57 & & 0.19 & 1.87 & B & 1.92 & 20.7 & & 0.96 & 25.4 & & 0.48 \\
\hline
\end{tabular}


Table C-8. 126-H-1 Coal Ash Pre-Leaching Quadruplicate Sample Results. (2 Pages)

\begin{tabular}{|c|c|c|c|c|c|c|c|c|c|c|c|c|c|c|}
\hline \multirow{2}{*}{ Sample Location } & \multirow{2}{*}{$\begin{array}{c}\text { HEIS } \\
\text { Number }\end{array}$} & \multirow{2}{*}{$\begin{array}{c}\text { Sample } \\
\text { Date }\end{array}$} & \multicolumn{3}{|c|}{ Manganese } & \multicolumn{3}{|c|}{ Mercury } & \multicolumn{3}{|c|}{ Molybdenum } & \multicolumn{3}{|c|}{ Nickel } \\
\hline & & & $\mathrm{mg} / \mathrm{kg}$ & $\mathbf{Q}$ & PQL & $\mathrm{mg} / \mathrm{kg}$ & Q & PQL & $\mathrm{mg} / \mathrm{kg}$ & Q & PQL & $\mathrm{mg} / \mathrm{kg}$ & Q & PQL \\
\hline 100-H-25 (Depth) & $\mathrm{J} 1 \mathrm{HJ} 80-1$ & $8 / 24 / 11$ & 95.1 & & 4.03 & 0.033 & & 0.03 & 1.35 & B & 1.61 & 6.25 & & 3.23 \\
\hline 100-H-25 (Depth) & $\mathrm{J} 1 \mathrm{HJ} 80-2$ & $8 / 24 / 11$ & 95.0 & & 3.68 & 0.101 & & 0.03 & 1.48 & & 1.47 & 7.28 & & 2.94 \\
\hline 100-H-25 (Depth) & J1HJ80-3 & $8 / 24 / 11$ & 94.3 & & 4.90 & 0.031 & & 0.03 & 1.34 & B & 1.96 & 6.66 & & 3.92 \\
\hline 100-H-25 (Depth) & J1HJ80-4 & $8 / 24 / 11$ & 103 & & 4.81 & 0.030 & & 0.03 & 1.25 & $B$ & 1.92 & 6.53 & & 3.85 \\
\hline 100-H-20 (Depth) & J1HJ81-1 & $8 / 24 / 11$ & 110 & & 3.79 & 1.65 & & 0.08 & 0.977 & B & 1.52 & 8.28 & & 3.03 \\
\hline 100-H-20 (Depth) & J1HJ81-2 & $8 / 24 / 11$ & 232 & & 4.17 & 0.802 & & 0.03 & 2.04 & & 1.67 & 12.8 & & 3.33 \\
\hline 100-H-20 (Depth) & J1HJ81-3 & $8 / 24 / 11$ & 144 & & 5.00 & 0.829 & & 0.03 & 1.59 & B & 2.00 & 9.36 & & 4.00 \\
\hline 100-H-20 (Depth) & J1HJ81-4 & $8 / 24 / 11$ & 275 & & 4.24 & 0.898 & & 0.03 & 1.54 & B & 1.69 & 9.52 & & 3.39 \\
\hline 100-H-17 (Depth) & J1HJ82-1 & $8 / 27 / 11$ & 73.0 & & 4.90 & 0.063 & & 0.03 & 0.879 & $\mathrm{~B}$ & 1.96 & 4.80 & & 3.92 \\
\hline 100-H-17 (Depth) & J1HJ82-2 & $8 / 27 / 11$ & 63.9 & & 4.81 & 0.070 & & 0.03 & 0.769 & B & 1.92 & 4.29 & & 3.85 \\
\hline 100-H-17 (Depth) & $\mathrm{J} 1 \mathrm{HJ} 82-3$ & $8 / 27 / 11$ & 70.8 & & 4.03 & 0.065 & & 0.03 & 0.830 & B & 1.61 & 4.81 & & 3.23 \\
\hline 100-H-17 (Depth) & $\mathrm{J} 1 \mathrm{HJ} 82-4$ & $8 / 27 / 11$ & 71.7 & & 4.81 & 0.051 & & 0.03 & 0.920 & $B$ & 1.92 & 5.38 & & 3.85 \\
\hline \multirow{2}{*}{ Sample Location } & \multirow{2}{*}{$\begin{array}{c}\text { HEIS } \\
\text { Number }\end{array}$} & \multirow{2}{*}{$\begin{array}{c}\text { Sample } \\
\text { Date }\end{array}$} & \multicolumn{3}{|c|}{ Silver } & \multicolumn{3}{|c|}{ Thallium } & \multicolumn{3}{|c|}{ Vanadium } & \multicolumn{3}{|c|}{ Zinc } \\
\hline & & & $\mathrm{mg} / \mathrm{kg}$ & $\mathbf{Q}$ & $\mathrm{PQL}$ & $\mathrm{mg} / \mathrm{kg}$ & $\mathbf{Q}$ & PQL & $\mathrm{mg} / \mathrm{kg}$ & $\mathbf{Q}$ & $\mathrm{PQL}$ & $\mathrm{mg} / \mathrm{kg}$ & $\mathbf{Q}$ & $\mathrm{PQL}$ \\
\hline 100-H-25 (Depth) & J1HJ80-1 & $8 / 24 / 11$ & 0.161 & $U$ & 0.16 & 0.403 & $U$ & 0.40 & 30.3 & & 2.02 & 38.2 & & 8.06 \\
\hline 100-H-25 (Depth) & $\mathrm{J} 1 \mathrm{HJ} 80-2$ & $8 / 24 / 11$ & 0.147 & $U$ & 0.15 & 0.368 & $\mathrm{U}$ & 0.37 & 30.3 & & 1.84 & 25.8 & & 7.35 \\
\hline 100-H-25 (Depth) & J1HJ80-3 & $8 / 24 / 11$ & 0.196 & $U$ & 0.20 & 0.490 & $U$ & 0.49 & 29.6 & & 2.45 & 21.6 & & 9.80 \\
\hline 100-H-25 (Depth) & $\mathrm{J} 1 \mathrm{HJ} 80-4$ & $8 / 24 / 11$ & 0.192 & $\mathrm{U}$ & 0.19 & 0.481 & $U$ & 0.48 & 29.5 & & 2.40 & 24.7 & & 9.62 \\
\hline 100-H-20 (Depth) & J1HJ81-1 & $8 / 24 / 11$ & 0.152 & $U$ & 0.15 & 0.379 & $\mathrm{U}$ & 0.38 & 28.5 & & 1.89 & 55.5 & & 7.58 \\
\hline 100-H-20 (Depth) & J1HJ81-2 & $8 / 24 / 11$ & 0.167 & U & 0.17 & 0.417 & $\mathrm{U}$ & 0.42 & 42.4 & & 2.08 & 31.8 & & 8.33 \\
\hline 100-H-20 (Depth) & J1HJ81-3 & $8 / 24 / 11$ & 0.200 & $\mathrm{U}$ & 0.20 & 0.500 & $\mathrm{U}$ & 0.50 & 45.6 & & 2.50 & 32.0 & & 10.0 \\
\hline 100-H-20 (Depth) & $\mathrm{J} 1 \mathrm{HJ} 81-4$ & $8 / 24 / 11$ & 0.169 & $\mathrm{U}$ & 0.17 & 0.424 & $\mathrm{U}$ & 0.42 & 35.7 & & 2.12 & 34.1 & & 8.47 \\
\hline 100-H-17 (Depth) & J1HJ82-1 & $8 / 27 / 11$ & 0.196 & $\mathrm{U}$ & 0.20 & 0.490 & $\mathrm{U}$ & 0.49 & 21.4 & & 2.45 & 42.4 & & 9.80 \\
\hline 100-H-17 (Depth) & J1HJ82-2 & $8 / 27 / 11$ & 0.192 & U & 0.19 & 0.481 & 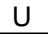 & 0.48 & 19.2 & & 2.40 & 26.7 & & 9.62 \\
\hline 100-H-17 (Depth) & J1HJ82-3 & $8 / 27 / 11$ & 0.161 & $U$ & 0.16 & 0.403 & $U$ & 0.40 & 22.0 & & 2.02 & 28.0 & & 8.06 \\
\hline 100-H-17 (Depth) & $\mathrm{J} 1 \mathrm{HJ} 82-4$ & $8 / 27 / 11$ & 0.192 & $U$ & 0.19 & 0.481 & $\mathrm{U}$ & 0.48 & 22.9 & & 2.40 & 28.5 & & 9.62 \\
\hline
\end{tabular}


Table C-9. 126-H-1 Coal Ash Leaching Sample Results. (2 Pages)

\begin{tabular}{|c|c|c|c|c|c|c|c|c|c|c|c|c|c|c|c|c|c|c|}
\hline \multirow{2}{*}{ Sample Location } & \multirow{2}{*}{$\begin{array}{l}\text { Coal Ash:Water } \\
\text { Ratio }\end{array}$} & \multirow{2}{*}{$\begin{array}{c}\text { HEIS } \\
\text { Number }\end{array}$} & \multirow{2}{*}{$\begin{array}{l}\text { Sample } \\
\text { Date }\end{array}$} & \multicolumn{3}{|c|}{ Antimony } & \multicolumn{3}{|c|}{ Arsenic } & \multicolumn{3}{|c|}{ Barium } & \multicolumn{3}{|c|}{ Beryllium } & \multicolumn{3}{|c|}{ Boron } \\
\hline & & & & $\mathrm{mg} / \mathrm{L}$ & Q & $\mathrm{PQL}$ & $\mathrm{mg} / \mathrm{L}$ & Q & $\mathrm{PQL}$ & $\mathrm{mg} / \mathrm{L}$ & Q & $\mathrm{PQL}$ & $\mathrm{mg} / \mathrm{L}$ & Q & $\overline{\mathrm{PQL}}$ & $\mathrm{mg} / \mathrm{L}$ & Q & $\mathrm{PQL}$ \\
\hline 100-H-25 (Depth) & $1: 1$ & J1HJ80-A1 & $8 / 24 / 11$ & 0.100 & $\bar{U}$ & 0.10 & 0.075 & $\bar{U}$ & 0.08 & 0.076 & & 0.01 & 0.005 & $\bar{U}$ & 0.01 & 13.4 & & 0.08 \\
\hline 100-H-25 (Depth) & $1: 2.5$ & J1HJ80-B1 & $8 / 24 / 11$ & 0.100 & $U$ & 0.10 & 0.075 & U & 0.08 & 0.075 & & 0.01 & 0.005 & $\mathrm{U}$ & 0.01 & 7.18 & & 0.08 \\
\hline $100-\mathrm{H}-25$ (Depth) & $1: 2.5$ & J1HJ80-B2 & $8 / 24 / 11$ & 0.100 & $U$ & 0.10 & 0.075 & $U$ & 0.08 & 0.077 & & 0.01 & 0.005 & $\mathrm{U}$ & 0.01 & 6.79 & & 0.08 \\
\hline 100-H-25 (Depth) & $1: 5$ & J1HJ80-C1 & $8 / 24 / 11$ & 0.100 & $U$ & 0.10 & 0.075 & $U$ & 0.08 & 0.077 & & 0.01 & 0.005 & $\mathrm{U}$ & 0.01 & 4.04 & & 0.08 \\
\hline 100-H-20 (Depth) & $1: 1$ & J1HJ81-A1 & $8 / 24 / 11$ & 0.100 & $U$ & 0.10 & 0.075 & $U$ & 0.08 & 0.066 & & 0.01 & 0.005 & $\mathrm{U}$ & 0.01 & 4.95 & & 0.08 \\
\hline 100-H-20 (Depth) & $1: 2.5$ & J1HJ81-B1 & $8 / 24 / 11$ & 0.100 & $U$ & 0.10 & 0.075 & $\mathrm{U}$ & 0.08 & 0.069 & & 0.01 & 0.005 & $\mathrm{U}$ & 0.01 & 2.82 & & 0.08 \\
\hline 100-H-20 (Depth) & $1: 5$ & $\mathrm{~J} 1 \mathrm{HJ} 81-\mathrm{C} 1$ & $8 / 24 / 11$ & 0.100 & $U$ & 0.10 & 0.075 & $U$ & 0.08 & 0.081 & & 0.01 & 0.005 & $\mathrm{U}$ & 0.01 & 1.75 & & 0.08 \\
\hline 100-H-20 (Depth) & $1: 5$ & J1HJ81-C2 & $8 / 24 / 11$ & 0.100 & $U$ & 0.10 & 0.075 & $U$ & 0.08 & 0.079 & & 0.01 & 0.005 & $\mathrm{U}$ & 0.01 & 1.75 & & 0.08 \\
\hline 100-H-17 (Depth) & $1: 1$ & J1HJ82-A1 & $8 / 27 / 11$ & 0.100 & $U$ & 0.10 & 0.075 & $U$ & 0.08 & 0.039 & & 0.01 & 0.005 & $\mathrm{U}$ & 0.01 & 10.2 & & 0.08 \\
\hline $100-\mathrm{H}-17$ (Depth) & $1: 1$ & J1HJ82-A2 & $8 / 27 / 11$ & 0.100 & $U$ & 0.10 & 0.075 & $U$ & 0.08 & 0.041 & & 0.01 & 0.005 & $\mathrm{U}$ & 0.01 & 11.2 & & 0.08 \\
\hline 100-H-17 (Depth) & $1: 2.5$ & $\mathrm{~J} 1 \mathrm{HJ} 82-\mathrm{B} 1$ & $8 / 27 / 11$ & 0.100 & $U$ & 0.10 & 0.075 & $U$ & 0.08 & 0.042 & & 0.01 & 0.005 & $U$ & 0.01 & 6.66 & & 0.08 \\
\hline 100-H-17 (Depth) & $1: 5$ & $\mathrm{~J} 1 \mathrm{HJ} 82-\mathrm{C} 1$ & $8 / 27 / 11$ & 0.100 & $U$ & 0.10 & 0.075 & U & 0.08 & 0.039 & & 0.01 & 0.005 & $U$ & 0.01 & 4.11 & & 0.08 \\
\hline \multirow{2}{*}{ Sample Location } & \multirow{2}{*}{$\begin{array}{c}\text { Coal Ash:Water } \\
\text { Ratio }\end{array}$} & \multirow{2}{*}{$\begin{array}{c}\text { HEIS } \\
\text { Number }\end{array}$} & \multirow{2}{*}{$\begin{array}{c}\text { Sample } \\
\text { Date }\end{array}$} & \multicolumn{3}{|c|}{ Cadmium } & \multicolumn{3}{|c|}{ Chromium } & \multicolumn{3}{|c|}{ Cobalt } & \multicolumn{3}{|c|}{ Copper } & \multicolumn{3}{|c|}{ Lead } \\
\hline & & & & $\mathrm{mg} / \mathrm{L}$ & Q & PQL & $\mathrm{mg} / \mathrm{L}$ & $\mathbf{Q}$ & $\mathrm{PQL}$ & $\mathrm{mg} / \mathrm{L}$ & $Q$ & $\mathrm{PQL}$ & $\mathrm{mg} / \mathrm{L}$ & $Q$ & $\mathrm{PQL}$ & $\mathrm{mg} / \mathrm{L}$ & Q & $\mathrm{PQL}$ \\
\hline 100-H-25 (Depth) & $1: 1$ & J1HJ80-A1 & $8 / 24 / 11$ & 0.015 & $\dot{U}$ & 0.02 & 0.005 & B & 0.03 & 0.010 & 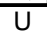 & 0.01 & 0.100 & $\dot{U}$ & 0.10 & 0.050 & 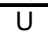 & 0.05 \\
\hline $100-\mathrm{H}-25$ (Depth) & $1: 2.5$ & J1HJ80-B1 & $8 / 24 / 11$ & 0.015 & $U$ & 0.02 & 0.003 & B & 0.03 & 0.010 & $U$ & 0.01 & 0.100 & U & 0.10 & 0.050 & $U$ & 0.05 \\
\hline $100-\mathrm{H}-25$ (Depth) & $1: 2.5$ & J1HJ80-B2 & $8 / 24 / 11$ & 0.015 & $U$ & 0.02 & 0.003 & B & 0.03 & 0.010 & $U$ & 0.01 & 0.100 & $U$ & 0.10 & 0.011 & B & 0.05 \\
\hline $100-\mathrm{H}-25$ (Depth) & $1: 5$ & J1HJ80-C1 & $8 / 24 / 11$ & 0.015 & $\mathrm{U}$ & 0.02 & 0.025 & $\bar{U}$ & 0.03 & 0.010 & $U$ & 0.01 & 0.100 & $U$ & 0.10 & 0.050 & $U$ & 0.05 \\
\hline $100-\mathrm{H}-20$ (Depth) & $1: 1$ & J1HJ81-A1 & $8 / 24 / 11$ & 0.015 & $\mathrm{U}$ & 0.02 & 0.008 & B & 0.03 & 0.010 & $\mathrm{U}$ & 0.01 & 0.100 & U & 0.10 & 0.050 & 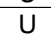 & 0.05 \\
\hline 100-H-20 (Depth) & $1: 2.5$ & J1HJ81-B1 & $8 / 24 / 11$ & 0.015 & $U$ & 0.02 & 0.025 & $U$ & 0.03 & 0.010 & $U$ & 0.01 & 0.100 & $U$ & 0.10 & 0.050 & $U$ & 0.05 \\
\hline 100-H-20 (Depth) & $1: 5$ & J1HJ81-C1 & $8 / 24 / 11$ & 0.015 & $U$ & 0.02 & 0.025 & $U$ & 0.03 & 0.010 & $U$ & 0.01 & 0.100 & U & 0.10 & 0.050 & $U$ & 0.05 \\
\hline 100-H-20 (Depth) & $1: 5$ & $\mathrm{~J} 1 \mathrm{HJ} 81-\mathrm{C} 2$ & $8 / 24 / 11$ & 0.015 & $\mathrm{U}$ & 0.02 & 0.025 & $\mathrm{U}$ & 0.03 & 0.010 & $U$ & 0.01 & 0.100 & $U$ & 0.10 & 0.050 & $U$ & 0.05 \\
\hline 100-H-17 (Depth) & $1: 1$ & J1HJ82-A1 & $8 / 27 / 11$ & 0.015 & $\mathrm{U}$ & 0.02 & 0.025 & $\mathrm{U}$ & 0.03 & 0.010 & $U$ & 0.01 & 0.100 & $U$ & 0.10 & 0.050 & $U$ & 0.05 \\
\hline 100-H-17 (Depth) & $1: 1$ & J1HJ82-A2 & $8 / 27 / 11$ & 0.015 & $\mathrm{U}$ & 0.02 & 0.025 & $U$ & 0.03 & 0.010 & $U$ & 0.01 & 0.100 & $\mathrm{U}$ & 0.10 & 0.050 & $U$ & 0.05 \\
\hline 100-H-17 (Depth) & $1: 2.5$ & J1HJ82-B1 & $8 / 27 / 11$ & 0.015 & $\mathrm{U}$ & 0.02 & 0.025 & $U$ & 0.03 & 0.010 & $U$ & 0.01 & 0.100 & $\mathrm{U}$ & 0.10 & 0.050 & $U$ & 0.05 \\
\hline $100-\mathrm{H}-17$ (Depth) & $1: 5$ & J1HJ82-C1 & $8 / 27 / 11$ & 0.015 & $\mathrm{U}$ & 0.02 & 0.025 & $\mathrm{U}$ & 0.03 & 0.010 & $\mathrm{U}$ & 0.01 & 0.100 & U & 0.10 & 0.050 & U & 0.05 \\
\hline
\end{tabular}


Table C-9. 126-H-1 Coal Ash Leaching Sample Results. (2 Pages)

\begin{tabular}{|c|c|c|c|c|c|c|c|c|c|c|c|c|c|c|c|c|c|c|c|}
\hline \multirow{2}{*}{ Sample Location } & \multirow{2}{*}{$\begin{array}{l}\text { Coal Ash:Water } \\
\text { Ratio }\end{array}$} & \multirow{2}{*}{$\begin{array}{c}\text { HEIS } \\
\text { Number }\end{array}$} & \multirow{2}{*}{$\begin{array}{c}\text { Sample } \\
\text { Date }\end{array}$} & \multicolumn{3}{|c|}{ Manganese } & \multicolumn{3}{|c|}{ Mercury } & \multicolumn{3}{|c|}{ Molybdenum } & \multicolumn{3}{|c|}{ Nickel } & \multicolumn{3}{|c|}{$\begin{array}{l}\text { Selenium } \\
\end{array}$} & \\
\hline & & & & $\mathrm{mg} / \mathrm{L}$ & $Q$ & PQL & $\mathrm{mg} / \mathrm{L}$ & Q & PQL & $\mathrm{mg} / \mathrm{L}$ & Q & PQL & $\mathrm{mg} / \mathrm{L}$ & Q & $\mathrm{PQL}$ & $\mathrm{mg} / \mathrm{L}$ & Q & PQL & \\
\hline 100-H-25 (Depth) & $1: 1$ & J1HJ80-A1 & $8 / 24 / 11$ & 0.025 & $\dot{U}$ & 0.03 & 0.000186 & $\mathrm{~B}$ & 0.0004 & 0.017 & & 0.02 & 0.100 & $\dot{U}$ & 0.10 & 0.100 & $\bar{U}$ & 0.10 & \\
\hline $100-\mathrm{H}-25$ (Depth) & $1: 2.5$ & J1HJ80-B1 & $8 / 24 / 11$ & 0.025 & $U$ & 0.03 & 0.000103 & B & 0.0002 & 0.008 & B & 0.02 & 0.100 & $U$ & 0.10 & 0.100 & $U$ & 0.10 & \\
\hline 100-H-25 (Depth) & $1: 2.5$ & J1HJ80-B2 & $8 / 24 / 11$ & 0.025 & $U$ & 0.03 & 0.000111 & B & 0.0002 & 0.009 & B & 0.02 & 0.003 & B & 0.10 & 0.100 & $U$ & 0.10 & \\
\hline 100-H-25 (Depth) & $1: 5$ & $\mathrm{~J} 1 \mathrm{HJ} 80-\mathrm{C} 1$ & $8 / 24 / 11$ & 0.025 & $U$ & 0.03 & 0.000100 & B & 0.0002 & 0.007 & B & 0.02 & 0.100 & $U$ & 0.10 & 0.100 & $U$ & 0.10 & \\
\hline 100-H-17 (Depth) & $1: 1$ & J1HJ81-A1 & $8 / 24 / 11$ & 0.025 & $\mathrm{U}$ & 0.03 & 0.000339 & $\mathrm{~B}$ & 0.0006 & 0.010 & $\mathrm{~B}$ & 0.02 & 0.100 & $\mathrm{U}$ & 0.10 & 0.100 & $\mathrm{U}$ & 0.10 & \\
\hline $100-\mathrm{H}-17$ (Depth) & $1: 2.5$ & J1HJ81-B1 & $8 / 24 / 11$ & 0.025 & $U$ & 0.03 & 0.000115 & $\mathrm{~B}$ & 0.0002 & 0.003 & $B$ & 0.02 & 0.100 & $U$ & 0.10 & 0.100 & $U$ & 0.10 & \\
\hline 100-H-17 (Depth) & $1: 5$ & $\mathrm{J1HJ81-C1}$ & $8 / 24 / 11$ & 0.025 & $\mathrm{U}$ & 0.03 & 0.000117 & $\mathrm{~B}$ & 0.0002 & 0.015 & $\mathrm{U}$ & 0.02 & 0.100 & $\mathrm{U}$ & 0.10 & 0.100 & $\bar{U}$ & 0.10 & \\
\hline 100-H-17 (Depth) & $1: 5$ & $\mathrm{~J} 1 \mathrm{HJ} 81-\mathrm{C} 2$ & $8 / 24 / 11$ & 0.025 & $\mathrm{U}$ & 0.03 & 0.000110 & $\mathrm{~B}$ & 0.0002 & 0.015 & $U$ & 0.02 & 0.100 & $U$ & 0.10 & 0.100 & $\mathrm{U}$ & 0.10 & \\
\hline $100-\mathrm{H}-20$ (Depth) & $1: 1$ & J1HJ82-A1 & $8 / 27 / 11$ & 0.025 & $U$ & 0.03 & 0.000366 & $\mathrm{~B}$ & 0.0006 & 0.018 & & 0.02 & 0.100 & $U$ & 0.10 & 0.019 & B & 0.10 & \\
\hline $100-\mathrm{H}-20$ (Depth) & $1: 1$ & J1HJ82-A2 & $8 / 27 / 11$ & 0.025 & $U$ & 0.03 & 0.000202 & $\mathrm{~B}$ & 0.0004 & 0.019 & & 0.02 & 0.100 & $U$ & 0.10 & 0.018 & $B$ & 0.10 & \\
\hline 100-H-20 (Depth) & $1: 2.5$ & J1HJ82-B1 & $8 / 27 / 11$ & 0.025 & $\mathrm{U}$ & 0.03 & 0.000115 & $\mathrm{~B}$ & 0.0002 & 0.008 & $\mathrm{~B}$ & 0.02 & 0.100 & $\mathrm{U}$ & 0.10 & 0.100 & $\bar{U}$ & 0.10 & \\
\hline $100-\mathrm{H}-20$ (Depth) & $1: 5$ & $\mathrm{~J} 1 \mathrm{HJ} 82-\mathrm{C} 1$ & $8 / 27 / 11$ & 0.025 & $\mathrm{U}$ & 0.03 & 0.000102 & $\mathrm{~B}$ & 0.0002 & 0.005 & $\mathrm{~B}$ & 0.02 & 0.100 & $U$ & 0.10 & 0.100 & $\mathrm{U}$ & 0.10 & \\
\hline \multirow{2}{*}{ Sample Location } & Coal Ash:Water & HEIS & Sample & \multicolumn{3}{|c|}{ Silver } & \multicolumn{3}{|c|}{$\begin{array}{l}\text { Thallium } \\
\end{array}$} & \multicolumn{3}{|c|}{ Uranium (KPA) } & \multicolumn{3}{|c|}{ Vanadium } & \multicolumn{3}{|c|}{ Zinc } & \\
\hline & Ratio & Number & Date & $\mathrm{mg} / \mathrm{L}$ & $\mathbf{Q}$ & $\mathrm{PQL}$ & $\mathrm{mg} / \mathrm{L}$ & $\mathbf{Q}$ & $\mathrm{PQL}$ & ug/L & Q & MDA & $\mathrm{mg} / \mathrm{L}$ & $\mathbf{Q}$ & $\mathrm{PQL}$ & $\mathrm{mg} / \mathrm{L}$ & Q & $\mathrm{PQL}$ & $\mathrm{pH}$ \\
\hline 100-H-25 (Depth) & $1: 1$ & J1HJ80-A1 & $8 / 24 / 11$ & 0.030 & $\dot{U}$ & 0.03 & 0.075 & $\dot{U}$ & 0.08 & 1.77 & & 0.032 & 0.046 & & 0.03 & 0.250 & $\dot{U}$ & 0.25 & 8.41 \\
\hline $100-\mathrm{H}-25$ (Depth) & $1: 2.5$ & J1HJ80-B1 & $8 / 24 / 11$ & 0.030 & $U$ & 0.03 & 0.075 & $U$ & 0.08 & 0.897 & & 0.032 & 0.034 & & 0.03 & 0.250 & $U$ & 0.25 & 9.05 \\
\hline 100-H-25 (Depth) & $1: 2.5$ & $\mathrm{~J} 1 \mathrm{HJ} 80-\mathrm{B} 2$ & $8 / 24 / 11$ & 0.030 & $U$ & 0.03 & 0.075 & $\mathrm{U}$ & 0.08 & 0.842 & & 0.032 & 0.032 & & 0.03 & 0.250 & $\bar{U}$ & 0.25 & 9.12 \\
\hline 100-H-25 (Depth) & $1: 5$ & $\mathrm{J1HJ80-C1}$ & $8 / 24 / 11$ & 0.030 & $\mathrm{U}$ & 0.03 & 0.075 & $U$ & 0.08 & 0.551 & & 0.032 & 0.027 & & 0.03 & 0.250 & $U$ & 0.25 & 9.31 \\
\hline $100-\mathrm{H}-20$ (Depth) & $1: 1$ & J1HJ81-A1 & $8 / 24 / 11$ & 0.030 & $U$ & 0.03 & 0.075 & $U$ & 0.08 & 1.09 & & 0.032 & 0.032 & & 0.03 & 0.250 & $U$ & 0.25 & 9.01 \\
\hline 100-H-20 (Depth) & $1: 2.5$ & J1HJ81-B1 & $8 / 24 / 11$ & 0.030 & $U$ & 0.03 & 0.075 & $U$ & 0.08 & 0.677 & & 0.032 & 0.028 & & 0.03 & 0.250 & $U$ & 0.25 & 9.17 \\
\hline $100-\mathrm{H}-20$ (Depth) & $1: 5$ & J1HJ81-C1 & $8 / 24 / 11$ & 0.030 & $U$ & 0.03 & 0.075 & $U$ & 0.08 & 0.322 & & 0.032 & 0.021 & B & 0.03 & 0.250 & $U$ & 0.25 & 9.35 \\
\hline 100-H-20 (Depth) & 1:5 & $\mathrm{J} 1 \mathrm{HJ} 81-\mathrm{C} 2$ & $8 / 24 / 11$ & 0.030 & $U$ & 0.03 & 0.075 & $U$ & 0.08 & 0.407 & & 0.032 & 0.020 & B & 0.03 & 0.250 & $U$ & 0.25 & 9.31 \\
\hline 100-H-17 (Depth) & $1: 1$ & J1HJ82-A1 & $8 / 27 / 11$ & 0.030 & $U$ & 0.03 & 0.075 & $U$ & 0.08 & 5.94 & & 0.032 & 0.033 & & 0.03 & 0.250 & $U$ & 0.25 & 8.46 \\
\hline 100-H-17 (Depth) & $1: 1$ & J1HJ82-A2 & $8 / 27 / 11$ & 0.030 & $U$ & 0.03 & 0.075 & $U$ & 0.08 & 5.96 & & 0.032 & 0.037 & & 0.03 & 0.250 & $U$ & 0.25 & 8.43 \\
\hline 100-H-17 (Depth) & $1: 2.5$ & J1HJ82-B1 & $8 / 27 / 11$ & 0.030 & $U$ & 0.03 & 0.075 & $U$ & 0.08 & 3.34 & & 0.032 & 0.025 & B & 0.03 & 0.250 & $U$ & 0.25 & 8.56 \\
\hline $100-\mathrm{H}-17$ (Depth) & $1: 5$ & $\mathrm{J1HJ82-C1}$ & $8 / 27 / 11$ & 0.030 & $\mathrm{U}$ & 0.03 & 0.075 & $U$ & 0.08 & 2.28 & & 0.032 & 0.019 & $\mathrm{~B}$ & 0.03 & 0.250 & $U$ & 0.25 & 8.64 \\
\hline
\end{tabular}


Table C-10. 300 Area Coal Ash Sample Results. (5 Pages)

\begin{tabular}{|c|c|c|c|c|c|c|c|c|c|c|c|c|c|c|c|c|c|}
\hline \multirow{2}{*}{$\begin{array}{c}\text { Sample } \\
\text { Location }\end{array}$} & \multirow{2}{*}{$\begin{array}{c}\text { HEIS } \\
\text { Number }\end{array}$} & \multirow{2}{*}{$\begin{array}{c}\text { Sample } \\
\text { Date }\end{array}$} & \multicolumn{3}{|c|}{ Antimony } & \multicolumn{3}{|c|}{ Arsenic } & \multicolumn{3}{|c|}{ Barium } & \multicolumn{3}{|c|}{ Beryllium } & \multicolumn{3}{|c|}{ Boron } \\
\hline & & & $\mathrm{mg} / \mathrm{kg}$ & $\mathbf{Q}$ & PQL & $\mathrm{mg} / \mathrm{kg}$ & $\mathbf{Q}$ & PQL & $\mathrm{mg} / \mathrm{kg}$ & $\mathbf{Q}$ & $\mathrm{PQL}$ & $\mathrm{mg} / \mathrm{kg}$ & $\mathbf{Q}$ & PQL & $\mathrm{mg} / \mathrm{kg}$ & $\mathbf{Q}$ & PQL \\
\hline $300-01$ & J1HJN8 & $8 / 26 / 11$ & 0.469 & UJ & 0.47 & 3.30 & & 0.78 & 486 & & 0.39 & 0.934 & & 0.16 & 129 & & 1.56 \\
\hline $300-02$ & J1HJN7 & $8 / 25 / 11$ & 0.448 & UJ & 0.45 & 2.89 & & 0.75 & 490 & & 0.37 & 0.784 & & 0.15 & 161 & & 1.49 \\
\hline 300-02 (Depth) & J1HJV1 & $8 / 25 / 11$ & 0.545 & UJ & 0.55 & 2.25 & & 0.91 & 527 & & 0.46 & 0.862 & & 0.18 & 288 & & 1.82 \\
\hline $300-03$ & J1HJN6 & $8 / 25 / 11$ & 0.455 & UJ & 0.46 & 3.33 & & 0.76 & 306 & & 0.38 & 0.544 & & 0.15 & 65.4 & & 1.52 \\
\hline 300-03 (Depth) & J1HJV2 & $8 / 25 / 11$ & 0.526 & UJ & 0.53 & 2.37 & & 0.88 & 526 & & 0.44 & 0.818 & & 0.18 & 256 & & 1.75 \\
\hline $300-04$ & J1HJN9 & $8 / 25 / 11$ & 0.588 & UJ & 0.59 & 2.71 & & 0.98 & 541 & & 0.49 & 1.14 & & 0.20 & 196 & & 1.96 \\
\hline $\begin{array}{c}\text { Duplicate of } \\
\text { J1HJN9 }\end{array}$ & J1HJT5 & $8 / 25 / 11$ & 0.484 & UJ & 0.48 & 2.66 & & 0.81 & 639 & & 0.40 & 1.03 & & 0.16 & 199 & & 1.61 \\
\hline 300-04 (Depth) & J1HJV0 & $8 / 25 / 11$ & 0.476 & UJ & 0.48 & 2.45 & & 0.79 & 629 & & 0.40 & 1.03 & & 0.16 & 360 & & 1.59 \\
\hline $300-05$ & J1HJP0 & $8 / 26 / 11$ & 0.577 & UJ & 0.58 & 3.10 & & 0.96 & 608 & & 0.48 & 0.995 & & 0.19 & 177 & & 1.92 \\
\hline $300-06$ & J1HJP1 & $8 / 26 / 11$ & 0.492 & UJ & 0.49 & 2.30 & & 0.82 & 521 & $\mathrm{~J}$ & 0.41 & 0.878 & & 0.16 & 459 & & 1.64 \\
\hline $\begin{array}{c}\text { Duplicate of } \\
\text { J1HJP1 }\end{array}$ & J1HJT6 & $8 / 26 / 11$ & 0.462 & UJ & 0.46 & 2.36 & & 0.77 & 564 & & 0.39 & 0.853 & & 0.15 & 492 & & 1.54 \\
\hline $300-07$ & J1HJP2 & $8 / 26 / 11$ & 0.492 & UJ & 0.49 & 3.07 & & 0.82 & 636 & $\mathrm{~J}$ & 0.41 & 1.22 & & 0.16 & 211 & & 1.64 \\
\hline $300-08$ & J1HJP3 & $8 / 26 / 11$ & 0.566 & UJ & 0.57 & 2.98 & & 0.94 & 754 & $\mathrm{~J}$ & 0.47 & 1.14 & & 0.19 & 257 & & 1.89 \\
\hline $300-09$ & J1HJP4 & $8 / 26 / 11$ & 0.484 & UJ & 0.48 & 1.60 & & 0.81 & 177 & $\mathrm{~J}$ & 0.40 & 0.588 & & 0.16 & 139 & & 1.61 \\
\hline $300-10$ & J1HJP5 & $8 / 26 / 11$ & 0.517 & UJ & 0.52 & 3.63 & & 0.86 & 782 & $\mathrm{~J}$ & 0.43 & 1.15 & & 0.17 & 253 & & 1.72 \\
\hline $300-11$ & J1HJP6 & $8 / 26 / 11$ & 0.508 & UJ & 0.51 & 3.08 & & 0.85 & 748 & $\mathrm{~J}$ & 0.42 & 1.09 & & 0.17 & 369 & & 1.69 \\
\hline $300-12$ & J1HJP7 & $8 / 26 / 11$ & 0.536 & UJ & 0.54 & 2.92 & & 0.89 & 552 & $\mathrm{~J}$ & 0.45 & 1.37 & & 0.18 & 193 & & 1.79 \\
\hline $300-13$ & J1HJP8 & $8 / 26 / 11$ & 0.476 & UJ & 0.48 & 2.41 & & 0.79 & 297 & $\mathrm{~J}$ & 0.40 & 0.948 & & 0.16 & 260 & & 1.59 \\
\hline $300-14$ & J1HJP9 & $8 / 26 / 11$ & 0.492 & UJ & 0.49 & 2.09 & & 0.82 & 204 & $\mathrm{~J}$ & 0.41 & 0.572 & & 0.16 & 135 & & 1.64 \\
\hline $300-15$ & J1HJR0 & $8 / 26 / 11$ & 0.484 & UJ & 0.48 & 2.41 & & 0.81 & 586 & $\mathrm{~J}$ & 0.40 & 0.783 & & 0.16 & 215 & & 1.61 \\
\hline $300-16$ & J1HJR1 & $8 / 26 / 11$ & 0.588 & UJ & 0.59 & 2.21 & & 0.98 & 374 & $\mathrm{~J}$ & 0.49 & 1.00 & & 0.20 & 148 & & 1.96 \\
\hline $300-17$ & J1HJR2 & $8 / 26 / 11$ & 0.500 & UJ & 0.50 & 3.36 & & 0.83 & 542 & $\mathrm{~J}$ & 0.42 & 0.919 & & 0.17 & 99 & & 1.67 \\
\hline $300-18$ & J1HJR3 & $8 / 26 / 11$ & 0.536 & UJ & 0.54 & 2.17 & & 0.89 & 244 & $\mathrm{~J}$ & 0.45 & 0.813 & & 0.18 & 208 & & 1.79 \\
\hline $300-19$ & J1HJR4 & $8 / 26 / 11$ & 0.517 & UJ & 0.52 & 2.28 & & 0.86 & 472 & $\mathrm{~J}$ & 0.43 & 0.817 & & 0.17 & 156 & & 1.72 \\
\hline $300-20$ & J1HJR5 & $8 / 26 / 11$ & 0.545 & UJ & 0.55 & 3.30 & & 0.91 & 651 & $\mathrm{~J}$ & 0.46 & 1.60 & & 0.18 & 190 & & 1.82 \\
\hline $300-21$ & J1HJR6 & $8 / 26 / 11$ & 0.556 & UJ & 0.56 & 3.87 & & 0.93 & 569 & $\mathrm{~J}$ & 0.46 & 0.926 & & 0.19 & 128 & & 1.85 \\
\hline $300-22$ & J1HJR7 & $8 / 26 / 11$ & 0.476 & UJ & 0.48 & 3.31 & & 0.79 & 407 & $\mathrm{~J}$ & 0.40 & 0.79 & & 0.16 & 74.5 & & 1.59 \\
\hline $300-23$ & J1HJR8 & $8 / 26 / 11$ & 0.536 & UJ & 0.54 & 3.00 & & 0.89 & 492 & $\mathrm{~J}$ & 0.45 & 1.30 & & 0.18 & 185 & & 1.79 \\
\hline $300-24$ & J1HJR9 & $8 / 26 / 11$ & 0.429 & UJ & 0.43 & 1.62 & & 0.71 & 208 & $\mathrm{~J}$ & 0.36 & 0.644 & & 0.14 & 137 & & 1.43 \\
\hline $300-25$ & J1HJT0 & $8 / 26 / 11$ & 0.441 & $\mathrm{UJ}$ & 0.44 & 3.34 & & 0.74 & 707 & $\mathrm{~J}$ & 0.37 & 1.30 & & 0.15 & 251 & & 1.47 \\
\hline $300-26$ & J1HJT1 & $8 / 26 / 11$ & 0.517 & $\mathrm{UJ}$ & 0.52 & 3.28 & & 0.86 & 680 & & 0.43 & 1.22 & & 0.17 & 181 & & 1.72 \\
\hline $300-27$ & J1HJT2 & $8 / 26 / 11$ & 0.56 & UJ & 0.56 & 3.70 & & 0.93 & 450 & & 0.46 & 1.95 & & 0.19 & 157 & & 1.85 \\
\hline $300-28$ & J1HJT3 & $8 / 26 / 11$ & 0.469 & UJ & 0.47 & 2.66 & & 0.78 & 273 & & 0.39 & 0.824 & & 0.16 & 104 & & 1.56 \\
\hline $300-29$ & J1HJT4 & $8 / 26 / 11$ & 0.526 & UJ & 0.53 & 3.91 & & 0.88 & 464 & & 0.44 & 1.66 & & 0.18 & 171 & & 1.75 \\
\hline Equip Blank & J1HJT7 & $8 / 25 / 11$ & 0.484 & UJ & 0.48 & 0.50 & B & 0.81 & 2.22 & & 0.40 & 0.046 & B & 0.16 & 1.61 & $\mathrm{U}$ & 1.61 \\
\hline
\end{tabular}




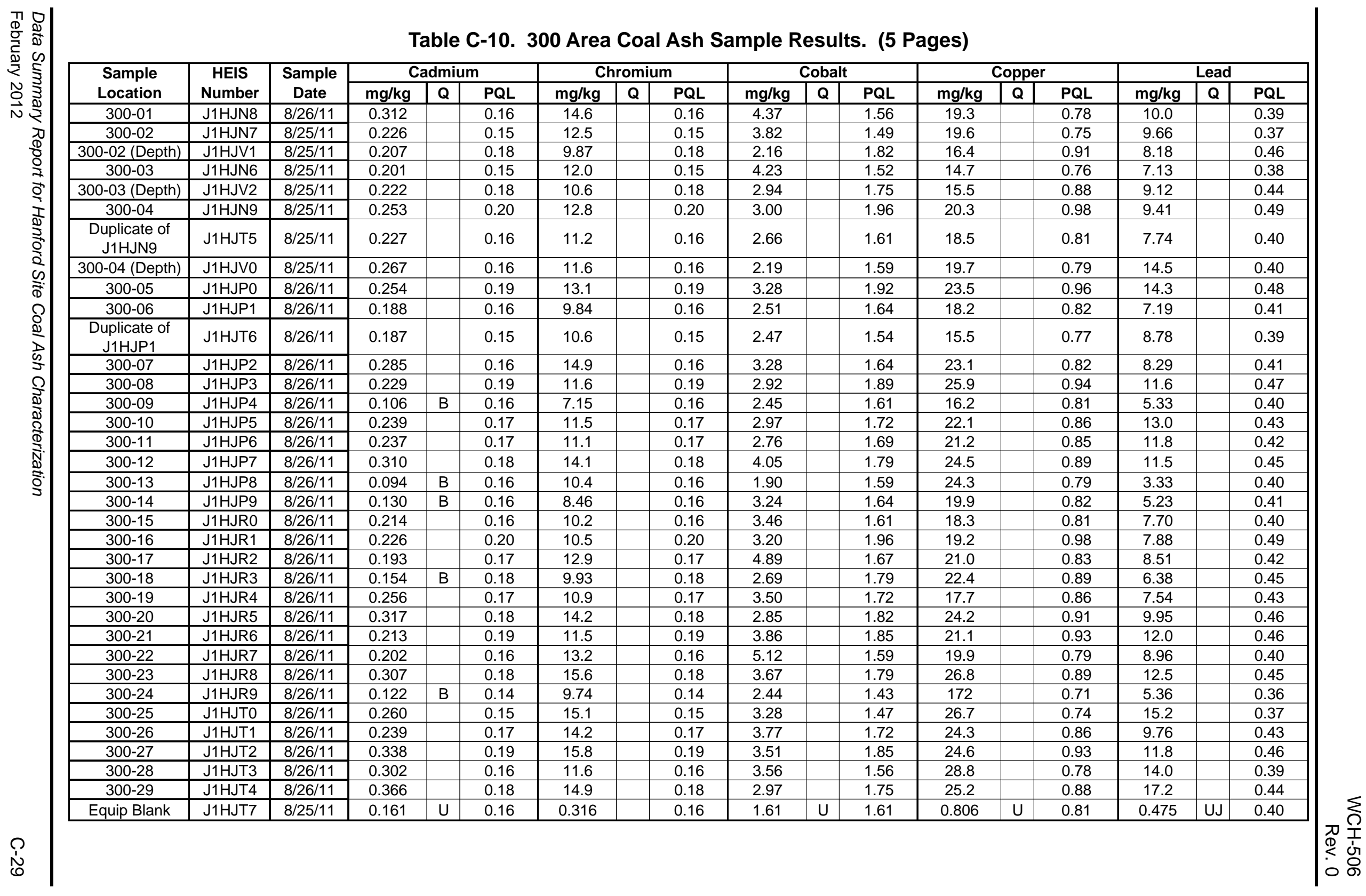


Table C-10. 300 Area Coal Ash Sample Results. (5 Pages)

\begin{tabular}{|c|c|c|c|c|c|c|c|c|c|c|c|c|c|c|c|c|c|}
\hline \multirow{2}{*}{$\begin{array}{c}\text { Sample } \\
\text { Location }\end{array}$} & \multirow{2}{*}{$\begin{array}{c}\text { HEIS } \\
\text { Number }\end{array}$} & \multirow{2}{*}{$\begin{array}{l}\text { Sample } \\
\text { Date }\end{array}$} & \multicolumn{3}{|c|}{ Manganese } & \multicolumn{3}{|c|}{ Mercury } & \multicolumn{3}{|c|}{ Molybdenum } & \multicolumn{3}{|c|}{ Nickel } & \multicolumn{3}{|c|}{ Selenium } \\
\hline & & & $\mathrm{mg} / \mathrm{kg}$ & Q & $\mathrm{PQL}$ & $\mathrm{mg} / \mathrm{kg}$ & $\mathbf{Q}$ & $\mathrm{PQL}$ & $\mathrm{mg} / \mathrm{kg}$ & $\mathbf{Q}$ & PQL & $\mathrm{mg} / \mathrm{kg}$ & $\mathbf{Q}$ & $\mathrm{PQL}$ & $\mathrm{mg} / \mathrm{kg}$ & $\mathbf{Q}$ & PQL \\
\hline $300-01$ & J1HJN8 & $8 / 26 / 11$ & 176 & & 3.91 & 0.211 & & 0.03 & 0.832 & $\mathrm{~B}$ & 1.56 & 9.09 & $\mathrm{~J}$ & 3.12 & 1.02 & $\mathrm{~J}$ & 0.23 \\
\hline $300-02$ & J1HJN7 & $8 / 25 / 11$ & 186 & & 3.73 & 0.713 & & 0.03 & 0.851 & $\mathrm{~B}$ & 1.49 & 8.37 & $\mathrm{~J}$ & 2.99 & 0.943 & & 0.22 \\
\hline 300-02 (Depth) & J1HJV1 & $8 / 25 / 11$ & 85.6 & & 4.55 & 3.32 & & 0.16 & 0.830 & $\mathrm{~B}$ & 1.82 & 5.84 & $\mathrm{~J}$ & 3.64 & 1.19 & & 0.27 \\
\hline $300-03$ & J1HJN6 & $8 / 25 / 11$ & 213 & & 3.79 & 0.060 & & 0.03 & 0.472 & $\mathrm{~B}$ & 1.52 & 9.21 & $\mathrm{~J}$ & 3.03 & 0.877 & & 0.23 \\
\hline 300-03 (Depth) & J1HJV2 & $8 / 25 / 11$ & 95.9 & & 4.39 & 0.291 & & 0.02 & 0.690 & $B$ & 1.75 & 6.53 & $\mathrm{~J}$ & 3.51 & 1.53 & & 0.26 \\
\hline $300-04$ & J1HJN9 & $8 / 25 / 11$ & 107 & & 4.90 & 0.365 & & 0.03 & 1.04 & $\mathrm{~B}$ & 1.96 & 8.97 & $\mathrm{~J}$ & 3.92 & 1.24 & & 0.29 \\
\hline $\begin{array}{c}\text { Duplicate of } \\
\text { J1HJN9 }\end{array}$ & J1HJT5 & $8 / 25 / 11$ & 91.7 & & 4.03 & 0.344 & & 0.03 & 0.946 & B & 1.61 & 8.07 & $\mathrm{~J}$ & 3.23 & 1.17 & & 0.24 \\
\hline 300-04 (Depth) & J1HJV0 & $8 / 25 / 11$ & 66.3 & & 3.97 & 0.501 & & 0.03 & 0.923 & B & 1.59 & 6.43 & $\mathrm{~J}$ & 3.17 & 1.77 & & 0.24 \\
\hline $300-05$ & J1HJP0 & $8 / 26 / 11$ & 131 & & 4.81 & 0.083 & & 0.03 & 1.02 & $\mathrm{~B}$ & 1.92 & 8.10 & $\mathrm{~J}$ & 3.85 & 1.27 & $\mathrm{~J}$ & 0.29 \\
\hline $300-06$ & J1HJP1 & $8 / 26 / 11$ & 106 & $\mathrm{~J}$ & 4.10 & 0.370 & & 0.02 & 0.968 & $\mathrm{~B}$ & 1.64 & 6.36 & & 3.28 & 1.16 & & 0.25 \\
\hline $\begin{array}{c}\text { Duplicate of } \\
\text { J1HJP1 }\end{array}$ & J1HJT6 & $8 / 26 / 11$ & 101 & & 3.85 & 0.706 & & 0.03 & 1.12 & B & 1.54 & 6.29 & $\mathrm{~J}$ & 3.08 & 1.14 & & 0.23 \\
\hline $300-07$ & J1HJP2 & $8 / 26 / 11$ & 87.5 & $\mathrm{~J}$ & 4.10 & 0.119 & & 0.02 & 1.26 & $\mathrm{~B}$ & 1.64 & 12.5 & & 3.28 & 1.460 & & 0.25 \\
\hline $300-08$ & J1HJP3 & $8 / 26 / 11$ & 92.8 & $\mathrm{~J}$ & 4.72 & 0.293 & & 0.03 & 1.23 & $\mathrm{~B}$ & 1.89 & 8.20 & & 3.77 & 1.34 & & 0.28 \\
\hline $300-09$ & J1HJP4 & $8 / 26 / 11$ & 69.5 & $\mathrm{~J}$ & 4.03 & 0.115 & & 0.02 & 0.717 & $B$ & 1.61 & 5.73 & & 3.23 & 0.67 & & 0.24 \\
\hline $300-10$ & J1HJP5 & $8 / 26 / 11$ & 96.6 & $\mathrm{~J}$ & 4.31 & 0.170 & & 0.03 & 0.952 & $B$ & 1.72 & 8.72 & & 3.45 & 1.63 & & 0.26 \\
\hline $300-11$ & J1HJP6 & $8 / 26 / 11$ & 101 & $\mathrm{~J}$ & 4.24 & 0.321 & & 0.02 & 1.43 & $B$ & 1.69 & 7.09 & & 3.39 & 1.65 & & 0.25 \\
\hline $300-12$ & J1HJP7 & $8 / 26 / 11$ & 94.5 & $\mathrm{~J}$ & 4.46 & 0.115 & & 0.03 & 1.11 & $\mathrm{~B}$ & 1.79 & 11.2 & & 3.57 & 1.62 & & 0.27 \\
\hline $300-13$ & J1HJP8 & $8 / 26 / 11$ & 93.3 & $\mathrm{~J}$ & 3.97 & 1.02 & & 0.03 & 1.50 & $B$ & 1.59 & 7.62 & & 3.17 & 0.491 & & 0.24 \\
\hline $300-14$ & J1HJP9 & $8 / 26 / 11$ & 142 & $\mathrm{~J}$ & 4.10 & 0.181 & & 0.03 & 0.706 & $\mathrm{~B}$ & 1.64 & 7.27 & & 3.28 & 0.688 & & 0.25 \\
\hline $300-15$ & J1HJR0 & $8 / 26 / 11$ & 132 & $\mathrm{~J}$ & 4.03 & 0.044 & & 0.02 & 1.17 & $B$ & 1.61 & 8.25 & & 3.23 & 0.911 & & 0.24 \\
\hline $300-16$ & J1HJR1 & $8 / 26 / 11$ & 111 & $\mathrm{~J}$ & 4.90 & 0.087 & & 0.03 & 0.879 & $B$ & 1.96 & 9.10 & & 3.92 & 1.10 & & 0.29 \\
\hline $300-17$ & J1HJR2 & $8 / 26 / 11$ & 195 & $\mathrm{~J}$ & 4.17 & 0.062 & & 0.02 & 0.701 & $B$ & 1.67 & 9.26 & & 3.33 & 0.884 & & 0.25 \\
\hline $300-18$ & J1HJR3 & $8 / 26 / 11$ & 101 & $\mathrm{~J}$ & 4.46 & 0.468 & & 0.02 & 0.981 & $B$ & 1.79 & 7.24 & & 3.57 & 0.840 & & 0.27 \\
\hline $300-19$ & J1HJR4 & $8 / 26 / 11$ & 146 & $\mathrm{~J}$ & 4.31 & 0.034 & & 0.02 & 0.916 & $B$ & 1.72 & 8.09 & & 3.45 & 0.815 & & 0.26 \\
\hline $300-20$ & J1HJR5 & $8 / 26 / 11$ & 35.5 & $\mathrm{~J}$ & 4.55 & 0.132 & & 0.03 & 1.03 & $\mathrm{~B}$ & 1.82 & 11.4 & & 3.64 & 2.46 & & 0.27 \\
\hline $300-21$ & J1HJR6 & $8 / 26 / 11$ & 134 & $\mathrm{~J}$ & 4.63 & 0.284 & & 0.03 & 0.812 & $\mathrm{~B}$ & 1.85 & 8.28 & & 3.70 & 1.09 & & 0.28 \\
\hline $300-22$ & $\mathrm{~J} 1 \mathrm{HJR} 7$ & 8/26/11 & 210 & $\mathrm{~J}$ & 3.97 & 0.052 & & 0.03 & 0.762 & B & 1.59 & 9.84 & & 3.17 & 0.929 & & 0.24 \\
\hline $300-23$ & J1HJR8 & 8/26/11 & 107 & $\mathrm{~J}$ & 4.46 & 0.068 & & 0.03 & 1.23 & B & 1.79 & 14.9 & & 3.57 & 1.08 & & 0.27 \\
\hline $300-24$ & J1HJR9 & $8 / 26 / 11$ & 81.4 & $\mathrm{~J}$ & 3.57 & 0.274 & & 0.03 & 2.12 & & 1.43 & 9.99 & & 2.86 & 0.571 & & 0.21 \\
\hline $300-25$ & J1HJT0 & $8 / 26 / 11$ & 81.1 & $\mathrm{~J}$ & 3.68 & 0.176 & & 0.02 & 1.38 & $\mathrm{~B}$ & 1.47 & 8.91 & & 2.94 & 1.63 & & 0.22 \\
\hline $300-26$ & J1HJT1 & $8 / 26 / 11$ & 144 & & 4.31 & 0.101 & & 0.03 & 1.18 & $\mathrm{~B}$ & 1.72 & 10.4 & $\mathrm{~J}$ & 3.45 & 1.40 & & 0.26 \\
\hline $300-27$ & J1HJT2 & $8 / 26 / 11$ & 61.8 & & 4.63 & 0.107 & & 0.03 & 1.07 & $\mathrm{~B}$ & 1.85 & 13.0 & $\mathrm{~J}$ & 3.70 & 2.12 & & 0.28 \\
\hline $300-28$ & J1HJT3 & $8 / 26 / 11$ & 136 & & 3.91 & 0.086 & & 0.03 & 0.708 & $\mathrm{~B}$ & 1.56 & 10.8 & $\mathrm{~J}$ & 3.12 & 1.18 & & 0.23 \\
\hline $300-29$ & J1HJT4 & $8 / 26 / 11$ & 43.9 & & 4.39 & 0.157 & & 0.03 & 0.807 & $\mathrm{~B}$ & 1.75 & 13.2 & $\mathrm{~J}$ & 3.51 & 2.65 & & 0.26 \\
\hline Equip Blank & J1HJT7 & $8 / 25 / 11$ & 5.6 & & 4.03 & 0.024 & $\mathrm{U}$ & 0.02 & 1.61 & $\mathrm{U}$ & 1.61 & 3.23 & $\mathrm{~J}$ & 3.23 & 0.346 & & 0.24 \\
\hline
\end{tabular}


Table C-10. 300 Area Coal Ash Sample Results. (5 Pages)

\begin{tabular}{|c|c|c|c|c|c|c|c|c|c|c|c|c|c|c|c|c|c|}
\hline \multirow{2}{*}{$\begin{array}{c}\text { Sample } \\
\text { Location }\end{array}$} & \multirow{2}{*}{$\begin{array}{c}\text { HEIS } \\
\text { Number }\end{array}$} & \multirow{2}{*}{$\begin{array}{c}\text { Sample } \\
\text { Date }\end{array}$} & \multicolumn{3}{|c|}{ Silver } & \multicolumn{3}{|c|}{ Thallium } & \multicolumn{3}{|c|}{ Uranium (KPA) } & \multicolumn{3}{|c|}{ Vanadium } & \multicolumn{3}{|c|}{ Zinc } \\
\hline & & & $\mathrm{mg} / \mathrm{kg}$ & $\mathbf{Q}$ & PQL & $\mathrm{mg} / \mathrm{kg}$ & Q & PQL & ug/g & Q & MDA & $\mathrm{mg} / \mathrm{kg}$ & Q & PQL & $\mathrm{mg} / \mathrm{kg}$ & $\mathbf{Q}$ & PQL \\
\hline 300-01 & J1HJN8 & $8 / 26 / 11$ & 0.156 & U & 0.16 & 0.391 & U & 0.390 & & & & 42.4 & & 1.95 & 53.0 & $\mathrm{~J}$ & 7.81 \\
\hline $300-02$ & J1HJN7 & $8 / 25 / 11$ & 0.149 & $U$ & 0.15 & 0.373 & U & 0.370 & 4.22 & & 0.132 & 42.8 & & 1.87 & 35.8 & $\mathrm{~J}$ & 7.46 \\
\hline 300-02 (Depth) & J1HJV1 & $8 / 25 / 11$ & 0.182 & $U$ & 0.18 & 0.455 & $U$ & 0.460 & 2.61 & & 0.132 & 25.1 & & 2.27 & 27.8 & $\mathrm{~J}$ & 9.09 \\
\hline $300-03$ & J1HJN6 & $8 / 25 / 11$ & 0.152 & $U$ & 0.15 & 0.379 & $\mathrm{U}$ & 0.380 & 3.70 & & 0.132 & 44.4 & & 1.89 & 35.3 & $\mathrm{~J}$ & 7.58 \\
\hline 300-03 (Depth) & J1HJV2 & $8 / 25 / 11$ & 0.175 & $U$ & 0.18 & 0.439 & $U$ & 0.440 & 2.86 & & 0.132 & 28.3 & & 2.19 & 26.7 & $\mathrm{~J}$ & 8.77 \\
\hline $300-04$ & $\mathrm{~J} 1 \mathrm{HJN} 9$ & $8 / 25 / 11$ & 0.196 & U & 0.20 & 0.490 & U & 0.490 & 3.36 & & 0.132 & 34.0 & & 2.45 & 27.2 & $\mathrm{~J}$ & 9.80 \\
\hline $\begin{array}{c}\text { Duplicate of } \\
\text { J1HJN9 }\end{array}$ & J1HJT5 & $8 / 25 / 11$ & 0.161 & $U$ & 0.16 & 0.403 & $U$ & 0.400 & 3.20 & & 0.132 & 31.5 & & 2.02 & 28.3 & $\mathrm{~J}$ & 8.06 \\
\hline 300-04 (Depth) & J1HJV0 & $8 / 25 / 11$ & 0.159 & $\mathrm{U}$ & 0.16 & 0.397 & U & 0.400 & 3.14 & & 0.132 & 24.3 & & 1.98 & 26.9 & $\mathrm{~J}$ & 7.94 \\
\hline $300-05$ & J1HJP0 & $8 / 26 / 11$ & 0.192 & $\mathrm{U}$ & 0.19 & 0.481 & $\mathrm{U}$ & 0.480 & & & & 36.8 & & 2.40 & 43.3 & $\mathrm{~J}$ & 9.62 \\
\hline $300-06$ & J1HJP1 & $8 / 26 / 11$ & 0.164 & $\mathrm{U}$ & 0.16 & 0.410 & $\mathrm{U}$ & 0.410 & & & & 26.4 & & 2.05 & 43.0 & $\mathrm{~J}$ & 8.20 \\
\hline $\begin{array}{c}\text { Duplicate of } \\
\text { J1HJP1 }\end{array}$ & J1HJT6 & $8 / 26 / 11$ & 0.154 & U & 0.15 & 0.385 & $U$ & 0.39 & & & & 28.3 & & 1.92 & 23.3 & $\mathrm{~J}$ & 7.69 \\
\hline $300-07$ & $\mathrm{~J} 1 \mathrm{HJP} 2$ & $8 / 26 / 11$ & 0.164 & $\mathrm{U}$ & 0.16 & 0.410 & $\mathrm{U}$ & 0.410 & & & & 39.9 & & 2.05 & 26.1 & $\mathrm{~J}$ & 8.20 \\
\hline $300-08$ & J1HJP3 & $8 / 26 / 11$ & 0.189 & $\mathrm{U}$ & 0.19 & 0.472 & U & 0.470 & & & & 29.6 & & 2.36 & 25.5 & $\mathrm{~J}$ & 9.43 \\
\hline $300-09$ & J1HJP4 & $8 / 26 / 11$ & 0.161 & $\mathrm{U}$ & 0.16 & 0.403 & U & 0.400 & & & & 21.3 & & 2.02 & 18.3 & $\mathrm{~J}$ & 8.06 \\
\hline $300-10$ & J1HJP5 & $8 / 26 / 11$ & 0.172 & 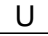 & 0.17 & 0.431 & U & 0.430 & & & & 27.8 & & 2.16 & 23.3 & $\mathrm{~J}$ & 8.62 \\
\hline $300-11$ & J1HJP6 & $8 / 26 / 11$ & 0.169 & $\mathrm{U}$ & 0.17 & 0.424 & $\mathrm{U}$ & 0.420 & & & & 29.9 & & 2.12 & 24.6 & $\mathrm{~J}$ & 8.47 \\
\hline $300-12$ & J1HJP7 & $8 / 26 / 11$ & 0.179 & $\mathrm{U}$ & 0.18 & 0.446 & $U$ & 0.450 & & & & 35.9 & & 2.23 & 30.3 & $\mathrm{~J}$ & 8.93 \\
\hline $300-13$ & J1HJP8 & $8 / 26 / 11$ & 0.159 & $U$ & 0.16 & 0.397 & $\mathrm{U}$ & 0.400 & & & & 22.1 & & 1.98 & 12.6 & $\mathrm{~J}$ & 7.94 \\
\hline $300-14$ & J1HJP9 & $8 / 26 / 11$ & 0.164 & $U$ & 0.16 & 0.410 & $\mathrm{U}$ & 0.410 & & & & 33.0 & & 2.05 & 26.0 & $\mathrm{~J}$ & 8.20 \\
\hline $300-15$ & J1HJR0 & $8 / 26 / 11$ & 0.161 & $U$ & 0.16 & 0.403 & $\mathrm{U}$ & 0.400 & & & & 32.8 & & 2.02 & 27.7 & $\mathrm{~J}$ & 8.06 \\
\hline $300-16$ & J1HJR1 & $8 / 26 / 11$ & 0.196 & $U$ & 0.20 & 0.490 & $\mathrm{U}$ & 0.490 & & & & 32.4 & & 2.45 & 29.7 & $\mathrm{~J}$ & 9.80 \\
\hline $300-17$ & J1HJR2 & $8 / 26 / 11$ & 0.167 & $U$ & 0.17 & 0.417 & $\mathrm{U}$ & 0.420 & & & & 46.2 & & 2.08 & 31.2 & $\mathrm{~J}$ & 8.33 \\
\hline $300-18$ & J1HJR3 & $8 / 26 / 11$ & 0.179 & $U$ & 0.18 & 0.446 & $\mathrm{U}$ & 0.450 & & & & 29.6 & & 2.23 & 22.2 & $\mathrm{~J}$ & 8.93 \\
\hline $300-19$ & J1HJR4 & $8 / 26 / 11$ & 0.172 & $\mathrm{U}$ & 0.17 & 0.431 & $\mathrm{U}$ & 0.430 & & & & 34.5 & & 2.16 & 34.4 & $\mathrm{~J}$ & 8.62 \\
\hline $300-20$ & J1HJR5 & $8 / 26 / 11$ & 0.182 & $U$ & 0.18 & 0.455 & $\mathrm{U}$ & 0.460 & & & & 34.1 & & 2.27 & 23.4 & $\mathrm{~J}$ & 9.09 \\
\hline $300-21$ & J1HJR6 & $8 / 26 / 11$ & 0.185 & $U$ & 0.19 & 0.463 & U & 0.460 & & & & 37.3 & & 2.31 & 28.5 & $\mathrm{~J}$ & 9.26 \\
\hline $300-22$ & J1HJR7 & $8 / 26 / 11$ & 0.159 & U & 0.16 & 0.397 & U & 0.400 & & & & 45.7 & & 1.98 & 34.7 & $\mathrm{~J}$ & 7.94 \\
\hline $300-23$ & J1HJR8 & $8 / 26 / 11$ & 0.179 & U & 0.18 & 0.446 & U & 0.450 & & & & 41.3 & & 2.23 & 36.0 & $\mathrm{~J}$ & 8.93 \\
\hline $300-24$ & J1HJR9 & $8 / 26 / 11$ & 0.143 & $U$ & 0.14 & 0.357 & $\mathrm{U}$ & 0.360 & & & & 24.8 & & 1.79 & 23.7 & $\mathrm{~J}$ & 7.14 \\
\hline $300-25$ & J1HJT0 & $8 / 26 / 11$ & 0.147 & $U$ & 0.15 & 0.368 & $\mathrm{U}$ & 0.370 & & & & 34.1 & & 1.84 & 25.8 & $\mathrm{~J}$ & 7.35 \\
\hline $300-26$ & J1HJT1 & $8 / 26 / 11$ & 0.172 & $U$ & 0.17 & 0.431 & $\mathrm{U}$ & 0.430 & & & & 40.4 & & 2.16 & 26.8 & $\mathrm{~J}$ & 8.62 \\
\hline $300-27$ & J1HJT2 & $8 / 26 / 11$ & 0.185 & $\mathrm{U}$ & 0.19 & 0.463 & $\mathrm{U}$ & 0.46 & & & & 38.2 & & 2.31 & 35.1 & $\mathrm{~J}$ & 9.26 \\
\hline $300-28$ & J1HJT3 & $8 / 26 / 11$ & 0.156 & $\mathrm{U}$ & 0.16 & 0.391 & $\mathrm{U}$ & 0.390 & & & & 36.7 & & 1.95 & 43.0 & $\mathrm{~J}$ & 7.81 \\
\hline $300-29$ & J1HJT4 & $8 / 26 / 11$ & 0.175 & $\mathrm{U}$ & 0.18 & 0.439 & $\mathrm{U}$ & 0.440 & & & & 34.6 & & 2.19 & 29.1 & $\mathrm{~J}$ & 8.77 \\
\hline Equip Blank & J1HJT7 & $8 / 25 / 11$ & 0.161 & U & 0.16 & 0.403 & U & 0.40 & & & & 0.581 & B & 2.02 & 2.00 & UJB & 8.06 \\
\hline
\end{tabular}


Table C-10. 300 Area Coal Ash Sample Results. (5 Pages)

\begin{tabular}{|c|c|c|c|c|c|c|c|c|c|c|c|c|c|c|c|c|c|c|c|c|}
\hline \multirow{2}{*}{$\begin{array}{c}\text { Sample } \\
\text { Location }\end{array}$} & \multirow{2}{*}{$\begin{array}{c}\text { HEIS } \\
\text { Number }\end{array}$} & \multirow{2}{*}{$\begin{array}{c}\text { Sample } \\
\text { Date }\end{array}$} & \multicolumn{3}{|c|}{ Acenaphthene } & \multicolumn{3}{|c|}{ Acenaphthylene } & \multicolumn{3}{|c|}{ Anthracene } & \multicolumn{3}{|c|}{ Benzo(a)anthracene } & \multicolumn{3}{|c|}{ Benzo(a)pyrene } & \multicolumn{3}{|c|}{ Benzo(b)fluoranthene } \\
\hline & & & ug/kg & Q & PQL & $u g / k g$ & Q & PQL & ug/kg & Q & $\mathrm{PQL}$ & ug/kg & Q & PQL & $u g / k g$ & Q & $\mathrm{PQL}$ & ug/kg & Q & PQL \\
\hline $300-02$ & J1HJN7 & $8 / 25 / 11$ & 338 & $\mathrm{U}$ & 338 & 338 & $\mathrm{U}$ & 338 & 338 & $\mathrm{U}$ & 338 & 338 & $\mathrm{U}$ & 338 & 338 & $\mathrm{U}$ & 338 & 338 & $\mathrm{U}$ & 338 \\
\hline $300-03$ & J1HJN6 & $8 / 25 / 11$ & 330 & $\mathrm{U}$ & 330 & 330 & $\mathrm{U}$ & 330 & 330 & 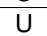 & 330 & 330 & $\bar{U}$ & 330 & 330 & $\mathrm{U}$ & 330 & 330 & $U$ & 330 \\
\hline $300-04$ & J1HJN9 & $8 / 25 / 11$ & 333 & $U$ & 333 & 333 & $U$ & 333 & 333 & 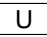 & 333 & 333 & $\mathrm{U}$ & 333 & 333 & $\bar{U}$ & 333 & 333 & $\bar{U}$ & 333 \\
\hline $\begin{array}{c}\text { Duplicate of } \\
\text { J1HJN9 }\end{array}$ & J1HJT5 & $8 / 25 / 11$ & 359 & UD & 359 & 359 & UD & 359 & 359 & UD & 359 & 359 & UD & 359 & 359 & UD & 359 & 359 & UD & 359 \\
\hline 300-02 (Depth) & J1HJV1 & $8 / 25 / 11$ & 328 & $\mathrm{U}$ & 328 & 328 & $\mathrm{U}$ & 328 & 328 & $\mathrm{U}$ & 328 & 328 & $\mathrm{U}$ & 328 & 328 & $\mathrm{U}$ & 328 & 328 & $\mathrm{U}$ & 328 \\
\hline 300-03 (Depth) & J1HJV2 & $8 / 25 / 11$ & 352 & UD & 352 & 352 & UD & 352 & 352 & UD & 352 & 352 & UD & 352 & 352 & UD & 352 & 352 & UD & 352 \\
\hline 300-04 (Depth) & J1HJV0 & $8 / 25 / 11$ & 334 & $U$ & 334 & 334 & $\mathrm{U}$ & 334 & 334 & $\mathrm{U}$ & 334 & 334 & $\mathrm{U}$ & 334 & 334 & $\mathrm{U}$ & 334 & 334 & $\mathrm{U}$ & 334 \\
\hline Equip Blank & J1HJT7 & $8 / 25 / 11$ & 324 & $U$ & 324 & 324 & $U$ & 324 & 324 & $U$ & 324 & 324 & $\mathrm{U}$ & 324 & 324 & $U$ & 324 & 324 & $U$ & 324 \\
\hline
\end{tabular}

\begin{tabular}{|c|c|c|c|c|c|c|c|c|c|c|c|c|c|c|c|c|c|c|c|c|}
\hline \multirow{2}{*}{$\begin{array}{c}\text { Sample } \\
\text { Location }\end{array}$} & \multirow{2}{*}{$\begin{array}{c}\text { HEIS } \\
\text { Number }\end{array}$} & \multirow{2}{*}{$\begin{array}{c}\text { Sample } \\
\text { Date }\end{array}$} & \multicolumn{3}{|c|}{ Benzo(ghi)perylene } & \multicolumn{3}{|c|}{ Benzo(k)fluoranthene } & \multicolumn{3}{|c|}{ Chrysene } & \multicolumn{3}{|c|}{ Dibenz(a,h)anthracene } & \multicolumn{3}{|c|}{ Fluoranthene } & \multicolumn{3}{|c|}{ Fluorene } \\
\hline & & & $u g / k g$ & Q & $\mathrm{PQL}$ & ug/kg & Q & $\mathrm{PQL}$ & $u g / \mathbf{k g}$ & $Q$ & $\mathrm{PQL}$ & ug/kg & Q & $\mathrm{PQL}$ & ug/kg & $\mathbf{Q}$ & $\mathrm{PQL}$ & ug/kg & Q & PQL \\
\hline $300-02$ & J1HJN7 & $8 / 25 / 11$ & 338 & $\mathrm{U}$ & 338 & 338 & $\mathrm{U}$ & 338 & 338 & $\bar{U}$ & 338 & 338 & $\bar{U}$ & 338 & 338 & $\mathrm{U}$ & 338 & 338 & $\mathrm{U}$ & 338 \\
\hline $300-03$ & J1HJN6 & $8 / 25 / 11$ & 330 & 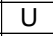 & 330 & 330 & 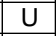 & 330 & 330 & $U$ & 330 & 330 & $U$ & 330 & 330 & $U$ & 330 & 330 & $\mathrm{U}$ & 330 \\
\hline $300-04$ & J1HJN9 & $8 / 25 / 11$ & 333 & $\mathrm{U}$ & 333 & 333 & $\mathrm{U}$ & 333 & 333 & $U$ & 333 & 333 & $U$ & 333 & 333 & $U$ & 333 & 333 & $\mathrm{U}$ & 333 \\
\hline $\begin{array}{c}\text { Duplicate of } \\
\text { J1HJN9 }\end{array}$ & J1HJT5 & 8/25/11 & 359 & UD & 359 & 359 & UD & 359 & 359 & UD & 359 & 359 & UD & 359 & 359 & UD & 359 & 359 & UD & 359 \\
\hline 300-02 (Depth) & J1HJV1 & $8 / 25 / 11$ & 328 & $\mathrm{U}$ & 328 & 328 & $\mathrm{U}$ & 328 & 328 & u & 328 & 328 & $\mathrm{U}$ & 328 & 328 & U & 328 & 328 & $\mathrm{U}$ & 328 \\
\hline 300-03 (Depth) & $\mathrm{J} 1 \mathrm{HJV} 2$ & $8 / 25 / 11$ & 352 & UD & 352 & 352 & UD & 352 & 352 & UD & 352 & 352 & UD & 352 & 352 & UD & 352 & 352 & UD & 352 \\
\hline 300-04 (Depth) & J1HJV0 & $8 / 25 / 11$ & 334 & $\mathrm{U}$ & 334 & 334 & $\mathrm{U}$ & 334 & 334 & $U$ & 334 & 334 & $\mathrm{U}$ & 334 & 334 & $\mathrm{U}$ & 334 & 334 & $\mathrm{U}$ & 334 \\
\hline Equip Blank & J1HJT7 & 8/25/11 & 324 & $U$ & 324 & 324 & $U$ & 324 & 324 & $U$ & 324 & 324 & $U$ & 324 & 324 & $U$ & 324 & 324 & $U$ & 324 \\
\hline
\end{tabular}

\begin{tabular}{|c|c|c|c|c|c|c|c|c|c|c|c|c|c|c|}
\hline \multirow{2}{*}{$\begin{array}{c}\text { Sample } \\
\text { Location }\end{array}$} & \multirow{2}{*}{$\begin{array}{c}\text { HEIS } \\
\text { Number }\end{array}$} & \multirow{2}{*}{$\begin{array}{c}\text { Sample } \\
\text { Date }\end{array}$} & \multicolumn{3}{|c|}{ Indeno(1,2,3-cd)pyrene } & \multicolumn{3}{|c|}{ Naphthalene } & \multicolumn{3}{|c|}{ Phenanthrene } & \multicolumn{3}{|c|}{ Pyrene } \\
\hline & & & ug/kg & Q & $\mathrm{PQL}$ & $u g / k g$ & Q & $\mathrm{PQL}$ & ug/kg & Q & $\mathrm{PQL}$ & $u g / k g$ & Q & $\mathrm{PQL}$ \\
\hline $300-02$ & J1HJN7 & $8 / 25 / 11$ & 338 & 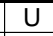 & 338 & 338 & U & 338 & 338 & U & 338 & 338 & U & 338 \\
\hline $300-03$ & J1HJN6 & $8 / 25 / 11$ & 330 & $U$ & 330 & 330 & $U$ & 330 & 330 & $U$ & 330 & 330 & $U$ & 330 \\
\hline $300-04$ & J1HJN9 & $8 / 25 / 11$ & 333 & $\mathrm{U}$ & 333 & 333 & $\mathrm{U}$ & 333 & 333 & $\mathrm{U}$ & 333 & 333 & $\mathrm{U}$ & 333 \\
\hline $\begin{array}{c}\text { Duplicate of } \\
\text { J1HJN9 }\end{array}$ & J1HJT5 & $8 / 25 / 11$ & 359 & UD & 359 & 359 & UD & 359 & 359 & UD & 359 & 359 & UD & 359 \\
\hline 300-02 (Depth) & J1HJV1 & $8 / 25 / 11$ & 328 & $\mathrm{U}$ & 328 & 124 & $\mathrm{~J}$ & 602 & 328 & $\mathrm{U}$ & 328 & 328 & $\mathrm{U}$ & 328 \\
\hline 300-03 (Depth) & J1HJV2 & $8 / 25 / 11$ & 352 & UD & 352 & 352 & UD & 352 & 352 & UD & 352 & 352 & UD & 352 \\
\hline 300-04 (Depth) & $\mathrm{J} 1 \mathrm{HJV} 0$ & $8 / 25 / 11$ & 334 & U & 334 & 334 & $\mathrm{U}$ & 334 & 334 & $\mathrm{U}$ & 334 & 334 & $\mathrm{U}$ & 334 \\
\hline Equip Blank & J1HJT7 & $8 / 25 / 11$ & 324 & $U$ & 324 & 324 & $u$ & 324 & 324 & $u$ & 324 & 324 & $\mathrm{U}$ & 324 \\
\hline
\end{tabular}




\begin{tabular}{|c|c|c|c|c|c|c|c|c|c|c|c|c|c|c|c|c|c|}
\hline \multirow{2}{*}{ 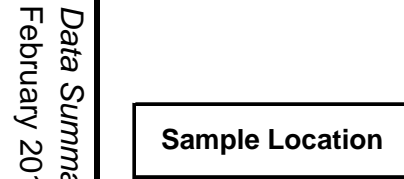 } & \multicolumn{16}{|c|}{ Table C-11. 300 Area Coal Ash Pre-Leaching Quadruplicate Sample Results. (2 Pages) } & \\
\hline & Number & Date & $\mathrm{mg} / \mathrm{kg}$ & Q & PQL & $\mathrm{mg} / \mathrm{kg}$ & Q & $\mathrm{PQL}$ & $\mathrm{mg} / \mathrm{kg}$ & $\mathbf{Q}$ & $\overline{P Q L}$ & $\mathrm{mg} / \mathrm{kg}$ & $\mathbf{Q}$ & PQL & $\mathrm{mg} / \mathrm{kg}$ & Q & $\mathrm{PQL}$ \\
\hline 岕 & J1HJV0-1 & $8 / 25 / 11$ & 0.526 & $\mathrm{U}$ & 0.53 & 2.03 & & 0.88 & 555 & & 0.44 & 0.906 & & 0.18 & 352 & & 1.75 \\
\hline 300-04 (Depth) & J1HJV0-2 & $8 / 25 / 11$ & 0.448 & $\mathrm{U}$ & 0.45 & 2.35 & & 0.75 & 548 & & 0.37 & 0.993 & & 0.15 & 367 & & 1.49 \\
\hline 300-04 (Depth) & J1HJV0-3 & $8 / 25 / 11$ & 0.400 & $\mathrm{U}$ & 0.40 & 1.98 & & 0.67 & 498 & & 0.33 & 0.905 & & 0.13 & 353 & & 1.33 \\
\hline 300-04 (Depth) & J1HJV0-4 & $8 / 25 / 11$ & 0.390 & $\mathrm{U}$ & 0.39 & 2.11 & & 0.65 & 558 & & 0.33 & 0.976 & & 0.13 & 392 & & 1.30 \\
\hline 300-02 (Depth) & J1HJV1-1 & $8 / 25 / 11$ & 0.423 & $U$ & 0.42 & 2.41 & & 0.70 & 542 & & 0.35 & 0.886 & & 0.14 & 273 & & 1.41 \\
\hline 300-02 (Depth) & J1HJV1-2 & $8 / 25 / 11$ & 0.435 & $\mathrm{U}$ & 0.44 & 2.05 & & 0.73 & 550 & & 0.36 & 0.845 & & 0.15 & 296 & & 1.45 \\
\hline 300-02 (Depth) & J1HJV1-3 & $8 / 25 / 11$ & 0.476 & $\mathrm{U}$ & 0.48 & 1.91 & & 0.79 & 699 & & 0.40 & 0.838 & & 0.16 & 283 & & 1.59 \\
\hline 300-02 (Depth) & J1HJV1-4 & $8 / 25 / 11$ & 0.517 & $\mathrm{U}$ & 0.52 & 2.16 & & 0.86 & 613 & & 0.43 & 0.903 & & 0.17 & 297 & & 1.72 \\
\hline 300-03 (Depth) & J1HJV2-1 & $8 / 25 / 11$ & 0.536 & $U$ & 0.54 & 2.55 & & 0.89 & 601 & & 0.45 & 0.846 & & 0.18 & 298 & & 1.79 \\
\hline 300-03 (Depth) & $\mathrm{J} 1 \mathrm{HJV} 2-2$ & $8 / 25 / 11$ & 0.536 & $\mathrm{U}$ & 0.54 & 2.60 & & 0.89 & 527 & & 0.45 & 0.922 & & 0.18 & 281 & & 1.79 \\
\hline 300-03 (Depth) & $\mathrm{J} 1 \mathrm{HJV} 2-3$ & $8 / 25 / 11$ & 0.469 & $U$ & 0.47 & 2.69 & & 0.78 & 513 & & 0.39 & 0.868 & & 0.16 & 294 & & 1.56 \\
\hline 300-03 (Depth) & $\mathrm{J} 1 \mathrm{HJV} 2-4$ & $8 / 25 / 11$ & 0.469 & $\mathrm{U}$ & 0.47 & 2.62 & & 0.78 & 561 & & 0.39 & 0.952 & & 0.16 & 298 & & 1.56 \\
\hline \multirow{2}{*}{ Sample Location } & \multirow{2}{*}{$\begin{array}{c}\text { HEIS } \\
\text { Number }\end{array}$} & \multirow{2}{*}{$\begin{array}{c}\text { Sample } \\
\text { Date }\end{array}$} & \multicolumn{3}{|c|}{ Cadmium } & \multicolumn{3}{|c|}{ Chromium } & \multicolumn{3}{|c|}{ Cobalt } & \multicolumn{3}{|c|}{ Copper } & \multicolumn{3}{|c|}{ Lead } \\
\hline & & & $\mathrm{mg} / \mathrm{kg}$ & Q & PQL & $\mathrm{mg} / \mathrm{kg}$ & Q & PQL & $\mathrm{mg} / \mathrm{kg}$ & Q & $\mathrm{PQL}$ & $\mathrm{mg} / \mathrm{kg}$ & $\mathrm{Q}$ & $\mathrm{PQL}$ & $\mathrm{mg} / \mathrm{kg}$ & Q & PQL \\
\hline 300-04 (Depth) & $\mathrm{J} 1 \mathrm{HJV} 0-1$ & $8 / 25 / 11$ & 0.236 & & 0.18 & 10.6 & & 0.18 & 2.53 & & 1.75 & 16.1 & & 0.88 & 11.9 & & 0.44 \\
\hline 300-04 (Depth) & J1HJV0-2 & $8 / 25 / 11$ & 0.233 & & 0.15 & 11.2 & & 0.15 & 2.41 & & 1.49 & 17.7 & & 0.75 & 13.0 & & 0.37 \\
\hline 300-04 (Depth) & $\mathrm{J} 1 \mathrm{HJV} 0-3$ & $8 / 25 / 11$ & 0.224 & & 0.13 & 9.76 & & 0.13 & 1.97 & & 1.33 & 16.0 & & 0.67 & 13.0 & & 0.33 \\
\hline 300-04 (Depth) & $\mathrm{J} 1 \mathrm{HJVO}-4$ & $8 / 25 / 11$ & 0.210 & & 0.13 & 11.7 & & 0.13 & 2.02 & & 1.30 & 17.7 & & 0.65 & 14.1 & & 0.33 \\
\hline 300-02 (Depth) & J1HJV1-1 & $8 / 25 / 11$ & 0.191 & & 0.14 & 9.67 & & 0.14 & 2.48 & & 1.41 & 16.8 & & 0.70 & 8.41 & & 0.35 \\
\hline 300-02 (Depth) & J1HJV1-2 & $8 / 25 / 11$ & 0.211 & & 0.15 & 9.33 & & 0.15 & 2.16 & & 1.45 & 16.1 & & 0.73 & 7.96 & & 0.36 \\
\hline 300-02 (Depth) & J1HJV1-3 & $8 / 25 / 11$ & 0.196 & & 0.16 & 9.23 & & 0.16 & 2.20 & & 1.59 & 16.3 & & 0.79 & 7.84 & & 0.40 \\
\hline 300-02 (Depth) & J1HJV1-4 & $8 / 25 / 11$ & 0.197 & & 0.17 & 9.51 & & 0.17 & 2.29 & & 1.72 & 19.6 & & 0.86 & 7.97 & & 0.43 \\
\hline 300-03 (Depth) & J1HJV2-1 & $8 / 25 / 11$ & 0.215 & & 0.18 & 11.1 & & 0.18 & 2.65 & & 1.79 & 17.0 & & 0.89 & 12.1 & & 0.45 \\
\hline 300-03 (Depth) & $\mathrm{J} 1 \mathrm{HJV} 2-2$ & $8 / 25 / 11$ & 0.232 & & 0.18 & 11.7 & & 0.18 & 3.04 & & 1.79 & 17.2 & & 0.89 & 10.5 & & 0.45 \\
\hline 300-03 (Depth) & J1HJV2-3 & $8 / 25 / 11$ & 0.217 & & 0.16 & 11.9 & & 0.16 & 3.13 & & 1.56 & 17.5 & & 0.78 & 10.4 & & 0.39 \\
\hline 300-03 (Depth) & $\mathrm{J} 1 \mathrm{HJV} 2-4$ & $8 / 25 / 11$ & 0.250 & & 0.16 & 11.6 & & 0.16 & 2.76 & & 1.56 & 17.4 & & 0.78 & 10.3 & & 0.39 \\
\hline
\end{tabular}


Table C-11. 300 Area Coal Ash Pre-Leaching Quadruplicate Sample Results. (2 Pages)

\begin{tabular}{|c|c|c|c|c|c|c|c|c|c|c|c|c|c|c|}
\hline \multirow{2}{*}{ Sample Location } & \multirow{2}{*}{$\begin{array}{c}\text { HEIS } \\
\text { Number }\end{array}$} & \multirow{2}{*}{$\begin{array}{c}\text { Sample } \\
\text { Date }\end{array}$} & \multicolumn{3}{|c|}{ Manganese } & \multicolumn{3}{|c|}{ Mercury } & \multicolumn{3}{|c|}{ Molybdenum } & \multicolumn{3}{|c|}{ Nickel } \\
\hline & & & $\mathrm{mg} / \mathrm{kg}$ & $\mathbf{Q}$ & PQL & $\mathrm{mg} / \mathrm{kg}$ & $\mathbf{Q}$ & PQL & $\mathrm{mg} / \mathrm{kg}$ & $\mathbf{Q}$ & PQL & $\mathrm{mg} / \mathrm{kg}$ & Q & $\begin{array}{l}\mathrm{PQL} \\
\end{array}$ \\
\hline 300-04 (Depth) & J1HJVO-1 & $8 / 25 / 11$ & 64.0 & & 4.39 & 0.447 & & 0.03 & 0.831 & $B$ & 1.75 & 6.38 & & 3.51 \\
\hline 300-04 (Depth) & J1HJV0-2 & $8 / 25 / 11$ & 56.0 & & 3.73 & 1.15 & & 0.03 & 0.863 & $B$ & 1.49 & 6.32 & & 2.99 \\
\hline 300-04 (Depth) & J1HJV0-3 & $8 / 25 / 11$ & 78.9 & & 3.33 & 0.563 & & 0.03 & 0.812 & $B$ & 1.33 & 5.63 & & 2.67 \\
\hline 300-04 (Depth) & J1HJV0-4 & $8 / 25 / 11$ & 72.2 & & 3.25 & 0.546 & & 0.03 & 1.02 & B & 1.30 & 5.90 & & 2.60 \\
\hline 300-02 (Depth) & J1HJV1-1 & $8 / 25 / 11$ & 96.5 & & 3.52 & 1.61 & & 0.08 & 0.735 & $\mathrm{~B}$ & 1.41 & 5.97 & & 2.82 \\
\hline 300-02 (Depth) & J1HJV1-2 & $8 / 25 / 11$ & 79.6 & & 3.62 & 1.46 & & 0.08 & 0.849 & $B$ & 1.45 & 5.58 & & 2.90 \\
\hline 300-02 (Depth) & J1HJV1-3 & $8 / 25 / 11$ & 80.7 & & 3.97 & 1.22 & & 0.03 & 0.764 & $B$ & 1.59 & 5.48 & & 3.17 \\
\hline 300-02 (Depth) & J1HJV1-4 & $8 / 25 / 11$ & 79.6 & & 4.31 & 1.55 & & 0.07 & 0.890 & B & 1.72 & 5.74 & & 3.45 \\
\hline 300-03 (Depth) & J1HJV2-1 & $8 / 25 / 11$ & 90.8 & & 4.46 & 0.604 & & 0.02 & 0.718 & $B$ & 1.79 & 6.47 & & 3.57 \\
\hline 300-03 (Depth) & J1HJV2-2 & $8 / 25 / 11$ & 104 & & 4.46 & 0.543 & & 0.03 & 0.688 & B & 1.79 & 7.24 & & 3.57 \\
\hline 300-03 (Depth) & J1HJV2-3 & $8 / 25 / 11$ & 110 & & 3.91 & 0.158 & & 0.03 & 0.802 & $B$ & 1.56 & 7.15 & & 3.12 \\
\hline 300-03 (Depth) & J1HJV2-4 & $8 / 25 / 11$ & 86.7 & & 3.91 & 0.159 & & 0.03 & 0.704 & $\mathrm{~B}$ & 1.56 & 6.85 & & 3.12 \\
\hline \multirow{2}{*}{ Sample Location } & \multirow{2}{*}{$\begin{array}{c}\text { HEIS } \\
\text { Number }\end{array}$} & \multirow{2}{*}{$\begin{array}{c}\text { Sample } \\
\text { Date }\end{array}$} & \multicolumn{3}{|c|}{ Silver } & \multicolumn{3}{|c|}{$\begin{array}{c}\text { Thallium } \\
\end{array}$} & \multicolumn{3}{|c|}{ Vanadium } & \multicolumn{3}{|c|}{ Zinc } \\
\hline & & & $\mathrm{mg} / \mathrm{kg}$ & $\mathbf{Q}$ & PQL & $\mathrm{mg} / \mathrm{kg}$ & $\mathbf{Q}$ & $\mathrm{PQL}$ & $\mathrm{mg} / \mathrm{kg}$ & $\mathbf{Q}$ & PQL & $\mathrm{mg} / \mathrm{kg}$ & Q & $\mathrm{PQL}$ \\
\hline 300-04 (Depth) & J1HJV0-1 & $8 / 25 / 11$ & 0.175 & $U$ & 0.18 & 0.439 & $U$ & 0.44 & 21.0 & & 2.19 & 25.6 & & 8.77 \\
\hline 300-04 (Depth) & J1HJV0-2 & $8 / 25 / 11$ & 0.149 & $\mathrm{U}$ & 0.15 & 0.373 & $\mathrm{U}$ & 0.37 & 22.8 & & 1.87 & 26.1 & & 7.46 \\
\hline 300-04 (Depth) & J1HJV0-3 & $8 / 25 / 11$ & 0.133 & $\mathrm{U}$ & 0.13 & 0.333 & $U$ & 0.33 & 21.1 & & 1.67 & 24.8 & & 6.67 \\
\hline 300-04 (Depth) & J1HJV0-4 & $8 / 25 / 11$ & 0.130 & $\mathrm{U}$ & 0.13 & 0.325 & $U$ & 0.33 & 23.7 & & 1.62 & 22.6 & & 6.49 \\
\hline 300-02 (Depth) & J1HJV1-1 & $8 / 25 / 11$ & 0.141 & $\mathrm{U}$ & 0.14 & 0.352 & $\mathrm{U}$ & 0.35 & 26.1 & & 1.76 & 27.8 & & 7.04 \\
\hline 300-02 (Depth) & J1HJV1-2 & $8 / 25 / 11$ & 0.145 & $U$ & 0.15 & 0.362 & $U$ & 0.36 & 23.8 & & 1.81 & 25.1 & & 7.25 \\
\hline 300-02 (Depth) & J1HJV1-3 & $8 / 25 / 11$ & 0.159 & $U$ & 0.16 & 0.397 & $\mathrm{U}$ & 0.40 & 23.1 & & 1.98 & 26.4 & & 7.94 \\
\hline 300-02 (Depth) & J1HJV1-4 & $8 / 25 / 11$ & 0.172 & $U$ & 0.17 & 0.431 & $\mathrm{U}$ & 0.43 & 23.8 & & 2.16 & 30.0 & & 8.62 \\
\hline 300-03 (Depth) & J1HJV2-1 & $8 / 25 / 11$ & 0.179 & $U$ & 0.18 & 0.446 & $\mathrm{U}$ & 0.45 & 28.1 & & 2.23 & 25.8 & & 8.93 \\
\hline 300-03 (Depth) & J1HJV2-2 & $8 / 25 / 11$ & 0.179 & U & 0.18 & 0.446 & U & 0.45 & 29.6 & & 2.23 & 30.3 & & 8.93 \\
\hline 300-03 (Depth) & J1HJV2-3 & $8 / 25 / 11$ & 0.156 & U & 0.16 & 0.391 & U & 0.39 & 29.9 & & 1.95 & 29.3 & & 7.81 \\
\hline 300-03 (Depth) & J1HJV2-4 & $8 / 25 / 11$ & 0.156 & $\mathrm{U}$ & 0.16 & 0.391 & $\mathrm{U}$ & 0.39 & 27.4 & & 1.95 & 31.5 & & 7.81 \\
\hline
\end{tabular}


Table C-12. 300 Area Coal Ash Leaching Sample Results. (2 Pages)

\begin{tabular}{|c|c|c|c|c|c|c|c|c|c|c|c|c|c|c|c|c|c|c|}
\hline \multirow{2}{*}{ Sample Location } & \multirow{2}{*}{$\begin{array}{c}\text { Coal Ash:Water } \\
\text { Ratio }\end{array}$} & \multirow{2}{*}{$\begin{array}{c}\text { HEIS } \\
\text { Number }\end{array}$} & \multirow{2}{*}{$\begin{array}{l}\text { Sample } \\
\text { Date }\end{array}$} & \multicolumn{3}{|c|}{ Antimony } & \multicolumn{3}{|c|}{ Arsenic } & \multicolumn{3}{|c|}{ Barium } & \multicolumn{3}{|c|}{ Beryllium } & \multicolumn{3}{|c|}{ Boron } \\
\hline & & & & $\mathrm{mg} / \mathrm{L}$ & $\mathrm{Q}$ & $\overline{P Q L}$ & $\mathrm{mg} / \mathrm{L}$ & Q & $\mathrm{PQL}$ & $\mathrm{mg} / \mathrm{L}$ & $\mathbf{Q}$ & $\mathrm{PQL}$ & $\mathrm{mg} / \mathrm{L}$ & $Q$ & $\mathrm{PQL}$ & $\mathrm{mg} / \mathrm{L}$ & Q & $\mathrm{PQL}$ \\
\hline 300-04 (Depth) & $1: 1$ & J1HJV0-A1 & $8 / 25 / 11$ & 0.100 & $U$ & 0.10 & 0.075 & $U$ & 0.08 & 0.042 & & 0.01 & 0.005 & $U$ & 0.01 & 77.8 & & 0.08 \\
\hline 300-04 (Depth) & $1: 1$ & J1HJV0-A2 & $8 / 25 / 11$ & 0.100 & $U$ & 0.10 & 0.075 & $U$ & 0.08 & 0.037 & & 0.01 & 0.005 & $\mathrm{U}$ & 0.01 & 70.0 & & 0.08 \\
\hline 300-04 (Depth) & $1: 2.5$ & J1HJV0-B1 & $8 / 25 / 11$ & 0.100 & $U$ & 0.10 & 0.075 & $U$ & 0.08 & 0.034 & & 0.01 & 0.005 & $\mathrm{U}$ & 0.01 & 40.4 & & 0.08 \\
\hline 300-04 (Depth) & $1: 5$ & $\mathrm{~J} 1 \mathrm{HJV} 0-\mathrm{C} 1$ & $8 / 25 / 11$ & 0.100 & $U$ & 0.10 & 0.075 & $\mathrm{U}$ & 0.08 & 0.033 & & 0.01 & 0.005 & $\mathrm{U}$ & 0.01 & 23.1 & & 0.08 \\
\hline 300-02 (Depth) & $1: 1$ & J1HJV1-A1 & $8 / 25 / 11$ & 0.100 & $U$ & 0.10 & 0.075 & $U$ & 0.08 & 0.045 & & 0.01 & 0.005 & $U$ & 0.01 & 43.4 & & 0.08 \\
\hline 300-02 (Depth) & $1: 2.5$ & J1HJV1-B1 & $8 / 25 / 11$ & 0.100 & $U$ & 0.10 & 0.075 & $U$ & 0.08 & 0.036 & & 0.01 & 0.005 & $U$ & 0.01 & 22.9 & & 0.08 \\
\hline 300-02 (Depth) & $1: 2.5$ & J1HJV1-B2 & $8 / 25 / 11$ & 0.100 & $U$ & 0.10 & 0.075 & $U$ & 0.08 & 0.037 & & 0.01 & 0.005 & $U$ & 0.01 & 21.6 & & 0.08 \\
\hline 300-02 (Depth) & $1: 5$ & J1HJV1-C1 & $8 / 25 / 11$ & 0.100 & $U$ & 0.10 & 0.075 & $U$ & 0.08 & 0.036 & & 0.01 & 0.005 & $U$ & 0.01 & 13.0 & & 0.08 \\
\hline 300-03 (Depth) & $1: 1$ & J1HJV2-A1 & $8 / 25 / 11$ & 0.100 & $\mathrm{U}$ & 0.10 & 0.075 & $\mathrm{U}$ & 0.08 & 0.048 & & 0.01 & 0.005 & $\mathrm{U}$ & 0.01 & 58.4 & & 0.08 \\
\hline 300-03 (Depth) & $1: 2.5$ & J1HJV2-B1 & $8 / 25 / 11$ & 0.100 & $U$ & 0.10 & 0.075 & $U$ & 0.08 & 0.037 & & 0.01 & 0.005 & $U$ & 0.01 & 33.7 & & 0.08 \\
\hline 300-03 (Depth) & $1: 5$ & J1HJV2-C1 & $8 / 25 / 11$ & 0.100 & $U$ & 0.10 & 0.075 & $U$ & 0.08 & 0.034 & & 0.01 & 0.005 & $U$ & 0.01 & 19.3 & & 0.08 \\
\hline 300-03 (Depth) & $1: 5$ & J1HJV2-C2 & $8 / 25 / 11$ & 0.100 & $U$ & 0.10 & 0.075 & $U$ & 0.08 & 0.034 & & 0.01 & 0.005 & $U$ & 0.01 & 20.2 & & 0.08 \\
\hline \multirow{2}{*}{ Sample Location } & Coal Ash:Water & HEIS & Sample & \multicolumn{3}{|c|}{ Cadmium } & \multicolumn{3}{|c|}{ Chromium } & \multicolumn{3}{|c|}{ Cobalt } & \multicolumn{3}{|c|}{ Copper } & \multicolumn{3}{|c|}{ Lead } \\
\hline & Ratio & Number & Date & $\mathrm{mg} / \mathrm{L}$ & $\mathrm{Q}$ & $\mathrm{PQL}$ & $\mathrm{mg} / \mathrm{L}$ & Q & $\mathrm{PQL}$ & $\mathrm{mg} / \mathrm{L}$ & $\mathrm{Q}$ & PQL & $\mathrm{mg} / \mathrm{L}$ & $\mathrm{Q}$ & $\mathrm{PQL}$ & $\mathrm{mg} / \mathrm{L}$ & Q & $\mathrm{PQL}$ \\
\hline 300-04 (Depth) & $1: 1$ & J1HJV0-A1 & $8 / 25 / 11$ & 0.015 & $\dot{U}$ & 0.02 & 0.003 & B & 0.03 & 0.010 & U & 0.01 & 0.100 & U & 0.10 & 0.050 & U & 0.05 \\
\hline 300-04 (Depth) & $1: 1$ & J1HJVO-A2 & $8 / 25 / 11$ & 0.015 & $U$ & 0.02 & 0.025 & $U$ & 0.03 & 0.010 & $U$ & 0.01 & 0.100 & $U$ & 0.10 & 0.050 & $U$ & 0.05 \\
\hline 300-04 (Depth) & $1: 2.5$ & J1HJV0-B1 & $8 / 25 / 11$ & 0.015 & $U$ & 0.02 & 0.025 & $U$ & 0.03 & 0.010 & $U$ & 0.01 & 0.100 & $U$ & 0.10 & 0.050 & $U$ & 0.05 \\
\hline 300-04 (Depth) & $1: 5$ & J1HJV0-C1 & $8 / 25 / 11$ & 0.015 & $U$ & 0.02 & 0.025 & $U$ & 0.03 & 0.010 & $U$ & 0.01 & 0.100 & $U$ & 0.10 & 0.050 & $U$ & 0.05 \\
\hline 300-02 (Depth) & $1: 1$ & J1HJV1-A1 & $8 / 25 / 11$ & 0.015 & $U$ & 0.02 & 0.003 & B & 0.03 & 0.010 & $U$ & 0.01 & 0.100 & $U$ & 0.10 & 0.050 & $U$ & 0.05 \\
\hline 300-02 (Depth) & $1: 2.5$ & J1HJV1-B1 & $8 / 25 / 11$ & 0.015 & $U$ & 0.02 & 0.025 & $U$ & 0.03 & 0.010 & $U$ & 0.01 & 0.100 & $U$ & 0.10 & 0.050 & $U$ & 0.05 \\
\hline 300-02 (Depth) & $1: 2.5$ & J1HJV1-B2 & $8 / 25 / 11$ & 0.015 & $U$ & 0.02 & 0.003 & B & 0.03 & 0.010 & U & 0.01 & 0.100 & $U$ & 0.10 & 0.050 & $U$ & 0.05 \\
\hline 300-02 (Depth) & $1: 5$ & J1HJV1-C1 & $8 / 25 / 11$ & 0.015 & $U$ & 0.02 & 0.025 & $U$ & 0.03 & 0.010 & 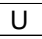 & 0.01 & 0.100 & $U$ & 0.10 & 0.050 & $U$ & 0.05 \\
\hline 300-03 (Depth) & $1: 1$ & J1HJV2-A1 & $8 / 25 / 11$ & 0.015 & $U$ & 0.02 & 0.003 & $\mathrm{~B}$ & 0.03 & 0.010 & $U$ & 0.01 & 0.100 & $U$ & 0.10 & 0.050 & $U$ & 0.05 \\
\hline 300-03 (Depth) & $1: 2.5$ & J1HJV2-B1 & $8 / 25 / 11$ & 0.015 & $U$ & 0.02 & 0.025 & $\mathrm{U}$ & 0.03 & 0.010 & $U$ & 0.01 & 0.100 & $U$ & 0.10 & 0.050 & $U$ & 0.05 \\
\hline 300-03 (Depth) & $1: 5$ & J1HJV2-C1 & $8 / 25 / 11$ & 0.015 & $U$ & 0.02 & 0.025 & $\mathrm{U}$ & 0.03 & 0.010 & $U$ & 0.01 & 0.100 & $U$ & 0.10 & 0.050 & $U$ & 0.05 \\
\hline 300-03 (Depth) & $1: 5$ & J1HJV2-C2 & $8 / 25 / 11$ & 0.015 & $U$ & 0.02 & 0.025 & $U$ & 0.03 & 0.010 & $U$ & 0.01 & 0.100 & $U$ & 0.10 & 0.050 & $U$ & 0.05 \\
\hline
\end{tabular}


Table C-12. 300 Area Coal Ash Leaching Sample Results. (2 Pages)

\begin{tabular}{|c|c|c|c|c|c|c|c|c|c|c|c|c|c|c|c|c|c|c|}
\hline \multirow{2}{*}{ Sample Location } & \multirow{2}{*}{$\begin{array}{l}\text { Coal Ash:Water } \\
\text { Ratio }\end{array}$} & \multirow{2}{*}{$\begin{array}{c}\text { HEIS } \\
\text { Number }\end{array}$} & \multirow{2}{*}{$\begin{array}{c}\text { Sample } \\
\text { Date }\end{array}$} & \multicolumn{3}{|c|}{ Manganese } & \multicolumn{3}{|c|}{ Mercury } & \multicolumn{3}{|c|}{ Molybdenum } & \multicolumn{3}{|c|}{ Nickel } & \multicolumn{3}{|c|}{ Selenium } \\
\hline & & & & $\mathrm{mg} / \mathrm{L}$ & $Q$ & $\mathrm{PQL}$ & $\mathrm{mg} / \mathrm{L}$ & $Q$ & $\mathrm{PQL}$ & $\mathrm{mg} / \mathrm{L}$ & $\mathrm{Q}$ & PQL & $\mathrm{mg} / \mathrm{L}$ & $Q$ & $\mathrm{PQL}$ & $\mathrm{mg} / \mathrm{L}$ & $Q$ & $\mathrm{PQL}$ \\
\hline 300-04 (Depth) & $1: 1$ & J1HJV0-A1 & $8 / 25 / 11$ & 0.025 & $\dot{U}$ & 0.03 & 0.00023 & $\mathrm{~B}$ & 0.00 & 0.133 & & 0.02 & 0.100 & $\dot{U}$ & 0.10 & 0.175 & & 0.10 \\
\hline 300-04 (Depth) & $1: 1$ & J1HJV0-A2 & $8 / 25 / 11$ & 0.025 & $U$ & 0.03 & 0.000236 & $B$ & 0.00 & 0.120 & & 0.02 & 0.100 & $U$ & 0.10 & 0.151 & & 0.10 \\
\hline 300-04 (Depth) & $1: 2.5$ & J1HJV0-B1 & $8 / 25 / 11$ & 0.025 & $\mathrm{U}$ & 0.03 & 0.00013 & B & 0.00 & 0.062 & & 0.02 & 0.100 & $U$ & 0.10 & 0.079 & $B$ & 0.10 \\
\hline 300-04 (Depth) & $1: 5$ & J1HJV0-C1 & $8 / 25 / 11$ & 0.025 & $U$ & 0.03 & 0.00012 & $\mathrm{~B}$ & 0.00 & 0.035 & & 0.02 & 0.100 & $U$ & 0.10 & 0.041 & $\mathrm{~B}$ & 0.10 \\
\hline $300-02$ (Depth) & $1: 1$ & J1HJV1-A1 & $8 / 25 / 11$ & 0.025 & $\bar{U}$ & 0.03 & 0.00023 & $B$ & 0.00 & 0.060 & & 0.02 & 0.100 & 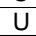 & 0.10 & 0.062 & $\mathrm{~B}$ & 0.10 \\
\hline 300-02 (Depth) & $1: 2.5$ & J1HJV1-B1 & $8 / 25 / 11$ & 0.025 & $U$ & 0.03 & 0.00013 & $B$ & 0.00 & 0.032 & & 0.02 & 0.100 & $U$ & 0.10 & 0.027 & $\mathrm{~B}$ & 0.10 \\
\hline 300-02 (Depth) & $1: 2.5$ & J1HJV1-B2 & $8 / 25 / 11$ & 0.025 & $U$ & 0.03 & 0.00011 & $B$ & 0.00 & 0.028 & & 0.02 & 0.100 & $U$ & 0.10 & 0.024 & $B$ & 0.10 \\
\hline 300-02 (Depth) & $1: 5$ & J1HJV1-C1 & $8 / 25 / 11$ & 0.025 & $U$ & 0.03 & 0.00013 & B & 0.00 & 0.016 & & 0.02 & 0.100 & $U$ & 0.10 & 0.100 & $U$ & 0.10 \\
\hline 300-03 (Depth) & $1: 1$ & J1HJV2-A1 & $8 / 25 / 11$ & 0.025 & $u$ & 0.03 & 0.00040 & B & 0.00 & 0.069 & & 0.02 & 0.100 & $u$ & 0.10 & 0.064 & B & 0.10 \\
\hline 300-03 (Depth) & $1: 2.5$ & J1HJV2-B1 & $8 / 25 / 11$ & 0.025 & $U$ & 0.03 & 0.00013 & $\mathrm{~B}$ & 0.00 & 0.035 & & 0.02 & 0.100 & $U$ & 0.10 & 0.042 & $\mathrm{~B}$ & 0.10 \\
\hline 300-03 (Depth) & $1: 5$ & J1HJV2-C1 & $8 / 25 / 11$ & 0.025 & $U$ & 0.03 & 0.00015 & B & 0.00 & 0.019 & & 0.02 & 0.100 & $U$ & 0.10 & 0.019 & $B$ & 0.10 \\
\hline 300-03 (Depth) & $1: 5$ & J1HJV2-C2 & $8 / 25 / 11$ & 0.025 & $U$ & 0.03 & 0.00013 & B & 0.00 & 0.019 & & 0.02 & 0.100 & $U$ & 0.10 & 0.019 & $B$ & 0.10 \\
\hline
\end{tabular}

\begin{tabular}{|c|c|c|c|c|c|c|c|c|c|c|c|c|c|c|c|c|c|c|c|}
\hline \multirow{2}{*}{ Sample Location } & \multirow{2}{*}{$\begin{array}{c}\text { Coal Ash:Water } \\
\text { Ratio }\end{array}$} & \multirow{2}{*}{$\begin{array}{c}\text { HEIS } \\
\text { Number }\end{array}$} & \multirow{2}{*}{$\begin{array}{c}\text { Sample } \\
\text { Date }\end{array}$} & \multicolumn{3}{|c|}{ Silver } & \multicolumn{3}{|c|}{ Thallium } & \multicolumn{3}{|c|}{ Uranium (KPA) } & \multicolumn{3}{|c|}{ Vanadium } & \multicolumn{3}{|c|}{ Zinc } & \multirow[b]{2}{*}{$\mathrm{pH}$} \\
\hline & & & & $\mathrm{mg} / \mathrm{L}$ & Q & $\mathrm{PQL}$ & $\mathrm{mg} / \mathrm{L}$ & Q & PQL & ug/L & Q & MDA & $\mathrm{mg} / \mathrm{L}$ & Q & PQL & $\mathrm{mg} / \mathrm{L}$ & Q & $\overline{\mathrm{PQL}}$ & \\
\hline 300-04 (Depth) & $1: 1$ & J1HJV0-A1 & $8 / 25 / 11$ & 0.030 & $\dot{U}$ & 0.03 & 0.075 & $\dot{U}$ & 0.08 & 27.3 & & 0.32 & 0.123 & & 0.03 & 0.250 & U & 0.25 & 8.17 \\
\hline 300-04 (Depth) & $1: 1$ & J1HJV0-A2 & $8 / 25 / 11$ & 0.030 & U & 0.03 & 0.075 & U & 0.08 & 27.0 & & 0.32 & 0.118 & & 0.03 & 0.250 & $U$ & 0.25 & 8.26 \\
\hline 300-04 (Depth) & $1: 2.5$ & J1HJV0-B1 & $8 / 25 / 11$ & 0.030 & $U$ & 0.03 & 0.075 & $U$ & 0.08 & 13.0 & & 0.03 & 0.083 & & 0.03 & 0.250 & U & 0.25 & 8.78 \\
\hline 300-04 (Depth) & $1: 5$ & J1HJV0-C1 & $8 / 25 / 11$ & 0.030 & $U$ & 0.03 & 0.075 & $U$ & 0.08 & 9.02 & & 0.03 & 0.057 & & 0.03 & 0.250 & $U$ & 0.25 & 8.69 \\
\hline 300-02 (Depth) & $1: 1$ & J1HJV1-A1 & $8 / 25 / 11$ & 0.030 & $\mathrm{U}$ & 0.03 & 0.075 & $\mathrm{U}$ & 0.08 & 18.6 & & 0.32 & 0.077 & & 0.03 & 0.250 & $\mathrm{U}$ & 0.25 & 8.41 \\
\hline $300-02$ (Depth) & $1: 2.5$ & J1HJV1-B1 & $8 / 25 / 11$ & 0.030 & U & 0.03 & 0.075 & U & 0.08 & 8.31 & & 0.03 & 0.049 & & 0.03 & 0.250 & $\mathrm{U}$ & 0.25 & 8.58 \\
\hline 300-02 (Depth) & $1: 2.5$ & J1HJV1-B2 & $8 / 25 / 11$ & 0.030 & $U$ & 0.03 & 0.075 & $U$ & 0.08 & 8.25 & & 0.03 & 0.048 & & 0.03 & 0.250 & U & 0.25 & 8.59 \\
\hline 300-02 (Depth) & $1: 5$ & J1HJV1-C1 & $8 / 25 / 11$ & 0.030 & $U$ & 0.03 & 0.075 & U & 0.08 & 5.27 & & 0.03 & 0.033 & & 0.03 & 0.250 & $U$ & 0.25 & 8.80 \\
\hline 300-03 (Depth) & $1: 1$ & J1HJV2-A1 & $8 / 25 / 11$ & 0.030 & $U$ & 0.03 & 0.075 & U & 0.08 & 28.9 & & 0.32 & 0.073 & & 0.03 & 0.250 & $U$ & 0.25 & 8.21 \\
\hline 300-03 (Depth) & $1: 2.5$ & J1HJV2-B1 & $8 / 25 / 11$ & 0.030 & $\bar{U}$ & 0.03 & 0.075 & $\mathrm{U}$ & 0.08 & 15.8 & & 0.03 & 0.049 & & 0.03 & 0.250 & $\mathrm{U}$ & 0.25 & 8.42 \\
\hline 300-03 (Depth) & $1: 5$ & J1HJV2-C1 & $8 / 25 / 11$ & 0.030 & $U$ & 0.03 & 0.075 & $U$ & 0.08 & 10.6 & & 0.03 & 0.033 & & 0.03 & 0.250 & $U$ & 0.25 & 8.61 \\
\hline 300-03 (Depth) & $1: 5$ & J1HJV2-C2 & $8 / 25 / 11$ & 0.030 & $U$ & 0.03 & 0.075 & $U$ & 0.08 & 10.9 & & 0.03 & 0.033 & & 0.03 & 0.250 & U & 0.25 & 8.63 \\
\hline
\end{tabular}


Table C-13. 600-207 Coal Ash Sample Results. (5 Pages)

\begin{tabular}{|c|c|c|c|c|c|c|c|c|c|c|c|c|c|c|c|c|c|}
\hline \multirow{2}{*}{$\begin{array}{c}\text { Sample } \\
\text { Location }\end{array}$} & \multirow{2}{*}{$\begin{array}{c}\text { HEIS } \\
\text { Number }\end{array}$} & \multirow{2}{*}{$\begin{array}{c}\text { Sample } \\
\text { Date }\end{array}$} & \multicolumn{3}{|c|}{ Antimony } & \multicolumn{3}{|c|}{ Arsenic } & \multicolumn{3}{|c|}{ Barium } & \multicolumn{3}{|c|}{ Beryllium } & \multicolumn{3}{|c|}{ Boron } \\
\hline & & & $\mathrm{mg} / \mathrm{kg}$ & $\mathrm{Q}$ & PQL & $\mathrm{mg} / \mathrm{kg}$ & $\mathbf{Q}$ & PQL & $\mathrm{mg} / \mathrm{kg}$ & $\mathbf{Q}$ & PQL & $\mathrm{mg} / \mathrm{kg}$ & Q & PQL & $\mathrm{mg} / \mathrm{kg}$ & $\mathrm{Q}$ & PQL \\
\hline $600-01$ & J1HHM3 & 9/28/11 & 0.526 & $\bar{U}$ & 0.53 & 3.73 & & 0.88 & 656 & & 0.44 & 0.691 & & 0.18 & 60.9 & & 1.75 \\
\hline $600-02$ & J1HHM4 & 9/28/11 & 0.536 & $U$ & 0.54 & 5.29 & & 0.89 & 673 & & 0.45 & 0.655 & & 0.18 & 42.7 & & 1.79 \\
\hline $600-03$ & J1HHM5 & 9/28/11 & 0.556 & $U$ & 0.56 & 5.19 & & 0.93 & 721 & & 0.46 & 0.730 & & 0.19 & 55.1 & & 1.85 \\
\hline $600-04$ & J1HHM6 & 9/28/11 & 0.517 & $U$ & 0.52 & 3.82 & & 0.86 & 552 & & 0.43 & 0.556 & & 0.17 & 44.3 & & 1.72 \\
\hline $600-05$ & J1HHM7 & 9/28/11 & 0.556 & $U$ & 0.56 & 4.91 & & 0.93 & 645 & & 0.46 & 0.619 & & 0.19 & 44.7 & & 1.85 \\
\hline $600-06$ & J1HHM8 & 9/28/11 & 0.526 & $\mathrm{U}$ & 0.53 & 3.63 & & 0.88 & 695 & & 0.44 & 0.592 & & 0.18 & 42.7 & & 1.75 \\
\hline $600-07$ & J1HHM9 & 9/27/11 & 0.577 & UJ & 0.58 & 6.60 & & 0.96 & 958 & $\mathrm{~J}$ & 0.48 & 0.903 & & 0.19 & 146 & $\mathrm{~J}$ & 1.92 \\
\hline 600-07 (depth) & J1HHT8 & 9/27/11 & 0.476 & UJ & 0.48 & 4.29 & & 0.79 & 711 & $\mathrm{~J}$ & 0.40 & 1.21 & & 0.16 & 147 & $\mathrm{~J}$ & 1.59 \\
\hline $600-08$ & J1HHNO & 9/28/11 & 0.492 & $\mathrm{U}$ & 0.49 & 5.18 & & 0.82 & 686 & & 0.41 & 0.676 & & 0.16 & 43.1 & & 1.64 \\
\hline $600-09$ & J1HHN1 & 9/28/11 & 0.545 & $\mathrm{U}$ & 0.55 & 5.42 & & 0.91 & 643 & & 0.46 & 0.800 & & 0.18 & 50.9 & & 1.82 \\
\hline $600-10$ & J1HHN2 & 9/28/11 & 0.508 & $U$ & 0.51 & 4.62 & & 0.85 & 608 & & 0.42 & 0.592 & & 0.17 & 44.7 & & 1.69 \\
\hline $600-11$ & J1HHN3 & 9/28/11 & 0.508 & $\mathrm{U}$ & 0.51 & 3.71 & & 0.85 & 379 & & 0.42 & 0.627 & & 0.17 & 31.2 & & 1.69 \\
\hline $600-12$ & $\mathrm{~J} 1 \mathrm{HHN} 4$ & $9 / 28 / 11$ & 0.492 & $U$ & 0.49 & 3.39 & & 0.82 & 632 & & 0.41 & 0.460 & & 0.16 & 41.6 & & 1.64 \\
\hline $600-13$ & J1HHN5 & $9 / 28 / 11$ & 0.517 & $\mathrm{U}$ & 0.52 & 3.90 & & 0.86 & 609 & & 0.43 & 0.529 & & 0.17 & 35.4 & & 1.72 \\
\hline $600-14$ & J1HHN6 & $9 / 28 / 11$ & 0.545 & $\mathrm{U}$ & 0.55 & 4.46 & & 0.91 & 606 & & 0.46 & 0.696 & & 0.18 & 44.8 & & 1.82 \\
\hline $600-15$ & J1HHN7 & 9/27/11 & 0.577 & UJ & 0.58 & 10.6 & & 0.96 & 1210 & $\mathrm{~J}$ & 0.48 & 1.43 & & 0.19 & 112 & $\mathrm{~J}$ & 1.92 \\
\hline $\begin{array}{c}\text { Duplicate of } \\
\text { J1HHN7 }\end{array}$ & J1HHR2 & 9/27/11 & 0.508 & UJ & 0.51 & 9.58 & & 0.85 & 1250 & $\mathrm{~J}$ & 0.42 & 1.46 & & 0.17 & 162 & $\mathrm{~J}$ & 1.69 \\
\hline 600-15 (depth) & J1HHT9 & 9/27/11 & 0.269 & BJ & 0.52 & 4.92 & & 0.86 & 653 & $\mathrm{~J}$ & 0.43 & 0.658 & & 0.17 & 168 & $\mathrm{~J}$ & 1.72 \\
\hline $600-16$ & J1HHN8 & 9/28/11 & 0.526 & $\mathrm{U}$ & 0.53 & 5.38 & & 0.88 & 767 & & 0.44 & 0.772 & & 0.18 & 60.6 & & 1.75 \\
\hline $600-17$ & J1HHN9 & 9/28/11 & 0.476 & $U$ & 0.48 & 5.02 & & 0.79 & 678 & & 0.40 & 0.629 & & 0.16 & 58.8 & & 1.59 \\
\hline $600-18$ & J1HHP0 & 9/28/11 & 0.484 & $U$ & 0.48 & 4.89 & & 0.81 & 683 & & 0.40 & 0.84 & & 0.16 & 50.2 & & 1.61 \\
\hline $600-19$ & J1HHP1 & 9/28/11 & 0.517 & $U$ & 0.52 & 4.56 & & 0.86 & 705 & & 0.43 & 0.737 & & 0.17 & 60.3 & & 1.72 \\
\hline $600-20$ & J1HHP2 & $9 / 28 / 11$ & 0.536 & $\mathrm{U}$ & 0.54 & 6.63 & & 0.89 & 805 & & 0.45 & 0.992 & & 0.18 & 57.1 & & 1.79 \\
\hline $600-21$ & J1HHP3 & 9/28/11 & 0.588 & UJ & 0.59 & 6.30 & & 0.98 & 1060 & $\mathrm{~J}$ & 0.49 & 1.01 & & 0.20 & 84.0 & $\mathrm{~J}$ & 1.96 \\
\hline $600-22$ & J1HHP4 & 9/27/11 & 0.500 & UJ & 0.50 & 6.19 & & 0.83 & 796 & $\mathrm{~J}$ & 0.42 & 0.957 & & 0.17 & 58.3 & $\mathrm{~J}$ & 1.67 \\
\hline $600-23$ & J1HHP5 & $9 / 27 / 11$ & 0.536 & UJ & 0.54 & 6.46 & & 0.89 & 915 & $\mathrm{~J}$ & 0.45 & 0.969 & & 0.18 & 103 & $\mathrm{~J}$ & 1.79 \\
\hline 600-23 (depth) & J1HHVO & 9/27/11 & 0.556 & UJ & 0.56 & 4.83 & & 0.93 & 680 & $\mathrm{~J}$ & 0.46 & 0.847 & & 0.19 & 155 & $\mathrm{~J}$ & 1.85 \\
\hline $600-24$ & J1HHP6 & 9/28/11 & 0.588 & UJ & 0.59 & 7.25 & & 0.98 & 910 & $\mathrm{~J}$ & 0.49 & 1.19 & & 0.20 & 69.8 & $\mathrm{~J}$ & 1.96 \\
\hline $\begin{array}{c}\text { Duplicate of } \\
\text { J1HHP6 }\end{array}$ & J1HHR3 & 9/27/11 & 0.500 & UJ & 0.50 & 6.57 & & 0.83 & 1030 & $\mathrm{~J}$ & 0.42 & 1.18 & & 0.17 & 81.7 & $\mathrm{~J}$ & 1.67 \\
\hline $600-25$ & J1HHP7 & $9 / 27 / 11$ & 0.600 & UJ & 0.60 & 5.52 & & 1.00 & 694 & $\mathrm{~J}$ & 0.50 & 0.907 & & 0.20 & 48.0 & $\mathrm{~J}$ & 2.00 \\
\hline $600-26$ & J1HHP8 & $9 / 28 / 11$ & 0.577 & UJ & 0.58 & 8.54 & & 0.96 & 1030 & & 0.48 & 1.18 & & 0.19 & 66.0 & $\mathrm{~J}$ & 1.92 \\
\hline $600-27$ & J1HHP9 & $9 / 27 / 11$ & 0.500 & UJ & 0.50 & 6.17 & & 0.83 & 944 & $\mathrm{~J}$ & 0.42 & 1.05 & & 0.17 & 63.7 & $\mathrm{~J}$ & 1.67 \\
\hline $600-28$ & J1HHRO & $9 / 27 / 11$ & 0.566 & UJ & 0.57 & 4.90 & & 0.94 & 979 & $\mathrm{~J}$ & 0.47 & 1.11 & & 0.19 & 71.3 & $\mathrm{~J}$ & 1.89 \\
\hline $600-29$ & J1HHR1 & 9/28/11 & 0.435 & UJ & 0.44 & 5.58 & & 0.73 & 802 & $\mathrm{~J}$ & 0.36 & 0.833 & & 0.15 & 57.4 & $\mathrm{~J}$ & 1.45 \\
\hline Equip blank & J1HHT7 & 9/27/11 & 0.395 & UJ & 0.40 & 0.658 & $U$ & 0.66 & 2.47 & UJ & 0.33 & 0.035 & B & 0.13 & 1.32 & UJ & 1.32 \\
\hline
\end{tabular}


Table C-13. 600-207 Coal Ash Sample Results. (5 Pages)

\begin{tabular}{|c|c|c|c|c|c|c|c|c|c|c|c|c|c|c|c|c|c|}
\hline \multirow{2}{*}{$\begin{array}{c}\text { Sample } \\
\text { Location }\end{array}$} & \multirow{2}{*}{$\begin{array}{c}\text { HEIS } \\
\text { Number }\end{array}$} & \multirow{2}{*}{$\begin{array}{c}\text { Sample } \\
\text { Date }\end{array}$} & \multicolumn{3}{|c|}{ Cadmium } & \multicolumn{3}{|c|}{ Chromium } & \multicolumn{3}{|c|}{ Cobalt } & \multicolumn{3}{|c|}{ Copper } & \multicolumn{3}{|c|}{ Lead } \\
\hline & & & $\mathrm{mg} / \mathrm{kg}$ & $\mathbf{Q}$ & PQL & $\mathrm{mg} / \mathrm{kg}$ & Q & PQL & $\mathrm{mg} / \mathrm{kg}$ & $\mathbf{Q}$ & $\mathrm{PQL}$ & $\mathrm{mg} / \mathrm{kg}$ & $\mathbf{Q}$ & $\mathrm{PQL}$ & $\mathrm{mg} / \mathrm{kg}$ & $\mathbf{Q}$ & PQL \\
\hline $600-01$ & J1HHM3 & $9 / 28 / 11$ & 0.178 & & 0.18 & 8.27 & & 0.18 & 3.88 & & 1.75 & 20.0 & & 0.88 & 4.69 & & 0.44 \\
\hline $600-02$ & $\mathrm{~J} 1 \mathrm{HHM} 4$ & 9/28/11 & 0.186 & & 0.18 & 8.66 & & 0.18 & 4.02 & & 1.79 & 20.4 & & 0.89 & 5.08 & & 0.45 \\
\hline $600-03$ & J1HHM5 & 9/28/11 & 0.187 & & 0.19 & 9.26 & & 0.19 & 4.05 & & 1.9 & 21.1 & & 0.9 & 7.21 & & 0.5 \\
\hline $600-04$ & $\mathrm{~J} 1 \mathrm{HHM} 6$ & 9/28/11 & 0.204 & & 0.17 & 7.82 & & 0.17 & 3.51 & & 1.7 & 17.1 & & 0.9 & 5.2 & & 0.4 \\
\hline $600-05$ & $\mathrm{~J} 1 \mathrm{HHM} 7$ & 9/28/11 & 0.188 & & 0.19 & 9.00 & & 0.19 & 3.94 & & 1.9 & 20.2 & & 0.9 & 6.07 & & 0.5 \\
\hline $600-06$ & $\mathrm{~J} 1 \mathrm{HHM} 8$ & $9 / 28 / 11$ & 0.211 & & 0.18 & 8.04 & & 0.18 & 3.30 & & 1.8 & 18.7 & & 0.9 & 5.77 & & 0.4 \\
\hline $600-07$ & $\mathrm{~J} 1 \mathrm{HHM} 9$ & 9/27/11 & 0.122 & B & 0.19 & 12.9 & $\mathrm{~J}$ & 0.19 & 5.74 & & 1.9 & 46.8 & $\mathrm{~J}$ & 1.0 & 5.28 & & 0.5 \\
\hline 600-07 (depth) & J1HHT8 & 9/27/11 & 0.111 & B & 0.16 & 7.50 & $\mathrm{~J}$ & 0.16 & 4.42 & & 1.59 & 21.9 & $\mathrm{~J}$ & 0.79 & 3.3 & & 0.40 \\
\hline $600-08$ & J1HHNO & 9/28/11 & 0.194 & & 0.16 & 8.91 & & 0.16 & 4.28 & & 1.64 & 22.2 & & 0.82 & 5.35 & & 0.41 \\
\hline $600-09$ & J1HHN1 & 9/28/11 & 0.211 & & 0.18 & 9.33 & & 0.18 & 4.49 & & 1.82 & 24.1 & & 0.91 & 5.75 & & 0.46 \\
\hline $600-10$ & J1HHN2 & 9/28/11 & 0.178 & & 0.17 & 8.09 & & 0.17 & 3.53 & & 1.69 & 19.2 & & 0.85 & 5.24 & & 0.42 \\
\hline $600-11$ & J1HHN3 & 9/28/11 & 0.166 & $B$ & 0.17 & 9.70 & & 0.17 & 4.83 & & 1.69 & 18.5 & & 0.85 & 4.26 & & 0.42 \\
\hline $600-12$ & $\mathrm{~J} 1 \mathrm{HHN} 4$ & $9 / 28 / 11$ & 0.182 & & 0.16 & 8.08 & & 0.16 & 3.24 & & 1.64 & 16.9 & & 0.82 & 4.23 & & 0.41 \\
\hline $600-13$ & J1HHN5 & $9 / 28 / 11$ & 0.153 & B & 0.17 & 6.81 & & 0.17 & 3.21 & & 1.72 & 19.4 & & 0.86 & 4.58 & & 0.43 \\
\hline $600-14$ & J1HHN6 & 9/28/11 & 0.173 & B & 0.18 & 8.29 & & 0.18 & 3.70 & & 1.82 & 20.1 & & 0.91 & 7 & & 0.46 \\
\hline $600-15$ & J1HHN7 & $9 / 27 / 11$ & 0.232 & & 0.19 & 15.5 & $\mathrm{~J}$ & 0.19 & 7.14 & & 1.92 & 35.4 & $\mathrm{~J}$ & 0.96 & 11.1 & & 0.48 \\
\hline $\begin{array}{c}\text { Duplicate of } \\
\text { J1HHN7 }\end{array}$ & J1HHR2 & $9 / 27 / 11$ & 0.213 & & 0.17 & 16.7 & $\mathrm{~J}$ & 0.17 & 7.08 & & 1.69 & 38.6 & $\mathrm{~J}$ & 0.85 & 7.5 & & 0.42 \\
\hline 600-15 (depth) & J1HHT9 & 9/27/11 & 0.186 & & 0.17 & 8.99 & $\mathrm{~J}$ & 0.17 & 3.56 & & 1.72 & 21.6 & $\mathrm{~J}$ & 0.86 & 5.61 & & 0.43 \\
\hline $600-16$ & J1HHN8 & 9/28/11 & 0.186 & & 0.18 & 9.14 & & 0.18 & 4.69 & & 1.75 & 20.3 & & 0.88 & 5.45 & & 0.44 \\
\hline $600-17$ & J1HHN9 & 9/28/11 & 0.202 & & 0.16 & 9.87 & & 0.16 & 4.70 & & 1.59 & 23.2 & & 0.79 & 7.1 & & 0.40 \\
\hline $600-18$ & J1HHP0 & 9/28/11 & 0.189 & & 0.16 & 8.23 & & 0.16 & 4.16 & & 1.61 & 20.6 & & 0.81 & 5.93 & & 0.40 \\
\hline $600-19$ & J1HHP1 & 9/28/11 & 0.172 & $B$ & 0.17 & 8.55 & & 0.17 & 4.31 & & 1.72 & 20.9 & & 0.86 & 4.99 & & 0.43 \\
\hline $600-20$ & J1HHP2 & 9/28/11 & 0.193 & & 0.18 & 11.4 & & 0.18 & 5.65 & & 1.79 & 26.7 & & 0.89 & 5.44 & & 0.45 \\
\hline $600-21$ & J1HHP3 & 9/28/11 & 0.127 & $B$ & 0.20 & 11.8 & $\mathrm{~J}$ & 0.20 & 5.46 & & 1.96 & 24.6 & $\mathrm{~J}$ & 0.98 & 4.94 & & 0.49 \\
\hline $600-22$ & J1HHP4 & 9/27/11 & 0.14 & B & 0.17 & 11.4 & $\mathrm{~J}$ & 0.17 & 5.52 & & 1.67 & 24.6 & $\mathrm{~J}$ & 0.83 & 6.92 & & 0.42 \\
\hline $600-23$ & J1HHP5 & 9/27/11 & 0.143 & $B$ & 0.18 & 11.5 & $\mathrm{~J}$ & 0.18 & 6.07 & & 1.79 & 28.2 & $\mathrm{~J}$ & 0.89 & 6.61 & & 0.45 \\
\hline 600-23 (depth) & J1HHV0 & $9 / 27 / 11$ & 0.139 & $\mathrm{~B}$ & 0.19 & 8.11 & $\mathrm{~J}$ & 0.19 & 3.98 & & 1.85 & 21.3 & $\mathrm{~J}$ & 0.93 & 5.08 & & 0.46 \\
\hline $600-24$ & J1HHP6 & 9/28/11 & 0.174 & $B$ & 0.20 & 12.3 & $\mathrm{~J}$ & 0.20 & 5.92 & & 1.96 & 32.3 & $\mathrm{~J}$ & 0.98 & 7.03 & & 0.49 \\
\hline $\begin{array}{c}\text { Duplicate of } \\
\text { J1HHP6 }\end{array}$ & J1HHR3 & $9 / 27 / 11$ & 0.157 & B & 0.17 & 11.4 & $\mathrm{~J}$ & 0.17 & 5.85 & & 1.67 & 28.0 & $\mathrm{~J}$ & 0.83 & 5.6 & & 0.42 \\
\hline $600-25$ & J1HHP7 & $9 / 27 / 11$ & 0.176 & B & 0.20 & 11.3 & $\mathrm{~J}$ & 0.20 & 5.38 & & 2.00 & 26.8 & $\mathrm{~J}$ & 1.00 & 6.06 & & 0.50 \\
\hline $600-26$ & J1HHP8 & $9 / 28 / 11$ & 0.149 & B & 0.19 & 12.8 & & 0.19 & 5.93 & & 1.92 & 29.9 & & 0.96 & 14.5 & & 0.48 \\
\hline $600-27$ & J1HHP9 & $9 / 27 / 11$ & 0.175 & & 0.17 & 11.1 & $\mathrm{~J}$ & 0.17 & 4.91 & & 1.67 & 25.2 & $\mathrm{~J}$ & 0.83 & 6.15 & & 0.42 \\
\hline $600-28$ & J1HHRO & 9/27/11 & 0.138 & B & 0.19 & 9.85 & $\mathrm{~J}$ & 0.19 & 4.83 & & 1.89 & 24.3 & $\mathrm{~J}$ & 0.94 & 5.47 & & 0.47 \\
\hline $600-29$ & J1HHR1 & $9 / 28 / 11$ & 0.151 & & 0.15 & 10.9 & $\mathrm{~J}$ & 0.15 & 4.96 & & 1.45 & 23.2 & $\mathrm{~J}$ & 0.73 & 5.3 & & 0.36 \\
\hline Equip blank & J1HHT7 & 9/27/11 & 0.132 & $U$ & 0.13 & 0.298 & $\mathrm{~J}$ & 0.13 & 1.32 & U & 1.32 & 0.658 & UJ & 0.66 & 0.528 & & 0.33 \\
\hline
\end{tabular}


Table C-13. 600-207 Coal Ash Sample Results. (5 Pages)

\begin{tabular}{|c|c|c|c|c|c|c|c|c|c|c|c|c|c|c|c|c|c|}
\hline \multirow{2}{*}{$\begin{array}{l}\text { Sample } \\
\text { Location }\end{array}$} & \multirow{2}{*}{$\begin{array}{c}\text { HEIS } \\
\text { Number }\end{array}$} & \multirow{2}{*}{$\begin{array}{c}\text { Sample } \\
\text { Date }\end{array}$} & \multicolumn{3}{|c|}{ Manganese } & \multicolumn{3}{|c|}{ Mercury } & \multicolumn{3}{|c|}{ Molybdenum } & \multicolumn{3}{|c|}{ Nickel } & \multicolumn{3}{|c|}{ Selenium } \\
\hline & & & $\mathrm{mg} / \mathrm{kg}$ & Q & $\mathrm{PQL}$ & $\mathrm{mg} / \mathrm{kg}$ & Q & PQL & $\mathrm{mg} / \mathrm{kg}$ & $\mathbf{Q}$ & PQL & $\mathrm{mg} / \mathrm{kg}$ & Q & PQL & $\mathrm{mg} / \mathrm{kg}$ & Q & $\mathrm{PQL}$ \\
\hline $600-01$ & J1HHM3 & 9/28/11 & 244 & & 4.39 & 0.026 & 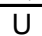 & 0.03 & 1.37 & B & 1.75 & 9.86 & & 3.51 & 1.33 & & 0.26 \\
\hline $600-02$ & J1HHM4 & 9/28/11 & 259 & & 4.46 & 0.027 & $U$ & 0.03 & 1.57 & $\mathrm{~B}$ & 1.79 & 10.3 & & 3.57 & 1.72 & & 0.27 \\
\hline $600-03$ & J1HHM5 & 9/28/11 & 293 & & 4.63 & 0.042 & & 0.03 & 1.58 & $\mathrm{~B}$ & 1.9 & 10.9 & & 3.70 & 1.76 & & 0.28 \\
\hline $600-04$ & J1HHM6 & 9/28/11 & 209 & & 4.31 & 0.026 & $U$ & 0.03 & 1.27 & $B$ & 1.7 & 8.92 & & 3.45 & 1.36 & & 0.26 \\
\hline $600-05$ & J1HHM7 & 9/28/11 & 266 & & 4.63 & 0.027 & $U$ & 0.03 & 1.59 & $B$ & 1.9 & 10.3 & & 3.70 & 1.42 & & 0.28 \\
\hline $600-06$ & J1HHM8 & 9/28/11 & 229 & & 4.39 & 0.009 & B & 0.03 & 1.25 & $B$ & 1.8 & 8.87 & & 3.51 & 1.29 & & 0.26 \\
\hline $600-07$ & J1HHM9 & 9/27/11 & 486 & $\mathrm{~J}$ & 4.81 & 0.027 & $U$ & 0.03 & 2.34 & & 1.9 & 16.7 & $\mathrm{~J}$ & 3.85 & 1.22 & & 0.29 \\
\hline $600-07$ (depth) & J1HHT8 & 9/27/11 & 327 & $\mathrm{~J}$ & 4.0 & 0.026 & $U$ & 0.03 & 1.91 & & 1.59 & 11.2 & $\mathrm{~J}$ & 3.2 & 1.29 & & 0.24 \\
\hline $600-08$ & J1HHNO & 9/28/11 & 270 & & 4.10 & 0.046 & & 0.03 & 1.42 & $B$ & 1.64 & 10.8 & & 3.28 & 1.5 & & 0.25 \\
\hline $600-09$ & J1HHN1 & 9/28/11 & 291 & & 4.55 & 0.027 & $U$ & 0.03 & 1.78 & $\mathrm{~B}$ & 1.82 & 12.7 & & 3.64 & 1.33 & & 0.27 \\
\hline $600-10$ & J1HHN2 & 9/28/11 & 227 & & 4.24 & 0.024 & U & 0.02 & 1.28 & $\mathrm{~B}$ & 1.69 & 9.12 & & 3.39 & 1.35 & & 0.25 \\
\hline $600-11$ & J1HHN3 & 9/28/11 & 237 & & 4.24 & 0.027 & $U$ & 0.03 & 1.38 & $B$ & 1.69 & 11.7 & & 3.39 & 0.946 & & 0.25 \\
\hline $600-12$ & J1HHN4 & 9/28/11 & 205 & & 4.10 & 0.027 & U & 0.03 & 1.01 & B & 1.64 & 7.95 & & 3.28 & 0.898 & & 0.25 \\
\hline $600-13$ & J1HHN5 & 9/28/11 & 209 & & 4.31 & 0.012 & B & 0.03 & 1.26 & $\mathrm{~B}$ & 1.72 & 8.10 & & 3.45 & 1.08 & & 0.26 \\
\hline $600-14$ & J1HHN6 & 9/28/11 & 260 & & 4.55 & 0.013 & B & 0.03 & 1.47 & $B$ & 1.82 & 10.0 & & 3.64 & 1.41 & & 0.27 \\
\hline $600-15$ & J1HHN7 & 9/27/11 & 481 & $\mathrm{~J}$ & 4.81 & 0.025 & U & 0.03 & 2.11 & & 1.92 & 18.9 & $\mathrm{~J}$ & 3.85 & 2.27 & & 0.29 \\
\hline $\begin{array}{c}\text { Duplicate of } \\
\text { J1HHN7 }\end{array}$ & J1HHR2 & 9/27/11 & 537 & $\mathrm{~J}$ & 4.2 & 0.017 & B & 0.02 & 2.49 & & 1.69 & 19.7 & $\mathrm{~J}$ & 3.4 & 1.73 & & 0.25 \\
\hline $600-15$ (depth) & J1HHT9 & 9/27/11 & 250 & $\mathrm{~J}$ & 4.3 & 0.025 & $U$ & 0.03 & 2.16 & & 1.72 & 9.42 & $\mathrm{~J}$ & 3.5 & 1.83 & & 0.26 \\
\hline $600-16$ & J1HHN8 & $9 / 28 / 11$ & 293 & & 4.39 & 0.010 & B & 0.03 & 1.75 & & 1.75 & 12.6 & & 3.51 & 1.37 & & 0.26 \\
\hline $600-17$ & J1HHN9 & $9 / 28 / 11$ & 264 & & 3.97 & 0.027 & $U$ & 0.03 & 1.62 & & 1.59 & 11.9 & & 3.17 & 1.30 & & 0.24 \\
\hline $600-18$ & J1HHP0 & 9/28/11 & 280 & & 4.03 & 0.027 & $U$ & 0.03 & 1.53 & $B$ & 1.61 & 10.9 & & 3.23 & 1.56 & & 0.24 \\
\hline $600-19$ & J1HHP1 & 9/28/11 & 271 & & 4.31 & 0.026 & $U$ & 0.03 & 1.74 & & 1.72 & 11.1 & & 3.45 & 1.51 & & 0.26 \\
\hline $600-20$ & J1HHP2 & 9/28/11 & 349 & & 4.46 & 0.025 & $U$ & 0.03 & 2.26 & & 1.79 & 14.6 & & 3.57 & 1.77 & & 0.27 \\
\hline $600-21$ & J1HHP3 & 9/28/11 & 357 & $\mathrm{~J}$ & 4.90 & 0.026 & $U$ & 0.03 & 2.35 & & 1.96 & 14.4 & $\mathrm{~J}$ & 3.92 & 1.51 & & 0.29 \\
\hline $600-22$ & J1HHP4 & 9/27/11 & 359 & $\mathrm{~J}$ & 4.2 & 0.027 & $U$ & 0.03 & 2.25 & & 1.67 & 15.8 & $\mathrm{~J}$ & 3.3 & 1.88 & & 0.25 \\
\hline $600-23$ & J1HHP5 & 9/27/11 & 410 & $\mathrm{~J}$ & 4.46 & 0.027 & B & 0.03 & 2.02 & & 1.79 & 16.2 & $\mathrm{~J}$ & 3.6 & 1.44 & & 0.27 \\
\hline $600-23$ (depth) & J1HHV0 & $9 / 27 / 11$ & 286 & $\mathrm{~J}$ & 4.63 & 0.026 & $U$ & 0.03 & 1.78 & $B$ & 1.85 & 10.6 & $\mathrm{~J}$ & 3.70 & 1.85 & & 0.28 \\
\hline $600-24$ & J1HHP6 & 9/28/11 & 373 & $\mathrm{~J}$ & 4.90 & 0.045 & & 0.03 & 2.69 & & 1.96 & 15.7 & $\mathrm{~J}$ & 3.9 & 2.10 & & 0.29 \\
\hline $\begin{array}{c}\text { Duplicate of } \\
\text { J1HHP6 }\end{array}$ & J1HHR3 & 9/27/11 & 390 & $\mathrm{~J}$ & 4.2 & 0.030 & & 0.03 & 2.46 & & 1.67 & 17.7 & $\mathrm{~J}$ & 3.3 & 1.64 & & 0.25 \\
\hline $600-25$ & J1HHP7 & 9/27/11 & 252 & $\mathrm{~J}$ & 5.00 & 0.021 & B & 0.03 & 1.88 & $B$ & 2.00 & 13.1 & $\mathrm{~J}$ & 4.0 & 1.95 & & 0.30 \\
\hline $600-26$ & J1HHP8 & 9/28/11 & 435 & & 4.8 & 0.022 & B & 0.03 & 3.13 & & 1.92 & 16.7 & & 3.9 & 2.06 & & 0.29 \\
\hline $600-27$ & J1HHP9 & 9/27/11 & 379 & $\mathrm{~J}$ & 4.2 & 0.028 & & 0.03 & 1.88 & & 1.67 & 14.5 & $\mathrm{~J}$ & 3.3 & 1.92 & & 0.25 \\
\hline $600-28$ & J1HHR0 & 9/27/11 & 401 & $\mathrm{~J}$ & 4.72 & 0.019 & B & 0.02 & 2.11 & & 1.89 & 14.3 & $\mathrm{~J}$ & 3.8 & 1.60 & & 0.28 \\
\hline $600-29$ & J1HHR1 & 9/28/11 & 315 & $\mathrm{~J}$ & 3.6 & 0.028 & & 0.02 & 1.89 & & 1.45 & 13.0 & $\mathrm{~J}$ & 2.9 & 1.61 & & 0.22 \\
\hline Equip blank & J1HHT7 & 9/27/11 & 7 & UJ & 3.3 & 0.011 & $B$ & 0.03 & 1.32 & $U$ & 1.32 & 2.63 & UJ & 2.6 & 0.197 & $U$ & 0.20 \\
\hline
\end{tabular}


Table C-13. 600-207 Coal Ash Sample Results. (5 Pages)

\begin{tabular}{|c|c|c|c|c|c|c|c|c|c|c|c|c|c|c|c|c|c|}
\hline \multirow{2}{*}{$\begin{array}{l}\text { Sample } \\
\text { Location }\end{array}$} & \multirow{2}{*}{$\begin{array}{c}\text { HEIS } \\
\text { Number }\end{array}$} & \multirow{2}{*}{$\begin{array}{c}\text { Sample } \\
\text { Date }\end{array}$} & \multicolumn{3}{|c|}{ Silver } & \multicolumn{3}{|c|}{ Thallium } & \multicolumn{3}{|c|}{ Uranium (KPA) } & \multicolumn{3}{|c|}{ Vanadium } & \multicolumn{3}{|c|}{ Zinc } \\
\hline & & & $\mathrm{mg} / \mathrm{kg}$ & Q & PQL & $\mathrm{mg} / \mathrm{kg}$ & $\mathbf{Q}$ & PQL & ug/g & Q & MDA & $\mathrm{mg} / \mathrm{kg}$ & Q & PQL & $\mathrm{mg} / \mathrm{kg}$ & Q & PQL \\
\hline $600-01$ & J1HHM3 & 9/28/11 & 0.175 & $\mathrm{U}$ & 0.18 & 0.439 & $U$ & 0.440 & & & & 32.0 & & 2.19 & 17.1 & & 8.77 \\
\hline $600-02$ & J1HHM4 & 9/28/11 & 0.179 & $U$ & 0.18 & 0.446 & $U$ & 0.450 & & & & 32.2 & & 2.23 & 17.3 & & 8.93 \\
\hline $600-03$ & J1HHM5 & 9/28/11 & 0.185 & $U$ & 0.19 & 0.463 & $U$ & 0.460 & & & & 33.0 & & 2.31 & 21.6 & & 9.26 \\
\hline $600-04$ & J1HHM6 & 9/28/11 & 0.172 & $U$ & 0.17 & 0.431 & $U$ & 0.430 & & & & 29.0 & & 2.16 & 26 & & 8.62 \\
\hline $600-05$ & J1HHM7 & 9/28/11 & 0.185 & $U$ & 0.19 & 0.463 & $U$ & 0.460 & & & & 30.6 & & 2.31 & 21.1 & & 9.26 \\
\hline $600-06$ & J1HHM8 & 9/28/11 & 0.175 & $U$ & 0.18 & 0.439 & $\mathrm{U}$ & 0.440 & & & & 28.2 & & 2.19 & 20.9 & & 8.77 \\
\hline $600-07$ & J1HHM9 & 9/27/11 & 0.192 & $U$ & 0.19 & 0.481 & UJ & 0.480 & 2.56 & & 0.136 & 43.7 & $\mathrm{~J}$ & 2.40 & 22.1 & & 9.62 \\
\hline 600-07 (depth) & J1HHT8 & 9/27/11 & 0.159 & $U$ & 0.16 & 0.397 & UJ & 0.40 & 2.64 & & 0.136 & 36.8 & $\mathrm{~J}$ & 1.98 & 17.7 & & 7.9 \\
\hline $600-08$ & J1HHNO & 9/28/11 & 0.164 & $U$ & 0.16 & 0.41 & $\mathrm{U}$ & 0.410 & & & & 32.3 & & 2.05 & 22.4 & & 8.20 \\
\hline $600-09$ & J1HHN1 & 9/28/11 & 0.182 & $\mathrm{U}$ & 0.18 & 0.455 & $U$ & 0.460 & & & & 33.2 & & 2.27 & 24.8 & & 9.09 \\
\hline $600-10$ & J1HHN2 & $9 / 28 / 11$ & 0.169 & $\mathrm{U}$ & 0.17 & 0.424 & $\mathrm{U}$ & 0.420 & & & & 26.7 & & 2.12 & 16.6 & & 8.47 \\
\hline $600-11$ & $\mathrm{~J} 1 \mathrm{HHN} 3$ & $9 / 28 / 11$ & 0.169 & U & 0.17 & 0.424 & $\mathrm{U}$ & 0.420 & & & & 38.7 & & 2.12 & 24.5 & & 8.47 \\
\hline $600-12$ & J1HHN4 & $9 / 28 / 11$ & 0.164 & 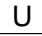 & 0.16 & 0.41 & $\mathrm{U}$ & 0.410 & & & & 26.2 & & 2.05 & 22.8 & & 8.20 \\
\hline $600-13$ & J1HHN5 & $9 / 28 / 11$ & 0.172 & $U$ & 0.17 & 0.431 & U & 0.430 & & & & 25.4 & & 2.16 & 15.8 & & 8.62 \\
\hline $600-14$ & J1HHN6 & $9 / 28 / 11$ & 0.182 & $U$ & 0.18 & 0.455 & U & 0.460 & & & & 29.8 & & 2.27 & 17.6 & & 9.09 \\
\hline 600-15 & J1HHN7 & 9/27/11 & 0.192 & $U$ & 0.19 & 0.481 & UJ & 0.480 & 4.18 & & 0.136 & 55.3 & $\mathrm{~J}$ & 2.40 & 28.5 & & 9.62 \\
\hline $\begin{array}{c}\text { Duplicate of } \\
\text { J1HHN7 }\end{array}$ & J1HHR2 & 9/27/11 & 0.169 & $U$ & 0.17 & 0.424 & UJ & 0.42 & 5.18 & & 0.136 & 102 & $\mathrm{~J}$ & 2.12 & 22.1 & & 8.5 \\
\hline 600-15 (depth) & J1HHT9 & 9/27/11 & 0.172 & $U$ & 0.17 & 0.431 & UJ & 0.43 & 1.76 & & 0.136 & 32.9 & $\mathrm{~J}$ & 2.16 & 18.1 & & 8.6 \\
\hline $600-16$ & J1HHN8 & 9/28/11 & 0.175 & $U$ & 0.18 & 0.439 & $\mathrm{U}$ & 0.440 & & & & 32.2 & & 2.19 & 19.1 & & 8.77 \\
\hline $600-17$ & J1HHN9 & 9/28/11 & 0.159 & $U$ & 0.16 & 0.397 & $U$ & 0.400 & & & & 36.2 & & 1.98 & 23.7 & & 7.94 \\
\hline $600-18$ & J1HHP0 & 9/28/11 & 0.161 & $U$ & 0.16 & 0.403 & $U$ & 0.400 & & & & 32.6 & & 2.02 & 19.2 & & 8.06 \\
\hline $600-19$ & J1HHP1 & 9/28/11 & 0.172 & $U$ & 0.17 & 0.431 & $U$ & 0.430 & & & & 32.1 & & 2.16 & 17.3 & & 8.62 \\
\hline $600-20$ & J1HHP2 & 9/28/11 & 0.179 & $U$ & 0.18 & 0.446 & $\mathrm{U}$ & 0.450 & & & & 40.4 & & 2.23 & 17.6 & & 8.93 \\
\hline $600-21$ & J1HHP3 & $9 / 28 / 11$ & 0.196 & $U$ & 0.20 & 0.49 & UJ & 0.490 & & & & 44.4 & $\mathrm{~J}$ & 2.45 & 19.3 & & 9.80 \\
\hline $600-22$ & J1HHP4 & 9/27/11 & 0.167 & $U$ & 0.17 & 0.417 & UJ & 0.42 & & & & 41.0 & $\mathrm{~J}$ & 2.08 & 22.3 & & 8.33 \\
\hline $600-23$ & J1HHP5 & $9 / 27 / 11$ & 0.179 & $U$ & 0.18 & 0.446 & UJ & 0.450 & 3.23 & & 0.136 & 45.3 & $\mathrm{~J}$ & 2.23 & 22.9 & & 8.93 \\
\hline 600-23 (depth) & J1HHV0 & 9/27/11 & 0.185 & $U$ & 0.19 & 0.463 & UJ & 0.460 & 3.28 & & 0.136 & 32.1 & $\mathrm{~J}$ & 2.31 & 16 & & 9.3 \\
\hline $600-24$ & J1HHP6 & $9 / 28 / 11$ & 0.196 & $U$ & 0.20 & 0.49 & UJ & 0.490 & & & & 47.9 & $\mathrm{~J}$ & 2.45 & 24.1 & & 9.80 \\
\hline $\begin{array}{c}\text { Duplicate of } \\
\text { J1HHP6 }\end{array}$ & J1HHR3 & 9/27/11 & 0.167 & $U$ & 0.17 & 0.417 & UJ & 0.42 & & & & 44.4 & $\mathrm{~J}$ & 2.08 & 19.2 & & 8.33 \\
\hline $600-25$ & J1HHP7 & 9/27/11 & 0.2 & $U$ & 0.20 & 0.5 & UJ & 0.500 & & & & 38.5 & $\mathrm{~J}$ & 2.50 & 20.2 & & 10.0 \\
\hline $600-26$ & J1HHP8 & 9/28/11 & 0.192 & $U$ & 0.19 & 0.481 & $\mathrm{U}$ & 0.48 & & & & 50.1 & & 2.40 & 26.9 & & 9.62 \\
\hline $600-27$ & J1HHP9 & 9/27/11 & 0.167 & $U$ & 0.17 & 0.417 & UJ & 0.42 & & & & 40.4 & $\mathrm{~J}$ & 2.08 & 27.1 & & 8.33 \\
\hline $600-28$ & J1HHR0 & 9/27/11 & 0.189 & $U$ & 0.19 & 0.472 & UJ & 0.470 & & & & 40.8 & $\mathrm{~J}$ & 2.36 & 19.3 & & 9.43 \\
\hline $600-29$ & J1HHR1 & 9/28/11 & 0.145 & $U$ & 0.15 & 0.362 & UJ & 0.36 & & & & 40.6 & $\mathrm{~J}$ & 1.81 & 22.1 & & 7.3 \\
\hline Equip blank & J1HHT7 & 9/27/11 & 0.132 & $U$ & 0.13 & 0.329 & UJ & 0.330 & & & & 0.392 & BJ & 1.64 & 1.52 & B & 6.6 \\
\hline
\end{tabular}


Table C-13. 600-207 Coal Ash Sample Results. (5 Pages)

\begin{tabular}{|c|c|c|c|c|c|c|c|c|c|c|c|c|c|c|c|c|c|c|c|c|}
\hline \multirow{2}{*}{$\begin{array}{c}\text { Sample } \\
\text { Location }\end{array}$} & \multirow{2}{*}{$\begin{array}{c}\text { HEIS } \\
\text { Number }\end{array}$} & \multirow{2}{*}{$\begin{array}{c}\text { Sample } \\
\text { Date }\end{array}$} & \multicolumn{3}{|c|}{ Acenaphthene } & \multicolumn{3}{|c|}{ Acenaphthylene } & \multicolumn{3}{|c|}{ Anthracene } & \multicolumn{3}{|c|}{ Benzo(a)anthracene } & \multicolumn{3}{|c|}{ Benzo(a)pyrene } & \multicolumn{3}{|c|}{ Benzo(b)fluoranthene } \\
\hline & & & ug/kg & $Q$ & $\mathrm{PQL}$ & ug/kg & Q & $\mathrm{PQL}$ & $u g / k g$ & Q & $\mathrm{PQL}$ & ug/kg & Q & $\mathrm{PQL}$ & ug/kg & $\mathrm{Q}$ & $\mathrm{PQL}$ & $u g / k g$ & Q & $\mathrm{PQL}$ \\
\hline $600-07$ & J1HHM9 & 9/27/11 & 328 & UD & 328 & 328 & UD & 328 & 328 & UD & 328 & 328 & UD & 328 & 328 & UDJ & 328 & 328 & UD & 328 \\
\hline $600-15$ & J1HHN7 & 9/27/11 & 326 & $\mathrm{U}$ & 326 & 326 & $U$ & 326 & 326 & $\mathrm{U}$ & 326 & 326 & $\mathrm{U}$ & 326 & 326 & UJ & 326 & 326 & $\mathrm{U}$ & 326 \\
\hline $\begin{array}{c}\text { Duplicate of } \\
\text { J1HHN7 }\end{array}$ & J1HHR2 & 9/27/11 & 328 & $\mathrm{U}$ & 328 & 328 & u & 328 & 328 & $u$ & 328 & 328 & U & 328 & 328 & UJ & 328 & 328 & $u$ & 328 \\
\hline $600-23$ & J1HHP5 & 9/27/11 & 329 & $\mathrm{U}$ & 329 & 329 & 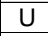 & 329 & 329 & $\mathrm{U}$ & 329 & 329 & $\mathrm{U}$ & 329 & 329 & UJ & 329 & 71 & $\mathrm{~J}$ & 329 \\
\hline $600-07$ (depth) & J1HHT8 & 9/27/11 & 329 & UD & 329 & 329 & UD & 329 & 329 & UD & 329 & 329 & UD & 329 & 329 & UDJ & 329 & 329 & UD & 329 \\
\hline 600-15 (depth) & J1HHT9 & 9/27/11 & 329 & $u$ & 329 & 329 & $u$ & 329 & 329 & $u$ & 329 & 329 & $u$ & 329 & 329 & UJ & 329 & 329 & $u$ & 329 \\
\hline $600-23$ (depth) & J1HHV0 & 9/27/11 & 326 & UD & 326 & 326 & UD & 326 & 326 & UD & 326 & 326 & UD & 326 & 326 & UDJ & 326 & 326 & UD & 326 \\
\hline Equip blank & J1HHT7 & 9/27/11 & 316 & $\mathrm{U}$ & 316 & 316 & $U$ & 316 & 316 & $\mathrm{U}$ & 316 & 316 & $\mathrm{U}$ & 316 & 316 & UJ & 316 & 316 & $\mathrm{U}$ & 316 \\
\hline
\end{tabular}

\begin{tabular}{|c|c|c|c|c|c|c|c|c|c|c|c|c|c|c|c|c|c|c|c|c|}
\hline \multirow{2}{*}{$\begin{array}{c}\text { Sample } \\
\text { Location }\end{array}$} & \multirow{2}{*}{$\begin{array}{c}\text { HEIS } \\
\text { Number }\end{array}$} & \multirow{2}{*}{$\begin{array}{c}\text { Sample } \\
\text { Date }\end{array}$} & \multicolumn{3}{|c|}{ Benzo(ghi)perylene } & \multicolumn{3}{|c|}{ Benzo(k)fluoranthene } & \multicolumn{3}{|c|}{ Chrysene } & \multicolumn{3}{|c|}{ Dibenz(a,h)anthracene } & \multicolumn{3}{|c|}{ Fluoranthene } & \multicolumn{3}{|c|}{ Fluorene } \\
\hline & & & ug/kg & Q & $\mathrm{PQL}$ & ug/kg & $\mathrm{Q}$ & $\mathrm{PQL}$ & $u g / k g$ & Q & $\mathrm{PQL}$ & $u g / \mathrm{kg}$ & $Q$ & PQL & $u g / k g$ & $\mathrm{Q}$ & $\mathrm{PQL}$ & ug/kg & Q & $\mathrm{PQL}$ \\
\hline $600-07$ & J1HHM9 & 9/27/11 & 328 & UDJ & 328 & 328 & UD & 328 & 328 & UD & 328 & 328 & UDJ & 328 & 224 & $\mathrm{JD}$ & 985 & 328 & UD & 328 \\
\hline $600-15$ & J1HHN7 & 9/27/11 & 326 & UJ & 326 & 326 & $\mathrm{U}$ & 326 & 326 & $\mathrm{U}$ & 326 & 326 & UJ & 326 & 56 & $\mathrm{~J}$ & 326 & 326 & $\mathrm{U}$ & 326 \\
\hline $\begin{array}{c}\text { Duplicate of } \\
\text { J1HHN7 }\end{array}$ & J1HHR2 & 9/27/11 & 328 & UJ & 328 & 328 & $u$ & 328 & 328 & $u$ & 328 & 328 & UJ & 328 & 58 & $\mathrm{~J}$ & 328 & 328 & $u$ & 328 \\
\hline $600-23$ & J1HHP5 & 9/27/11 & 329 & UJ & 329 & 65 & $\mathrm{~J}$ & 329 & 100 & $\mathrm{~J}$ & 329 & 329 & UJ & 329 & 259 & $\mathrm{~J}$ & 329 & 329 & $\mathrm{U}$ & 329 \\
\hline 600-07 (depth) & J1HHT8 & 9/27/11 & 329 & UDJ & 329 & 329 & UD & 329 & 329 & UD & 329 & 329 & UDJ & 329 & 195 & $\mathrm{JD}$ & 986 & 329 & UD & 329 \\
\hline 600-15 (depth) & J1HHT9 & 9/27/11 & 329 & UJ & 329 & 329 & $u$ & 329 & 329 & $u$ & 329 & 329 & UJ & 329 & 329 & $u$ & 329 & 329 & $u$ & 329 \\
\hline 600-23 (depth) & J1HHV0 & 9/27/11 & 326 & UDJ & 326 & 326 & UD & 326 & 326 & UD & 326 & 326 & UDJ & 326 & 326 & UD & 326 & 326 & UD & 326 \\
\hline Equip blank & J1HHT7 & 9/27/11 & 316 & UJ & 316 & 316 & $\mathrm{U}$ & 316 & 316 & U & 316 & 316 & UJ & 316 & 316 & U & 316 & 316 & $U$ & 316 \\
\hline
\end{tabular}

\begin{tabular}{|c|c|c|c|c|c|c|c|c|c|c|c|c|c|c|}
\hline \multirow{2}{*}{$\begin{array}{c}\text { Sample } \\
\text { Location }\end{array}$} & \multirow{2}{*}{$\begin{array}{c}\text { HEIS } \\
\text { Number }\end{array}$} & \multirow{2}{*}{$\begin{array}{c}\text { Sample } \\
\text { Date }\end{array}$} & \multicolumn{3}{|c|}{ Indeno(1,2,3-cd)pyrene } & \multicolumn{3}{|c|}{ Naphthalene } & \multicolumn{3}{|c|}{ Phenanthrene } & \multicolumn{3}{|c|}{ Pyrene } \\
\hline & & & ug/kg & Q & PQL & ug/kg & Q & PQL & ug/kg & Q & PQL & ug/kg & Q & PQL \\
\hline $600-07$ & J1HHM9 & 9/27/11 & 328 & UDJ & 328 & 429 & $\mathrm{JD}$ & 985 & 316 & JD & 985 & 328 & UD & 328 \\
\hline $600-15$ & J1HHN7 & 9/27/11 & 326 & UJ & 326 & 263 & $\mathrm{~J}$ & 326 & 94 & $\mathrm{~J}$ & 326 & 326 & $\mathrm{U}$ & 326 \\
\hline $\begin{array}{c}\text { Duplicate of } \\
\text { J1HHN7 }\end{array}$ & J1HHR2 & 9/27/11 & 328 & UJ & 328 & 310 & $\mathrm{~J}$ & 328 & 96 & $\mathrm{~J}$ & 328 & 328 & U & 328 \\
\hline $600-23$ & J1HHP5 & 9/27/11 & 329 & UJ & 329 & 417 & & 329 & 306 & $\mathrm{~J}$ & 329 & 115 & $\mathrm{~J}$ & 329 \\
\hline 600-07 (depth) & J1HHT8 & 9/27/11 & 329 & UDJ & 329 & 451 & $\mathrm{JD}$ & 986 & 302 & $J \mathrm{~J}$ & 986 & 329 & UD & 329 \\
\hline 600-15 (depth) & J1HHT9 & 9/27/11 & 329 & UJ & 329 & 51 & $\mathrm{~J}$ & 329 & 329 & U & 329 & 329 & u & 329 \\
\hline $600-23$ (depth) & J1HHV0 & 9/27/11 & 326 & UDJ & 326 & 326 & UD & 326 & 326 & UD & 326 & 326 & UD & 326 \\
\hline Equip blank & J1HHT7 & 9/27/11 & 316 & UJ & 316 & 316 & $\mathrm{U}$ & 316 & 316 & $\mathrm{U}$ & 316 & 316 & $U$ & 316 \\
\hline
\end{tabular}




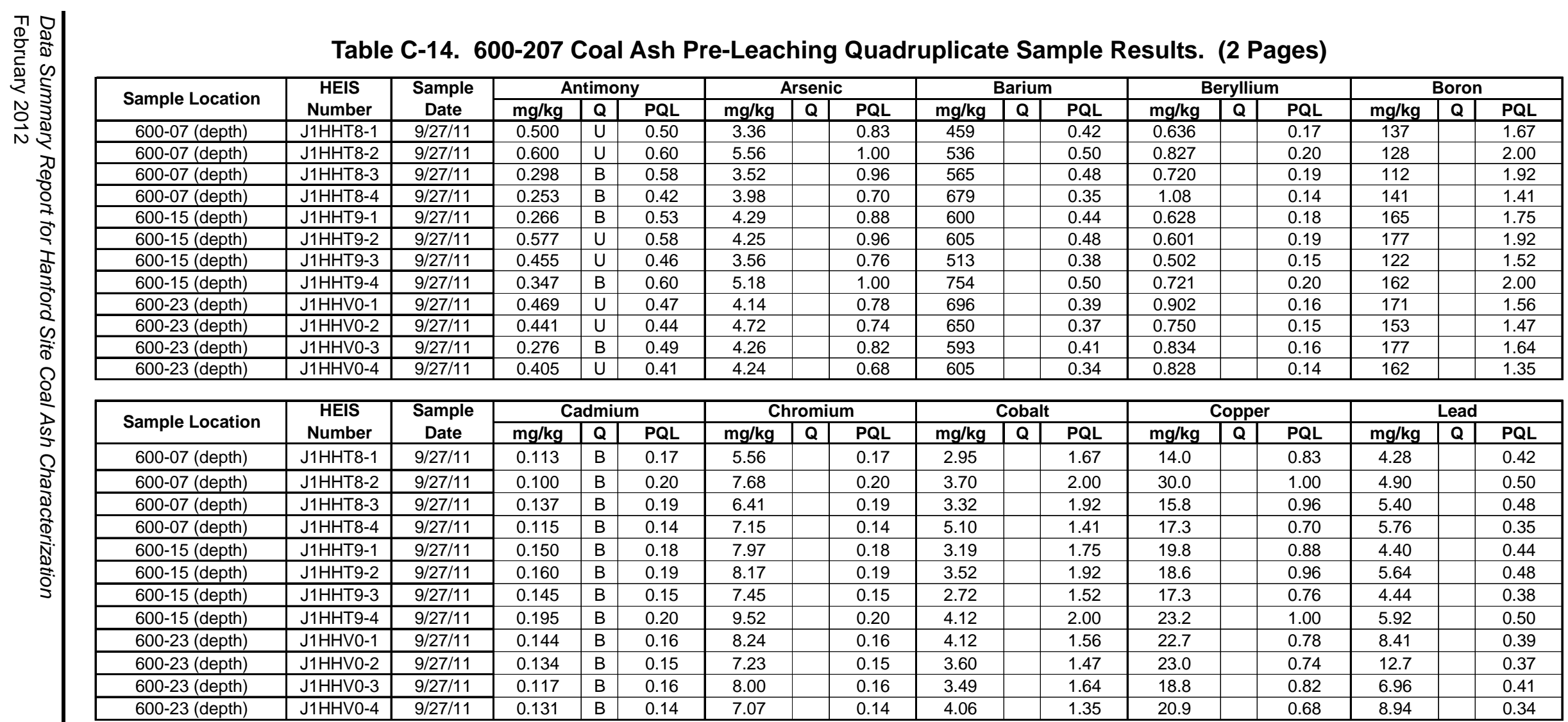


Table C-14. 600-207 Coal Ash Pre-Leaching Quadruplicate Sample Results. (2 Pages)

\begin{tabular}{|c|c|c|c|c|c|c|c|c|c|c|c|c|c|c|}
\hline \multirow{2}{*}{ Sample Location } & \multirow{2}{*}{$\begin{array}{c}\text { HEIS } \\
\text { Number }\end{array}$} & \multirow{2}{*}{$\begin{array}{c}\text { Sample } \\
\text { Date }\end{array}$} & \multicolumn{3}{|c|}{ Manganese } & \multicolumn{3}{|c|}{ Mercury } & \multicolumn{3}{|c|}{ Molybdenum } & \multicolumn{3}{|c|}{ Nickel } \\
\hline & & & $\mathrm{mg} / \mathrm{kg}$ & Q & PQL & $\mathrm{mg} / \mathrm{kg}$ & Q & $\mathrm{PQL}$ & $\mathrm{mg} / \mathrm{kg}$ & Q & $\mathrm{PQL}$ & $\mathrm{mg} / \mathrm{kg}$ & $\mathbf{Q}$ & $\mathrm{PQL}$ \\
\hline 600-07 (depth) & J1HHT8-1 & 9/27/11 & 203 & & 4.17 & 0.027 & $U$ & 0.03 & 1.21 & $B$ & 1.67 & 7.88 & & 3.33 \\
\hline 600-07 (depth) & J1HHT8-2 & 9/27/11 & 270 & & 5.00 & 0.026 & $\mathrm{U}$ & 0.03 & 1.70 & $\mathrm{~B}$ & 2.00 & 10.2 & & 4.00 \\
\hline 600-07 (depth) & J1HHT8-3 & 9/27/11 & 245 & & 4.81 & 0.026 & $U$ & 0.03 & 1.37 & $\mathrm{~B}$ & 1.92 & 8.23 & & 3.85 \\
\hline 600-07 (depth) & J1HHT8-4 & 9/27/11 & 306 & & 3.52 & 0.024 & $U$ & 0.02 & 1.64 & & 1.41 & 11.0 & & 2.82 \\
\hline $600-15$ (depth) & J1HHT9-1 & 9/27/11 & 242 & & 4.39 & 0.027 & $U$ & 0.03 & 2.02 & & 1.75 & 8.34 & & 3.51 \\
\hline $600-15$ (depth) & J1HHT9-2 & $9 / 27 / 11$ & 225 & & 4.81 & 0.027 & $\mathrm{U}$ & 0.03 & 2.00 & & 1.92 & 9.21 & & 3.85 \\
\hline $600-15$ (depth) & J1HHT9-3 & 9/27/11 & 177 & & 3.79 & 0.026 & $U$ & 0.03 & 1.57 & & 1.52 & 7.33 & & 3.03 \\
\hline 600-15 (depth) & J1HHT9-4 & 9/27/11 & 257 & & 5.00 & 0.026 & $U$ & 0.03 & 2.38 & & 2.00 & 10.5 & & 4.00 \\
\hline $600-23$ (depth) & J1HHV0-1 & 9/27/11 & 294 & & 3.91 & 0.024 & $U$ & 0.02 & 1.91 & & 1.56 & 10.1 & & 3.12 \\
\hline $600-23$ (depth) & J1HHVO-2 & 9/27/11 & 275 & & 3.68 & 0.026 & $\mathrm{U}$ & 0.03 & 1.57 & & 1.47 & 9.73 & & 2.94 \\
\hline $600-23$ (depth) & J1HHV0-3 & 9/27/11 & 241 & & 4.10 & 0.026 & $\mathrm{U}$ & 0.03 & 1.99 & & 1.64 & 10.1 & & 3.28 \\
\hline $600-23$ (depth) & J1HHVO-4 & $9 / 27 / 11$ & 274 & & 3.38 & 0.024 & $\mathrm{U}$ & 0.02 & 1.72 & & 1.35 & 9.84 & & 2.70 \\
\hline \multirow{2}{*}{ Sample Location } & \multirow{2}{*}{$\begin{array}{c}\text { HEIS } \\
\text { Number }\end{array}$} & \multirow{2}{*}{$\begin{array}{c}\text { Sample } \\
\text { Date }\end{array}$} & \multicolumn{3}{|c|}{ Silver } & \multicolumn{3}{|c|}{ Thallium } & \multicolumn{3}{|c|}{ Vanadium } & \multicolumn{3}{|c|}{ Zinc } \\
\hline & & & $\mathrm{mg} / \mathrm{kg}$ & 0 & PQL & $\mathrm{mg} / \mathrm{kg}$ & 0 & PQL & $\mathrm{mg} / \mathrm{kg}$ & 0 & PQL & $\mathrm{mg} / \mathrm{kg}$ & Q & PQL \\
\hline 600-07 (depth) & J1HHT8-1 & 9/27/11 & 0.167 & $\dot{U}$ & 0.17 & 0.417 & $U$ & 0.42 & 21.1 & & 2.08 & 12.8 & & 8.33 \\
\hline $600-07$ (depth) & J1HHT8-2 & 9/27/11 & 0.200 & $U$ & 0.20 & 0.500 & $U$ & 0.50 & 29.5 & & 2.50 & 14.6 & & 10.0 \\
\hline 600-07 (depth) & J1HHT8-3 & $9 / 27 / 11$ & 0.192 & $U$ & 0.19 & 0.481 & $U$ & 0.48 & 24.1 & & 2.40 & 15.7 & & 9.62 \\
\hline 600-07 (depth) & J1HHT8-4 & $9 / 27 / 11$ & 0.141 & $\mathrm{U}$ & 0.14 & 0.352 & $U$ & 0.35 & 27.5 & & 1.76 & 15.6 & & 7.04 \\
\hline 600-15 (depth) & J1HHT9-1 & 9/27/11 & 0.175 & $\mathrm{U}$ & 0.18 & 0.439 & $\mathrm{U}$ & 0.44 & 31.0 & & 2.19 & 15.6 & & 8.77 \\
\hline $600-15$ (depth) & J1HHT9-2 & $9 / 27 / 11$ & 0.192 & $\mathrm{U}$ & 0.19 & 0.481 & $U$ & 0.48 & 29.1 & & 2.40 & 16.1 & & 9.62 \\
\hline $600-15$ (depth) & J1HHT9-3 & 9/27/11 & 0.152 & $U$ & 0.15 & 0.379 & $U$ & 0.38 & 25.1 & & 1.89 & 14.3 & & 7.58 \\
\hline $600-15$ (depth) & J1HHT9-4 & 9/27/11 & 0.200 & $\mathrm{U}$ & 0.20 & 0.500 & $U$ & 0.50 & 35.8 & & 2.50 & 19.4 & & 10.0 \\
\hline $600-23$ (depth) & J1HHV0-1 & 9/27/11 & 0.156 & $U$ & 0.16 & 0.391 & $U$ & 0.39 & 36.1 & & 1.95 & 18.3 & & 7.81 \\
\hline $600-23$ (depth) & J1HHV0-2 & 9/27/11 & 0.147 & $U$ & 0.15 & 0.368 & $U$ & 0.37 & 29.4 & & 1.84 & 14.8 & & 7.35 \\
\hline $600-23$ (depth) & J1HHV0-3 & 9/27/11 & 0.164 & $U$ & 0.16 & 0.410 & $U$ & 0.41 & 34.5 & & 2.05 & 12.8 & & 8.20 \\
\hline $600-23$ (depth) & J1HHV0-4 & 9/27/11 & 0.135 & $U$ & 0.14 & 0.338 & $\mathrm{U}$ & 0.34 & 30.8 & & 1.69 & 13.9 & & 6.76 \\
\hline
\end{tabular}


Table C-15. 600-207 Coal Ash Leaching Sample Results. (2 Pages)

\begin{tabular}{|c|c|c|c|c|c|c|c|c|c|c|c|c|c|c|c|c|c|c|}
\hline \multirow{2}{*}{$\begin{array}{c}\text { Sample } \\
\text { Location }\end{array}$} & \multirow{2}{*}{$\begin{array}{c}\text { Coal Ash:Water } \\
\text { Ratio }\end{array}$} & \multirow{2}{*}{$\begin{array}{c}\text { HEIS } \\
\text { Number }\end{array}$} & \multirow{2}{*}{$\begin{array}{c}\text { Sample } \\
\text { Date }\end{array}$} & \multicolumn{3}{|c|}{ Antimony } & \multicolumn{3}{|c|}{ Arsenic } & \multicolumn{3}{|c|}{ Barium } & \multicolumn{3}{|c|}{ Beryllium } & \multicolumn{3}{|c|}{ Boron } \\
\hline & & & & $\mathrm{mg} / \mathrm{L}$ & $\mathrm{Q}$ & $\mathrm{PQL}$ & $\mathrm{mg} / \mathrm{L}$ & Q & $\mathrm{PQL}$ & $\mathrm{mg} / \mathrm{L}$ & Q & $\mathrm{PQL}$ & $\mathrm{mg} / \mathrm{L}$ & Q & $\mathrm{PQL}$ & $\mathrm{mg} / \mathrm{L}$ & Q & $\mathrm{PQL}$ \\
\hline $600-07$ (depth) & $1: 1$ & J1HHT8-A1 & 9/27/11 & 0.10 & $\dot{U}$ & 0.10 & 0.075 & $\dot{U}$ & 0.08 & 0.039 & & 0.01 & 0.005 & $\dot{U}$ & 0.01 & 24.8 & & 0.08 \\
\hline $600-07$ (depth) & $1: 1$ & J1HHT8-A2 & 9/27/11 & 0.10 & $U$ & 0.10 & 0.075 & 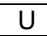 & 0.08 & 0.041 & & 0.01 & 0.005 & $U$ & 0.01 & 26.2 & & 0.08 \\
\hline $600-07$ (depth) & $1: 2.5$ & J1HHT8-B1 & $9 / 27 / 11$ & 0.02 & $U$ & 0.02 & 0.002 & $\mathrm{~B}$ & 0.02 & 0.036 & & 0.00 & 0.001 & $U$ & 0.00 & 15.1 & & 0.02 \\
\hline $600-07$ (depth) & $1: 5$ & J1HHT8-C1 & 9/27/11 & 0.02 & $U$ & 0.02 & 0.003 & B & 0.02 & 0.039 & & 0.00 & 0.001 & $U$ & 0.00 & 10.4 & & 0.02 \\
\hline $600-15$ (depth) & $1: 1$ & J1HHT9-A1 & 9/27/11 & 0.10 & $U$ & 0.10 & 0.075 & $U$ & 0.08 & 0.027 & & 0.01 & 0.005 & $U$ & 0.01 & 11.7 & & 0.08 \\
\hline $600-15$ (depth) & $1: 2.5$ & J1HHT9-B1 & 9/27/11 & 0.02 & $U$ & 0.02 & 0.003 & $\mathrm{~B}$ & 0.02 & 0.026 & & 0.00 & 0.001 & $U$ & 0.00 & 7.66 & & 0.02 \\
\hline $600-15$ (depth) & $1: 2.5$ & J1HHT9-B2 & 9/27/11 & 0.02 & $U$ & 0.02 & 0.004 & B & 0.02 & 0.029 & & 0.00 & 0.001 & $U$ & 0.00 & 8.71 & & 0.02 \\
\hline $600-15$ (depth) & $1: 5$ & J1HHT9-C1 & 9/27/11 & 0.02 & $U$ & 0.02 & 0.015 & $U$ & 0.02 & 0.029 & & 0.00 & 0.001 & $U$ & 0.00 & 5.48 & & 0.02 \\
\hline $600-23$ (depth) & $1: 1$ & J1HHV0-A1 & 9/27/11 & 0.10 & $U$ & 0.10 & 0.075 & $U$ & 0.08 & 0.037 & & 0.01 & 0.005 & $U$ & 0.01 & 29.0 & & 0.08 \\
\hline $600-23$ (depth) & $1: 2.5$ & J1HHV0-B1 & 9/27/11 & 0.02 & $U$ & 0.02 & 0.015 & $U$ & 0.02 & 0.033 & & 0.00 & 0.001 & $U$ & 0.00 & 19.0 & & 0.02 \\
\hline $600-23$ (depth) & $1: 5$ & J1HHV0-C1 & 9/27/11 & 0.02 & $U$ & 0.02 & 0.002 & B & 0.02 & 0.035 & & 0.00 & 0.001 & $U$ & 0.00 & 12.5 & & 0.02 \\
\hline $600-23$ (depth) & $1: 5$ & J1HHVO-C2 & 9/27/11 & 0.02 & $\mathrm{U}$ & 0.02 & 0.015 & $U$ & 0.02 & 0.034 & & 0.00 & 0.001 & $\mathrm{U}$ & 0.00 & 11.3 & & 0.02 \\
\hline \multirow{2}{*}{$\begin{array}{c}\text { Sample } \\
\text { Location }\end{array}$} & \multirow{2}{*}{$\begin{array}{c}\text { Coal Ash:Water } \\
\text { Ratio }\end{array}$} & \multirow{2}{*}{$\begin{array}{c}\text { HEIS } \\
\text { Number }\end{array}$} & \multirow{2}{*}{$\begin{array}{c}\text { Sample } \\
\text { Date }\end{array}$} & \multicolumn{3}{|c|}{ Cadmium } & \multicolumn{3}{|c|}{ Chromium } & \multicolumn{3}{|c|}{ Cobalt } & \multicolumn{3}{|c|}{ Copper } & \multicolumn{3}{|c|}{ Lead } \\
\hline & & & & $\mathrm{mg} / \mathrm{L}$ & Q & $\mathrm{PQL}$ & $\mathrm{mg} / \mathrm{L}$ & Q & $\mathrm{PQL}$ & $\mathrm{mg} / \mathrm{L}$ & $\mathrm{Q}$ & $\mathrm{PQL}$ & $\mathrm{mg} / \mathrm{L}$ & Q & $\mathrm{PQL}$ & $\mathrm{mg} / \mathrm{L}$ & Q & $\mathrm{PQL}$ \\
\hline 600-07 (depth) & $1: 1$ & J1HHT8-A1 & 9/27/11 & 0.0020 & $B$ & 0.02 & 0.025 & $u$ & 0.03 & 0.010 & $\mathrm{U}$ & 0.01 & 0.100 & $U$ & 0.10 & 0.022 & $B$ & 0.05 \\
\hline 600-07 (depth) & $1: 1$ & J1HHT8-A2 & 9/27/11 & 0.0020 & B & 0.02 & 0.003 & $B$ & 0.03 & 0.010 & $\mathrm{U}$ & 0.01 & 0.100 & $U$ & 0.10 & 0.023 & $B$ & 0.05 \\
\hline $600-07$ (depth) & $1: 2.5$ & J1HHT8-B1 & 9/27/11 & \begin{tabular}{|l|}
0.000474 \\
\end{tabular} & $\mathrm{~B}$ & 0.00 & 0.001 & $\mathrm{~B}$ & 0.01 & 0.002 & $\mathrm{U}$ & 0.00 & 0.020 & $U$ & 0.02 & 0.014 & & 0.01 \\
\hline 600-07 (depth) & $1: 5$ & J1HHT8-C1 & 9/27/11 & 0.00049 & $B$ & 0.00 & 0.001 & B & 0.01 & 0.002 & $\mathrm{U}$ & 0.00 & 0.003 & $B$ & 0.02 & 0.009 & B & 0.01 \\
\hline $600-15$ (depth) & $1: 1$ & J1HHT9-A1 & 9/27/11 & 0.0020 & $\mathrm{~B}$ & 0.02 & 0.004 & $B$ & 0.03 & 0.010 & $\mathrm{U}$ & 0.01 & 0.100 & $U$ & 0.10 & 0.05 & $U$ & 0.05 \\
\hline $600-15$ (depth) & $1: 2.5$ & J1HHT9-B1 & 9/27/11 & 0.0010 & $B$ & 0.00 & 0.001 & B & 0.01 & 0.002 & $\mathrm{U}$ & 0.00 & 0.020 & $U$ & 0.02 & 0.003 & $B$ & 0.01 \\
\hline $600-15$ (depth) & $1: 2.5$ & J1HHT9-B2 & 9/27/11 & 0.0010 & $\mathrm{~B}$ & 0.00 & 0.002 & $\mathrm{~B}$ & 0.01 & 0.001 & $\mathrm{~B}$ & 0.00 & 0.020 & $U$ & 0.02 & 0.004 & $B$ & 0.01 \\
\hline $600-15$ (depth) & $1: 5$ & J1HHT9-C1 & 9/27/11 & 0.0010 & $B$ & 0.00 & 0.002 & B & 0.01 & 0.002 & $\mathrm{U}$ & 0.00 & 0.020 & $U$ & 0.02 & 0.003 & $B$ & 0.01 \\
\hline $600-23$ (depth) & $1: 1$ & J1HHV0-A1 & 9/27/11 & 0.0020 & $\mathrm{~B}$ & 0.02 & 0.004 & $B$ & 0.03 & 0.010 & $\mathrm{U}$ & 0.01 & 0.100 & $U$ & 0.10 & 0.022 & $\mathrm{~B}$ & 0.05 \\
\hline $600-23$ (depth) & $1: 2.5$ & J1HHV0-B1 & 9/27/11 & 0.000514 & B & 0.00 & 0.001 & B & 0.01 & 0.002 & $\mathrm{U}$ & 0.00 & 0.020 & $U$ & 0.02 & 0.018 & & 0.01 \\
\hline $600-23$ (depth) & $1: 5$ & J1HHV0-C1 & 9/27/11 & 0.0010 & $B$ & 0.00 & 0.001 & $B$ & 0.01 & 0.002 & $\mathrm{U}$ & 0.00 & 0.020 & $U$ & 0.02 & 0.012 & & 0.01 \\
\hline $600-23$ (depth) & $1: 5$ & $\mathrm{~J} 1 \mathrm{HHVO}-\mathrm{C} 2$ & 9/27/11 & 0.0010 & $\mathrm{~B}$ & 0.00 & 0.005 & $U$ & 0.01 & 0.002 & $\mathrm{U}$ & 0.00 & 0.020 & $U$ & 0.02 & 0.010 & & 0.01 \\
\hline
\end{tabular}


Table C-15. 600-207 Coal Ash Leaching Sample Results. (2 Pages)

\begin{tabular}{|c|c|c|c|c|c|c|c|c|c|c|c|c|c|c|c|c|c|c|}
\hline \multirow{2}{*}{$\begin{array}{c}\text { Sample } \\
\text { Location }\end{array}$} & \multirow{2}{*}{$\begin{array}{c}\text { Coal Ash:Water } \\
\text { Ratio }\end{array}$} & \multirow{2}{*}{$\begin{array}{l}\text { HEIS } \\
\text { Number }\end{array}$} & \multirow{2}{*}{$\begin{array}{c}\text { Sample } \\
\text { Date }\end{array}$} & \multicolumn{3}{|c|}{ Manganese } & \multicolumn{3}{|c|}{ Mercury } & \multicolumn{3}{|c|}{ Molybdenum } & \multicolumn{3}{|c|}{ Nickel } & \multicolumn{3}{|c|}{ Selenium } \\
\hline & & & & $\mathrm{mg} / \mathrm{L}$ & Q & $\mathrm{PQL}$ & $\mathrm{mg} / \mathrm{L}$ & $\mathbf{Q}$ & $\mathrm{PQL}$ & $\mathrm{mg} / \mathrm{L}$ & $\mathbf{Q}$ & $\mathrm{PQL}$ & $\mathrm{mg} / \mathrm{L}$ & $\mathbf{Q}$ & $\mathrm{PQL}$ & $\mathrm{mg} / \mathrm{L}$ & $\mathbf{Q}$ & $\mathrm{PQL}$ \\
\hline 600-07 (depth) & $1: 1$ & J1HHT8-A1 & 9/27/11 & 0.424 & & 0.03 & 0.002 & $u$ & 0.00 & 0.003 & B & 0.02 & 0.100 & $u$ & 0.10 & 0.100 & $u$ & 0.10 \\
\hline $600-07$ (depth) & $1: 1$ & J1HHT8-A2 & 9/27/11 & 0.443 & & 0.03 & 0.0020 & $\mathrm{U}$ & 0.00 & 0.003 & B & 0.02 & 0.100 & $\mathrm{U}$ & 0.10 & 0.100 & $U$ & 0.10 \\
\hline 600-07 (depth) & $1: 2.5$ & J1HHT8-B1 & 9/27/11 & 0.341 & & 0.01 & 0.0002 & $\mathrm{U}$ & 0.00 & 0.001 & B & 0.00 & 0.001 & B & 0.02 & 0.020 & $U$ & 0.02 \\
\hline 600-07 (depth) & $1: 5$ & J1HHT8-C1 & 9/27/11 & 0.303 & & 0.01 & 0.0002 & $U$ & 0.00 & 0.001 & B & 0.00 & 0.001 & B & 0.02 & 0.020 & $U$ & 0.02 \\
\hline $600-15$ (depth) & $1: 1$ & J1HHT9-A1 & 9/27/11 & 0.099 & & 0.03 & 0.0020 & $U$ & 0.00 & 0.197 & & 0.02 & 0.100 & $U$ & 0.10 & 0.416 & & 0.10 \\
\hline $600-15$ (depth) & $1: 2.5$ & J1HHT9-B1 & 9/27/11 & 0.066 & & 0.01 & 0.0002 & $U$ & 0.00 & 0.103 & & 0.00 & 0.020 & $U$ & 0.02 & 0.210 & & 0.02 \\
\hline $600-15$ (depth) & $1: 2.5$ & J1HHT9-B2 & $9 / 27 / 11$ & 0.072 & & 0.01 & 0.0002 & $U$ & 0.00 & 0.112 & & 0.00 & 0.020 & U & 0.02 & 0.239 & & 0.02 \\
\hline $600-15$ (depth) & $1: 5$ & J1HHT9-C1 & $9 / 27 / 11$ & 0.048 & & 0.01 & 0.0002 & $U$ & 0.00 & 0.057 & & 0.00 & 0.020 & $U$ & 0.02 & 0.112 & & 0.02 \\
\hline 600-23 (depth) & $1: 1$ & J1HHV0-A1 & 9/27/11 & 0.165 & & 0.03 & 0.0020 & $U$ & 0.00 & 0.008 & B & 0.02 & 0.004 & B & 0.10 & 0.100 & $U$ & 0.10 \\
\hline 600-23 (depth) & $1: 2.5$ & J1HHV0-B1 & 9/27/11 & 0.140 & & 0.01 & 0.0002 & $U$ & 0.00 & 0.004 & & 0.00 & 0.001 & B & 0.02 & 0.003 & B & 0.02 \\
\hline $600-23$ (depth) & 1:5 & $\mathrm{J} 1 \mathrm{HHVO}-\mathrm{C} 2$ & $9 / 27 / 11$ & 0.120 & & 0.01 & 0.0002 & $U$ & 0.00 & 0.003 & B & 0.00 & 0.001 & B & 0.02 & 0.020 & $U$ & 0.02 \\
\hline
\end{tabular}

\begin{tabular}{|c|c|c|c|c|c|c|c|c|c|c|c|c|c|c|c|c|c|c|c|}
\hline \multirow{2}{*}{$\begin{array}{c}\text { Sample } \\
\text { Location }\end{array}$} & \multirow{2}{*}{$\begin{array}{l}\text { Coal Ash:Water } \\
\text { Ratio }\end{array}$} & \multirow{2}{*}{$\begin{array}{c}\text { HEIS } \\
\text { Number }\end{array}$} & \multirow{2}{*}{$\begin{array}{c}\text { Sample } \\
\text { Date }\end{array}$} & \multicolumn{3}{|c|}{ Silver } & \multicolumn{3}{|c|}{ Thallium } & \multicolumn{3}{|c|}{ Uranium (KPA) } & \multicolumn{3}{|c|}{ Vanadium } & \multicolumn{3}{|c|}{ Zinc } & \multirow[b]{2}{*}{$\mathrm{pH}$} \\
\hline & & & & $\mathrm{mg} / \mathrm{L}$ & Q & $\mathrm{PQL}$ & $\mathrm{mg} / \mathrm{L}$ & Q & $\mathrm{PQL}$ & ug/L & Q & MDA & $\mathrm{mg} / \mathrm{L}$ & $Q$ & $\mathrm{PQL}$ & $\mathrm{mg} / \mathrm{L}$ & $\mathrm{Q}$ & $\mathrm{PQL}$ & \\
\hline $600-07$ (depth) & $1: 1$ & J1HHT8-A1 & 9/27/11 & 0.03 & $U^{U}$ & 0.03 & 0.075 & U & 0.08 & 0.030 & & 0.025 & 0.090 & & 0.03 & 0.25 & $\mathrm{U}$ & 0.25 & 7.68 \\
\hline $600-07$ (depth) & $1: 1$ & J1HHT8-A2 & $9 / 27 / 11$ & 0.03 & $\mathrm{U}$ & 0.03 & 0.075 & $U$ & 0.08 & 0.013 & $U$ & 0.025 & 0.089 & & 0.03 & 0.25 & $U$ & 0.25 & 6.99 \\
\hline 600-07 (depth) & $1: 2.5$ & J1HHT8-B1 & 9/27/11 & 0.006 & $U$ & 0.01 & 0.015 & $U$ & 0.02 & 0.008 & $U$ & 0.025 & 0.015 & & 0.01 & 0.05 & $U$ & 0.05 & 7.16 \\
\hline 600-07 (depth) & $1: 5$ & J1HHT8-C1 & 9/27/11 & 0.006 & $U$ & 0.01 & 0.015 & $U$ & 0.02 & 0 & $U$ & 0.025 & 0.018 & & 0.01 & 0.05 & $U$ & 0.05 & 7.09 \\
\hline 600-15 (depth) & $1: 1$ & J1HHT9-A1 & 9/27/11 & 0.03 & $\mathrm{U}$ & 0.03 & 0.075 & $U$ & 0.08 & 0.897 & & 0.025 & 0.073 & & 0.03 & 0.25 & $U$ & 0.25 & 7.44 \\
\hline $600-15$ (depth) & $1: 2.5$ & J1HHT9-B1 & 9/27/11 & 0.006 & U & 0.01 & 0.015 & $U$ & 0.02 & 0.520 & & 0.025 & 0.005 & $u$ & 0.01 & 0.05 & $U$ & 0.05 & 8.02 \\
\hline $600-15$ (depth) & $1: 2.5$ & J1HHT9-B2 & 9/27/11 & 0.006 & U & 0.01 & 0.015 & $U$ & 0.02 & 0.491 & & 0.025 & 0.005 & U & 0.01 & 0.05 & $U$ & 0.05 & 8.23 \\
\hline $600-15$ (depth) & $1: 5$ & J1HHT9-C1 & $9 / 27 / 11$ & 0.006 & $U$ & 0.01 & 0.015 & $U$ & 0.02 & 0.354 & & 0.025 & 0.013 & & 0.01 & 0.05 & $U$ & 0.05 & 8.41 \\
\hline $600-23$ (depth) & $1: 1$ & J1HHVO-A1 & 9/27/11 & 0.03 & $U$ & 0.03 & 0.075 & $U$ & 0.08 & 0.015 & $U$ & 0.025 & 0.092 & & 0.03 & 0.25 & $U$ & 0.25 & 7.61 \\
\hline $600-23$ (depth) & $1: 2.5$ & J1HHVO-B1 & 9/27/11 & 0.006 & $U$ & 0.01 & 0.015 & $U$ & 0.02 & 0.014 & $U$ & 0.025 & 0.007 & & 0.01 & 0.05 & $U$ & 0.05 & 7.63 \\
\hline $600-23$ (depth) & $1: 5$ & $\mathrm{~J} 1 \mathrm{HHVO}-\mathrm{C} 1$ & 9/27/11 & 0.006 & $U$ & 0.01 & 0.015 & $U$ & 0.02 & 0 & $U$ & 0.025 & 0.012 & & 0.01 & 0.05 & $U$ & 0.05 & 7.58 \\
\hline $600-23$ (depth) & $1: 5$ & $\mathrm{~J} 1 \mathrm{HHVO}-\mathrm{C} 2$ & $9 / 27 / 11$ & 0.006 & $U$ & 0.01 & 0.015 & $U$ & 0.02 & 0.006 & $U$ & 0.025 & 0.014 & & 0.01 & 0.05 & $U$ & 0.05 & 7.49 \\
\hline
\end{tabular}




\begin{tabular}{|c|c|c|c|c|c|c|c|c|c|c|c|c|c|c|c|c|c|}
\hline \multirow{2}{*}{ 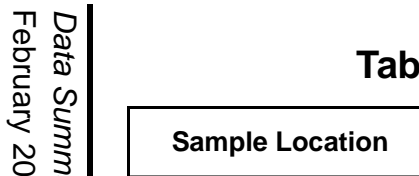 } & \multirow{2}{*}{$\begin{array}{c}\text { HEIS } \\
\text { Number }\end{array}$} & \multirow{2}{*}{$\begin{array}{c}\text { Sample } \\
\text { Date }\end{array}$} & \multicolumn{3}{|c|}{ Antimony } & \multirow{2}{*}{\multicolumn{2}{|c|}{ 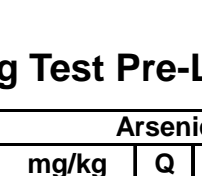 }} & eachir & \multicolumn{7}{|c|}{ Quadruplicate Sample Results. (4 Pages) } & \multicolumn{2}{|c|}{ 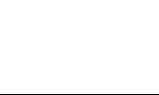 } \\
\hline & & & $\mathrm{mg} / \mathrm{kg}$ & $Q$ & $\mathrm{PQL}$ & & & $\mathrm{PQL}$ & $\mathrm{mg} / \mathrm{kg}$ & $Q$ & $\mathrm{PQL}$ & $\mathrm{mg} / \mathrm{kg}$ & $Q$ & $\mathrm{PQL}$ & $\mathrm{mg} / \mathrm{kg}$ & Q & $\overline{P Q L}$ \\
\hline 600-207 SURFACE & J1HHN7-1 & $9 / 27 / 11$ & 0.926 & $U$ & 0.93 & 10.9 & & 0.93 & 1100 & & 0.46 & 1.45 & & 0.19 & 114 & & 1.85 \\
\hline 600-207 SURFACE & J1HHN7-2 & $9 / 27 / 11$ & 0.820 & $\mathrm{U}$ & 0.82 & 11.7 & & 0.82 & 1110 & & 0.41 & 1.39 & & 0.16 & 116 & & 1.64 \\
\hline 600-207 SURFACE & J1HHN7-3 & $9 / 27 / 11$ & 0.288 & $\mathrm{~B}$ & 0.77 & 11.6 & & 0.77 & 1090 & & 0.39 & 1.36 & & 0.15 & 115 & & 1.54 \\
\hline 600-207 SURFACE & J1HHN7-4 & 9/27/11 & 0.847 & $\mathrm{U}$ & 0.85 & 10.7 & & 0.85 & 1070 & & 0.42 & 1.32 & & 0.17 & 109 & & 1.69 \\
\hline 600-207 SURFACE & J1HHN7-5 & 9/27/11 & 0.330 & $B$ & 0.94 & 10.8 & & 0.94 & 1140 & & 0.47 & 1.5 & & 0.19 & 120 & & 1.89 \\
\hline 600-207 SURFACE & J1HHN7-6 & $9 / 27 / 11$ & 0.893 & $\mathrm{U}$ & 0.89 & 9.81 & & 0.89 & 1030 & & 0.45 & 1.36 & & 0.18 & 112 & & 1.79 \\
\hline 600-207 SURFACE & J1HHN7-7 & 9/27/11 & 0.806 & $\mathrm{U}$ & 0.81 & 11.1 & & 0.81 & 1050 & & 0.40 & 1.36 & & 0.16 & 114 & & 1.61 \\
\hline 600-207 SURFACE & J1HHN7-8 & $9 / 27 / 11$ & 0.943 & $U$ & 0.94 & 11.3 & & 0.94 & 1100 & & 0.47 & 1.41 & & 0.19 & 113 & & 1.89 \\
\hline 126-B-1 SURFACE & $\mathrm{J} 1 \mathrm{HHX8-1}$ & $4 / 25 / 11$ & 0.909 & $\mathrm{U}$ & 0.91 & 6.23 & & 0.91 & 1010 & & 0.46 & 1.12 & & 0.18 & 84.4 & & 1.82 \\
\hline 126-B-1 SURFACE & $\mathrm{J} 1 \mathrm{HH} \times 8-2$ & $4 / 25 / 11$ & 0.847 & $U$ & 0.85 & 7.04 & & 0.85 & 1380 & & 0.42 & 1.6 & & 0.17 & 143 & & 1.69 \\
\hline 126-B-1 SURFACE & $\mathrm{J} 1 \mathrm{HH} \times 8-3$ & $4 / 25 / 11$ & 0.926 & $\mathrm{U}$ & 0.93 & 6.68 & & 0.93 & 1170 & & 0.46 & 1.31 & & 0.19 & 101 & & 1.85 \\
\hline 126-B-1 SURFACE & J1HHX8-4 & $4 / 25 / 11$ & 0.345 & $\mathrm{~B}$ & 0.94 & 6.43 & & 0.94 & 1920 & & 0.47 & 1.19 & & 0.19 & 89.8 & & 1.89 \\
\hline 126-B-1 SURFACE & $\mathrm{J} 1 \mathrm{HHX8-5}$ & $4 / 25 / 11$ & 0.372 & $\mathrm{~B}$ & 0.78 & 7.24 & & 0.78 & 1300 & & 0.39 & 1.75 & & 0.16 & 101 & & 1.56 \\
\hline 126-B-1 SURFACE & J1HHX8-6 & $4 / 25 / 11$ & 0.847 & $\mathrm{U}$ & 0.85 & 6.85 & & 0.85 & 1300 & & 0.42 & 1.33 & & 0.17 & 110 & & 1.69 \\
\hline 126-B-1 SURFACE & $\mathrm{J} 1 \mathrm{HHX8-7}$ & $4 / 25 / 11$ & 0.877 & $\mathrm{U}$ & 0.88 & 6.71 & & 0.88 & 1040 & & 0.44 & 1.35 & & 0.18 & 98.6 & & 1.75 \\
\hline 126-B-1 SURFACE & $\mathrm{J} 1 \mathrm{HHX8-8}$ & $4 / 25 / 11$ & 0.962 & $\mathrm{U}$ & 0.96 & 6.87 & & 0.96 & 1120 & & 0.48 & 1.370 & & 0.19 & 108 & & 1.92 \\
\hline 126-B-1 DEPTH & J1HJ08-5 & $4 / 26 / 11$ & 0.909 & $\mathrm{U}$ & 0.91 & 8.08 & & 0.91 & 1210 & & 0.46 & 0.976 & & 0.18 & 38.4 & & 1.82 \\
\hline 126-B-1 DEPTH & $\mathrm{J} 1 \mathrm{HJ} 08-6$ & $4 / 26 / 11$ & 1 & $\mathrm{U}$ & 1.00 & 7.34 & & 1.00 & 1230 & & 0.50 & 0.968 & & 0.20 & 50.1 & & 2.00 \\
\hline 126-B-1 DEPTH & $\mathrm{J} 1 \mathrm{HJ} 08-7$ & $4 / 26 / 11$ & 0.277 & $\mathrm{~B}$ & 0.73 & 7.67 & & 0.73 & 1110 & & 0.36 & 0.979 & & 0.15 & 34.9 & & 1.45 \\
\hline 126-B-1 DEPTH & $\mathrm{J} 1 \mathrm{HJ} 08-8$ & $4 / 26 / 11$ & 0.35 & $\mathrm{~B}$ & 0.89 & 7.44 & & 0.89 & 1070 & & 0.45 & 0.979 & & 0.18 & 41 & & 1.79 \\
\hline $126-\mathrm{H}-1$ DEPTH & $\mathrm{J} 1 \mathrm{HJ} 80-5$ & $8 / 24 / 11$ & 0.862 & $U$ & 0.86 & 2.73 & & 0.86 & 1190 & & 0.43 & 1.5 & & 0.17 & 393 & & 1.72 \\
\hline 126-H-1 DEPTH & J1HJ80-6 & $8 / 24 / 11$ & 0.962 & $\mathrm{U}$ & 0.96 & 2.49 & & 0.96 & 1200 & & 0.48 & 1.61 & & 0.19 & 448 & & 1.92 \\
\hline 126-H-1 DEPTH & J1HJ80-7 & $8 / 24 / 11$ & 0.893 & $\mathrm{U}$ & 0.89 & 2.53 & & 0.89 & 1110 & & 0.45 & 1.370 & & 0.18 & 391 & & 1.79 \\
\hline 126-H-1 DEPTH & J1HJ80-8 & $8 / 24 / 11$ & 0.839 & & 0.71 & 2.47 & & 0.71 & 990 & & 0.36 & 1.46 & & 0.14 & 411 & & 1.43 \\
\hline
\end{tabular}


Table C-16. Coal Ash Follow on Leaching Test Pre-Leaching Quadruplicate Sample Results. (4 Pages)

\begin{tabular}{|c|c|c|c|c|c|c|c|c|c|c|c|c|c|c|c|c|c|}
\hline \multirow{2}{*}{ Sample Location } & \multirow{2}{*}{$\begin{array}{c}\text { HEIS } \\
\text { Number }\end{array}$} & \multirow{2}{*}{$\begin{array}{c}\text { Sample } \\
\text { Date }\end{array}$} & \multicolumn{3}{|c|}{ Cadmium } & \multicolumn{3}{|c|}{ Chromium } & \multicolumn{3}{|c|}{ Cobalt } & \multicolumn{3}{|c|}{ Copper } & \multicolumn{3}{|c|}{ Lead } \\
\hline & & & $\mathrm{mg} / \mathrm{kg}$ & $\mathbf{Q}$ & PQL & $\mathrm{mg} / \mathrm{kg}$ & $\mathbf{Q}$ & $\mathrm{PQL}$ & $\mathrm{mg} / \mathrm{kg}$ & $\mathbf{Q}$ & PQL & $\mathrm{mg} / \mathrm{kg}$ & Q & $\mathrm{PQL}$ & $\mathrm{mg} / \mathrm{kg}$ & $\mathbf{Q}$ & PQL \\
\hline 600-207 SURFACE & J1HHN7-1 & $9 / 27 / 11$ & 0.286 & & 0.23 & 15.0 & & 0.9 & 6.85 & & 2.78 & 35.9 & & 1.85 & 9.53 & & 0.93 \\
\hline 600-207 SURFACE & J1HHN7-2 & 9/27/11 & 0.177 & B & 0.21 & 15.7 & & 0.8 & 7.28 & & 2.46 & 35.0 & & 1.64 & 9.67 & & 0.82 \\
\hline 600-207 SURFACE & J1HHN7-3 & 9/27/11 & 0.192 & & 0.19 & 15.5 & & 0.8 & 6.91 & & 2.31 & 36.0 & & 1.54 & 8.48 & & 0.77 \\
\hline 600-207 SURFACE & J1HHN7-4 & $9 / 27 / 11$ & 0.192 & B & 0.21 & 14.7 & & 0.9 & 6.78 & & 2.54 & 33.6 & & 1.69 & 8.15 & & 0.85 \\
\hline 600-207 SURFACE & J1HHN7-5 & 9/27/11 & 0.176 & B & 0.24 & 15.2 & & 0.9 & 6.94 & & 2.83 & 34.3 & & 1.89 & 8.32 & & 0.94 \\
\hline 600-207 SURFACE & J1HHN7-6 & 9/27/11 & 0.169 & B & 0.22 & 14.1 & & 0.9 & 6.53 & & 2.68 & 32.6 & & 1.79 & 7.99 & & 0.89 \\
\hline 600-207 SURFACE & J1HHN7-7 & 9/27/11 & 0.182 & B & 0.20 & 15.3 & & 0.8 & 6.80 & & 2.42 & 34.9 & & 1.61 & 8.67 & & 0.81 \\
\hline 600-207 SURFACE & J1HHN7-8 & 9/27/11 & 0.157 & B & 0.24 & 14.4 & & 0.9 & 6.70 & & 2.83 & 34.3 & & 1.89 & 7.92 & & 0.94 \\
\hline 126-B-1 SURFACE & J1HHX8-1 & $4 / 25 / 11$ & 0.245 & & 0.23 & 8.8 & & 0.9 & 3.29 & & 2.73 & 22.0 & & 1.82 & 10.4 & & 0.91 \\
\hline 126-B-1 SURFACE & J1HHX8-2 & $4 / 25 / 11$ & 0.258 & & 0.21 & 11.1 & & 0.9 & 4.62 & & 2.54 & 34.2 & & 1.69 & 10.1 & & 0.85 \\
\hline 126-B-1 SURFACE & $\mathrm{J} 1 \mathrm{HH} \times 8-3$ & $4 / 25 / 11$ & 0.269 & & 0.23 & 10.2 & & 0.9 & 3.81 & & 2.78 & 25.4 & & 1.85 & 10.8 & & 0.93 \\
\hline 126-B-1 SURFACE & J1HHX8-4 & $4 / 25 / 11$ & 0.253 & & 0.24 & 9.1 & & 0.9 & 3.54 & & 2.83 & 22.6 & & 1.89 & 10.3 & & 0.94 \\
\hline 126-B-1 SURFACE & J1HHX8-5 & 4/25/11 & 0.241 & & 0.20 & 9.7 & & 0.8 & 3.90 & & 2.34 & 30.3 & & 1.56 & 9.08 & & 0.78 \\
\hline 126-B-1 SURFACE & $\mathrm{J} 1 \mathrm{HHX8-6}$ & $4 / 25 / 11$ & 0.234 & & 0.21 & 10.3 & & 0.9 & 4.12 & & 2.54 & 25.5 & & 1.69 & 9.90 & & 0.85 \\
\hline 126-B-1 SURFACE & J1HHX8-7 & $4 / 25 / 11$ & 0.234 & & 0.22 & 12.3 & & 0.9 & 4.15 & & 2.63 & 23.5 & & 1.75 & 8.98 & & 0.88 \\
\hline 126-B-1 SURFACE & J1HHX8-8 & $4 / 25 / 11$ & 0.252 & & 0.24 & 9.8 & & 1.0 & 3.97 & & 2.88 & 25.5 & & 1.92 & 9.75 & & 0.96 \\
\hline 126-B-1 DEPTH & $\mathrm{J} 1 \mathrm{HJ} 08-5$ & $4 / 26 / 11$ & 0.094 & $B$ & 0.23 & 10.3 & & 0.9 & 4.60 & & 2.73 & 26.9 & & 1.82 & 3.92 & & 0.91 \\
\hline 126-B-1 DEPTH & $\mathrm{J} 1 \mathrm{HJ} 08-6$ & $4 / 26 / 11$ & 0.075 & B & 0.25 & 9.8 & & 1.0 & 4.40 & & 3.00 & 23.9 & & 2.00 & 3.56 & & 1.00 \\
\hline 126-B-1 DEPTH & J1HJ08-7 & $4 / 26 / 11$ & 0.105 & B & 0.18 & 10.7 & & 0.7 & 4.02 & & 2.17 & 24.9 & & 1.45 & 3.97 & & 0.73 \\
\hline 126-B-1 DEPTH & J1HJ08-8 & $4 / 26 / 11$ & 0.087 & B & 0.22 & 10.0 & & 0.9 & 4.26 & & 2.68 & 24.4 & & 1.79 & 3.87 & & 0.89 \\
\hline 126-H-1 DEPTH & $\mathrm{J} 1 \mathrm{HJ} 80-5$ & $8 / 24 / 11$ & 0.085 & $B$ & 0.22 & 13.0 & & 0.9 & 2.70 & & 2.59 & 24.1 & & 1.72 & 13.6 & & 0.86 \\
\hline 126-H-1 DEPTH & J1HJ80-6 & $8 / 24 / 11$ & 0.218 & B & 0.24 & 13.7 & & 1.0 & 2.33 & B & 2.88 & 31.1 & & 1.92 & 14.5 & & 0.96 \\
\hline 126-H-1 DEPTH & J1HJ80-7 & $8 / 24 / 11$ & 0.16 & B & 0.22 & 12.0 & & 0.9 & 2.51 & B & 2.68 & 39.8 & & 1.79 & 10.1 & & 0.89 \\
\hline 126-H-1 DEPTH & $\mathrm{J} 1 \mathrm{HJ} 80-8$ & $8 / 24 / 11$ & 0.147 & $B$ & 0.18 & 10.5 & & 0.7 & 2.14 & & 2.14 & 60.6 & & 1.43 & 267 & & 0.71 \\
\hline
\end{tabular}




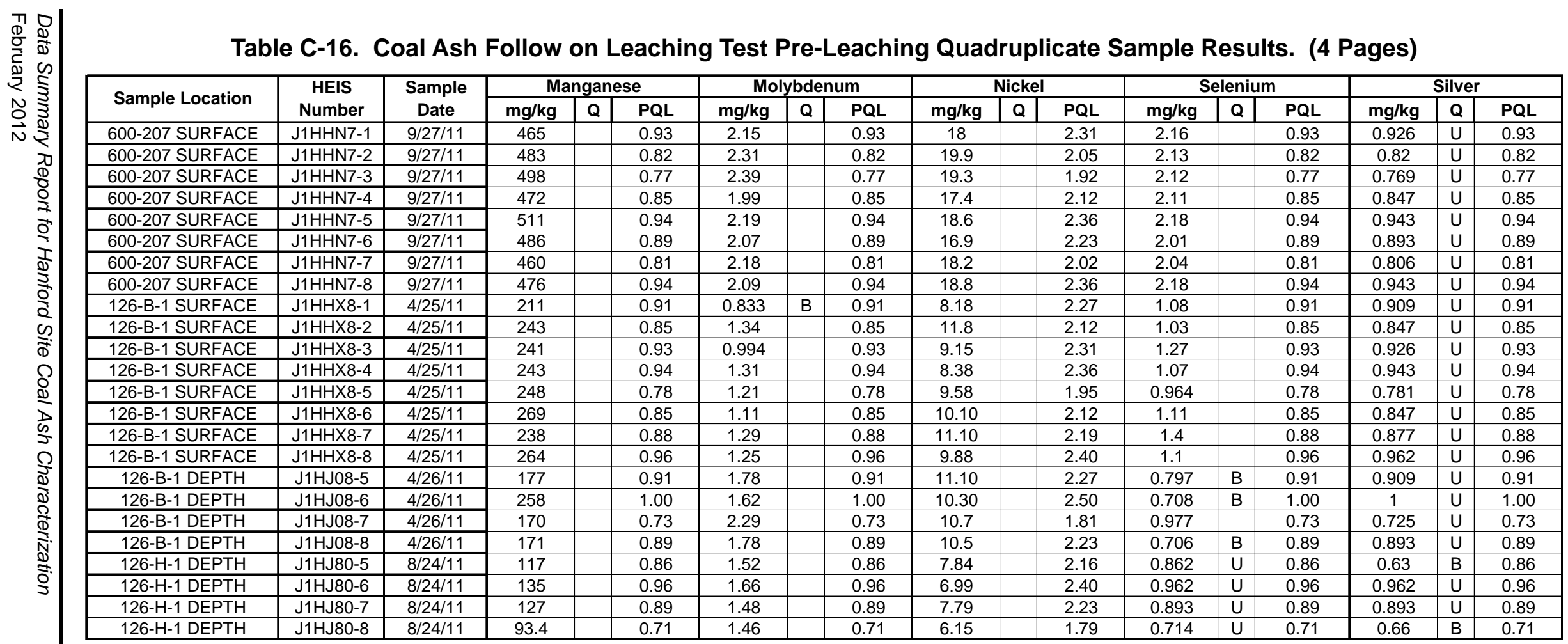


Table C-16. Coal Ash Follow on Leaching Test Pre-Leaching Quadruplicate Sample Results. (4 Pages)

\begin{tabular}{|c|c|c|c|c|c|c|c|c|c|c|c|}
\hline \multirow{2}{*}{ Sample Location } & \multirow{2}{*}{$\begin{array}{c}\text { HEIS } \\
\text { Number }\end{array}$} & \multirow{2}{*}{$\begin{array}{c}\text { Sample } \\
\text { Date }\end{array}$} & \multicolumn{3}{|c|}{ Thallium } & \multicolumn{3}{|c|}{ Vanadium } & \multicolumn{3}{|c|}{ Zinc } \\
\hline & & & $\mathrm{mg} / \mathrm{kg}$ & $\mathbf{Q}$ & PQL & mg/kg & $\mathbf{Q}$ & PQL & $\mathrm{mg} / \mathrm{kg}$ & $\mathbf{Q}$ & PQL \\
\hline 600-207 SURFACE & J1HHN7-1 & $9 / 27 / 11$ & 0.926 & $\mathrm{U}$ & 0.93 & 54.4 & & 0.93 & 25.4 & & 2.8 \\
\hline 600-207 SURFACE & J1HHN7-2 & $9 / 27 / 11$ & 0.82 & $\mathrm{U}$ & 0.82 & 55.8 & & 0.82 & 25.6 & & 2.5 \\
\hline 600-207 SURFACE & J1HHN7-3 & $9 / 27 / 11$ & 0.769 & $U$ & 0.77 & 54.4 & & 0.77 & 26.8 & & 2.3 \\
\hline 600-207 SURFACE & J1HHN7-4 & $9 / 27 / 11$ & 0.847 & $\mathrm{U}$ & 0.85 & 53.3 & & 0.85 & 27.1 & & 2.5 \\
\hline 600-207 SURFACE & J1HHN7-5 & $9 / 27 / 11$ & 0.943 & $\mathrm{U}$ & 0.94 & 55 & & 0.94 & 24 & & 2.8 \\
\hline 600-207 SURFACE & J1HHN7-6 & $9 / 27 / 11$ & 0.893 & $\mathrm{U}$ & 0.89 & 53.2 & & 0.89 & 23.9 & & 2.7 \\
\hline 600-207 SURFACE & J1HHN7-7 & $9 / 27 / 11$ & 0.806 & $\mathrm{U}$ & 0.81 & 54.7 & & 0.81 & 24.8 & & 2.4 \\
\hline 600-207 SURFACE & J1HHN7-8 & $9 / 27 / 11$ & 0.943 & $\mathrm{U}$ & 0.94 & 53.3 & & 0.94 & 23.2 & & 2.8 \\
\hline 126-B-1 SURFACE & J1HHX8-1 & $4 / 25 / 11$ & 0.909 & $\mathrm{U}$ & 0.91 & 28 & & 0.91 & 30.6 & & 2.7 \\
\hline 126-B-1 SURFACE & $\mathrm{J} 1 \mathrm{HH}$ X8-2 & $4 / 25 / 11$ & 0.847 & $\mathrm{U}$ & 0.85 & 49.4 & & 0.85 & 34.1 & & 2.5 \\
\hline 126-B-1 SURFACE & J1HHX8-3 & $4 / 25 / 11$ & 0.926 & $\mathrm{U}$ & 0.93 & 32.7 & & 0.93 & 37.2 & & 2.8 \\
\hline 126-B-1 SURFACE & J1HHX8-4 & $4 / 25 / 11$ & 0.943 & $\mathrm{U}$ & 0.94 & 28.9 & & 0.94 & 30 & & 2.8 \\
\hline 126-B-1 SURFACE & $\mathrm{J} 1 \mathrm{HH} \times 8-5$ & $4 / 25 / 11$ & 0.781 & $\mathrm{U}$ & 0.78 & 34.9 & & 0.78 & 33.9 & & 2.3 \\
\hline 126-B-1 SURFACE & J1HHX8-6 & $4 / 25 / 11$ & 0.847 & $\mathrm{U}$ & 0.85 & 30.800 & & 0.85 & 35.1 & & 2.5 \\
\hline 126-B-1 SURFACE & J1HHX8-7 & $4 / 25 / 11$ & 0.877 & $\mathrm{U}$ & 0.88 & 32.100 & & 0.88 & 36.5 & & 2.6 \\
\hline 126-B-1 SURFACE & $\mathrm{J1HHX8-8}$ & $4 / 25 / 11$ & 0.962 & $\mathrm{U}$ & 0.96 & 32.600 & & 0.96 & 37.3 & & 2.9 \\
\hline 126-B-1 DEPTH & J1HJ08-5 & $4 / 26 / 11$ & 0.909 & $\mathrm{U}$ & 0.91 & 35.300 & & 0.91 & 23.6 & & 2.7 \\
\hline 126-B-1 DEPTH & J1HJ08-6 & $4 / 26 / 11$ & 1.000 & $\mathrm{U}$ & 1.00 & 35.100 & & 1.00 & 22.7 & & 3.0 \\
\hline 126-B-1 DEPTH & $\mathrm{J} 1 \mathrm{HJ} 08-7$ & $4 / 26 / 11$ & 0.725 & $\mathrm{U}$ & 0.73 & 32.900 & & 0.73 & 22.7 & & 2.2 \\
\hline 126-B-1 DEPTH & $\mathrm{J} 1 \mathrm{HJ} 08-8$ & $4 / 26 / 11$ & 0.893 & $\mathrm{U}$ & 0.89 & 34.600 & & 0.89 & 23.3 & & 2.7 \\
\hline 126-H-1 DEPTH & J1HJ80-5 & $8 / 24 / 11$ & 0.862 & $\mathrm{U}$ & 0.86 & 34.500 & & 0.86 & 23.1 & & 2.6 \\
\hline 126-H-1 DEPTH & J1HJ80-6 & $8 / 24 / 11$ & 0.962 & $\mathrm{U}$ & 0.96 & 34.200 & & 0.96 & 42.7 & & 2.9 \\
\hline 126-H-1 DEPTH & J1HJ80-7 & $8 / 24 / 11$ & 0.893 & $\mathrm{U}$ & 0.89 & 31.100 & & 0.89 & 31.6 & & 2.7 \\
\hline 126-H-1 DEPTH & J1HJ80-8 & $8 / 24 / 11$ & 0.714 & $\mathrm{U}$ & 0.71 & 29.4 & & 0.71 & 74 & & 2.1 \\
\hline
\end{tabular}


Table C-17. Coal Ash Follow on Leaching Test Coal Ash Leaching Sample Results. (4 Pages)

\begin{tabular}{|c|c|c|c|c|c|c|c|c|c|c|c|c|c|c|c|c|c|c|}
\hline \multirow{2}{*}{ Sample Location } & \multirow{2}{*}{$\begin{array}{l}\text { Coal Ash:Water } \\
\text { Ratio }\end{array}$} & \multirow{2}{*}{$\begin{array}{c}\text { HEIS } \\
\text { Number } \\
\end{array}$} & \multirow{2}{*}{$\begin{array}{c}\text { Sample } \\
\text { Date }\end{array}$} & \multicolumn{3}{|c|}{ Antimony } & \multicolumn{3}{|c|}{ Arsenic } & \multicolumn{3}{|c|}{ Barium } & \multicolumn{3}{|c|}{ Beryllium } & \multicolumn{3}{|c|}{ Boron } \\
\hline & & & & $\mathrm{mg} / \mathrm{L}$ & $\mathbf{Q}$ & $\mathrm{PQL}$ & $\mathrm{mg} / \mathrm{L}$ & $\mathbf{Q}$ & PQL & $\mathrm{mg} / \mathrm{L}$ & $\mathbf{Q}$ & $\mathrm{PQL}$ & $\mathrm{mg} / \mathrm{L}$ & $\mathbf{Q}$ & PQL & $\mathrm{mg} / \mathrm{L}$ & $\mathbf{Q}$ & PQL \\
\hline 600-207 SURFACE & $1: 1$ & J1HHN7-A1 & 9/27/11 & 0.10 & U & 0.10 & 0.075 & U & 0.08 & 0.081 & & 0.01 & 0.005 & $\mathrm{U}$ & 0.01 & 2.78 & & 0.08 \\
\hline 600-207 SURFACE & $1: 2.5$ & J1HHN7-B1 & 9/27/11 & 0.02 & $U$ & 0.02 & 0.003 & $B$ & 0.02 & 0.087 & & 0.00 & 0.001 & $U$ & 0.00 & 2.1 & & 0.02 \\
\hline 600-207 SURFACE & $1: 2.5$ & J1HHN7-B2 & 9/27/11 & 0.02 & $U$ & 0.02 & 0.005 & B & 0.02 & 0.085 & & 0.00 & 0.001 & U & 0.00 & 1.89 & & 0.02 \\
\hline 600-207 SURFACE & $1: 5$ & J1HHN7-C1 & 9/27/11 & 0.02 & $U$ & 0.02 & 0.003 & $B$ & 0.02 & 0.132 & & 0.00 & 0.001 & $U$ & 0.00 & 1.42 & & 0.02 \\
\hline 600-207 SURFACE & $1: 1$ & J1HHN7-D1 & 9/27/11 & 0.10 & $U$ & 0.10 & 0.075 & $U$ & 0.08 & 0.097 & & 0.01 & 0.005 & $U$ & 0.01 & 2.58 & & 0.08 \\
\hline 600-207 SURFACE & $1: 2.5$ & J1HHN7-E1 & 9/27/11 & 0.02 & $\mathrm{U}$ & 0.02 & 0.002 & $\mathrm{~B}$ & 0.02 & 0.092 & & 0.00 & 0.001 & $U$ & 0.00 & 2.14 & & 0.02 \\
\hline 600-207 SURFACE & $1: 5$ & J1HHN7-F1 & 9/27/11 & 0.02 & $U$ & 0.02 & 0.015 & $\mathrm{U}$ & 0.02 & 0.079 & & 0.00 & 0.001 & U & 0.00 & 1.42 & & 0.02 \\
\hline 600-207 SURFACE & $1: 5$ & J1HHN7-F2 & 9/27/11 & 0.02 & $U$ & 0.02 & 0.002 & B & 0.02 & 0.079 & & 0.00 & 0.001 & $\mathrm{U}$ & 0.00 & 1.45 & & 0.02 \\
\hline 126-B-1 SURFACE & $1: 1$ & J1HHX8-A1 & 4/25/11 & 0.10 & $U$ & 0.10 & 0.015 & B & 0.08 & 0.068 & & 0.01 & 0.005 & $u$ & 0.01 & 1.86 & & 0.08 \\
\hline 126-B-1 SURFACE & $1: 2.5$ & J1HHX8-B1 & $4 / 25 / 11$ & 0.02 & $\mathrm{U}$ & 0.02 & 0.008 & B & 0.02 & 0.054 & & 0.00 & 0.001 & 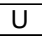 & 0.00 & 1.22 & & 0.02 \\
\hline 126-B-1 SURFACE & $1: 5$ & $\mathrm{~J} 1 \mathrm{HH} \times 8-\mathrm{C} 1$ & $4 / 25 / 11$ & 0.02 & $U$ & 0.02 & 0.008 & $B$ & 0.02 & 0.047 & & 0.00 & 0.001 & $\mathrm{U}$ & 0.00 & 0.766 & & 0.02 \\
\hline 126-B-1 SURFACE & $1: 5$ & J1HHX8-C2 & $4 / 25 / 11$ & 0.02 & $U$ & 0.02 & 0.007 & $B$ & 0.02 & 0.041 & & 0.00 & 0.001 & $\mathrm{U}$ & 0.00 & 0.85 & & 0.02 \\
\hline 126-B-1 SURFACE & $1: 1$ & J1HHX8-D1 & $4 / 25 / 11$ & 0.10 & $\mathrm{U}$ & 0.10 & 0.075 & $\mathrm{U}$ & 0.08 & 0.073 & & 0.01 & 0.005 & $U$ & 0.01 & 2.07 & & 0.08 \\
\hline 126-B-1 SURFACE & $1: 2.5$ & J1HHX8-E1 & $4 / 25 / 11$ & 0.02 & $\mathrm{U}$ & 0.02 & 0.007 & B & 0.02 & 0.057 & & 0.00 & 0.001 & $U$ & 0.00 & 1.3 & & 0.02 \\
\hline 126-B-1 SURFACE & $1: 2.5$ & J1HHX8-E2 & $4 / 25 / 11$ & 0.02 & $U$ & 0.02 & 0.006 & $B$ & 0.02 & 0.054 & & 0.00 & 0.001 & $U$ & 0.00 & 1.27 & & 0.02 \\
\hline 126-B-1 SURFACE & $1: 5$ & J1HHX8-F1 & 4/25/11 & 0.02 & $U$ & 0.02 & 0.006 & B & 0.02 & 0.042 & & 0.00 & 0.001 & $u$ & 0.00 & 0.826 & & 0.02 \\
\hline 126-B-1 DEPTH & $1: 1$ & J1HJ08-D1 & $4 / 26 / 11$ & 0.10 & $U$ & 0.10 & 0.075 & $U$ & 0.08 & 0.031 & & 0.01 & 0.005 & 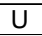 & 0.01 & 3.34 & & 0.08 \\
\hline 126-B-1 DEPTH & $1: 1$ & J1HJ08-D2 & $4 / 26 / 11$ & 0.10 & $\mathrm{U}$ & 0.10 & 0.075 & $\mathrm{U}$ & 0.08 & 0.032 & & 0.01 & 0.005 & $U$ & 0.01 & 3.11 & & 0.08 \\
\hline 126-B-1 DEPTH & $1: 2.5$ & J1HJ08-E1 & $4 / 26 / 11$ & 0.02 & $U$ & 0.02 & 0.002 & $\mathrm{~B}$ & 0.02 & 0.036 & & 0.00 & 0.001 & $U$ & 0.00 & 1.9 & & 0.02 \\
\hline 126-B-1 DEPTH & $1: 5$ & J1HJ08-F1 & $4 / 26 / 11$ & 0.02 & $U$ & 0.02 & 0.015 & $U$ & 0.02 & 0.037 & & 0.00 & 0.001 & $u$ & 0.00 & 1.19 & & 0.02 \\
\hline 126-H-1 DEPTH & $1: 1$ & J1HJ80-D1 & $8 / 24 / 11$ & 0.10 & $U$ & 0.10 & 0.075 & $U$ & 0.08 & 0.071 & & 0.01 & 0.005 & $u$ & 0.01 & 13.6 & & 0.08 \\
\hline 126-H-1 DEPTH & $1: 2.5$ & J1HJ80-E1 & $8 / 24 / 11$ & 0.02 & $U$ & 0.02 & 0.006 & $B$ & 0.02 & 0.072 & & 0.00 & 0.001 & $U$ & 0.00 & 7.2 & & 0.02 \\
\hline 126-H-1 DEPTH & $1: 2.5$ & J1HJ80-E2 & $8 / 24 / 11$ & 0.02 & $U$ & 0.02 & 0.006 & B & 0.02 & 0.075 & & 0.00 & 0.001 & 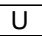 & 0.00 & 7.4 & & 0.02 \\
\hline 126-H-1 DEPTH & $1: 5$ & J1HJ80-F1 & $8 / 24 / 11$ & 0.02 & $\mathrm{U}$ & 0.02 & 0.005 & $B$ & 0.02 & 0.077 & & 0.00 & 0.001 & & 0.00 & 4.44 & & 0.02 \\
\hline
\end{tabular}

126-H-1 DEPTH 
Table C-17. Coal Ash Follow on Leaching Test Coal Ash Leaching Sample Results. (4 Pages)

\begin{tabular}{|c|c|c|c|c|c|c|c|c|c|c|c|c|c|c|c|c|c|c|}
\hline \multirow{2}{*}{ Sample Location } & \multirow{2}{*}{$\begin{array}{c}\text { Coal Ash:Water } \\
\text { Ratio }\end{array}$} & \multirow{2}{*}{$\begin{array}{c}\text { HEIS } \\
\text { Number }\end{array}$} & \multirow{2}{*}{$\begin{array}{c}\text { Sample } \\
\text { Date }\end{array}$} & \multicolumn{3}{|c|}{ Cadmium } & \multicolumn{3}{|c|}{ Chromium } & \multicolumn{3}{|c|}{ Cobalt } & \multicolumn{3}{|c|}{ Copper } & \multicolumn{3}{|c|}{ Lead } \\
\hline & & & & $\mathrm{mg} / \mathrm{L}$ & Q & PQL & $\mathrm{mg} / \mathrm{L}$ & $\mathbf{Q}$ & PQL & $\mathrm{mg} / \mathrm{L}$ & $\mathbf{Q}$ & $\mathrm{PQL}$ & $\mathrm{mg} / \mathrm{L}$ & $\mathbf{Q}$ & PQL & $\mathrm{mg} / \mathrm{L}$ & Q & PQL \\
\hline 600-207 SURFACE & $1: 1$ & J1HHN7-A1 & 9/27/11 & 0.0150 & $\bar{U}$ & 0.02 & 0.025 & $\bar{U}$ & 0.03 & 0.010 & $\bar{U}$ & 0.01 & 0.100 & $\bar{U}$ & 0.10 & 0.05 & $\bar{U}$ & 0.05 \\
\hline 600-207 SURFACE & $1: 2.5$ & J1HHN7-B1 & 9/27/11 & 0.0004 & $\mathrm{~B}$ & 0.00 & 0.005 & $U$ & 0.01 & 0.002 & $U$ & 0.00 & 0.020 & $U$ & 0.02 & 0.01 & $U$ & 0.01 \\
\hline 600-207 SURFACE & $1: 2.5$ & J1HHN7-B2 & 9/27/11 & 0.0002 & $\mathrm{~B}$ & 0.00 & 0.001 & $\mathrm{~B}$ & 0.01 & 0.002 & $U$ & 0.00 & 0.020 & $U$ & 0.02 & 0.01 & $U$ & 0.01 \\
\hline 600-207 SURFACE & $1: 5$ & J1HHN7-C1 & 9/27/11 & 0.0030 & $U$ & 0.00 & 0.001 & $\mathrm{~B}$ & 0.01 & 0.002 & $U$ & 0.00 & 0.020 & $U$ & 0.02 & 0.01 & $U$ & 0.01 \\
\hline 600-207 SURFACE & $1: 1$ & J1HHN7-D1 & 9/27/11 & 0.0150 & $\mathrm{U}$ & 0.02 & 0.025 & $U$ & 0.03 & 0.010 & $U$ & 0.01 & 0.100 & $\mathrm{U}$ & 0.10 & 0.05 & $\mathrm{U}$ & 0.05 \\
\hline 600-207 SURFACE & $1: 2.5$ & J1HHN7-E1 & 9/27/11 & 0.0004 & B & 0.00 & 0.005 & $U$ & 0.01 & 0.002 & $U$ & 0.00 & 0.020 & $U$ & 0.02 & 0.01 & $U$ & 0.01 \\
\hline 600-207 SURFACE & $1: 5$ & J1HHN7-F1 & 9/27/11 & 0.0003 & $\mathrm{~B}$ & 0.00 & 0.002 & $\mathrm{~B}$ & 0.01 & 0.002 & $U$ & 0.00 & 0.020 & $U$ & 0.02 & 0.01 & $U$ & 0.01 \\
\hline 600-207 SURFACE & $1: 5$ & J1HHN7-F2 & 9/27/11 & 0.0003 & $\mathrm{~B}$ & 0.00 & 0.005 & $U$ & 0.01 & 0.002 & $U$ & 0.00 & 0.020 & $\mathrm{U}$ & 0.02 & 0.01 & $\mathrm{U}$ & 0.01 \\
\hline 126-B-1 SURFACE & $1: 1$ & J1HHX8-A1 & $4 / 25 / 11$ & 0.0150 & $\mathrm{U}$ & 0.02 & 0.003 & B & 0.03 & 0.010 & $U$ & 0.01 & 0.100 & $U$ & 0.10 & 0.05 & $\mathrm{U}$ & 0.05 \\
\hline 126-B-1 SURFACE & $1: 2.5$ & J1HHX8-B1 & $4 / 25 / 11$ & 0.0030 & $\mathrm{U}$ & 0.00 & 0.001 & $\mathrm{~B}$ & 0.01 & 0.002 & $\mathrm{U}$ & 0.00 & 0.020 & $\mathrm{U}$ & 0.02 & 0.01 & $U$ & 0.01 \\
\hline 126-B-1 SURFACE & $1: 5$ & $\mathrm{J1HHX8-C1}$ & $4 / 25 / 11$ & 0.0030 & $\mathrm{U}$ & 0.00 & 0.001 & $\mathrm{~B}$ & 0.01 & 0.002 & 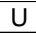 & 0.00 & 0.020 & $U$ & 0.02 & 0.01 & $\mathrm{U}$ & 0.01 \\
\hline 126-B-1 SURFACE & $1: 5$ & J1HHX8-C2 & $4 / 25 / 11$ & 0.0030 & $\mathrm{U}$ & 0.00 & 0.001 & $B$ & 0.01 & 0.002 & $U$ & 0.00 & 0.020 & $U$ & 0.02 & 0.01 & $U$ & 0.01 \\
\hline 126-B-1 SURFACE & $1: 1$ & J1HHX8-D1 & $4 / 25 / 11$ & 0.0150 & $U$ & 0.02 & 0.025 & $U$ & 0.03 & 0.010 & $U$ & 0.01 & 0.100 & $U$ & 0.10 & 0.05 & $U$ & 0.05 \\
\hline 126-B-1 SURFACE & $1: 2.5$ & J1HHX8-E1 & $4 / 25 / 11$ & 0.0030 & $\mathrm{U}$ & 0.00 & 0.001 & $\mathrm{~B}$ & 0.01 & 0.002 & $U$ & 0.00 & 0.002 & $B$ & 0.02 & 0.01 & $U$ & 0.01 \\
\hline 126-B-1 SURFACE & $1: 2.5$ & J1HHX8-E2 & $4 / 25 / 11$ & 0.0030 & $\mathrm{U}$ & 0.00 & 0.001 & $\mathrm{~B}$ & 0.01 & 0.002 & $U$ & 0.00 & 0.002 & $\mathrm{~B}$ & 0.02 & 0.01 & $\mathrm{U}$ & 0.01 \\
\hline 126-B-1 SURFACE & $1: 5$ & J1HHX8-F1 & $4 / 25 / 11$ & 0.003 & $\mathrm{U}$ & 0.00 & 0.001 & B & 0.01 & 0.002 & U & 0.00 & 0.020 & U & 0.02 & 0.01 & $\mathrm{U}$ & 0.01 \\
\hline 126-B-1 DEPTH & $1: 1$ & J1HJ08-D1 & 4/26/11 & 0.015 & $U$ & 0.02 & 0.025 & $U$ & 0.03 & 0.010 & $U$ & 0.01 & 0.100 & $U$ & 0.10 & 0.05 & $U$ & 0.05 \\
\hline 126-B-1 DEPTH & $1: 1$ & J1HJ08-D2 & $4 / 26 / 11$ & 0.0150 & $U$ & 0.02 & 0.025 & $\mathrm{U}$ & 0.03 & 0.010 & $U$ & 0.01 & 0.100 & $\mathrm{U}$ & 0.10 & 0.05 & $\mathrm{U}$ & 0.05 \\
\hline 126-B-1 DEPTH & $1: 2.5$ & J1HJ08-E1 & $4 / 26 / 11$ & 0.0002 & B & 0.00 & 0.005 & $U$ & 0.01 & 0.002 & $U$ & 0.00 & 0.020 & $U$ & 0.02 & 0.01 & $U$ & 0.01 \\
\hline 126-B-1 DEPTH & $1: 5$ & J1HJ08-F1 & $4 / 26 / 11$ & 0.0030 & $U$ & 0.00 & 0.005 & $U$ & 0.01 & 0.002 & $U$ & 0.00 & 0.020 & $\mathrm{U}$ & 0.02 & 0.01 & $U$ & 0.01 \\
\hline 126-H-1 DEPTH & $1: 1$ & J1HJ80-D1 & $8 / 24 / 11$ & 0.0150 & $U$ & 0.02 & 0.006 & $\mathrm{~B}$ & 0.03 & 0.010 & $U$ & 0.01 & 0.100 & $U$ & 0.10 & 0.05 & $\mathrm{U}$ & 0.05 \\
\hline 126-H-1 DEPTH & $1: 2.5$ & J1HJ80-E1 & $8 / 24 / 11$ & 0.0030 & $\mathrm{U}$ & 0.00 & 0.004 & $\mathrm{~B}$ & 0.01 & 0.002 & U & 0.00 & 0.020 & $\mathrm{U}$ & 0.02 & 0.01 & $\mathrm{U}$ & 0.01 \\
\hline 126-H-1 DEPTH & $1: 2.5$ & J1HJ80-E2 & $8 / 24 / 11$ & 0.003 & $\mathrm{U}$ & 0.00 & 0.004 & B & 0.01 & 0.002 & $U$ & 0.00 & 0.020 & $U$ & 0.02 & 0.01 & $U$ & 0.01 \\
\hline 126-H-1 DEPTH & $1: 5$ & J1HJ80-F1 & $8 / 24 / 11$ & 0.0030 & $U$ & 0.00 & 0.002 & B & 0.01 & 0.002 & $U$ & 0.00 & 0.020 & $U$ & 0.02 & 0.010 & $U$ & 0.01 \\
\hline
\end{tabular}

126-H-1 DEPTH 
Table C-17. Coal Ash Follow on Leaching Test Coal Ash Leaching Sample Results. (4 Pages)

\begin{tabular}{|c|c|c|c|c|c|c|c|c|c|c|c|c|c|c|c|c|c|c|}
\hline \multirow{2}{*}{ Sample Location } & \multirow{2}{*}{$\begin{array}{c}\text { Coal Ash:Water } \\
\text { Ratio }\end{array}$} & \multirow{2}{*}{$\begin{array}{c}\text { HEIS } \\
\text { Number }\end{array}$} & \multirow{2}{*}{$\begin{array}{c}\text { Sample } \\
\text { Date }\end{array}$} & \multicolumn{3}{|c|}{ Manganese } & \multicolumn{3}{|c|}{ Molybdenum } & \multicolumn{3}{|c|}{ Nickel } & \multicolumn{3}{|c|}{ Selenium } & \multicolumn{3}{|c|}{ Silver } \\
\hline & & & & $\mathrm{mg} / \mathrm{L}$ & $\mathbf{Q}$ & PQL & $\mathrm{mg} / \mathrm{L}$ & $\mathbf{Q}$ & PQL & $\mathrm{mg} / \mathrm{L}$ & $\mathbf{Q}$ & PQL & $\mathrm{mg} / \mathrm{L}$ & Q & PQL & $\mathrm{mg} / \mathrm{L}$ & Q & PQL \\
\hline 600-207 SURFACE & $1: 1$ & J1HHN7-A1 & 9/27/11 & 0.01 & $\mathrm{~B}$ & 0.03 & 0.0130 & $\mathrm{~B}$ & 0.02 & 0.1 & $U$ & 0.10 & 0.018 & $\mathrm{~B}$ & 0.10 & 0.030 & $U$ & 0.03 \\
\hline 600-207 SURFACE & $1: 2.5$ & J1HHN7-B1 & 9/27/11 & 0.006 & & 0.01 & 0.0080 & & 0.00 & 0.02 & $U$ & 0.02 & 0.008 & B & 0.02 & 0.006 & $U$ & 0.01 \\
\hline 600-207 SURFACE & $1: 2.5$ & J1HHN7-B2 & 9/27/11 & 0.006 & & 0.01 & 0.0080 & & 0.00 & 0.02 & $U$ & 0.02 & 0.007 & $\mathrm{~B}$ & 0.02 & 0.006 & $U$ & 0.01 \\
\hline 600-207 SURFACE & $1: 5$ & J1HHN7-C1 & 9/27/11 & 0.036 & & 0.01 & 0.0080 & & 0.00 & 0.02 & $U$ & 0.02 & 0.006 & B & 0.02 & 0.006 & $U$ & 0.01 \\
\hline 600-207 SURFACE & $1: 1$ & J1HHN7-D1 & 9/27/11 & 0.003 & B & 0.03 & 0.0150 & & 0.02 & 0.1 & $U$ & 0.10 & 0.100 & $U$ & 0.10 & 0.030 & $U$ & 0.03 \\
\hline 600-207 SURFACE & $1: 2.5$ & J1HHN7-E1 & 9/27/11 & 0.006 & & 0.01 & 0.0100 & & 0.00 & 0.02 & 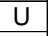 & 0.02 & 0.010 & $\mathrm{~B}$ & 0.02 & 0.006 & $\mathrm{U}$ & 0.01 \\
\hline 600-207 SURFACE & $1: 5$ & J1HHN7-F1 & 9/27/11 & 0.003 & B & 0.01 & 0.0070 & & 0.00 & 0.001 & $\mathrm{~B}$ & 0.02 & 0.007 & $\mathrm{~B}$ & 0.02 & 0.006 & $U$ & 0.01 \\
\hline 600-207 SURFACE & $1: 5$ & J1HHN7-F2 & 9/27/11 & 0.003 & B & 0.01 & 0.0070 & & 0.00 & 0.02 & $U$ & 0.02 & 0.005 & $\mathrm{~B}$ & 0.02 & 0.006 & $\mathrm{U}$ & 0.01 \\
\hline 126-B-1 SURFACE & $1: 1$ & J1HHX8-A1 & $4 / 25 / 11$ & 0.005 & B & 0.03 & 0.0100 & B & 0.02 & 0.1 & $\mathrm{U}$ & 0.10 & 0.015 & B & 0.10 & 0.030 & $U$ & 0.03 \\
\hline 126-B-1 SURFACE & $1: 2.5$ & $\mathrm{~J} 1 \mathrm{HHX} 8-\mathrm{B} 1$ & $4 / 25 / 11$ & 0.001 & B & 0.01 & 0.0040 & & 0.00 & 0.001 & $\mathrm{~B}$ & 0.02 & 0.007 & $\mathrm{~B}$ & 0.02 & 0.006 & $\mathrm{U}$ & 0.01 \\
\hline 126-B-1 SURFACE & $1: 5$ & $\mathrm{~J} 1 \mathrm{HHX} 8-\mathrm{C} 1$ & $4 / 25 / 11$ & 0.001 & B & 0.01 & 0.0030 & & 0.00 & 0.001 & $B$ & 0.02 & 0.005 & $B$ & 0.02 & 0.006 & $U$ & 0.01 \\
\hline 126-B-1 SURFACE & $1: 5$ & $\mathrm{~J} 1 \mathrm{HHX} 8-\mathrm{C} 2$ & $4 / 25 / 11$ & 0.001 & $\mathrm{~B}$ & 0.01 & 0.0020 & B & 0.00 & 0.001 & B & 0.02 & 0.007 & B & 0.02 & 0.006 & $U$ & 0.01 \\
\hline 126-B-1 SURFACE & $1: 1$ & J1HHX8-D1 & $4 / 25 / 11$ & 0.025 & $U$ & 0.03 & 0.0080 & $\mathrm{~B}$ & 0.02 & 0.004 & $\mathrm{~B}$ & 0.10 & 0.100 & $\mathrm{U}$ & 0.10 & 0.030 & $U$ & 0.03 \\
\hline 126-B-1 SURFACE & $1: 2.5$ & J1HHX8-E1 & 4/25/11 & 0.001 & B & 0.01 & 0.0040 & & 0.00 & 0.001 & B & 0.02 & 0.007 & $\mathrm{~B}$ & 0.02 & 0.006 & $U$ & 0.01 \\
\hline 126-B-1 SURFACE & $1: 2.5$ & J1HHX8-E2 & $4 / 25 / 11$ & 0.001 & B & 0.01 & 0.0040 & & 0.00 & 0.001 & $B$ & 0.02 & 0.007 & $B$ & 0.02 & 0.006 & $U$ & 0.01 \\
\hline 126-B-1 SURFACE & $1: 5$ & J1HHX8-F1 & 4/25/11 & 0.001 & B & 0.01 & 0.0020 & B & 0.00 & 0.001 & B & 0.02 & 0.007 & $\mathrm{~B}$ & 0.02 & 0.006 & $U$ & 0.01 \\
\hline 126-B-1 DEPTH & $1: 1$ & J1HJ08-D1 & $4 / 26 / 11$ & 0.149 & & 0.03 & 0.0070 & B & 0.02 & 0.004 & B & 0.10 & 0.100 & $\mathrm{U}$ & 0.10 & 0.030 & U & 0.03 \\
\hline 126-B-1 DEPTH & $1: 1$ & J1HJ08-D2 & 4/26/11 & 0.108 & & 0.03 & 0.0070 & B & 0.02 & 0.1 & $U$ & 0.10 & 0.100 & $U$ & 0.10 & 0.030 & $U$ & 0.03 \\
\hline 126-B-1 DEPTH & $1: 2.5$ & J1HJ08-E1 & 4/26/11 & 0.056 & & 0.01 & 0.0060 & & 0.01 & 0.02 & 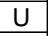 & 0.02 & 0.005 & $\mathrm{~B}$ & 0.02 & 0.006 & $U$ & 0.01 \\
\hline 126-B-1 DEPTH & $1: 5$ & J1HJ08-F1 & $4 / 26 / 11$ & 0.031 & & 0.01 & 0.0060 & & 0.00 & 0.02 & $\mathrm{U}$ & 0.02 & 0.004 & B & 0.02 & 0.006 & U & 0.01 \\
\hline 126-H-1 DEPTH & $1: 1$ & J1HJ80-D1 & $8 / 24 / 11$ & 0.025 & $\mathrm{U}$ & 0.03 & 0.0210 & & 0.02 & 0.1 & $\mathrm{U}$ & 0.10 & 0.1 & $U$ & 0.10 & 0.030 & $U$ & 0.03 \\
\hline 126-H-1 DEPTH & $1: 2.5$ & J1HJ80-E1 & $8 / 24 / 11$ & 0.005 & $\mathrm{U}$ & 0.01 & 0.0090 & & 0.00 & 0.02 & $U$ & 0.02 & 0.005 & $\mathrm{~B}$ & 0.02 & 0.006 & $U$ & 0.01 \\
\hline 126-H-1 DEPTH & $1: 2.5$ & J1HJ80-E2 & $8 / 24 / 11$ & 0.001 & $\mathrm{~B}$ & 0.01 & 0.0100 & & 0.00 & 0.02 & 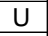 & 0.02 & 0.006 & $\mathrm{~B}$ & 0.02 & 0.006 & $U$ & 0.01 \\
\hline 126-H-1 DEPTH & $1: 5$ & J1HJ80-F1 & $8 / 24 / 11$ & 0.005 & $\mathrm{U}$ & 0.01 & 0.0080 & & 0.00 & 0.02 & $\mathrm{U}$ & 0.02 & 0.005 & $\mathrm{~B}$ & 0.02 & 0.006 & $U$ & 0.01 \\
\hline
\end{tabular}


Table C-17. Coal Ash Follow on Leaching Test Coal Ash Leaching Sample Results. (4 Pages)

\begin{tabular}{|c|c|c|c|c|c|c|c|c|c|c|c|c|c|}
\hline \multirow{2}{*}{ Sample Location } & \multirow{2}{*}{$\begin{array}{c}\text { Coal Ash:Water } \\
\text { Ratio }\end{array}$} & \multirow{2}{*}{$\begin{array}{c}\text { HEIS } \\
\text { Number }\end{array}$} & \multirow{2}{*}{$\begin{array}{l}\text { Sample } \\
\text { Date }\end{array}$} & \multicolumn{3}{|c|}{$\begin{array}{c}\text { Thallium } \\
\end{array}$} & \multicolumn{3}{|c|}{ Vanadium } & \multicolumn{3}{|c|}{ Zinc } & \multirow[b]{2}{*}{ pH } \\
\hline & & & & $\mathrm{mg} / \mathrm{L}$ & $\mathbf{Q}$ & $\overline{P Q L}$ & $\mathrm{mg} / \mathrm{L}$ & $\mathbf{Q}$ & $\begin{array}{l}\text { PQL } \\
\end{array}$ & $\mathrm{mg} / \mathrm{L}$ & $\mathbf{Q}$ & PQL & \\
\hline 600-207 SURFACE & $1: 1$ & J1HHN7-A1 & $9 / 27 / 11$ & 0.075 & $\mathrm{U}$ & 0.08 & 0.063 & & 0.03 & 0.25 & $\mathrm{U}$ & 0.3 & 7.60 \\
\hline 600-207 SURFACE & $1: 2.5$ & J1HHN7-B1 & $9 / 27 / 11$ & 0.015 & $\mathrm{U}$ & 0.02 & 0.037 & & 0.01 & 0.05 & $\mathrm{U}$ & 0.1 & 7.68 \\
\hline 600-207 SURFACE & $1: 2.5$ & J1HHN7-B2 & $9 / 27 / 11$ & 0.015 & $U$ & 0.02 & 0.039 & & 0.01 & 0.05 & $\mathrm{U}$ & 0.1 & 7.70 \\
\hline 600-207 SURFACE & $1: 5$ & J1HHN7-C1 & $9 / 27 / 11$ & 0.015 & $\mathrm{U}$ & 0.02 & 0.035 & & 0.01 & 0.05 & $\mathrm{U}$ & 0.1 & 7.62 \\
\hline 600-207 SURFACE & $1: 1$ & J1HHN7-D1 & $9 / 27 / 11$ & 0.075 & $\mathrm{U}$ & 0.08 & 0.063 & & 0.03 & 0.25 & $U$ & 0.3 & 7.80 \\
\hline 600-207 SURFACE & $1: 2.5$ & J1HHN7-E1 & $9 / 27 / 11$ & 0.015 & $\mathrm{U}$ & 0.02 & 0.037 & & 0.01 & 0.05 & $\mathrm{U}$ & 0.1 & 7.82 \\
\hline 600-207 SURFACE & $1: 5$ & J1HHN7-F1 & $9 / 27 / 11$ & 0.015 & $\mathrm{U}$ & 0.02 & 0.032 & & 0.01 & 0.05 & $\mathrm{U}$ & 0.1 & 7.79 \\
\hline 600-207 SURFACE & $1: 5$ & J1HHN7-F2 & $9 / 27 / 11$ & 0.015 & $U$ & 0.02 & 0.032 & & 0.01 & 0.05 & $\mathrm{U}$ & 0.1 & 7.89 \\
\hline 126-B-1 SURFACE & $1: 1$ & J1HHX8-A1 & $4 / 25 / 11$ & 0.075 & $\mathrm{U}$ & 0.08 & 0.03 & & 0.03 & 0.25 & $U$ & 0.3 & 8.50 \\
\hline 126-B-1 SURFACE & $1: 2.5$ & $\mathrm{~J} 1 \mathrm{HH} \times 8-\mathrm{B} 1$ & $4 / 25 / 11$ & 0.015 & $\mathrm{U}$ & 0.02 & 0.028 & & 0.01 & 0.05 & $\mathrm{U}$ & 0.1 & 8.71 \\
\hline 126-B-1 SURFACE & $1: 5$ & $\mathrm{~J} 1 \mathrm{HH} \times 8-\mathrm{C} 1$ & $4 / 25 / 11$ & 0.015 & $U$ & 0.02 & 0.027 & & 0.01 & 0.05 & $U$ & 0.1 & 8.72 \\
\hline 126-B-1 SURFACE & $1: 5$ & $\mathrm{~J} 1 \mathrm{HH} \times 8-\mathrm{C} 2$ & $4 / 25 / 11$ & 0.015 & $\mathrm{U}$ & 0.02 & 0.029 & & 0.01 & 0.013 & $\mathrm{~B}$ & 0.1 & 8.71 \\
\hline 126-B-1 SURFACE & $1: 1$ & J1HHX8-D1 & $4 / 25 / 11$ & 0.075 & $U$ & 0.08 & 0.03 & & 0.03 & 0.25 & U & 0.3 & 8.50 \\
\hline 126-B-1 SURFACE & $1: 2.5$ & J1HHX8-E1 & $4 / 25 / 11$ & 0.015 & $\mathrm{U}$ & 0.02 & 0.027 & & 0.01 & 0.05 & $\mathrm{U}$ & 0.1 & 8.60 \\
\hline 126-B-1 SURFACE & $1: 2.5$ & J1HHX8-E2 & $4 / 25 / 11$ & 0.015 & $\mathrm{U}$ & 0.02 & 0.027 & & 0.01 & 0.05 & $U$ & 0.1 & 8.64 \\
\hline 126-B-1 SURFACE & $1: 5$ & J1HHX8-F1 & $4 / 25 / 11$ & 0.015 & $\mathrm{U}$ & 0.02 & 0.025 & & 0.01 & 0.05 & $\mathrm{U}$ & 0.1 & 8.71 \\
\hline 126-B-1 DEPTH & $1: 1$ & $\mathrm{~J} 1 \mathrm{HJ} 08-\mathrm{D} 1$ & $4 / 26 / 11$ & 0.075 & $U$ & 0.08 & 0.044 & & 0.03 & 0.25 & U & 0.3 & 6.71 \\
\hline 126-B-1 DEPTH & $1: 1$ & J1HJ08-D2 & $4 / 26 / 11$ & 0.075 & $\mathrm{U}$ & 0.08 & 0.036 & & 0.03 & 0.25 & $\mathrm{U}$ & 0.3 & 6.79 \\
\hline 126-B-1 DEPTH & $1: 2.5$ & J1HJ08-E1 & $4 / 26 / 11$ & 0.015 & $U$ & 0.02 & 0.019 & & 0.01 & 0.05 & U & 0.1 & 6.88 \\
\hline 126-B-1 DEPTH & $1: 5$ & J1HJ08-F1 & $4 / 26 / 11$ & 0.015 & $\mathrm{U}$ & 0.02 & 0.015 & & 0.01 & 0.05 & $\mathrm{U}$ & 0.1 & 6.93 \\
\hline 126-H-1 DEPTH & $1: 1$ & J1HJ80-D1 & $8 / 24 / 11$ & 0.075 & $U$ & 0.08 & 0.053 & & 0.03 & 0.25 & $\mathrm{U}$ & 0.3 & 9.36 \\
\hline 126-H-1 DEPTH & $1: 2.5$ & J1HJ80-E1 & $8 / 24 / 11$ & 0.015 & $\mathrm{U}$ & 0.02 & 0.037 & & 0.01 & 0.05 & $\mathrm{U}$ & 0.1 & 9.51 \\
\hline 126-H-1 DEPTH & $1: 2.5$ & J1HJ80-E2 & $8 / 24 / 11$ & 0.015 & $U$ & 0.02 & 0.039 & & 0.01 & 0.05 & $U$ & 0.1 & 9.59 \\
\hline 126-H-1 DEPTH & $1: 5$ & J1HJ80-F1 & $8 / 24 / 11$ & 0.015 & $\mathrm{U}$ & 0.02 & 0.03 & & 0.01 & 0.05 & $\mathrm{U}$ & 0.1 & 9.71 \\
\hline
\end{tabular}


WCH-506

Rev. 0 


\section{APPENDIX D}

\section{COAL ASH CALCULATIONS}


WCH-506

Rev. 0 


\section{APPENDIX D}

\section{CALCULATION BRIEFS}

The calculations in this appendix are kept in the active Washington Closure Hanford project files and are available upon request. When the project is completed, the file will be stored in a U.S. Department of Energy, Richland Operations Office, repository. These calculations have been prepared in accordance with ENG-1, Engineering Services, ENG-1-4.5, "Project Calculation," Washington Closure Hanford, Richland, Washington. The following calculations are provided in this appendix.

Coal Ash Relative Percent Difference (RPD) Calculations, 0100X-CA-V0070, Rev. 0, Washington Closure Hanford, Richland, Washington.

Coal Ash Characterization 90 $0^{\text {th }}$ Percentile and Median Equality Calculations, 0100X-CA-V0073, Rev. 0, Washington Closure Hanford, Richland, Washington.

Coal Ash Depth and Corresponding Surface Sample Confidence Limit Calculations, 0100X-CA-V0075, Rev. 0, Washington Closure Hanford, Richland, Washington. 
WCH-506

Rev. 0 


\section{CALCULATION COVER SHEET}

Project Title: 100 and 300 Areas Coal Ash Characterization

Job No. 14655

Area: $100-B, 100-D, 100-H, 100-[U-6$, and 300 Areas

Discipline: Environmental

${ }^{*}$ Calculation No: 0100X-CA-V0070

Subject: Coal Ash Relative Percent Difference (RPD) Calculations

Computer Program: Excel

Program No: Excel 2003

The attached calculations have been generated for a specific purpose and task. Use of the calculations by persons who do not have access to all pertinent facts may lead to incorrect conclusions and/or results. Before applying these calculations to your work, the underlying basis, rationale, and other pertinent information relevant to these calculations must be thoroughly reviewed with appropriate Washington Closure Hanford LLC $(W C H)$ officials or other authorized personnel. WCH is not responsible for the use of a calculation not under its direct control.

Committed Calculation $\bigotimes \quad$ Preliminary $\square \quad$ Superseded $\square \quad$ Voided $\square$

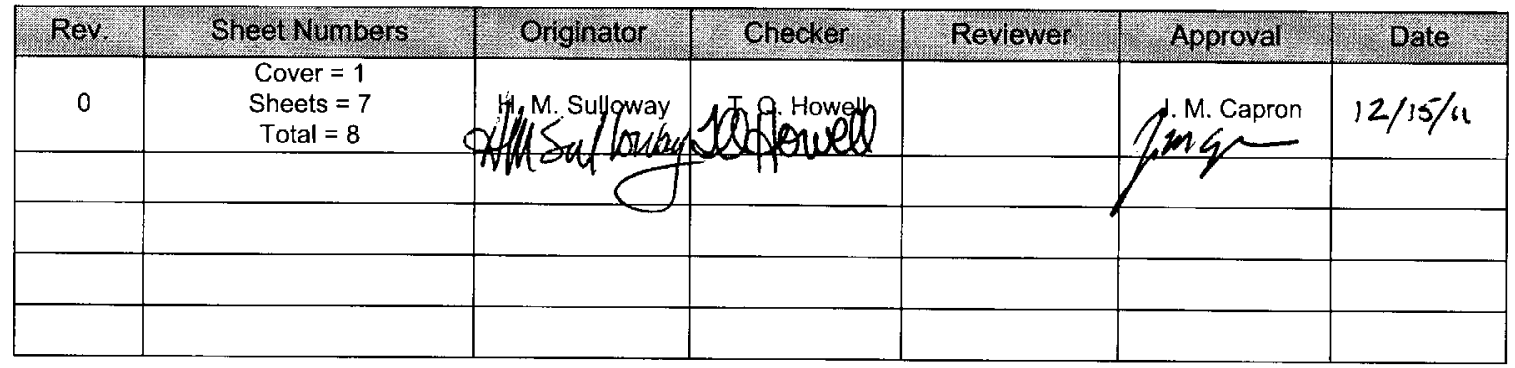

SUMMARY OF REVISION

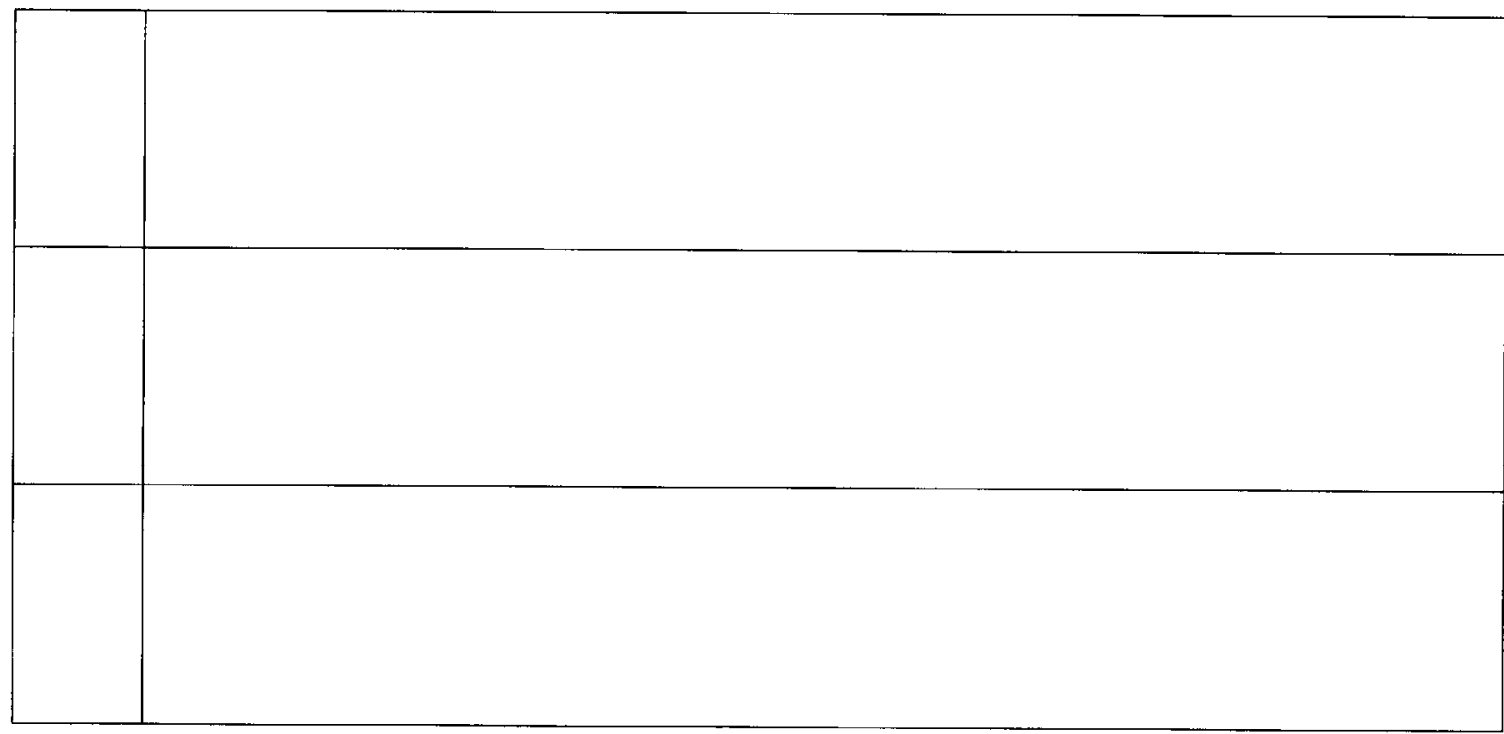




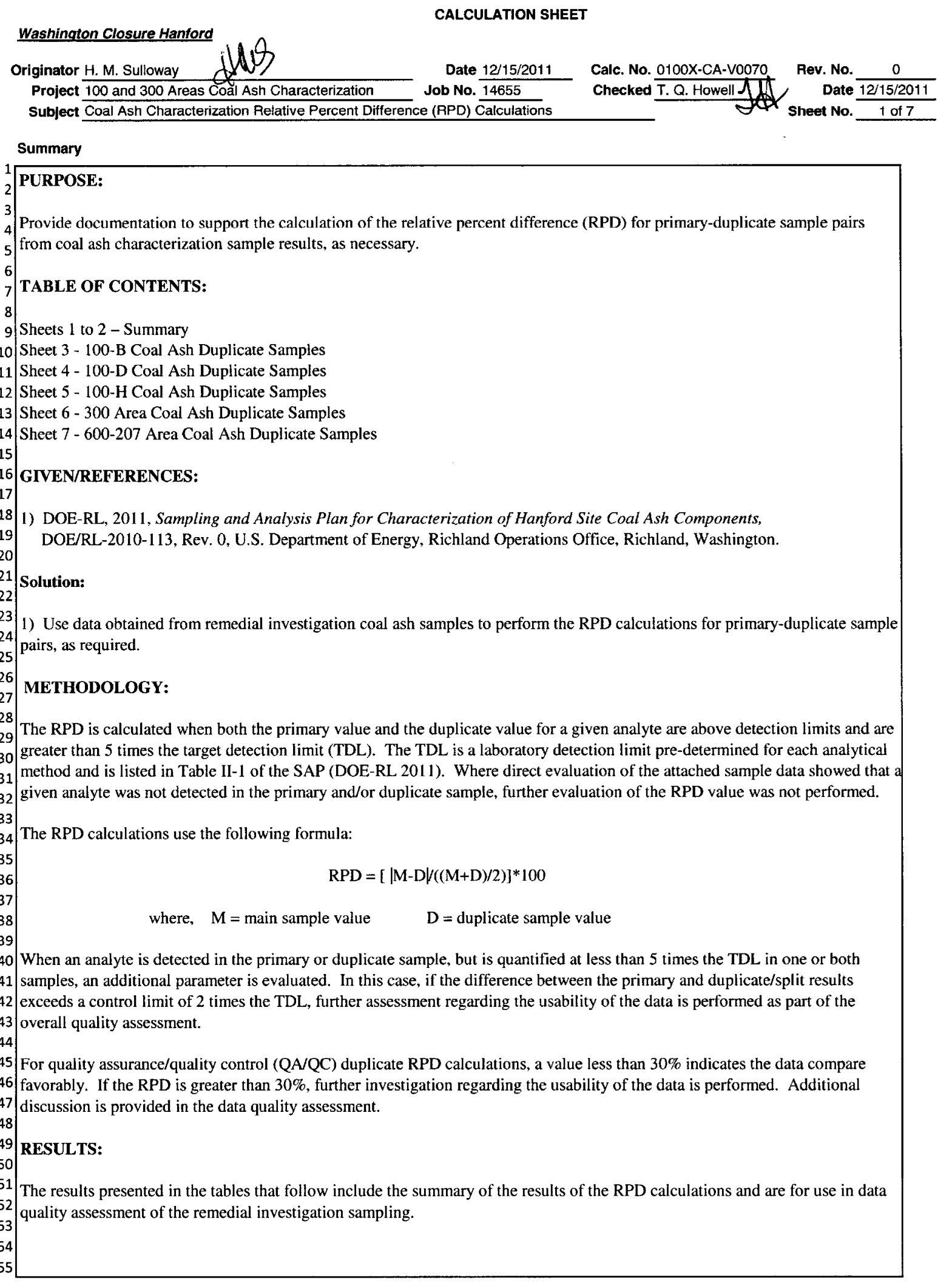


Washington Closure Hanford

Originator H.M. Sulloway

Project 100 and 300 Areas Coal Ash Characterization

Subject Coal Ash Characterization Relative Percent Difference (RPD) Calculation
CALCULATION SHEET

Date 12/15/2011 Calc. No. 0100X-CA-V0070 Job No.

$$
14655
$$

Calc. No. 0100X-CA-V0070 Checked T. Q. Howell A

Rev. No.

Date 12/15/2011 Sheet No.

2 of 7

\section{Summary (continued)}

3 Relative Percent Difference Results and QAVQC Analysis

\section{Sample Location}

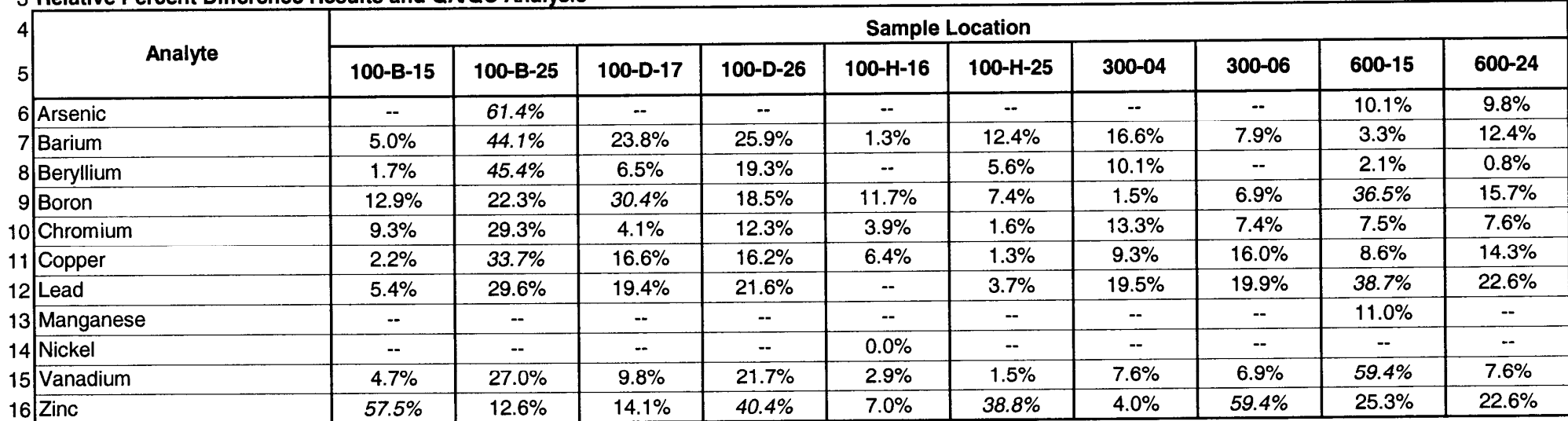

16 Zinc

$57.5 \%$

18 -- = RPD analysis not required 
WCH-506

Rev. 0 
Washington Closure Hanford ypus

dx

Subject Coal Ash Characterization Relative Percention Difference (RPD) Calculations
Duplicate Analysis at Sample Location 100-B-15

Date $12 / 15 / 2011$
Job No. Calc. No. $0100 X-C A-V 0070$
Checked T. Q. Howell AN

$\begin{aligned} \text { Rev. No. } & \frac{0}{\text { Date }} \\ \text { Sheet No. } & \frac{1215 / 2011}{3 \text { of } 7}\end{aligned}$

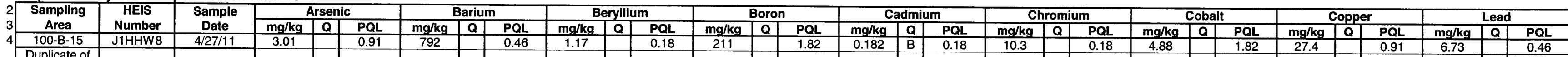

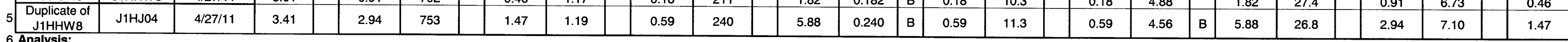

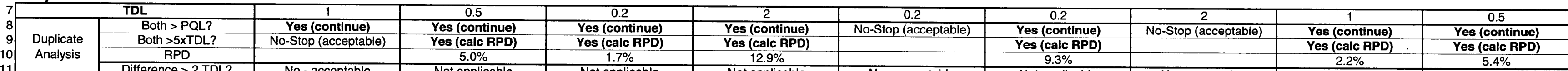

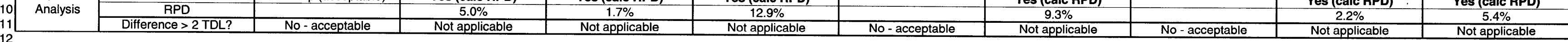

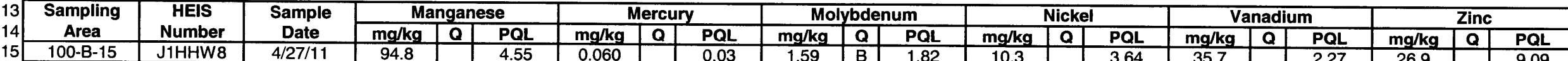

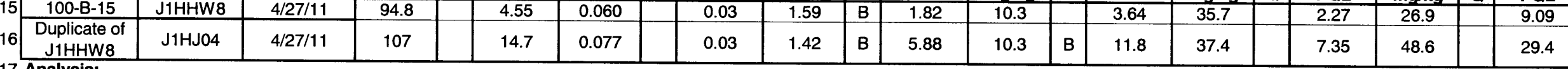

\begin{tabular}{|c|c|c|c|c|c|c|c|}
\hline \multicolumn{5}{|c|}{ TDL } & & & \\
\hline & $\begin{array}{l}\text { Both > PQL? } \\
\text { Both >5xTDL? }\end{array}$ & $\frac{\text { Yes (continue) }}{\text { No-Stop (acceptable) }}$ & $\begin{array}{c}\text { Yes (continue) } \\
\text { No-Stop (acceotable) }\end{array}$ & No-Stop (acceptable) & No-Stop (acceptable) & Yes (continue) & Yes (continue) \\
\hline Analysis & RPD & (t) & (1) & & & $\begin{array}{c}4.7 \% \\
\end{array}$ & $\begin{array}{l}\text { Yes (calc RPD) } \\
57.5 \%\end{array}$ \\
\hline
\end{tabular}

23 Duplicate Analysis at Sample Location 100-B-25

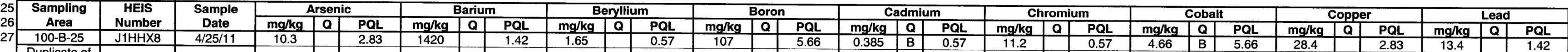

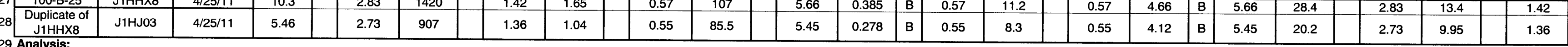

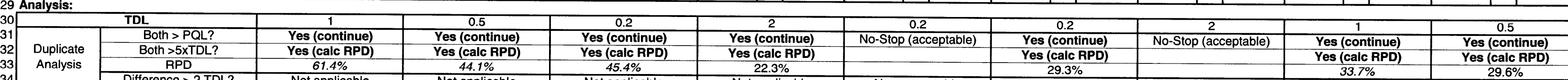

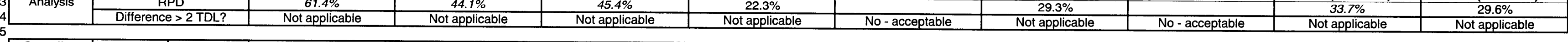

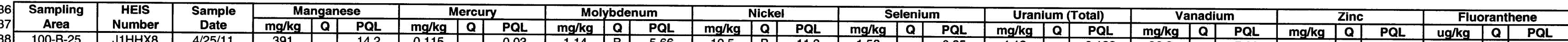

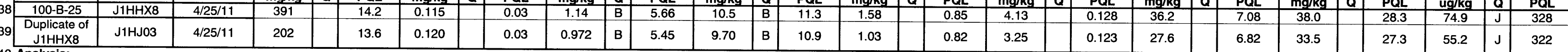

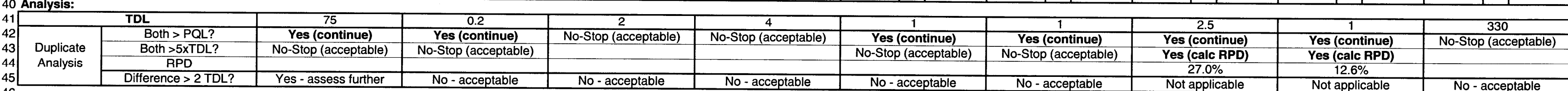

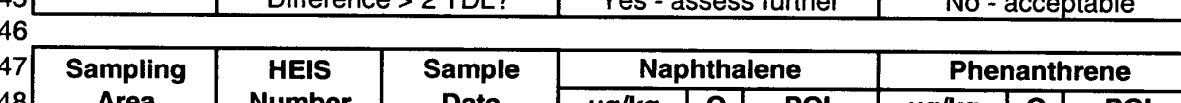

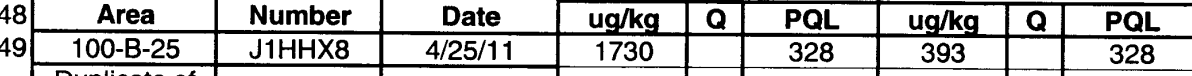

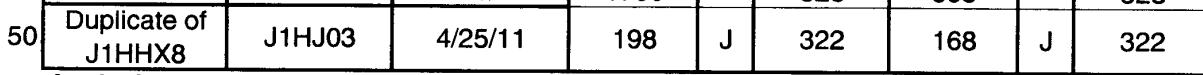

\begin{tabular}{|l|c|c|c|}
51 & & & \\
\hline 50 & 330 & 330 \\
\hline
\end{tabular}

\begin{tabular}{l|l|c|c|c|}
\hline 54 & & & \\
54 & Duplicate & Both $>$ PQL? & No-Stop (acceptable) & No-Stop (acceptable) \\
\cline { 2 - 4 } 5 & Bnalysis & Both $>$ SxTDL? & & \\
\cline { 3 - 5 } & SPD & &
\end{tabular}




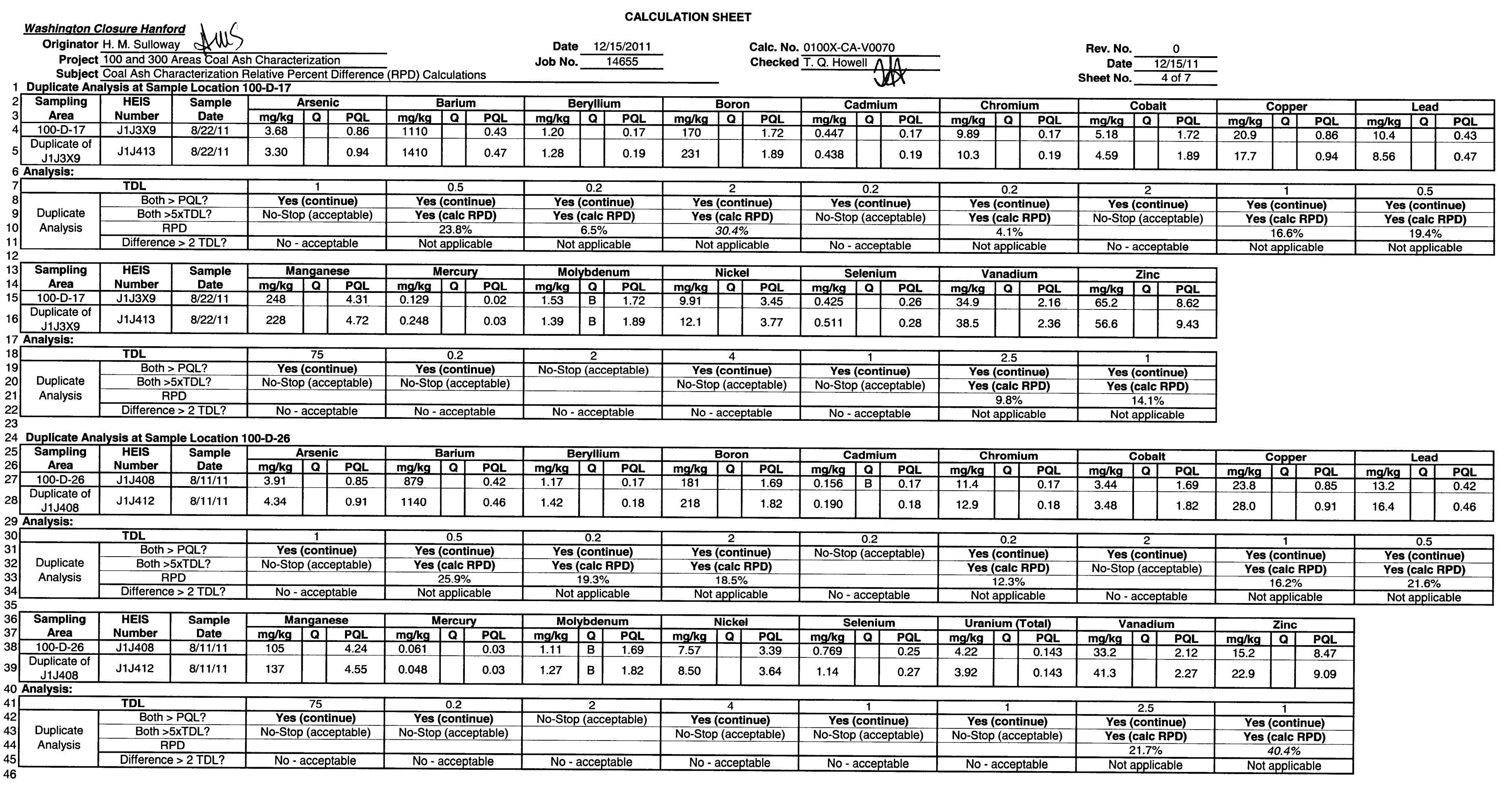


$\frac{\text { Washington Closure Hanford }}{\text { Originator H. M. Sulloway }}$

UNB

Project 100 and 300 Areas Coal Ash Chara

Duplicate Analysis at Sample Location Relative $100-\mathrm{H}-1$

Job No. $\frac{1215 / 2011}{14655}$

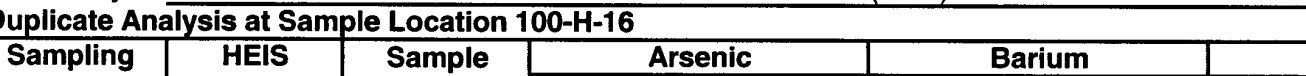

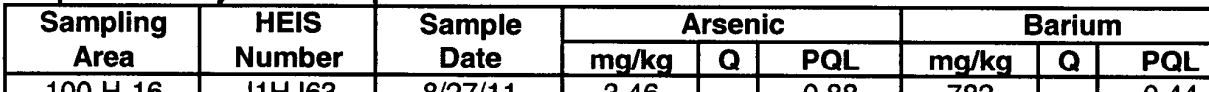
\begin{tabular}{|c|c|c|c|}
\hline $\begin{array}{c}\text { Duplicate of } \\
\mathrm{J} 1 \mathrm{HJ} 63\end{array}$ & $\mathrm{~J} 1 \mathrm{HJ} 78$ & $8 / 27 / 11$ & 3.01 \\
\hline
\end{tabular} 6 Analysis:

\begin{tabular}{|c|c|c|c|c|c|c|c|c|c|c|}
\hline \multirow{4}{*}{\begin{tabular}{|l|} 
Duplicate \\
Analysis
\end{tabular}} & & & \multirow{2}{*}{$\begin{array}{c}0.5 \\
\text { Yes (continue) } \\
\text { Yes (calc RPD) }\end{array}$} & & \multirow{2}{*}{$\frac{2}{2}$} & & \multirow{2}{*}{$\begin{array}{c}0.2 \\
\text { Yes (continue) }\end{array}$} & & \multirow{2}{*}{$\frac{1}{\text { Yes (continue) }}$} & \\
\hline & & & & & & & & & & \\
\hline & \multirow{2}{*}{$\begin{array}{c}\text { Both > PQL? } \\
\text { Both >5xTDL? } \\
\text { RPD } \\
\text { Difference }>2 \text { TDL? }\end{array}$} & $\begin{array}{c}\text { Yes (continue) } \\
\text { No-Stop (acceptable) }\end{array}$ & \multirow{2}{*}{$\begin{array}{l}\text { Yes (calc RPD) } \\
1.3 \% \\
\text { Not apolicable }\end{array}$} & \multirow{2}{*}{$\begin{array}{l}\text { Yes (continue) } \\
\text { No-Stop (acceptable) }\end{array}$} & \multirow{2}{*}{$\begin{array}{l}\text { Yes (calc RPD) } \\
\frac{11.7 \%}{\text { Not applicable }}\end{array}$} & \multirow{2}{*}{ No-Stop (acceptable) } & \multirow{2}{*}{$\begin{array}{c}\text { Yes (calc RPD) } \\
\frac{3.9 \%}{\text { Not applicable }}\end{array}$} & \multirow{2}{*}{$\begin{array}{c}\text { Yes continue) } \\
\text { No-Stop (acceptable) } \\
\text { No - acceptable }\end{array}$} & \multirow{2}{*}{$\begin{array}{c}\text { Yes (calc RPD) } \\
6.4 \% \\
\end{array}$} & $\begin{array}{c}\text { Yes (continue) } \\
\text { No-Stop (acceptable } \\
\end{array}$ \\
\hline & & No - acceptable & & & & & & & & No - acceptable \\
\hline
\end{tabular}

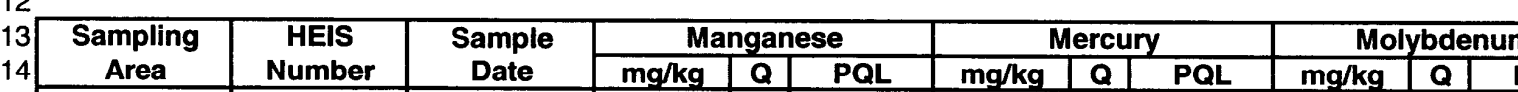

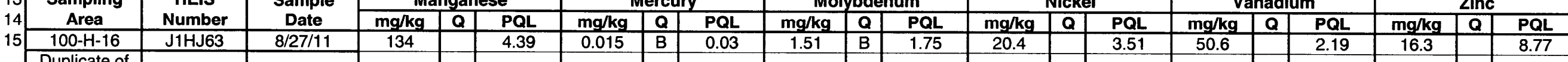

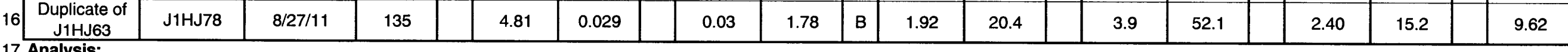

17 Analysis:

\begin{tabular}{|c|c|c|c|c|c|c|c|}
\hline \multirow{3}{*}{$\begin{array}{l}\text { Duplicate } \\
\text { Analysis }\end{array}$} & TDL & 75 & 0.2 & & 4 & 2.5 & \\
\hline & $\begin{array}{l}\text { Both > PQL? } \\
\text { Both > 5xTDL? }\end{array}$ & $\begin{array}{c}\text { Yes (continue) } \\
\text { No-Ston (acceotable) }\end{array}$ & No-Stop (acceptable) & No-Stop (acceptable) & Yes (continue) & Yes (continue) & Yes (continue) \\
\hline & & & & & $0.0 \%$ & $2.9 \%$ & $70 \%$ \\
\hline
\end{tabular}

22

24 Duplicate Analysis at Sample Location 100-H-25

25 Sampling Analysis at Sample Location 100-H-25

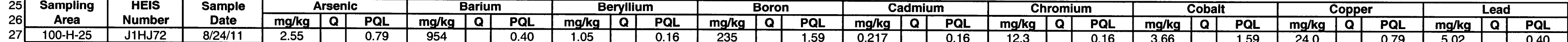

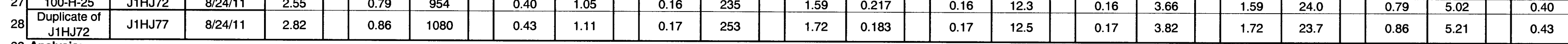

\begin{tabular}{|c|c|c|c|c|c|c|c|c|c|c|}
\hline \multirow{3}{*}{$\begin{array}{l}\text { Duplicate } \\
\text { Analysis }\end{array}$} & TDL & 1 & 0.5 & 0.2 & 2 & 0.2 & 0.2 & 2 & 1 & \\
\hline & $\begin{array}{c}\text { Both > PQL? } \\
\text { Both > 5xTDL? }\end{array}$ & $\begin{array}{c}\text { Yes (continue) } \\
\text { No-Stop (acceptable) }\end{array}$ & $\begin{array}{l}\text { Yes (continue) } \\
\text { Yes (calc RPD) }\end{array}$ & Yes (continue) & Yes (continue) & Yes (continue) & Yes (continue) & Yes (continue) & Yes (continue) & Yes (continue) \\
\hline & $\begin{array}{c}\text { Bon >xSIDL? } \\
\text { RPD }\end{array}$ & No-Stop (acceptable) & $\begin{array}{c}\text { Yes ( (alc RPD) } \\
12.4 \%\end{array}$ & $\begin{array}{c}\text { Yes (calc RPD) } \\
5.6 \%\end{array}$ & $\begin{array}{l}\text { Yes (calc RPD) } \\
7.4 \%\end{array}$ & No-Stop (acceptable) & $\begin{array}{c}\text { Yes (calc RPD) } \\
1.6 \% \\
\end{array}$ & No-Stop (acceptable) & $\begin{array}{c}\text { Yes (calc RPD) } \\
1.3 \%\end{array}$ & $\begin{aligned} \text { Yes } \\
3.7 \% \\
\end{aligned}$ \\
\hline
\end{tabular}

Sampling 1 HEIS

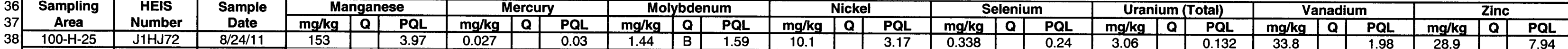

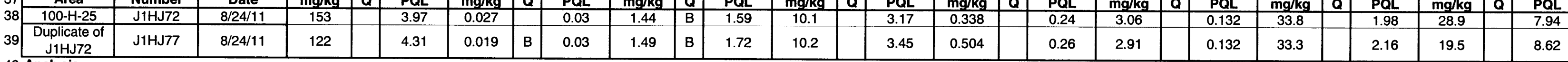

Analysis:

\begin{tabular}{l|l}
42 & Duplicat \\
43 & Analysis \\
44 &
\end{tabular}

\begin{tabular}{|c|c|c|}
\hline TDL & 75 & \\
\hline Both $>$ PQL? & Yes (continue) & No-S1 \\
\hline Both $>5 \times T D L ?$ & No-Stop (acceptable) & \\
\hline RPD & \\
\hline
\end{tabular}

\begin{tabular}{c|c|c|c|c|}
0.2 & 2 & \\
\hline o-Stop (acceptable) & No-Stop (acceptable) & (1) & \\
\hline
\end{tabular}

\begin{tabular}{c|c|c|c|}
\hline Yes (continue) & Yes (continue) & Yes (continue) & \\
\hline No-Stop (acceptable) & No-Stop (acceptable) & No-Stop (acceptable) & $Y$ \\
\hline
\end{tabular}

\begin{tabular}{l|l|l|l|l|l}
2.5 & 1 \\
\hline
\end{tabular}

\begin{tabular}{|c|c|}
\hline Yes (continue) & Yes (continue) \\
\hline Yes (calc RPD) & Yes (calc RPD) \\
\hline
\end{tabular}

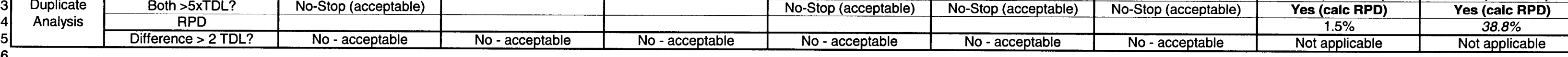




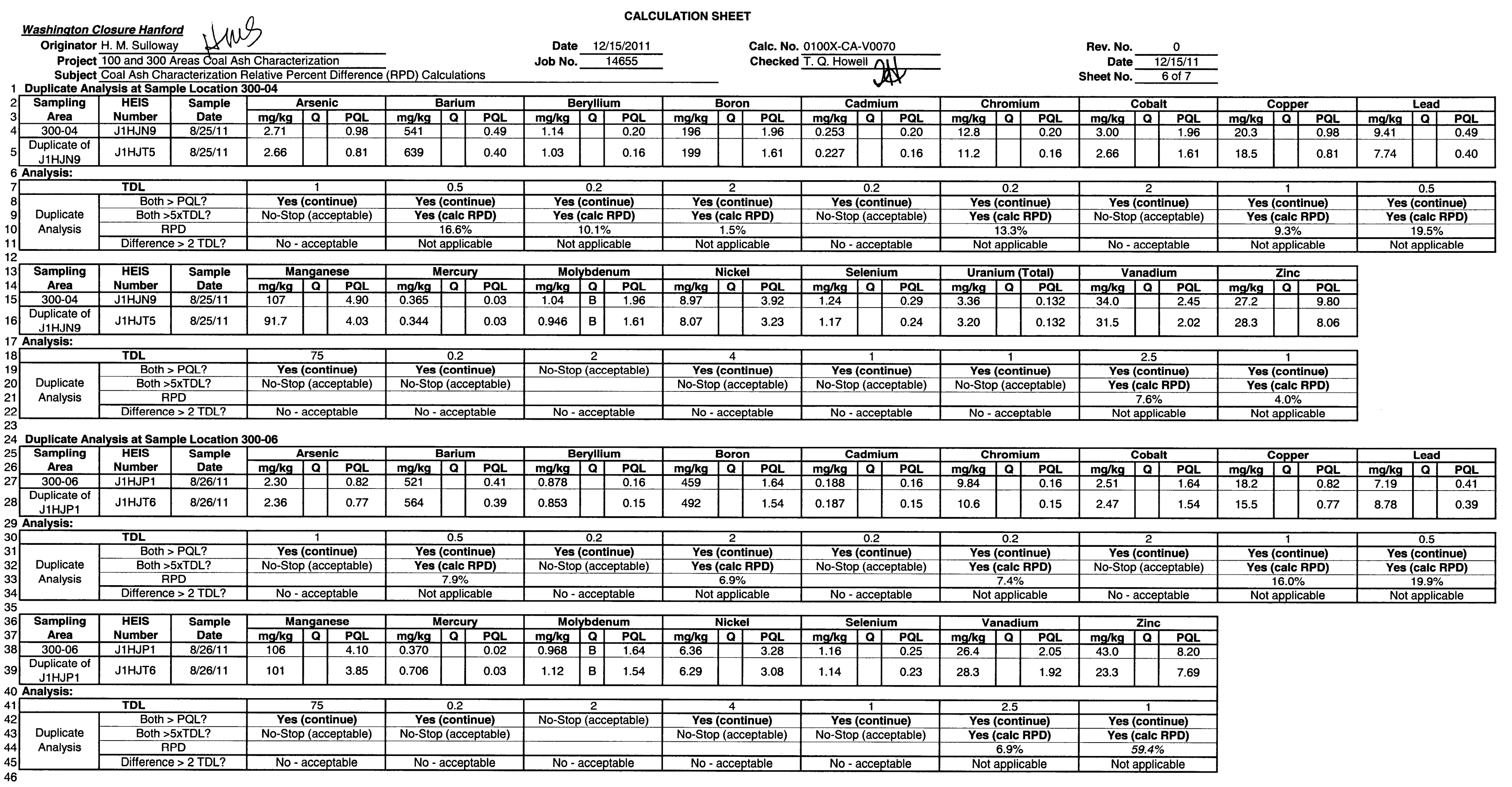


$\frac{\text { Washington Closure Hanford }}{\text { Originator H. M. Sulloway }}$ dups

Project 100 and 300 Areas Coal Ash

12011 Subject $\frac{100 \text { al }}{\text { Coal }}$ Ash Characterization Relative Percent Difference (RPD) Calculations
Duplicate Analysis at Sample Location 600-15 $\begin{aligned} \text { Date } & 12 / 15 / 2011 \\ \text { Job No. } & 14655\end{aligned}$

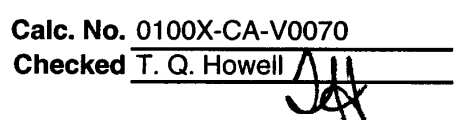
Rev. No. $\frac{0}{\text { Date }}$
Sheet No. $\frac{12 / 15 / 11}{7 \text { of } 7}$

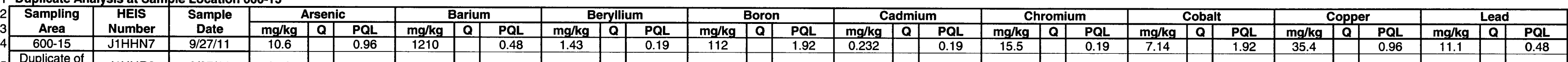

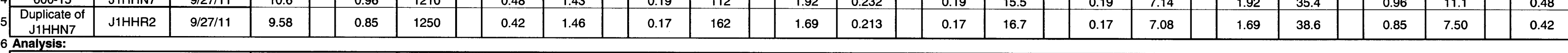

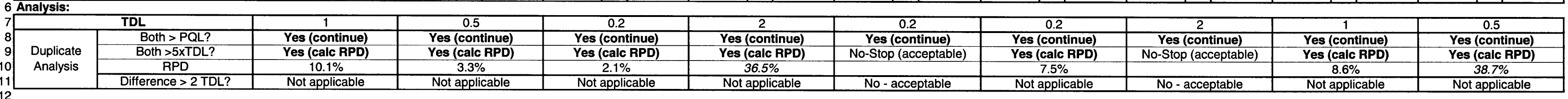

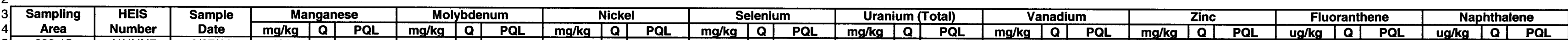

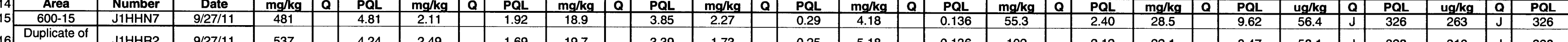

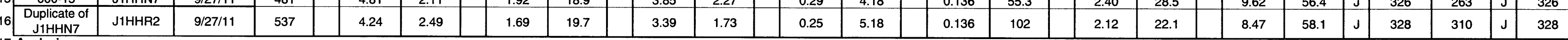

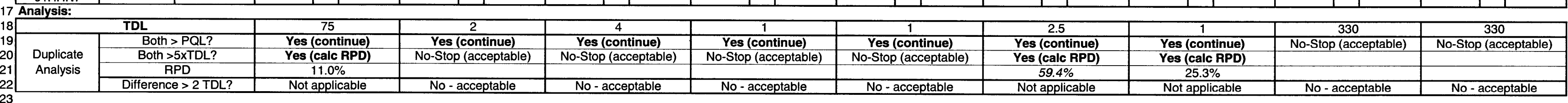

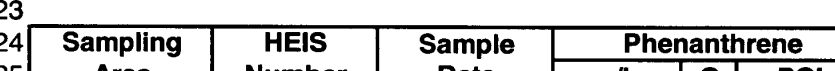

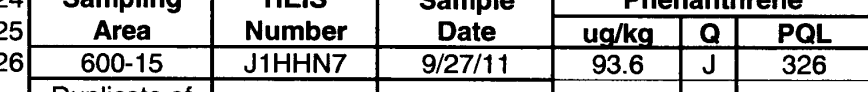
\begin{tabular}{|c|c|c|c|c|c|}
\hline Duplicate of & J1HHR2 & $9 / 27 / 11$ & 95.5 & $\mathrm{~J}$ & 328 \\
\hline
\end{tabular}

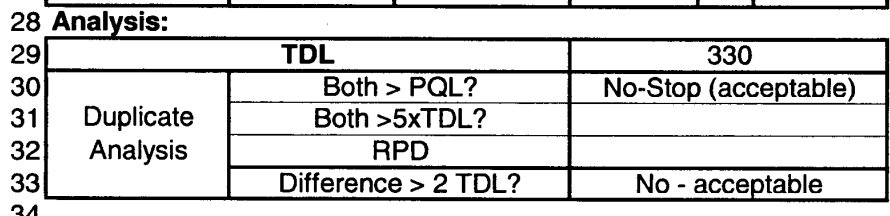

Duplicate Analysis at Sample Location 600-24

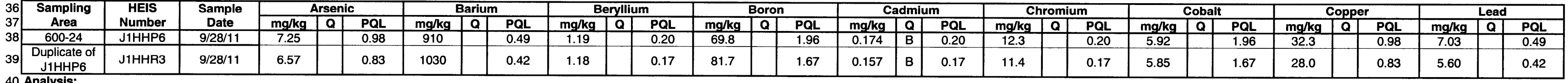

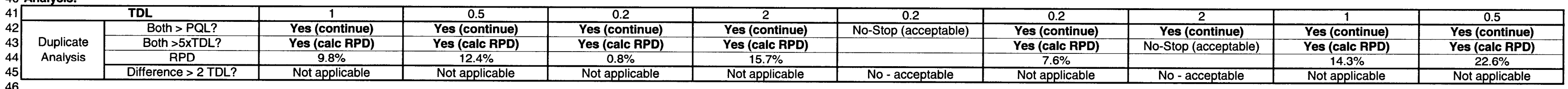

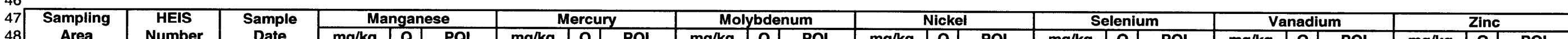

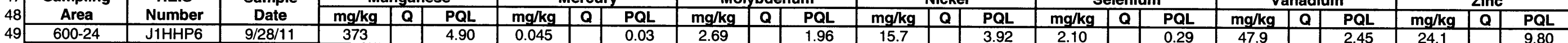

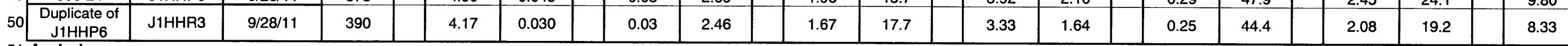

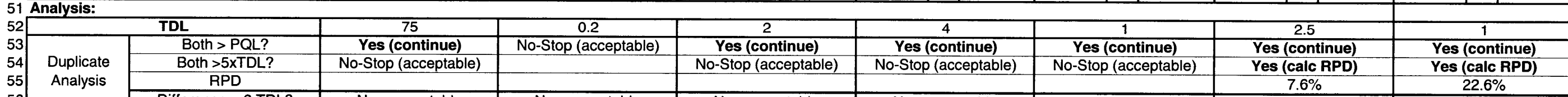




\section{CALCULATION COVER SHEET}

Area: $100-\mathrm{B}, 100-\mathrm{D}, 100-\mathrm{H}, 100-\mathrm{JU}-6$, and 300 Areas

Discipline: Environmental

${ }^{\star}$ Calculation No: 0100X-CA-V0073

Subject: Coal Ash Characterization 90th Percentile and Median Equality Calculations

Computer Program: Excel

Program No: Excel 2003

The attached calculations have been generated for a specific purpose and task. Use of the calculations by persons who do not have access to all pertinent facts may lead to incorrect conclusions and/or results. Before applying these calculations to your work, the underlying basis, rationale, and other pertinent information relevant to these calculations must be thoroughly reviewed with appropriate Washington Closure Hanford LLC (WCH) officials or other authorized personnel. WCH is not responsible for the use of a calculation not under its direct control.

Committed Calculation $\bigotimes \quad$ Preliminary $\square \quad$ Superseded $\square \quad$ Voided

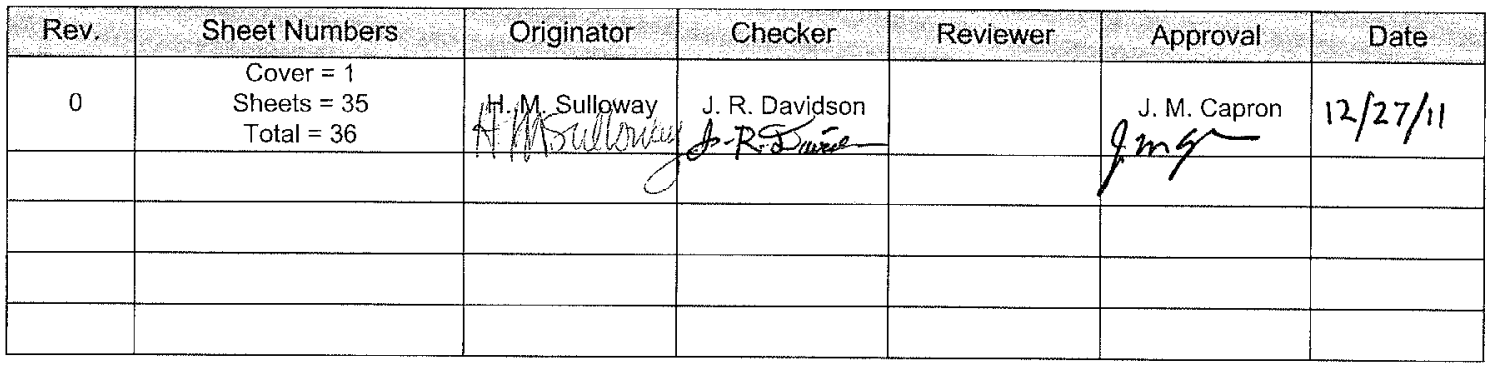

SUMMARY OF REVISION

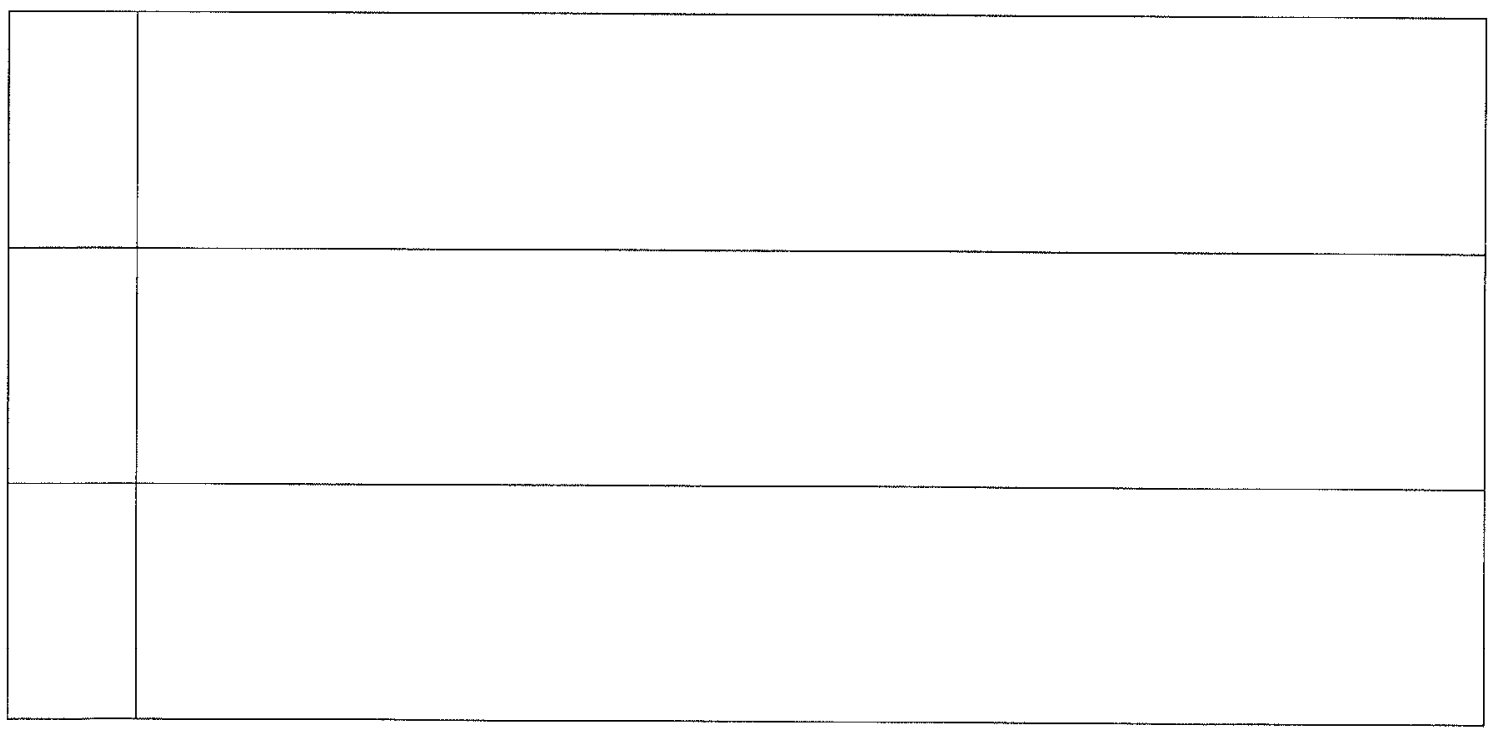


Washington Closure Hanford, Inc.

\begin{tabular}{|c|c|c|c|c|c|c|c|}
\hline Originator: & H. M. Sulloway IIM? & Date: & $12 / 20 / 11$ & Calc. No: & $0100 \mathrm{X}-\mathrm{CA}-\mathrm{V} 0073$ & Rev.: & 0 \\
\hline Project: & $\begin{array}{l}100 \text { and } 300 \text { Areas Coal Ash } \\
\text { Characterization }\end{array}$ & Job No: & 14655 & Checked: & J. R. Davidson & Date: & $12 / 20 / 11$ \\
\hline Subject: & \multicolumn{5}{|c|}{ Coal Ash Characterization 90th Percentile and Median Equality Calculations } & \multicolumn{2}{|c|}{ Sheet No. 1 of 35} \\
\hline
\end{tabular}

\title{
PURPOSE:
}

\begin{abstract}
The calculation provides documentation to support the calculation of the $90^{\text {th }}$ percentile values, $90^{\text {th }}$ percentile upper tolerance limit (UTL) values, and statistical analysis of median equality using the nonparametric Kruskal-Wallis one-way analysis of variance test on coal ash surface sample data from five sample sites. ProUCL 4.1 (EPA 2010) software was used for all calculations.
\end{abstract}

\section{TABLE OF CONTENTS:}

Sheets 1 to 4 -Summary

Sheets 5 to 9 - ProUCL output including $90^{\text {th }}$ Percentile and $90^{\text {th }}$ Percentile UTL

Sheets 10 to 15 - ProUCL output of Kruskal-Wallis test

Sheets 16 to 35 - Coal Ash Surface Sample Data

\section{GIVEN/REFERENCES:}

1) DOE-RL, 2011, Sampling and Analysis Plan for Characterization of Hanford Site Coal Ash Components, DOE/RL-2010-113, Rev 0, U.S. Department of Energy, Richland Operations Office, Richland, Washington.

2) Ecology, 1993, Statistical Guidance for Ecology Site Managers, Supplement S-6, Analyzing Site or Background Data with Below-detection Limit or Below-PQL Values (Censored Data Sets),

Publication \#92-54, Washington Department of Ecology, Olympia, Washington.

3) EPA, 2010, ProUCL, Version 4.1, U.S. Environmental Protection Agency, Washington, D.C. <http://www.epa.gov/osp/hst1/tsc/software.htm>.

\section{SOLUTION:}

The calculation of $90^{\text {th }}$ percentile values, $90^{\text {th }}$ percentile UTL values, and Kruskal-Wallis test for coal ash constituents was performed using ProUCL 4.1 with the attached data.

\section{METHODOLOGY:}

Five sample sites underwent statistical sampling of coal ash as described in DOE-RL, 2011. A total of 29 surface samples were collected at each of the five sample sites and analyzed for metals. Analytes with at least one detection within a given sample site data set were chosen for $90^{\text {th }}$ percentile, $90^{\text {th }}$ percentile UTL, and potential Kruskal-Wallis test calculations. The $90^{\text {th }}$ percentile UTL represents the $95 \%$ upper confidence level on the $90^{\text {th }}$ percentile value. Nonparametric methods were used for the $90^{\text {th }}$ percentile and $90^{\text {th }}$ percentile UTL calculations. All data reported as being undetected were set to $1 / 2$ the detection limit value for calculation of the statistics (Ecology 1993). For the statistical evaluation of primary-duplicate sample pairs, the sample results were averaged before being included in the data set, after adjustments for censored data as described above. All statistical analyses with ProUCL 4.1 (EPA 2010) were performed using these adjusted data sets (sheets 17 to 26). 
Washington Closure Hanford Ind

CALCULATION SHEET

\begin{tabular}{|c|c|c|c|c|c|c|c|}
\hline Originator: & H. M. Sulloway (X) & Date: & $12 / 20 / 11$ & Calc. No.: & $0100 \mathrm{X}-\mathrm{CA}-\mathrm{V} 0073$ & Rev.: & 0 \\
\hline Project: & $\begin{array}{l}100 \text { and } 300 \text { Areas Coal Ash } \\
\text { Characterization }\end{array}$ & Job No: & 14655 & Checked: & J. R. Davidson & Date: & $12 / 20 / 11$ \\
\hline Subject: & \multicolumn{5}{|c|}{ Coal Ash Characterization 90th Percentile and Median Equality Calculations } & \multicolumn{2}{|c|}{ Sheet No. 2 of 35} \\
\hline
\end{tabular}

\section{RESULTS:}

2

ProUCL output from $90^{\text {th }}$ percentile analysis using nonparametric methods provides additional statistics. This output generated by ProUCL including these additional statistics is presented, but only the 90th percentile and UTL values are summarized. ProUCL data output on sheets 5 through 9 is presented exactly as generated from the software. Arrangement of the ProUCL data output into formatted tables was performed for this calculation to optimize data presentation for each sample site.

Analysis for median equality by the Kruskal-Wallis test was performed for analytes with detections in three or more sample sites. Of the analytes with detections in coal ash, all met this threshold for analysis except for thallium which was detected at only a single sample site.

The null hypothesis for the Kruskal-Wallis test is that the medians of the data sets (sample sites) are equal. The Kruskal-Wallis tests were conducted at a $5 \%$ significance level. The $p$-value calculated by the analysis determines if the null hypothesis is accepted or rejected. If the $p$-value is greater than 0.05 the null hypothesis is accepted (medians are equal), but if the $p$-value is less than or equal to 0.05 the null hypothesis is rejected (medians are not equal). Of the analytes that met the threshold for analysis (detections in three or more sample sites), the null hypothesis is rejected for all except vanadium. Therefore, vanadium is the only analyte for which the medians of data from the sample sites are equal at a $5 \%$ significance level. 
Date 12/27/2011

Project 100 and 300 Areas Coal Ash Characterization

Subject Coal Ash Characterization 90th Percentile and Median Equality Calculations
Calc. No. 0100X-CA-V0073 Checked J.R. Davidson JPV
Rev. No.

Date 12/27/2011

Sheet No. 3 of 35 1 Summary

3 90th Percentile and 90th Percentile UTL Summary

\begin{tabular}{|c|c|c|c|c|c|c|c|c|c|c|}
\hline \multirow[b]{2}{*}{ Analyte } & \multicolumn{5}{|c|}{ 90th Percentile } & \multicolumn{5}{|c|}{ 90th Percentile UTL } \\
\hline & $126-B-1$ & $126-D-1$ & $126-\mathrm{H}-1$ & $\begin{array}{c}300 \text { Area } \\
\text { Ash }\end{array}$ & $600-207$ & 126-B-1 & 126-D-1 & $126-\mathrm{H}-1$ & $\begin{array}{c}300 \text { Area } \\
\text { Ash }\end{array}$ & $600-207$ \\
\hline Antimony & 0.844 & 0.633 & 0.290 & -- & -- & 1.26 & 0.894 & 0.295 & -- & -- \\
\hline Arsenic & 7.46 & 7.78 & 3.80 & 3.64 & 6.69 & 8.45 & 13.6 & 4.34 & 3.87 & 8.54 \\
\hline Barium & 1988 & 1480 & 1024 & 715 & 989 & 2250 & 1750 & 1250 & 754 & 1060 \\
\hline Beryllium & 1.94 & 1.88 & 1.31 & 1.42 & 1.12 & 2.39 & 2.51 & 1.44 & 1.66 & 1.19 \\
\hline Boron & 271 & 318 & 300 & 258 & 87.8 & 327 & 410 & 367 & 369 & 137 \\
\hline Cadmium & 0.508 & 0.383 & 0.353 & 0.313 & 0.205 & 0.899 & 0.443 & 0.388 & 0.338 & 0.211 \\
\hline Chromium & 13.5 & 12.2 & 13.6 & 14.9 & 12.0 & 16.2 & 12.5 & 14.4 & 15.6 & 12.9 \\
\hline Cobalt & 7.02 & 9.19 & 8.53 & 4.26 & 5.89 & 8.22 & 11.6 & 9.81 & 4.89 & 6.07 \\
\hline Copper & 39.3 & 31.6 & 41.1 & 26.7 & 30.0 & 94.3 & 34.8 & 43.5 & 28.8 & 37.0 \\
\hline Lead & 16.4 & 11.0 & 6.31 & 14.1 & 7.12 & 19.0 & 15.1 & 7.43 & 15.2 & 9.30 \\
\hline Manganese & 350 & 458 & 246 & 188 & 415 & 396 & 689 & 283 & 210 & 486 \\
\hline Mercury & 0.246 & 0.115 & 0.132 & 0.482 & 0.030 & 0.338 & 0.139 & 0.176 & 0.713 & 0.042 \\
\hline Molybdenum & 2.58 & 2.86 & 2.06 & 1.39 & 2.34 & 3.85 & 3.54 & 2.44 & 1.50 & 2.58 \\
\hline Nickel & 15.0 & 15.3 & 19.5 & 12.6 & 16.7 & 15.8 & 17.5 & 20.4 & 13.2 & 16.7 \\
\hline Selenium & 1.32 & 0.927 & 1.51 & 1.74 & 1.93 & 1.44 & 0.955 & 1.63 & 2.46 & 2.00 \\
\hline Thallium & -- & 0.243 & -- & -- & -- & -- & 0.245 & -- & -- & -- \\
\hline Vanadium & 49.8 & 53.8 & 53.0 & 43.1 & 45.5 & 64.0 & 59.7 & 58.4 & 45.7 & 50.1 \\
\hline Zinc & 60.5 & 56.6 & 48.9 & 37.4 & 25.4 & 94.8 & 69.7 & 53.6 & 43.3 & 26.9 \\
\hline
\end{tabular}

22 -- = Value not determined as no detections in subject data set 
Originator H.M. Sulloway

Project 100 and 300 Areas Coal Ash Characterization

Subject Coal Ash Characterization 90th Percentile and Median Equality Calculations
Date 12/27/2011 Calc. No. 0100X-CA-V0073 Job No.
Calc. No. $0100 X-C A-V 0073$
Rev. No.

Date $\overline{12 / 27 / 2011}$ 4 of 35

Summary (continued)

3 Kruskal-Wallis test results (surface samples)

\begin{tabular}{r|l|c|c|}
\hline \multicolumn{2}{|c|}{ Analyte } & p-Value & Accept/Reject Null \\
\cline { 2 - 4 } 5 & Antimony & 0.0013 & Reject \\
\hline 6 & Arsenic & $2.4 \mathrm{E}-11$ & Reject \\
\hline 7 & Barium & $3.0 \mathrm{E}-11$ & Reject \\
\hline 8 & Beryllium & $3.3 \mathrm{E}-10$ & Reject \\
\hline 9 & Boron & $1.4 \mathrm{E}-09$ & Reject \\
\hline 10 & Cadmium & $1.6 \mathrm{E}-05$ & Reject \\
\hline 11 & Chromium & $2.6 \mathrm{E}-04$ & Reject \\
\hline 12 & Cobalt & $7.3 \mathrm{E}-06$ & Reject \\
13 & Copper & $1.9 \mathrm{E}-04$ & Reject \\
\hline 14 & Lead & $6.1 \mathrm{E}-09$ & Reject \\
\hline 15 & Manganese & $7.0 \mathrm{E}-12$ & Reject \\
\hline 16 & Mercury & $1.1 \mathrm{E}-15$ & Reject \\
\hline 17 & Molybdenum & $5.5 \mathrm{E}-07$ & Reject \\
\hline 18 & Nickel & $1.2 \mathrm{E}-04$ & Reject \\
\hline 19 & Selenium & $3.3 \mathrm{E}-15$ & Reject \\
\hline 20 & Thallium & $\mathrm{NA}$ & NA \\
19 & Vanadium & 0.16 & Accept \\
\hline 20 & Zinc & $5.3 \mathrm{E}-06$ & Reject \\
\hline
\end{tabular}

$21 \mathrm{NA}=$ not applicable, insufficient number of data sets for test 
Washington Closure Hanford

Originator H.M. Sulloway LWS

Subject Coal Ash Characte $\begin{aligned} \text { Date } & 12 / 20 / 11 \\ \text { Job No. } & \frac{14655}{1}\end{aligned}$

\section{CALCULATION SHEET}

Calc. No. $0100 x-C A-V 0073$
Checked J. R. Davidson J RD
Rev. No. $\frac{0}{12 / 20 / 11}$
Date
Sheet No.
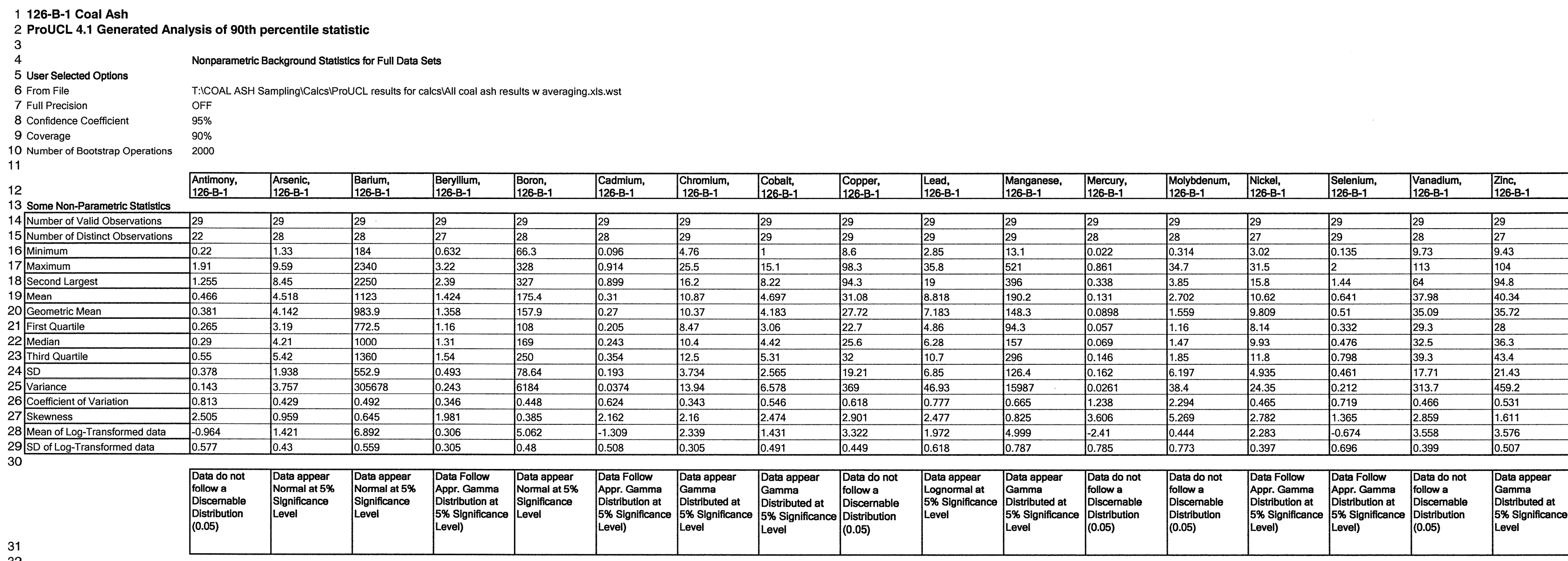

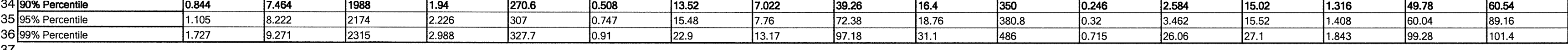
38 95\% UTL with 90\% Coverage

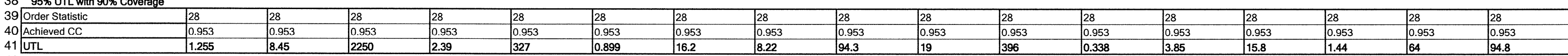

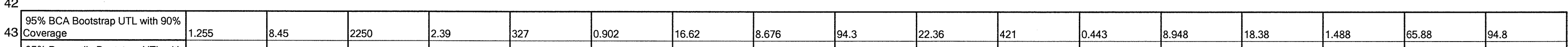

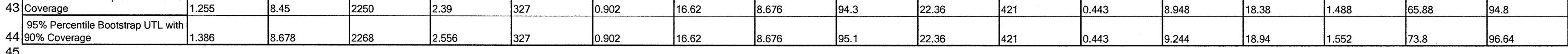

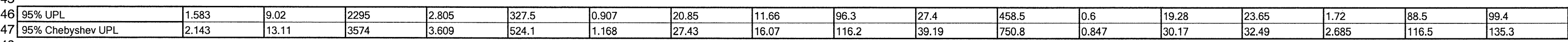
\begin{tabular}{l}
48 \\
49 Upper Limit Based upon IQR \\
\hline
\end{tabular} 
Washington Closure Hanford

Originator H. M. Sulloway $\$$ WW Project 100 and 300 Areas Coal Ash Characterization
Subject Coal Ash Characterization 90 th Percentile and Median Equality Calculations

\section{CALCULATION SHEET}

Calc. No. $0100 X-C A-V 0073$
Checked J.R. Davidson SRD $\begin{aligned} \text { Rev. No. } & \frac{0}{12 / 20 / 11} \\ \text { Date } & \frac{12 / 20 \text { of } 35}{\text { Sheet No. }}\end{aligned}$

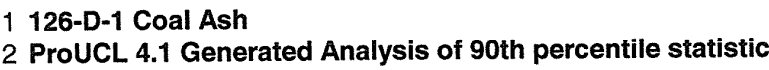

\section{4 Nonparametic Background Statistics for Full Data Sets}

User Selectedo

T:ICOAL ASH Sampling ICalcsiP roUCL results for calcsilll coal ash results w averaging.xls.wS

6 From File

T:ICOA
OFF
$95 \%$

8 Confidence Coefficient

$95 \%$
$90 \%$
2000

10 Number of Bootstrap Operations $\quad 2000$

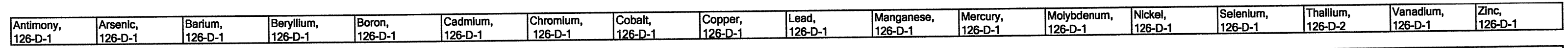

3 Some Non-Paramericic Statlstics 14 Number of Valid observations

\begin{tabular}{ll}
17 & Minimum \\
18 & Maximum \\
\hline Second Largest & \\
\hline
\end{tabular}

Geometric Mean

22 Median

23 Third Quartile

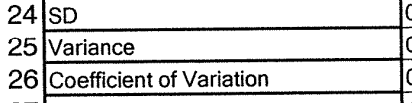

28 Meann of Log-Transformed data
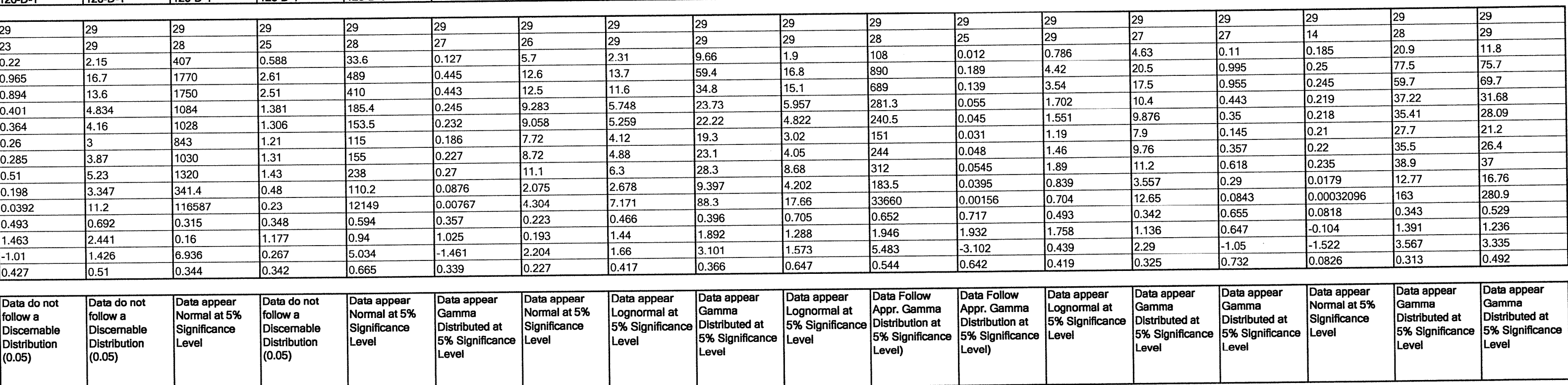

"

\begin{tabular}{|c|c|c|c|c|c|c|c|c|c|c|c|c|c|c|c|c|c|}
\hline 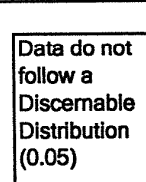 & \begin{tabular}{|l} 
Data do not \\
follow a \\
Discemable \\
Distributition \\
(0.05)
\end{tabular} & $\begin{array}{l}\text { Data appear } \\
\text { Nomal at } 5 \% \\
\text { Signififance } \\
\text { Level }\end{array}$ & \begin{tabular}{|l} 
Data do not \\
follow a \\
Discematle \\
Distributition \\
(0.05)
\end{tabular} & \begin{tabular}{|l} 
Data appear \\
Nomat at $5 \%$ \\
Slignificance \\
Level
\end{tabular} & $\begin{array}{l}\text { Data appear } \\
\text { Gamma } \\
\text { Distrituded at } \\
5 \% \text { Significance } \\
\text { Level }\end{array}$ & 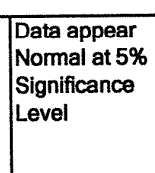 & $\begin{array}{l}\text { Data appear } \\
\text { Lognormal at } \\
5 \% \text { Significance } \\
\text { Level }\end{array}$ & \begin{tabular}{|l} 
Data appear \\
Gamma \\
Distrited at \\
$5 \%$ Significance \\
Level
\end{tabular} & $\begin{array}{l}\text { Data appear } \\
\text { Lognommal at } \\
\text { 5\% Significano } \\
\text { Level }\end{array}$ & $\begin{array}{l}\text { Data Follow } \\
\text { Apppr. Gamma } \\
\text { Dlstrituon at } \\
\text { 5\% Signifificance } \\
\text { Level) }\end{array}$ & $\begin{array}{l}\text { Data Follow } \\
\text { Appr. Gamma } \\
\text { Distributon at } \\
5 \% \text { Stignificance } \\
\text { Level) }\end{array}$ & 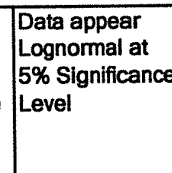 & \begin{tabular}{|l} 
Data appear \\
Gamma \\
alstrbuted at \\
5\% Shignificanc \\
Level
\end{tabular} & $\begin{array}{l}\text { Data appear } \\
\text { Gamma } \\
\text { Distrituted at } \\
5 \% \text { Shlynifcanc } \\
\text { Level }\end{array}$ & $\begin{array}{l}\text { Data appear } \\
\text { Nomal at } 5 \% \\
\text { Slongifecance } \\
\text { Level }\end{array}$ & \begin{tabular}{|l|} 
Data appear \\
Gamma \\
Distrited at \\
5s Signififance \\
Level
\end{tabular} & \begin{tabular}{|l} 
Data appear \\
Gamma \\
Distrited at \\
$5 \%$ Significance \\
Level
\end{tabular} \\
\hline
\end{tabular}

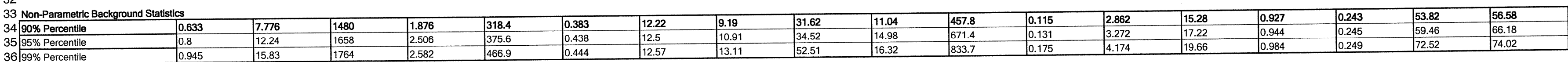
38 95\% UTL with $90 \%$ Coverage

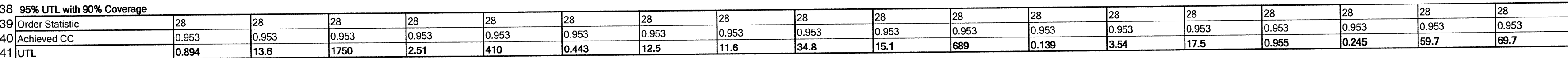

\begin{tabular}{|c|c|c|c|c|c|c|c|c|c|c|c|c|c|c|c|c|c|c|}
\hline $95 \%$ BCA Bootstrap UTL with $90 \%$ & 0.894 & 14.22 & 1750 & 2.53 & 410 & 0.443 & 12.52 & 11.6 & 39.72 & 15.44 & 694 & 0.139 & 3.54 & 18.1 & 0.955 & 0.246 & 63.26 & 70.9 \\
\hline $\begin{array}{l}95 \% \text { Percentile Bootstrap UTL with } \\
90 \% \text { Coverage }\end{array}$ & 0.908 & 14.22 & 1754 & 2.522 & 425.8 & 0.443 & 12.52 & 12.02 & 39.72 & 15.2 & 729.2 & 0.149 & 3.716 & 18.1 & 0.963 & 0.246 & 63.26 & 69.7 \\
\hline $\begin{array}{l}46995 \% \text { UPL } \\
4795 \% \text { Chebyshey UPL }\end{array}$ & 0.93 & 15.15 & 1760 & 2.56 & 449.5 & 0.444 & 12.55 & 12.65 & 47.1 & 15.95 & 789.5 & 0.164 & 3.98 & $\frac{19}{19}$ & 0.975 & 0.248 & $\frac{68.6}{63.62}$ & $\frac{70.9}{697}$ \\
\hline $47895 \%$ Chebyshev UPL & 1.279 & 19.67 & 2598 & 3.509 & I674.1 & 10.634 & 18.48 & 17.62 & 165.39 & 24.59 & {$[1095$} & 10.23 & 15.422 & 126.17 & 7.13 & & & \\
\hline Upper Limit Based upon $1 Q$ & 0.885 & 8.575 & 2036 & 11.76 & $\overline{422.5}$ & To.396 & 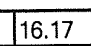 & 9.57 & $\sqrt{41.8}$ & 117.17 & 1553.5 & 10.0898 & 2.94 & 16.15 & 11.328 & 0.273 & 155.7 & 160.7 \\
\hline
\end{tabular}


Washington Closure Hanford

Originator H. M. Sulloway LUW

Subject Coal Ash Chareas Coal Ash Characterization

1 126-H-1 Coal Ash

作

3

5 User Selected Options Nonparametric Background Statistics for Full Data Sets

6 From File 1 T:COAL ASH SamplingICalcs|ProUCL results for calcsill coal ash results w weraging.xls.wst

7 Full Precision

$95 \%$
$90 \%$

Number of Bootstrap Operations $\quad 2000$

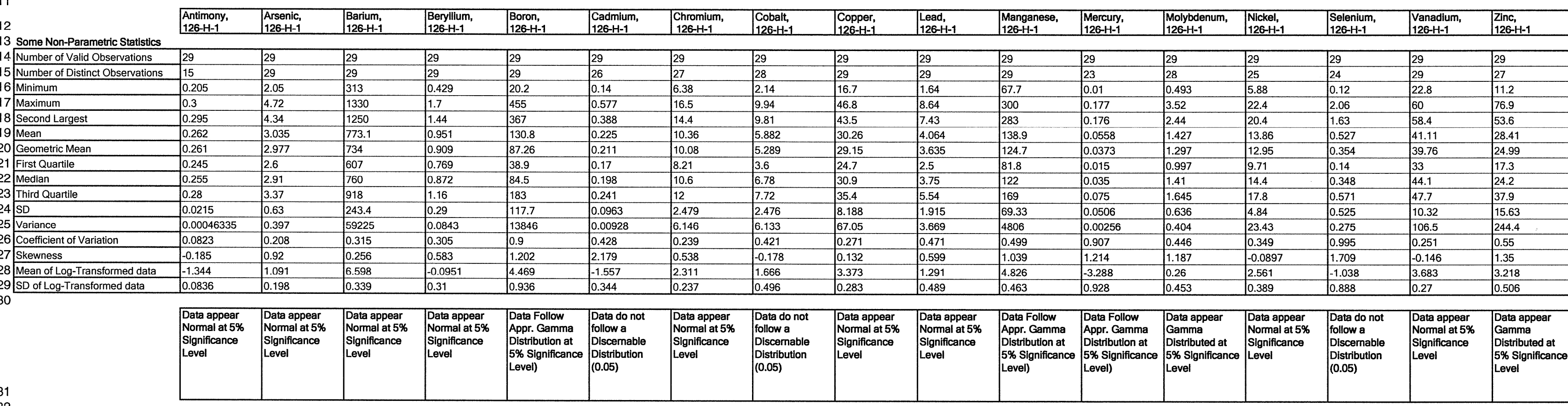

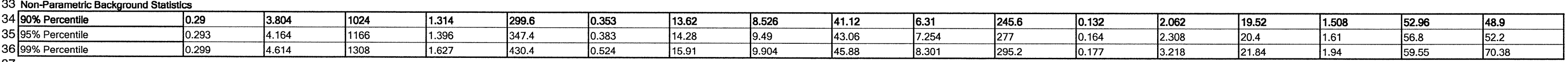
38 95\% UTL with $90 \%$ Coverage

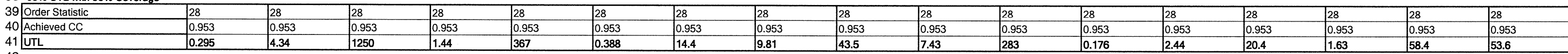

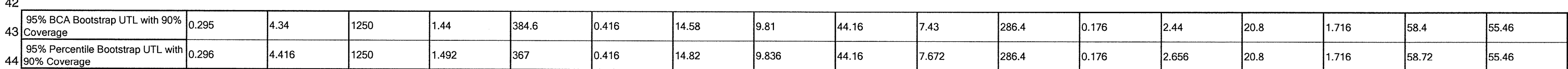

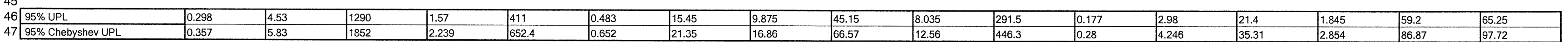

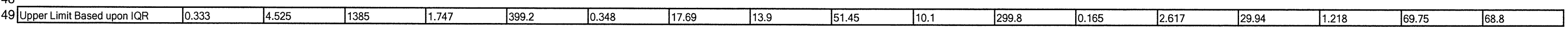


Washington Closure Hanford

Originator H. M. Sulloway LXIJ Dato $\frac{12 / 20 / 11}{14655}$
CALCULATION SHEET Checked J. R. Davidson JRD
Rev. No. $\frac{0}{12 / 20 / 11}$
Date
Sheet No. $\frac{12 / 20+35}{8}$

\section{ProUCL 4.1 Generated Analysis of 90th percentile statistic}

3
4
5

$4 \quad$ Nonparametric Background Statistics for Full Data Sets

5 User Selected 0 .
6 From File

7 Full Precision

8 Confidence Coefficien

$\begin{array}{cc}8 \text { Confdence Coefficient } & 95 \% \\ 10 \text { Number of Bootstrap Operations } & 90 \% \\ 11 & \end{array}$

T:ICOAL ASH SamplingICalcsIProuCL results for calcs IAll coal ash results w averaging XXs wst

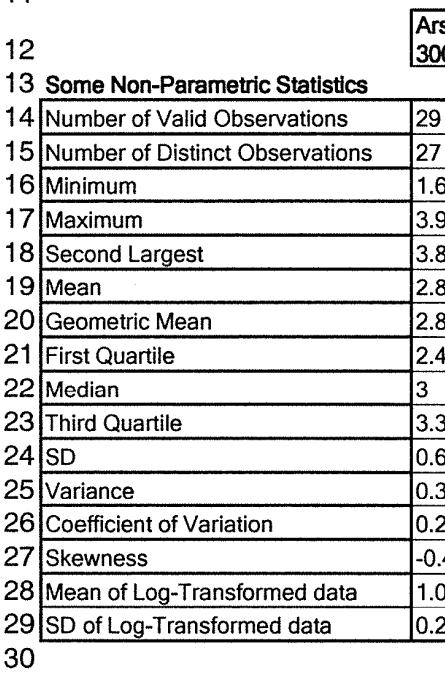

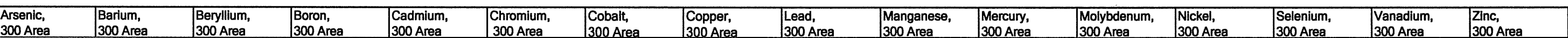

33 Non-Parametric Background Statistics

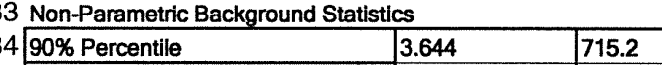
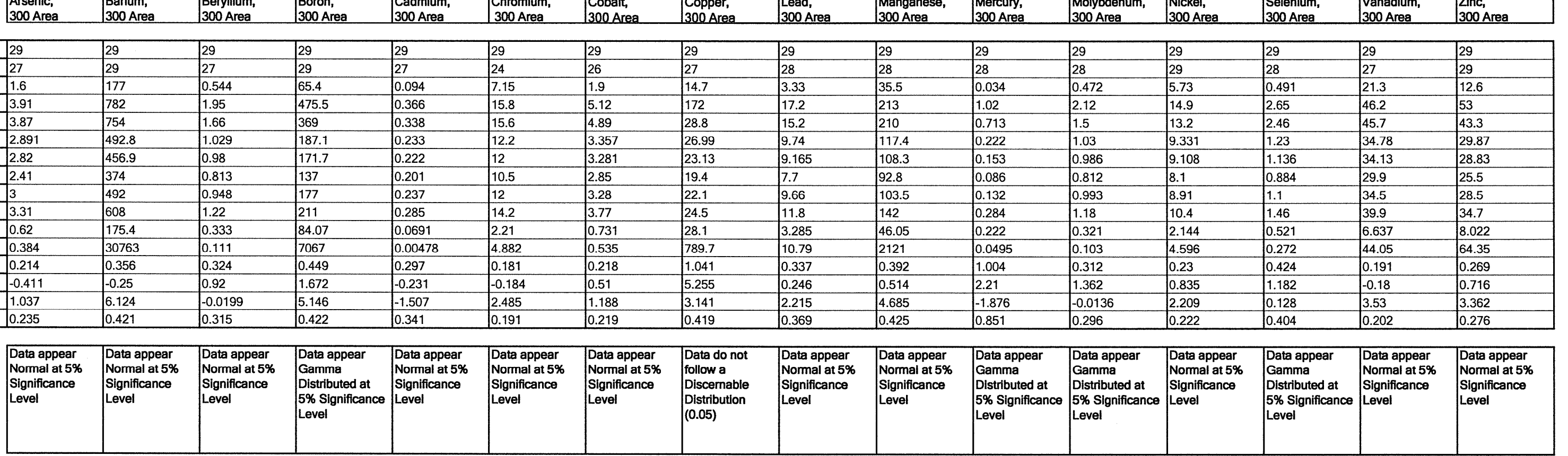

\begin{tabular}{l|l|l|l}
0.0136 & 2.209 \\
\hline 296 & 0.222 \\
\hline
\end{tabular}

\begin{tabular}{|l|l|l|l|}
\hline .182 & -0.18 & .176 \\
\hline 0.128 & 3.53 & 3.362 \\
\hline 0.404 & 0.202 & 0.276 \\
\hline
\end{tabular}

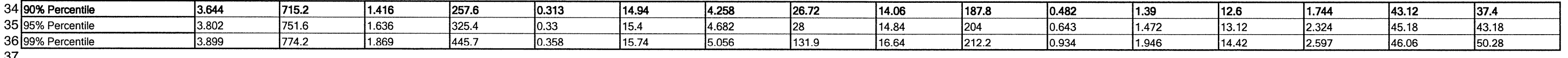

38 95\% UTL with 90\% Coverage

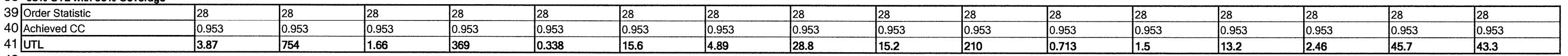

\begin{tabular}{|c|c|c|c|c|c|c|c|c|c|c|c|c|c|c|c|c|}
\hline \begin{tabular}{|l|l}
$95 \%$ BCA Bootstrap UTL with 90\% & 3 \\
Coverage
\end{tabular} & 3.878 & 754.8 & 1.718 & 390.3 & 0.338 & 15.6 & 4.936 & 57.44 & 15.6 & 210 & 0.713 & 1.568 & 13.38 & 2.46 & 45.8 & 45 \\
\hline \begin{tabular}{|l|}
$95 \%$ Percentile Bootstrap UTL with \\
$90 \%$
\end{tabular} & 3.878 & 759.6 & 1.718 & 369 & 0.344 & 15.64 & 4.89 & 57.44 & 15.6 & 210.6 & 0.774 & 1.624 & 13.38 & 2.46 & 45.8 & 45.24 \\
\hline
\end{tabular}

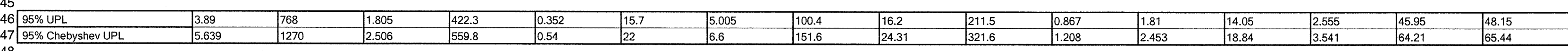

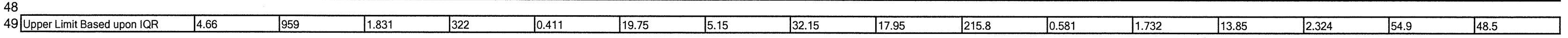


Washington Closure Hanford

Originator H. M. Sulloway 4 Wh

(1)

Subject Coal Ash Characterization 90th Percentile and Median Equality Calculations
Date $\frac{12 / 20 / 11}{14655}$
Job No.

\section{CALCULATION SHEET}

Calc. No. $\frac{0100 X-C A-V 0073}{\text { Checked J. R. Davidson J PD }}$
J
Rev. No. $\frac{0}{12 / 20 / 11}$
Date
Sheet No. $\frac{9 \text { of } 35}{}$

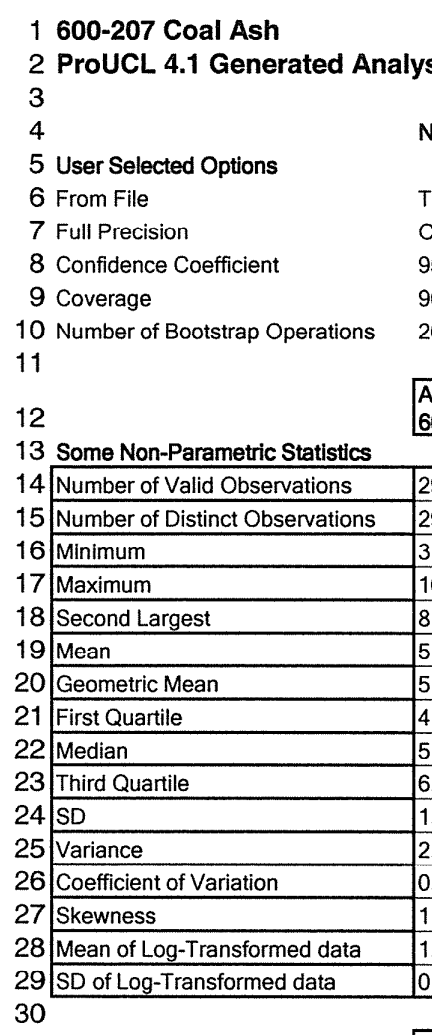

31
32
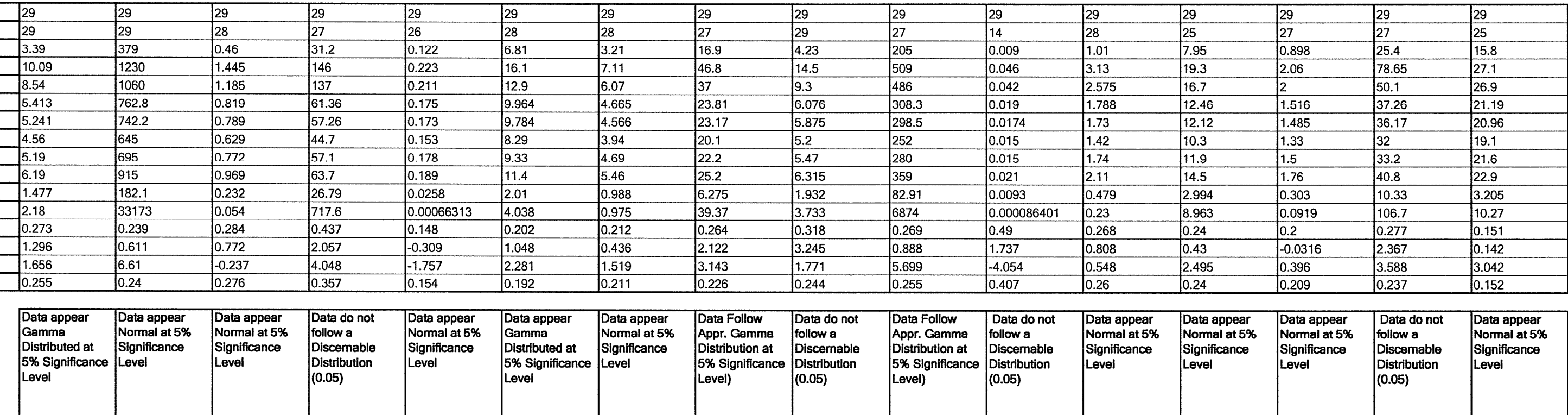

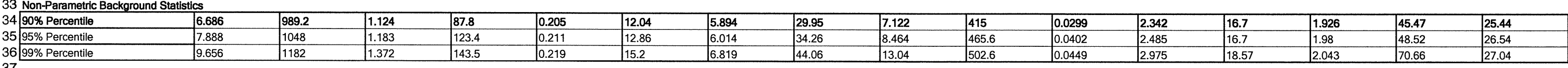
38 95\% UTL with 90\% Coverage

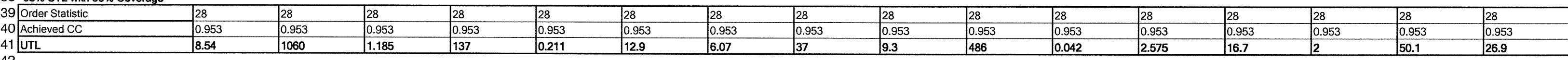

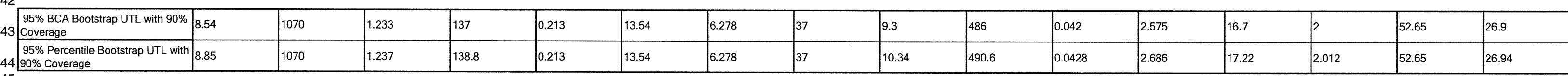
45 (1155

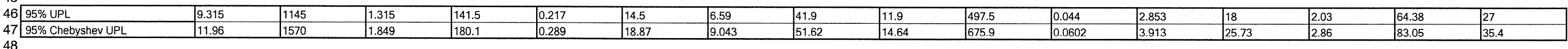

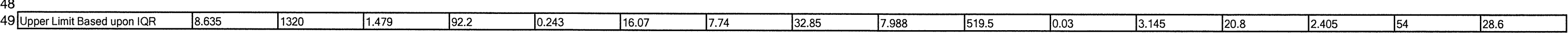


Washington Closure Hanford

Originator H. M. Sulloway if Wh Project 100 and 300 Areas Coal Ash Characterization Job No. 14655 Checked J.R. Davidson JQD Date 12/20/11 Subject Coal Ash Characterization 90th Percentile and Median Equality Calculations

1 Coal Ash Data from all Sample Areas

2 ProUCL 4.1 Generated Analysis of Kruskal-Wallis Test of Median Equality

3

4

5 User Selected Options

6 Date/Time of Computation

7 From File

8 Full Precision

9

10

11 Arsenic

12

13 Group

14

15

16

17

18

19 Overall

20

$21 \mathrm{~K}-\mathrm{W}$ (H-Stat)

22

23

24

26 Barium

27

28 Group

29

30

31

32
33

34 Overall

35

$36 \mathrm{~K}-\mathrm{W}$ (H-Stat)

37

38

39

40

41 Beryllium

42

43 Group

44

45

46

47

48

49 Overall

50

$51 \mathrm{~K}-\mathrm{W}$ (H-Stat)

52

53

\section{CALCULATION SHEET}

Date 12/20/11 Calc. No. 0100X-CA-V0073 Rev. No. 0 Sheet No. 10 of 35
Nonparametric One Way ANOVA (Kruskal-Wallis Test)

12/8/2011 8:06:56 AM

ANOVA_NP

OFF

Obs Median Ave Rank

\begin{tabular}{rr} 
& \multicolumn{1}{c}{$Z$} \\
85.07 & 1.73 \\
78.97 & 0.855 \\
46.28 & -3.831 \\
42.34 & -4.394 \\
112.3 & 5.64 \\
73 &
\end{tabular}

P-Value (Approx. Chisquare)

$4 \quad 2.416 \mathrm{E}-11$

4 2.412E-11 (Adjusted for Ties)

55.62

\begin{tabular}{|c|c|c|c|c|}
\hline & & Median & Ave Rank & $z$ \\
\hline 1 & 29 & 1000 & 95.45 & 3.218 \\
\hline 2 & 29 & 1030 & 102.7 & 4.256 \\
\hline 3 & 29 & 760 & 69.91 & -0.442 \\
\hline 4 & 29 & 492 & 28.98 & -6.31 \\
\hline \multirow[t]{2}{*}{5} & 29 & 695 & 67.97 & -0.722 \\
\hline & 145 & 760 & 73 & \\
\hline
\end{tabular}

$\begin{array}{llll}55.2 & \text { DOF } & & \text { P-Value } \\ 4 & 2.955 \mathrm{E}-11\end{array}$ (Approx. Chisquare)

55.2

4 2.953E-11

(Adjusted for Ties)

\begin{tabular}{rrrrr} 
& Obs & \multicolumn{1}{c}{ Median } & Ave Rank & \multicolumn{1}{c}{ Z } \\
1 & 29 & 1.31 & 103.1 & 4.313 \\
2 & 29 & 1.31 & 100.2 & 3.9 \\
3 & 29 & 0.872 & 57.07 & -2.284 \\
4 & 29 & 0.948 & 64.55 & -1.211 \\
5 & 29 & 0.772 & 40.09 & -4.718 \\
& 145 & 1.085 & 73 &
\end{tabular}

DOF P-Value (Approx. Chisquare)

50.2

4 3.278E-10

4 3.269E-10 (Adjusted for Ties) 


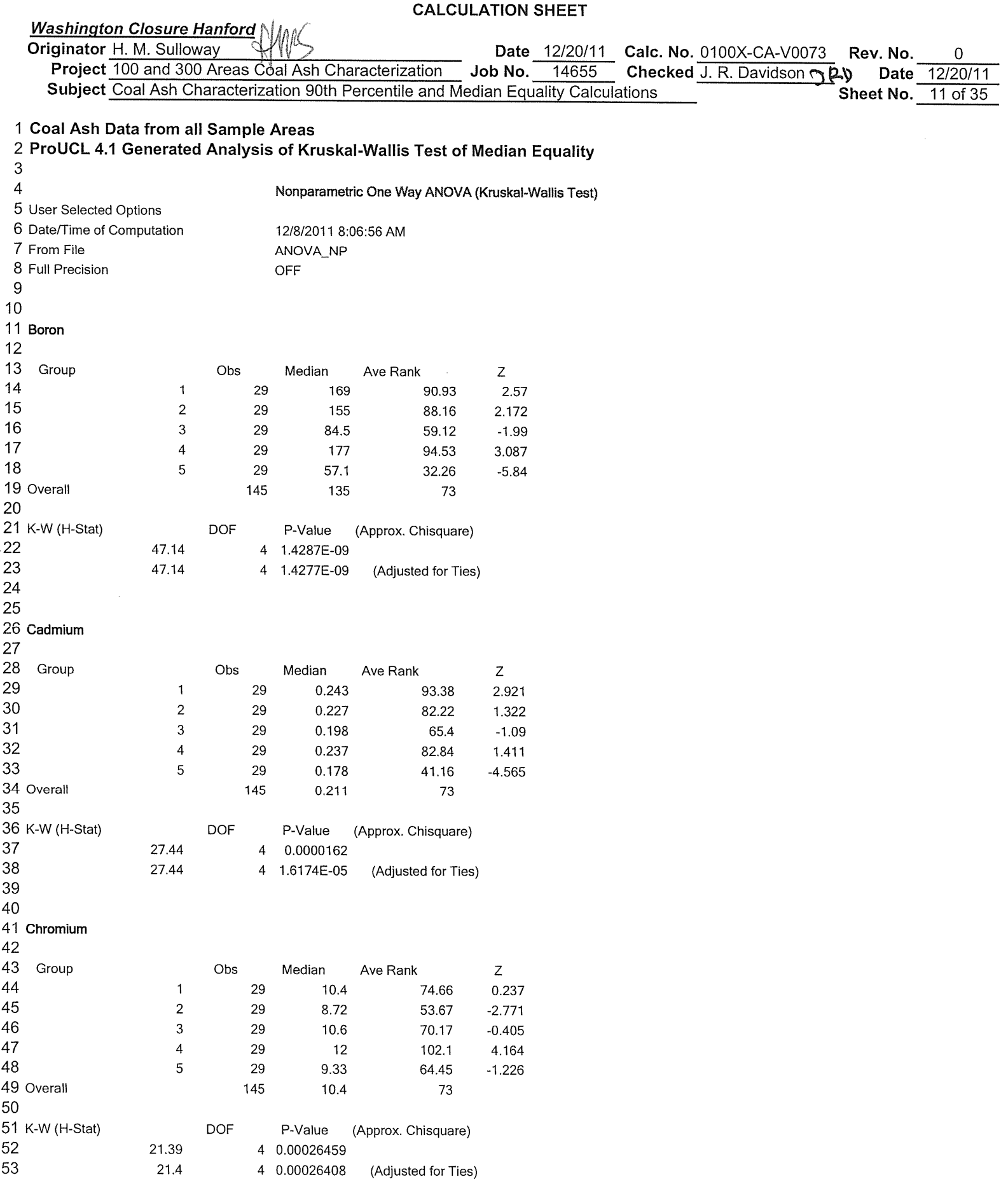




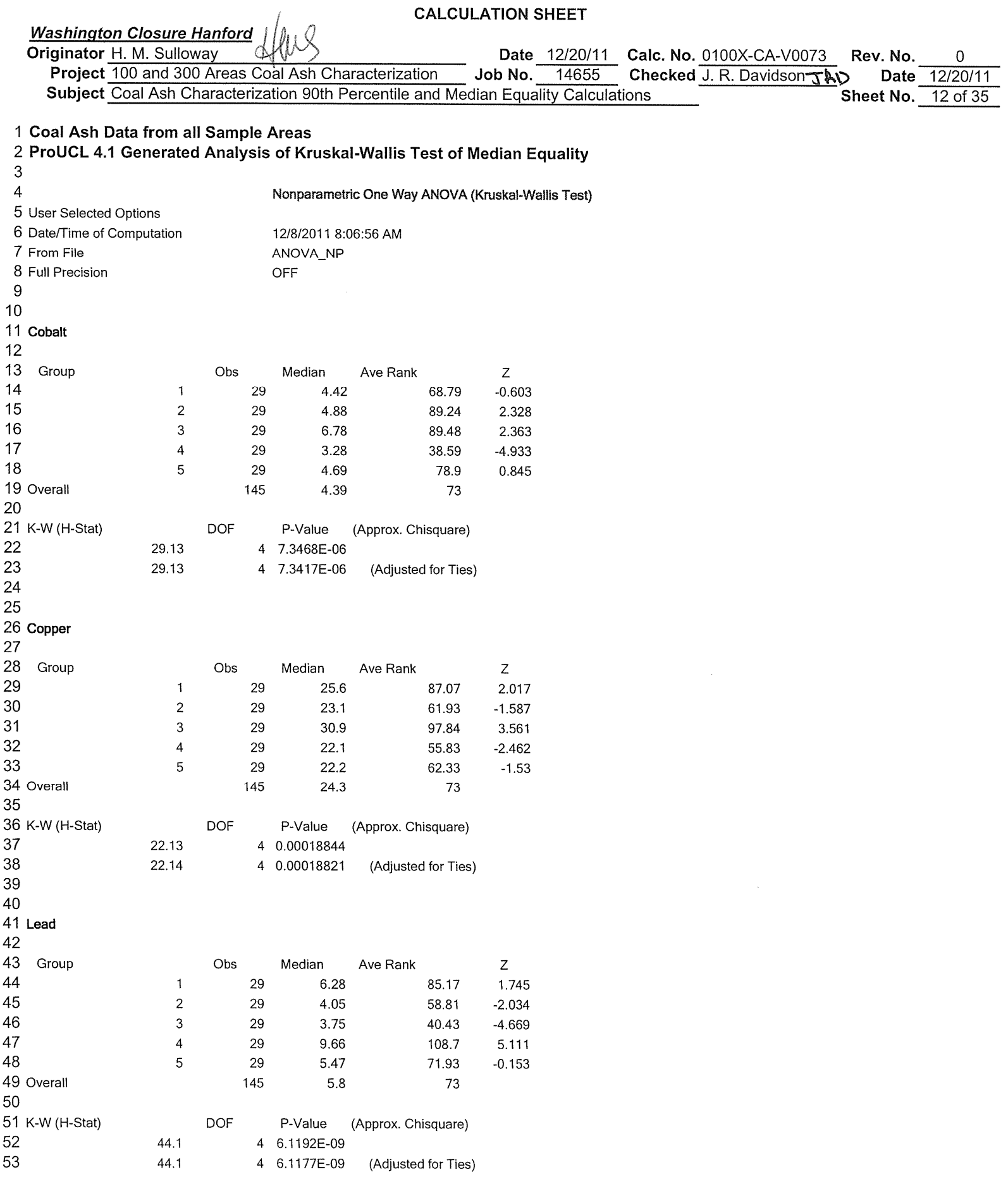




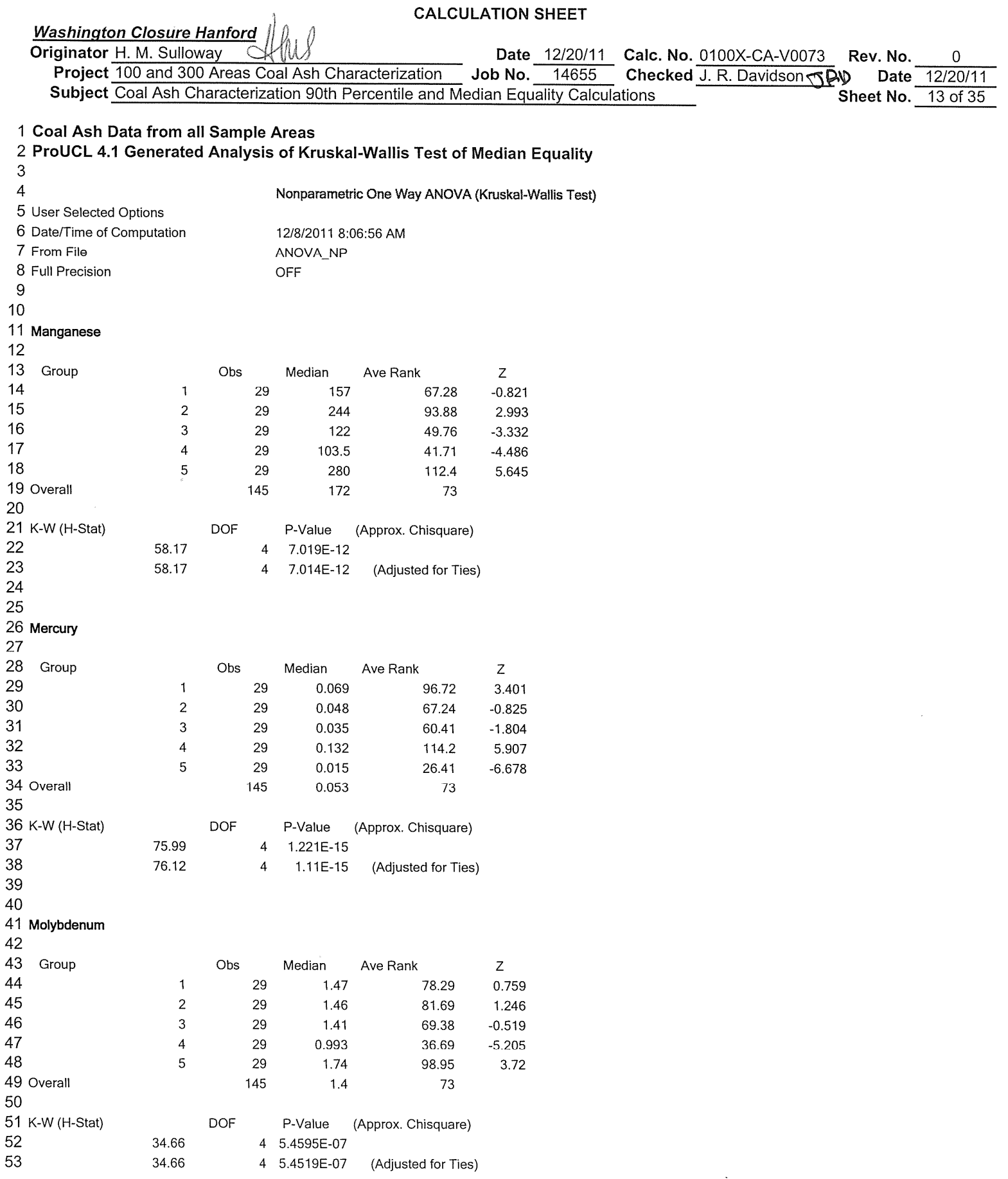




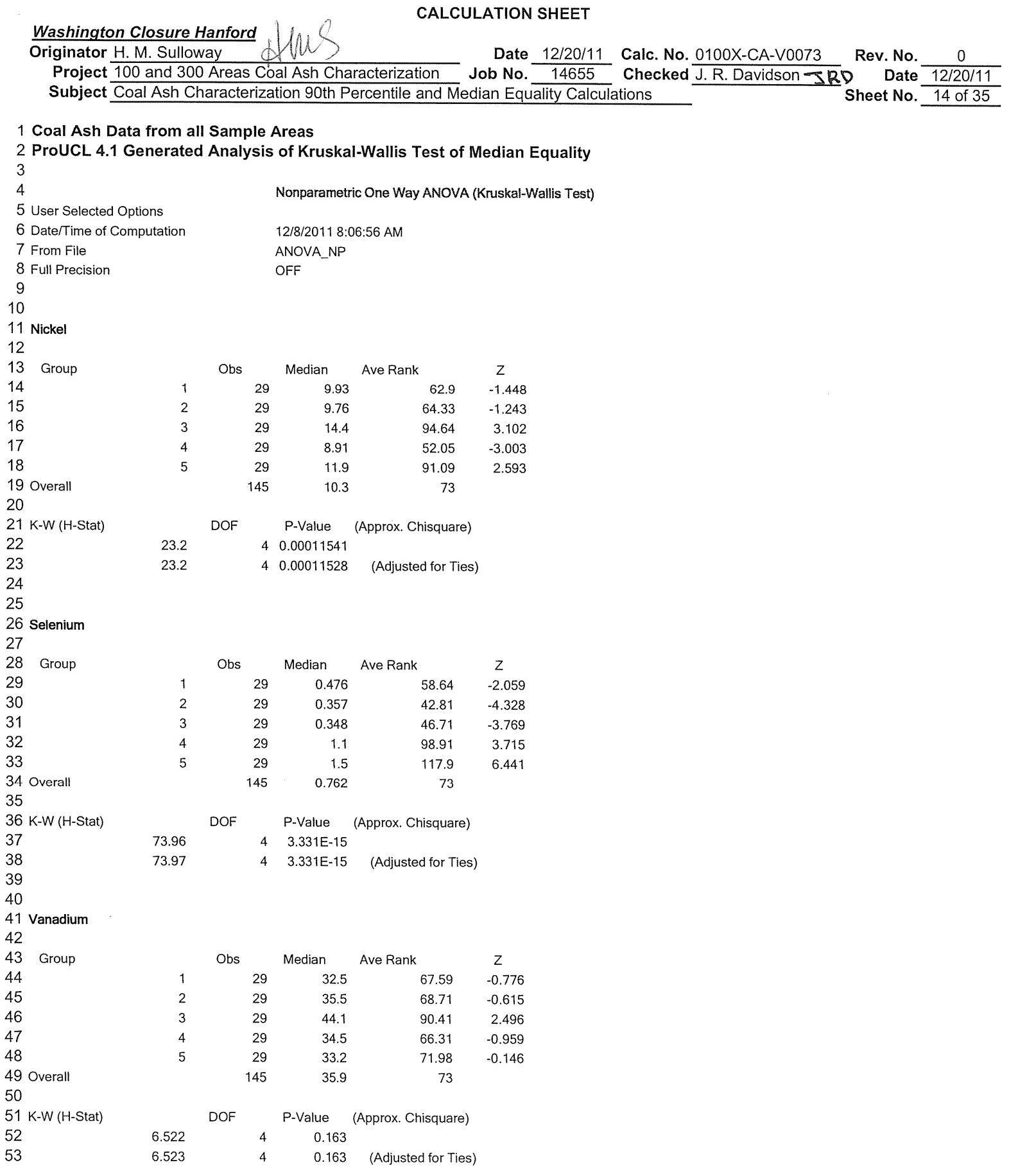




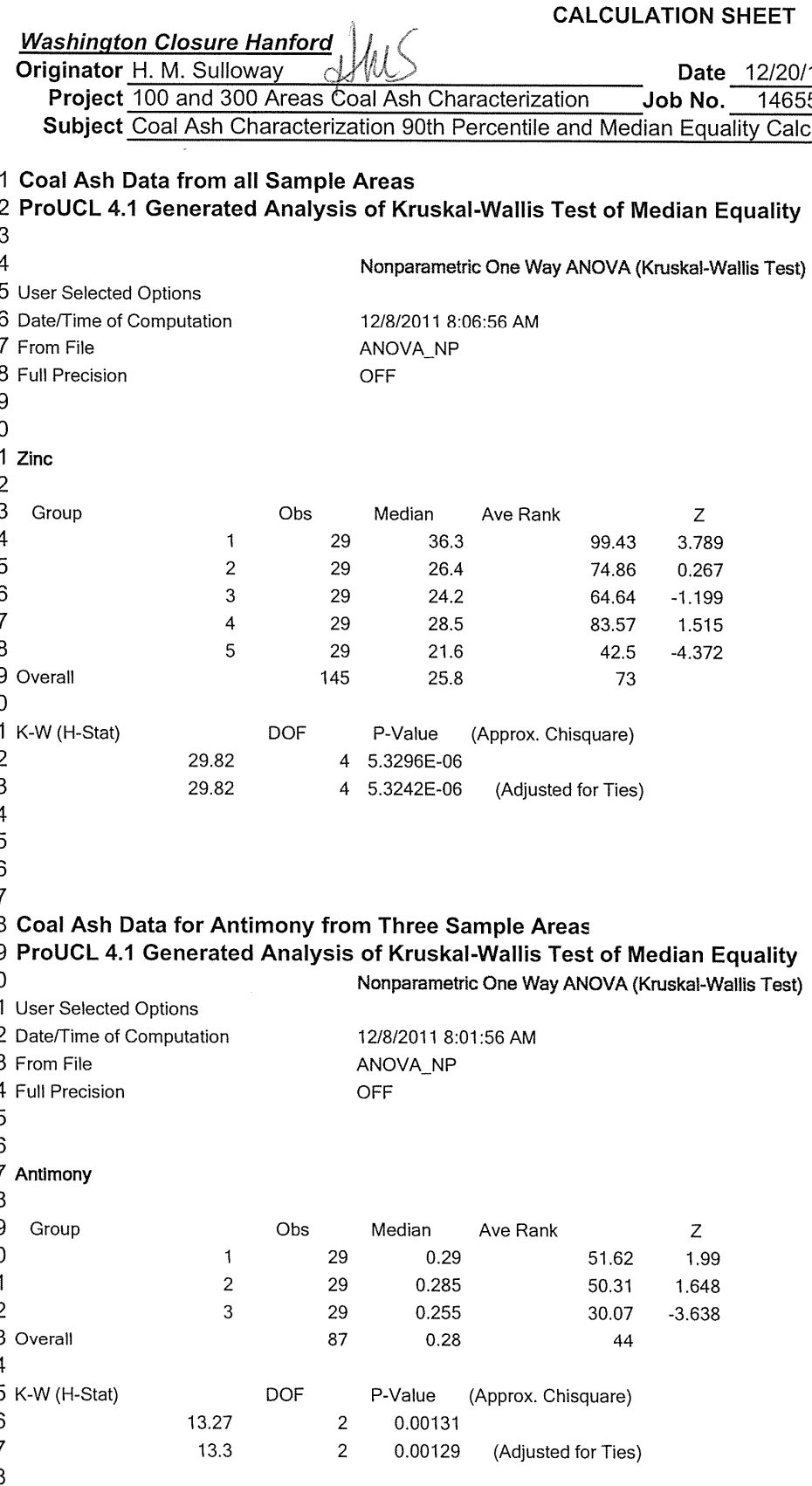


Washington Closure Hanford

\section{Originator H. M. Sulloway Wh}

Subject Coal Ash Characterization 90 oth Percentile and Median Equality Calculations
CALCULATION SHEET

Date $12 / 20 / 11$
Calc. No. 0100X-CA-V0073

Calc. No. $\frac{0100 X-C A-V 0073}{\text { Checked J. R. Davidson } 5 \text { RD }}$
Rev. No. $\frac{0}{12 / 20 / 1}$

\begin{tabular}{|c|c|c|c|c|c|c|c|c|c|c|c|c|c|c|c|c|c|c|c|c|c|c|c|c|c|c|c|c|}
\hline \multirow{3}{*}{$\begin{array}{l}2 \\
3\end{array}$} & \multirow{2}{*}{$\begin{array}{l}\text { Sample } \\
\text { Location }\end{array}$} & \multirow{2}{*}{\begin{tabular}{c|} 
HEIS \\
Number
\end{tabular}} & \multirow{2}{*}{$\begin{array}{c}\text { Sample } \\
\text { Date }\end{array}$} & \multicolumn{3}{|c|}{ Antimony } & \multicolumn{2}{|c|}{ Arsenic } & \multicolumn{2}{|c|}{ Barium } & \multicolumn{2}{|c|}{ Beryllium } & \multicolumn{3}{|c|}{ Boron } & \multirow{2}{*}{\multicolumn{3}{|c|}{ Cadmium }} & \multicolumn{2}{|c|}{ Chromium } & \multirow{2}{*}{\multicolumn{3}{|c|}{ Cobalt }} & \multicolumn{2}{|c|}{ Copper } & \multicolumn{3}{|c|}{ Lead } \\
\hline & & & & $\mathrm{mg} / \mathrm{kg}$ & & $\mathrm{PQL}$ & $\mathrm{mg} / \mathrm{kg}$ & \begin{tabular}{l|l}
$Q$ & $P Q L$ \\
\end{tabular} & $\mathrm{mg} / \mathrm{kg}$ & \begin{tabular}{l|l|}
$Q$ & $P Q L$ \\
\end{tabular} & $\mathrm{mg} / \mathrm{kg}$ & \begin{tabular}{l|l}
$Q$ & $P Q L$ \\
\end{tabular} & $\mathrm{mg} / \mathrm{kg}$ & Q & PQL & $\mathrm{mg} / \mathrm{kg}$ & & $\mathrm{PQL}$ & $\mathrm{mg} / \mathrm{kg}$ & \begin{tabular}{|l|l|}
$Q$ & $P Q L$ \\
\end{tabular} & $\mathrm{mg} / \mathrm{kg}$ & & & $\mathrm{mg} / \mathrm{kg}$ & \begin{tabular}{l|l}
$Q$ & $P Q L$ \\
\end{tabular} & $\mathrm{mg} / \mathrm{kg}$ & & $P Q L$ \\
\hline & & & & 0.588 & $\mathrm{U}$ & 0.59 & 5.85 & 0.98 & 1970 & 0.49 & 1.93 & 0.20 & 109 & & 1.96 & 0.198 & & 0.20 & 8.33 & 0.20 & 8.22 & & 1.96 & 39.2 & 0.98 & 2.89 & & 0.49 \\
\hline 5 & $100-\mathrm{B}-02$ & J1HHV5 & $4 / 27 / 11$ & 0.265 & B & 0.52 & 4.21 & 0.86 & 1660 & 0.43 & 1.44 & 0.17 & 88.4 & & 1.72 & 0.311 & & 0.17 & 8.03 & 0.17 & 4.49 & & 1.72 & 22.7 & 0.86 & 4.19 & & 0.43 \\
\hline 6 & $100-\mathrm{B}-03$ & J1HHV6 & $4 / 27 / 11$ & 0.566 & $U$ & 0.57 & 4.62 & 0.94 & 1620 & 0.47 & 1.21 & 0.19 & 130 & & 1.89 & 0.293 & & 0.19 & 9.87 & 0.19 & 6.47 & & 1.89 & 32.0 & 0.94 & 4.86 & & 0.5 \\
\hline & $100-\mathrm{B}-04$ & J1HHV7 & $4 / 27 / 11$ & 0.588 & $\mathrm{U}$ & 0.59 & 2.41 & 0.98 & 793 & 0.49 & 1.15 & 0.20 & 161 & & 1.96 & 0.175 & B & 0.20 & 8.46 & 0.20 & 4.68 & & 1.96 & 35.4 & 0.98 & 35.8 & & 0.5 \\
\hline 8 & $100-\mathrm{B}-05$ & J1HHV8 & $4 / 27 / 11$ & 0.304 & B & 0.57 & 4.46 & 0.94 & 824 & 0.47 & 1.23 & 0.19 & 328 & & 1.89 & 0.506 & & 0.19 & 16.2 & 0.19 & 4.12 & & 1.89 & 98.3 & 0.94 & 13.5 & & 0.5 \\
\hline 9 & $100-B-06$ & J1HHVg & $4 / 27 / 11$ & 0.566 & U & 0.57 & 5.37 & 0.94 & 1450 & 0.47 & 1.32 & 0.19 & 108 & & 1.89 & 0.243 & & 0.19 & 9.83 & 0.19 & 7.07 & & 1.89 & 28.6 & 0.94 & 3.84 & & 0.5 \\
\hline 10 & $100-B-07$ & J1HHWO & $4 / 27 / 11$ & 0.536 & U & 0.54 & 3.43 & 0.89 & 1160 & 0.45 & 1.16 & 0.18 & 76.5 & & 1.79 & 0.224 & & 0.18 & 8.47 & 0.18 & 5.31 & & 1.79 & 24.6 & 0.89 & 5.55 & & 0.5 \\
\hline 11 & $100-\mathrm{B}-08$ & J1HHW1 & $4 / 27 / 11$ & 0.517 & U & 0.52 & 4.76 & 0.86 & 2060 & 0.43 & 1.86 & 0.17 & 277 & & 1.72 & 0.215 & & 0.17 & 8.17 & 0.17 & 7.01 & & 1.72 & 39.5 & 0.86 & 2.85 & & 0.43 \\
\hline 12 & $100-B-09$ & J1HHW2 & $8 / 23 / 11$ & 0.469 & U & 0.47 & 4.28 & 0.78 & 1200 & 0.39 & 1.48 & 0.16 & 250 & & 1.56 & 0.205 & & 0.16 & 12.5 & 0.16 & 5.41 & & 1.56 & 32.4 & 0.78 & 6.08 & & 0.39 \\
\hline 13 & $100-\mathrm{B}-10$ & J1HHW3 & $4 / 27 / 11$ & 0.566 & U & 0.57 & 2.98 & 0.94 & 597 & 0.47 & 1.03 & 0.19 & 169 & & 1.89 & 0.117 & $B$ & 0.19 & 8.75 & 0.19 & 4.42 & & 1.89 & 28.8 & 0.94 & 5.59 & & 0.47 \\
\hline 14 & $100-\mathrm{B}-11$ & J1HHW4 & $4 / 27 / 11$ & 0.577 & U & 0.58 & 3.20 & 0.96 & 958 & 0.48 & 1.56 & 0.19 & 265 & & 1.92 & 0.221 & & 0.19 & 12.9 & 0.19 & 3.10 & & 1.92 & 23.7 & 0.96 & 6.05 & & 0.48 \\
\hline 15 & $100-B-12$ & J1HHW5 & $4 / 27 / 11$ & 0.577 & U & 0.58 & 2.63 & 0.96 & 696 & 0.48 & 1.54 & 0. & 256 & & 1.92 & 0.167 & B & 0.19 & 12.7 & 0.19 & 2.98 & & 1.92 & 22.2 & 0.96 & 5.80 & & 0.48 \\
\hline 16 & $100-B-13$ & J1HHW6 & $4 / 27 / 11$ & 0.505 & B & 0.54 & 7.36 & 0.89 & 2340 & 0.45 & 2.39 & 0.18 & 93.8 & & 1.79 & 0.518 & & 0.18 & 8.91 & 0.18 & 4.87 & & 1.79 & 25.4 & 0.89 & 3.72 & & 0.45 \\
\hline 17 & $100-\mathrm{B}-14$ & J1HHW7 & $8 / 23 / 11$ & 0.600 & $\bar{U}$ & 0.60 & 8.45 & 1.00 & 1170 & 0.50 & 1.24 & 0.20 & 169 & & 2.00 & 0.36 & & 0.20 & 11.5 & 0.20 & 4.59 & & 2.00 & 27.3 & 1.00 & 7.72 & & 0.50 \\
\hline 18 & $100-B-15$ & J1HHW 8 & $4 / 27 / 11$ & 0.545 & $U$ & 0.55 & 3.01 & 0.91 & 792 & 0.46 & 1.17 & 0.18 & 211 & & 1.82 & 0.182 & B & 0.18 & 10.3 & 0.18 & 4.88 & & 1.82 & 27.4 & 0.91 & 6.73 & & 0.46 \\
\hline 19 & Duplicate of & $\mathrm{J} 1 \mathrm{HJO4}$ & $4 / 27 / 11$ & 1.76 & u & 1.76 & 3.41 & 2.94 & 753 & 1.47 & 1.19 & 0.59 & 240 & & 5.88 & 0.240 & B & 0.59 & 11.3 & 0.59 & 4.56 & B & 5.88 & 26.8 & 2.94 & 7.10 & & 1.47 \\
\hline 20 & $\begin{array}{l}\text { JHAWW } \\
100-\mathrm{B}-16\end{array}$ & J1HHW9 & $4 / 26 / 11$ & 0.376 & U & 0.46 & 4.07 & 0.76 & 1360 & 0.38 & 1.50 & 0.15 & 129 & & 1.52 & 0.182 & & 0.15 & 6.74 & 0.15 & 3.55 & & 1.52 & 19.1 & 0.76 & 3.18 & & 0.38 \\
\hline 21 & $100-B-17$ & $\mathrm{~J} 1 \mathrm{HHXO}$ & $4 / 27 / 11$ & 0.484 & UJ & 0.48 & 2.50 & 0.81 & 556 & 0.40 & 1.31 & & 269 & & 1.61 & 0.221 & & 0.16 & 10.6 & & 2.51 & & 1.61 & 21.9 & 0.81 & 6.28 & & 0.40 \\
\hline 22 & $100-\mathrm{B}-18$ & $\mathrm{~J} 1 \mathrm{HHX} 1$ & $4 / 27 / 11$ & 0.818 & UJB & 1.58 & 5.69 & 2.63 & 599 & 1.32 & 1.36 & 0.5 & 156 & & 5.26 & 0.337 & $B$ & 0.53 & 10.5 & 0.53 & 3.97 & B & 5.26 & 25.6 & 2.63 & 6.58 & & 1.32 \\
\hline 23 & $100-\mathrm{B}-19$ & J1HHX2 & $8 / 23 / 11$ & 0.469 & u & 0.47 & 2.74 & 0.78 & 783 & 0.39 & 1.16 & 0.16 & 205 & & 1.56 & 0.265 & & 0.16 & 9.66 & 0.16 & 2.79 & & 1.56 & 20.9 & 0.78 & 10.7 & & 0.39 \\
\hline 24 & $100-\mathrm{B}-20$ & J1HHX3 & $8 / 23 / 11$ & 0.588 & U & 0.59 & 3.40 & 0.98 & 866 & 0.49 & 1.22 & 0.20 & 194 & & 1.96 & 0.187 & B & 0.20 & 11.1 & 0.20 & 4.88 & & 1.96 & 29.1 & 0.98 & 5.77 & & 0.49 \\
\hline 25 & $100-\mathrm{B}-21$ & J1HHX4 & $4 / 25 / 11$ & 0.918 & UJB & 1.55 & 4.76 & 2.59 & 406 & 1.29 & 1.12 & 0.52 & 145 & & 5.17 & 0.914 & & 0.52 & 14.4 & 0.52 & 3.06 & B & 5.17 & 25.0 & 2.59 & 18.4 & & 1.29 \\
\hline 26 & $100-B-22$ & J1HHX5 & $4 / 26 / 11$ & 0.416 & UJB & 0.57 & 1.33 & 0.94 & 184 & 0.47 & 0.632 & 0.19 & 66.3 & & 1.89 & 0.096 & $B$ & 0.19 & 4.76 & 0.19 & 1.00 & B & 1.89 & 8.60 & 0.94 & 6.50 & & 0.47 \\
\hline 27 & $100-\mathrm{B}-23$ & $\mathrm{~J} 1 \mathrm{HHX} 6$ & $4 / 26 / 11$ & 1.91 & $\mathrm{~J}$ & 1.70 & 9.59 & 2.83 & 2250 & 1.42 & 3.22 & 0.57 & 327 & & 5.66 & 0.899 & & 0.57 & 25.5 & 0.57 & 15.1 & & 5.66 & 94.3 & 2.83 & 19.0 & & 1.42 \\
\hline 28 & $100-\mathrm{B}-24$ & J1HHX7 & $4 / 26 / 11$ & 1.00 & UJB & 1.76 & 5.42 & 2.94 & 1310 & 1.47 & 1.60 & 0.59 & 192 & & 5.88 & 0.219 & $B$ & 0.59 & 13.3 & 0.59 & 5.84 & B & 5.88 & 36.7 & 2.94 & 10.6 & & 1.47 \\
\hline & $100-B-25$ & J1HHX8 & $4 / 25 / 11$ & 1.69 & BJ & 1.70 & 10.3 & 2.83 & 1420 & 1.42 & 1.65 & 0.57 & 107 & & 5.66 & 0.385 & B & 0.57 & 11.2 & 0.57 & 4.66 & B & 5.66 & 28.4 & 2.83 & 13.4 & & 1.42 \\
\hline 30 & Duplicate of & J1HJO3 & $4 / 25 / 11$ & 1.64 & u & 1.64 & 5.46 & 2.73 & 907 & 1.36 & 1.04 & 0.55 & 85.5 & & 5.45 & 0.278 & $B$ & 0.55 & 8.34 & 0.55 & 4.12 & B & 5.45 & 20.2 & 2.73 & 9.95 & & 1.36 \\
\hline 31 & $100-B-26$ & J1HHX9 & $8 / 23 / 11$ & 0.441 & $U$ & 0.44 & 3.14 & 0.74 & 1000 & 0.37 & 1.06 & 0.15 & 174 & & 1.47 & 0.354 & & 0.15 & 12.3 & 0.15 & 2.18 & & 1.47 & 17.7 & 0.74 & 12.2 & & 0.37 \\
\hline 32 & $100-B-27$ & J1HJOO & $4 / 27 / 11$ & 1.67 & U & 1.67 & 3.19 & 2.78 & 497 & 1.39 & 0.894 & & 92.6 & & 5.56 & 0.397 & B & 0.56 & 10.4 & 0.56 & 5.56 & U & 5.56 & 22.0 & 2.78 & 9.69 & & 1.39 \\
\hline 33 & $100-\mathrm{B}-28$ & J1HJO1 & $8 / 23 / 11$ & 0.259 & B & 0.48 & 4.16 & 0.79 & 969 & 0.40 & 1.18 & 0.16 & 267 & & 1.59 & 0.373 & & 0.16 & 13.2 & 0.16 & 2.69 & & 1.59 & 26.2 & 0.79 & 15.9 & & 40 \\
\hline 34 & $100-B-29$ & J1HJ02 & $8 / 23 / 11$ & 0.550 & & 0.44 & 5.94 & 0.73 & 1360 & 0.36 & 1.98 & 0.15 & 68.1 & & 1.45 & 0.251 & & 0.15 & 7.66 & 0.15 & 4.02 & & 1.45 & 22.8 & 0.73 & 3.87 & & 0.36 \\
\hline
\end{tabular}


Washinaton Closure Hanford

Originator H. M. Sulloway DVS

Project 100 and 300 Areas Coal Ash Characterization

Equality Calculations
CALCULATION SHEET

Date $\frac{12 / 20 / 11}{14655}$
Job No.

Calc. No. $0100 \mathrm{X}$-CA-V0073

Checked J.R. Davidson 5 (RD)

Rev. No.

Date $\frac{0}{12 / 20 / 1}$

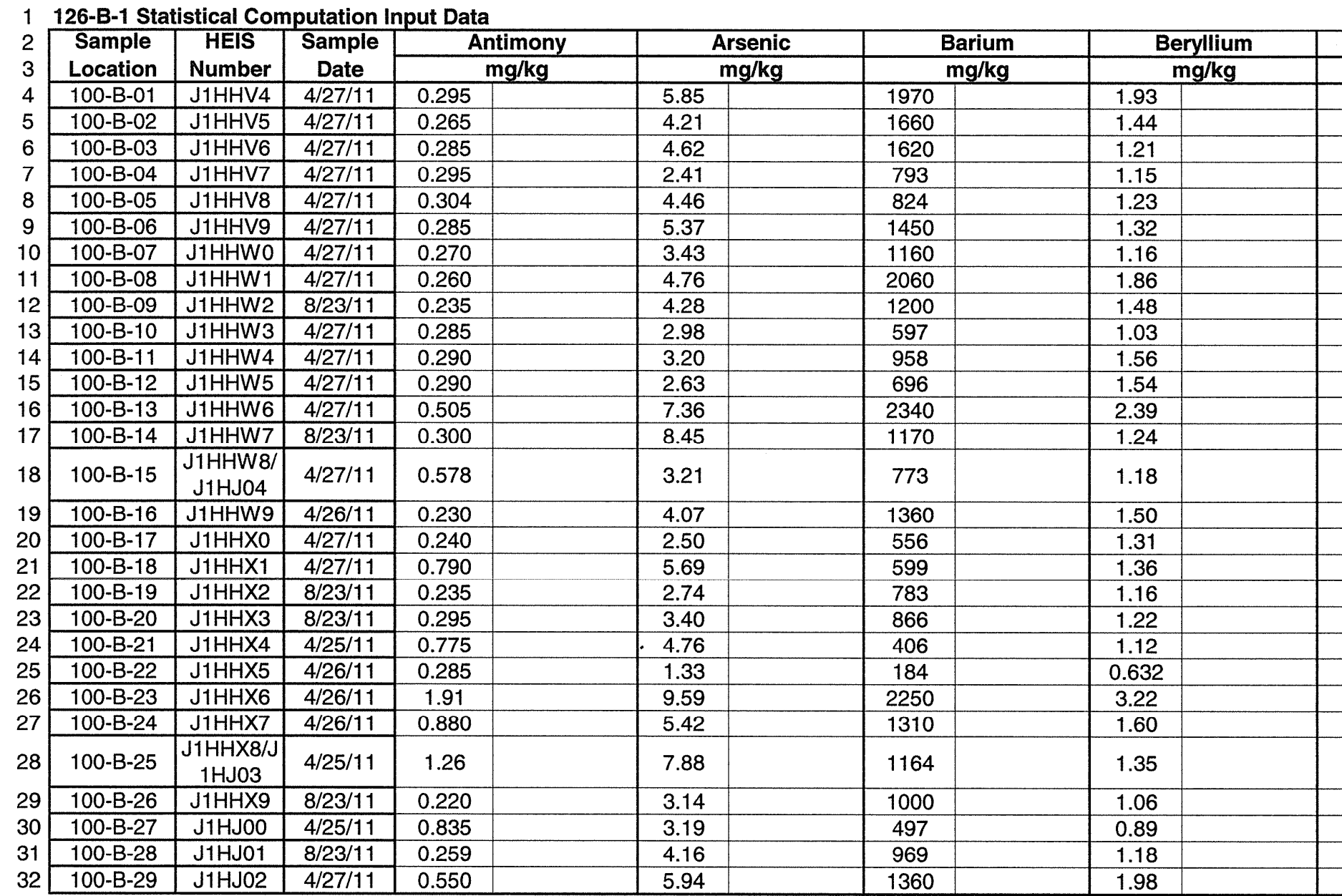

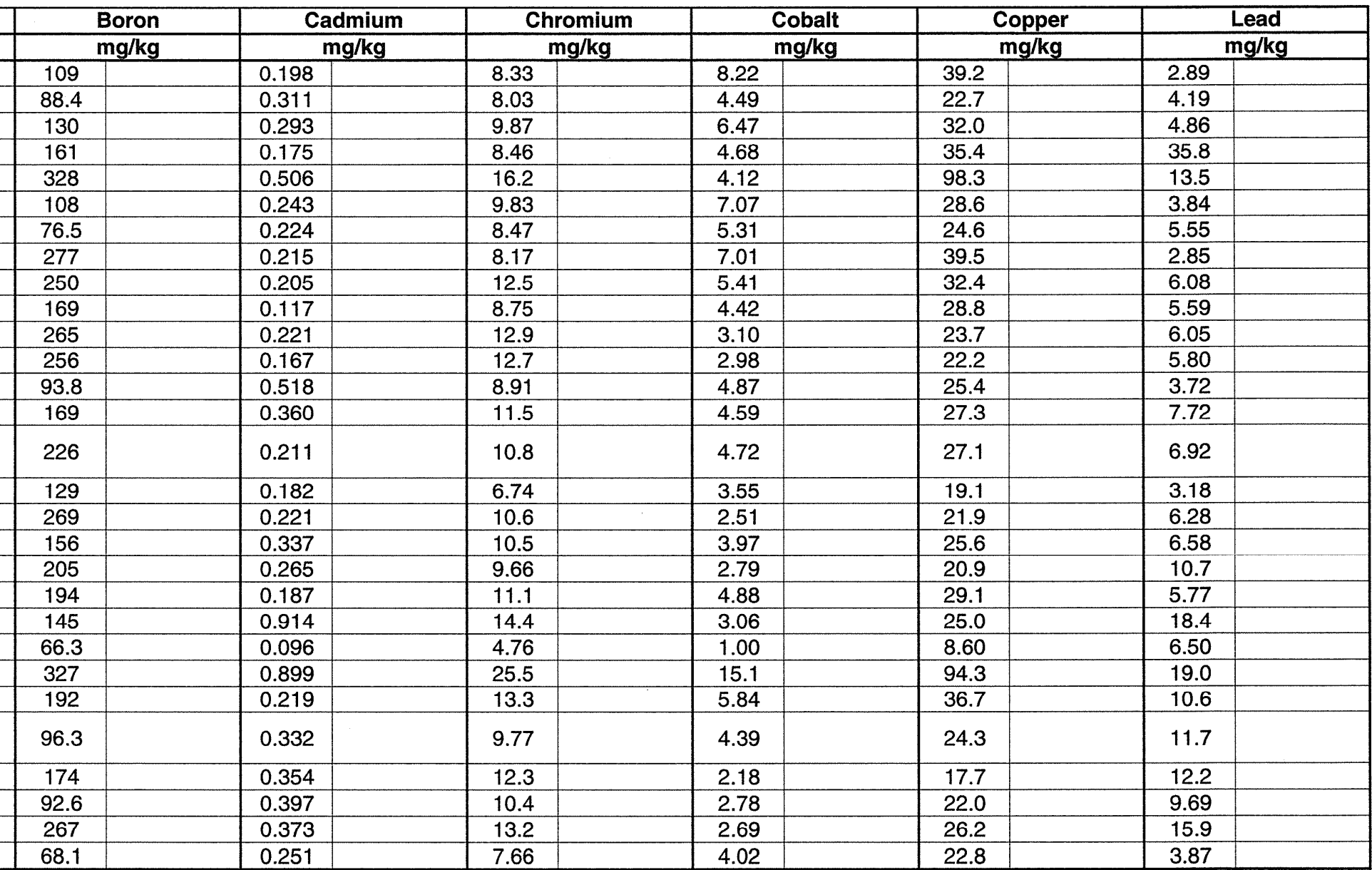


Washington Closure Hanford

CALCULATION SHEET

Originator H. M. Sulloway WW

Project 100 and 300 Areas Coal Ash Characterization

Subject Coal Ash Characterization 90th Percentile and Median Equality Calculations

Job No. 14655
Calc. No. 0100X-CA-V0073 Checked J. R. Davidson $5 R Q$
Rev. No. $\quad 0$

Date $12 / 20 / 11$

Sheet No. 18 of 35

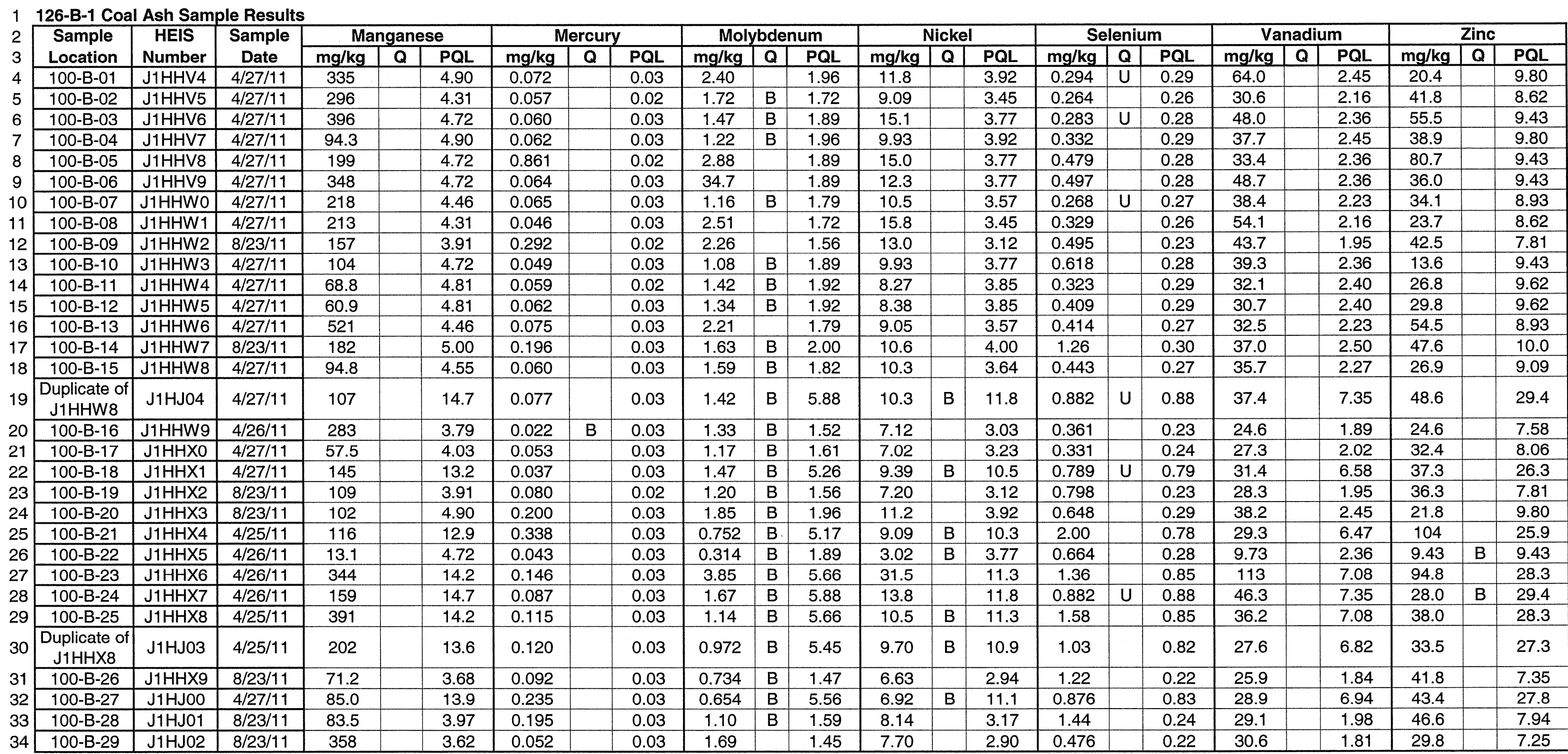


Washington Closure Hanford

Originator H. M. Sulloway dhes

Project 100 and 300 Areas Coal Ash Characterization

Subject Coal Ash Characterization 90th Percentile and Median Equality Calculations

Subject Coal Ash Characteriz

\begin{tabular}{l|l|l|l|l}
1 & \multicolumn{1}{c}{ 126-B-1 Statistical Computation Input Data } \\
\hline & Sample & HEIS & Sample & Manganese
\end{tabular}

\begin{tabular}{l|c|c|c|c|c}
\cline { 4 - 6 } 3 & Location & Number & Date & \multicolumn{2}{|c}{$\mathrm{mg} / \mathrm{kg}$} \\
\cline { 2 - 6 } & $100-\mathrm{B}-01$ & $\mathrm{J1HHV} 4$ & $4 / 27 / 11$ & 335 &
\end{tabular}

\begin{tabular}{|c|c|c|c|}
\hline $100-\mathrm{B}-02$ & J1HHV5 & $4 / 27 / 11$ & 296 \\
\hline
\end{tabular}

\begin{tabular}{|c|c|c|c|}
\hline 100-B-03 & J1HHV6 & $4 / 27 / 11$ & 396 \\
\hline
\end{tabular}

\begin{tabular}{|c|c|c|c|}
\hline 100-B-04 & J1HHV7 & $4 / 27 / 11$ & 94.3 \\
\hline
\end{tabular}

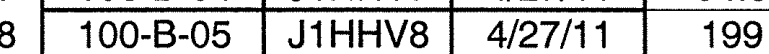

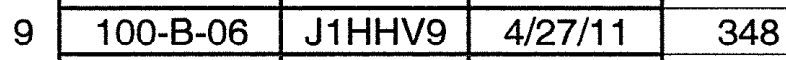

\begin{tabular}{|c|c|c|c|}
\hline $100-B-07$ & J1HHWO & $4 / 27 / 11$ & 218 \\
\hline
\end{tabular}

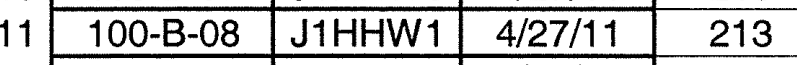

\begin{tabular}{l|c|c|c|c|}
12 & $100-\mathrm{B}-09$ & $\mathrm{~J} 1 \mathrm{HHW} 2$ & $8 / 23 / 11$ & 157 \\
\cline { 2 - 5 } 13 & $100-\mathrm{B}-10$ & $\mathrm{~J} 1 \mathrm{HHW} 3$ & $4 / 27 / 11$ & 104 \\
\hline
\end{tabular}

\begin{tabular}{l|c|c|c|c|}
13 & $100-\mathrm{B}-10$ & J1HHW3 & $4 / 27 / 11$ & 104 \\
\cline { 2 - 5 } 14 & $100-\mathrm{B}-11$ & J1HHW4 & $4 / 27 / 11$ & 68.8 \\
\hline & $100-\mathrm{B}-12$ & J1HHW5 & $4 / 27 / 11$ & 60.9 \\
\hline
\end{tabular}

\begin{tabular}{c|c|c|c|c|}
14 & $100-\mathrm{B}-11$ & J1HHW4 & $4 / 27 / 11$ & 68.8 \\
\hline $100-\mathrm{B}-12$ & J1HHW5 & $4 / 27 / 11$ & 60. \\
\hline
\end{tabular}

\begin{tabular}{l|c|c|c|c|}
15 & $100-B-12$ & J1HHW5 & $4 / 27 / 11$ & 60.9 \\
\cline { 2 - 5 } 16 & $100-B-13$ & J1HHW6 & $4 / 27 / 11$ & 521 \\
\hline
\end{tabular}

\begin{tabular}{c|c|c|c|c|}
\hline 17 & $100-B-14$ & J1HHW7 & $8 / 23 / 11$ & 182 \\
\hline
\end{tabular}

\begin{tabular}{l|c|c|c|c|}
\hline 18 & $100-\mathrm{B}-15$ & $\begin{array}{c}\text { J1HHW8/ } \\
\text { J1HJ04 }\end{array}$ & $4 / 27 / 11$ & 101 \\
\hline & &
\end{tabular}

\begin{tabular}{|c|c|c|c|}
\hline $100-B-16$ & J1HHW9 & $4 / 26 / 11$ & 283 \\
\hline $100-B-17$ & J1HHX0 & $4 / 27 / 11$ & 57.5 \\
\hline
\end{tabular}

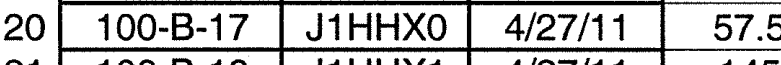

\begin{tabular}{|c|c|c|c|}
\hline $100-B-18$ & $J 1 H H X 1$ & $4 / 27 / 11$ & 145 \\
\hline
\end{tabular}

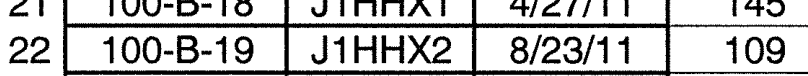

\begin{tabular}{l|c|c|c|c|}
\hline $100-\mathrm{B}-20$ & $\mathrm{~J} 1 \mathrm{HHX} 3$ & $8 / 23 / 11$ & 102 \\
\hline & $100-\mathrm{B}-21$ & $\mathrm{~J} 1 \mathrm{HHX}$ & $4 / 25 / 1$ & 116 \\
\hline
\end{tabular}

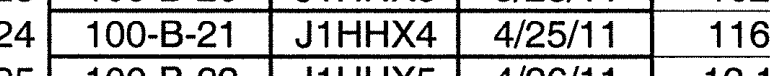

\begin{tabular}{l|c|c|c|c|}
25 & $100-B-22$ & J1HHX5 & $4 / 26 / 11$ & 13.1 \\
\hline $100-B-23$ & J1HHX6 & $4 / 26 / 11$ & 344 \\
\hline
\end{tabular}

\begin{tabular}{l|c|c|c|c|}
26 & $100-\mathrm{B}-23$ & $\mathrm{~J} 1 \mathrm{HHX} 6$ & $4 / 26 / 11$ & 344 \\
\cline { 2 - 5 } & $100-\mathrm{B}-24$ & $\mathrm{~J} 1 \mathrm{HHX7}$ & $4 / 26 / 11$ & 159
\end{tabular}

\begin{tabular}{l|c|c|c|c}
28 & $100-B-25$ & J1HHX8/J & $4 / 25 / 11$ & 297
\end{tabular}

\begin{tabular}{c|c|c|c|} 
& $1 \mathrm{HJO3}$ & & \\
\hline $100-\mathrm{B}-26$ & $\mathrm{~J} 1 \mathrm{HHX9}$ & $8 / 23 / 11$ & 71.2 \\
\hline $100-\mathrm{B}-27$ & $\mathrm{~J} H \mathrm{HJO0}$ & $4 / 25 / 11$ & 85.0
\end{tabular}

30 \begin{tabular}{|c|c|c|c|}
\hline $100-\mathrm{B}-27$ & $\mathrm{~J} 1 \mathrm{HJ} 00$ & $4 / 25 / 11$ & 85.0 \\
\hline $100-\mathrm{B}$ & $\mathrm{J} 1 \mathrm{HJ01}$ & $8 / 23 / 11$ & 83.5
\end{tabular}

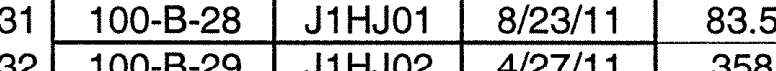
33

34

1.69

CALCULATION SHEET

Date 12/20/11 Job No. 14655
Calc. No. 0100X-CA-V0073 Checked J.R. Davidson JRY
Rev. No. 0

Date $12 / 20111$ Sheet No. $\frac{12 / 20 / 11}{19 \text { of } 35}$

\begin{tabular}{|c|c|c|c|c|c|c|}
\hline \multirow[t]{2}{*}{$\frac{\text { Mercury }}{\mathrm{mg} / \mathrm{kg}}$} & $\begin{array}{c}\text { Molybdenum } \\
\mathrm{mg} / \mathrm{kg}\end{array}$ & $\frac{\text { Nickel }}{\mathrm{ma} / \mathrm{kg}}$ & $\frac{\text { Selenium }}{\mathrm{ma} / \mathrm{kg}}$ & $\frac{\text { Vanadium }}{\mathrm{ma} / \mathrm{kg}}$ & \multirow{2}{*}{\multicolumn{2}{|c|}{$\frac{\text { Zinc }}{\mathrm{mg} / \mathrm{kg}}$}} \\
\hline & 240 & 118 & 10 & 610 & & \\
\hline & & & & & & \\
\hline $0.05 \pi$ & 1.72 & 9.09 & 0.264 & 30.6 & 41.8 & \\
\hline 0.060 & 1.47 & 15.1 & 0.140 & 48.0 & 55.5 & \\
\hline 0.062 & 1.22 & 9.93 & 0.332 & 37.7 & 38.9 & \\
\hline 0.861 & 2.88 & 15.0 & 0.479 & 33.4 & 80.7 & \\
\hline 0.064 & 34.7 & 12.3 & 0.497 & 48.7 & 36.0 & \\
\hline 0.065 & 1.16 & 10.5 & 0.135 & 38.4 & 34.1 & \\
\hline 0.046 & 2.51 & 15.8 & 0.329 & 54.1 & 23.7 & \\
\hline 0.292 & 2.26 & 13.0 & 0.495 & 43.7 & 42.5 & \\
\hline 0.049 & 1.08 & 9.93 & 0.618 & 39.3 & 13.6 & \\
\hline 0.059 & 1.42 & 8.27 & 0.323 & 32.1 & 26.8 & \\
\hline 0.062 & 1.34 & 8.38 & 0.409 & 30.7 & 29.8 & \\
\hline 0.075 & 2.21 & 9.05 & 0.414 & 32.5 & 54.5 & \\
\hline 0.196 & 1.63 & 10.6 & 1.26 & 37.0 & 47.6 & \\
\hline 0.069 & 1.51 & 10.3 & 0.442 & 36.6 & 37.8 & \\
\hline 0.022 & 1.33 & 7.12 & 0.361 & 24.6 & 24.6 & \\
\hline 0.053 & 1.17 & 7.02 & 0.331 & 27.3 & 32.4 & \\
\hline 0.037 & 1.47 & 9.39 & 0.395 & 31.4 & 37.3 & \\
\hline 0.080 & 1.20 & 7.20 & 0.798 & 28.3 & 36.3 & \\
\hline 0.200 & 1.85 & 11.2 & 0.648 & 38.2 & 21.8 & \\
\hline 0.338 & 0.752 & 9.09 & 2.00 & 29.3 & 104 & \\
\hline 0.043 & 0.314 & 3.02 & 0.664 & 9.73 & 9.43 & \\
\hline 0.146 & 3.85 & 31.5 & 1.36 & 113 & 94.8 & \\
\hline 0.087 & 1.67 & 13.8 & 0.440 & 46.3 & 28.0 & \\
\hline 0.118 & 1.06 & 10.1 & 1.31 & 31.9 & 35.8 & \\
\hline 0.092 & 0.734 & 6.63 & 1.22 & 25.9 & 41.8 & \\
\hline 0.235 & 0.654 & 6.92 & 0.876 & 28.9 & 43.4 & \\
\hline 0.195 & 1.10 & 8.14 & 1.44 & 29.1 & 46.6 & \\
\hline 0.052 & 1.69 & 7.70 & 0.476 & 30.6 & 29.8 & \\
\hline
\end{tabular}

1.44
0.476

30.1

46.6
29.8 
Washington Closure Hanford

Originator H. M. Sulloway U/WS

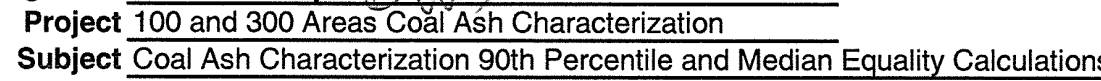

CALCULATION SHEET

$\begin{aligned} & \text { Date } 12 / 20 / 11 \\ & \text { Job No. } \frac{14655}{145}\end{aligned}$ Checked J. R. Davidson RQ R

Rev. No. 0

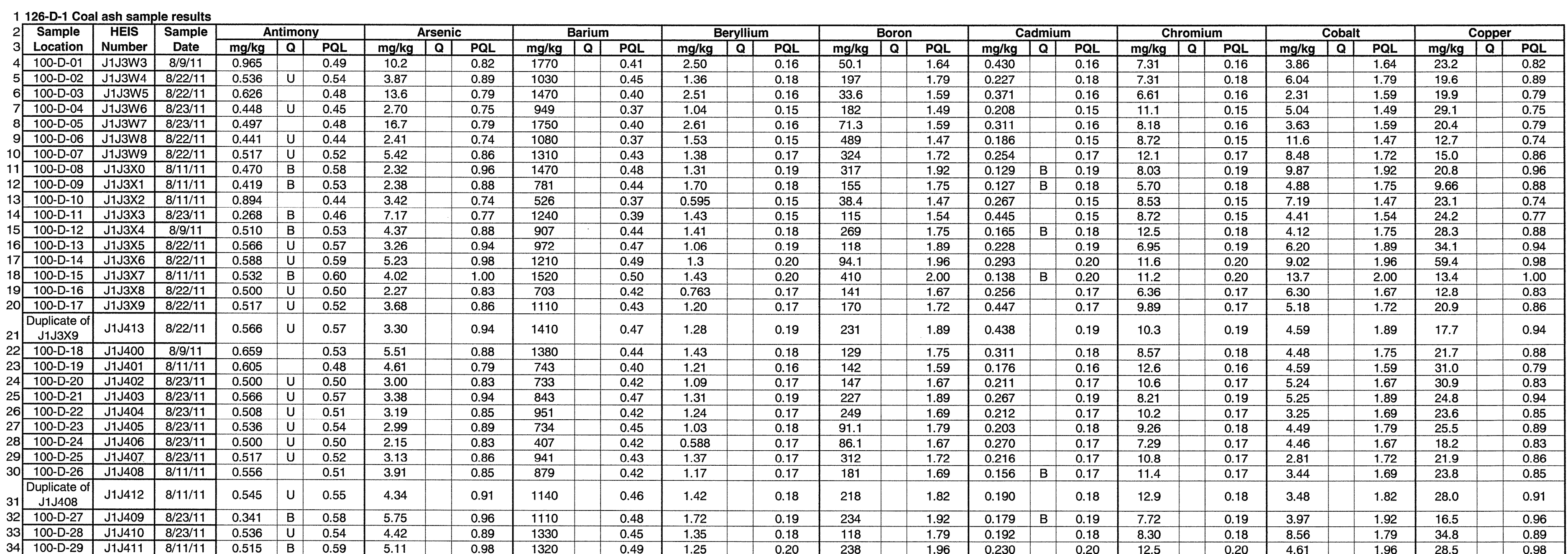


Washington Closure Hanford

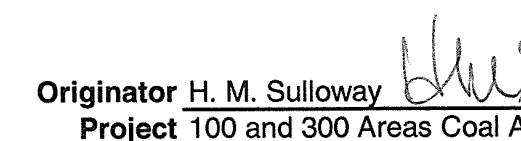

Project 100 and 300 Areas Coal Ash Characterization $\begin{array}{ll}\text { Date } \frac{12 / 20 / 11}{14655} & \begin{array}{l}\text { Calc. No. } 0100 X-C A-V 0073 \\ \text { Checked J. R. Davidson } \Sigma\end{array} \\ \text { Job No. } & \end{array}$

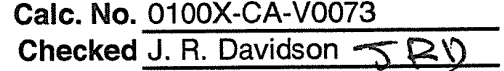

Rev. No. $\frac{0}{\text { Date }} \frac{12 / 20 / 11}{21 \text { of } 35}$

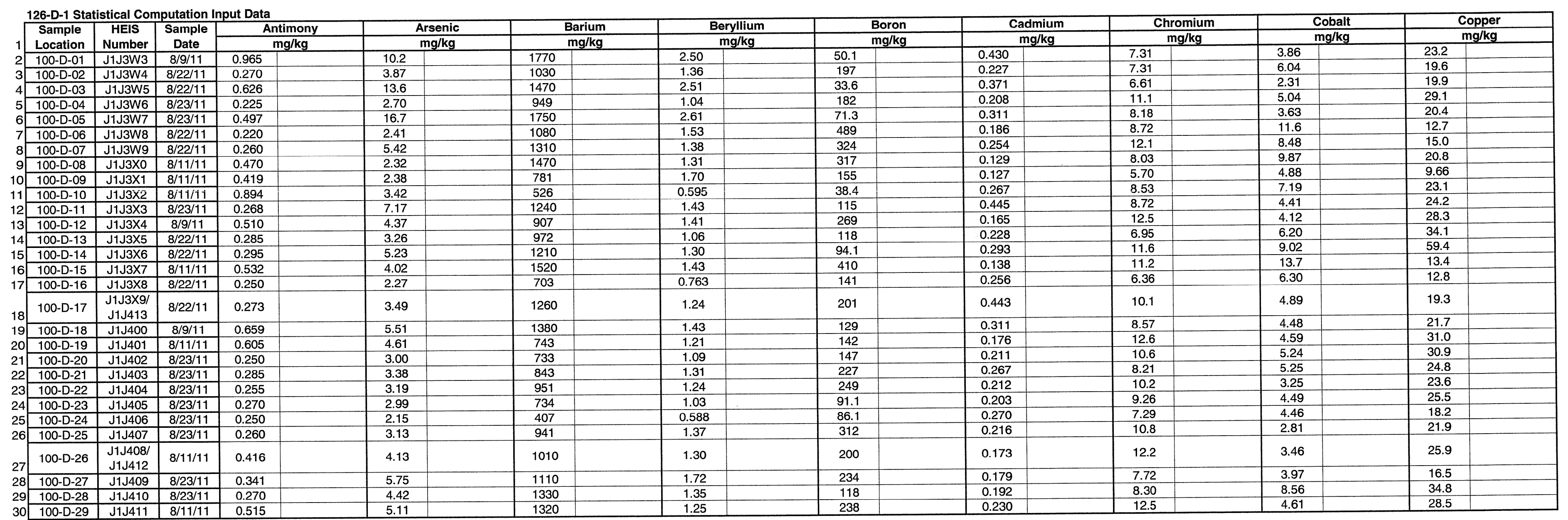


Washington Closure Hanford

Originator H.M. Sulloway $\mathrm{NWS}$

Project
Subject $\frac{100 \text { and } 300 \text { Areas Coal Ash Characterization }}{\text { Coal Ash Characterization } 90 \text { th Percentile and Median Equality Calculations }}$
CALCULATION SHEET

Date $\frac{12 / 20 / 11}{14655} \quad$ Calc. No. 0100X-CA-V0073

Job No. $\frac{14675}{14655} \quad$ Checked J. R. Davidson I R
Rev. No.

Date $\frac{12 / 20 / 11}{2200}$

\begin{tabular}{|c|c|c|c|c|c|c|c|c|c|c|c|c|c|c|c|c|c|c|c|c|c|c|c|c|c|c|c|c|c|c|}
\hline \multirow{3}{*}{\multicolumn{2}{|c|}{\begin{tabular}{l|l}
2 & Sample \\
3 & Location \\
\end{tabular}}} & \multirow{2}{*}{\begin{tabular}{c|} 
HEIS \\
Numbe \\
\end{tabular}} & \multirow{3}{*}{$\begin{array}{c}\text { Sample } \\
\text { Date } \\
\end{array}$} & \multicolumn{3}{|c|}{ Lead } & \multicolumn{3}{|c|}{ Manganese } & \multicolumn{3}{|c|}{ Mercury } & \multirow{2}{*}{\multicolumn{3}{|c|}{\begin{tabular}{c|c|c|}
\multicolumn{3}{c}{ Molybdenum } \\
$\mathrm{ma} / \mathrm{kg}$
\end{tabular}}} & \multirow{2}{*}{\multicolumn{3}{|c|}{\begin{tabular}{|c|c|c|}
\multicolumn{3}{|c}{ Nickel } \\
$\mathrm{mg} / \mathrm{kg}$ & $\mathrm{Q}$ & $\mathrm{PQL}$ \\
\end{tabular}}} & \multicolumn{3}{|c|}{$\begin{array}{l}\text { Selenium } \\
\end{array}$} & \multicolumn{3}{|c|}{$\begin{array}{l}\text { Thallium } \\
\end{array}$} & \multicolumn{3}{|c|}{ Vanadium } & \multicolumn{3}{|c|}{ Zinc } \\
\hline & & & & $\mathrm{mg} / \mathrm{kg}$ & $Q$ & PQL & $\mathrm{mg} / \mathrm{kg}$ & Q & $P Q L$ & $\mathrm{mg} / \mathrm{kg}$ & $\mathbf{Q}$ & PQL & & Q & $\mathrm{PQL}$ & & Q & & $\mathrm{mg} / \mathrm{kg}$ & Q & $\mathrm{PQL}$ & $\mathrm{mg} / \mathrm{kg}$ & Q & $\mathrm{PQL}$ & $\mathrm{mg} / \mathrm{kg}$ & Q & $\mathrm{PQL}$ & $\mathrm{mg} / \mathrm{kg}$ & & $P Q L$ \\
\hline 4 & & & & 3.91 & & 0.41 & 689 & & 4.10 & 0.014 & $B$ & 0.03 & 1.89 & & 1.64 & 6.33 & & 3.28 & 0.246 & $u$ & 0.25 & 0.410 & $\bar{U}$ & 0.41 & 27.9 & & 2.05 & 75.7 & & 8.20 \\
\hline & $100-D-02$ & J1J3W4 & $8 / 22 / 11$ & 3.02 & & 0.45 & 287 & & 4.46 & 0.050 & & 0.02 & 1.75 & B & 1.79 & 9.76 & & 3.57 & 0.280 & & 0.27 & 0.446 & $\mathrm{U}$ & 0.45 & 33.4 & & 2.23 & 24.4 & & 8.93 \\
\hline & 100-D-03 & J1J3W5 & $8 / 22 / 11$ & 3.71 & & 0.40 & 890 & & 3.97 & 0.031 & & 0.03 & 1.54 & B & 1.59 & 4.63 & & 3.17 & 0.398 & & 0.24 & 0.397 & $U$ & 0.40 & 22.2 & & 1.98 & 31.4 & & 7.94 \\
\hline & $100-D-04$ & J1J3W6 & $8 / 23 / 11$ & 6.19 & & 0.37 & 163 & & 3.73 & 0.024 & $B$ & 0.03 & 1.37 & B & 1.49 & 10.4 & & 2.99 & 0.286 & & 0.22 & 0.373 & $U$ & 0.37 & 47.6 & & 1.87 & 33.9 & & 7.46 \\
\hline 8 & $100-D-05$ & J1J3W7 & $8 / 23 / 11$ & 4.05 & & 0.40 & 645 & & 3.97 & 0.030 & & 0.03 & 4.42 & & 1.59 & 6.83 & & 3.17 & 0.357 & & 0.24 & 0.397 & $U$ & 0.40 & 26.4 & & 1.98 & 43.8 & & 7.94 \\
\hline 9 & $100-D-06$ & J1J3W8 & $8 / 22 / 11$ & 1.91 & & 0.37 & 399 & & 3.68 & 0.139 & & 0.03 & 2.87 & & 1.47 & 16.8 & & 2.94 & 0.221 & $U$ & 0.22 & 0.368 & $u$ & 0.37 & 25.7 & & 1.84 & 16.5 & & 7.35 \\
\hline 10 & $100-D-07$ & J1J3W9 & $8 / 22 / 11$ & 3.61 & & 0.43 & 271 & & 4.31 & 0.034 & & 0.03 & 1.57 & B & 1.72 & 13.8 & & 3.45 & 0.892 & & 0.26 & 0.431 & $U$ & 0.43 & 31.8 & & 2.16 & 19.0 & & 8.62 \\
\hline $\begin{array}{l}11 \\
12\end{array}$ & $100-D-08$ & J1J3X0 & $8 / 11 / 11$ & 1.90 & & 0.48 & 244 & & 4.81 & 0.119 & & 0.03 & 2.10 & & 1.92 & 14.9 & & 3.85 & 0.288 & $\mathrm{U}$ & 0.29 & 0.481 & $U$ & 0.48 & 27.6 & & 2.40 & 12.1 & & 9.62 \\
\hline 12 & 100-D-09 & J1 $133 \times 1$ & $8 / 11 / 11$ & 2.57 & & 0.44 & 161 & & 4.39 & 0.020 & $B$ & 0.03 & 1.16 & B & 1.75 & 7.42 & & 3.51 & 0.570 & & 0.26 & 0.439 & U & 0.44 & 20.9 & & 2.19 & 15.0 & & 8.77 \\
\hline & $100-D-10$ & $\mathrm{~J} 1 \mathrm{~J} 3 \mathrm{X}$ & $8 / 11 / 11$ & 9.50 & & 0.37 & 262 & & 3.68 & 0.012 & B & 0.02 & 0.86 & $B$ & 1.47 & 10. & & 2.94 & 0.221 & $U$ & 0.22 & 0.242 & B & 0.37 & 52.5 & & 1.84 & 69.7 & & 7.35 \\
\hline 14 & $100-D-11$ & $\mathrm{J1} \sqrt{\mathrm{J} X 3}$ & $8 / 23 / 11$ & 4.09 & & 0.39 & 366 & & 3.85 & 0.054 & & 0.03 & 1.49 & $B$ & 1.5 & & & 3.08 & 0.708 & & & 0.385 & $U$ & 0.39 & 36.4 & & 1.92 & 50.5 & & 7.69 \\
\hline 15 & $100-D-12$ & $\sqrt{\mathrm{J} 1 \mathrm{~J} 3 \mathrm{X} 4}$ & $8 / 9 / 11$ & 15.1 & & 0.44 & 108 & & 4.39 & 0.054 & & 0.03 & 1.39 & B & 1.75 & 9.17 & & 3.51 & 0.454 & & 0.26 & 0.439 & U & 0.44 & 38.9 & & 2.19 & 23.4 & & 8.77 \\
\hline 16 & $100-D-13$ & $\sqrt{\mathrm{J} 1 \mathrm{~J} 3 \times 5}$ & $8 / 22 / 11$ & 2.42 & & 0.47 & 208 & & 4.72 & 0.046 & & 0.03 & 1.66 & B & 1.89 & 11.8 & & 3.77 & 0.283 & $\mathrm{U}$ & 0.28 & 0.472 & U & 0.47 & 59.7 & & 2.36 & 26.4 & & 9.43 \\
\hline 17 & $100-D-14$ & J1J3X6 & $8 / 22 / 11$ & 3.59 & & 0.49 & 254 & & 4.90 & 0.053 & & 0.03 & 2.69 & & 1.96 & 20.5 & & 3.92 & 0.294 & $\mathrm{U}$ & 0.29 & 0.490 & $U$ & 0.49 & 77.5 & & 2.45 & 27.4 & & 9.80 \\
\hline & $100-D-15$ & \begin{tabular}{|l|l}
$11 \sqrt{ } 3 \times 7$ \\
\end{tabular} & $8 / 11 / 11$ & 3.15 & & 0.50 & 411 & & 5.00 & 0.054 & & 0.03 & 2.86 & & 2.00 & 17.5 & & 4.00 & 0.353 & & 0.30 & 0.500 & $U$ & 0.50 & 26.6 & & 2.50 & 11.8 & & 10.00 \\
\hline 19 & $100-D-16$ & J1J3X8 & $8 / 22 / 11$ & 2.70 & & 0.42 & 216 & & 4.17 & 0.042 & & 0.03 & 1.06 & $B$ & 1.67 & 11.2 & & 3.33 & 0.926 & & 0.25 & 0.417 & U & 0.42 & 27.7 & & 2.08 & 31.6 & & 8.33 \\
\hline 20 & $100-D-17$ & J1J3X9 & $8 / 22 / 11$ & 10.4 & & 0.43 & 248 & & 4.31 & 0.129 & & 0.02 & 1.53 & $B$ & 1.72 & 9.91 & & 3.45 & 0.425 & & 0.26 & 0.431 & $U$ & 0.43 & 34.9 & & 2.16 & 65.2 & & 8.62 \\
\hline 21 & $\begin{array}{c}\text { Duplicate of } \\
\text { J1J3 } \times 9\end{array}$ & J1J413 & $8 / 22 / 11$ & 8.56 & & 0.47 & 228 & & 4.72 & 0.248 & & 0.03 & 1.39 & $B$ & 1.89 & 12.1 & & 3.77 & 0.511 & & 0.28 & 0.472 & u & 0.47 & 38.5 & & 2.36 & 56.6 & & 9.43 \\
\hline 22 & $100-D-18$ & $\mathrm{~J} 1 \mathrm{~J} 400$ & $8 / 9 / 11$ & 4.27 & & 0.44 & 295 & & 4.39 & 0.040 & & 0.03 & 1.43 & B & 1.75 & 9.23 & & 3.51 & 0.263 & $U$ & 0.26 & 0.439 & $U$ & 0.44 & 31.8 & & 2.19 & 55.5 & & 8.77 \\
\hline & 100-D-19 & $\mathrm{J1} J 401$ & $8 / 11 / 11$ & 16.8 & & 0.40 & 122 & & 3.97 & 0.074 & & 0.03 & 1.03 & B & 1.59 & 10.5 & & 3.17 & 0.995 & & 0.24 & 0.198 & $B$ & 0.40 & 38.5 & & & & & 7.94 \\
\hline & $100-\mathrm{D}-20$ & $\mathrm{~J} 1 \mathrm{~J} 402$ & $8 / 23 / 11$ & 8.68 & & 0.42 & 141 & & 4.1 & 0.056 & & 0.03 & 1.1 & $B$ & 1.6 & 10. & & 3.33 & 0.519 & & 0.25 & 0.41 & $U$ & 0.42 & 45.1 & & 2.0 & & & 8.33 \\
\hline 25 & $100-D-21$ & $\mathrm{J1} \mathrm{J} 403$ & $8 / 23 / 11$ & 3.65 & & 0.47 & 265 & & 4.7 & 0.034 & & 0.03 & 1.5 & B & 1.8 & 10. & & 3.77 & 0.355 & & 0.28 & & $U$ & 0.47 & & & 2.3 & 39 & & 9.43 \\
\hline 26 & 100-D-22 & $\mathrm{J} 1 \mathrm{~J} 404$ & $8 / 23 / 11$ & 10.1 & & 0.42 & 135 & & 4.24 & 0.114 & & 0.03 & 1.3 & B & 1.6 & 7.9 & & 3.39 & 0.749 & & 0.25 & 0.4 & $U$ & 0.42 & 33.6 & & 2.12 & & & 8.47 \\
\hline 27 & $100-D-23$ & $\mathrm{~J} 1 \mathrm{~J} 405$ & $8 / 23 / 11$ & 6.44 & & 0.45 & 151 & & 4.46 & 0.05 & & 0.03 & 0.863 & B & 1.7 & 7.8 & & 3.57 & 0.929 & & 0.27 & 0.4 & $U$ & 0.45 & & & 2.23 & & & 8.93 \\
\hline 28 & $100-D-24$ & J1J406 & $8 / 23 / 11$ & 5.01 & & 0.42 & 208 & & 4.1 & & & & 0.7 & $B$ & 1.6 & 8.4 & & 3.33 & 0.306 & & 0.25 & 0.417 & U & & 35.5 & & 2.08 & & & 8.33 \\
\hline 29 & 100-D-25 & $\mathrm{J} 1 \mathrm{~J} 407$ & $8 / 23 / 11$ & 9.61 & & 0.43 & 134 & & 4.3 & 0.018 & $B$ & 0.0 & 1.2 & B & & 6.7 & & 3.45 & 0.392 & & 0.26 & 0.431 & U & 0.43 & 33.3 & & 2.16 & 21.8 & & 8.62 \\
\hline 30 & $100-D-26$ & $\mathrm{J1J} 408$ & $8 / 11 / 11$ & 13.2 & & 0.42 & 105 & & 4.24 & 0.061 & & 0.03 & 1.11 & $B$ & 1.69 & 7.57 & & 3.39 & 0.769 & & 0.25 & 0.424 & $U$ & 0.42 & 33.2 & & 2.12 & 15.2 & & 8.47 \\
\hline 31 & $\begin{array}{l}\text { Duplicate of } \\
\mathrm{J} 1 \mathrm{~J} 408\end{array}$ & $\mathrm{~J} 1 \mathrm{~J} 412$ & $8 / 11 / 11$ & 16.4 & & 0.46 & 137 & & 4.55 & 0.048 & & 0.03 & 1.27 & B & 1.82 & 8.50 & & 3.64 & 1.14 & & 0.27 & 0.455 & $u$ & 0.46 & 41.3 & & 2.27 & 22.9 & & 9.09 \\
\hline 32 & $100-\mathrm{D}-27$ & $\mathrm{~J} 1 \mathrm{~J} 409$ & $8 / 23 / 11$ & 2.18 & & 0.48 & 331 & & 4.81 & 0.048 & & 0.03 & 3.54 & & 1.92 & 7.4 & & 3.85 & 0.296 & & 0.29 & 0.481 & $U$ & 0.48 & 22.5 & & 2.40 & 16.7 & & 9.62 \\
\hline & 100-D-28 & $\mathrm{J} 1 \mathrm{~J} 410$ & $8 / 23 / 11$ & 2.34 & & 0.45 & 312 & & 4.46 & 0.069 & & 0.03 & 1.90 & & 1.79 & 12.8 & & 3.57 & 0.268 & $U$ & 0.27 & 0.446 & $U$ & 0.45 & 59.1 & & 2.23 & 22.5 & & 8.93 \\
\hline & 10 & & 0 & 707 & & 0 & & & & 0.028 & & 003 & 128 & $B_{B}$ & 196 & 9.52 & & 3.92 & 0.618 & & 0.29 & 0.490 & & 0.49 & 37.6 & & 2.45 & 37.0 & & 9.80 \\
\hline
\end{tabular}


Washington Closure Hanford

thus

Originator H. M. Sulloway 9

Subject Coal Ash Characterization 90th Percentile and Median Equality Calculations
CALCULATION SHEET
$\begin{aligned} & \text { Date } \frac{12 / 20 / 11}{14655} \quad \text { Calc. No. } 0100 X-C A-V 0073 \\ & \text { Check No. }\end{aligned}$
Date $\frac{0}{12 / 20 / 11}$

\begin{tabular}{|c|c|c|c|c|c|c|c|c|c|c|c|c|c|c|c|c|c|c|c|c|c|c|c|c|c|c|c|c|c|c|}
\hline \multirow{2}{*}{$\begin{array}{l}2 \\
3 \\
3\end{array}$} & \multirow{2}{*}{$\begin{array}{l}\text { Sample } \\
\text { Location } \\
\end{array}$} & \multirow{2}{*}{$\begin{array}{c}\text { HEIS } \\
\text { Number }\end{array}$} & \multirow{2}{*}{$\begin{array}{c}\text { Sample } \\
\text { Date }\end{array}$} & \multicolumn{3}{|c|}{ Antimony } & \multicolumn{3}{|c|}{$\begin{array}{l}\text { Arsenic } \\
\end{array}$} & \multicolumn{3}{|c|}{ Barium } & \multicolumn{3}{|c|}{ Beryllium } & \multicolumn{3}{|c|}{ Boron } & \multicolumn{3}{|c|}{ Cadmium } & \multicolumn{3}{|c|}{ Chromium } & \multicolumn{3}{|c|}{ Cobalt } & \multicolumn{3}{|c|}{ Copper } \\
\hline & & & & $\mathrm{mg} / \mathrm{kg}$ & Q & PQL & $\mathrm{mg} / \mathrm{kg}$ & $Q$ & PQL & $\mathrm{mg} / \mathrm{kg}$ & Q & PQL & $\mathrm{mg} / \mathrm{kg}$ & Q & PQL & $\mathrm{mg} / \mathrm{kg}$ & Q & $\mathrm{PQL}$ & $\mathrm{mg} / \mathrm{kg}$ & \begin{tabular}{l|l}
$Q$ \\
\end{tabular} & $\mathrm{PQL}$ & $\mathrm{mg} / \mathrm{kg}$ & 0 & $\mathrm{PQL}$ & $\mathrm{mg} / \mathrm{kg}$ & Q & $\mathrm{PQL}$ & $\mathrm{mg} / \mathrm{kg}$ & Q & $\mathrm{PQL}$ \\
\hline & & & $8 / 27 / 11$ & 0.566 & $\bar{U}$ & 0.57 & 2.53 & & 0.94 & 736 & & 0.47 & 0.828 & & 0.19 & 61.8 & & 1.89 & 0.198 & & 0.19 & 7.95 & & 0.19 & 7.03 & & 1.89 & 30.9 & & 0.94 \\
\hline 5 & $100-\mathrm{H}-02$ & J1HJ49 & $8 / 27 / 11$ & 0.508 & u & 0.51 & 2.60 & & 0.85 & 437 & & 0.42 & 0.610 & & 0.17 & 37.2 & & 1.69 & 0.170 & & 0.17 & 8.92 & & 0.17 & 6.68 & & 1.69 & 26.8 & & 0.85 \\
\hline & $100-\mathrm{H}-03$ & J1HJ50 & $8 / 28 / 11$ & 0.577 & U & 0.58 & 4.34 & & 0.96 & 607 & & 0.48 & 0.857 & & 0.19 & 90.6 & & 1.92 & 0.388 & & 0.19 & 11.9 & & 0.19 & 3.85 & & 1.92 & 26.9 & & 0.96 \\
\hline & $100-\mathrm{H}-04$ & J1H.J51 & $8 / 28 / 11$ & 0.484 & $\mathrm{U}$ & 0.48 & 2.93 & & 0.81 & 979 & & 0.40 & 1.70 & & 0.16 & 167 & & 1.61 & 0.219 & & 0.16 & 8.15 & & 0.16 & 7.93 & & 1.61 & 24.8 & & 0.81 \\
\hline 8 & $100-\mathrm{H}-05$ & J1HJ52 & $8 / 27 / 11$ & 0.500 & $u$ & 0.50 & 2.25 & & 0.83 & 589 & & 0.42 & 0.769 & & 0.17 & 38.9 & & 1.67 & 0.186 & & 0.17 & 7.62 & & 0.17 & 6.80 & & 1.67 & 32.4 & & 0.83 \\
\hline & $100-\mathrm{H}-06$ & J1HJ53 & $8 / 28 / 11$ & 0.526 & $u$ & 0.53 & 3.51 & & 0.88 & 681 & & 0.44 & 1.16 & & 0.18 & 155 & & 1.75 & 0.376 & & 0.18 & 12.0 & & 0.18 & 2.59 & & 1.75 & 23.0 & & 0.88 \\
\hline 10 & $100-\mathrm{H}-07$ & J1HJ54 & $8 / 28 / 11$ & 0.484 & $u$ & 0.48 & 2.91 & & 0.81 & 721 & & 0.40 & 1.12 & & 0.16 & 235 & & 1.61 & 0.264 & & 0.16 & 12.0 & & 0.16 & 3.26 & & 1.61 & 24.7 & & 0.81 \\
\hline & $100-\mathrm{H}-08$ & J1HJ55 & $8 / 28 / 11$ & 0.500 & $U$ & 0.50 & 2.74 & & 0.83 & 830 & & 0.42 & 0.764 & & 0.17 & 31.8 & & 1.67 & 0.162 & B & 0.17 & 8.16 & & 0.17 & 7.20 & & 1.67 & 37.2 & & 0.83 \\
\hline & $100-\mathrm{H}-09$ & J1HJ56 & $8 / 28 / 11$ & 0.536 & $U$ & 0.54 & 2.77 & & 0.89 & 918 & & 0.45 & 0.777 & & 0.18 & 31.5 & & 1.79 & 0.155 & B & 0.18 & 7.62 & & 0.18 & 7.51 & & 1.79 & 39.3 & & 0.89 \\
\hline & $100-\mathrm{H}-10$ & J1HJ57 & $8 / 27 / 11$ & 0.588 & $U$ & 0.59 & 2.76 & & 0.98 & 617 & & 0.49 & 0.771 & & 0.20 & 27.5 & & 1.96 & 0.145 & B & 0.20 & 8.35 & & 0.20 & 7.99 & & 1.96 & 40.8 & & 0.98 \\
\hline & $100-\mathrm{H}-11$ & J1HJ58 & $8 / 27 / 11$ & 0.577 & U & 0.58 & 3.37 & & 0.96 & 411 & & 0.48 & 0.884 & & 0.19 & 75.8 & & 1.92 & 0.198 & & 0.19 & 9.85 & & 0.19 & 2.31 & & 1.92 & 17.7 & & 0.96 \\
\hline & $100-\mathrm{H}-12$ & J1HJ59 & $8 / 28 / 11$ & 0.411 & $U$ & 0.41 & 3.03 & & 0.69 & 1040 & & 0.34 & 1.19 & & 0.14 & 295 & & 1.37 & 0.577 & & 0.14 & 11.1 & & 0.14 & 3.60 & & 1.37 & 33.2 & & 0.69 \\
\hline & $100-\mathrm{H}-13$ & & $8 / 28 / 11$ & 0.492 & $U$ & 0.49 & 2.61 & & 0.82 & 982 & & 0.41 & 1.33 & & 0.16 & 367 & & 1.64 & 0.332 & & 0.16 & 12.6 & & 0.16 & 2.92 & & 1.64 & 26.4 & & 0.82 \\
\hline & $100-\mathrm{H}-14$ & J1HJ61 & $8 / 28 / 11$ & 0.508 & $U$ & 0.51 & 2.78 & & 0.85 & 878 & & 0.42 & 0.738 & & 0.17 & 42.0 & & 1.69 & 0.172 & & 0.17 & 8.3 & & 0.17 & 7.38 & & 1.69 & 37.1 & & 0.85 \\
\hline & $100-\mathrm{H}-15$ & J1HJ62 & $8 / 28 / 11$ & 0.492 & U & 0.49 & 2.55 & & 0.82 & 476 & & 0.41 & 0.632 & & 0.16 & 44.3 & & 1.64 & 0.174 & & 0.16 & 14.4 & & 0.16 & 6.78 & & 1.64 & 26.0 & & 0.82 \\
\hline 9 & 100-H-16 & J1HJ63 & $8 / 27 / 11$ & 0.526 & U & 0.53 & 3.46 & & 0.88 & 783 & & 0.44 & 0.842 & & 0.18 & 46.7 & & 1.75 & 0.180 & & 0.18 & 9.38 & & 0.18 & 7.98 & & 1.75 & 42.1 & & 0.88 \\
\hline 20 & $\begin{array}{c}\text { Duplicate of } \\
\text { J1HJJ63 }\end{array}$ & J1HJ78 & $8 / 27 / 11$ & 0.296 & B & 0.58 & 3.01 & & 0.96 & 773 & & 0.48 & 0.901 & & 0.19 & 52.5 & & 1.92 & 0.176 & B & 0.19 & 9.75 & & 0.19 & 8.83 & & 1.92 & 44.9 & & 0.96 \\
\hline & $100-\mathrm{H}-17$ & $\mathrm{J1HJ64}$ & $8 / 27 / 11$ & 0.492 & U & 0.49 & 2.51 & & 0.82 & 527 & & 0.41 & 0.833 & & 0.16 & 145 & & 1.64 & 0.148 & B & 0.16 & 8.57 & & 0.16 & 2.14 & & 1.64 & 16.7 & & 0.82 \\
\hline & $100-\mathrm{H}-18$ & J1HJ65 & $8 / 27 / 11$ & 0.545 & U & 0.55 & 3.34 & & 0.91 & 506 & & 0.46 & 1.00 & & & 150 & & & 0.212 & & & 10 & & & 2.53 & & & 20.6 & & 0.91 \\
\hline & $100-\mathrm{H}-19$ & & $8 / 27 / 111$ & 0.566 & $U$ & 0.57 & 3.90 & & & 760 & & 0.47 & 0.941 & & & & & & 0.176 & B & & & & & & & & 46.8 & & 0.94 \\
\hline & $100-\mathrm{H}-20$ & J1HJ67 & $8 / 24 / 11$ & 0.508 & U & 0.51 & 2.94 & & 0.85 & 916 & & 0.42 & 1.31 & & 0. & 45 & & 1.6 & 0.178 & & & & & 0. & 3.77 & & 1.69 & 33.8 & & 0.85 \\
\hline & $100-\mathrm{H}-21$ & J1HJ68 & $8 / 28 / 11$ & 0.492 & U & 0.49 & 3.11 & & 0.82 & 313 & & 0.41 & 0.429 & & 0.16 & 31.6 & & 1.6 & 0.200 & & 0.1 & 13 & & 0.1 & 6.20 & & 1.64 & 19.1 & & 0.82 \\
\hline 6 & $100-\mathrm{H}-22$ & J1HJJ69 & $8 / 27 / 11$ & 0.500 & U & 0.50 & 4.72 & & 0.83 & 1250 & & 0.42 & 1.25 & & 0.17 & 183 & & 1.6 & 0.241 & & 0.1 & 8.2 & & 0.1 & 9.81 & & 1.67 & 35.0 & & 0.83 \\
\hline & $100-\mathrm{H}-23$ & J1HJ & $8 / 27 / 111$ & 0.556 & $U$ & 0.56 & 2.05 & & 0.93 & 718 & & 0.46 & 0.562 & & 0.19 & 20.2 & & 1.85 & 0.140 & $B$ & 0.19 & 6.38 & & 0.1 & 6.00 & & 1.85 & 32.5 & & 0.93 \\
\hline & $100-\mathrm{H}-24$ & J1HJ71 & $8 / 27 / 11$ & 0.600 & $U$ & 0.60 & 2.49 & & 1.00 & 722 & & 0.50 & 1.22 & & 0.20 & 318 & & 2.00 & 0.150 & $B$ & 0.20 & 11.2 & & 0.2 & 2.42 & & 2.00 & 18.4 & & 1.00 \\
\hline 29 & $100-\mathrm{H}-25$ & $\mathrm{~J} 1 \mathrm{H} \mathrm{J} 72$ & $8 / 24 / 11$ & 0.476 & $U$ & 0.48 & 2.55 & & 0.79 & 954 & & 0.40 & 1.05 & & 0.16 & 235 & & 1.59 & 0.217 & & 0.16 & 12.3 & & 0.16 & 3.66 & & 1.59 & 24.0 & & 0.79 \\
\hline 30 & $\begin{array}{l}\text { Duplicate of } \\
\text { J1HJ72 }\end{array}$ & J1HJ77 & $8 / 24 / 11$ & 0.517 & u & 0.52 & 2.82 & & 0.86 & 1080 & & 0.43 & 1.11 & & 0.17 & 253 & & 1.72 & 0.183 & & 0.17 & 12.5 & & 0.17 & 3.82 & & 1.72 & 23.7 & & 0.86 \\
\hline & $100-H-26$ & J1HJ73 & $8 / 28 / 11$ & 0.517 & $U$ & 0.52 & 3.78 & & 0.86 & 846 & & 0.43 & 0.83 & & 0.17 & 102 & & 1.72 & 0.244 & & 0.17 & 14.1 & & 0.17 & 8.25 & & 1.72 & 30.7 & & 0.86 \\
\hline & $100-\mathrm{H}-27$ & J1HJ74 & $8 / 27 / 111$ & 0.476 & $U$ & 0.48 & 2.28 & & 0.79 & 816 & & 0.40 & 0.65 & & 0.16 & 24.0 & & 1.59 & 0.159 & B & 0.16 & 7.39 & & 0.1 & 6.80 & & 1.59 & 35.4 & & 0.79 \\
\hline & $100-\mathrm{H}-28$ & J1HJ75 & $8 / 27 / 11$ & 0.556 & $U$ & 0.56 & 3.70 & & 0.93 & 1020 & & 0.46 & 1.04 & & 0.19 & 84.5 & & 1.85 & 0.233 & & 0.19 & 11.5 & & 0.19 & 7.72 & & 1.85 & 42.4 & & 0.93 \\
\hline & $100-\mathrm{H}-29$ & $\mathrm{J1HJ76}$ & $8 / 28 / 11$ & 0.517 & $U$ & 0.52 & 3.60 & & 0.86 & 1330 & & & 1.44 & & 0.17 & 243 & & 1.72 & 0.347 & & 0.17 & 10.7 & & 0.17 & 9.94 & & 172 & & & 086 \\
\hline
\end{tabular}


CALCULATION SHEET

Originator H. M. Sulloway $1 / 103$

Subject Coal Ash Characterization 9oth Percentilie and Median Equality Calculations

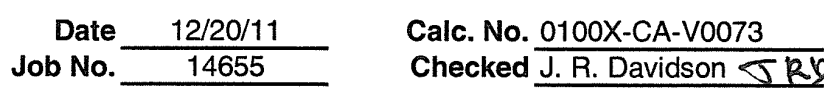

Rev. No. $\frac{0}{12 / 20111}$

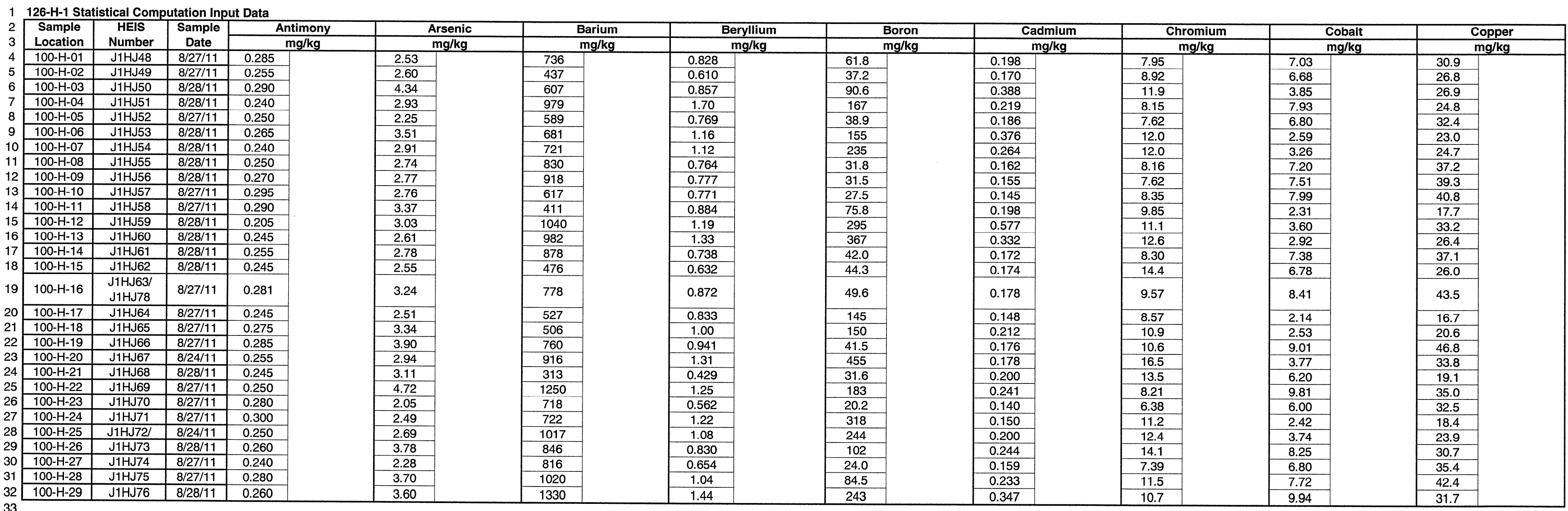


Washington Closure Hanford

HWS

\section{CALCULATION SHEET}

Project $\frac{H . M}{100 \text { and } 300 \text { Areas Coal Ash Characterization }}$

Date $\frac{12 / 20 / 11}{1405}$

Job No. $\frac{12641}{14655}$
Calc. No. $0100 X-C A-V 0073$
Checked J. R. Davidson J (2U
Rev. No. $\frac{0}{12 / 20 / 11}$ Sheet No. $\frac{12 / 20 / 11}{26 \text { of } 35}$

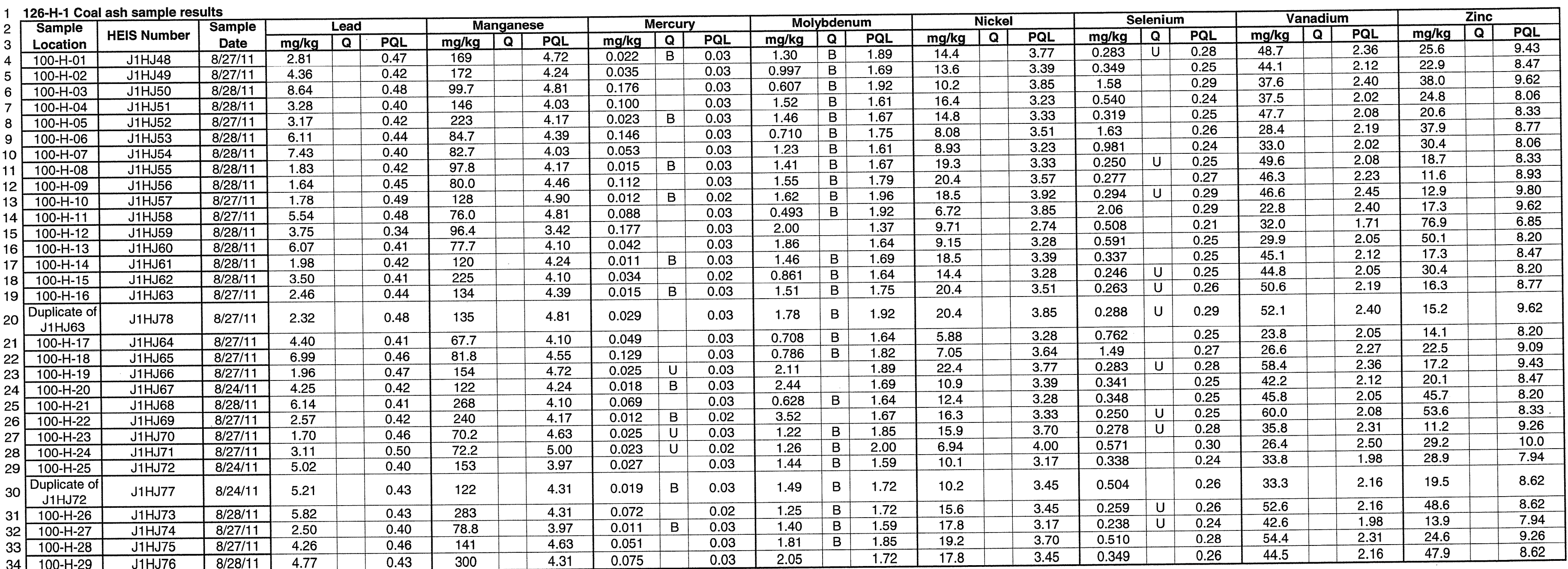


Washington Closure Hanford

CALCULATION SHEET

Originator H.M. Sulloway WUS

Date $\frac{12 / 20 / 11}{14655}$
Job No.

Subject Coal Ash Characterization 90th Percentile and Median Equality Calculations

Calc. No. $0100 X-C A-V 0073$
Checked J. R. Davidson S 2 (1)

Rev. No. $\frac{0}{12(20 / 11}$

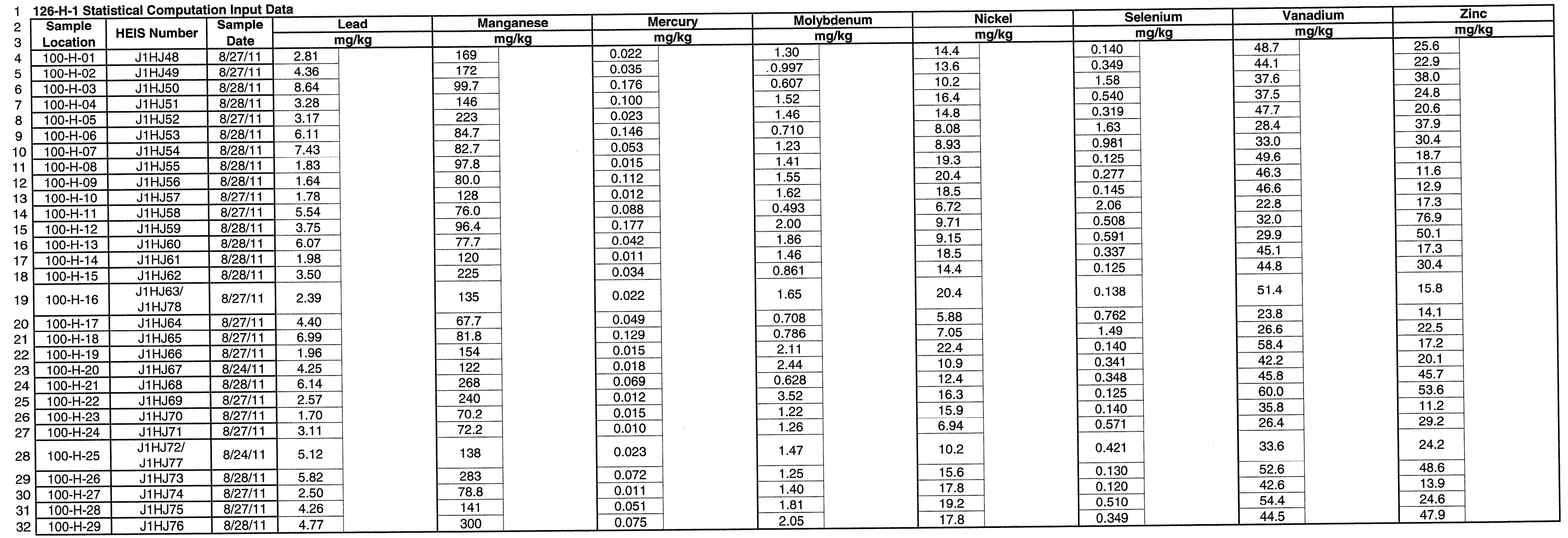


Originator H. M. Sulloway duW

Project 100 and 300 Areas Coal Ash Characterization

Equality Calculations Date $\frac{12 / 20 / 11}{14655}$
Job No. Calc. No. $0100 x-C A-V 0073$
Checked J. R. Davidson J RQ
Rev. No. $\frac{0}{\text { Date }} \frac{0}{12 / 20 / 11}$

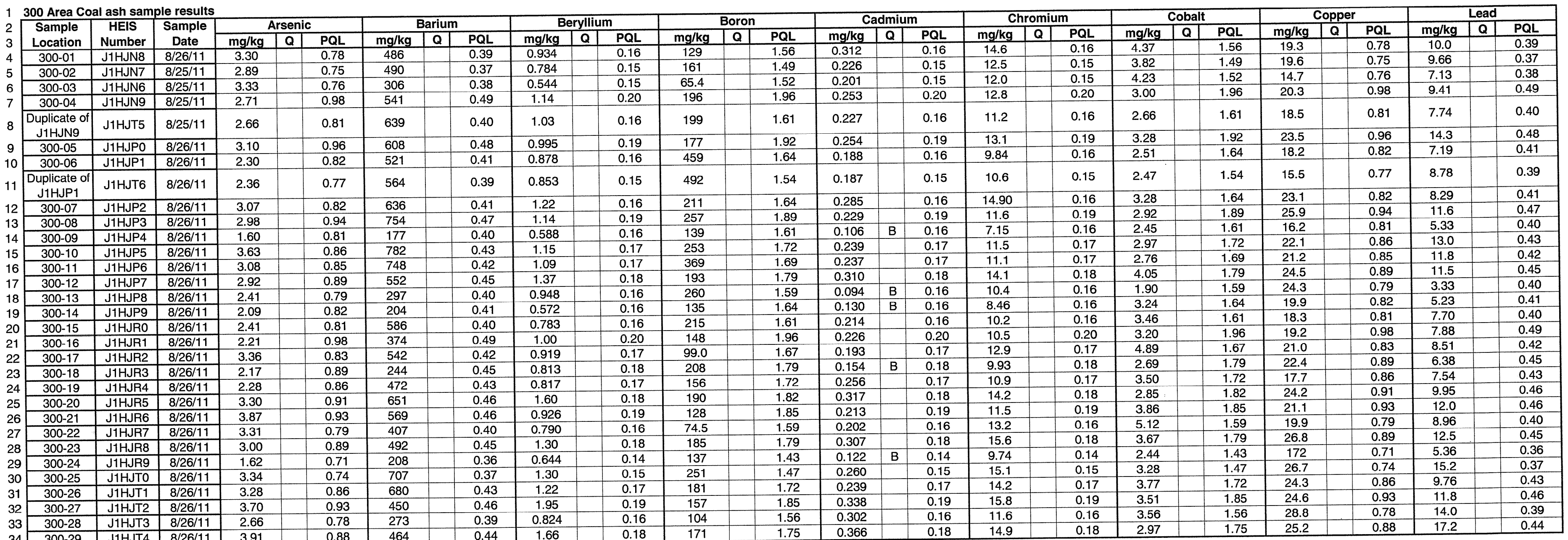


Washington Closure Hanford

$$
\begin{aligned}
& \text { Originator H. M. Sulloway dWW } \\
& \text { 作 }
\end{aligned}
$$

CALCULATION SHEET
Date $12 / 20 / 11$
Job No. 14655
Calc. No. $0100 \mathrm{X}-\mathrm{CA}-\mathrm{V} 0073$ Checked J. R. Davidson SRY
Rev. No. $\frac{0}{12 / 20 / 11}$

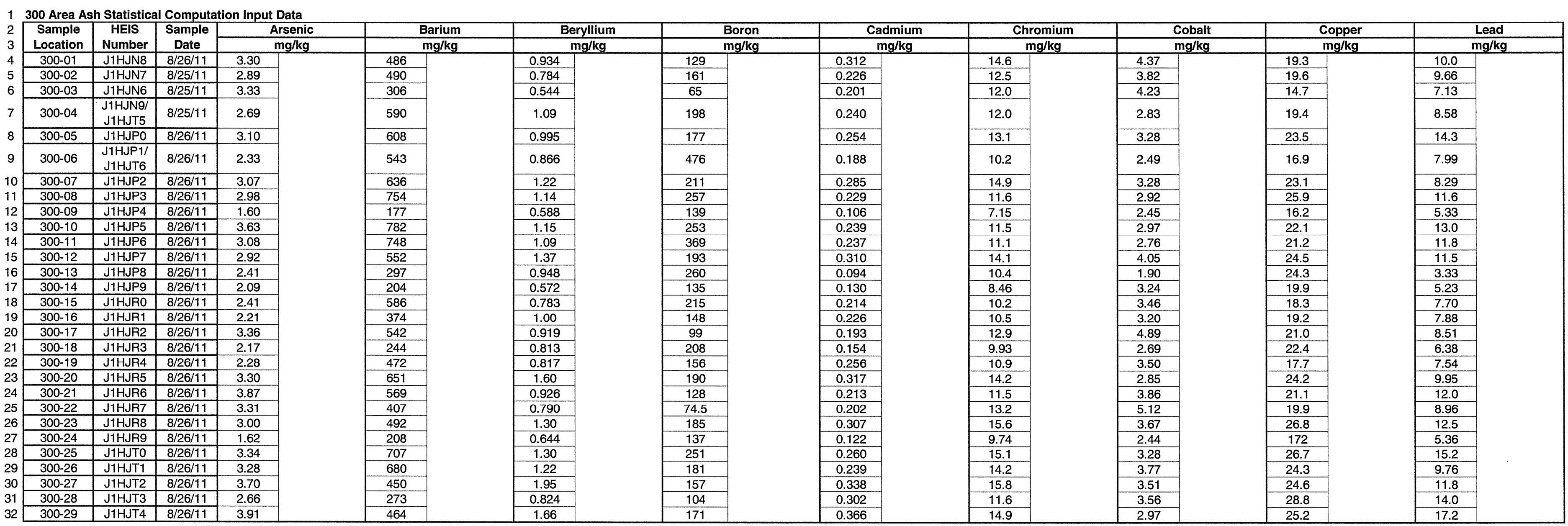


Washington Closure Hanford

Originator H.M. Sulloway $\mathrm{XWS}$

roject 100 and 300 Areads Coal Ash Characterization Subject Coal Ash Characterization 90th Percentile and Median Equality Calculations
CALCULATION SHEET

Date $\frac{12 / 20 / 11}{14655}$ Job No. 14655
Calc. No. 0100X-CA-V0073

Checked J.R. Davidson JRU
Rev. No. Date. $\frac{0}{12 / 20 / 11}$

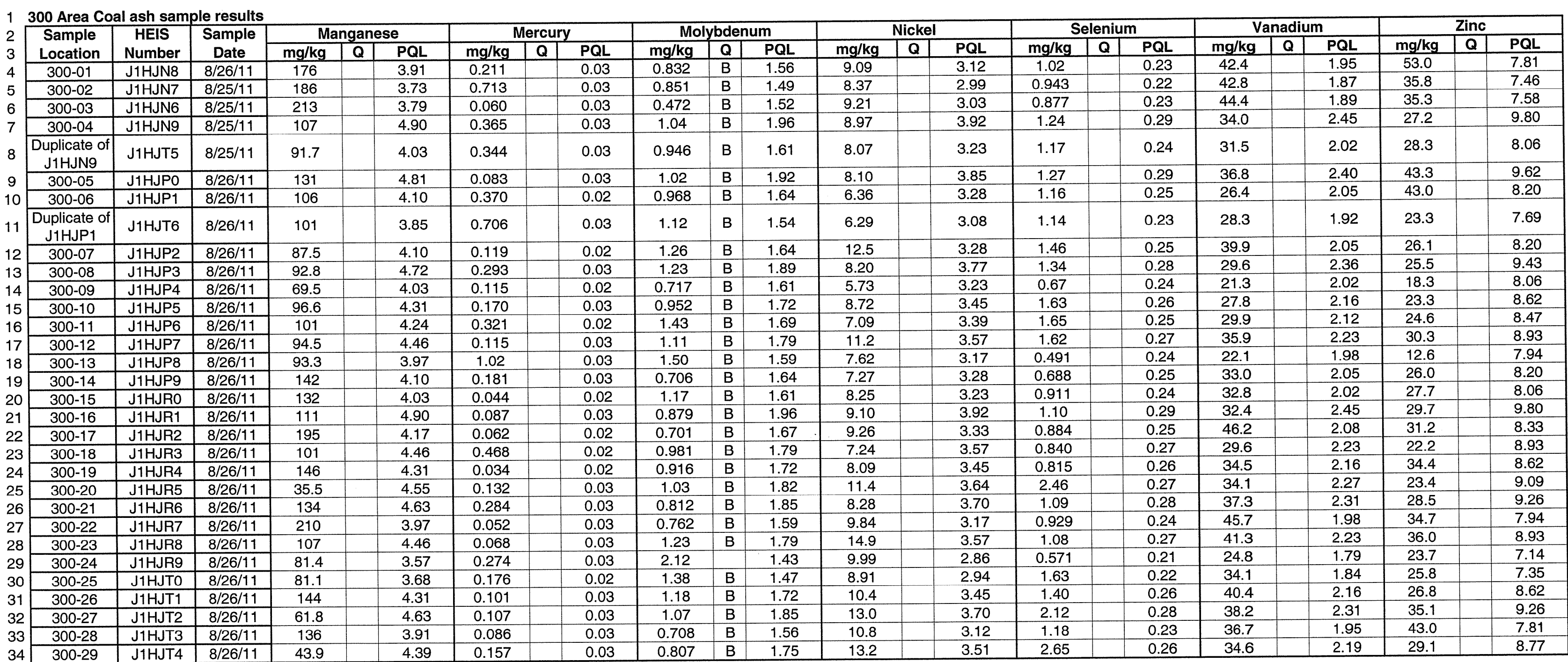


Washington Closure Hanford Itus

Project 100 and 300 Areas Coal Ash Characterization

Subject Coal Ash Characterization 90th Percentile and Median Equality Calculations
CALCULATION SHEET

Date $\frac{12 / 20 / 11}{14655}$
Job No.
Calc. No. $0100 X-C A-V 0073$
Checked $\frac{\text { J.R. Davidson T R1) }}{}$

$\begin{aligned} \text { Rev. No. } & \frac{0}{12 / 20 / 11} \\ \text { Date } & \frac{12 \text { of } 35}{\text { Sheet No. }}\end{aligned}$

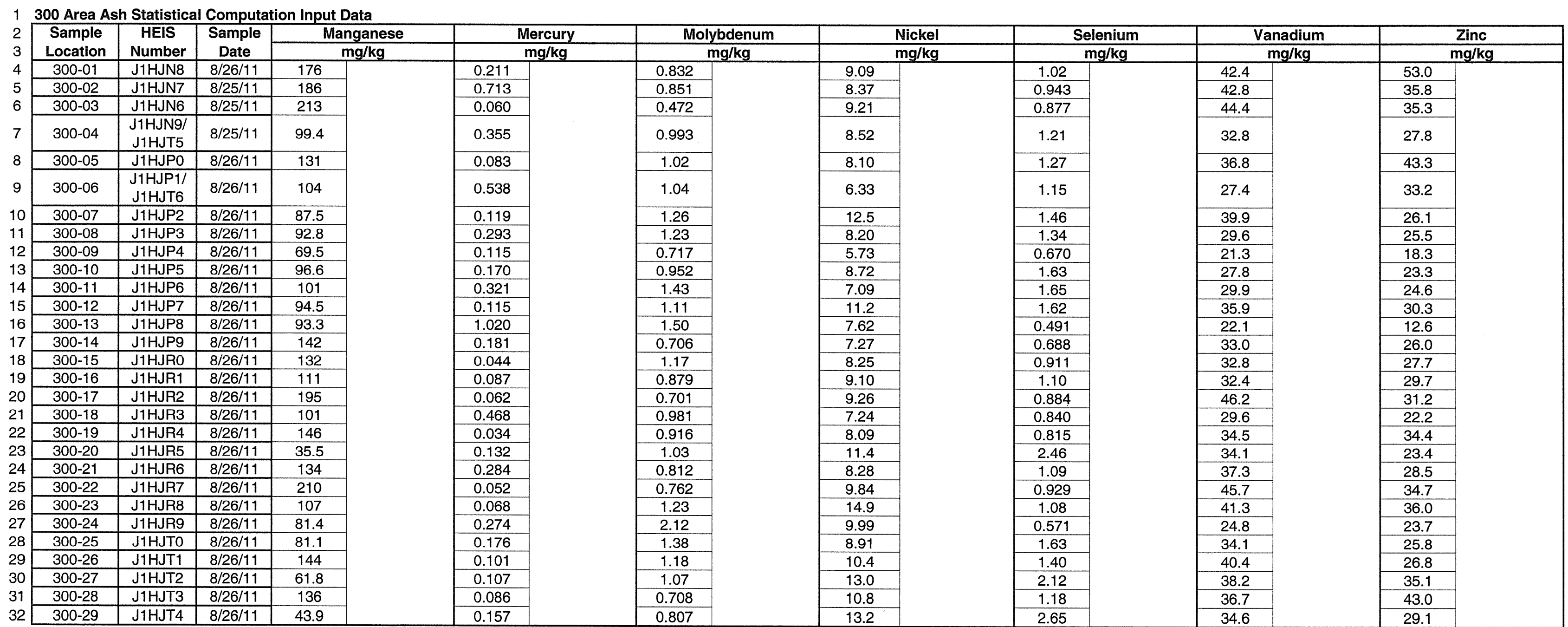


Washington Closure Hanford

riginator H. M. Sulloway U UhS
CALCULATION SHEET

$\begin{aligned} & \text { Date } \frac{12 / 20 / 11}{14655} \\ & \text { Job No. }\end{aligned}$
Calc. No. $0100 \mathrm{X}-\mathrm{CA}-\mathrm{V} 0073$

on $5 R$ $\begin{aligned} \text { Rev. No. } & \frac{0}{\text { Date }} \\ \text { Sheet No. } & \frac{12 / 20 / 11}{32 \text { of } 35}\end{aligned}$

\begin{tabular}{|c|c|c|c|c|c|c|c|c|c|c|c|c|c|c|c|c|c|c|c|c|c|c|c|c|c|c|c|c|c|c|}
\hline \multirow{3}{*}{\multicolumn{2}{|c|}{\begin{tabular}{l|c|}
1 & $600-207$ Coa \\
2 & Sample \\
3 & Location \\
\cline { 2 - 3 } & $600-01$
\end{tabular}}} & \multirow{2}{*}{$\begin{array}{c}\text { al ash sam } \\
\begin{array}{|c|}\text { HIIS } \\
\text { Number }\end{array} \\
\end{array}$} & \multirow{2}{*}{$\begin{array}{c}\text { e results } \\
\text { Sample } \\
\text { Date }\end{array}$} & & \multirow{2}{*}{\multicolumn{3}{|c|}{ Barium }} & \multirow{2}{*}{\multicolumn{3}{|c|}{ Beryllium }} & \multirow{2}{*}{\multicolumn{3}{|c|}{ Boron }} & \multirow{2}{*}{\multicolumn{3}{|c|}{ Cadmium }} & \multirow{2}{*}{\multicolumn{3}{|c|}{ Chromium }} & \multirow{2}{*}{\multicolumn{3}{|c|}{ Cobalt }} & \multirow{2}{*}{\multicolumn{3}{|c|}{ Copper }} & \multirow{2}{*}{\multicolumn{3}{|c|}{ Lead }} \\
\hline & & & & $\mathrm{mg} / \mathrm{kg}$ & $\frac{1}{10}$ & $\frac{c}{P Q L}$ & & & & & & & & & & & & & & & & & & & & & & & & \\
\hline & & \begin{tabular}{|l}
$\mathrm{J} 1 \mathrm{HHM} 3$ \\
\end{tabular} & $9 / 28 / 11$ & 3.73 & & 0.88 & 656 & & 0.44 & $\frac{m g / k 9}{0.691}$ & & $\frac{18}{0.18}$ & $\frac{\mathrm{mg} / \mathrm{kg}}{60.9}$ & & $\frac{P Q L}{1.75}$ & $\frac{\mathrm{mg} / \mathrm{kg}}{0.178}$ & $a_{1}$ & $\begin{array}{l}\text { PQL } \\
0.18 \\
\end{array}$ & $\frac{m g / k g}{8.27}$ & & $\frac{P Q L}{0.18}$ & $\frac{\operatorname{mog} / \mathrm{kg}}{3.88}$ & & $\frac{P \mathrm{PL}}{1.75}$ & $\frac{\mathrm{mg} / \mathrm{kg}}{20.0}$ & & $\frac{\text { PQL }}{0.88}$ & $\frac{\mathrm{m} / \mathrm{kg}}{4.69}$ & & 044 \\
\hline & $\begin{array}{l}600-02 \\
\end{array}$ & $\begin{array}{l}\text { J1HHM4 } \\
\end{array}$ & $9 / 28 / 11$ & 5.29 & & 0.89 & 673 & & $\begin{array}{l}0.44 \\
0.45\end{array}$ & 0.655 & & 0.18 & 42.7 & & $\frac{1.10}{1.79}$ & 0.186 & & $\begin{array}{l}0.10 \\
0.18\end{array}$ & 8.66 & & 0.18 & $\frac{.002}{4.02}$ & & $\frac{1.10}{1.79}$ & 20.4 & & 0.00 & $\frac{4.09}{5.08}$ & & 0.44 \\
\hline & $600-03$ & J1HHM5 & $9 / 28 / 11$ & 5.19 & & 0.93 & 721 & & 0.46 & 0.730 & & 0.19 & 55.1 & & 1.85 & 0.187 & & 0.19 & 9.26 & & 0.19 & 4.05 & & 1.85 & $\frac{20.4}{21.1}$ & & 0.09 & $\frac{0.00}{721}$ & & 0.45 \\
\hline & $600-04$ & J1HHM6 & $9 / 28 / 11$ & 3.82 & & 0.86 & 552 & & 0.43 & 0.556 & & 0.17 & 40.13 & & $\frac{7.85}{1.72}$ & 0.187 & & $\begin{array}{l}0.19 \\
0.17\end{array}$ & $\begin{array}{l}9.26 \\
7.82 \\
-1\end{array}$ & & $\begin{array}{l}0.19 \\
0.17\end{array}$ & $\frac{4.05}{3.51}$ & & $\frac{7.85}{1.72}$ & $\frac{21.1}{17.1}$ & & $\begin{array}{l}0.9 \\
0.9\end{array}$ & 5.21 & & 0.46 \\
\hline & $600-05$ & J1HHM7 & $9 / 28 / 11$ & 4.91 & & 0.03 & $\frac{52}{645}$ & & 0.46 & 0.619 & & 0.19 & 44.7 & & $\frac{1.16}{1.85}$ & 0.188 & & 0.19 & 9.00 & & 0.19 & $\begin{array}{l}0.01 \\
3.94\end{array}$ & & $\frac{1.12}{1.85}$ & 20.2 & & 0.9 & $\begin{array}{ll}0.207 \\
6.07\end{array}$ & & 0.46 \\
\hline & $600-06$ & J1HHM8 & $9 / 28 / 11$ & 3.63 & & 0.88 & 695 & & 0.44 & 0.592 & & 0.18 & 42.7 & & $\frac{1.00}{1.75}$ & 0.211 & & 0.18 & 8.04 & & 0.18 & $\frac{0.54}{3.30}$ & & $\frac{1.00}{175}$ & 18. & & 0.5 & 5.77 & & 0.44 \\
\hline & $600-07$ & J1HHM9 & $9 / 27 / 11$ & 6.60 & & 0.96 & 958 & & 0.48 & 0.903 & & 0.19 & 146 & & 1.92 & 0.122 & B & 0.19 & 12.9 & & 0.19 & 5.74 & & $\frac{1.92}{1.92}$ & 46.8 & & $\frac{.00}{10}$ & 5.28 & & 0.48 \\
\hline & $600-08$ & J1HHNO & $9 / 28 / 11$ & 5.18 & & 0.82 & 686 & & 0.41 & 0.676 & & 0.16 & 43.1 & & 1.64 & 0.194 & & 0.16 & 8.91 & & 0.16 & 4.28 & & $\frac{1.64}{1.64}$ & 22.2 & & 0.82 & 5.35 & & 0.41 \\
\hline 12 & $600-09$ & J1HHN1 & $9 / 28 / 11$ & 5.42 & & 0.91 & 643 & & 0.46 & 0.800 & & 0.18 & 50.9 & & 1.82 & 0.211 & & 0.18 & 9.33 & & 0.18 & 4.49 & & 1.82 & 24.1 & & 0.91 & 5.75 & & 0.46 \\
\hline & $600-10$ & J1HHN2 & $9 / 28 / 11$ & 4.62 & & 0.85 & 608 & & 0.42 & 0.592 & & 0.17 & 44.7 & & 1.69 & 0.178 & & 0.17 & 8.09 & & 0.17 & 3.53 & & 1.69 & 19.2 & & 0.85 & 5.24 & & 0.42 \\
\hline & $600-11$ & J1HHN3 & $9 / 28 / 11$ & 3.71 & & 0.85 & 379 & & 0.42 & 0.627 & & 0.17 & 31.2 & & 1.69 & 0.166 & B & 0.17 & 9.70 & & 0.17 & 4.83 & & 1.69 & 18.5 & & 0.85 & 4.26 & & 0.42 \\
\hline & $600-12$ & J1HHN4 & $9 / 28 / 11$ & 3.39 & & 0.82 & 632 & & 0.41 & 0.460 & & 0.16 & 41.6 & & 1.64 & 0.182 & & 0.16 & 8.08 & & 0.16 & 3.24 & & 1.64 & 16.9 & & 0.82 & 4.23 & & 0.41 \\
\hline & $600-13$ & J1HHN5 & $9 / 28 / 11$ & 3.90 & & 0.86 & 609 & & 0.43 & 0.529 & & 0.17 & 35.4 & & 1.72 & 0.153 & B & 0.17 & 6.81 & & 0.17 & 3.21 & & 1.72 & 19.4 & & 0.86 & 4.58 & & 0.43 \\
\hline & $600-14$ & J1HHN6 & $9 / 28 / 11$ & 4.46 & & 0.91 & 606 & & 0.46 & 0.696 & & 0.18 & 44.8 & & 1.82 & 0.173 & B & 0.18 & 8.29 & & 0.18 & 3.70 & & 1.82 & 20.1 & & 0.91 & 7.00 & & 0.46 \\
\hline & $600-15$ & J1HHN7 & $9 / 27 / 11$ & 10.6 & & 0.96 & 1210 & & 0.48 & 1.43 & & 0.19 & 112 & & 1.92 & 0.232 & & 0.19 & 15.5 & & 0.19 & 7.14 & & 1.92 & 35.4 & & 0.96 & 11.1 & & 0.48 \\
\hline & Duplicate of & J1HHR2 & 9/27/11 & 9.58 & & 0.85 & 1250 & & 0.42 & 1.46 & & 0.17 & 162 & & 1.69 & 0.213 & & 0.17 & 16.7 & & 0.17 & 7.08 & & 1.69 & 38.6 & & 0.85 & 7.5 & & 0.42 \\
\hline & $600-16$ & \begin{tabular}{|l} 
J1HHN8 \\
\end{tabular} & $9 / 28 / 11$ & 5.38 & & 0.88 & 767 & & 0.44 & 0.772 & & 0.18 & 60.6 & & 1.75 & 0.186 & & 0.18 & 9.14 & & 0.18 & 4.69 & & 1.75 & 20.3 & & 0.88 & 5.45 & & 0.44 \\
\hline & $600-17$ & J1HHN9 & 9/28/11 & 5.02 & & 0.79 & 678 & & 0.40 & 0.629 & & 0.16 & 58.8 & & 1.59 & 0.202 & & 0.16 & 9.87 & & 0.16 & 4.70 & & 1.59 & 23.2 & & 0.79 & 7.10 & & 0.40 \\
\hline & $600-18$ & J1HHPO & 9/28/11 & 4.89 & & 0.81 & 683 & & 0.40 & 0.84 & & 0.16 & 50.2 & & 1.61 & 0.189 & & 0.16 & 8.23 & & 0.16 & 4.16 & & 1.61 & 20.6 & & 0.81 & 5.93 & & 0.40 \\
\hline & $600-19$ & J1HHP1 & $9 / 28 / 11$ & 4.56 & & 0.86 & 705 & & 0.43 & 0.737 & & 0.17 & 60.3 & & 1.72 & 0.172 & B & 0.17 & 8.55 & & 0.17 & 4.31 & & 1.72 & 20.9 & & 0.86 & 4.99 & & 0.43 \\
\hline $24-2$ & $600-20$ & J1HHP2 & $9 / 28 / 11$ & 6.63 & & 0.89 & 805 & & 0.45 & 0.992 & & 0.18 & 57.1 & & 1.79 & 0.193 & & 0.18 & 11.4 & & 0. & 5.65 & & 1.79 & 26.7 & & 0.89 & 5.44 & & 0.45 \\
\hline & $600-21$ & J1HHP3 & $9 / 28 / 11$ & 6.30 & & 0.98 & 1060 & & 0.49 & 1.01 & & 0.20 & 84.0 & & 1.96 & 0.127 & B & 0.20 & 11.8 & & 0. & 5.46 & & 1.96 & 24.6 & & 0.98 & 4.94 & & 0.49 \\
\hline & 600-22 & J1HHP4 & $9 / 27 / 11$ & 6.19 & & 0.83 & 796 & & 0.42 & 0.957 & & 0.17 & 58.3 & & 1.67 & 0.140 & B & 0.17 & 11.4 & & 0. & 5.52 & & 1.67 & 24.6 & & 0.8 & 6.92 & & 0.42 \\
\hline & $600-23$ & J1HHP5 & 9/27/11 & 6.46 & & 0.89 & 915 & & 0.45 & 0.969 & & 0.18 & 103 & & 1.79 & 0.143 & B & 0.18 & 11.5 & & 0.18 & 6.07 & & 1.79 & 28.2 & & 0.8 & 6.61 & & 0.45 \\
\hline & \begin{tabular}{|l|}
$600-24$ \\
\end{tabular} & JIHHPG & $9 / 28 / 11$ & 7.25 & & 0.98 & 910 & & 0.49 & 1.19 & & 0.20 & 69.8 & & 1.96 & 0.174 & B & 0.20 & 12.3 & & 0.20 & 5.92 & & 1.96 & 32.3 & & 0.98 & 7.03 & & 0.49 \\
\hline 29 & $\begin{array}{l}\text { Duplicate of } \\
\text { J1HHP6 }\end{array}$ & J1HHR3 & $9 / 27 / 11$ & 6.57 & & 0.83 & 1030 & & 0.42 & 1.18 & & 0.17 & 81.7 & & 1.67 & 0.157 & B & 0.17 & 11.4 & & 0.17 & 5.85 & & 1.67 & 28.0 & & 0.83 & 5.60 & & 0.42 \\
\hline 30 & $600-25$ & J1HHP7 & 9/27/11 & 5.52 & & 1.00 & 694 & & 0.50 & 0.907 & & 0.20 & 48.0 & & 2.00 & 0.176 & B & 0.20 & 11.3 & & 0.20 & 5.38 & & 2.00 & 26.8 & & 1.00 & 6.06 & & 0.50 \\
\hline 31 & $600-26$ & J1HHP8 & $9 / 28 / 11$ & 8.54 & & 0.96 & 1030 & & 0.48 & 1.18 & & 0.19 & 66.0 & & 1.92 & 0.149 & $B$ & 0.19 & 12.8 & & 0.19 & 5.93 & & 1.92 & 29.9 & & 0.96 & 14.5 & & 0.48 \\
\hline 32 & $600-27$ & J1HHP9 & $9 / 27 / 11$ & 6.17 & & 0.83 & 944 & & 0.42 & 1.05 & & 0.17 & 63.7 & & 1.67 & 0.175 & & 0.17 & 11.1 & & 0.17 & 4.91 & & 1.67 & 25.2 & & 0.83 & 6.15 & & 0.42 \\
\hline 33 & $600-28$ & J1HHRO & $9 / 27 / 11$ & 4.90 & & 0.94 & 979 & & 0.47 & 1.11 & & 0.19 & 71.3 & & 1.89 & 0.138 & B & 0.19 & 9.85 & & 0.19 & 4.83 & & 1.89 & 24.3 & & 0.94 & 5.47 & & 0.47 \\
\hline 34 & $600-29$ & \begin{tabular}{|l|l}
$\mathrm{J} 1 \mathrm{HHR} 1$ \\
\end{tabular} & $9 / 28 / 11$ & 5.58 & & 0.73 & 802 & & 0.36 & 0.833 & & 0.15 & 57.4 & & 1.45 & 0.151 & & 0.15 & 10.9 & & 0.15 & 4.96 & & 1.45 & 23.2 & & 0.73 & 5.32 & & 0.36 \\
\hline
\end{tabular}


Washington Closure Hanford

Whos

CALCULATION SHEET

Originator H. M. Sulloway
Project $\frac{100 \text { and } 300 \text { Areas Coal Ash Characterization }}{100 \text { Subject }}$ Coal Ash Characterization 90th Percentile and Median Equality Calculations

$\begin{aligned} & \text { Date } 12 / 20 / 11 \\ & \text { Job No. } \frac{14655}{145}\end{aligned}$

Calc. No. $0100 X-C A-V 0073$
Checked J. R. Davidson TRQ

Rev. No. 0

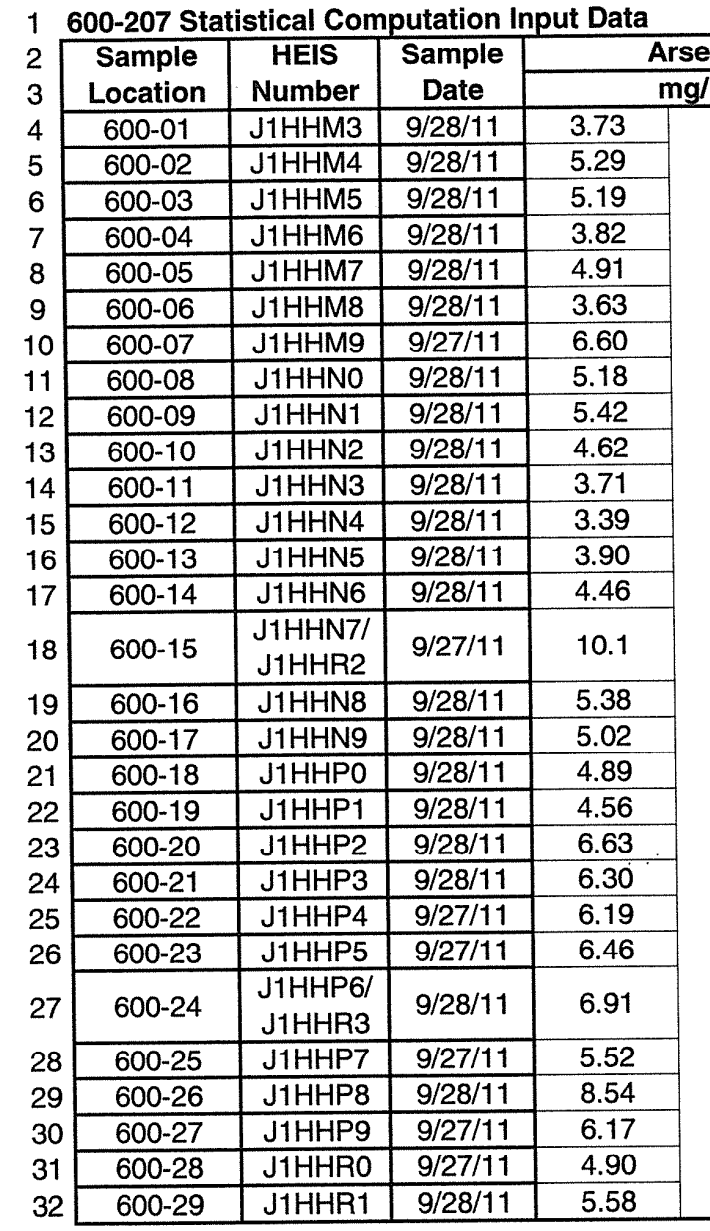

\begin{tabular}{|c|c|}
\hline \multirow{25}{*}{$\begin{array}{c}\text { Arsenic } \\
\mathrm{mg} / \mathrm{kg}\end{array}$} & \multirow{2}{*}{$\begin{array}{c}\text { Barium } \\
\mathrm{mg} / \mathrm{kg} \\
\end{array}$} \\
\hline & \\
\hline & $\begin{array}{ll}656 \\
673\end{array}$ \\
\hline & 721 \\
\hline & 552 \\
\hline & 645 \\
\hline & 695 \\
\hline & 958 \\
\hline & 643 \\
\hline & 608 \\
\hline & 379 \\
\hline & 632 \\
\hline & $\frac{609}{606}$ \\
\hline & 1230 \\
\hline & 767 \\
\hline & 678 \\
\hline & 683 \\
\hline & $\begin{array}{l}705 \\
885\end{array}$ \\
\hline & 1060 \\
\hline & $\begin{array}{l}796 \\
915\end{array}$ \\
\hline & 970 \\
\hline & \\
\hline & 1030 \\
\hline & \\
\hline & 979 \\
\hline
\end{tabular}

\begin{tabular}{l|l} 
Beryllium & Boron \\
\hline S & . \\
\hline
\end{tabular}

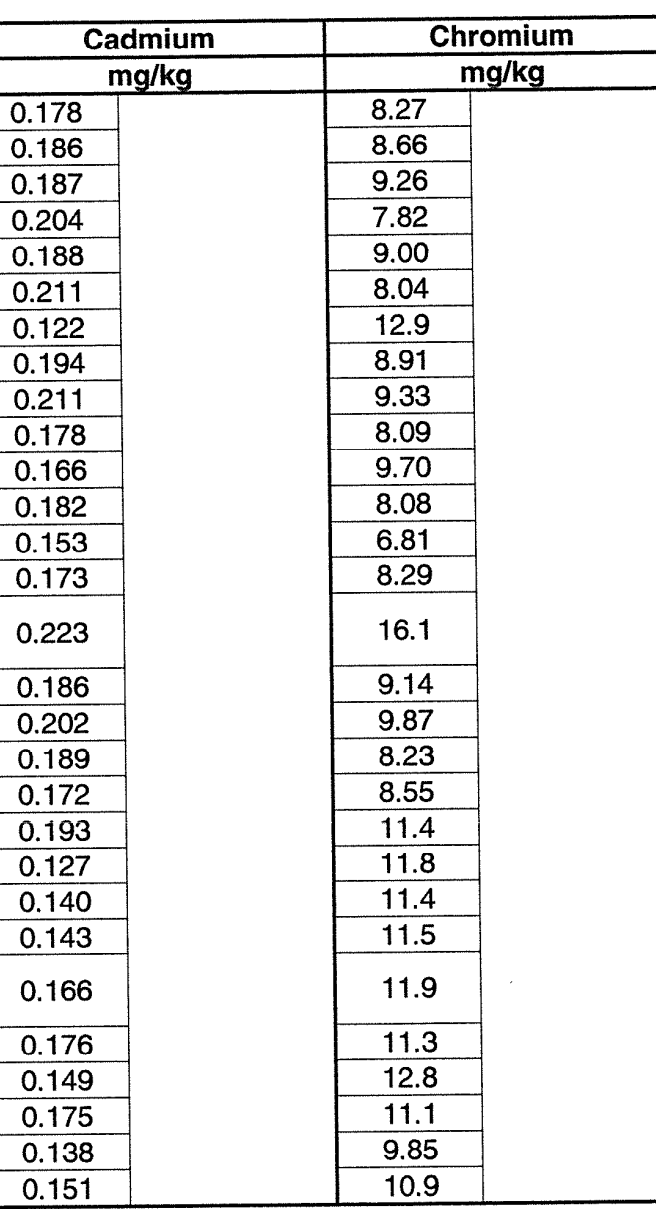

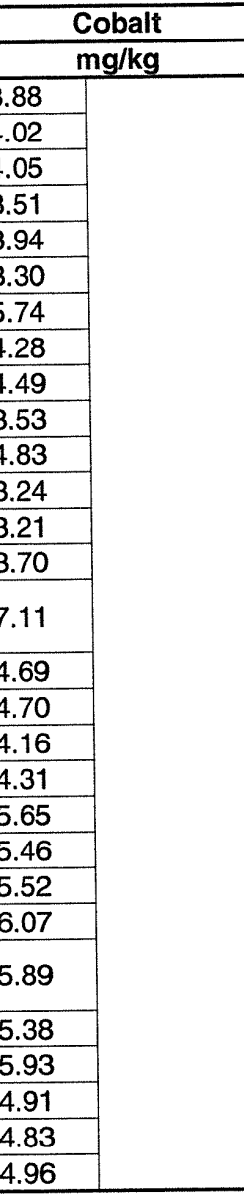

Sheet No. 33 of 35

\begin{tabular}{|c|c|c|}
\hline \multirow{2}{*}{\multicolumn{3}{|c|}{\begin{tabular}{l|}
\multicolumn{2}{c}{$\mathrm{mg} / \mathrm{kg}$} \\
0.691
\end{tabular}}} \\
\hline & & \\
\hline $\begin{array}{l}0.655 \\
0.730\end{array}$ & & $\begin{array}{l}42.7 \\
55.7\end{array}$ \\
\hline$\frac{0.730}{0.556}$ & & $\begin{array}{l}55.1 \\
44.3\end{array}$ \\
\hline 0.619 & & \\
\hline 0.592 & & \begin{tabular}{|l|l}
42.7 \\
1.5
\end{tabular} \\
\hline $\begin{array}{l}0.903 \\
0.676\end{array}$ & & 146 \\
\hline $\begin{array}{l}0.676 \\
0.800\end{array}$ & & $\begin{array}{l}43.1 \\
5.1\end{array}$ \\
\hline $\begin{array}{l}0.800 \\
0.592\end{array}$ & & 50.9 \\
\hline 0.592 & & 44. \\
\hline 0.627 & & 31.2 \\
\hline 0.460 & & 41.6 \\
\hline 0.529 & & $\begin{array}{l}35.4 \\
448\end{array}$ \\
\hline 0.696 & & 44.8 \\
\hline 1.45 & & 137 \\
\hline 0.772 & & 60. \\
\hline 0.629 & & 58. \\
\hline $\begin{array}{l}0.839 \\
0.773\end{array}$ & & 50 \\
\hline $\begin{array}{l}0.737 \\
0.992\end{array}$ & & $\begin{array}{ll}60 \\
57\end{array}$ \\
\hline$\frac{0.992}{1.01}$ & & $\frac{57}{84}$ \\
\hline 0.957 & & $5 \varepsilon$ \\
\hline 0.969 & & 103 \\
\hline 1.19 & & $75 . \varepsilon$ \\
\hline 0.907 & & \\
\hline 1.18 & & \\
\hline$\frac{1.05}{1.11}$ & & \\
\hline 1.11 & & $\frac{1.4}{57}$ \\
\hline
\end{tabular}

\begin{tabular}{|l|}
\hline \multicolumn{1}{|c|}{$\mathrm{mg} / \mathrm{kg}$} \\
\hline 60.9 \\
\hline 42.7 \\
\hline 55.1 \\
\hline 44.3 \\
\hline 44.7 \\
\hline 42.7 \\
\hline 146 \\
\hline 43.1 \\
\hline 50.9 \\
\hline 44.7 \\
\hline 31.2 \\
\hline 41.6 \\
\hline 35.4 \\
\hline 44.8 \\
\hline 137 \\
\hline 60.6 \\
\hline 58.8 \\
\hline 50.2 \\
\hline 60.3 \\
\hline 57.1 \\
\hline 84.0 \\
\hline 58.3 \\
\hline 103 \\
\hline 75.8 \\
\hline 48.0 \\
\hline 66.0 \\
\hline 63.7 \\
\hline 71.3 \\
\hline 57.4 \\
\hline
\end{tabular}

0.151

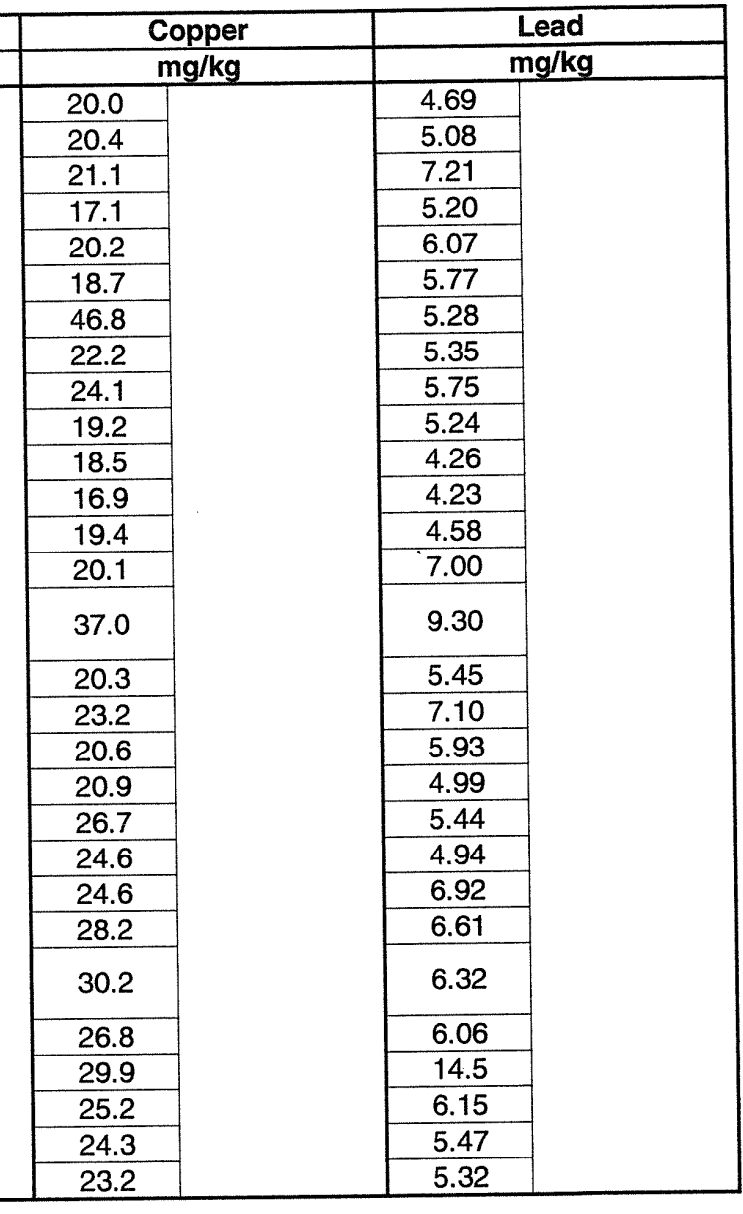


riginator H. M. Sulloway UUS

Date $12 / 20 / 11$

\section{0-207 Coal ash sample results}

\begin{tabular}{|c|c|c|c|}
\hline Sample & HEIS & Sample \\
\hline
\end{tabular}

\begin{tabular}{|l|c|c|c|c|c|}
\hline Location & Number & Sample & \multicolumn{3}{|c|}{ Manganese } \\
\cline { 3 - 5 } & Date & $\mathrm{mg} / \mathrm{kg}$ & $\mathrm{Q}$ & $\mathrm{PO}$ \\
\hline
\end{tabular}

\begin{tabular}{l|l|l|l|l} 
& & & \\
Manganese & Mercury & Molybdenum \\
\hline
\end{tabular}

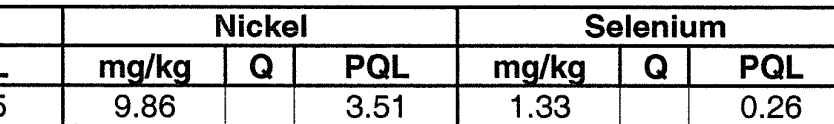

\begin{tabular}{|c|c|c|}
\multicolumn{4}{c}{$\mathrm{mg} / \mathrm{kg}$} & $\mathbf{Q}$ & $\mathrm{PQL}$ \\
\hline 1.33 & & 0.26 \\
\hline 172 & & 0.27
\end{tabular}

Vanadium

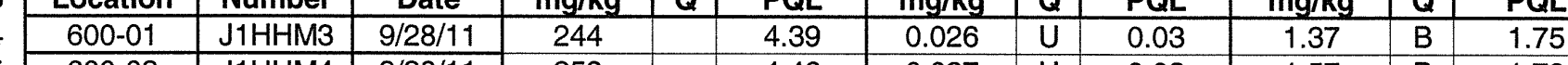

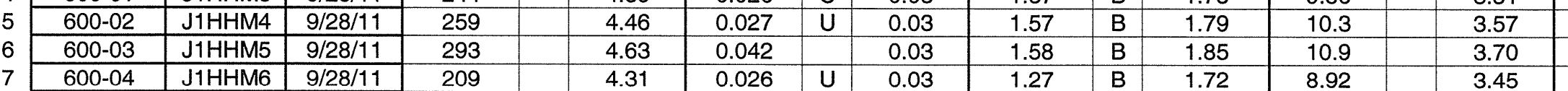

\begin{tabular}{|c|c|c|c|c|}
\hline $600-04$ & J1HHM6 & $9 / 28 / 11$ & 209 \\
\hline $600-05$ & J1HHM7 & $9 / 28 / 11$ & 206 \\
\hline
\end{tabular}

\begin{tabular}{|l|l|l|l|l|l}
\hline 266 & 4.63 & 0.027 & $U$ & 0.03 \\
\hline
\end{tabular}

\begin{tabular}{|c|c|c|c|c|c|c|c|}
\hline $600-07$ & J1HHM9 & $9 / 27 / 11$ & 486 & 4.39 & 0.009 & B & 0.03 \\
\hline
\end{tabular}

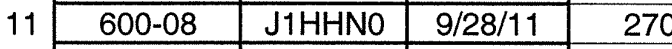

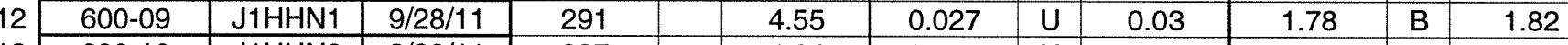

\begin{tabular}{|c|c|c|c|}
$600-10$ & J1HHN2 & $9 / 28 / 11$ & 227 \\
\hline $600-11$ & J1HHN3 & 92811 & 23 \\
\hline
\end{tabular}

\begin{tabular}{|l|l|l|l|l|l|l|l|}
\hline $600-11$ & J1HHN3 & $9 / 28111$ & 237 & 4.24 & 0.027 & $\mathrm{U}$ & 0.03 \\
\hline $600-12$ & J1HHN4 & 928 & 205 & 4.10 & 0.027 & $\mathrm{U}$ & 0.03 \\
\hline
\end{tabular}

\begin{tabular}{|c|c|c|c|c|c|c|c|c|}
\hline 16 & $600-13$ & J1HHN5 & $9 / 28 / 11$ & 209 & 4.31 & 0.027 & B & 0.03 \\
\hline
\end{tabular}

\begin{tabular}{c|c|c|c|c}
\hline 17 & $600-14$ & J1HHNG & $9 / 28 / 11$ & 200 \\
\hline
\end{tabular}

\begin{tabular}{|c|c|c|c|c|}
\hline $600-15$ & J1HHN7 & $9 / 27 / 11$ & 481 & 4.55 \\
\hline
\end{tabular}

19 \begin{tabular}{c|c|c|c|}
\hline $\begin{array}{c}\text { Duplicate of } \\
\text { J1HHN7 }\end{array}$ & J1HHR2 & $9 / 27 / 11$ & 537 \\
\hline
\end{tabular}

\begin{tabular}{c|c|c|c|c|c|}
\hline 20 & J1HAO-16 & J1HHN8 & $9 / 28 / 11$ & 293 & 4.39 \\
\hline
\end{tabular}

\begin{tabular}{l|c|c|c|c|}
21 & $600-17$ & J1HHN9 & $9 / 28 / 11$ & 264 \\
22 & $600-18$ & J1HHPO & $9 / 28 / 11$ & 280 \\
\cline { 2 - 5 } 23 & $600-19$ & J1HHP1 & $9 / 28 / 11$ & 271 \\
\hline
\end{tabular}

\begin{tabular}{|l|l|l|l|l|l|l|l}
\hline & & 0.027 & $\mathrm{U}$ & 0.03 \\
\hline
\end{tabular}

\begin{tabular}{l|l|l|l|l|}
24 & $600-20$ & J1HHP2 & $9 / 28 / 11$ & 349 \\
\hline & $600-21$ & J1HHP3 & $9 / 28 / 11$ & 357 \\
\hline
\end{tabular}

\begin{tabular}{|c|c|c|c|c|c|c|c|}
\hline & 24 & 4.00 & 0.026 & $U$ & 0.03 \\
\hline
\end{tabular}

\begin{tabular}{l|l|l|l|l|l|l|l|l|}
27 & $600-23$ & J1HHP5 & $9 / 27 / 11$ & 410 & 4.46 & 0.027 & B & 0.03 \\
\hline
\end{tabular}

\begin{tabular}{|c|c|c|c|}
\hline $600-24$ & J1HHP6 & $9 / 28 / 11$ & 373 \\
\hline Duplicate of & J1HHHB & $0 / 27 / 11$ & 300 \\
\hline
\end{tabular}

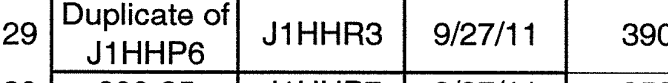

\begin{tabular}{l|c|c|c|c|}
30 & $600-25$ & J1HHP7 & $9 / 27 / 11$ & 252 \\
\cline { 2 - 5 } 31 & $600-26$ & J1HHP8 & $9 / 28 / 11$ & 435 \\
\cline { 2 - 5 } 3 & $600-27$ & J1HHP9 & $9 / 27 / 11$ & 379 \\
\hline
\end{tabular}

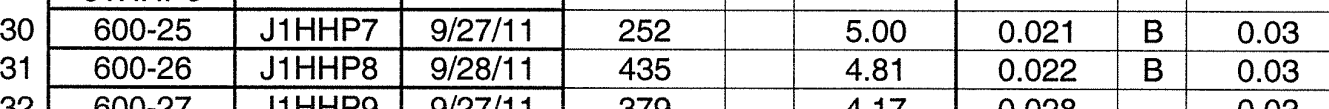

\begin{tabular}{c|c|c|c|c|}
33 & $600-28$ & J1HHRO & $9 / 27 / 11$ & 401 \\
\hline
\end{tabular}

\begin{tabular}{|l|l|r|}
\hline 1.28 & $\mathrm{~B}$ & 1.69 \\
\hline 1.38 & $\mathrm{~B}$ & 1.69 \\
\hline 1.01 & $\mathrm{~B}$ & 1.64 \\
\hline 1.26 & $\mathrm{~B}$ & 1.72 \\
\hline
\end{tabular}

1.36
1.42
1.29

\begin{tabular}{l|l|l}
1.42 & 0.28 \\
\hline 1.29 & 0.26 \\
1.22 & & 0.29
\end{tabular}

\begin{tabular}{|l|l|l|l|l|}
\hline 8.87 & 3.51 & 1.29 & & 0.26 \\
\hline 16.7 & 3.85 & 1.22 & & 0.29 \\
\hline 10.8 & 3.28 & 1.50 & 0.25 \\
\hline
\end{tabular}

\begin{tabular}{|l|l|}
\hline 8.87 & 3.85 \\
\hline 10.8 & 3.28 \\
\hline
\end{tabular}

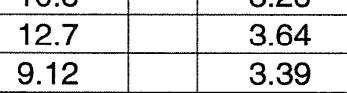

\begin{tabular}{l|l|l|}
1.22 & 0.29 \\
\hline 1.50 & & 0.25 \\
\hline 1.33 & & 0.27 \\
\hline
\end{tabular}

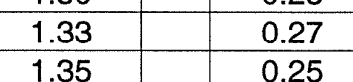

\begin{tabular}{l|l|l|}
\hline 11.7 & 3.39 & 1.35 \\
\hline 11.7 & 3.39 & 0.946 \\
\hline 7.5 & 3.28 & 0.898 \\
\hline
\end{tabular}

\begin{tabular}{|l|l|l|l|l|}
\hline 7.95 & 3.28 & 0.898 & & 0.25 \\
\hline 8.10 & 3.45 & 1.08 & & 0.26 \\
\hline 10.0 & 3.04 & 1.41 & & 0.27 \\
\hline
\end{tabular}

\begin{tabular}{l|l|l|l|}
1.26 & $\mathrm{~B}$ & 1.72 \\
\hline 1.47 & $\mathrm{~B}$ & 1.82
\end{tabular}

3.45

1.08

\begin{tabular}{l|l|l}
\hline 2.11 & & 1.92 \\
\hline 2.49 & & 1.69
\end{tabular}

\begin{tabular}{|l|l|}
\hline 18.9 & 3.64 \\
\hline 18.9 & 3.85 \\
\hline
\end{tabular}

\begin{tabular}{l|l}
1.75 & 1.75 \\
\hline
\end{tabular}

\begin{tabular}{l|r}
19.7 & 3.39 \\
\hline 12.6 & 3.51 \\
\hline 11.9 & 3.9 \\
\hline
\end{tabular}

11.9

\begin{tabular}{l|l|r|}
\hline 1.53 & B & 1.61 \\
\hline 1.74 & & 1.72 \\
\hline 2.26
\end{tabular}

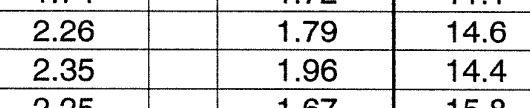

\begin{tabular}{|l|l|l|l|l|}
\hline 11.1 & 3.45 & 1.51 & & 0.26 \\
\hline 14.6 & 3.57 & 1.77 & & 0.27 \\
\hline 14.4 & 3.92 & 1.51 & & 0.29 \\
\hline 15.8 & & 3.33 & 1.88 &
\end{tabular}

\begin{tabular}{|l|l|l|l|l|l|}
2.02 & 1.79 & 16.2 & 3.37 & 1.88 & 0.25 \\
\hline & 1.44 & 0.27
\end{tabular}

\begin{tabular}{|l|r|}
\hline 2.69 & 1.79 \\
\hline 2.96
\end{tabular}

16.2
15.7
17.7

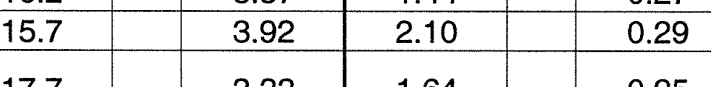

\begin{tabular}{l|l|l|}
1.88 & B & 2.00 \\
\hline 3
\end{tabular}

\begin{tabular}{|l|l|}
\hline 13.1 & 4.00 \\
\hline 16.7 & 3.85 \\
\hline 14.5 &
\end{tabular}

1.95

\begin{tabular}{|l|l|l|}
\hline 14.5 & 3.85 & 2.06 \\
\hline 14.3 & 3.33 & 1.92 \\
\hline 13.0 & 3.77 & 1.00 \\
\hline
\end{tabular}

\begin{tabular}{l|l}
1.92 & 0.20 \\
\hline 1.92 & 0.29 \\
\hline
\end{tabular}

\begin{tabular}{|l|l|}
\hline 1.88 & 1.67 \\
\hline 2.11 & 1.89 \\
\hline
\end{tabular}

\begin{tabular}{l|l|}
1.89 & 14.3 \\
\hline 1.45 & 13.0
\end{tabular} 
Washington Closure Hanford

Originator H. M. Sulloway LUA S

roject 100 and 300 Areas Coal Ash Characterization

Subject Coal Ash Characterization 90th Percentile and Median Equality Calculations
CALCULATION SHEET Date $\frac{12 / 20 / 11}{14655}$
Job No.
Calc. No. $0100 X-C A-V 0073$

Checked J. R. Davidson TR
Rev. No.

Sheet No. $\frac{12 / 20 / 11}{35 \text { of } 35}$ 1 600-207 Statistical Computation Input Da

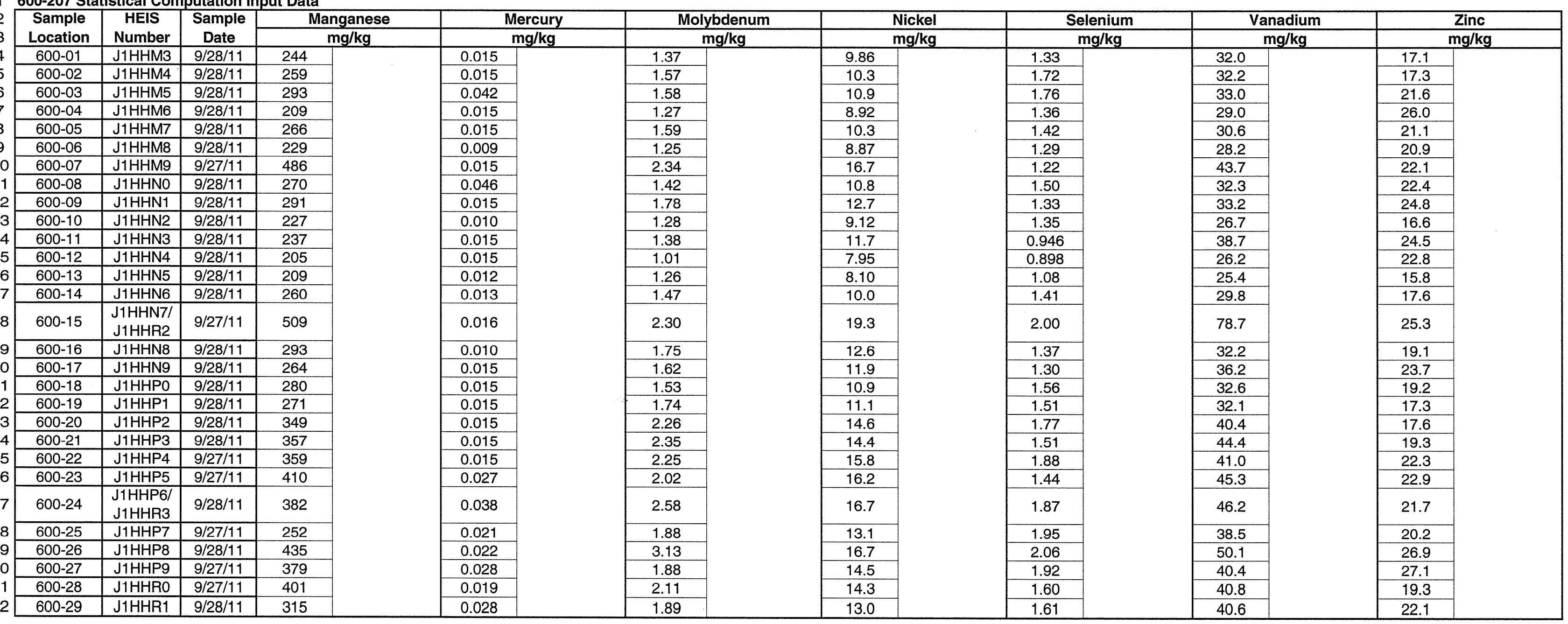




\section{CALCULATION COVER SHEET}

Area: 100-B, 100-D, 100-H, 100-IU-6, and 300 Areas

Discipline: Environmental

*Calculation No: 0100X-CA-V0075

Subject: Coal Ash Depth and Corresponding Surface Sample Confidence Limit Calculations

Computer Program: Excel

Program No: Excel 2003

The attached calculations have been generated for a specific purpose and task. Use of the calculations by persons who do not have access to all pertinent facts may lead to incorrect conclusions and/or results. Before applying these calculations to your work, the underlying basis, rationale, and other pertinent information relevant to these calculations must be thoroughly reviewed with appropriate Washington Closure Hanford LLC (WCH) officials or other authorized personnel. WCH is not responsible for the use of a calculation not under its direct control.

Committed Calculation $\bigotimes \quad$ Preliminary $\square \quad$ Superseded $\square \quad$ Voided

\begin{tabular}{|c|c|c|c|c|c|c|}
\hline Rev. & Sheet Numbers & Originator & Checker & Reviewer & Approval & Date \\
\hline 0 & $\begin{array}{c}\text { Cover }=1 \\
\text { Sheets }=13 \\
\text { Total }=14\end{array}$ & 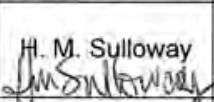 & $\begin{array}{l}\text { J.R. Davidson } \\
\text { J.R. D fuibsor }\end{array}$ & & J.M. Capron & $2 / 21 / 12$ \\
\hline & & 0 & & & & \\
\hline & & & & & & \\
\hline & & & & & & \\
\hline & & & & & & \\
\hline
\end{tabular}

\section{SUMMARY OF REVISION}

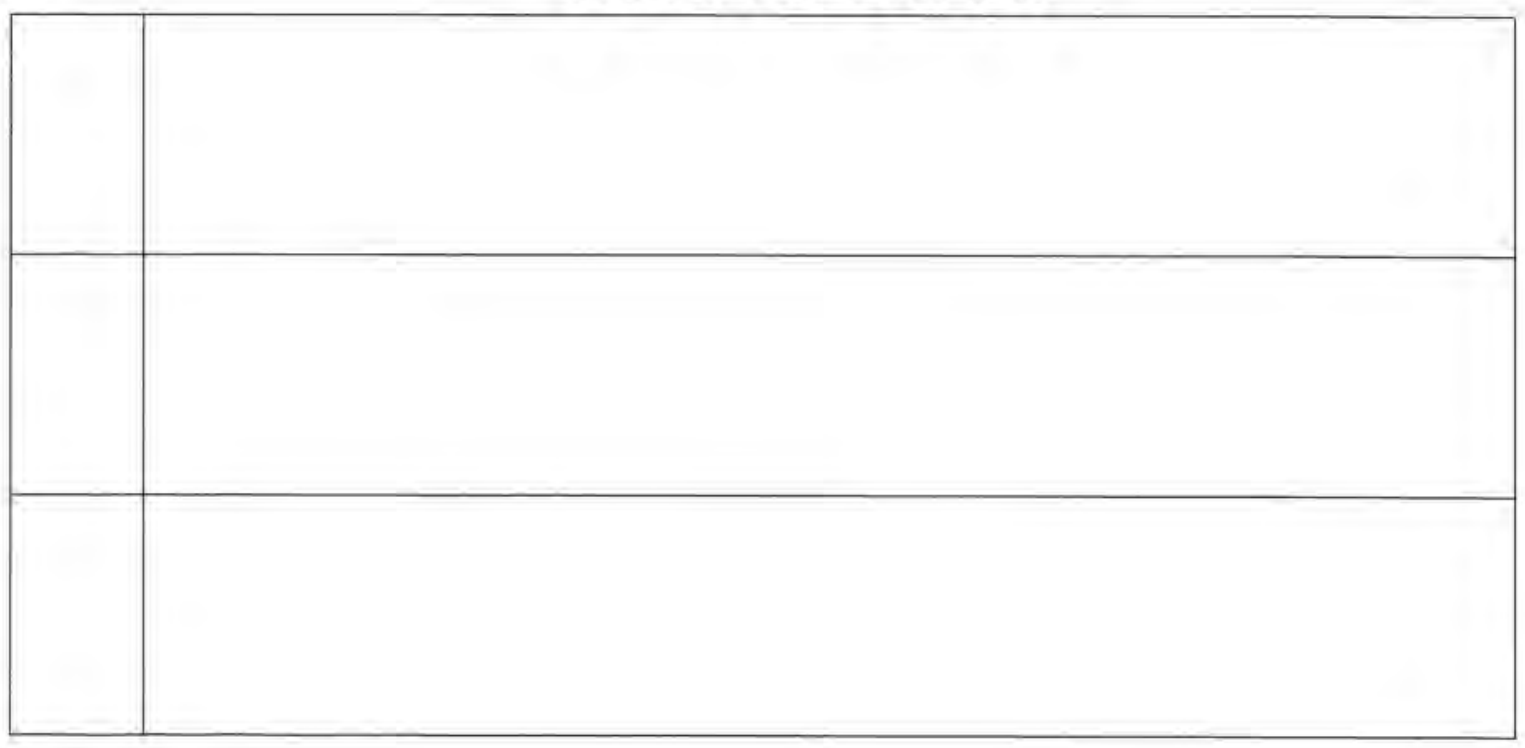




\begin{tabular}{|c|c|c|c|c|c|c|c|}
\hline \multicolumn{2}{|c|}{ Washington Closure Hanford, Inc. } & \multicolumn{6}{|c|}{ CALCULATION SHEET } \\
\hline Originator: & H. M. Sulloway Yhl S & Date: & 212112 & Calc. No.: & $0100 X-C A-V 0075$ & Rev:: & 0 \\
\hline Project: & $\begin{array}{l}100 \text { and } 300 \text { Areas Coal Ash } \\
\text { Characterization }\end{array}$ & Job No: & 14655 & Checked: & J. R. Davidson \& $Q$ & Date: & $2 / 21 / 12$ \\
\hline
\end{tabular}

\section{PURPOSE:}

The calculation provides documentation to support the calculation of the lower confidence limit (LCL), upper confidence limit (UCL), mean, and standard deviation on a subset of coal ash samples. Each of the five coal ash sample sites had three sample locations from which material was collected at both the surface and at a depth of $0.9 \mathrm{~m}(3 \mathrm{ft})$. Sample data from the three surface sample locations at all five sample sites $(\mathrm{N}=15)$ was compiled. A second data compilation was prepared for all depth sample data $(\mathrm{N}=15)$.

\section{TABLE OF CONTENTS:}

Sheets 1 to 3 - Summary

Sheets 4 to 8 - Coal ash surface samples calculations

Sheets 9 to 13 - Coal ash depth samples calculations

\section{GIVEN/REFERENCES:}

1) DOE-RL, 2011a, Sampling and Analysis Plan for Characterization of Hanford Site Coal Ash Components, DOE/RL-2010-113, Rev 0, U.S. Department of Energy, Richland Operations Office, Richland, Washington.

2) DOE-RL, 2011b, Tri-Party Agreement Change Notice, TPA-CN-431, Modify Sampling and Analysis Plan for Characterization of Hanford Site Coal Ash Components to add Additional Analysis for Total Uranium on a Subset of Coal Ash Samples, (DOE/RL-2010-113, Rev, 0), U.S. Department of Energy, Richland Operations Office, Richland, Washington.

3) DOE-RL, 2011c, Tri-Party Agreement Change Notice, TPA-CN-451, Modify Sampling and Analysis Plan for Characterization of Hanford Site Coal Ash Components to 1) Update Sample Area of 126-D-1 to Exclude an Area Containing Minimal Coal Ash and 2) Identify Waste Management Approach for Waste Related to Coal Ash Sampling Activities (DOE/RL-2010-113, Rev. 0), U.S. Department of Energy, Richland Operations Office, Richland, Washington.

4) Ecology, 1993, Statistical Guidance for Ecology Site Managers, Supplement S-6, Analyzing Site or Background Data with Below-detection Limit or Below-PQL Values (Censored Data Sets), Publication \#92-54, Washington Department of Ecology, Olympia, Washington.

5) Gilbert, R. O., 1987, Statistical Methods for Environmental Pollution Monitoring, John Wiley \& Sons, Inc., New York, New York.

\section{SOLUTION:}

The calculation of data mean, standard deviation, LCL, and UCL was performed as described in the Methodology section using the data provided. 
Washington Closure Hanford, Inc.

CALCULATION SHEET

\begin{tabular}{|c|c|c|c|c|c|c|c|}
\hline Originator: & H. M. Sulloway H(v) & Date: & 221,2 & Calc No.: & $0100 \mathrm{X}-\mathrm{CA}-\mathrm{V} 0075$ & Rev.. & 0 \\
\hline Project: & $\begin{array}{l}100 \text { and } 300 \text { Areas Coal Ash } \\
\text { Characterization }\end{array}$ & Job No: & 14655 & Checked: & 1. R. Davidson JeQ & Date: & $2 / 21)(2$ \\
\hline Subject: & \multicolumn{5}{|c|}{ Coal Ash Depth and Corresponding Surface Sample Confidence Limit Calculations } & \multicolumn{2}{|c|}{ Sheet No. 2 of 13} \\
\hline
\end{tabular}

\section{METHODOLOGY:}

Five coal ash sample sites underwent sampling as described in Sampling and Analysis Plan for Characterization of Hanford Site Coal Ash Components and subsequent change notices

(DOE-RL 2011a, 2011b, and 2011c). Amongst the five sample sites, a total of 15 surface samples were collected at a depth of $3 \mathrm{ft}(0.9 \mathrm{~m})$. A surface sample was also collected at each of the 15 depth sample locations. All samples were analyzed for metals, mercury, and total uranium.

All analytes were detected in at least one sample, with the exception of silver. All data reported as being undetected were set to $1 / 2$ the detection limit value for calculation of the statistics (Ecology 1993). For the statistical evaluation of primary-duplicate sample pairs, the sample results were averaged before being included in the data set, after adjustments for censored data as described above. All statistical analyses were performed using these adjusted data sets. Data means and standard deviations were determined using preset Excel functions.

The LCL and UCL were determined based on formulae provided in Gilbert (1987).

LCL $=$ lower confidence limit for a two-sided $95 \%$ confidence interval for the population mean. $\mathrm{UCL}=$ upper confidence limit for a two-sided $95 \%$ confidence interval for the population mean.

Formulas

where

$$
L C L=\bar{x}-t_{1-\alpha / 2, N-1} \frac{s}{\sqrt{N}}
$$

$$
U C L=\bar{x}+t_{1-\alpha / 2, N-1} \frac{s}{\sqrt{N}}
$$

$$
\bar{x}=\frac{\sum_{i=1}^{i=N} x_{i}}{N} \quad=\text { sample mean }
$$

$N \quad=$ number of values in data set, sample size;

$t_{t-x / 3, N-1}=$ Student's $t$-score where $\alpha=0.05$ in this case for a $95 \%$ confidence interval, $N-1$ is the degrees of freedom;

$\alpha$ $=$ long run proportion of confidence intervals that may not contain population mean, $\alpha=0.05$ in this case;

$$
s=\sqrt{\frac{\sum_{i=1}^{i=N}\left(x_{i}-\bar{x}\right)^{2}}{N-1}} \quad=\text { sample standard deviation. }
$$


Washington Closure Hanford

Originator $\mathrm{H}$. M. Sulloway

Project 100 and 300 Areas Coal Ash

$$
\text { Characterization }
$$

Subject Coal Ash Depth and Corresponding Surface Sample Confidence Limit Calculations

\section{CALCULATION SHEET}

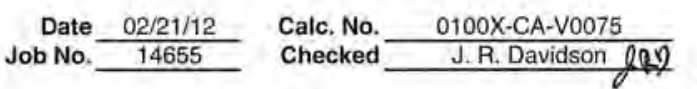
Checked J.R. Davidson len

Rev. No. Date $02 / 21 / 12$ Sheet No. 3 of 13

1 Summary

2 Results:

4 The results presented in the following table summarizes the average, standard deviation, LCL, and UCL for each of the respective

5 data sets.

6 Statistics and Confidence Limits for Depth and Corresponding Surface Coal Ash Samples

\begin{tabular}{|c|c|c|c|c|c|c|c|c|c|}
\hline \multirow[t]{2}{*}{7} & \multirow[b]{2}{*}{ Analyte } & \multicolumn{4}{|c|}{ Surface Concentrations and Confidence Limits } & \multicolumn{4}{|c|}{ Depth Concentrations and Confidence Limits } \\
\hline & & $\begin{array}{c}\text { Mean } \\
(\mathrm{mg} / \mathrm{kg})\end{array}$ & $\begin{array}{c}\text { Standard } \\
\text { Deviation } \\
(\mathrm{mg} / \mathrm{kg})\end{array}$ & $\begin{array}{c}\text { LCL } \\
(\mathrm{mg} / \mathrm{kg})\end{array}$ & $\begin{array}{c}\text { UCL } \\
\text { (mg/kg) }\end{array}$ & $\begin{array}{c}\text { Mean } \\
(\mathrm{mg} / \mathrm{kg})\end{array}$ & $\begin{array}{c}\text { Standard } \\
\text { Deviation } \\
(\mathrm{mg} / \mathrm{kg})\end{array}$ & $\begin{array}{c}\mathrm{LCL} \\
(\mathrm{mg} / \mathrm{kg})\end{array}$ & $\begin{array}{c}\text { UCL } \\
\text { (mg/kg) }\end{array}$ \\
\hline 9 & Antimony & 0.372 & 0.267 & 0.224 & 0.519 & 0.296 & 0.142 & 0.218 & 0.375 \\
\hline 0 & Arsenic & 4.31 & 2.37 & 3.00 & 5.62 & 4.20 & 2.67 & 2.72 & 5.68 \\
\hline 1 & Barium & 833 & 322 & 654 & 1011 & 803 & 271 & 653 & 953 \\
\hline 4 & Cadmium & 0.189 & 0.056 & 0.157 & 0.220 & 0.187 & 0.053 & 0.158 & 0.216 \\
\hline 5 & Chromium & 11.8 & 2,44 & 10.4 & 13.1 & 9.43 & 1.76 & 8.45 & 10.4 \\
\hline 6 & Cobalt & 4.58 & 1.98 & 3.49 & 5.67 & 3.65 & 1.42 & 2.86 & 4.44 \\
\hline 7 & Copper & 26.2 & 8.46 & 21.5 & 30.9 & 21.2 & 4.56 & 18.7 & 23.8 \\
\hline & Lead & 7.82 & 4.08 & 5.57 & 10.1 & 17.0 & 32.5 & -1.06 & 35.0 \\
\hline & Nickel & 11.0 & 3.91 & 8.88 & 13.2 & 8.78 & 2.33 & 7,48 & 10.1 \\
\hline & Selenium & 0.904 & 0.493 & 0.631 & 1.18 & 0.938 & 0.632 & 0.588 & 1.29 \\
\hline & Silver & - & - & - & - & - & $*$ & - & - \\
\hline & Thallium & 0.247 & 0.125 & 0.177 & 0.316 & $*$ & $\cdots$ & $*$ & - \\
\hline & Uranium & 3.62 & 0.832 & 3.16 & 4,08 & 3.60 & 1.16 & 2.96 & 4.24 \\
\hline & Vanadium & 39.3 & 12.8 & 32.2 & 46.4 & 29.9 & 5.29 & 27.0 & 32.9 \\
\hline & Zinc & 24.1 & 7.98 & 19.7 & 28.5 & 23.6 & 6.92 & 19.8 & 27.5 \\
\hline
\end{tabular}

$29-=$ Value not reported as no detections in subject data set

$30 \mathrm{LCL}=$ lower confidence limit

$31 \mathrm{UCL}=$ upper confidence limit 
Washington Closure Hanford

Originator H.M. Sulloway 1 .

Project 100 and 300 Areas Coal Ash

Characterization

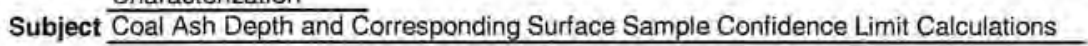

\section{CALCULATION SHEET}

Date $02 / 21 / 12$ Calc. No. 0100X-CA-V0075 Rev. No. 0 Job No. $\frac{14655}{1}$ Checked J.R. Davidson $/ 74$ Date $02 / 21 / 12$

\begin{tabular}{|c|c|c|c|c|c|c|c|c|c|c|c|c|c|c|c|}
\hline \multirow[t]{2}{*}{2} & \multirow{2}{*}{$\begin{array}{c}\text { Sample } \\
\text { Location }\end{array}$} & \multirow{2}{*}{$\begin{array}{c}\text { HEIS } \\
\text { Number }\end{array}$} & \multirow{2}{*}{$\begin{array}{c}\text { Sample } \\
\text { Date }\end{array}$} & \multicolumn{3}{|c|}{ Antimony } & \multicolumn{3}{|c|}{ Arsenic } & \multicolumn{3}{|c|}{ Barium } & \multicolumn{3}{|c|}{ Beryllium } \\
\hline & & & & $\mathrm{mg} / \mathrm{kg}$ & $Q$ & $P Q L$ & $\mathrm{mg} / \mathrm{kg}$ & $\mathbf{Q}$ & PQL & $\mathrm{mg} / \mathrm{kg}$ & Q & $\mathrm{PQL}$ & $\mathrm{mg} / \mathrm{kg}$ & $Q$ & $P Q L$ \\
\hline 4 & $100-B-10$ & J1HHW3 & $4 / 27 / 2011$ & 0.566 & $\mathrm{U}$ & 0.57 & 2.98 & & 0.94 & 597 & & 0.47 & 1.03 & & 0.19 \\
\hline 5 & $100-B-17$ & J1HHXO & $4 / 27 / 2011$ & 0.484 & UJ & 0.48 & 2.50 & & 0.81 & 556 & & 0.40 & 1.31 & & 0.16 \\
\hline 6 & $100-B-25$ & J1HHX8 & $4 / 25 / 2011$ & 1.69 & BJ & 1.70 & 10.3 & & 2.83 & 1420 & & 1.42 & 1.65 & & 0.57 \\
\hline 7 & $\begin{array}{c}\text { Duplicate of } \\
\text { J1HHX8 }\end{array}$ & J1HJO3 & $4 / 25 / 2011$ & 1.64 & U & 1.64 & 5.46 & & 2.73 & 907 & & 1.36 & 1.04 & & 0.55 \\
\hline 8 & $100-D-08$ & $51 \sqrt{3} \times 0$ & $8 / 11 / 2011$ & 0.470 & BJ & 0.58 & 2.32 & & 0.96 & 1470 & & 0.48 & 1.31 & & 0.19 \\
\hline 9 & $100-D-19$ & $\mathrm{J1} J 401$ & $8 / 11 / 2011$ & 0.605 & $\mathrm{~J}$ & 0.48 & 4.61 & & 0.79 & 743 & & 0.40 & 1.21 & & 0.16 \\
\hline 10 & $100-D-26$ & $J 1 J 408$ & $8 / 11 / 2011$ & 0.556 & $\mathrm{~J}$ & 0.51 & 3.91 & & 0.85 & 879 & & 0.42 & 1.17 & & 0.17 \\
\hline 11 & $\begin{array}{c}\text { Duplicate of } \\
\mathrm{J} 1 \mathrm{~J} 408\end{array}$ & $\mathrm{~J} 1 \mathrm{~J} 412$ & $8 / 11 / 2011$ & 0.545 & UJ & 0.55 & 4.34 & & 0.91 & 1140 & & 0.46 & 1.42 & & 0.18 \\
\hline 12 & $100-\mathrm{H}-17$ & J1HJ64 & $8 / 27 / 2011$ & 0.492 & UJ & 0.49 & 2.51 & & 0.82 & 527 & & 0.41 & 0.833 & & 0.16 \\
\hline 13 & $100-\mathrm{H}-20$ & J1HJ67 & $8 / 24 / 2011$ & 0.508 & UJ & 0.51 & 2.94 & & 0.85 & 916 & & 0.42 & 1.31 & & 0.17 \\
\hline 14 & $100-\mathrm{H}-25$ & J1HJ72 & $8 / 24 / 2011$ & 0.476 & UJ & 0.48 & 2.55 & & 0.79 & 954 & & 0.40 & 1.05 & & 0.16 \\
\hline 15 & $\begin{array}{c}\text { Duplicate of } \\
\text { J1HJ } 22\end{array}$ & J1HJ77 & $8 / 24 / 2011$ & 0.517 & UJ & 0.52 & 2.82 & & 0.86 & 1080 & & 0.43 & 1.11 & & 0.17 \\
\hline 16 & $300-02$ & J1HJN7 & $8 / 25 / 2011$ & 0.448 & UJ & 0.45 & 2.89 & & 0.75 & 490 & & 0.37 & 0.784 & & 0.15 \\
\hline 17 & $300-03$ & J1HJN6 & $8 / 25 / 2011$ & 0.455 & UJ & 0.46 & 3.33 & & 0.76 & 306 & & 0.38 & 0.544 & & 0.15 \\
\hline 18 & $300-04$ & J1HJN9 & $8 / 25 / 2011$ & 0.588 & UJ & 0.59 & 2.71 & & 0.98 & 541 & & 0.49 & 1.14 & & 0.20 \\
\hline 19 & $\begin{array}{c}\text { Duplicate of } \\
\text { J1HJN9 }\end{array}$ & J1HJT5 & $8 / 25 / 2011$ & 0.484 & UJ & 0.48 & 2.66 & & 0.81 & 639 & & 0.40 & 1.03 & & 0.16 \\
\hline 20 & $600-07$ & J1HHM9 & 9/27/2011 & 0.577 & UJ & 0.58 & 6.60 & & 0.96 & 958 & $\mathrm{~J}$ & 0.48 & 0.903 & & 0.19 \\
\hline & $600-15$ & J1HHN7 & 9/27/2011 & 0.577 & UJ & 0.58 & 10.6 & & 0.96 & 1210 & $J$ & 0.48 & 1.43 & & 0.19 \\
\hline 22 & $\begin{array}{c}\text { Duplicate of } \\
\text { JHHHN7 }\end{array}$ & J1HHR2 & 9/27/2011 & 0.508 & UJ & 0.51 & 9.58 & & 0.85 & 1250 & $J$ & 0.42 & 1.46 & & 0.17 \\
\hline 23 & $600-23$ & J1HHP5 & 9/27/2011 & 0.536 & UJ & 0.54 & 6.46 & & 0.89 & 915 & $J$ & 0.45 & 0.969 & & 0.18 \\
\hline
\end{tabular}

25 Statistical Computation Input Data

\begin{tabular}{|c|c|c|c|c|c|c|c|}
\hline 26 & Sample & HEIS & Sample & Antimony & Arsenic & Barium & Beryllium \\
\hline 27 & Location & Number & Date & $\mathrm{mg} / \mathrm{kg}$ & $\mathrm{mg} / \mathrm{kg}$ & $\mathrm{mg} / \mathrm{kg}$ & $\mathrm{mg} / \mathrm{kg}$ \\
\hline 28 & $100-8-10$ & J1HHW3 & $4 / 27 / 2011$ & 0.285 & 2.98 & 597 & 1.03 \\
\hline 29 & $100-B-17$ & J1HHXO & $4 / 27 / 2011$ & 0.240 & 2.50 & 556 & 1.31 \\
\hline 30 & 100-B-25 & $\begin{array}{c}\mathrm{J} 1 \mathrm{HHX8/} \\
\mathrm{J} 1 \mathrm{HJ} 03 \\
\end{array}$ & $4 / 25 / 2011$ & 1.26 & 7.88 & 1164 & 1.35 \\
\hline 31 & $100-D-08$ & $\mathrm{J1} J 3 \times 0$ & $8 / 11 / 2011$ & 0.470 & 2.32 & 1470 & 1.31 \\
\hline 32 & $100-D-19$ & J1J401 & $8 / 11 / 2011$ & 0.605 & 4.61 & 743 & 1.21 \\
\hline 33 & $100-D-26$ & $\begin{array}{l}J 1 J 408 / \\
J 1 J 412\end{array}$ & $8 / 11 / 2011$ & 0.416 & 4.13 & 1010 & 1.30 \\
\hline 34 & $100-\mathrm{H}-17$ & J1HJ64 & $8 / 27 / 2011$ & 0.245 & 2.51 & 527 & 0.833 \\
\hline 35 & $100-\mathrm{H}-20$ & J1HJ67 & $8 / 24 / 2011$ & 0.255 & 2.94 & 916 & 1.31 \\
\hline 36 & $100-\mathrm{H}-25$ & $\begin{array}{l}\mathrm{J} 1 \mathrm{HJ} 72 / \\
\mathrm{J} 1 \mathrm{HJ} 77 \\
\end{array}$ & $8 / 24 / 2011$ & 0.250 & 2,69 & 1017 & 1.08 \\
\hline 37 & $300-02$ & J1HJN7 & $8 / 25 / 2011$ & 0.225 & 2.89 & 490 & 0.784 \\
\hline 38 & $300-03$ & J1HJNG & $8 / 25 / 2011$ & 0.230 & 3.33 & 306 & 0.544 \\
\hline 39 & 300-04 & $\begin{array}{l}\text { J1HJN9/ } \\
\text { J1HJT5 }\end{array}$ & $8 / 25 / 2011$ & 0.268 & 2.69 & 590 & 1.09 \\
\hline 40 & $600-07$ & J1HHM9 & 9/27/2011 & 0.290 & 6.60 & 958 & 0.903 \\
\hline 41 & $600-15$ & $\begin{array}{l}\text { J1HHN7/ } \\
\text { J1HHR2 }\end{array}$ & 9/27/2011 & 0.273 & 10.1 & 1230 & 1.45 \\
\hline 42 & $600-23$ & J1HHP5 & 9/27/2011 & 0.270 & 6.46 & 915 & 0.969 \\
\hline
\end{tabular}

43

44 Computations

\begin{tabular}{|c|c|c|c|c|c|}
\hline 45[ & $\mathrm{N}$ & 15 & 15 & 15 & 15 \\
\hline 46 & Percent below detection limit & $73 \%$ & $0 \%$ & $0 \%$ & $0 \%$ \\
\hline 47 & Mean & 0.372 & 4.31 & 833 & 1.10 \\
\hline 48 & Standard Deviation & 0.267 & 2,37 & 322 & 0.254 \\
\hline 49 & Lower Confidence Limit & 0.224 & 3.00 & 654 & 0.956 \\
\hline 50 & Upper Confidence Limit & 0.519 & 5.62 & 1011 & 1.24 \\
\hline $\begin{array}{l}51 \\
52\end{array}$ & $\begin{array}{l}\text { lank contamination } \\
=\text { Hanford Environmental Inforn }\end{array}$ & 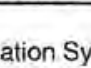 & $\begin{array}{l}=\text { estin } \\
Q \mathrm{LL}=p\end{array}$ & on limi & $\begin{array}{l}\text { er } \\
\text { ected }\end{array}$ \\
\hline
\end{tabular}


Washington Closure Hanford

Originator H.M. Sulloway d.

Project 100 and 300 Areas' ${ }^{\prime} \mathrm{Coal}$ Ash Characterization

Subject Coal Ash Depth and Corresponding Surface Sample Confidence Limit Calculations

\section{CALCULATION SHEET}

Date $\underline{02 / 21 / 12}$ Calc. No. 0100X-CA-V0075, Rey. No. 0 Job No. 14655 Checked J.R. Davidson (2) Date 02/21/12 Sheet No. 5 of 13

1 Coal Ash Surface Sample Data

\begin{tabular}{|c|c|c|c|c|c|c|c|c|c|c|c|c|c|c|}
\hline \multirow{2}{*}{$\begin{array}{c}\text { Sample } \\
\text { Location }\end{array}$} & \multirow{2}{*}{$\begin{array}{c}\text { HEIS } \\
\text { Number }\end{array}$} & \multirow{2}{*}{$\begin{array}{c}\text { Sample } \\
\text { Date }\end{array}$} & \multicolumn{3}{|c|}{ Boron } & \multicolumn{3}{|c|}{ Cadmium } & \multicolumn{3}{|c|}{ Chromium } & \multicolumn{3}{|c|}{ Cobalt } \\
\hline & & & $\mathrm{mg} / \mathrm{kg}$ & $Q$ & $\mathrm{PQL}$ & $\mathrm{mg} / \mathrm{kg}$ & $\mathbf{Q}$ & PQL & $\mathrm{mg} / \mathrm{kg}$ & $\mathbf{Q}$ & $\mathrm{PQL}$ & $\mathrm{mg} / \mathrm{kg}$ & $Q$ & PQL \\
\hline $100-\mathrm{B}-10$ & J1HHW3 & $4 / 27 / 2011$ & 169 & & 1.89 & 0.117 & B & 0.19 & 8.75 & & 0.19 & 4.42 & & 1.89 \\
\hline $100-B-17$ & J1HHXO & $4 / 27 / 2011$ & 269 & & 1.61 & 0.221 & & 0.16 & 10.6 & & 0.16 & 2.51 & & 1.61 \\
\hline $100-B-25$ & J1HHX8 & $4 / 25 / 2011$ & 107 & & 5.66 & 0.385 & B & 0.57 & 11.2 & & 0.57 & 4.66 & B & 5.66 \\
\hline $\begin{array}{c}\text { Duplicate of } \\
\mathrm{J} 1 \mathrm{HHX8}\end{array}$ & J1HJ03 & $4 / 25 / 2011$ & 85.5 & & 5.45 & 0.278 & B & 0.55 & 8.34 & & 0.55 & 4.12 & B & 5.45 \\
\hline $100-D-08$ & $J 1 \mathrm{~J} 3 \times 0$ & $8 / 11 / 2011$ & 317 & $\mathrm{~J}$ & 1.92 & 0.129 & B & 0.19 & 8.03 & & 0.19 & 9.87 & & 1.92 \\
\hline $100-D-19$ & J1J401 & $8 / 11 / 2011$ & 142 & $J$ & 1.59 & 0.176 & & 0.16 & 12.6 & & 0.16 & 4.59 & & 1.59 \\
\hline $100-D-26$ & J1J408 & $8 / 11 / 2011$ & 181 & $\mathrm{~J}$ & 1.69 & 0.156 & B & 0.17 & 11.4 & & 0.17 & 3.44 & & 1.69 \\
\hline $\begin{array}{c}\text { Duplicate of } \\
\text { J1J408 }\end{array}$ & J1J412 & $8 / 11 / 2011$ & 218 & $J$ & 1.82 & 0.190 & & 0.18 & 12.9 & & 0.18 & 3,48 & & 1.82 \\
\hline $100-\mathrm{H}-17$ & J1HJ64 & $8 / 27 / 2011$ & 145 & $\mathrm{~J}$ & 1.64 & 0.148 & B & 0.16 & 8.57 & & 0.16 & 2.14 & & 1.64 \\
\hline $100-\mathrm{H}-20$ & J1HJ67 & $8 / 24 / 2011$ & 455 & $\mathrm{~J}$ & 1.69 & 0.178 & & 0.17 & 16.5 & & 0.17 & 3.77 & & 1.69 \\
\hline $100-\mathrm{H}-25$ & J1HJ72 & $8 / 24 / 2011$ & 235 & $\mathrm{~J}$ & 1.59 & 0.217 & & 0.16 & 12.3 & & 0.16 & 3.66 & & 1.59 \\
\hline $\begin{array}{c}\text { Duplicate of } \\
\text { J1HJ72 }\end{array}$ & J1HJ77 & $8 / 24 / 2011$ & 253 & J & 1.72 & 0.183 & & 0.17 & 12.5 & & 0.17 & 3.82 & & 1.72 \\
\hline $300-02$ & J1HJN7 & $8 / 25 / 2011$ & 161 & & 1.49 & 0.226 & & 0.15 & 12.5 & & 0.15 & 3.82 & & 1.49 \\
\hline $300-03$ & J1HJNG & $8 / 25 / 2011$ & 65.4 & & 1.52 & 0.201 & & 0.15 & 12.0 & & 0.15 & 4.23 & & 1.52 \\
\hline $300-04$ & J1HJN9 & $8 / 25 / 2011$ & 196 & & 1.96 & 0.253 & & 0.20 & 12.8 & & 0.20 & 3.00 & & 1.96 \\
\hline $\begin{array}{c}\text { Duplicate of } \\
\text { J1HJN9 }\end{array}$ & J1HJT5 & $8 / 25 / 2011$ & 199 & & 1.61 & 0.227 & & 0.16 & 11.2 & & 0.16 & 2.66 & & 1.61 \\
\hline $600-07$ & J1HHM9 & $9 / 27 / 2011$ & 146 & $\mathrm{~J}$ & 1.92 & 0.122 & B & 0.19 & 12.9 & $J$ & 0.19 & 5.74 & & 1.9 \\
\hline $600-15$ & J1HHN7 & 9/27/2011 & 112 & $J$ & 1.92 & 0.232 & & 0.19 & 15.5 & $J$ & 0.19 & 7.14 & & 1.92 \\
\hline $\begin{array}{c}\text { Duplicate of } \\
\text { J1HHN7 }\end{array}$ & J1HHR2 & 9/27/2011 & 162 & $J$ & 1.69 & 0.213 & & 0.17 & 16.7 & $J$ & 0.17 & 7.08 & & 1.69 \\
\hline $600-23$ & J1HHP5 & $9 / 27 / 2011$ & 103 & $J$ & 1.79 & 0.143 & B & 0.18 & 11.5 & $J$ & 0.18 & 6.07 & & 1.79 \\
\hline
\end{tabular}
24

25 Statistical Computation Input Data

\begin{tabular}{|c|c|c|c|c|c|c|c|}
\hline \multirow{2}{*}{$\begin{array}{l}26 \\
27\end{array}$} & \multirow{2}{*}{$\begin{array}{c}\text { Sample } \\
\text { Location }\end{array}$} & \multirow{2}{*}{\begin{tabular}{|c|c|} 
HEIS \\
Number
\end{tabular}} & \multirow{2}{*}{$\begin{array}{c}\text { Sample } \\
\text { Date }\end{array}$} & \multirow{2}{*}{$\begin{array}{l}\text { Boron } \\
\mathrm{mg} / \mathrm{kg}\end{array}$} & \multirow{2}{*}{$\begin{array}{c}\text { Cadmium } \\
\mathrm{mg} / \mathrm{kg}\end{array}$} & \multirow{2}{*}{$\begin{array}{c}\text { Chromium } \\
\mathrm{mg} / \mathrm{kg}\end{array}$} & \multirow{2}{*}{$\frac{\text { Cobalt }}{\mathrm{mg} / \mathrm{kg}}$} \\
\hline & & & & & & & \\
\hline 28 & $100-\mathrm{B}-10$ & \begin{tabular}{|l|} 
J1HHW3 \\
\end{tabular} & $4 / 27 / 2011$ & 169 & 0.117 & 8.75 & 4.42 \\
\hline 29 & $100-B-17$ & J1HHXO & $4 / 27 / 2011$ & 269 & 0.221 & 10.6 & 2.51 \\
\hline 30 & $100-B-25$ & $\begin{array}{c}\mathrm{J} 1 \mathrm{HHX8/} \\
\mathrm{J} 1 \mathrm{HJO3}\end{array}$ & $4 / 25 / 2011$ & 96.3 & 0.332 & 9.77 & 4.39 \\
\hline 31 & $100-D-08$ & J1J3X0 & $8 / 11 / 2011$ & 317 & 0.129 & 8.03 & 9.87 \\
\hline 32 & $100-D-19$ & J1J401 & $8 / 11 / 2011$ & 142 & 0.176 & 12.6 & 4.59 \\
\hline 33 & 100-D-26 & $\begin{array}{l}\mathrm{J} 1 \mathrm{~J} 408 / \\
\mathrm{J} 1 \mathrm{~J} 412 \\
\end{array}$ & $8 / 11 / 2011$ & 200 & 0.173 & 12.2 & 3.46 \\
\hline 34 & $100-\mathrm{H}-17$ & J1HJ64 & $8 / 27 / 2011$ & 145 & 0.148 & 8.57 & 2.14 \\
\hline 35 & $100-\mathrm{H}-20$ & J1HJ67 & $8 / 24 / 2011$ & 455 & 0.178 & 16.5 & 3.77 \\
\hline 36 & $100-\mathrm{H}-25$ & $\begin{array}{l}\mathrm{J} 1 \mathrm{H} J 72 / \\
\mathrm{J} 1 \mathrm{HJ} 77\end{array}$ & 8/24/2011 & 244 & 0.200 & 12.4 & 3.74 \\
\hline 37 & $300-02$ & J1HJN7 & $8 / 25 / 2011$ & 161 & 0.226 & 12.5 & 3.82 \\
\hline 38 & $300-03$ & \begin{tabular}{|l|} 
J1HJN6 \\
\end{tabular} & $8 / 25 / 2011$ & 65 & 0.201 & 12.0 & 4.23 \\
\hline 39 & $300-04$ & \begin{tabular}{|l|} 
J1HJN9/ \\
J1HJT5 \\
\end{tabular} & $8 / 25 / 2011$ & 198 & 0.240 & 12.0 & 2.83 \\
\hline 40 & $600-07$ & J1HHM9 & 9/27/2011 & 146 & 0.122 & 12.9 & 5.74 \\
\hline 41 & $600-15$ & \begin{tabular}{|l|} 
J1HHN7/ \\
J1HHR2 \\
\end{tabular} & $9 / 27 / 2011$ & 137 & 0.223 & 16.1 & 7.11 \\
\hline 42[ & $600-23$ & J1HHP5 & 9/27/2011 & 103 & 0.143 & 11.5 & 6.07 \\
\hline \multicolumn{8}{|c|}{43 Computations } \\
\hline 45 & & & $N$ & 15 & 15 & 15 & 15 \\
\hline 46 & Percent & below dete & ction limit & $0 \%$ & $0 \%$ & $0 \%$ & $0 \%$ \\
\hline 47 & & & Mean & 190 & 0.189 & 11.8 & 4.58 \\
\hline 48 & & Standard & Deviation & 99.2 & 0.056 & 2.44 & 1.98 \\
\hline 49 & & ver Confid & ence Limit & 135 & 0.157 & 10.4 & 3.49 \\
\hline 50 & & per Confid & ence Limit & 245 & 0.220 & 13.1 & 5.67 \\
\hline
\end{tabular}


Washington Closure Hanford

Originator H. M. Sulloway of (U)

Project 100 and 300 Areas Coal Ash Characterization

Subject Coal Ash Depth and Corresponding Suriace Sample Contidence Limit Calculations

\section{CALCULATION SHEET}

Date 02/21/12 Calc. No. 0100X-CA-V0075 Rev. No. 0 Job No, $\frac{14655}{145}$ Checked $\frac{0}{\text { J.R.Davidson } / \text { DS Date }}$

Coal Ash Surface Sample Data

\begin{tabular}{|c|c|c|c|c|c|c|c|c|c|c|c|c|c|c|}
\hline \multirow{2}{*}{$\begin{array}{l}\text { Sample } \\
\text { Location }\end{array}$} & \multirow{2}{*}{$\begin{array}{c}\text { HEIS } \\
\text { Number }\end{array}$} & \multirow{2}{*}{$\begin{array}{c}\text { Sample } \\
\text { Date }\end{array}$} & \multicolumn{3}{|c|}{ Copper } & \multicolumn{3}{|c|}{ Lead } & \multicolumn{3}{|c|}{ Manganese } & \multicolumn{3}{|c|}{ Mercury } \\
\hline & & & $\mathrm{mg} / \mathrm{kg}$ & Q & PQL & $\mathrm{mg} / \mathrm{kg}$ & $Q$ & PQL & $\mathrm{mg} / \mathrm{kg}$ & $\mathbf{Q}$ & $P Q L$ & $\mathrm{mg} / \mathrm{kg}$ & $\mathbf{Q}$ & PQL \\
\hline $100-B-10$ & J1HHW3 & $4 / 27 / 2011$ & 28.8 & & 0.94 & 5.59 & & 0.47 & 104 & & 4.72 & 0.049 & & 0.03 \\
\hline $100-B-17$ & $\mathrm{~J} 1 \mathrm{HHXO}$ & $4 / 27 / 2011$ & 21.9 & & 0.81 & 6.28 & & 0.40 & 57.5 & & 4.03 & 0.053 & & 0.03 \\
\hline $100-B-25$ & $\mathrm{~J} 1 \mathrm{HH} \times 8$ & $4 / 25 / 2011$ & 28.4 & & 2.83 & 13.4 & & 1.42 & 391 & & 14.2 & 0.115 & & 0.03 \\
\hline $\begin{array}{c}\text { Duplicate of } \\
\mathrm{J} 1 \mathrm{HHX8}\end{array}$ & J1HJO3 & $4 / 25 / 2011$ & 20.2 & & 2.73 & 9.95 & & 1.36 & 202 & & 13.6 & 0.120 & & 0.03 \\
\hline $100-D-08$ & $\mathrm{~J} 1 \mathrm{~J} 3 \times 0$ & $8 / 11 / 2011$ & 20.8 & & 0.96 & 1.90 & $J$ & 0.48 & 244 & & 4.81 & 0.119 & & 0.03 \\
\hline $100-D-19$ & $J 1 \sqrt{401}$ & $8 / 11 / 2011$ & 31.0 & & 0.79 & 16.8 & J & 0.40 & 122 & & 3.97 & 0.074 & & 0.03 \\
\hline 100-D-26 & $J 1 J 408$ & $8 / 11 / 2011$ & 23.8 & & 0.85 & 13.2 & $J$ & 0.42 & 105 & & 4.24 & 0.061 & & 0.03 \\
\hline $\begin{array}{c}\text { Duplicate of } \\
\text { J1 } 4408\end{array}$ & J1J412 & $8 / 11 / 2011$ & 28.0 & & 0.91 & 16.4 & J & 0.46 & 137 & J & 4.55 & 0.048 & & 0.03 \\
\hline $100-\mathrm{H}-17$ & J1HJ64 & $8 / 27 / 2011$ & 16.7 & & 0.82 & 4.40 & $\mathrm{~J}$ & 0.41 & 67.7 & $\mathrm{~J}$ & 4.10 & 0.049 & & 0.03 \\
\hline $100-\mathrm{H}-20$ & $\mathrm{~J} 1 \mathrm{HJ} 67$ & $8 / 24 / 2011$ & 33.8 & & 0.85 & 4.25 & $\mathrm{~J}$ & 0.42 & 122 & $\mathrm{~J}$ & 4.24 & 0.018 & B & 0.03 \\
\hline $100-\mathrm{H}-25$ & $\mathrm{J1HJ72}$ & $8 / 24 / 2011$ & 24.0 & & 0.79 & 5.02 & $\mathrm{~J}$ & 0.40 & 153 & $\mathrm{~J}$ & 3.97 & 0.027 & & 0.03 \\
\hline $\begin{array}{c}\text { Duplicate of } \\
\text { J1HJ72 }\end{array}$ & J1HJ77 & $8 / 24 / 2011$ & 23.7 & & 0.86 & 5.21 & $J$ & 0.43 & 122 & J & 4.31 & 0.019 & B & 0.03 \\
\hline $300-02$ & J1HJN7 & $8 / 25 / 2011$ & 19.6 & & 0.75 & 9.66 & & 0.37 & 186 & & 3.73 & 0.713 & & 0.03 \\
\hline $300-03$ & J1HJN6 & $8 / 25 / 2011$ & 14.7 & & 0.76 & 7.13 & & 0.38 & 213 & & 3.79 & 0.060 & & 0.03 \\
\hline $300-04$ & J1HJN9 & $8 / 25 / 2011$ & 20.3 & & 0.98 & 9.41 & & 0.49 & 107 & & 4.90 & 0.365 & & 0.03 \\
\hline $\begin{array}{c}\text { Duplicate of } \\
\text { JHHJN9 }\end{array}$ & J1HJT5 & $8 / 25 / 2011$ & 18.5 & & 0.81 & 7.74 & & 0.40 & 91.7 & & 4.03 & 0.344 & & 0.03 \\
\hline $600-07$ & J1HHM9 & 9/27/2011 & 46.8 & $\mathrm{~J}$ & 1.0 & 5.28 & & 0.5 & 486 & $J$ & 4.81 & 0.027 & U & 0.03 \\
\hline $600-15$ & J1HHN7 & 9/27/2011 & 35.4 & J & 0.96 & 11.1 & & 0.48 & 481 & $J$ & 4.81 & 0.025 & U & 0.03 \\
\hline $\begin{array}{c}\text { Duplicate of } \\
\text { J1HHN7 }\end{array}$ & J1HHR2 & $9 / 27 / 2011$ & 38.6 & J & 0.85 & 7.5 & & 0.42 & 537 & $J$ & 4.2 & 0.017 & B & 0.02 \\
\hline $600-23$ & J1HHP5 & $9 / 27 / 2011$ & 28.2 & $\mathrm{~J}$ & 0.89 & 6.61 & & 0.45 & 410 & $J$ & 4.46 & 0.027 & B & 0.03 \\
\hline
\end{tabular}
24

25 Statistical Computation Input Data

\begin{tabular}{|c|c|c|c|c|c|c|c|}
\hline 26 & \multirow{2}{*}{$\begin{array}{l}\text { Sample } \\
\text { Location }\end{array}$} & \multirow{2}{*}{$\begin{array}{l}\text { HEIS } \\
\text { Number }\end{array}$} & \multirow{2}{*}{$\begin{array}{c}\text { Sample } \\
\text { Date }\end{array}$} & Copper & Lead & \multirow{2}{*}{$\frac{\text { Manganese }}{\mathrm{mg} / \mathrm{kg}}$} & \multirow{2}{*}{$\begin{array}{c}\text { Mercury } \\
\mathrm{mg} / \mathrm{kg} \\
\end{array}$} \\
\hline 27 & & & & $\mathrm{mg} / \mathrm{kg}$ & $\mathrm{mg} / \mathrm{kg}$ & & \\
\hline 28 & $100-B-10$ & J1HHW3 & $4 / 27 / 2011$ & 28.8 & 5.59 & 104 & 0.049 \\
\hline 29 & $100-B-17$ & J1HHXO & $4 / 27 / 2011$ & 21.9 & 6.28 & 57.5 & 0.053 \\
\hline 30 & $100-B-25$ & $\begin{array}{c}\mathrm{J} 1 \mathrm{HHX8/} \\
\mathrm{J} 1 \mathrm{HJO3}\end{array}$ & $4 / 25 / 2011$ & 24.3 & 11.7 & 297 & 0.118 \\
\hline 31 & $100-D-08$ & $\mathrm{~J} 1 \mathrm{~J} 3 \times 0$ & $8 / 11 / 2011$ & 20.8 & 1.90 & 244 & 0.119 \\
\hline 32 & $100-D-19$ & $\sqrt{11} 4401$ & $8 / 11 / 2011$ & 31.0 & 16.8 & 122 & 0.074 \\
\hline 33 & $100-D-26$ & $\begin{array}{l}\mathrm{J} 1 \mathrm{~J} 408 / \\
\mathrm{J} 1 \mathrm{~J} 412 \\
\end{array}$ & $8 / 11 / 2011$ & 25.9 & 14.8 & 121 & 0.055 \\
\hline 34 & $100-\mathrm{H}-17$ & J1HJ64 & $8 / 27 / 2011$ & 16.7 & 4.40 & 67.7 & 0.049 \\
\hline 35 & $100-\mathrm{H}-20$ & J1HJ67 & $8 / 24 / 2011$ & 33.8 & 4.25 & 122 & 0.018 \\
\hline 36 & $100-\mathrm{H}-25$ & $\begin{array}{l}\mathrm{J} 1 \mathrm{HJ} 72 / \\
\mathrm{J} 1 \mathrm{HJ} 77\end{array}$ & $8 / 24 / 2011$ & 23.9 & 5.12 & 138 & 0.023 \\
\hline 37 & $300-02$ & J1HJN7 & $8 / 25 / 2011$ & 19.6 & 9.66 & 186 & 0.713 \\
\hline 38 & $300-03$ & J1HJN6 & $8 / 25 / 2011$ & 14.7 & 7.13 & 213 & 0.060 \\
\hline 39 & $300-04$ & $\begin{array}{l}\text { J1HJN9/ } \\
\text { J1HJT5 }\end{array}$ & $8 / 25 / 2011$ & 19.4 & 8.58 & 99.4 & 0.355 \\
\hline 40 & $600-07$ & J1HHM9 & $9 / 27 / 2011$ & 46.8 & 5.28 & 486 & 0.015 \\
\hline 41 & $600-15$ & $\begin{array}{l}\text { J1HHN7/ } \\
\text { J1HHR2 }\end{array}$ & $9 / 27 / 2011$ & 37.0 & 9.30 & 509 & 0.016 \\
\hline 42\lfloor & $600-23$ & J1HHPS & $9 / 27 / 2011$ & 28.2 & 6.61 & 410 & 0.027 \\
\hline $\begin{array}{l}43 \\
44\end{array}$ & inputano & & & & & & \\
\hline 45 & & & $\mathrm{~N}$ & 15 & 15 & 15 & 15 \\
\hline 46 & Percen & below dete & ction limit & $0 \%$ & $0 \%$ & $0 \%$ & $7 \%$ \\
\hline 47 & & & Mean & 26.2 & 7.82 & 212 & 0.116 \\
\hline 48 & & Standarc & Deviation & 8.46 & 4.08 & 149 & 0.185 \\
\hline 49 & & Ner Confid & ence Limit & 21.5 & 5.57 & 129 & 0.013 \\
\hline 50 & & per Confid & ence Limit & 30.9 & 10.1 & 294 & 0.219 \\
\hline
\end{tabular}


Washington Closure Hanford

Originator H.M. Sulloway A W

Project 100 and 300 Areas Coal Ash Characterization

Subject Coal Ash Depth and Corresponding Surface Sample Confidence Limit Calculations

\section{CALCULATION SHEET}

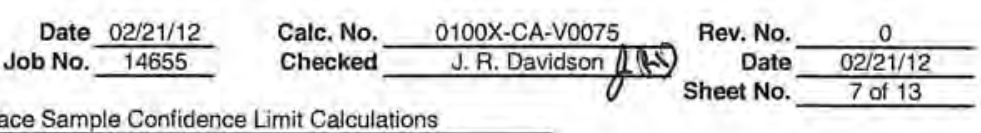

1 Coal Ash Surface Sample Data

\begin{tabular}{|c|c|c|c|c|c|c|c|c|c|c|c|c|c|c|c|}
\hline \multirow{2}{*}{$\begin{array}{l}2 \\
3\end{array}$} & \multirow{2}{*}{$\begin{array}{l}\text { Sample } \\
\text { Location }\end{array}$} & \multirow{2}{*}{$\begin{array}{c}\text { HEIS } \\
\text { Number }\end{array}$} & \multirow{2}{*}{$\begin{array}{c}\text { Sample } \\
\text { Date }\end{array}$} & \multicolumn{3}{|c|}{ Molybdenum } & \multicolumn{3}{|c|}{ Nickel } & \multicolumn{3}{|c|}{ Selenium } & \multicolumn{3}{|c|}{ Silver } \\
\hline & & & & $\mathrm{mg} / \mathrm{kg}$ & Q & PQL & $\mathrm{mg} / \mathrm{kg}$ & Q & PQL & $\mathrm{mg} / \mathrm{kg}$ & $\mathbf{Q}$ & PQL & $\mathrm{mg} / \mathrm{kg}$ & Q & PQL \\
\hline 4 & $100-B-10$ & J1HHW3 & $4 / 27 / 2011$ & 1.08 & $\mathrm{~B}$ & 1.89 & 9.93 & & 3.77 & 0.618 & & 0.28 & 0.189 & $\mathrm{U}$ & 0.19 \\
\hline 5 & $100-B-17$ & J1HHXO & $4 / 27 / 2011$ & 1.17 & B & 1.61 & 7.02 & & 3.23 & 0.331 & & 0.24 & 0.161 & U & 0.16 \\
\hline 6 & $100-B-25$ & J1HHX8 & $4 / 25 / 2011$ & 1.14 & B & 5.66 & 10.5 & B & 11.3 & 1.58 & & 0.85 & 0.566 & $\mathrm{U}$ & 0.57 \\
\hline 7 & $\begin{array}{c}\text { Duplicate of } \\
\mathrm{J} 1 \mathrm{HH} \times 8\end{array}$ & J1HJO3 & $4 / 25 / 2011$ & 0.972 & B & 5.45 & 9.70 & B & 10.9 & 1.03 & & 0.82 & 0.545 & $\mathrm{U}$ & 0.55 \\
\hline 8 & 100-D-08 & $\mathrm{J1J} 3 \times 0$ & $8 / 11 / 2011$ & 2.10 & & 1.92 & 14.9 & & 3.85 & 0.288 & U & 0.29 & 0.192 & $U$ & 0.19 \\
\hline 9 & $100-D-19$ & $J 1 J 401$ & $8 / 11 / 2011$ & 1.03 & B & 1.59 & 10.5 & & 3.17 & 0.995 & & 0.24 & 0.159 & $\mathrm{U}$ & 0.16 \\
\hline 10 & $100-D-26$ & J1J408 & $8 / 11 / 2011$ & 1.11 & B & 1.69 & 7.57 & & 3.4 & 0.769 & & 0.25 & 0.169 & $\mathrm{U}$ & 0.17 \\
\hline 11 & \begin{tabular}{|c|}
$\begin{array}{c}\text { Duplicate of } \\
\mathrm{J} 1 \mathrm{~J} 408\end{array}$ \\
\end{tabular} & J1J412 & $8 / 11 / 2011$ & 1.27 & B & 1.82 & 8.50 & & 3.6 & 1.14 & & 0.27 & 0.182 & $U$ & 0.18 \\
\hline 12 & $100-\mathrm{H}-17$ & J1HJ64 & $8 / 27 / 2011$ & 0.708 & $B$ & 1.64 & 5.88 & $J$ & 3.28 & 0.762 & & 0.25 & 0.164 & $U$ & 0.16 \\
\hline 13 & $100 \cdot \mathrm{H}-20$ & J1HJ67 & $8 / 24 / 2011$ & 2.44 & & 1.69 & 10.9 & $\mathrm{~J}$ & 3.39 & 0.341 & & 0.25 & 0.169 & U & 0.17 \\
\hline 14 & $100-\mathrm{H}-25$ & J1HJ72 & $8 / 24 / 2011$ & 1.44 & $\mathrm{~B}$ & 1.59 & 10.1 & $J$ & 3.17 & 0.338 & & 0.24 & 0.159 & U & 0.16 \\
\hline 15 & \begin{tabular}{|c|}
$\begin{array}{c}\text { Duplicate of } \\
\text { J1HJ72 }\end{array}$ \\
\end{tabular} & J1HJ77 & $8 / 24 / 2011$ & 1.49 & B & 1.72 & 10.2 & $\mathrm{~J}$ & 3.45 & 0.504 & & 0.26 & 0.172 & $U$ & 0.17 \\
\hline 16 & $300-02$ & J1HJN7 & $8 / 25 / 2011$ & 0.851 & B & 1.49 & 8.37 & $\mathrm{~J}$ & 2.99 & 0.943 & & 0.22 & 0.149 & U & 0.15 \\
\hline 17 & $300-03$ & J1HJN6 & $8 / 25 / 2011$ & 0.472 & B & 1.52 & 9.21 & $\mathrm{~J}$ & 3.03 & 0.877 & & 0.23 & 0.152 & U & 0.15 \\
\hline 18 & $300-04$ & J1HJN9 & $8 / 25 / 2011$ & 1.04 & B & 1.96 & 8.97 & $\mathrm{~J}$ & 3.92 & 1.24 & & 0.29 & 0.196 & U & 0.20 \\
\hline 19 & $\begin{array}{c}\begin{array}{c}\text { Duplicate of } \\
\text { J1H.JN9 }\end{array} \\
\end{array}$ & J1HJT5 & $8 / 25 / 2011$ & 0.946 & B & 1.61 & 8.07 & $J$ & 3.23 & 1.17 & & 0.24 & 0.161 & $U$ & 0.16 \\
\hline 20 & \begin{tabular}{|c|}
$600-07$ \\
\end{tabular} & J1HHM9 & 9/27/2011 & 2.34 & & 1.9 & 16.7 & $\mathrm{~J}$ & 3.85 & 1.22 & & 0.29 & 0.192 & $U$ & 0.19 \\
\hline 21 & $600-15$ & J1HHN7 & 9/27/2011 & 2.11 & & 1.92 & 18.9 & $J$ & 3.85 & 2.27 & & 0.29 & 0.192 & $U$ & 0.19 \\
\hline 22 & $\begin{array}{c}\begin{array}{c}\text { Duplicate of } \\
\text { J1HHN7 }\end{array} \\
\end{array}$ & J1HHR2 & 9/27/2011 & 2.49 & & 1.69 & 19.7 & $J$ & 3.4 & 1.73 & & 0.25 & 0.169 & $U$ & 0.17 \\
\hline 23[ & $600-23$ & J1HHP5 & 9/27/2011 & 2.02 & & 1.79 & 16.2 & $J$ & 3.6 & 1.44 & & 0.27 & 0.179 & $U$ & 0.18 \\
\hline $\begin{array}{l}24 \\
25\end{array}$ & \multicolumn{15}{|c|}{5 Statistical Computation Input Data } \\
\hline 26[ & Sample & HEIS & Sample & \multicolumn{3}{|c|}{ Molybdenum } & \multicolumn{3}{|c|}{ Nickel } & \multicolumn{3}{|c|}{ Selenium } & \multicolumn{3}{|c|}{ Silver } \\
\hline 27 & Location & Number & Date & \multicolumn{3}{|c|}{$\mathrm{mg} / \mathrm{kg}$} & \multicolumn{3}{|c|}{$\mathrm{mg} / \mathrm{kg}$} & \multicolumn{3}{|c|}{$\mathrm{mg} / \mathrm{kg}$} & \multicolumn{3}{|c|}{$\mathrm{mg} / \mathrm{kg}$} \\
\hline 28 & $100-B-10$ & J1HHW3 & $4 / 27 / 2011$ & \multicolumn{3}{|l|}{1.08} & 9.93 & & & 0.618 & & & 0.095 & & \\
\hline 29 & $100-B-17$ & J1HHXO & $4 / 27 / 2011$ & 1.17 & & & 7.02 & & & 0.331 & & & 0.080 & & \\
\hline 30 & $100-B-25$ & $\begin{array}{c}\text { J1HHX8/ } \\
\text { J1HJO3 }\end{array}$ & $4 / 25 / 2011$ & 1.06 & & & 10.1 & & & 1.31 & & & 0.280 & & \\
\hline 31 & $100-D-08$ & $\mathrm{~J} 1 \mathrm{~J} 3 \times 0$ & $8 / 11 / 2011$ & 2.10 & & & 14.9 & & & 0.145 & & & 0.095 & & \\
\hline 32 & $100-D-19$ & $\mathrm{~J} 1 \mathrm{~J} 401$ & $8 / 11 / 2011$ & 1.03 & & & 10.5 & & & 0.995 & & & 0.080 & & \\
\hline 33 & $100-D-26$ & $\begin{array}{l}J 1 J 408 / \\
J 1 J 412\end{array}$ & $8 / 11 / 2011$ & 1.19 & & & 8.04 & & & 0.955 & & & 0.088 & & \\
\hline 34 & $100-\mathrm{H}-17$ & J1HJ64 & $8 / 27 / 2011$ & 0.708 & & & 5.88 & & & 0.762 & & & 0.080 & & \\
\hline 35 & $100-\mathrm{H}-20$ & J1HJ67 & $8 / 24 / 2011$ & 2.44 & & & 10.9 & & & 0.341 & & & 0.085 & & \\
\hline 36 & $100-\mathrm{H}-25$ & $\begin{array}{l}\text { J1HJ72/ } \\
\text { J1HJ77 }\end{array}$ & $8 / 24 / 2011$ & 1.47 & & & 10,2 & & & 0.421 & & & 0.083 & & \\
\hline 37 & $300 \cdot 02$ & J1HJN7 & $8 / 25 / 2011$ & 0.851 & & & 8.37 & & & 0.943 & & & 0.075 & & \\
\hline 38 & $300-03$ & J1HJN6 & $8 / 25 / 2011$ & 0.472 & & & 9.21 & & & 0.877 & & & 0.075 & & \\
\hline 39 & $300-04$ & $\begin{array}{l}\text { J1HJN9/ } \\
\text { J1HJT5 }\end{array}$ & $8 / 25 / 2011$ & 0.993 & & & 8.52 & & & 1.21 & & & 0.090 & & \\
\hline 40 & $600-07$ & J1HHM9 & $9 / 27 / 2011$ & 2.34 & & & 16.7 & & & 1.22 & & & 0.095 & & \\
\hline 41 & $600-15$ & $\begin{array}{l}\text { J1HHN7/ } \\
\text { J1HHR2 }\end{array}$ & 9/27/2011 & 2.30 & & & 19.3 & & & 2.00 & & & 0.090 & & \\
\hline 42 & $600-23$ & J1HHP5 & $9 / 27 / 2011$ & 2.02 & & & 16.2 & & & 1.44 & & & 0.090 & & \\
\hline $\begin{array}{l}43 \\
44\end{array}$ & jimputano & & & & & & & & & & & & & & \\
\hline 45 & & & $\mathrm{~N}$ & 15 & & & 15 & & & 15 & & & 15 & & \\
\hline 46 & Percent & below dete & action limit & $0 \%$ & & & $0 \%$ & & & $7 \%$ & & & $100 \%$ & & \\
\hline 47 & & & Mean & 1.41 & & & 11.0 & & & 0.904 & & & 0.099 & & \\
\hline 48 & - & Standarc & Deviation & 0.650 & & & 3.91 & & & 0.493 & & & 0.051 & & \\
\hline 49 & Lol & Wer Confid & ence Limit & 1.05 & & & 8.88 & & & 0.631 & & & 0.071 & & \\
\hline 50 & Up & per Confid & ence Limit & 1.77 & & & 13.2 & & & 1.18 & & & 0.127 & & \\
\hline
\end{tabular}




\section{Washington Closure Hanford \\ Originator H.M. Sulloway 1 W} Characterization

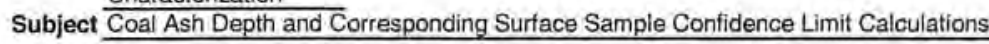

Project 100 and 300 Areas Coal Ash

\section{CALCULATION SHEET}

Date 02/21/12 Calc. No. 0100X-CA-V0075 Rev. No. 0

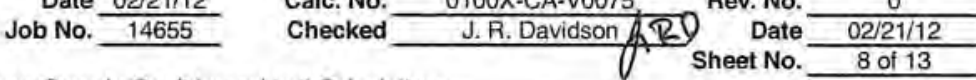

\begin{tabular}{|c|c|c|c|c|c|c|c|c|c|c|c|c|c|c|}
\hline \multirow{2}{*}{$\sqrt[2]{3}$} & \multirow{2}{*}{$\begin{array}{l}\text { Sample } \\
\text { Location }\end{array}$} & \multirow{2}{*}{$\begin{array}{c}\text { HEIS } \\
\text { Number }\end{array}$} & \multirow{2}{*}{\begin{tabular}{|c|} 
Sample \\
Date \\
\end{tabular}} & \multicolumn{3}{|c|}{$\begin{array}{l}\text { Thallium } \\
\end{array}$} & \multicolumn{2}{|c|}{ Uranium } & \multicolumn{3}{|c|}{$\begin{array}{l}\text { Vanadium } \\
\end{array}$} & \multicolumn{3}{|c|}{ Zinc } \\
\hline & & & & $\mathrm{mg} / \mathrm{kg}$ & $Q$ & $P Q L$ & $\mathrm{mg} / \mathrm{kg}$ & \begin{tabular}{l|l}
$Q$ & $P Q L$ \\
\end{tabular} & $\mathrm{mg} / \mathrm{kg}$ & $Q$ & $\mathrm{PQL}$ & $\mathrm{mg} / \mathrm{kg}$ & $Q$ & PQL \\
\hline 4 & $100-B-10$ & J1HHW3 & \begin{tabular}{|l|}
$4 / 27 / 2011$ \\
\end{tabular} & 0.472 & $\mathrm{U}$ & 0.472 & 2.67 & 0.123 & 39.3 & & 2.36 & 13.6 & & 9.43 \\
\hline 5 & $100 \cdot \mathrm{B} \cdot 17$ & J1HHXO & 4/27/2011 & 0.403 & $\mathrm{U}$ & 0.403 & 5.39 & 0.128 & 27.3 & & 2.02 & 32.4 & & 8.06 \\
\hline 6 & $100-B-25$ & J1HHX8 & $4 / 25 / 2011$ & 1.42 & $\mathrm{U}$ & 1.42 & 4.13 & 0.128 & 36.2 & & 7.08 & 38.0 & & 28.3 \\
\hline 7 & $\begin{array}{l}\text { Duplicate of } \\
\mathrm{J} 1 \mathrm{HHX8}\end{array}$ & J1HJO3 & $4 / 25 / 2011$ & 1.36 & $u$ & 1.36 & 3.25 & 0.123 & 27.6 & & 6.82 & 33.5 & & 27.3 \\
\hline 8 & $100-D-08$ & $51 \sqrt{3} \times 0$ & $8 / 11 / 2011$ & 0.481 & UJ & 0.48 & 3.66 & 0.143 & 27.6 & & 2.40 & 12.1 & $J$ & 9.62 \\
\hline 9 & $100-D-19$ & $\mathrm{J1} J 401$ & $8 / 11 / 2011$ & 0.198 & BJ & 0.40 & 3.95 & 0.143 & 38.5 & & 1.98 & 21.2 & $\mathrm{~J}$ & 7.94 \\
\hline 10 & $100-D-26$ & $\mathrm{~J} 1 \mathrm{~J} 408$ & $8 / 11 / 2011$ & 0.424 & UJ & 0.42 & 4.22 & 0.143 & 33.2 & & 2.12 & 15.2 & $J$ & 8.47 \\
\hline 11 & $\begin{array}{c}\text { Duplicate of } \\
\mathrm{J} 1 \mathrm{~J} 408\end{array}$ & J1J412 & $8 / 11 / 2011$ & 0.455 & UJ & 0.46 & 3.92 & 0.143 & 41.3 & & 2.27 & 22.9 & $J$ & 9.09 \\
\hline 12 & $100-\mathrm{H}-17$ & J1HJ64 & $8 / 27 / 2011$ & 0.410 & UJ & 0.41 & 2.24 & 0.132 & 23.8 & & 2.05 & 14.1 & & 8.20 \\
\hline 13 & $100-\mathrm{H}-20$ & J1HJ67 & $8 / 24 / 2011$ & 0.424 & UJ & 0.42 & 4.01 & 0.132 & 42.2 & & 2.12 & 20.1 & & 8.47 \\
\hline 14 & $100-\mathrm{H}-25$ & J1H 72 & $8 / 24 / 2011$ & 0.397 & UJ & 0.40 & 3.06 & 0.132 & 33.8 & & 1.98 & 28.9 & & 7.9 \\
\hline 15 & $\begin{array}{c}\text { Duplicate of } \\
\text { J1HJ72 }\end{array}$ & J1HJ77 & $8 / 24 / 2011$ & 0.431 & UJ & 0.43 & 2.91 & 0.132 & 33.3 & & 2.16 & 19.5 & & 8.6 \\
\hline 16 & $300-02$ & J1HJN7 & $8 / 25 / 2011$ & 0.373 & U & 0.370 & 4.22 & 0.132 & 42.8 & & 1.87 & 35.8 & $J$ & 7.46 \\
\hline 17 & $300-03$ & J1HJN6 & $8 / 25 / 2011$ & 0.379 & U & 0.380 & 3.70 & 0.132 & 44.4 & & 1.89 & 35.3 & $J$ & 7.58 \\
\hline 18 & $300-04$ & J1HJN9 & $8 / 25 / 2011$ & 0.490 & U & 0.490 & 3.36 & 0.132 & 34.0 & & 2.45 & 27.2 & $J$ & 9.80 \\
\hline 19 & $\begin{array}{c}\text { Duplicate of } \\
\text { J1HJN9 }\end{array}$ & J1HJT5 & $8 / 25 / 2011$ & 0.403 & $u$ & 0.400 & 3.20 & 0.132 & 31.5 & & 2.02 & 28.3 & $J$ & 8.06 \\
\hline 20 & $600-07$ & J1HHM9 & 9/27/2011 & 0.481 & UJ & 0.480 & 2.56 & 0.136 & 43.7 & $\mathrm{~J}$ & 2.40 & 22.1 & & 9.62 \\
\hline 21 & $600-15$ & J1HHN7 & $9 / 27 / 2011$ & 0.481 & UJ & 0.480 & 4.18 & 0.136 & 55.3 & $\mathrm{~J}$ & 2.40 & 28.5 & & 9.62 \\
\hline 22. & $\begin{array}{c}\text { Duplicate of } \\
\text { J1HHN7 }\end{array}$ & J1HHR2 & 9/27/2011 & 0.424 & UJ & 0.42 & 5.18 & 0.136 & 102 & J & 2.12 & 22.1 & & 8.5 \\
\hline 23 & $600-23$ & J1HHP5 & 9/27/2011 & 0.446 & UJ & 0.450 & 3.23 & 0.136 & 45.3 & $J$ & 2.23 & 22.9 & & 8.93 \\
\hline \multicolumn{15}{|c|}{24 Statistical Computation Input Data } \\
\hline 26 & Sample & HEIS & Sample & & & & \multicolumn{3}{|c|}{ Zinc } \\
\hline 27 & Location & Number & Date & & & & $\mathrm{mg} / \mathrm{kg}$ & & \multicolumn{2}{|c|}{$\mathrm{mg} / \mathrm{kg}$} & & \multicolumn{3}{|c|}{$\mathrm{mg} / \mathrm{kg}$} \\
\hline 28. & $100-B-10$ & J1HHW3 & $4 / 27 / 2011$ & \multicolumn{3}{|c|}{$\begin{array}{ll}\mathrm{mg} / \mathrm{kg} \\
0.236\end{array}$} & 2.67 & & 39.3 & & & 13.6 & & \\
\hline 29 & $100-\mathrm{B} \cdot 17$ & J1HHXO & $4 / 27 / 2011$ & 0.202 & & & 5.39 & & 27.3 & & & 32.4 & & \\
\hline 30 & $100-B-25$ & $\begin{array}{c}\mathrm{J} 1 \mathrm{HHX8/} \\
\mathrm{J} 1 \mathrm{HJO3}\end{array}$ & $4 / 25 / 2011$ & 0.695 & & & 3.69 & & 31.9 & & & 35.8 & & \\
\hline 31 & $100-D-08$ & $\mathrm{~J} 1 \mathrm{~J} 3 \times 0$ & $8 / 11 / 2011$ & 0.240 & & & 3.66 & & 27.6 & & & 12.1 & & \\
\hline 32 & $100-D-19$ & $\sqrt{1} 1 \mathrm{~J} 401$ & $8 / 11 / 2011$ & 0.198 & & & 3.95 & & 38.5 & & & 21.2 & & \\
\hline 33 & $100-D-26$ & $\begin{array}{l}\mathrm{J} 1 \mathrm{~J} 408 / \\
\mathrm{J} 1 \mathrm{~J} 412\end{array}$ & $8 / 11 / 2011$ & 0.220 & & & 4.07 & & 37.3 & & & 19.1 & & \\
\hline 34 & $100-\mathrm{H}-17$ & J1HJ64 & $8 / 27 / 2011$ & 0.205 & & & 2.24 & & 23.8 & & & 14.1 & & \\
\hline 35 & $100-\mathrm{H}-20$ & J1HJ67 & $8 / 24 / 2011$ & 0.210 & & & 4.01 & & 42.2 & & & 20.1 & & \\
\hline 36 & $100-\mathrm{H}-25$ & $\begin{array}{l}\text { J1HJ72/ } \\
\text { J1HJ77 }\end{array}$ & $8 / 24 / 2011$ & 0.208 & & & 2.99 & & 33.6 & & & 24.2 & & \\
\hline 37 & $300-02$ & J1HJN7 & $8 / 25 / 2011$ & 0.185 & & & 4.22 & & 42.8 & & & 35.8 & & \\
\hline 38 & $300-03$ & J1HJN6 & $8 / 25 / 2011$ & 0.190 & & & 3.70 & & 44.4 & & & 35.3 & & \\
\hline 39 & $300-04$ & $\begin{array}{l}\text { J1HJN9/ } \\
\text { J1HJT5 }\end{array}$ & $8 / 25 / 2011$ & 0.223 & & & 3.28 & & 32.8 & & & 27.8 & & \\
\hline 40 & $600-07$ & J1HHM9 & 9/27/2011 & 0.240 & & & 2.56 & & 43.7 & & & 22.1 & & \\
\hline 41 & $600-15$ & $\begin{array}{l}\text { J1HHN7/ } \\
\text { J1HHR2 }\end{array}$ & 9/27/2011 & 0.225 & & & 4.68 & & 78.7 & & & 25.3 & & \\
\hline 42 & $600-23$ & J1HHP5 & 9/27/2011 & 0.225 & & & 3.23 & & 45.3 & & & 22.9 & & \\
\hline 43 & Computation & & & & & & & & & & & & & \\
\hline 45 & & & $\mathrm{~N}$ & 15 & & & 15 & & 15 & & & 15 & & \\
\hline 46 & Percent & elow dete & ction limit & $93 \%$ & & & $0 \%$ & & $0 \%$ & & & $0 \%$ & & \\
\hline 47. & & & Mean & 0.247 & & & 3.62 & & 39.3 & & & 24.1 & & \\
\hline 48 & & Standard & Deviation & 0.125 & & & 0.832 & & 12.8 & & & 7.98 & & \\
\hline 49 & & Ier Confid & ence Limit & 0.177 & & & 3.16 & & 32.2 & & & 19.7 & & \\
\hline 50 & Up & er Confid & ence Limit & 0.316 & & & 4.08 & & 46.4 & & & 28.5 & & \\
\hline
\end{tabular}


Washington Closure Hanford

Originator $\mathrm{H}$. M. Sulloway $\mathrm{H}$ (U)

Project 100 and 300 Areas Coal Ash Characterization

Subject Coal Ash Depth and Corresponding Surface Sample Confidence Limit Calculations

\section{CALCULATION SHEET}

Date 02/21/12 Calc. No. 0100X-CA-V0075 Rev. No. 0 Job No. $\frac{14655}{145}$ Checked J.R. Davidson 100 Date $\frac{02 / 21 / 12}{9}$ Sheet No. $\frac{9 \text { of } 13}{9}$

1 Coal Ash Depth Sample Data

\begin{tabular}{|c|c|c|c|c|c|c|c|c|c|c|c|c|c|c|c|}
\hline \multirow[t]{2}{*}{ 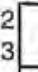 } & \multirow{2}{*}{$\begin{array}{c}\text { Sample } \\
\text { Location }\end{array}$} & \multirow{2}{*}{$\begin{array}{c}\text { HEIS } \\
\text { Number }\end{array}$} & \multirow{2}{*}{$\begin{array}{c}\text { Sample } \\
\text { Date }\end{array}$} & \multicolumn{3}{|c|}{ Antimony } & \multicolumn{3}{|c|}{ Arsenic } & \multicolumn{3}{|c|}{ Barium } & \multicolumn{3}{|c|}{ Beryllium } \\
\hline & & & & $\mathrm{mg} / \mathrm{kg}$ & Q & PQL & $\mathrm{mg} / \mathrm{kg}$ & $Q$ & $P Q L$ & $\mathrm{mg} / \mathrm{kg}$ & Q & PQL & $\mathrm{mg} / \mathrm{kg}$ & 0 & PQL \\
\hline & $100-B-10(D)$ & J1HJ10 & $4 / 27 / 2011$ & 0.556 & $U$ & 0.56 & 2.87 & & 0.93 & 916 & & 0.46 & 1.18 & & 0.19 \\
\hline & 100-B-17 (D) & J1HJ09 & $4 / 27 / 2011$ & 0.469 & UJ & 0.47 & 2.50 & & 0.78 & 681 & & 0.39 & 1.68 & & 0.16 \\
\hline & $100-B-25(D)$ & J1HJ08 & $4 / 26 / 2011$ & 0.956 & UJB & 1.61 & 8.09 & & 2.68 & 1130 & & 1.34 & 1.14 & & 0.54 \\
\hline & $100-D-08(D)$ & $51 J 444$ & $8 / 11 / 2011$ & 0.476 & UJ & 0.48 & 2.52 & & 0.79 & 1060 & & 0.40 & 1.45 & & 0.16 \\
\hline & $100-D-19(\mathrm{D})$ & $\mathrm{J} 1 \mathrm{~J} 443$ & $8 / 11 / 2011$ & 0.545 & UJ & 0.55 & 6.00 & & 0.91 & 1470 & & 0.46 & 1.62 & & 0.18 \\
\hline & $100-D-26(D)$ & $\mathrm{J1J442}$ & $8 / 11 / 2011$ & 0.435 & UJ & 0.44 & 3.16 & & 0.73 & 871 & & 0.36 & 1.10 & & 0.15 \\
\hline & $100-\mathrm{H}-17(\mathrm{D})$ & J1HJ82 & $8 / 27 / 2011$ & 0.566 & US & 0.57 & 2.79 & & 0.94 & 487 & & 0.47 & 1.15 & & 0.19 \\
\hline & $100-\mathrm{H}-20$ (D) & J1HJ81 & $8 / 24 / 2011$ & 0.545 & US & 0.55 & 2.24 & & 0.91 & 709 & & 0.46 & 0.691 & & 0.18 \\
\hline & $100-\mathrm{H}-25(\mathrm{D})$ & J1HJ80 & $8 / 24 / 2011$ & 0.517 & UJ & 0.52 & 11.7 & & 0.86 & 1000 & & 0.43 & 1.28 & & 0.17 \\
\hline & $300-02$ (D) & J1HJV1 & $8 / 25 / 2011$ & 0.545 & UJ & 0.55 & 2.25 & & 0.91 & 527 & & 0.46 & 0.862 & & 0.18 \\
\hline & $300-03(D)$ & J1HJV2 & $8 / 25 / 2011$ & 0.526 & UJ & 0.53 & 2.37 & & 0.88 & 526 & & 0.44 & 0.818 & & 0.18 \\
\hline & $300-04$ (D) & J1HJVO & $8 / 25 / 2011$ & 0.476 & UJ & 0.48 & 2.45 & & 0.79 & 629 & & 0.40 & 1.03 & & 0.16 \\
\hline & $600-07(\mathrm{D})$ & JHHTS & 9/27/2011 & 0.476 & UJ & 0.48 & 4.29 & & 0.79 & 711 & $J$ & 0.40 & 1.21 & & 0.16 \\
\hline & $600-15$ (D) & J1HHT9 & 9/27/2011 & 0.269 & BJ & 0.52 & 4.92 & & 0.86 & 653 & $J$ & 0.43 & 0.658 & & 0.17 \\
\hline & $600-23$ (D) & J1HHVO & 9/27/2011 & 0.556 & UJ & 0.56 & 4.83 & & 0.93 & 680 & $J$ & 0.46 & 0.847 & & 0.19 \\
\hline
\end{tabular}
19

20 Statistical Computation Input Data

\begin{tabular}{|c|c|c|c|c|c|c|}
\hline \multirow{2}{*}{$\begin{array}{c}\text { Sample } \\
\text { Location }\end{array}$} & \multirow{2}{*}{$\begin{array}{c}\text { HEIS } \\
\text { Number }\end{array}$} & \multirow{2}{*}{$\begin{array}{c}\text { Sample } \\
\text { Date }\end{array}$} & Antimony & Arsenic & \multirow{2}{*}{$\begin{array}{c}\text { Barium } \\
\mathrm{mg} / \mathrm{kg}\end{array}$} & \multirow{2}{*}{$\frac{\text { Beryllium }}{\mathrm{mg} / \mathrm{kg}}$} \\
\hline & & & $\mathrm{mg} / \mathrm{kg}$ & $\mathrm{mg} / \mathrm{kg}$ & & \\
\hline $100-B-10(D)$ & J1HJ10 & $4 / 27 / 2011$ & 0.280 & 2.87 & 916 & 1.18 \\
\hline 100-B-17(D) & J1HJ09 & 4/27/2011 & 0.235 & 2.50 & 681 & 1.68 \\
\hline $100-B-25(D)$ & J1HJOB & $4 / 26 / 2011$ & 0.805 & 8.09 & 1130 & 1.14 \\
\hline $100-D-08(D)$ & $\mathrm{J1J444}$ & $8 / 11 / 2011$ & 0.240 & 2.52 & 1060 & 1.45 \\
\hline $100-D-19(D)$ & $\mathrm{J1J443}$ & $8 / 11 / 2011$ & 0.275 & 6.00 & 1470 & 1.62 \\
\hline $100-D-26(D)$ & $\mathrm{J} 1 \mathrm{~J} 442$ & $8 / 11 / 2011$ & 0.220 & 3.16 & 871 & 1.10 \\
\hline $100-\mathrm{H}-17$ (D) & J1HJ82 & $8 / 27 / 2011$ & 0.285 & 2.79 & 487 & 1.150 \\
\hline $100-\mathrm{H}-20$ (D) & J1HJ81 & $8 / 24 / 2011$ & 0.275 & 2.24 & 709 & 0.69 \\
\hline $100-\mathrm{H}-25$ (D) & J1HJ80 & $8 / 24 / 2011$ & 0.260 & 11.7 & 1000 & 1.28 \\
\hline $300-02(D)$ & J1HJV1 & $8 / 25 / 2011$ & 0.275 & 2.25 & 527 & 0.862 \\
\hline $300-03(\mathrm{D})$ & J1HJV2 & $8 / 25 / 2011$ & 0.265 & 2.37 & 526 & 0.818 \\
\hline 300-04 (D) & JAHJVO & $8 / 25 / 2011$ & 0.240 & 2.45 & 629 & 1.03 \\
\hline $600-07$ (D) & J1HHT8 & $9 / 27 / 2011$ & 0.240 & 4.29 & 711 & 1.21 \\
\hline $600-15(\mathrm{D})$ & J1HHT9 & $9 / 27 / 2011$ & 0.269 & 4.92 & 653 & 0.66 \\
\hline $600-23(\mathrm{D})$ & J1HHVO & 9/27/2011 & 0.280 & 4.83 & 680 & 0.847 \\
\hline \multicolumn{7}{|c|}{ Computations } \\
\hline & & $\mathrm{N}$ & 15 & 15 & 15 & 15 \\
\hline Percent & jelow dete & ection limit & $67 \%$ & $0 \%$ & $0 \%$ & $0 \%$ \\
\hline & & Mean & 0.296 & 4.20 & 803 & 1.11 \\
\hline & Standard & Deviation & 0.142 & 2.67 & 271 & 0.310 \\
\hline Lov & ver Confid & ence Limit & 0.218 & 2.72 & 653 & 0.943 \\
\hline Upr & Jer Confid & ence Limit & 0.375 & 5.68 & 953 & 1.29 \\
\hline
\end{tabular}


Washington Closure Hanford

Originator $\mathrm{H}$. M. Sulloway $\mathrm{Al}$ S Project 100 and 300 Areas Coal Ash Characterization

\section{CALCULATION SHEET}

Date 02/21/12 Calc. No. 0100X-CA-V0075, Rev. No. 0 Job No. 14655 Checked J.R. Davidson (N) Date 02/21/12 Sheet No. 10 of 13 Subject Coal Ash Depth and Corresponding Surface Sample Confidence Limit Calculations

1 Coal Ash Depth Sample Data

\begin{tabular}{|c|c|c|c|c|c|c|c|c|c|c|c|c|c|c|c|}
\hline & \multirow{2}{*}{$\begin{array}{l}\text { Sample } \\
\text { Location }\end{array}$} & \multirow{2}{*}{$\begin{array}{l}\text { HEIS } \\
\text { Number }\end{array}$} & \multirow{2}{*}{$\begin{array}{c}\text { Sample } \\
\text { Date }\end{array}$} & \multicolumn{3}{|c|}{ Boron } & \multicolumn{3}{|c|}{ Cadmium } & \multicolumn{3}{|c|}{ Chromium } & \multicolumn{3}{|c|}{ Cobalt } \\
\hline & & & & $\mathrm{mg} / \mathrm{kg}$ & Q & $P Q L$ & $\mathrm{mg} / \mathrm{kg}$ & Q & PQL & $\mathrm{mg} / \mathrm{kg}$ & $Q$ & PQL & $\mathrm{mg} / \mathrm{kg}$ & Q & $P Q L$ \\
\hline & $100-\mathrm{B}-10(\mathrm{D})$ & J1HJ10 & $4 / 27 / 2011$ & 279 & & 1.85 & 0.239 & & 0.19 & 7.34 & & 0.19 & 3.44 & & 1.85 \\
\hline & $100-\mathrm{B}-17(\mathrm{D})$ & J1HJ09 & $4 / 27 / 2011$ & 262 & & 1.56 & 0.135 & B & 0.16 & 13.3 & & 0.16 & 2.64 & & 1.56 \\
\hline & $100-\mathrm{B}-25$ (D) & J1HJ08 & $4 / 26 / 2011$ & 35.6 & & 5.36 & 0.202 & B & 0.54 & 10.6 & & 0.54 & 5.12 & B & 5.36 \\
\hline & 100-D-08 (D) & J1J444 & $8 / 11 / 2011$ & 419 & $J$ & 1.59 & 0.178 & & 0.16 & 7.64 & & 0.16 & 6.82 & & 1.59 \\
\hline 3 & $100-\mathrm{D}-19$ (D) & $J 1 \sqrt{443}$ & $8 / 11 / 2011$ & 195 & $J$ & 1.82 & 0.208 & & 0.18 & 7.76 & & 0.18 & 5.63 & & 1.82 \\
\hline${ }^{2}$ & $100-D-26(\mathrm{D})$ & $\mathrm{J} 1 \mathrm{~J} 442$ & $8 / 11 / 2011$ & 169 & $J$ & 1.45 & 0.074 & B & 0.15 & 8.73 & & 0.15 & 4,21 & & 1.45 \\
\hline & $100-\mathrm{H}-17(\mathrm{D})$ & J1H.J82 & $8 / 27 / 2011$ & 245 & $J$ & 1.89 & 0.252 & & 0.19 & 8.52 & & 0.19 & 1.72 & B & 1.89 \\
\hline & $100-\mathrm{H}-20$ (D) & J1HJ81 & $8 / 24 / 2011$ & 199 & $J$ & 1.82 & 0.184 & & 0.18 & 9.62 & & 0.18 & 3.52 & & 1.82 \\
\hline & $100-\mathrm{H}-25$ (D) & J1HJ80 & $8 / 24 / 2011$ & 404 & $\mathrm{~J}$ & 1.72 & 0.200 & & 0.17 & 11.2 & & 0.17 & 2.42 & & 1.72 \\
\hline & $300-02(\mathrm{D})$ & J1HJV1 & $8 / 25 / 2011$ & 288 & & 1.82 & 0.207 & & 0.18 & 9.87 & & 0.18 & 2.16 & & 1.82 \\
\hline & $300-03(\mathrm{D})$ & J1HJV2 & $8 / 25 / 2011$ & 256 & & 1.75 & 0.222 & & 0.18 & 10.6 & & 0.18 & 2.94 & & 1.75 \\
\hline & $300-04$ (D) & J1HJVO & $8 / 25 / 2011$ & 360 & & 1.59 & 0.267 & & 0.16 & 11.6 & & 0.16 & 2.19 & & 1.59 \\
\hline & $600-07$ (D) & J1HHT8 & 9/27/2011 & 147 & $J$ & 1.59 & 0.111 & B & 0.16 & 7.50 & $J$ & 0.16 & 4.42 & & 1.59 \\
\hline & $600-15(\mathrm{D})$ & JHHTS & 9/27/2011 & 168 & $J$ & 1.72 & 0.186 & & 0.17 & 8.99 & $\mathrm{~J}$ & 0.17 & 3.56 & & 1.72 \\
\hline & $600-23(\mathrm{D})$ & J1HHVO & \begin{tabular}{|l|} 
9/27/2011 \\
\end{tabular} & 155 & $J$ & 1.85 & 0.139 & B & 0.19 & 8.11 & $\mathrm{~J}$ & 0.19 & 3.98 & & 1.85 \\
\hline
\end{tabular}
19

20 Statistical Computation Input Data

\begin{tabular}{|c|c|c|c|c|c|c|c|}
\hline \multirow{2}{*}{$\begin{array}{l}21 \\
22\end{array}$} & \multirow{2}{*}{$\begin{array}{c}\text { Sample } \\
\text { Location }\end{array}$} & \multirow{2}{*}{$\begin{array}{c}\text { HEIS } \\
\text { Number }\end{array}$} & \multirow{2}{*}{$\begin{array}{c}\text { Sample } \\
\text { Date }\end{array}$} & \multirow{2}{*}{$\begin{array}{l}\text { Boron } \\
\mathrm{mg} / \mathrm{kg}\end{array}$} & \multirow{2}{*}{$\begin{array}{c}\text { Cadmium } \\
\mathrm{mg} / \mathrm{kg}\end{array}$} & \multirow{2}{*}{$\begin{array}{c}\text { Chromium } \\
\mathrm{mg} / \mathrm{kg}\end{array}$} & \multirow{2}{*}{$\begin{array}{l}\text { Cobalt } \\
\mathrm{mg} / \mathrm{kg}\end{array}$} \\
\hline & & & & & & & \\
\hline 3 & $100-B-10$ (D) & J1HJ10 & $4 / 27 / 2011$ & 279 & 0.239 & 7.34 & 3.44 \\
\hline 4 & $100-\mathrm{B}-17$ (D) & J1HJO9 & $4 / 27 / 2011$ & 262 & 0,135 & 13.3 & 2.64 \\
\hline 5 & $100-B-25(D)$ & J1HJ08 & $4 / 26 / 2011$ & 35.6 & 0.202 & 10.6 & 5.12 \\
\hline 6 & 100-D-08 (D) & $\mathrm{J1J} 444$ & $8 / 11 / 2011$ & 419 & 0.178 & 7.64 & 6.82 \\
\hline 7 & $100-D-19(D)$ & $J 1 J 443$ & $8 / 11 / 2011$ & 195 & 0.208 & 7.76 & 5.63 \\
\hline 8 & $100-D-26(D)$ & $\mathrm{J} 1 \mathrm{~J} 442$ & $8 / 11 / 2011$ & 169 & 0.074 & 8.73 & 4.21 \\
\hline 9 & $100-\mathrm{H}-17$ (D) & J1HJ82 & 8/27/2011 & 245 & 0.252 & 8.52 & 1.72 \\
\hline 0 & $100-H-20(D)$ & J1HJ81 & $8 / 24 / 2011$ & 199 & 0.184 & 9.62 & 3.52 \\
\hline 1 & $100-\mathrm{H}-25$ (D) & J1HJ80 & $8 / 24 / 2011$ & 404 & 0.200 & 11.2 & 2.42 \\
\hline 2 & $300-02(\mathrm{D})$ & J1HJV1 & $8 / 25 / 2011$ & 288 & 0.207 & 9.87 & 2.16 \\
\hline 3 & $300-03(D)$ & J1HJV2 & $8 / 25 / 2011$ & 256 & 0.222 & 10.6 & 2.94 \\
\hline 4 & $300-04$ (D) & J1HJVO & $8 / 25 / 2011$ & 360 & 0.267 & 11.6 & 2.19 \\
\hline & $600-07$ (D) & J1HHT8 & 9/27/2011 & 147 & 0.111 & 7.50 & 4.42 \\
\hline & $600-15(D)$ & J1HHT9 & 9/27/2011 & 168 & 0.186 & 8.99 & 3.56 \\
\hline & $600-23(\mathrm{D})$ & J1HHVO & 9/27/2011 & 155 & 0.139 & 8.11 & 3.98 \\
\hline
\end{tabular}

39 Computations

\begin{tabular}{|c|c|c|c|c|c|}
\hline 40[ & $\mathrm{N}$ & 15 & 15 & 15 & 15 \\
\hline 41 & Percent below detection limit & $0 \%$ & $0 \%$ & $0 \%$ & $0 \%$ \\
\hline 42 & Mean & 239 & 0.187 & 9.43 & 3.65 \\
\hline 43 & Standard Deviation & 103 & 0.053 & 1.76 & 1.42 \\
\hline 44 & Lower Confidence Limit & 182 & 0.158 & 8.45 & 2.86 \\
\hline 45 & Upper Confidence Limit & 296 & 0.216 & 10.4 & 4.44 \\
\hline
\end{tabular}


Washinaton Closure Hanford

Originator $\mathrm{H}$.M. Sulloway $\mathrm{CX}$

Project 100 and 300 Areas Coal Ash Characterization

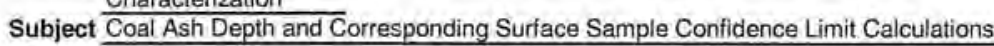

\section{CALCULATION SHEET}

Date 02/21/12 Calc. No. 0100X-CA-V0075 Rev. No. Job No. $\frac{02121 / 12}{14655}$ Checked J.R. Davidson LRב [102) Date

Date $02 / 21 / 12$

No. 11 of 13

1 Coal Ash Depth Sample Data

\begin{tabular}{|c|c|c|c|c|c|c|c|c|c|c|c|c|c|c|c|}
\hline \multirow{2}{*}{\multicolumn{2}{|c|}{\begin{tabular}{l|c|}
2 & Sample \\
3 & Location \\
\end{tabular}}} & \multirow{2}{*}{$\begin{array}{c}\text { HEIS } \\
\text { Number }\end{array}$} & \multirow{2}{*}{$\begin{array}{c}\text { Sample } \\
\text { Date }\end{array}$} & \multicolumn{3}{|c|}{ Copper } & \multicolumn{3}{|c|}{ Lead } & \multicolumn{3}{|c|}{ Manganese } & \multicolumn{3}{|c|}{ Mercury } \\
\hline & & & & $\mathrm{mg} / \mathrm{kg}$ & Q & PQL & $\mathrm{mg} / \mathrm{kg}$ & Q & PQL & $\mathrm{mg} / \mathrm{kg}$ & Q & PQL & $\mathrm{mg} / \mathrm{kg}$ & $Q$ & $\mathrm{PQL}$ \\
\hline & $100-B-10$ (D) & J1HJ10 & 4/27/2011 & 21.0 & & 0.93 & 11.8 & & 0.46 & 110 & & 4.63 & 0.042 & & 0.03 \\
\hline 5 & $100-B-17$ (D) & J1HJO9 & $4 / 27 / 2011$ & 19.6 & & 0.78 & 4.33 & & 0.39 & 38.5 & & 3.91 & 0.080 & & 0.03 \\
\hline 5 & $100-B-25$ (D) & J1HJ08 & $4 / 26 / 2011$ & 26.2 & & 2.68 & 4.91 & & 1.34 & 187 & & 13.4 & 0.026 & B & 0.03 \\
\hline & $100-D-08$ (D) & J1J444 & $8 / 11 / 2011$ & 13.6 & & 0.79 & 3.50 & $J$ & 0.40 & 255 & $\mathrm{~J}$ & 3.97 & 0.047 & & 0.03 \\
\hline 8 & 100-D-19 (D) & $J 1 J 443$ & $8 / 11 / 2011$ & 23.7 & & 0.91 & 3.24 & $\mathrm{~J}$ & 0.46 & 234 & $J$ & 4.55 & 0.052 & & 0.03 \\
\hline 9 & $100-D-26(D)$ & $J 1 J 442$ & $8 / 11 / 2011$ & 31.8 & & 0.73 & 3.35 & $\mathrm{~J}$ & 0.36 & 91.4 & $J$ & 3.62 & 0.036 & & 0.03 \\
\hline 0 & $100-\mathrm{H}-17$ (D) & J1HJ82 & $8 / 27 / 2011$ & 18.1 & & 0.94 & 27.3 & $J$ & 0.47 & 82.1 & $\mathrm{~J}$ & 4.72 & 0.053 & & 0.03 \\
\hline 1 & $100-\mathrm{H}-20(\mathrm{D})$ & J1HJ81 & $8 / 24 / 2011$ & 25.3 & & 0.91 & 18.3 & $J$ & 0.46 & 148 & $\mathrm{~J}$ & 4.55 & 0.863 & & 0.02 \\
\hline 2 & $100 \cdot \mathrm{H}-25$ (D) & J1HJ80 & $8 / 24 / 2011$ & 22.7 & & 0.86 & 132 & $\mathrm{~J}$ & 0.43 & 90,9 & $J$ & 4.31 & 0.035 & & 0.03 \\
\hline & $300-02(D)$ & J1HJV1 & $8 / 25 / 2011$ & 16.4 & & 0.91 & 8.18 & & 0.46 & 85.6 & & 4.55 & 3.32 & & 0.16 \\
\hline & $300-03(D)$ & J1HJV2 & $8 / 25 / 2011$ & 15.5 & & 0.88 & 9.12 & & 0.44 & 95.9 & & 4.39 & 0.291 & & 0.02 \\
\hline & $300-04$ (D) & J1HJVO & $8 / 25 / 2011$ & 19.7 & & 0.79 & 14.5 & & 0.40 & 66.3 & & 3.97 & 0.501 & & 0.03 \\
\hline & 600.07 (D) & J1HHT8 & 9/27/2011 & 21.9 & $J$ & 0.79 & 3.30 & & 0.40 & 327 & $\mathrm{~J}$ & 4.0 & 0.026 & U & 0.03 \\
\hline & $600-15(D)$ & ग1HHT9 & 9/27/2011 & 21.6 & $J$ & 0.86 & 5.61 & & 0.43 & 250 & $\mathrm{~J}$ & 4.3 & 0.025 & $U$ & 0.03 \\
\hline & $600-23(D)$ & J1HHVO & 9/27/2011 & 21.3 & $J$ & 0.93 & 5.08 & & 0.46 & 286 & $\mathrm{~J}$ & 4.63 & 0.026 & U & 0.03 \\
\hline
\end{tabular}
19

20 Statistical Computation Input Data

\begin{tabular}{|c|c|c|c|c|c|c|c|}
\hline \multirow{2}{*}{\multicolumn{2}{|c|}{$\begin{array}{l}\text { Sample } \\
\text { Location }\end{array}$}} & \multirow{2}{*}{$\begin{array}{l}\text { HEIS } \\
\text { Number }\end{array}$} & \multirow{2}{*}{$\begin{array}{c}\text { Sample } \\
\text { Date }\end{array}$} & Copper & \multirow{2}{*}{$\frac{\text { Lead }}{\mathrm{ma} / \mathrm{kg}}$} & \multirow{2}{*}{$\begin{array}{c}\text { Manganese } \\
\mathrm{mg} / \mathrm{kg}\end{array}$} & \multirow{2}{*}{$\frac{\text { Mercury }}{\mathrm{mg} / \mathrm{kg}}$} \\
\hline & & & & $\mathrm{mg} / \mathrm{kg}$ & & & \\
\hline 3 & $100-B-10(D)$ & J1HJ10 & $4 / 27 / 2011$ & 21.0 & 11.8 & 110 & 0.042 \\
\hline 4 & $100-B-17(D)$ & J1HJ09 & $4 / 27 / 2011$ & 19.6 & 4.33 & 38.5 & 0.080 \\
\hline & $100-B-25(D)$ & J1HJ08 & $4 / 26 / 2011$ & 26.2 & 4.91 & 187 & 0.026 \\
\hline 6 & $100-\mathrm{D}-08(\mathrm{D})$ & $J 1 J 444$ & $8 / 11 / 2011$ & 13.6 & 3.50 & 255 & 0.047 \\
\hline 7 & $100-D-19(D)$ & $J 1 J 443$ & $8 / 11 / 2011$ & 23.7 & 3.24 & 234 & 0.052 \\
\hline & $100-D-26(D)$ & $J 1 J 442$ & $8 / 11 / 2011$ & 31.8 & 3.35 & 91.4 & 0.036 \\
\hline 9 & $100-\mathrm{H}-17$ (D) & J1HJ82 & $8 / 27 / 2011$ & 18.1 & 27.3 & 82.1 & 0.053 \\
\hline 0 & $100-\mathrm{H}-20(\mathrm{D})$ & J1HJ81 & $8 / 24 / 2011$ & 25.3 & 18.3 & 148 & 0.863 \\
\hline 1 & $100-\mathrm{H}-25(\mathrm{D})$ & J1HJ 80 & $8 / 24 / 2011$ & 22.7 & 132 & 90.9 & 0.035 \\
\hline 2 & $300-02$ (D) & J1HJV1 & $8 / 25 / 2011$ & 16.4 & 8,18 & 85.6 & 3.32 \\
\hline 3 & $300-03(\mathrm{D})$ & J1HJV2 & $8 / 25 / 2011$ & 15.5 & 9.12 & 95.9 & 0.291 \\
\hline & $300-04(D)$ & J1HJV0 & $8 / 25 / 2011$ & 19.7 & 14.5 & 66.3 & 0.501 \\
\hline & $600.07(\mathrm{D})$ & J1HHTS & 9/27/2011 & 21.9 & 3.30 & 327 & 0.015 \\
\hline 6 & $600-15$ (D) & J1HHT9 & 9/27/2011 & 21.6 & 5,61 & 250 & 0.015 \\
\hline & $600-23$ (D) & J1HHVO & 9/27/2011 & 21.3 & 5.08 & 286 & 0.015 \\
\hline
\end{tabular}
38

39 Computations

\begin{tabular}{|c|c|c|c|c|c|}
\hline 40 & $\mathrm{~N}$ & 15 & 15 & 15 & 15 \\
\hline 41 & Percent below detection limit & $0 \%$ & $0 \%$ & $0 \%$ & $13 \%$ \\
\hline 42 & Mean & 21,2 & 17.0 & 157 & 0.359 \\
\hline 43 & Standard Deviation & 4.56 & 32.5 & 91.9 & 0.853 \\
\hline 44. & Lower Confidence Limit & 18.7 & -1.06 & 106 & -0.113 \\
\hline 45[ & Upper Confidence Limit & 23.8 & 35.0 & 207 & 0.832 \\
\hline
\end{tabular}


Washington Closure Hanford

Originator $\mathrm{H}$. M. Sulloway

Project 100 and 300 Areas Co

Characterization

Subject Coal Ash Depth

1 Coal Ash Depth Sample Data

\begin{tabular}{|c|c|c|c|c|c|c|c|c|c|c|c|c|c|c|}
\hline \multirow{2}{*}{$\begin{array}{c}\text { Sample } \\
3 \\
\text { Location }\end{array}$} & \multirow{2}{*}{$\begin{array}{c}\text { HEIS } \\
\text { Number }\end{array}$} & \multirow{2}{*}{$\begin{array}{c}\text { Sample } \\
\text { Date }\end{array}$} & \multicolumn{3}{|c|}{ Molybdenum } & \multicolumn{3}{|c|}{ Nickel } & \multicolumn{3}{|c|}{ Selenium } & \multicolumn{3}{|c|}{ Silver } \\
\hline & & & $\mathrm{mg} / \mathrm{kg}$ & $Q$ & PQL & $\mathrm{mg} / \mathrm{kg}$ & Q & PQL & $\mathrm{mg} / \mathrm{kg}$ & $Q$ & PQL & $\mathrm{mg} / \mathrm{kg}$ & $\mathbf{Q}$ & PQL \\
\hline $4100-B-10(D)$ & J1HJ10 & 4/27/2011 & 1.40 & $B$ & 1.85 & 7.29 & & 3.70 & 0.278 & $\bar{U}$ & 0.28 & 0.185 & $\mathrm{U}$ & 0,19 \\
\hline$5 \longdiv { 1 0 0 - B - 1 7 ( D ) }$ & J1HJ09 & $4 / 27 / 2011$ & 1.31 & B & 1.56 & 7.67 & & 3.12 & 0.367 & & 0.23 & 0.156 & $U$ & 0.16 \\
\hline $6100-B-25(D)$ & J1HJO8 & $4 / 26 / 2011$ & 1.59 & B & 5.36 & 11.2 & & 10.7 & 0.804 & $U$ & 0.80 & 0.536 & $u$ & 0.54 \\
\hline $7100-D-08(D)$ & J1J444 & $8 / 11 / 2011$ & 1.47 & B & 1.59 & 11.5 & & 3.17 & 0.837 & & 0.24 & 0.159 & U & 0.16 \\
\hline 8 & $\mathrm{J1J} 443$ & $8 / 11 / 2011$ & 1.67 & B & 1.82 & 11.0 & & 3.6 & 0.728 & & 0.27 & 0.182 & $u$ & 0.18 \\
\hline $100-D-26(\mathrm{D})$ & $\sqrt{1} \mathrm{~J} 442$ & $8 / 11 / 2011$ & 1.49 & & 1.45 & 10.6 & & 2.9 & 0.686 & & 0.22 & 0.145 & $u$ & 0.15 \\
\hline $100-\mathrm{H}-17(\mathrm{D})$ & $\mathrm{J1HJ82}$ & $8 / 27 / 2011$ & 0.916 & B & 1.89 & 4.79 & $J$ & 3.77 & 1.18 & & 0.28 & 0.189 & $U$ & 0.19 \\
\hline $100-\mathrm{H}-20(\mathrm{D})$ & J1HJ81 & $8 / 24 / 2011$ & 1.96 & & 1.82 & 10.6 & $\mathrm{~J}$ & 3.64 & 0.273 & $\mathrm{U}$ & 0.27 & 0.182 & $u$ & 0.18 \\
\hline $100-\mathrm{H}-25$ (D) & J1HJ80 & $8 / 24 / 2011$ & 1.41 & B & 1.72 & 6.96 & $J$ & 3.45 & 0.259 & $U$ & 0.26 & 0.172 & $U$ & 0.17 \\
\hline \begin{tabular}{|c|}
$300-02(\mathrm{D})$ \\
\end{tabular} & J1HJV1 & $8 / 25 / 2011$ & 0.830 & B & 1.82 & 5.84 & $J$ & 3.64 & 1.19 & & 0.27 & 0.182 & $u$ & 0.18 \\
\hline $300-03(\mathrm{D})$ & J1HJV2 & $8 / 25 / 2011$ & 0.690 & B & 1.75 & 6.53 & $\mathrm{~J}$ & 3.51 & 1.53 & & 0.26 & 0.175 & $U$ & 0.18 \\
\hline $300-04$ (D) & J1HJV0 & $8 / 25 / 2011$ & 0.923 & B & 1.59 & 6.43 & $J$ & 3.17 & 1.77 & & 0.24 & 0.159 & $U$ & 0.16 \\
\hline $600-07$ (D) & J1HHT8 & 9/27/2011 & 1.91 & & 1.59 & 11.2 & $J$ & 3.2 & 1.29 & & 0.24 & 0.159 & $U$ & 0.16 \\
\hline $600-15$ (D) & J1HHT9 & 9/27/2011 & 2.16 & & 1.72 & 9.42 & $J$ & 3.5 & 1.83 & & 0.26 & 0.172 & $U$ & 0.17 \\
\hline $600-23(\mathrm{D})$ & J1HHVO & 9/27/2011 & 1.78 & B & 1.85 & 10.6 & $J$ & 3.70 & 1.85 & & 0.28 & 0.185 & $U$ & 0.19 \\
\hline
\end{tabular}

19

20 Statistical Computation Input Data

\begin{tabular}{|c|c|c|c|c|c|c|c|}
\hline \multirow{2}{*}{$\begin{array}{l}21 \\
22\end{array}$} & \multirow{2}{*}{$\begin{array}{c}\text { Sample } \\
\text { Location }\end{array}$} & \multirow{2}{*}{$\begin{array}{l}\text { HEIS } \\
\text { Number }\end{array}$} & \multirow{2}{*}{$\begin{array}{c}\text { Sample } \\
\text { Date }\end{array}$} & \multirow{2}{*}{$\begin{array}{c}\text { Molybdenum } \\
\mathrm{mg} / \mathrm{kg}\end{array}$} & \multirow{2}{*}{$\begin{array}{l}\text { Nickel } \\
\mathrm{mg} / \mathrm{kg}\end{array}$} & \multirow{2}{*}{$\begin{array}{c}\text { Selenium } \\
\mathrm{mg} / \mathrm{kg}\end{array}$} & \multirow{2}{*}{$\begin{array}{l}\text { Silver } \\
\mathrm{mg} / \mathrm{kg}\end{array}$} \\
\hline & & & & & & & \\
\hline 23 & $100-B-10(D)$ & J1HJ10 & 4/27/2011 & 1.40 & 7.29 & 0.140 & 0.095 \\
\hline 24 & $100-B-17(D)$ & J1H.J09 & $4 / 27 / 2011$ & 1.31 & 7.67 & 0.367 & 0.080 \\
\hline 25 & $100-B-25(D)$ & J1HJ08 & $4 / 26 / 2011$ & 1.59 & 11.2 & 0.400 & 0.270 \\
\hline 26 & $100-D-08(D)$ & J1J444 & $8 / 11 / 2011$ & 1.47 & 11.5 & 0.837 & 0.080 \\
\hline 27 & 100-D-19(D) & $\sqrt{1 \sqrt{443}}$ & $8 / 11 / 2011$ & 1.67 & 11.0 & 0.728 & 0.090 \\
\hline 28 & $100-D-26(D)$ & $J 1 J 442$ & $8 / 11 / 2011$ & 1.49 & 10.6 & 0.686 & 0.075 \\
\hline 29 & $100-\mathrm{H}-17(\mathrm{D})$ & J1HJ82 & $8 / 27 / 2011$ & 0.916 & 4,79 & 1.18 & 0.095 \\
\hline 30 & $100-H-20(D)$ & J1HJ81 & $8 / 24 / 2011$ & 1.96 & 10.6 & 0.135 & 0.090 \\
\hline 31 & $100-\mathrm{H}-25$ (D) & J1HJBO & $8 / 24 / 2011$ & 1.41 & 6.96 & 0.130 & 0.085 \\
\hline 32 & $300-02(\mathrm{D})$ & J1HJV1 & $8 / 25 / 2011$ & 0.830 & 5.84 & 1.19 & 0.090 \\
\hline 33 & $300-03$ (D) & J1HJV2 & $8 / 25 / 2011$ & 0.690 & 6.53 & 1.53 & 0.090 \\
\hline 34 & 300.04 (D) & J1HJVO & $8 / 25 / 2011$ & 0.923 & 6.43 & 1.77 & 0.080 \\
\hline 35 & $600-07$ (D) & J1HHT8 & 9/27/2011 & 1.91 & 11.2 & 1.29 & 0.080 \\
\hline 36 & $600-15(\mathrm{D})$ & J1HHT9 & 9/27/2011 & 2.16 & 9.42 & 1.83 & 0.085 \\
\hline 37 & $600-23(\mathrm{D})$ & J1HHVO & 9/27/2011 & 1.78 & 10.6 & 1.85 & 0.095 \\
\hline
\end{tabular}

39 Computations

\begin{tabular}{|c|c|c|c|c|c|}
\hline 40 & $\mathrm{~N}$ & 15 & 15 & 15 & 15 \\
\hline 41 & Percent below detection limit & $0 \%$ & $0 \%$ & $13 \%$ & $73 \%$ \\
\hline 42 & Mean & 1.43 & 8.78 & 0.938 & 0.099 \\
\hline 43 & Standard Deviation & 0.439 & 2.33 & 0.632 & 0.048 \\
\hline 44 & Lower Confidence Limit & 1.19 & 7.48 & 0.588 & 0.072 \\
\hline 45 & Upper Confidence Limit & 1.68 & 10.1 & 1.29 & 0.125 \\
\hline
\end{tabular}

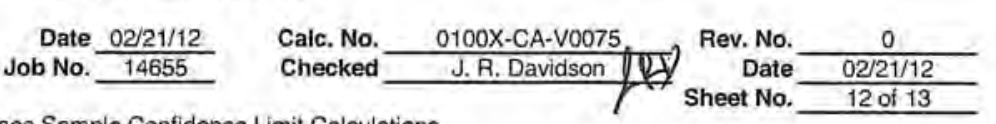




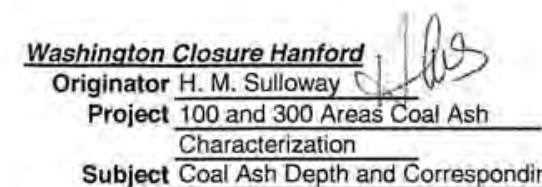

Subject Coal Ash Depth and Corresponding Surface Sample Confidence Limit Calculations

\section{CALCULATION SHEET}

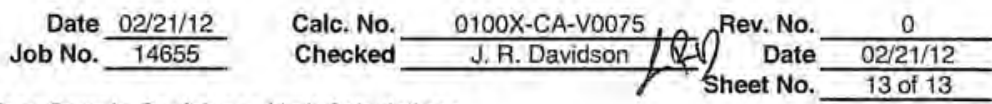

1 Coal Ash Depth Sample Data

\begin{tabular}{|c|c|c|c|c|c|c|c|c|c|c|c|c|c|c|}
\hline \multirow{2}{*}{\begin{tabular}{|c|c|} 
Sample \\
Location \\
\end{tabular}} & \multirow{2}{*}{$\begin{array}{l}\text { HEIS } \\
\text { Number }\end{array}$} & \multirow{2}{*}{$\begin{array}{c}\text { Sample } \\
\text { Date }\end{array}$} & \multicolumn{3}{|c|}{ Thallium } & \multicolumn{3}{|c|}{ Uranium } & \multicolumn{3}{|c|}{ Vanadium } & \multicolumn{3}{|c|}{ Zinc } \\
\hline & & & $\mathrm{mg} / \mathrm{kg}$ & Q & PQL & $\mathrm{mg} / \mathrm{kg}$ & $\mathbf{Q}$ & PQL & $\mathrm{mg} / \mathrm{kg}$ & $Q$ & PQL & $\mathrm{mg} / \mathrm{kg}$ & $Q$ & $\mathrm{PQL}$ \\
\hline $100-\mathrm{B}-10(\mathrm{D})$ & J1HJ10 & $4 / 27 / 2011$ & 0.463 & $U$ & 0.463 & 4.28 & & 0.123 & 28.7 & & 2.31 & 29.7 & & 9.26 \\
\hline 5 100-B-17(D) & J1HJ09 & $4 / 27 / 2011$ & 0.391 & $U$ & 0.391 & 6.75 & & 0.128 & 29.2 & & 1.95 & 15.1 & & 7.81 \\
\hline $100-B-25(D)$ & J1HJ08 & $4 / 26 / 2011$ & 1.34 & U & 1.34 & 3.56 & & 0.128 & 33.5 & & 6.70 & 23.8 & B & 26.8 \\
\hline $700-\mathrm{D}-08(\mathrm{D})$ & $51 J 444$ & $8 / 11 / 2011$ & 0.397 & UJ & 0.400 & 3.56 & & 0.143 & 31.2 & & 1.98 & 30.8 & $\mathrm{~J}$ & 7.94 \\
\hline$3 \longdiv { 1 0 0 - D - 1 9 ( \mathrm { D } ) }$ & $J 1 J 443$ & $8 / 11 / 2011$ & 0.455 & UJ & 0.460 & 4.46 & & 0.143 & 29.7 & & 2.27 & 33.6 & $J$ & 9.09 \\
\hline $100-D-26(\mathrm{D})$ & $J 1 J 442$ & $8 / 11 / 2011$ & 0.362 & UJ & 0.360 & 3.85 & & 0.143 & 41.8 & & 1.81 & 10.3 & $J$ & 7.25 \\
\hline $100-\mathrm{H}-17$ (D) & J1HJ82 & $8 / 27 / 2011$ & 0.472 & UJ & 0.470 & 4.18 & & 0.132 & 19.6 & & 2.36 & 25.7 & & 9.43 \\
\hline $100-\mathrm{H}-20$ (D) & J1HJ81 & $8 / 24 / 2011$ & 0.455 & UJ & 0.460 & 2.78 & & 0.132 & 28.9 & & 2.27 & 21.0 & & 9.09 \\
\hline $100-\mathrm{H}-25$ (D) & J1HJ80 & $8 / 24 / 2011$ & 0.431 & UJ & 0.430 & 4,25 & & 0.132 & 27.0 & & 2.16 & 31.3 & & 8.62 \\
\hline $300-02(\mathrm{D})$ & J1HJV1 & $8 / 25 / 2011$ & 0.455 & U & 0.460 & 2.61 & & 0.132 & 25.1 & & 2.27 & 27,8 & $\mathrm{~J}$ & 9.09 \\
\hline $300.03(\mathrm{D})$ & J1HJV2 & $8 / 25 / 2011$ & 0.439 & $U$ & 0.440 & 2.86 & & 0.132 & 28.3 & & 2.19 & 26.7 & $\mathrm{~J}$ & 8.77 \\
\hline $300-04(D)$ & J1HJVO & $8 / 25 / 2011$ & 0.397 & $U$ & 0.400 & 3.14 & & 0.132 & 24.3 & & 1.98 & 26.9 & $J$ & 7.94 \\
\hline $600-07$ (D) & J1HHT8 & 9/27/2011 & 0.397 & UJ & 0.400 & 2.64 & & 0.136 & 36.8 & $J$ & 1.98 & 17.7 & & 7.94 \\
\hline $600-15(D)$ & J1HHT9 & 9/27/2011 & 0.431 & UJ & 0.430 & 1.76 & & 0.136 & 32.9 & $\mathrm{~J}$ & 2.16 & 18.1 & & 8.62 \\
\hline $600-23(\mathrm{D})$ & J1HHVO & $9 / 27 / 2011$ & 0.463 & US & 0.460 & 3.28 & & 0.136 & 32.1 & $J$ & 2.31 & 16.0 & & 9.26 \\
\hline
\end{tabular}
19

20 Statistical Computation Input Data

\begin{tabular}{|c|c|c|c|c|c|c|c|}
\hline \multirow{2}{*}{$\begin{array}{l}21 \\
22 \\
\end{array}$} & \multirow{2}{*}{$\begin{array}{c}\text { Sample } \\
\text { Location } \\
\end{array}$} & \multirow{2}{*}{$\begin{array}{c}\text { HEIS } \\
\text { Number }\end{array}$} & \multirow{2}{*}{$\begin{array}{c}\text { Sample } \\
\text { Date }\end{array}$} & \multirow{2}{*}{$\begin{array}{c}\text { Thallium } \\
\mathrm{mg} / \mathrm{kg}\end{array}$} & \multirow{2}{*}{$\begin{array}{c}\text { Uranium } \\
\mathrm{mg} / \mathrm{kg}\end{array}$} & \multirow{2}{*}{$\begin{array}{c}\text { Vanadium } \\
\mathrm{mg} / \mathrm{kg}\end{array}$} & \multirow{2}{*}{$\begin{array}{r}\text { Zinc } \\
\mathrm{mg} / \mathrm{kc}\end{array}$} \\
\hline & & & & & & & \\
\hline 23 & $100-\mathrm{B}-10(\mathrm{D})$ & J1HJ10 & 4/27/2011 & 0.232 & 4.28 & 28.7 & 29.7 \\
\hline 24 & 100-B-17 (D) & J1HJ09 & 4/27/2011 & 0.196 & 6.75 & 29.2 & 15.1 \\
\hline 25 & $100-\mathrm{B}-25(\mathrm{D})$ & J1HJ08 & $4 / 26 / 2011$ & 0.670 & 3.56 & 33.5 & 23.8 \\
\hline 26 & 100-D-08(D) & $\mathrm{J} 1 \mathrm{~J} 444$ & $8 / 11 / 2011$ & 0.200 & 3.56 & 31.2 & 30.8 \\
\hline 27 & 100-D-19(D) & $\mathrm{J} 1 \mathrm{~J} 443$ & $8 / 11 / 2011$ & 0.230 & 4.46 & 29.7 & 33.6 \\
\hline 28 & $100-D-26(D)$ & J1J442 & $8 / 11 / 2011$ & 0.180 & 3.85 & 41.8 & 10.3 \\
\hline 29 & $100-\mathrm{H}-17(\mathrm{D})$ & J1HJ82 & $8 / 27 / 2011$ & 0.235 & 4.18 & 19.6 & 25.7 \\
\hline 30 & $100-\mathrm{H}-20(\mathrm{D})$ & J1HJ81 & $8 / 24 / 2011$ & 0.230 & 2.78 & 28.9 & 21.0 \\
\hline 31 & $100-\mathrm{H}-25(\mathrm{D})$ & J1HJ80 & $8 / 24 / 2011$ & 0.215 & 4.25 & 27.0 & 31.3 \\
\hline 32 & $300-02(\mathrm{D})$ & J1HJV1 & $8 / 25 / 2011$ & 0.230 & 2.61 & 25.1 & 27.8 \\
\hline 33 & $300-03(D)$ & J1HJV2 & $8 / 25 / 2011$ & 0.220 & 2.86 & 28.3 & 26.7 \\
\hline 34 & $300-04$ (D) & J1HJVO & $8 / 25 / 2011$ & 0.200 & 3,14 & 24.3 & 26.9 \\
\hline 35 & $600-07(D)$ & J1HHT8 & 9/27/2011 & 0.200 & 2.64 & 36.8 & 17.7 \\
\hline 36 & $600-15(D)$ & J1HHT9 & 9/27/2011 & 0.215 & 1,76 & 32.9 & 18.1 \\
\hline 37 & $600-23(D)$ & J1HHVO & 9/27/2011 & 0.230 & 3.28 & 32.1 & 16.0 \\
\hline
\end{tabular}
38

39 Computations

\begin{tabular}{|c|c|c|c|c|c|}
\hline 40 & $\mathrm{~N}$ & 15 & 15 & 15 & 15 \\
\hline 41 & Percent below detection limit & $73 \%$ & $0 \%$ & $0 \%$ & $0 \%$ \\
\hline 42 & Mean & 0.245 & 3.60 & 29.9 & 23.6 \\
\hline 43 & Standard Deviation & 0.119 & 1.16 & 5.29 & 6.92 \\
\hline 44] & Lower Confidence Limit & 0.180 & 2.96 & 27.0 & 19.8 \\
\hline 45 & Upper Confidence Limit & 0.311 & 4.24 & 32.9 & 27.5 \\
\hline
\end{tabular}




\section{APPENDIX E}

\section{COAL ASH SURFACE SAMPLES BOXPLOTS}


WCH-506

Rev. 0 
Figure E-1. Boxplots of Antimony Results from 126-B-1, 126-E-1, and 126-H-1 Coal Ash Surface Samples ( $=29$ ). ${ }^{\text {a }}$

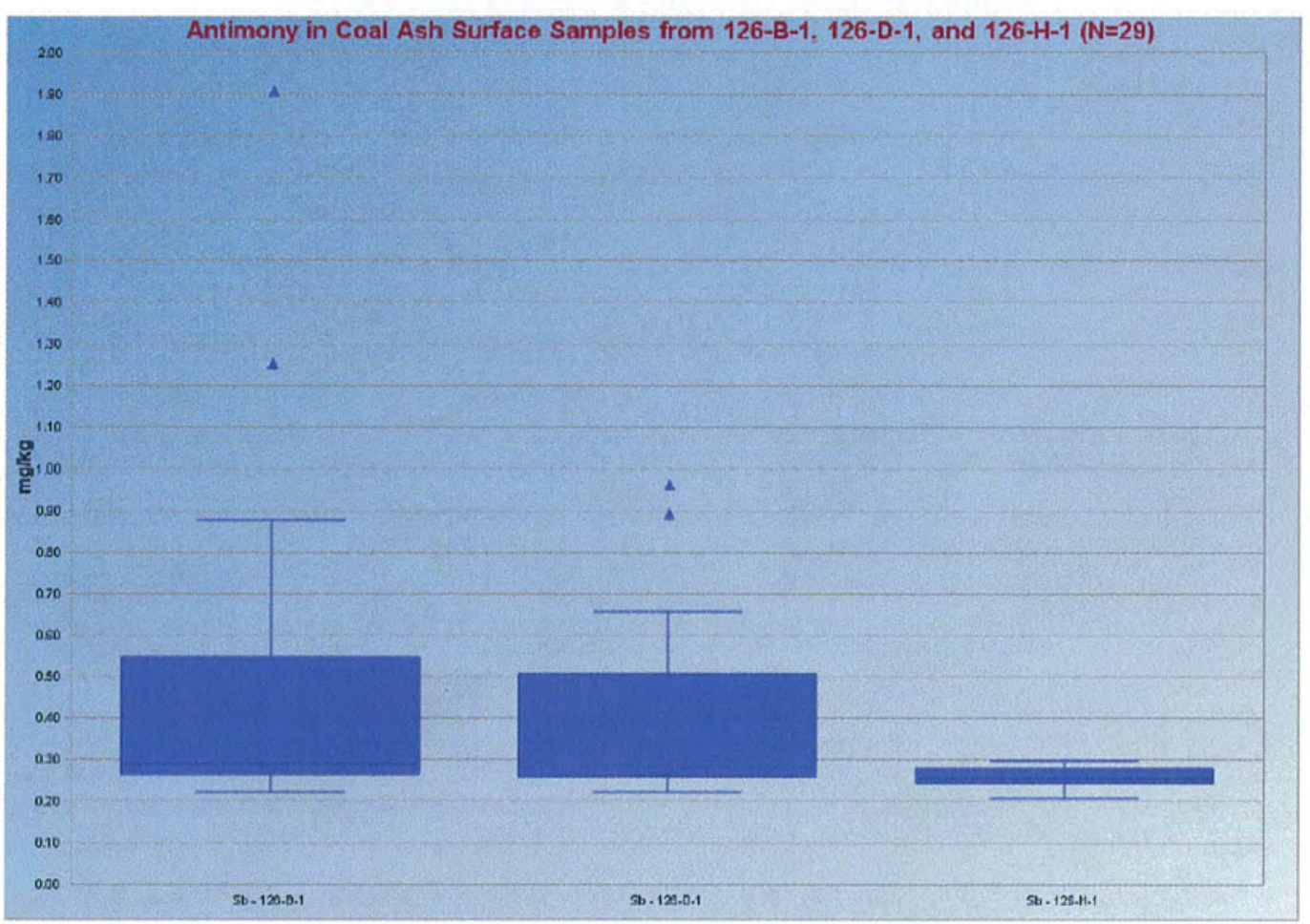

a Antimony was detected at only three of the five sample sites.

Figure E-2. Boxplots of Arsenic Results from Coal Ash Surface Samples (N=29).

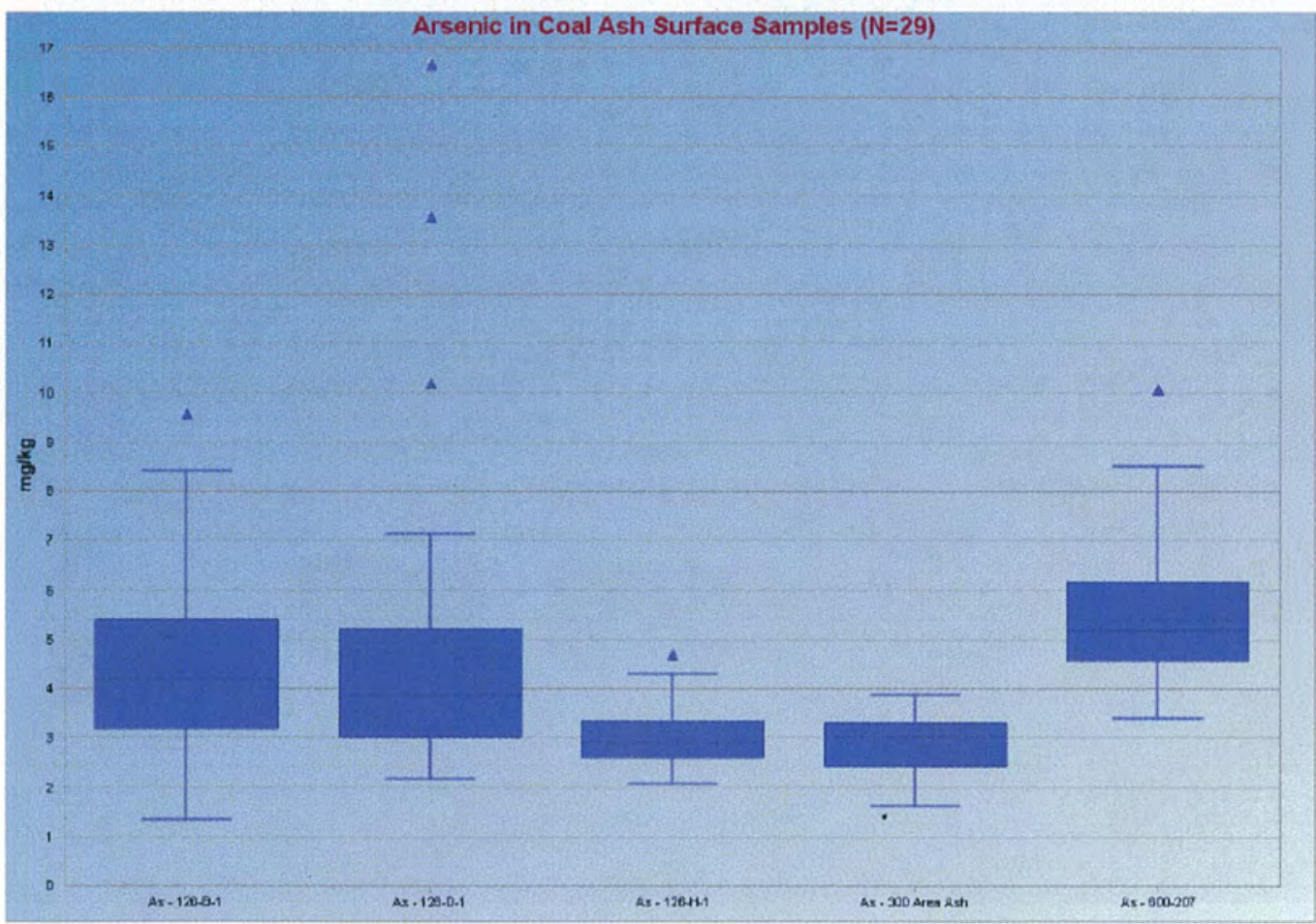


Figure E-3. Boxplots of Barium Results from Coal Ash Surface Samples ( $N=29$ ).

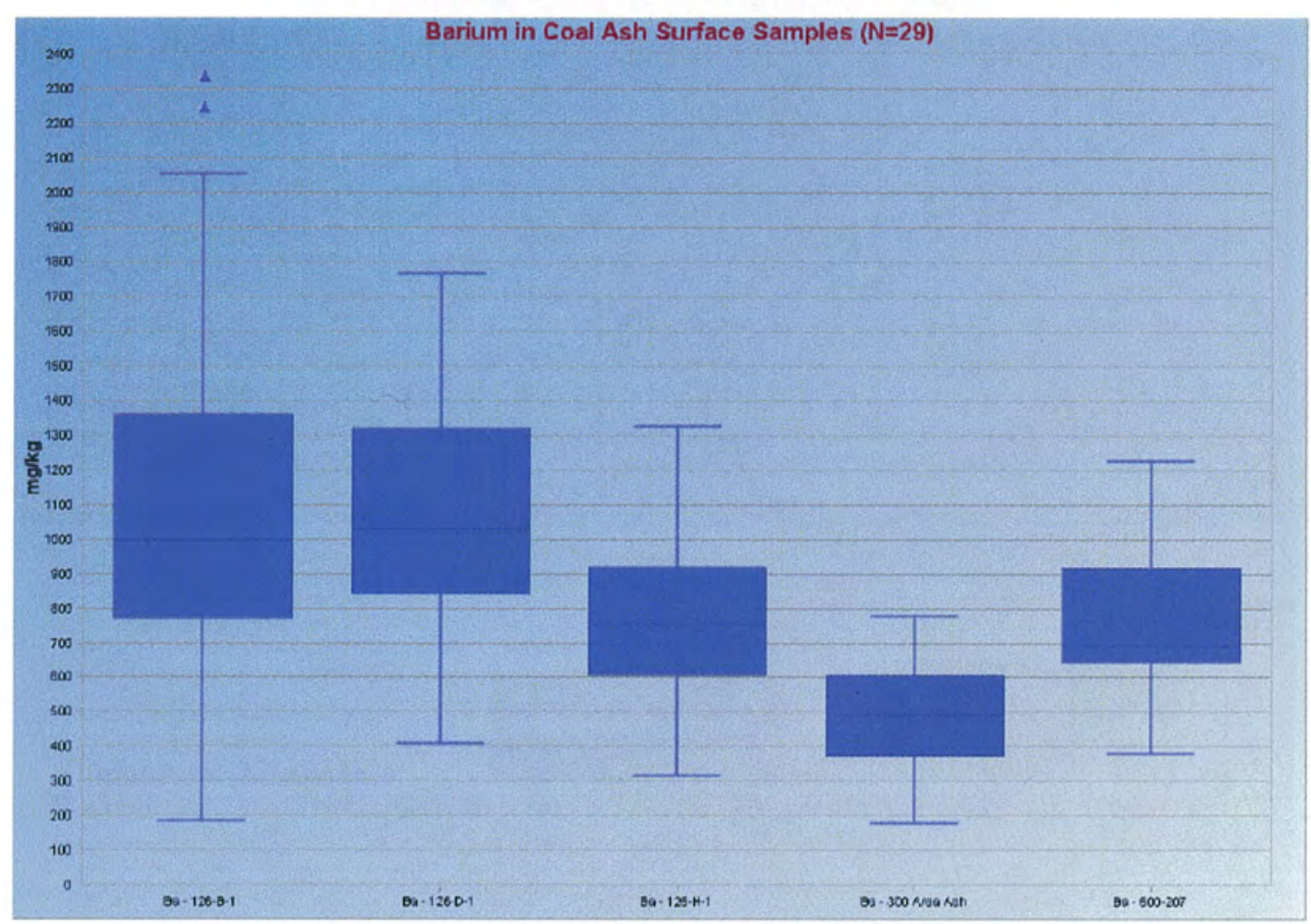

Figure E-4. Boxplots of Beryllium Results from Coal Ash Surface Samples ( $N=29)$.

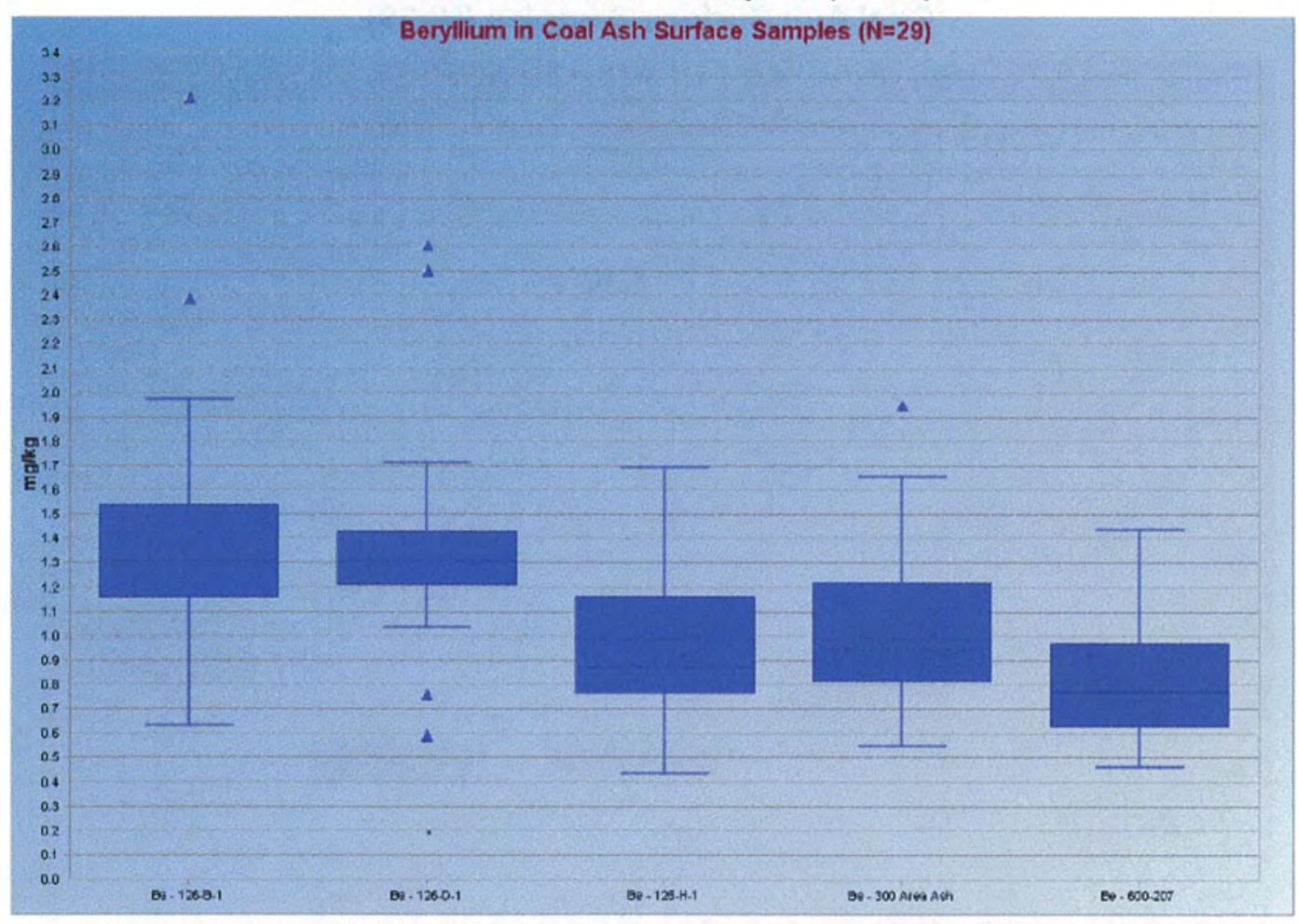


Figure E-5. Boxplots of Boron Results from Coal Ash Surface Samples $(\mathrm{N}=29)$.

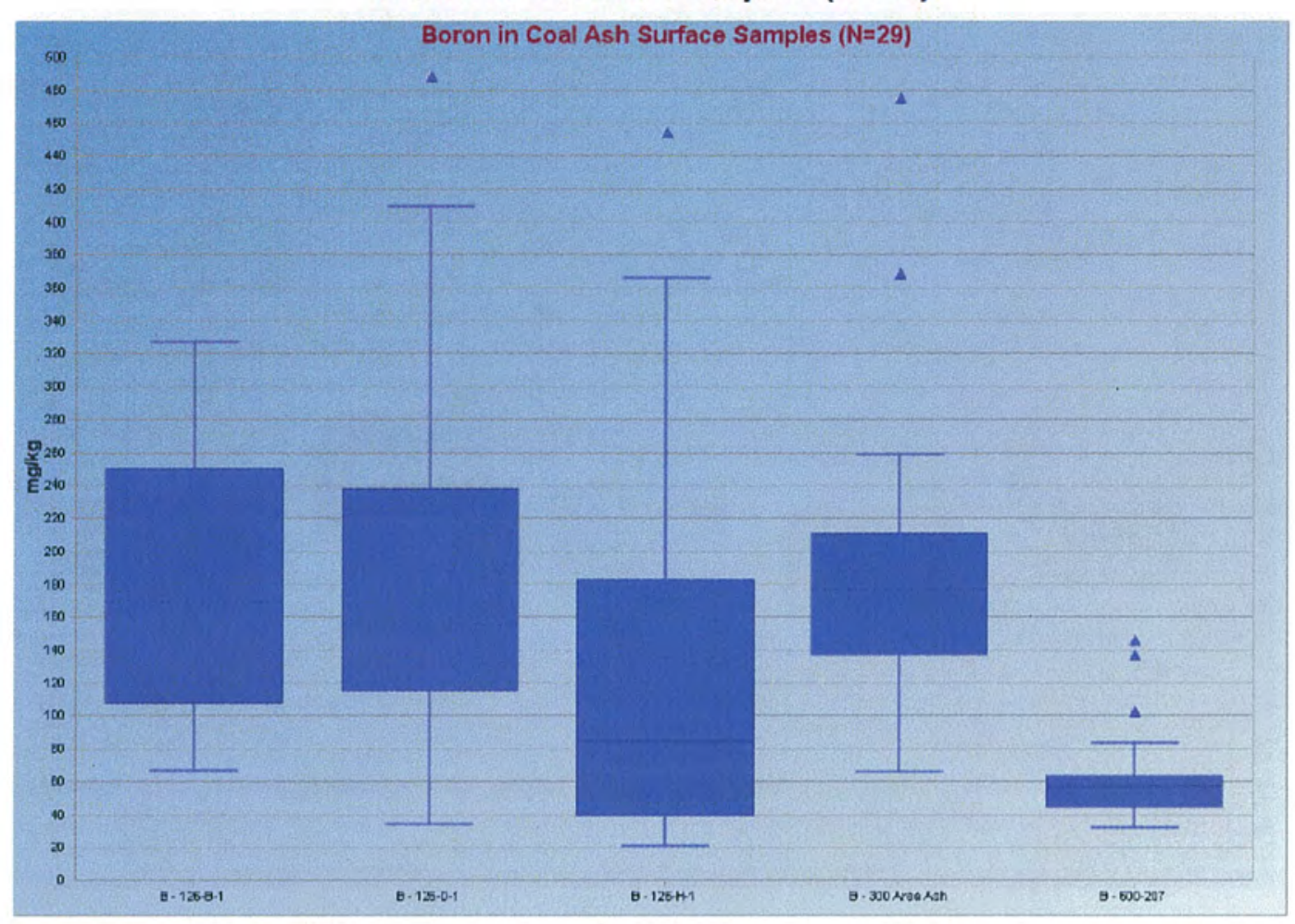

Figure E-6. Boxplots of Cadmium Results from Coal Ash Surface Samples ( $\mathrm{N}=29)$.

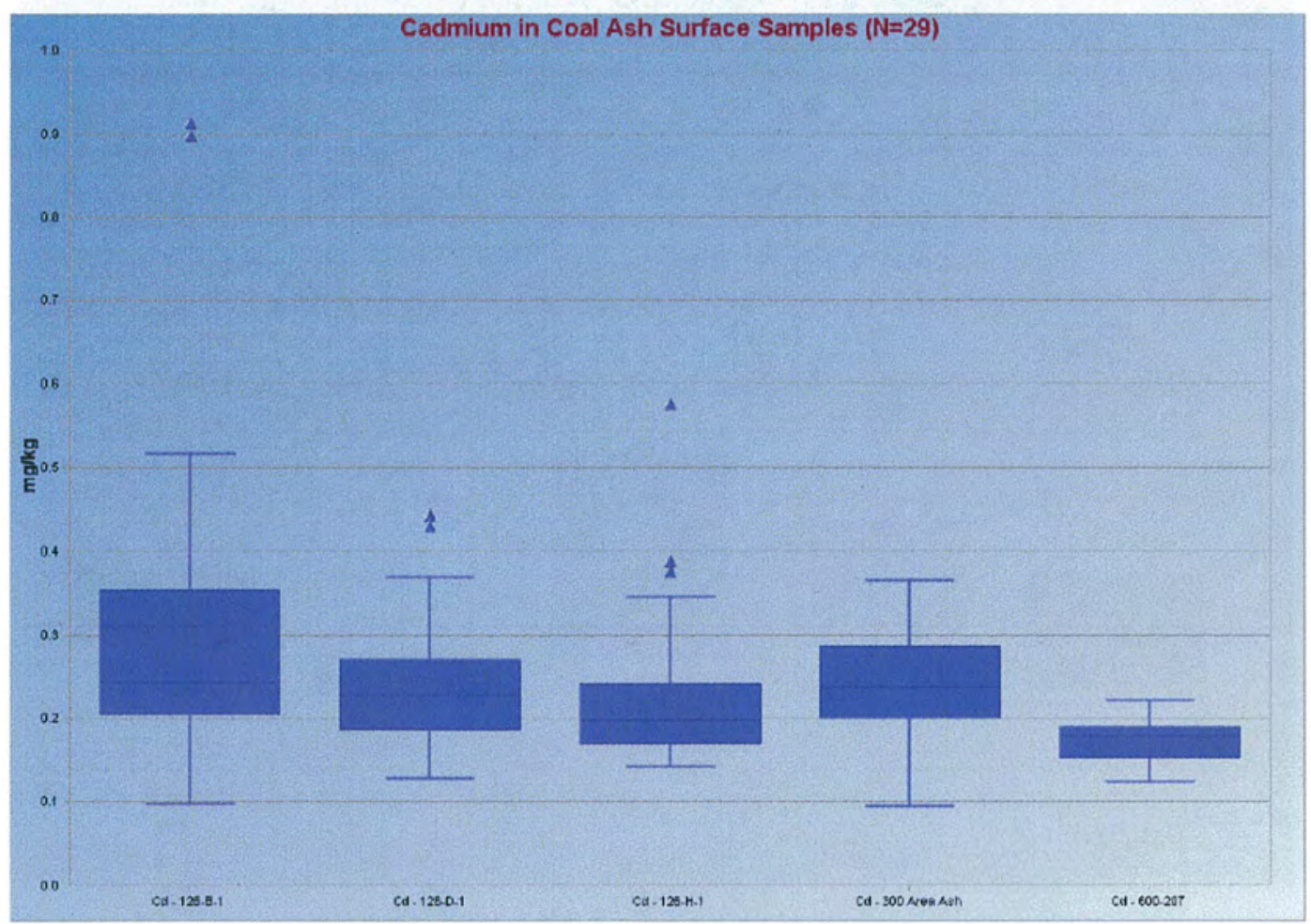


Figure E-7. Boxplots of Total Chromium Results from Coal Ash Surface Samples $(\mathrm{N}=29)$.

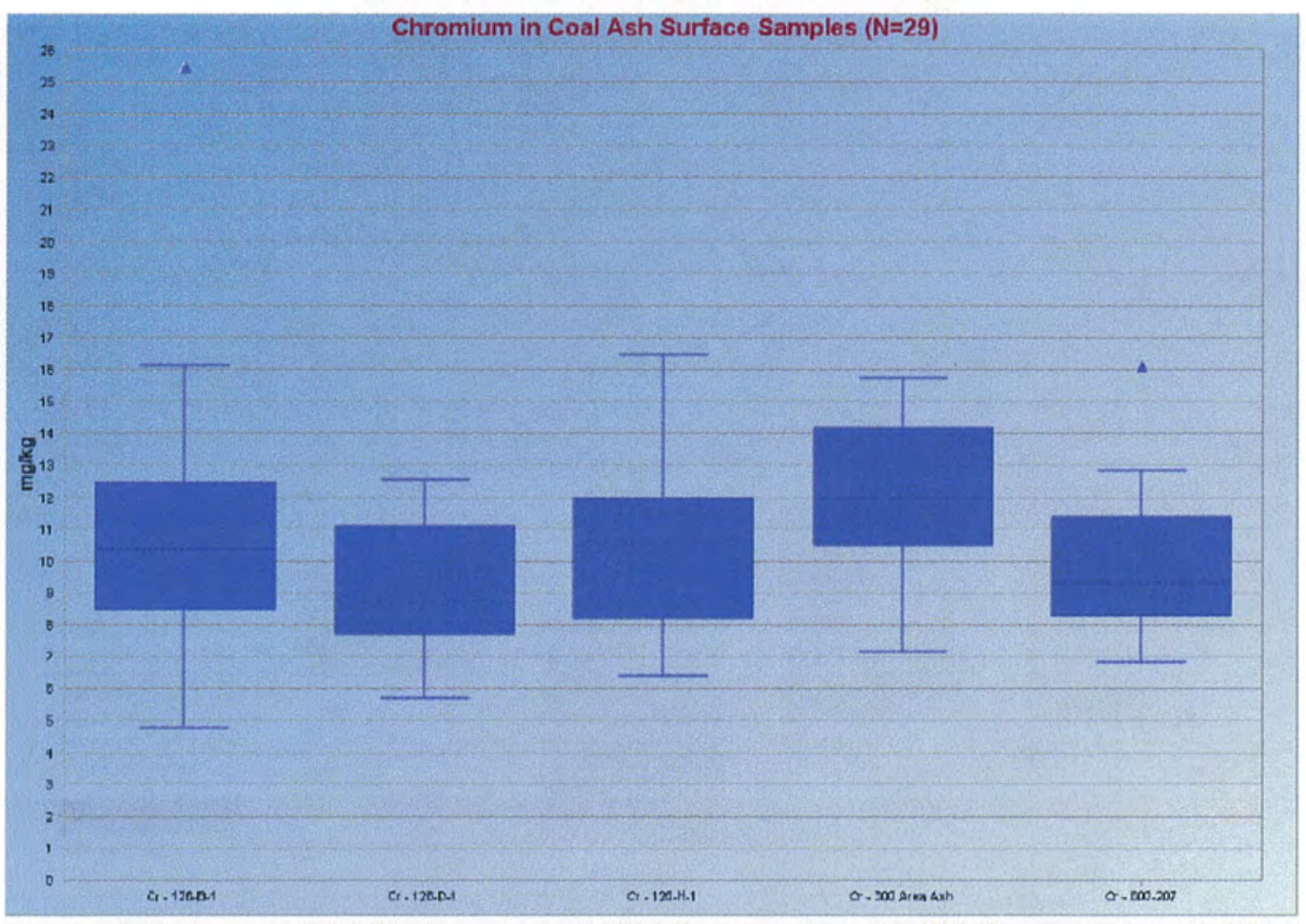

Figure E-8. Boxplots of Cobalt Results from

Coal Ash Surface Samples ( $\mathrm{N}=29$ ).

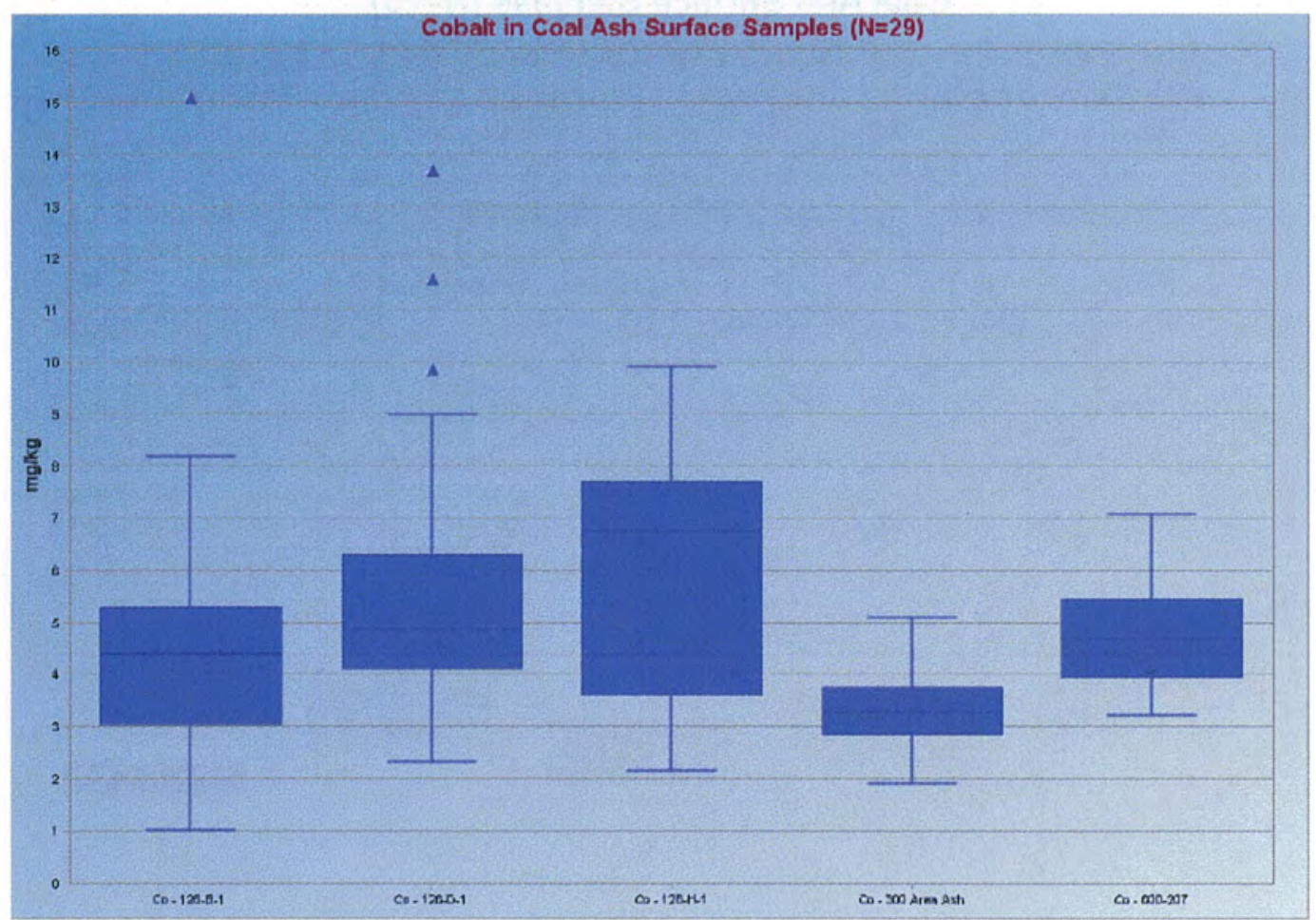


Figure E-9. Boxplots of Copper Results from Coal Ash Surface Samples ( $\mathrm{N}=29)$.

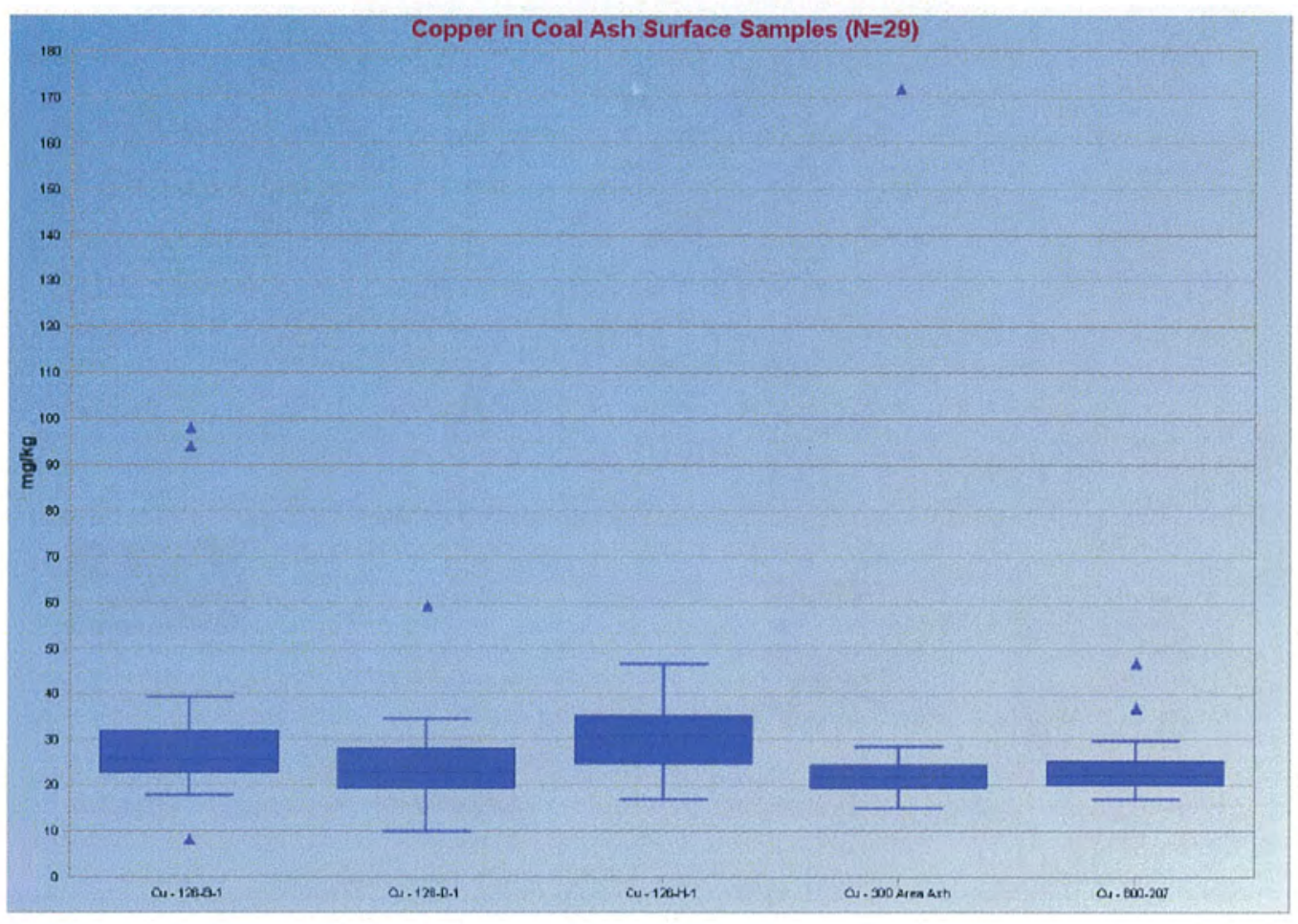

Figure E-10. Boxplots of Lead Results from

Coal Ash Surface Samples $(\mathrm{N}=29)$.

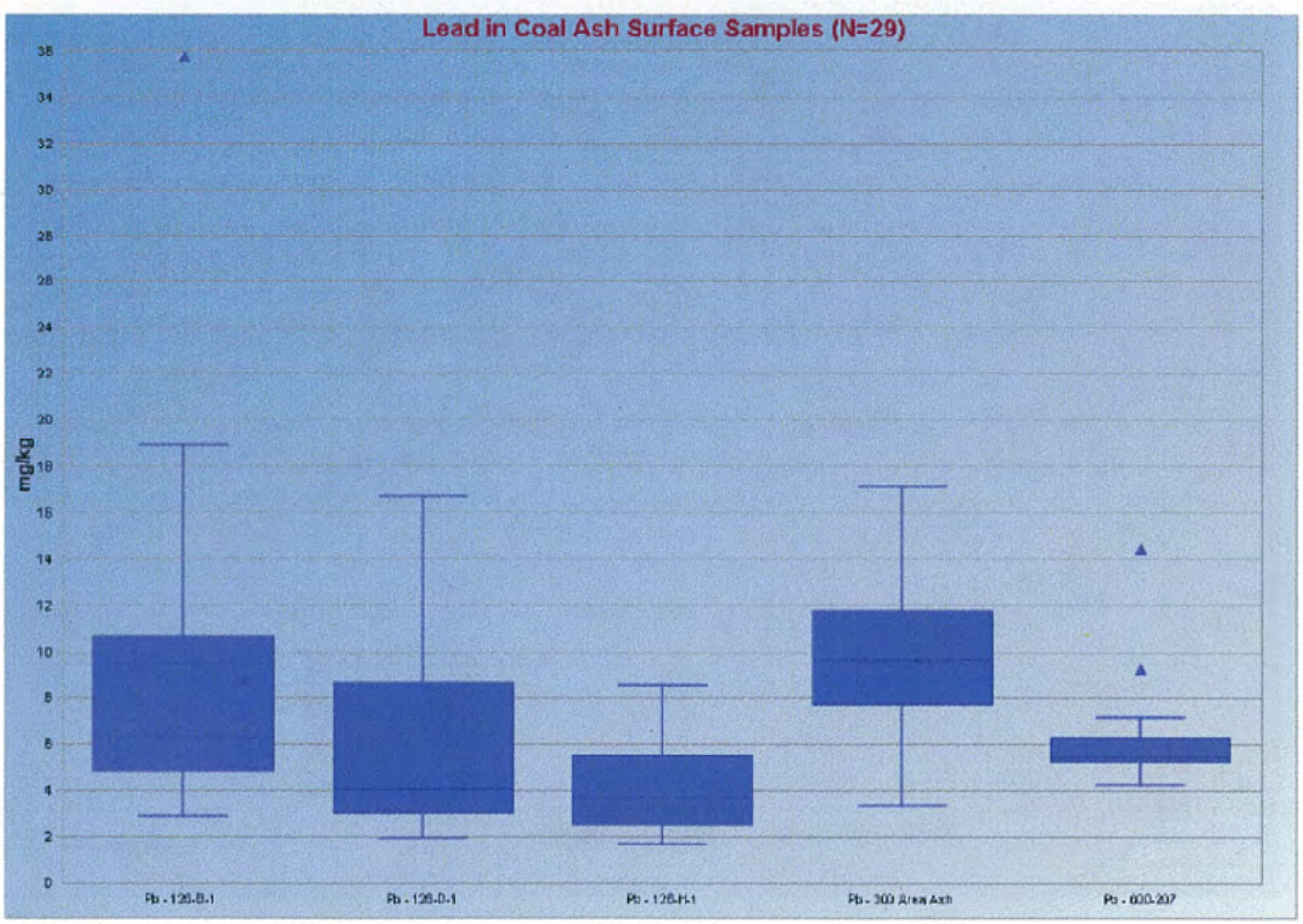


Figure E-11. Boxplots of Manganese Results from

Coal Ash Surface Samples ( $\mathrm{N}=29$ ).

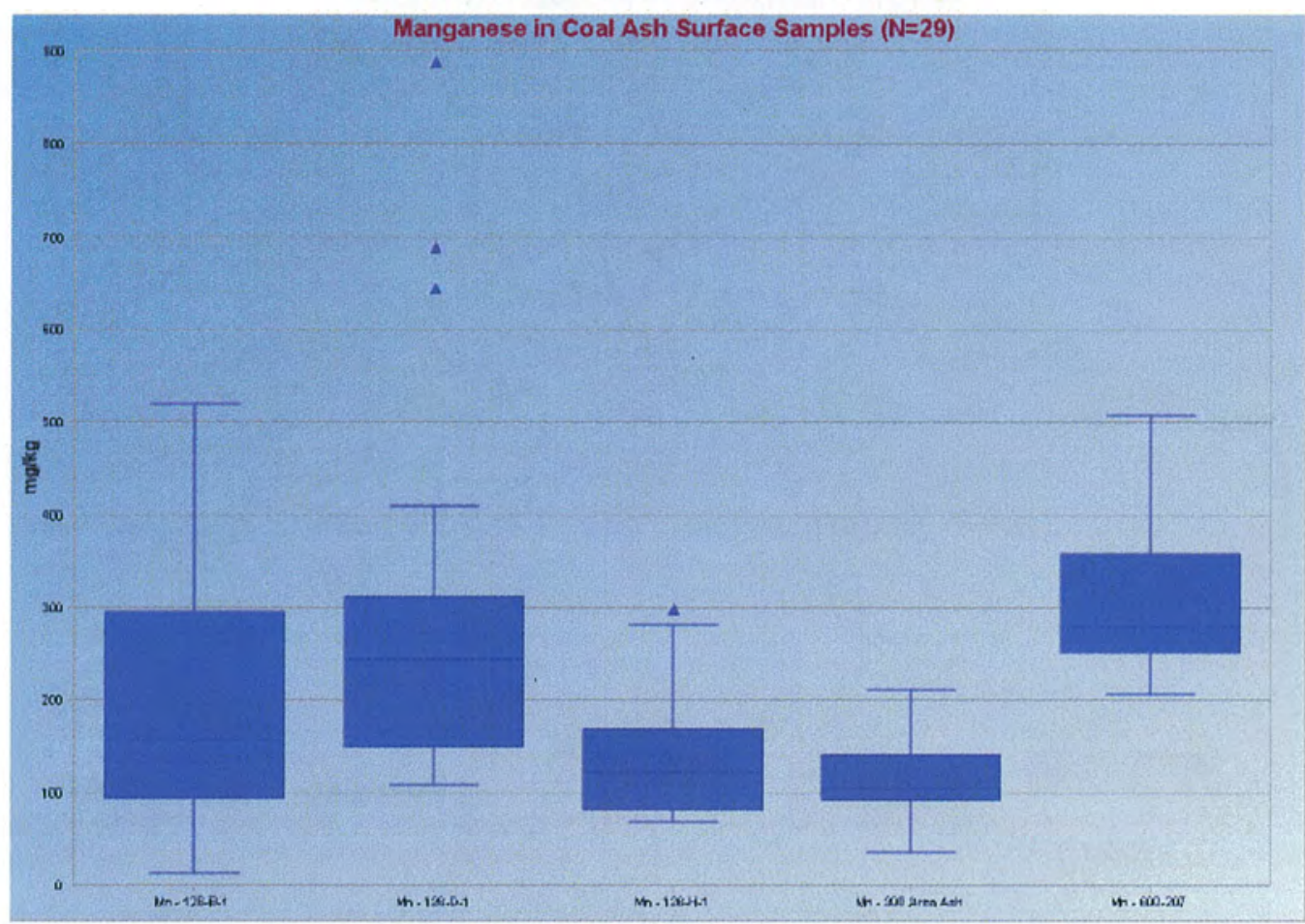

Figure E-12. Boxplots of Mercury Results from Coal Ash Surface Samples ( $N=29)$.

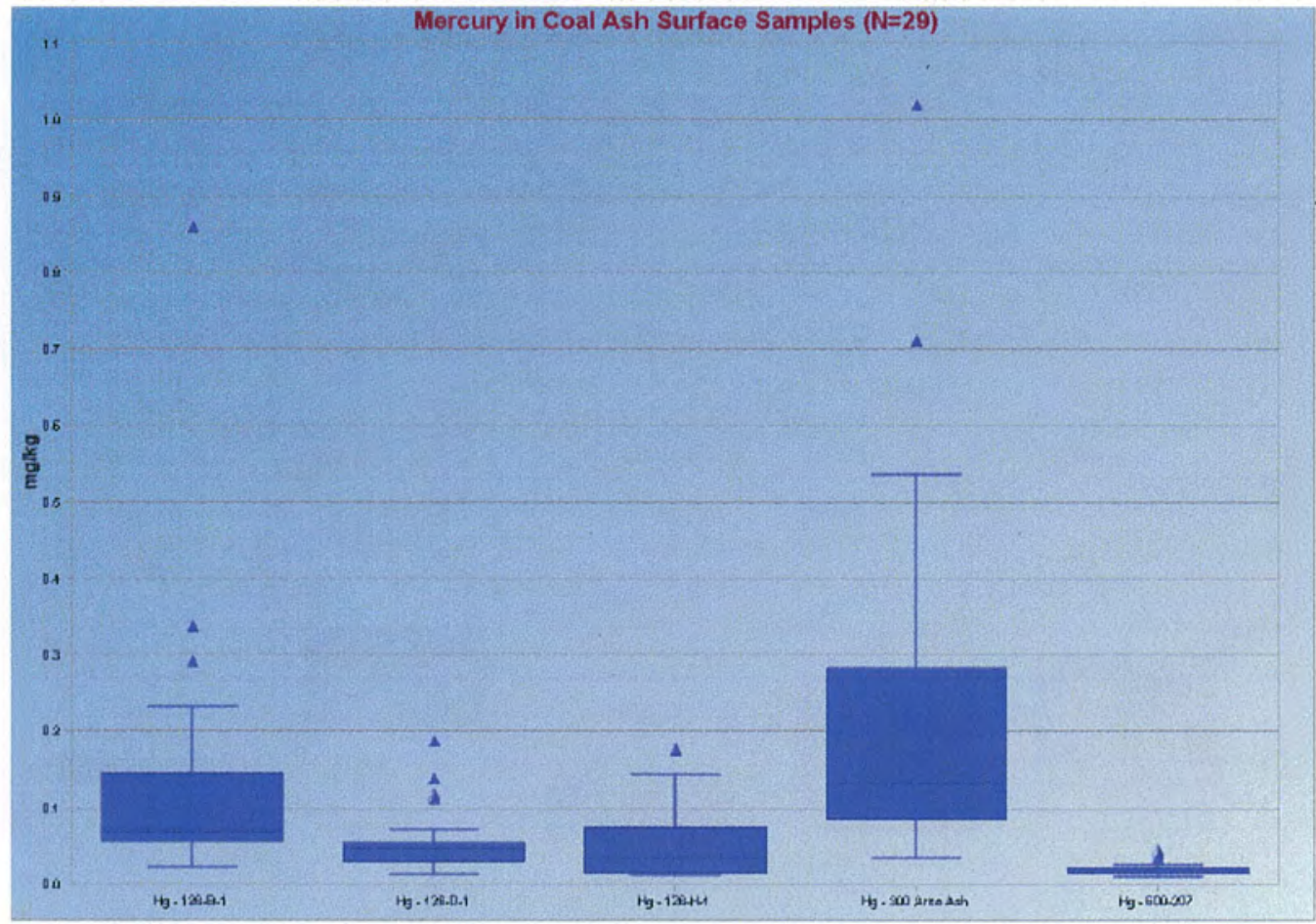


Figure E-13. Boxplots of Molybdenum Results from Coal Ash Surface Samples ( $\mathrm{N}=29)$.

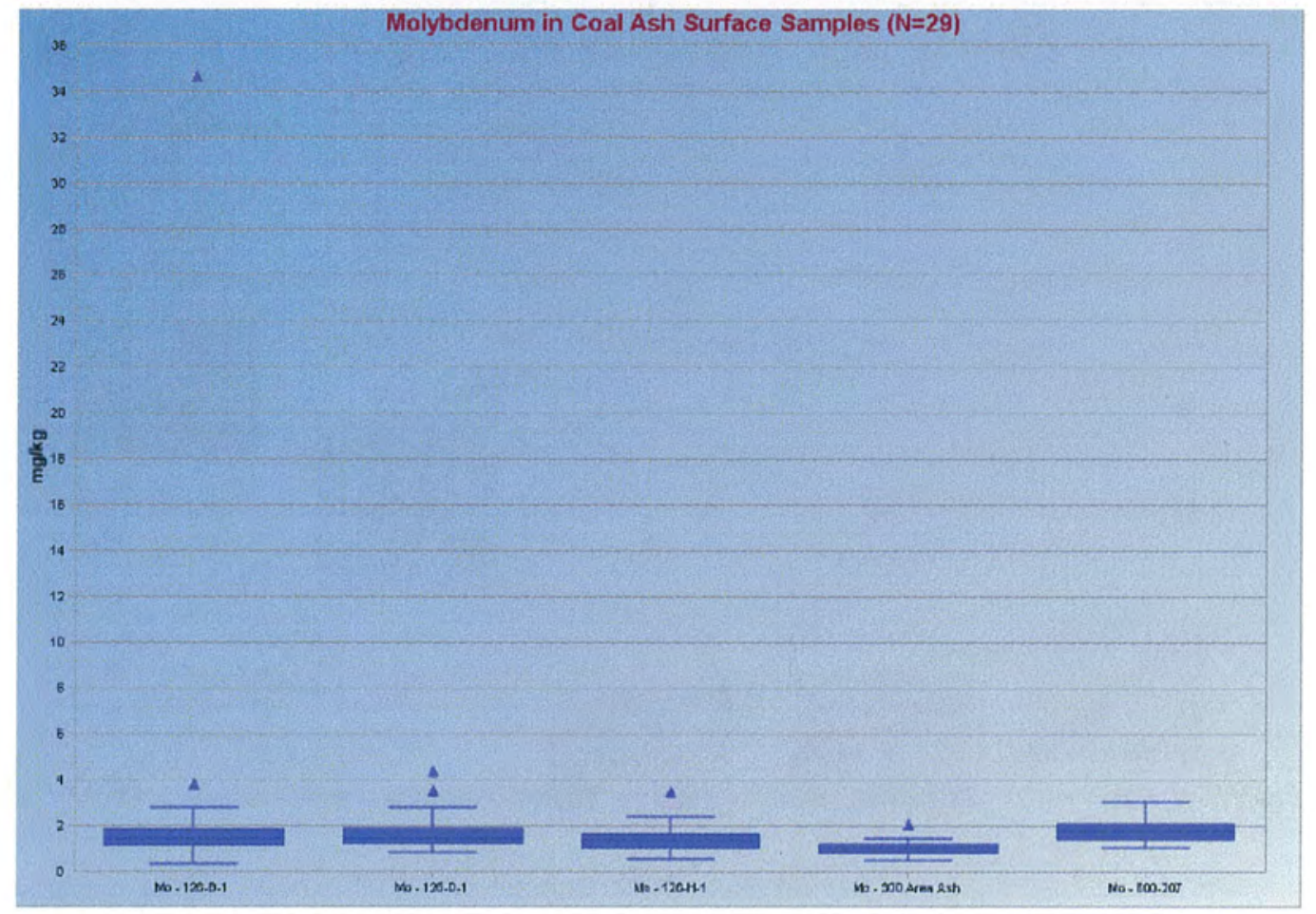

Figure E-14. Boxplots of Nickel Results from Coal Ash Surface Samples ( $N=29$ ).

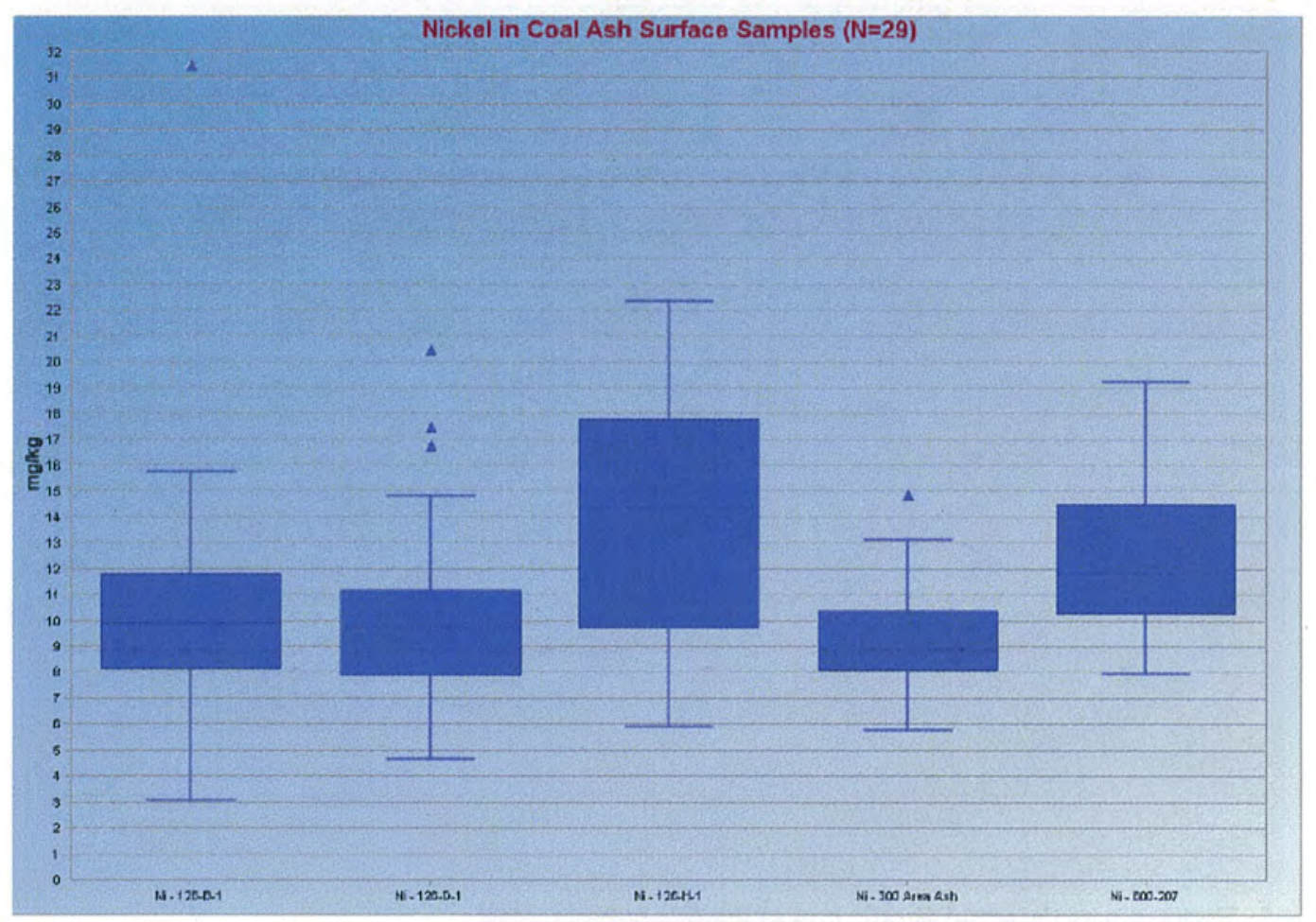


Figure E-15. Boxplots of Selenium Results from Coal Ash Surface Samples ( $N=29$ ).

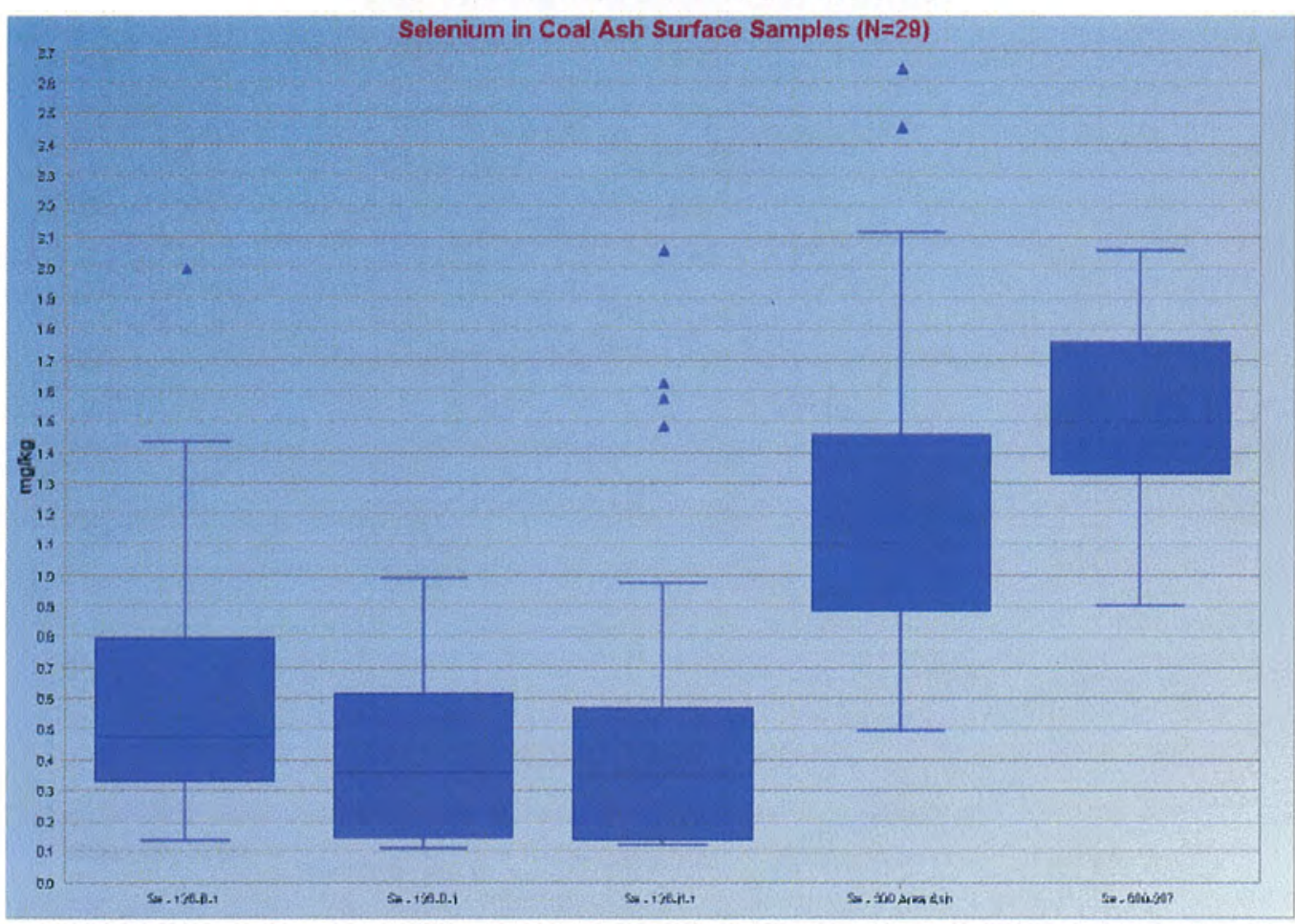

Figure E-16. Boxplots of Thallium Results from Coal Ash Surface Samples ( $N=29)$.

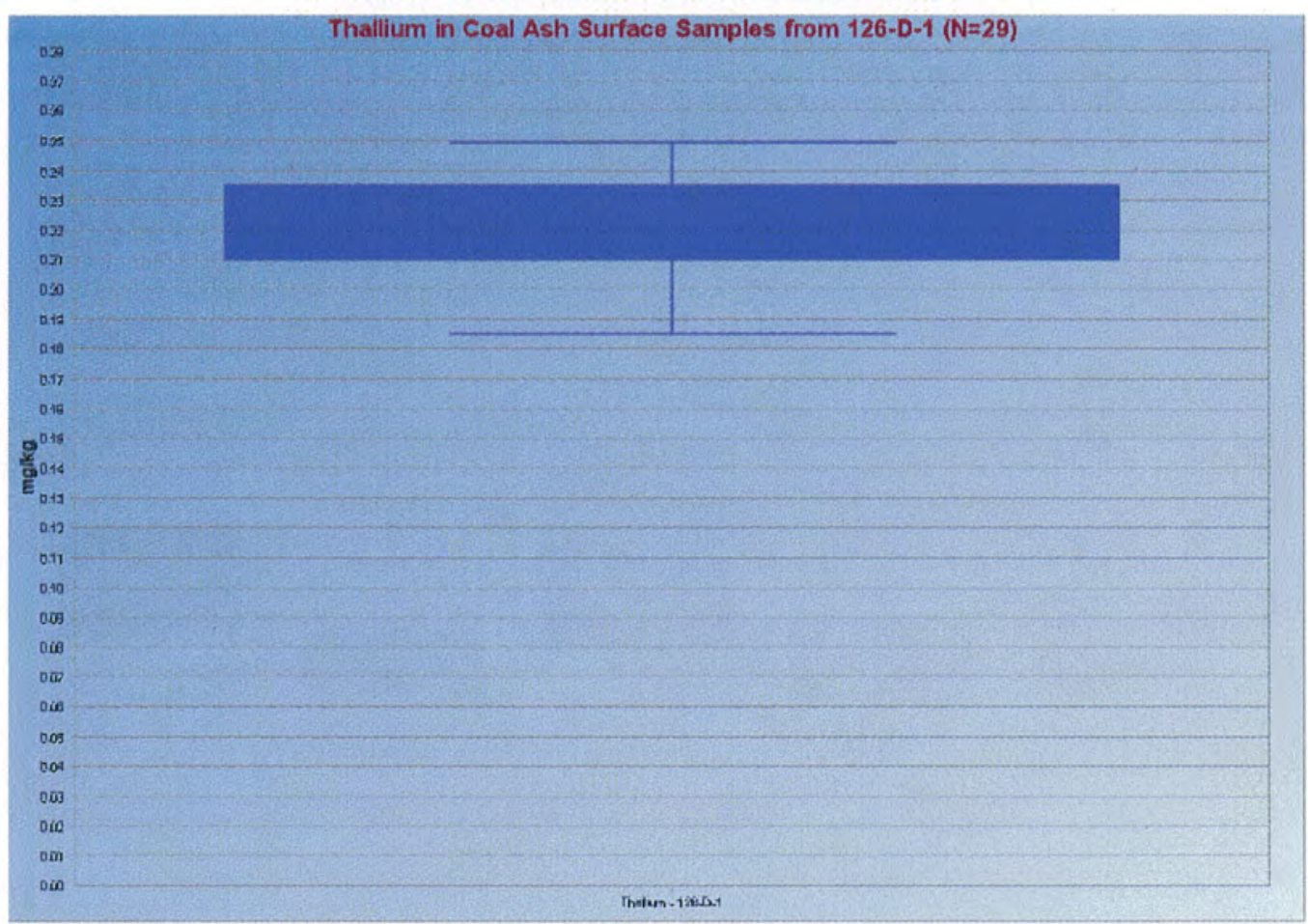

a Thallium was detected at only one of the five sample sites. 
Figure E-17. Boxplots of Vanadium Results from Coal Ash Surface Samples ( $\mathrm{N}=29$ ).

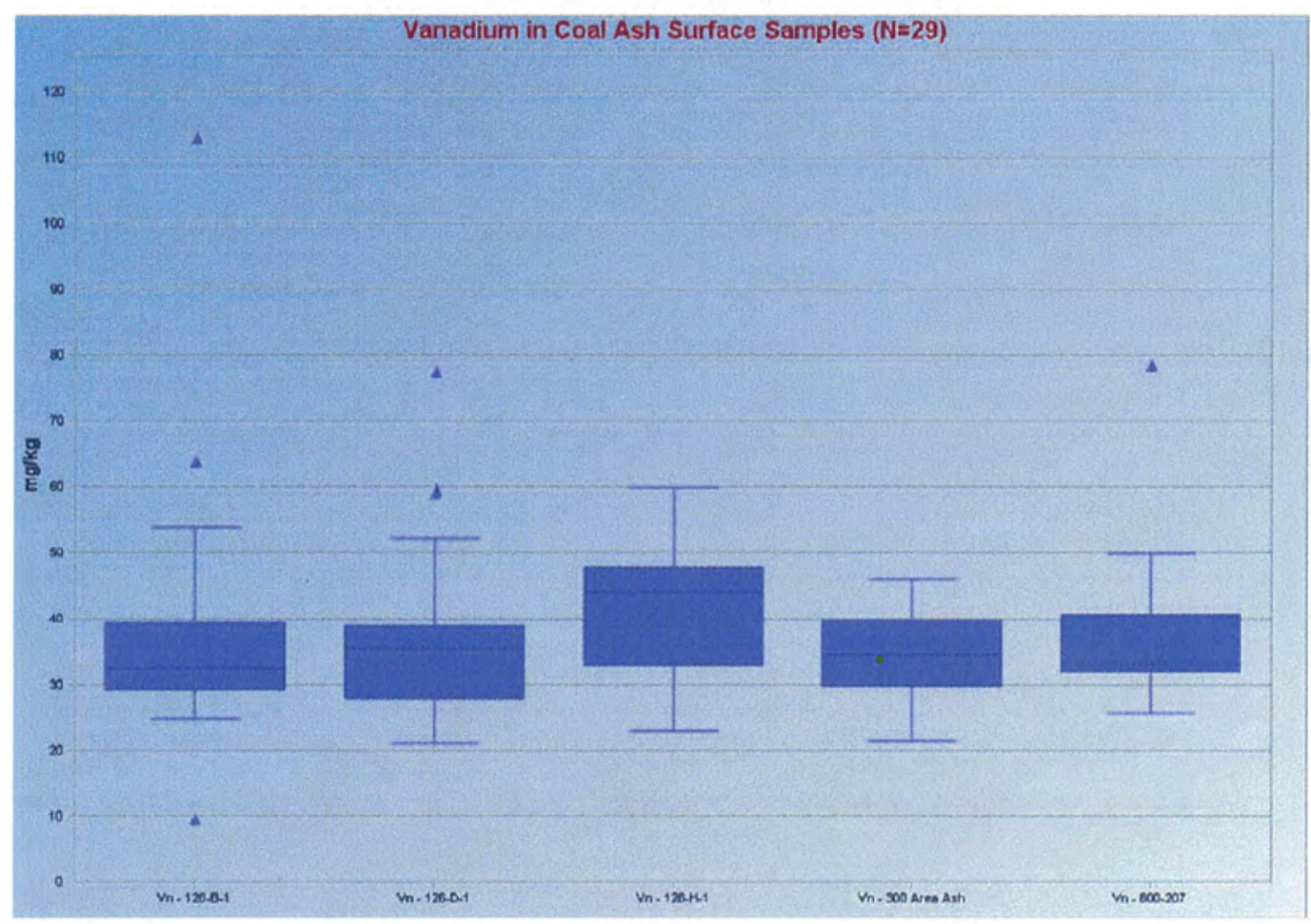

Figure E-18. Boxplots of Zinc Results from Coal Ash Surface Samples ( $\mathrm{N}=29$ ).

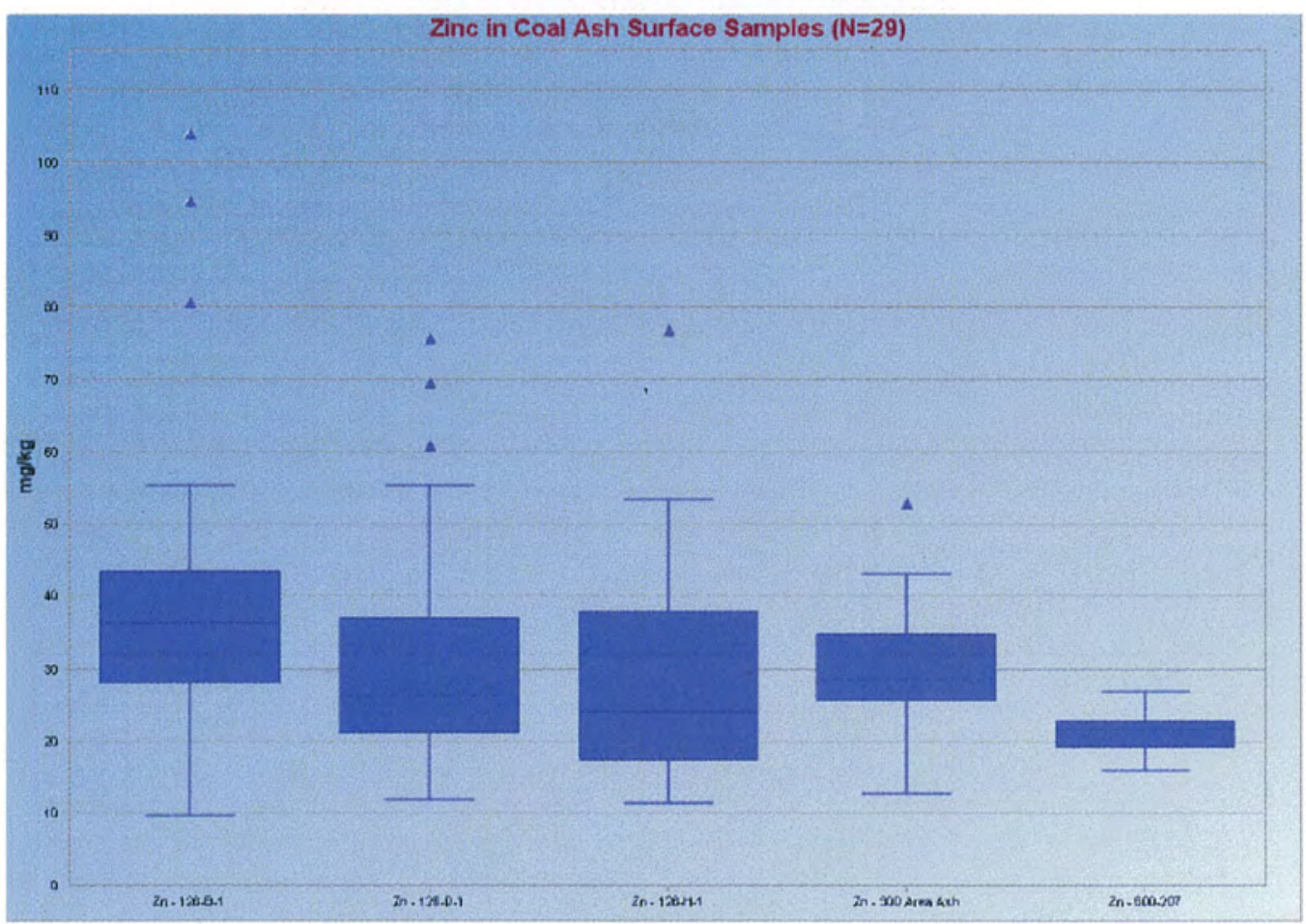


WCH-506

Rev. 0

\section{APPENDIX F}

COAL ASH SURFACE AND DEPTH SAMPLE BOXPLOTS 
WCH-506

Rev. 0 
Figure F-1. Boxplots of Antimony Results from 126-B-1, 126-D-1, and 600-207 Coal Ash Surface (S) and Depth (D) Samples ( $N=3$ ).

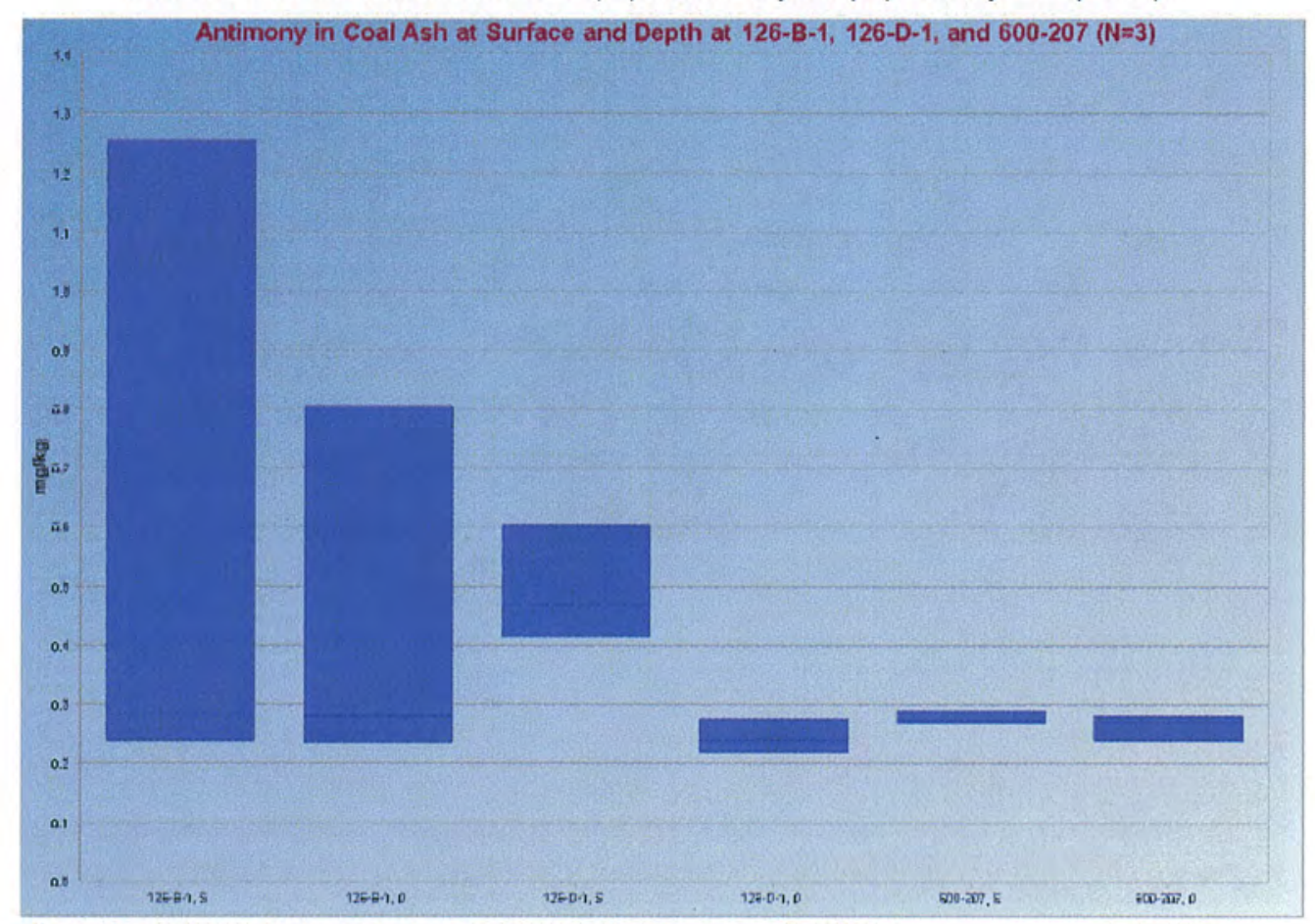

Antimony was detected at only three of the five sample sites.

Figure F-2. Boxplots of Arsenic Results from Coal Ash Surface (S) and Depth (D) Samples ( $N=3$ ).

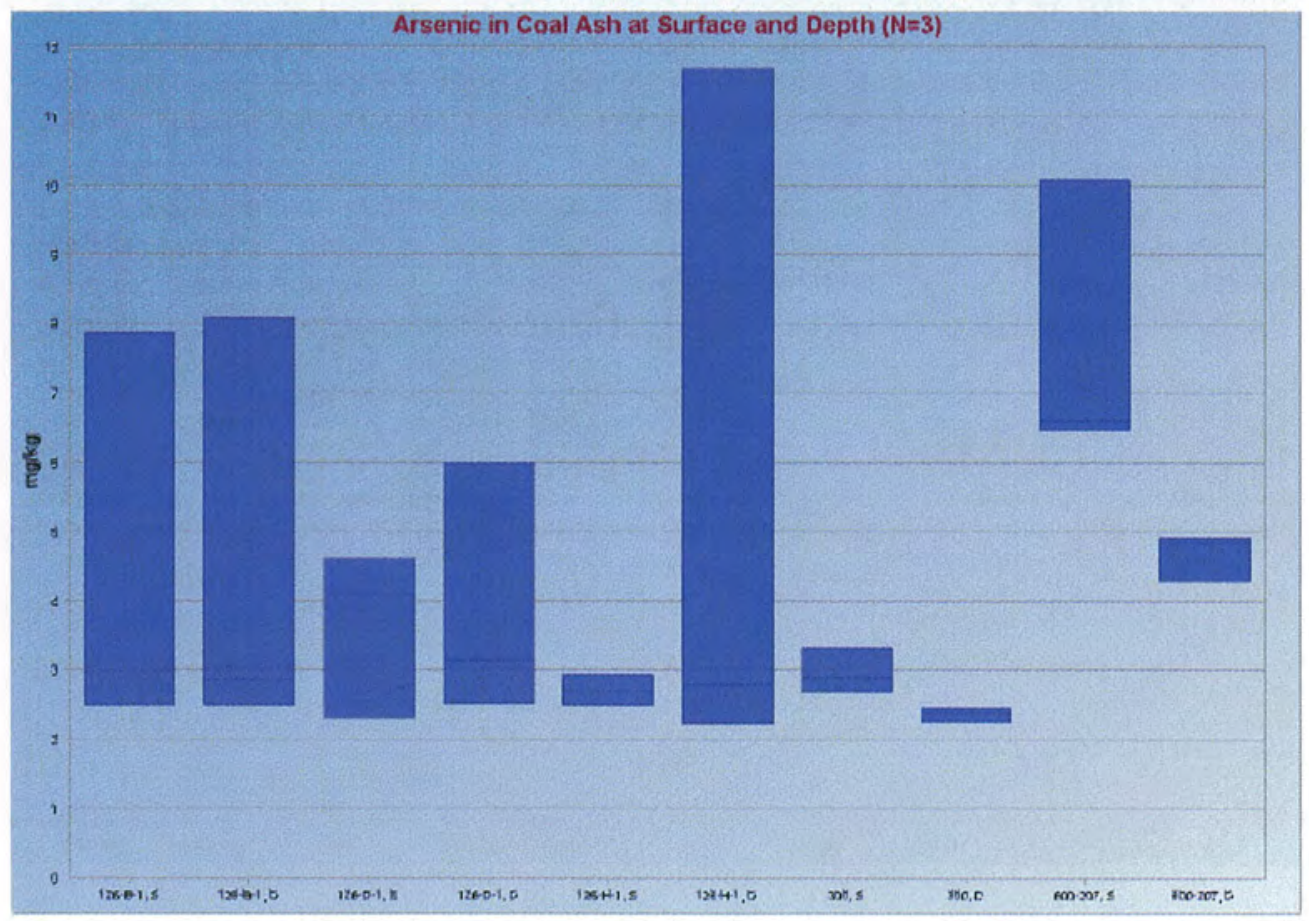


Figure F-3. Boxplots of Barium Results from Coal Ash Surface (S) and Depth (D) Samples ( $N=3$ ).

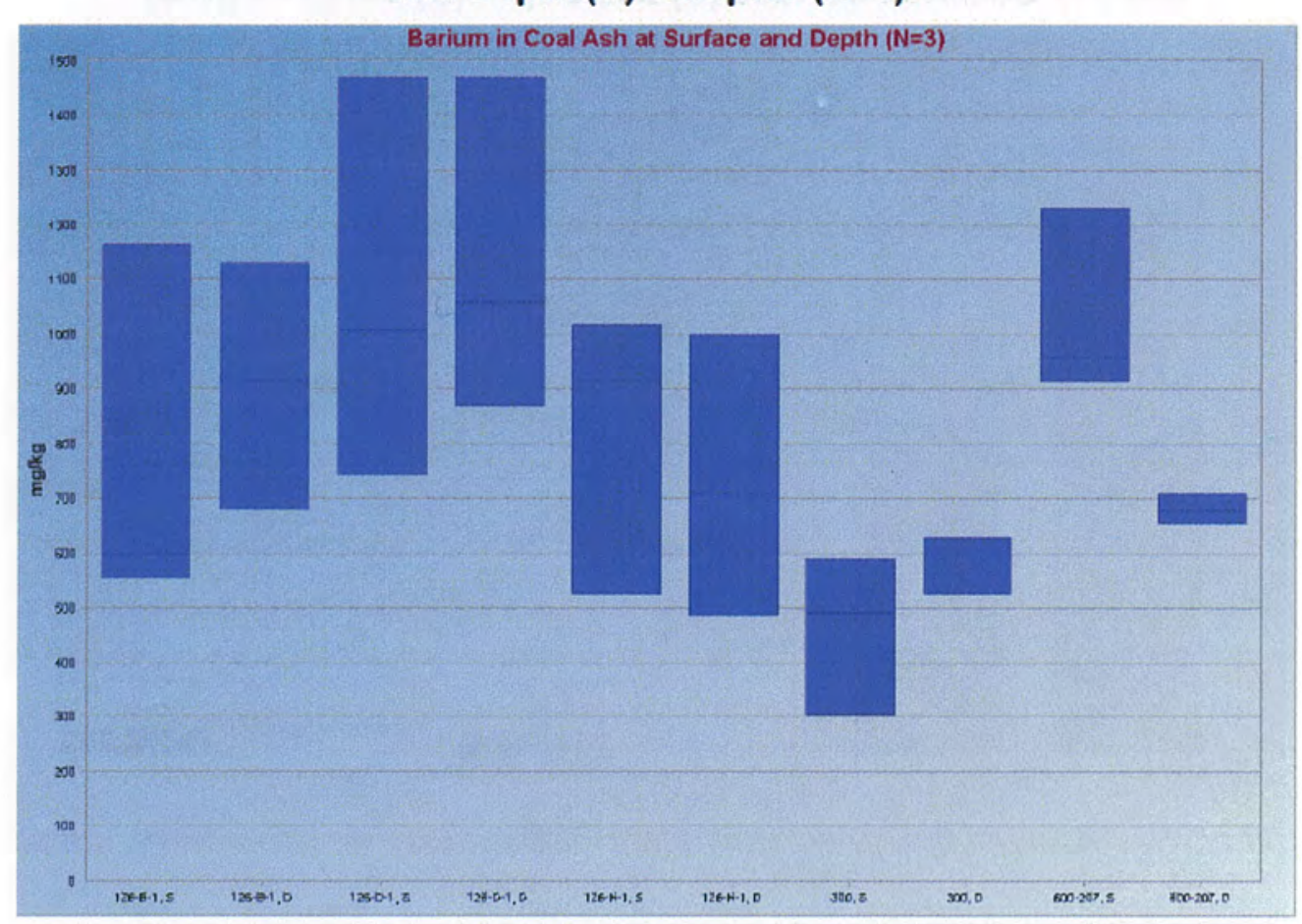

Figure F-4. Boxplots of Beryllium Results from Coal Ash Surface (S) and Depth (D) Samples ( $N=3$ ).

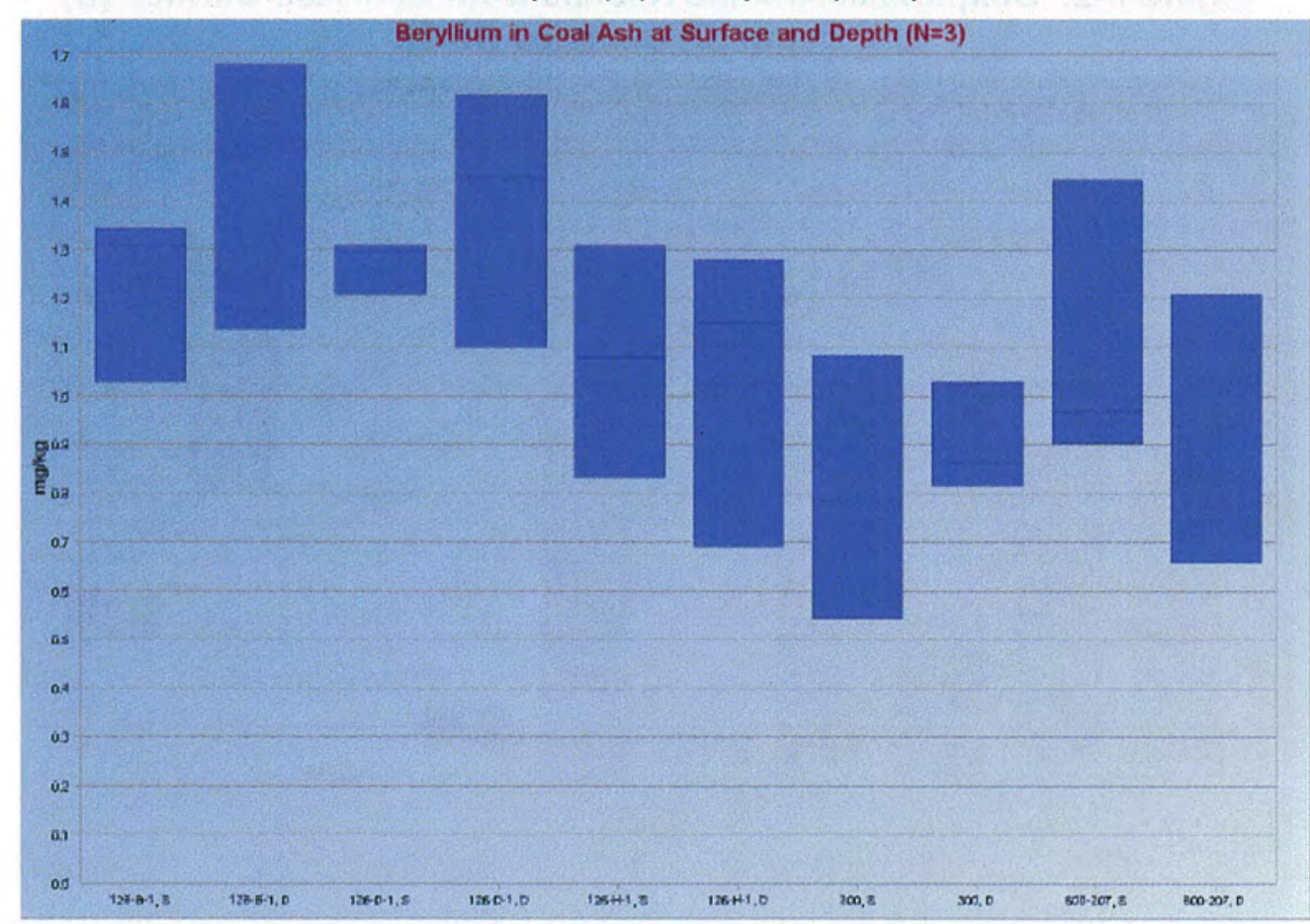


Figure F-5. Boxplots of Boron Results from Coal Ash Surface (S) and Depth (D) Samples ( $N=3$ ).

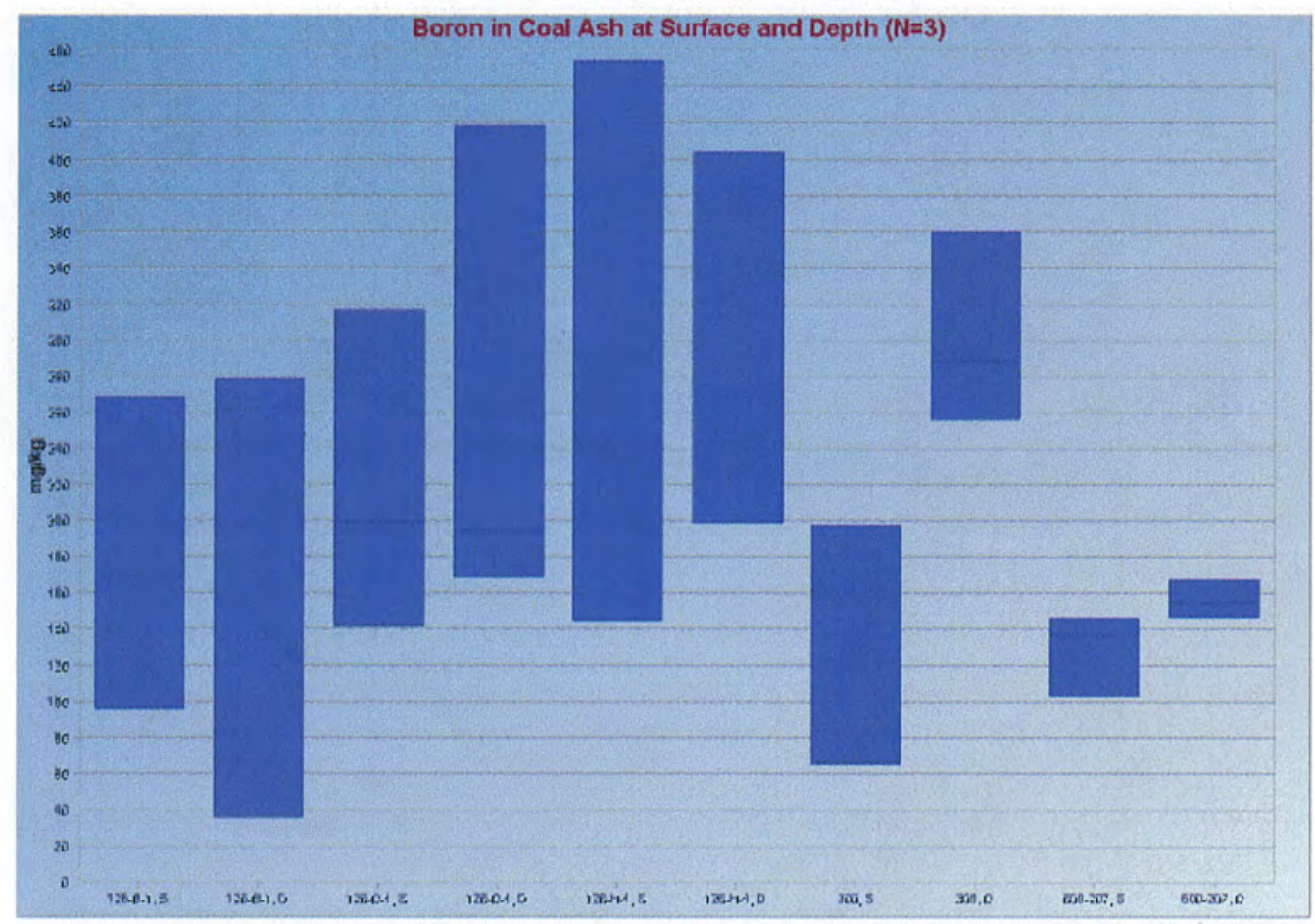

Figure F-6. Boxplots of Cadmium Results from Coal Ash Surface (S) and Depth (D) Samples ( $N=3$ ).

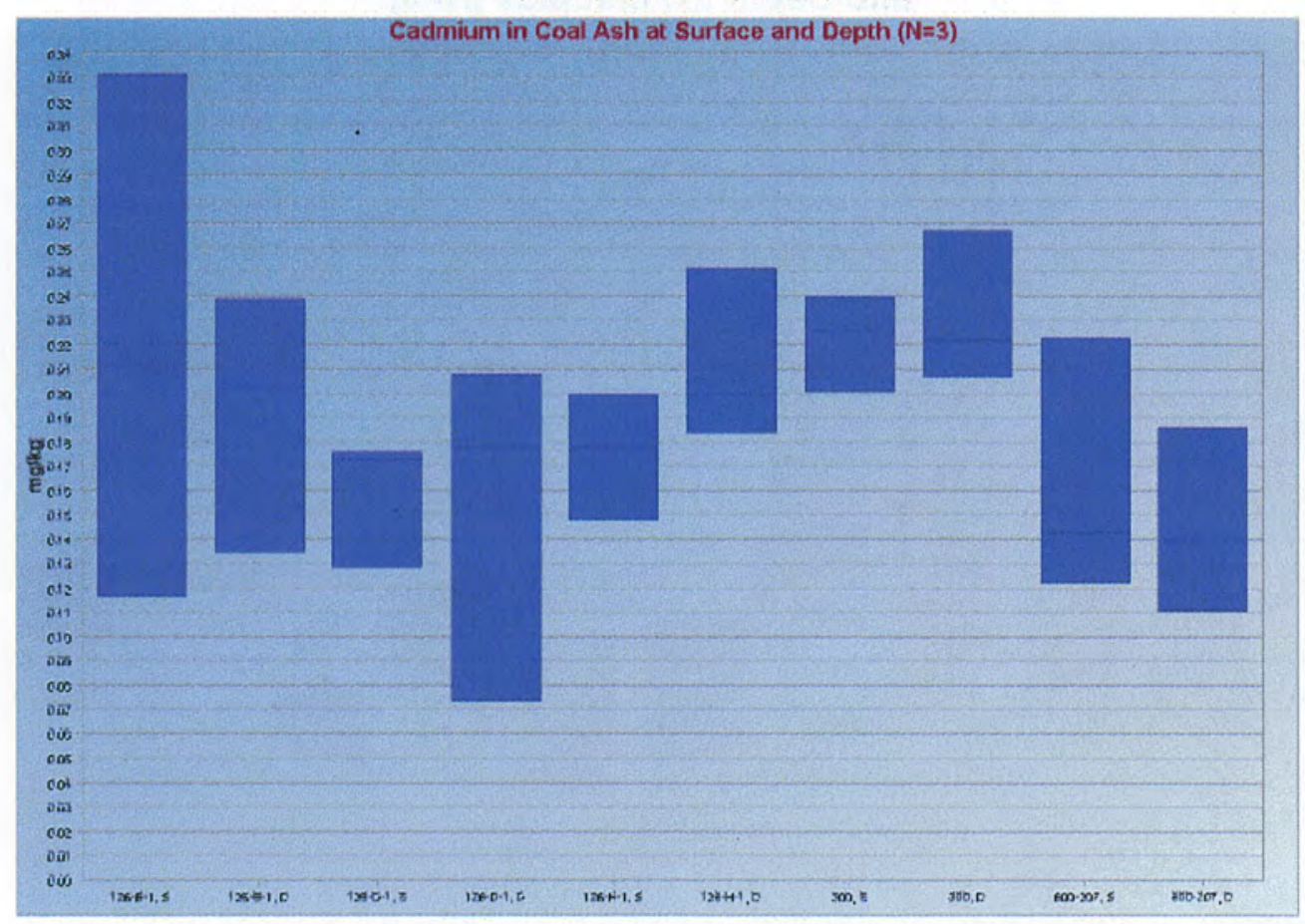


Figure F-7. Boxplots of Total Chromium Results from Coal Ash Surface (S) and Depth (D) Samples ( $N=3$ ).

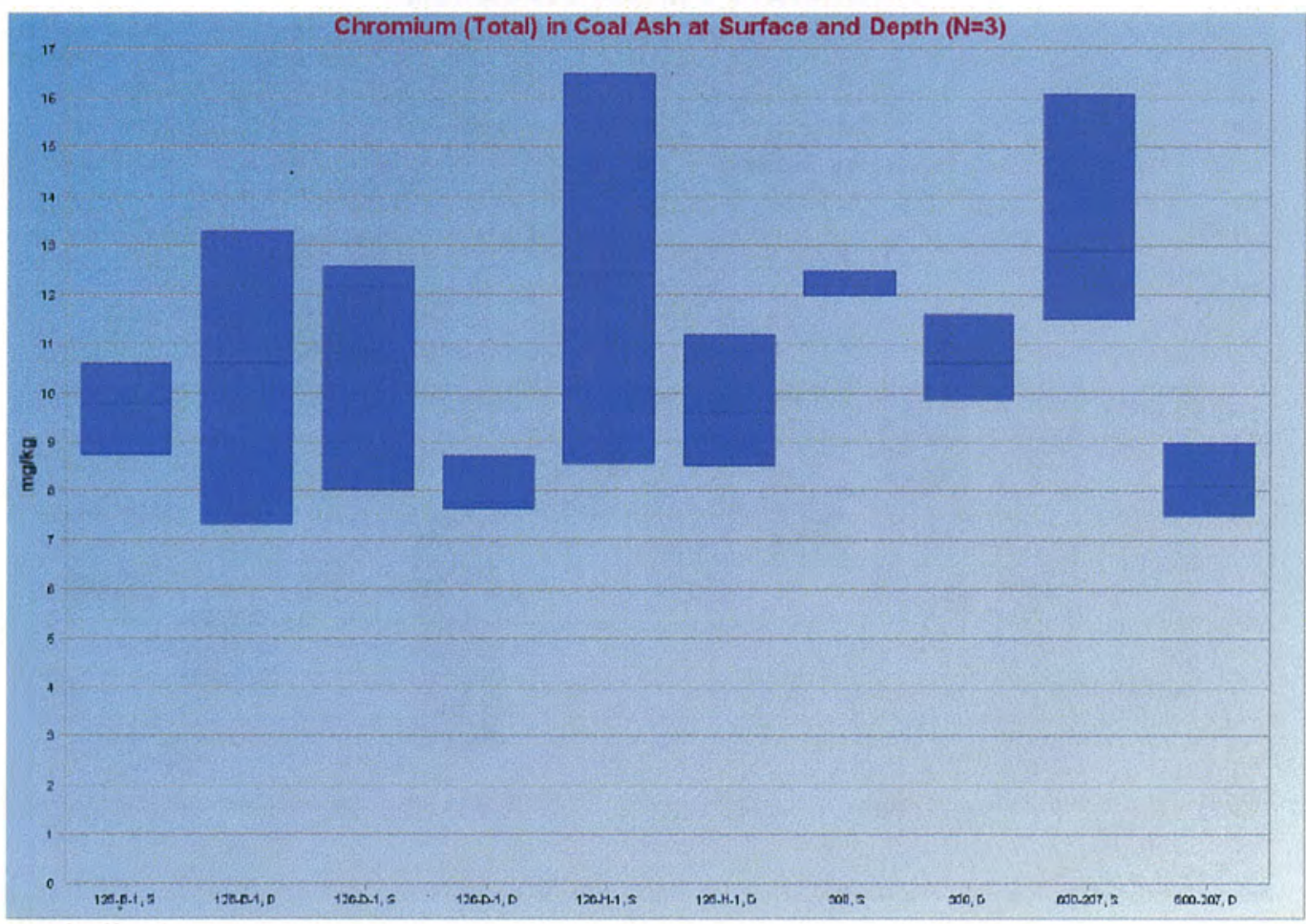

Figure F-8. Boxplots of Cobalt Results from Coal Ash Surface (S) and Depth (D) Samples ( $N=3$ ).

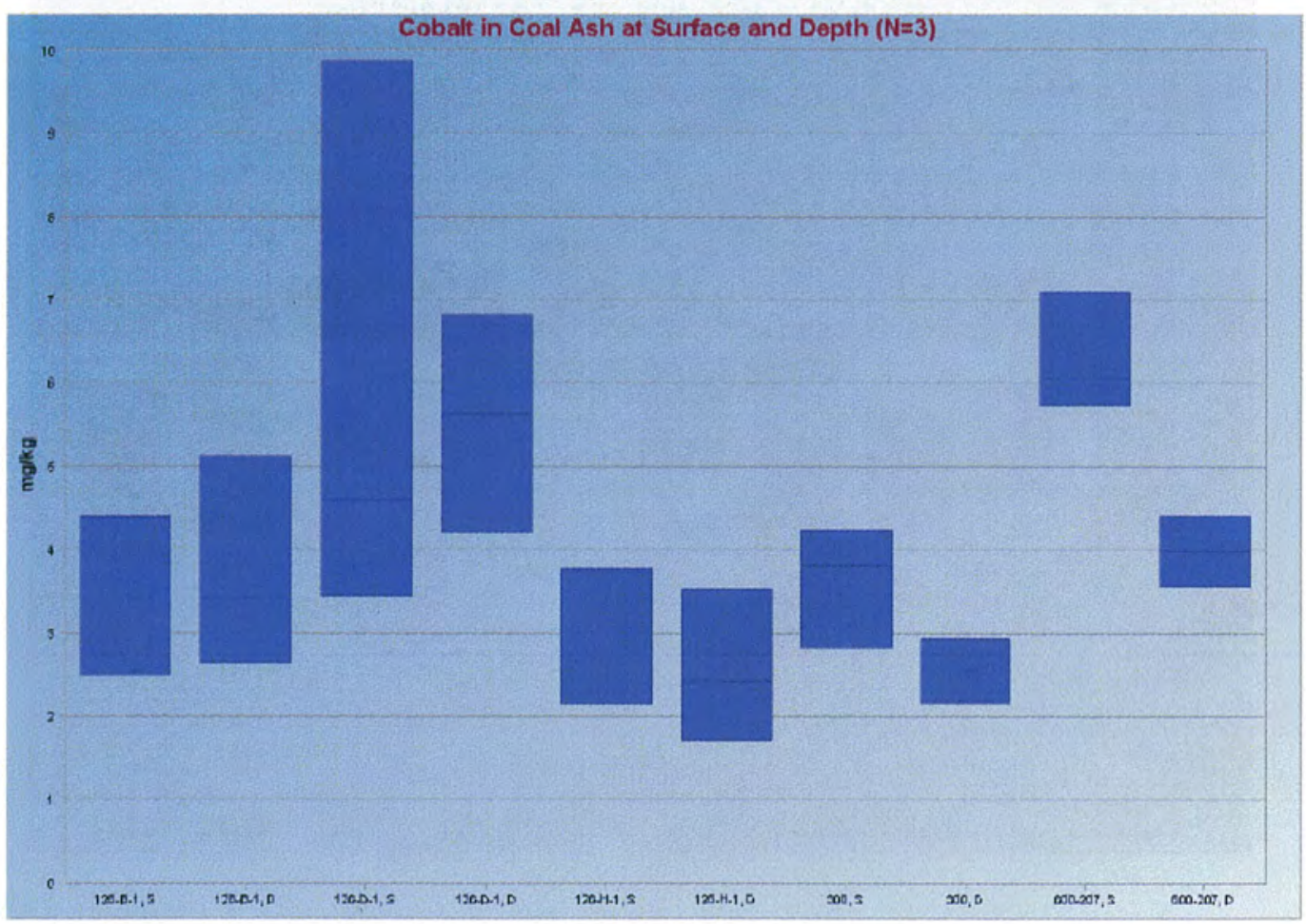


Figure F-9. Boxplots of Copper Results from Coal Ash Surface (S) and Depth (D) Samples ( $N=3$ ).

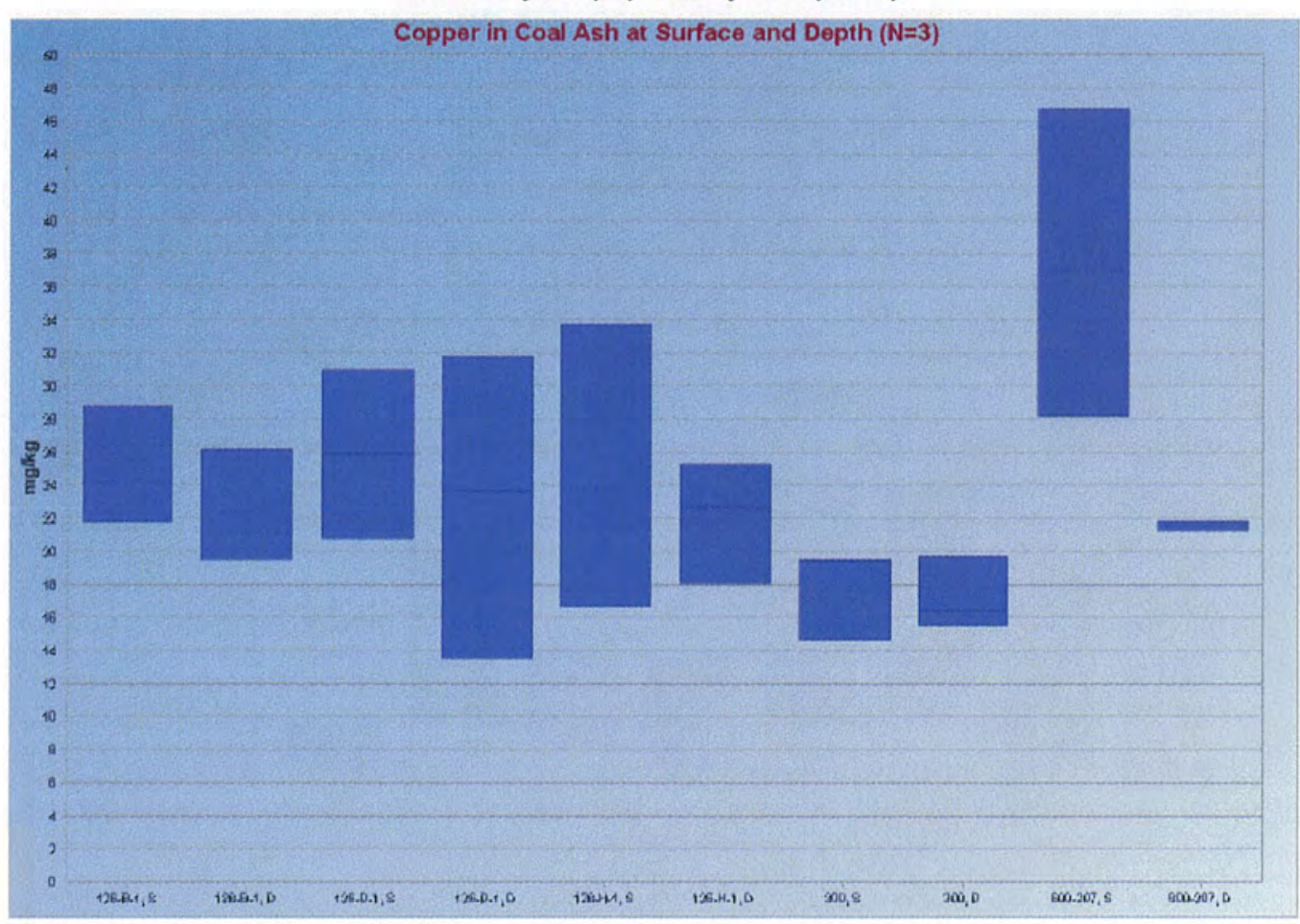

Figure F-10. Boxplots of Lead Results from Coal Ash Surface (S) and Depth (D) Samples ( $N=3$ ).

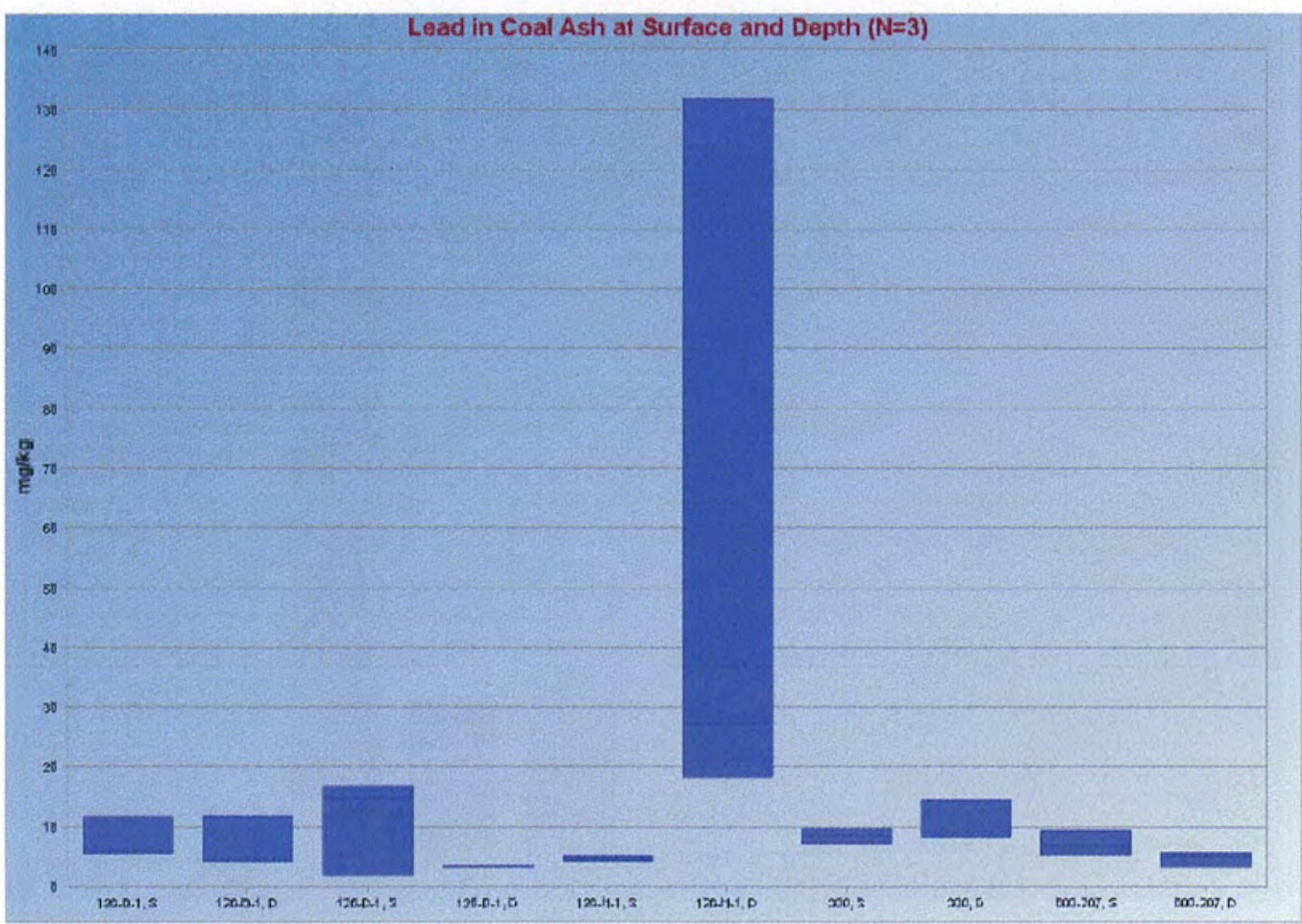


Figure F-11. Boxplots of Manganese Results from Coal Ash Surface (S) and Depth (D) Samples ( $N=3)$.

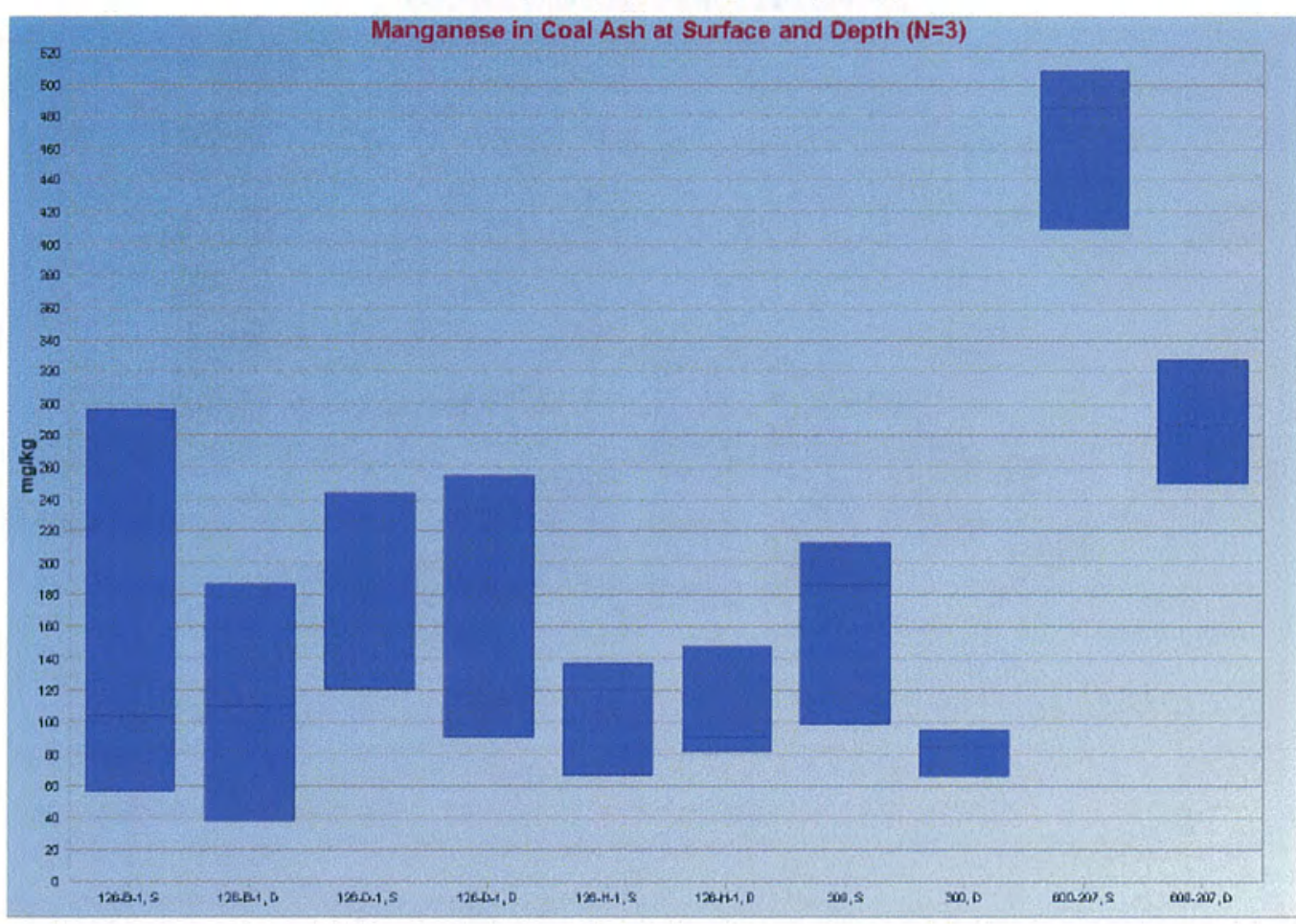

Figure F-12. Boxplots of Mercury Results from Coal Ash Surface (S) and Depth (D) Samples ( $N=3$ ).

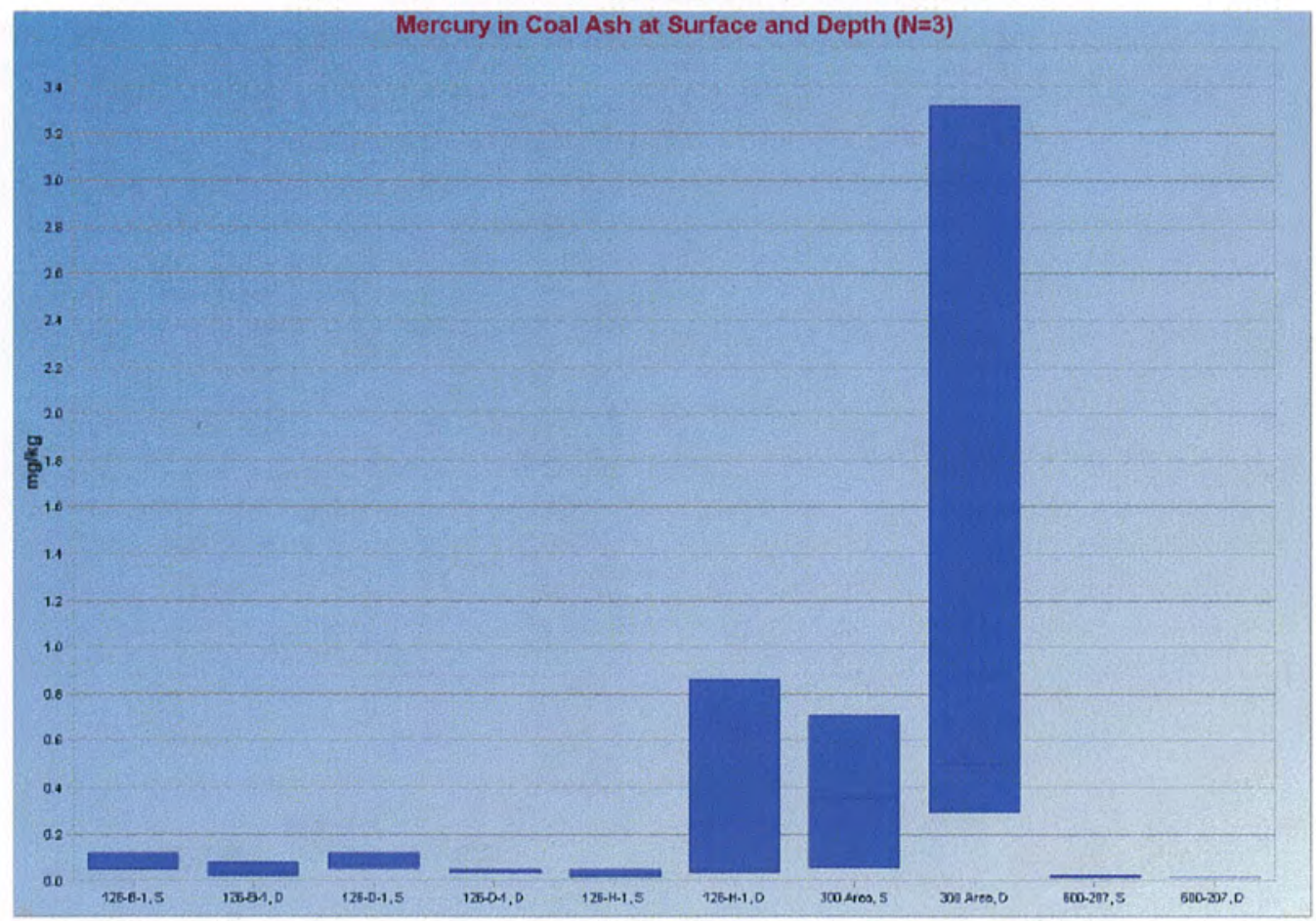


Figure F-13. Boxplots of Molybdenum Results from Coal Ash Surface (S) and Depth (D) Samples ( $N=3$ ).

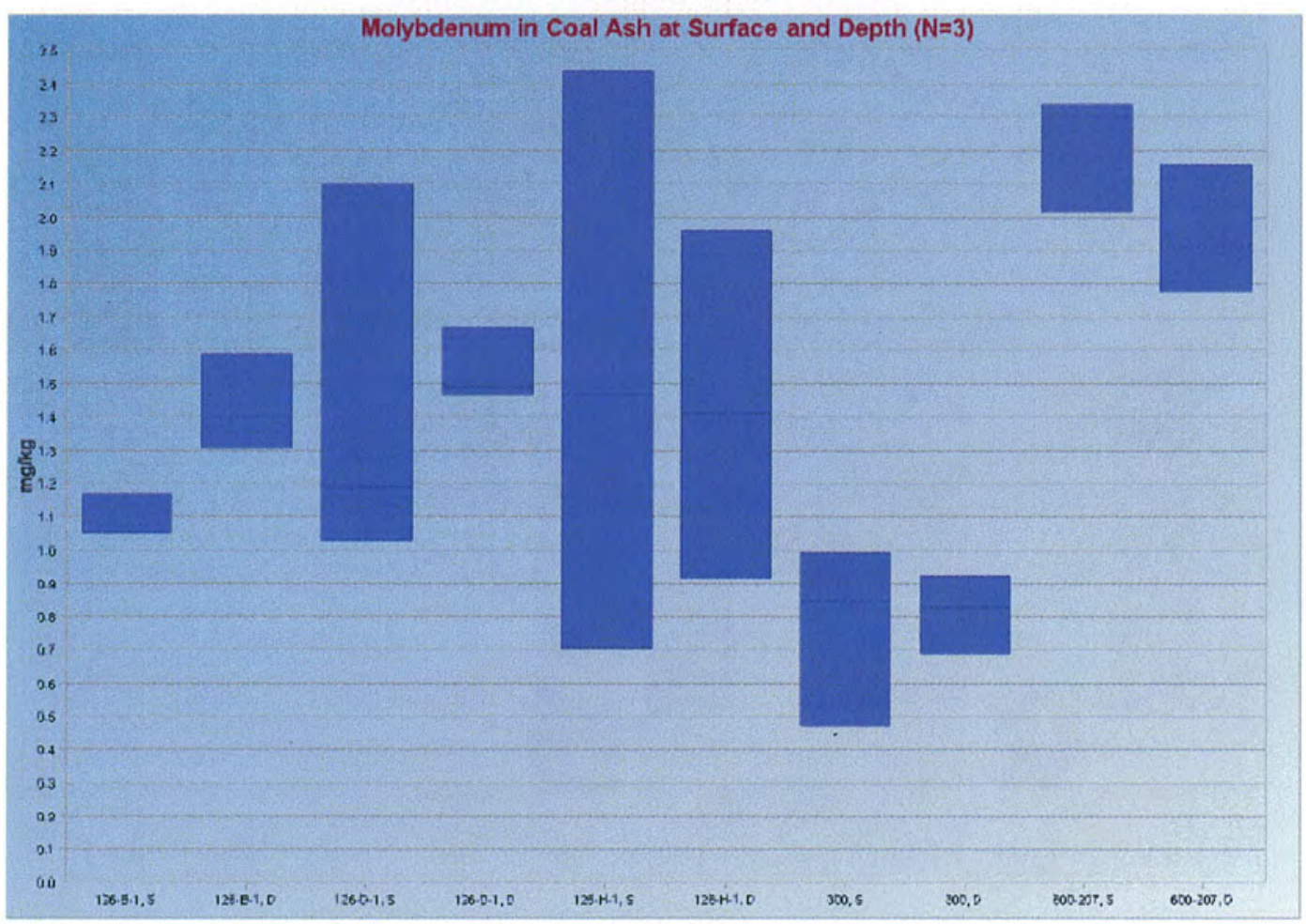

Figure F-14. Boxplots of Nickel Results from Coal Ash Surface (S) and Depth (D) Samples ( $N=3$ ).

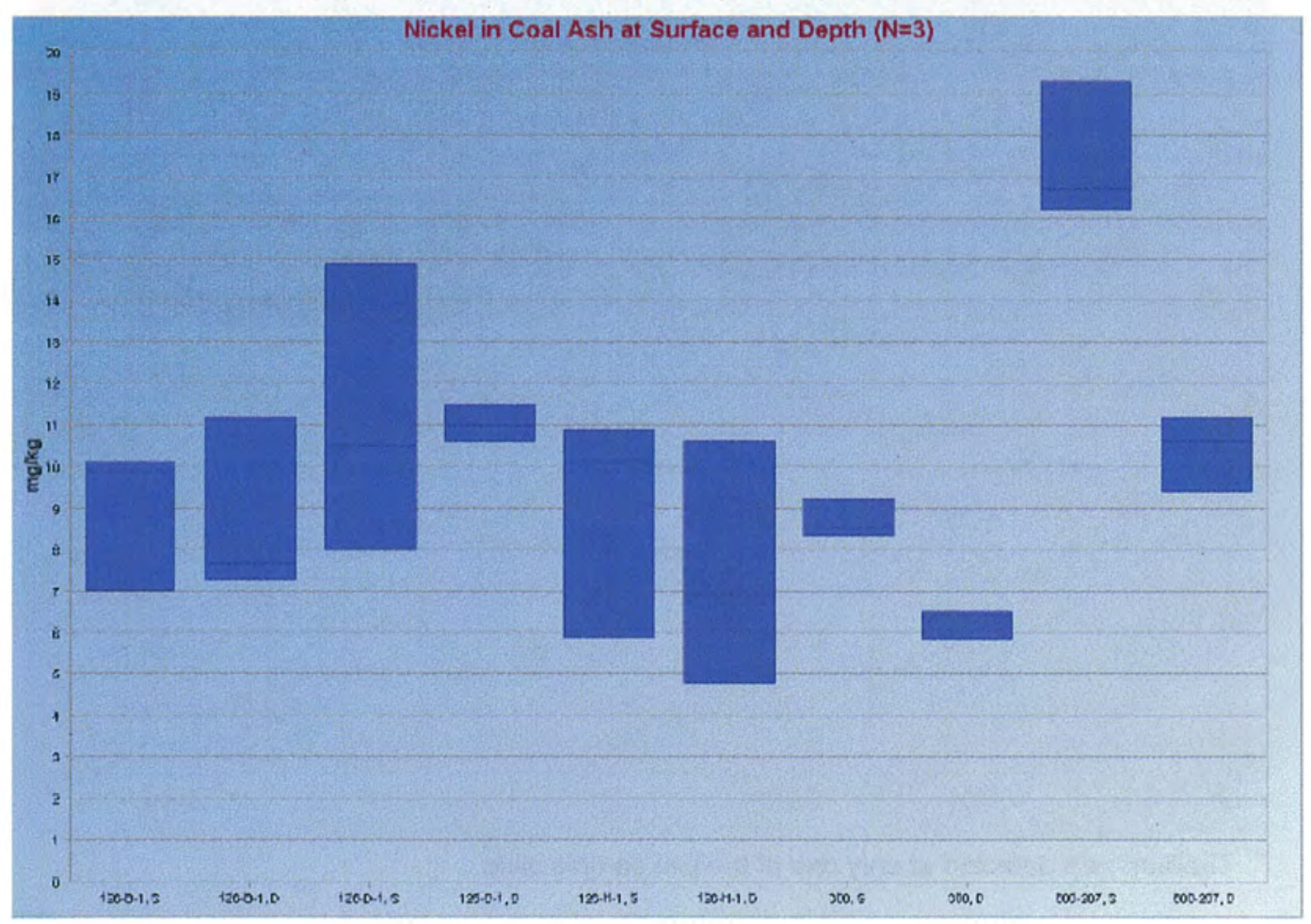


Figure F-15. Boxplots of Selenium Results from Coal Ash Surface (S) and Depth (D) Samples ( $\mathrm{N}=3$ ).

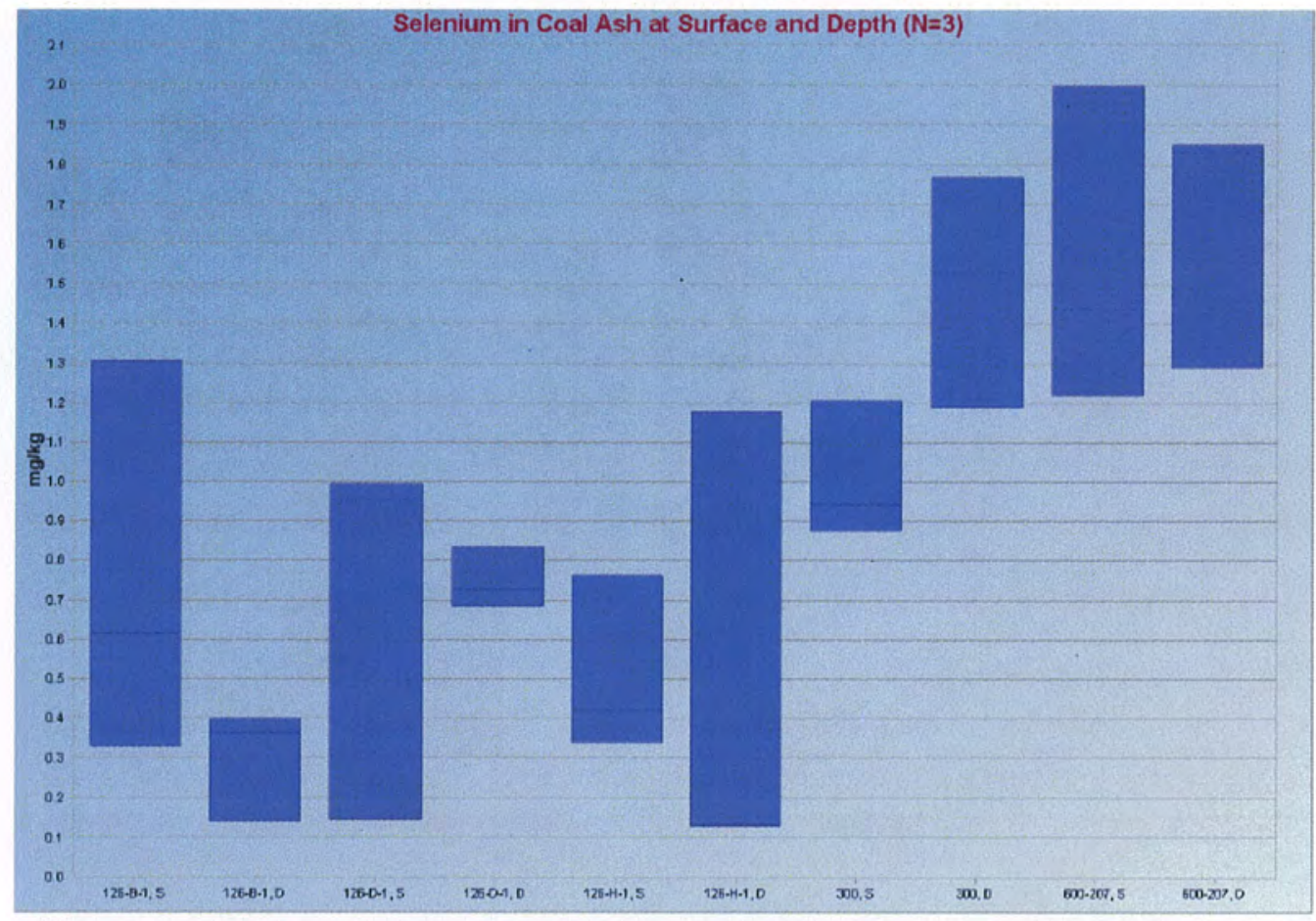

Figure F-16. Boxplots of Thallium Results from Coal Ash Surface (S) and Depth (D) Samples at 126-D-1 (N=3). ${ }^{\text {a }}$

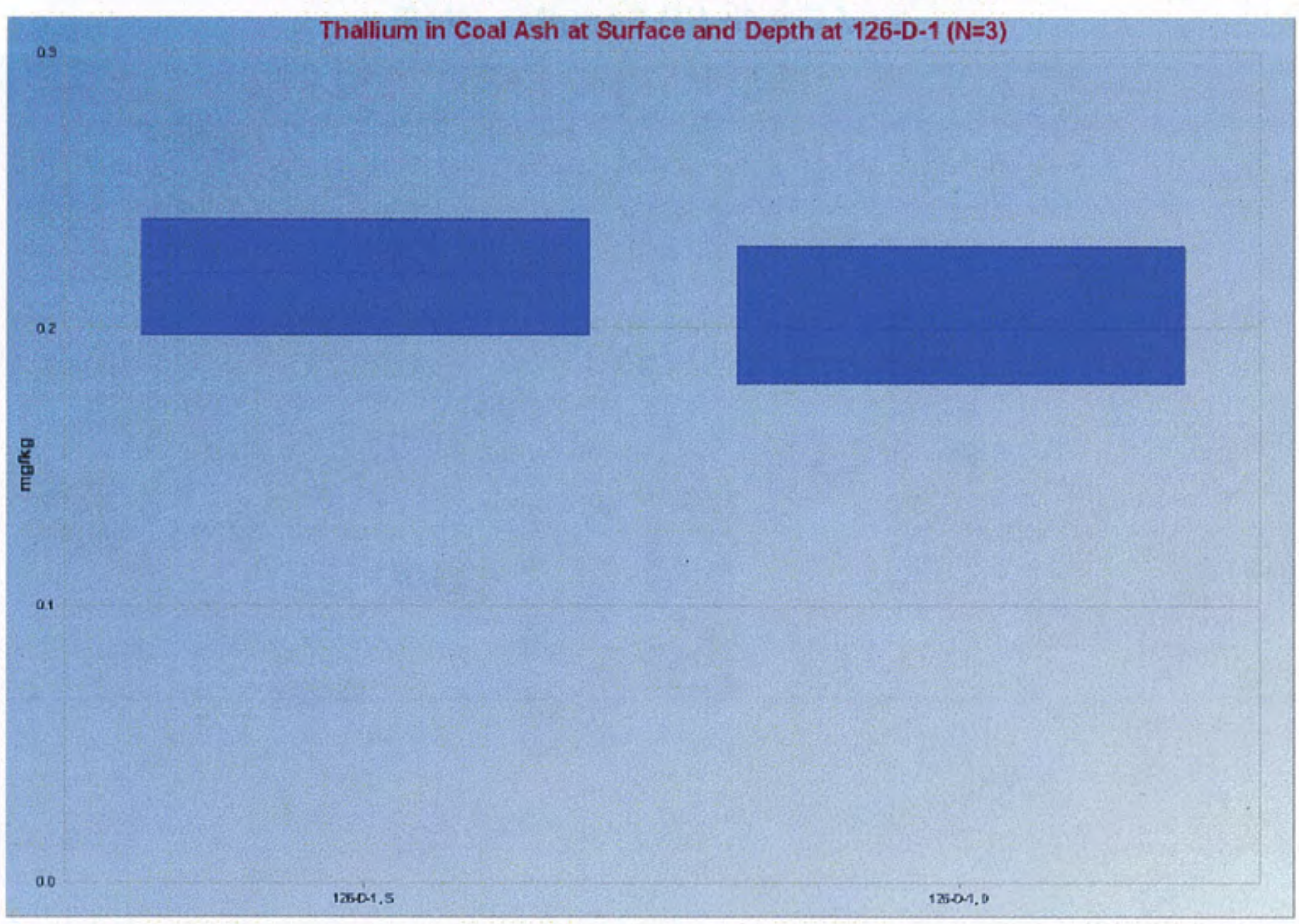

a Thallium was detected at only one of the five sample sites. 
Figure F-17. Boxplots of Uranium Results from Coal Ash Surface (S) and Depth (D) Samples ( $N=3$ ).

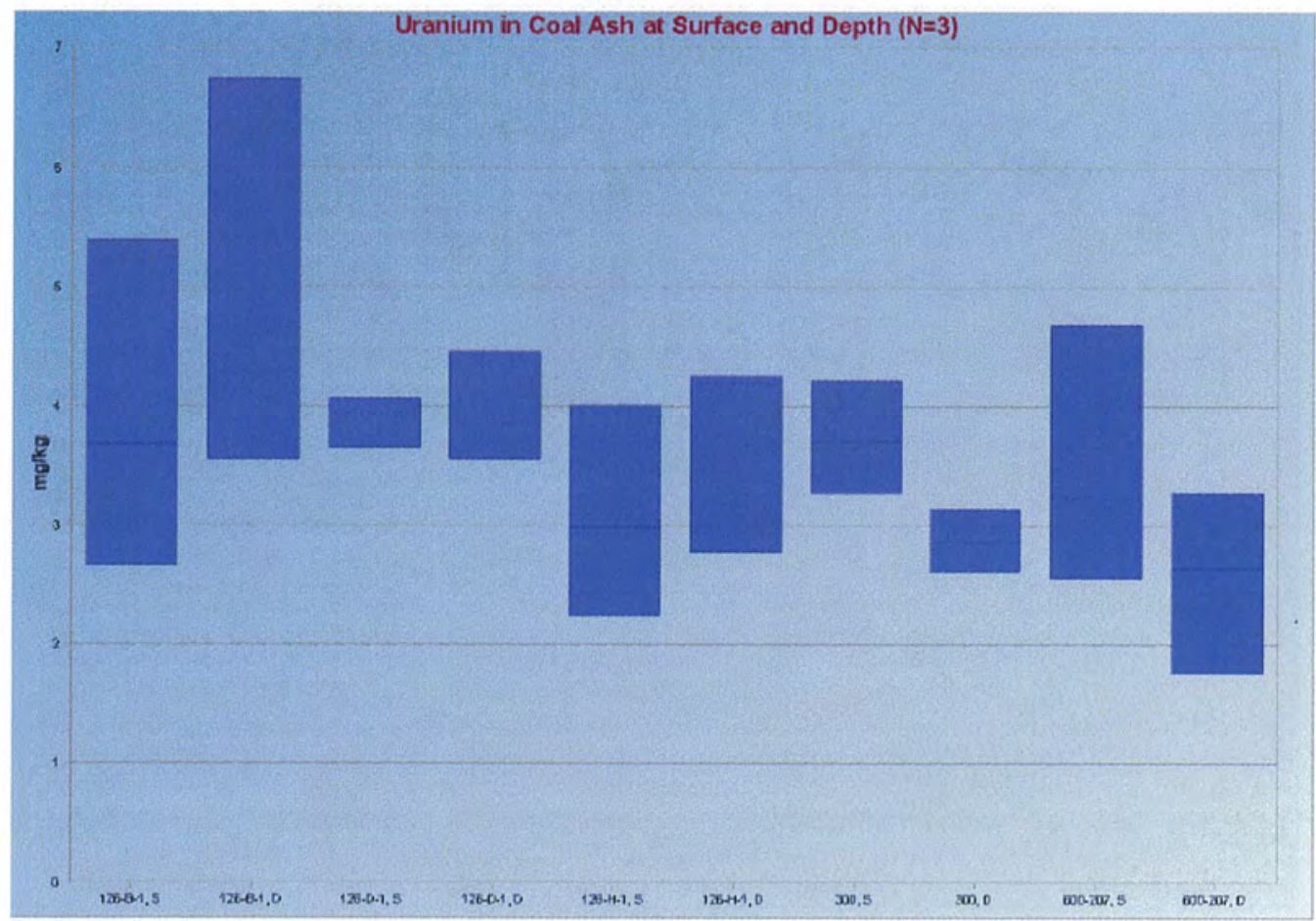

Figure F-18. Boxplots of Vanadium Results from Coal Ash Surface (S) and Depth (D) Samples ( $N=3$ ).

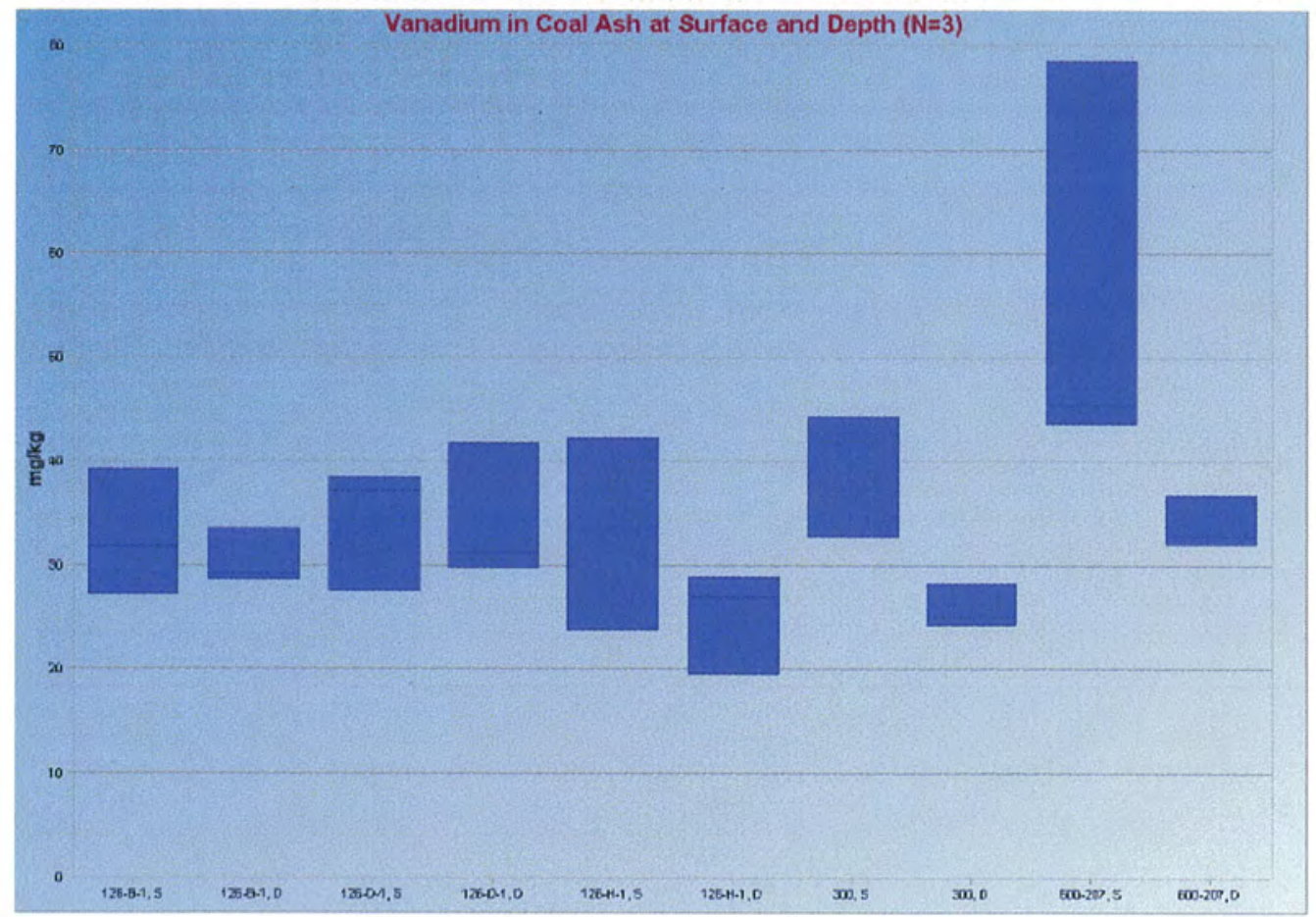


Figure F-19. Boxplots of Zinc Results from Coal Ash Surface (S) and Depth (D) Samples ( $N=3$ ).

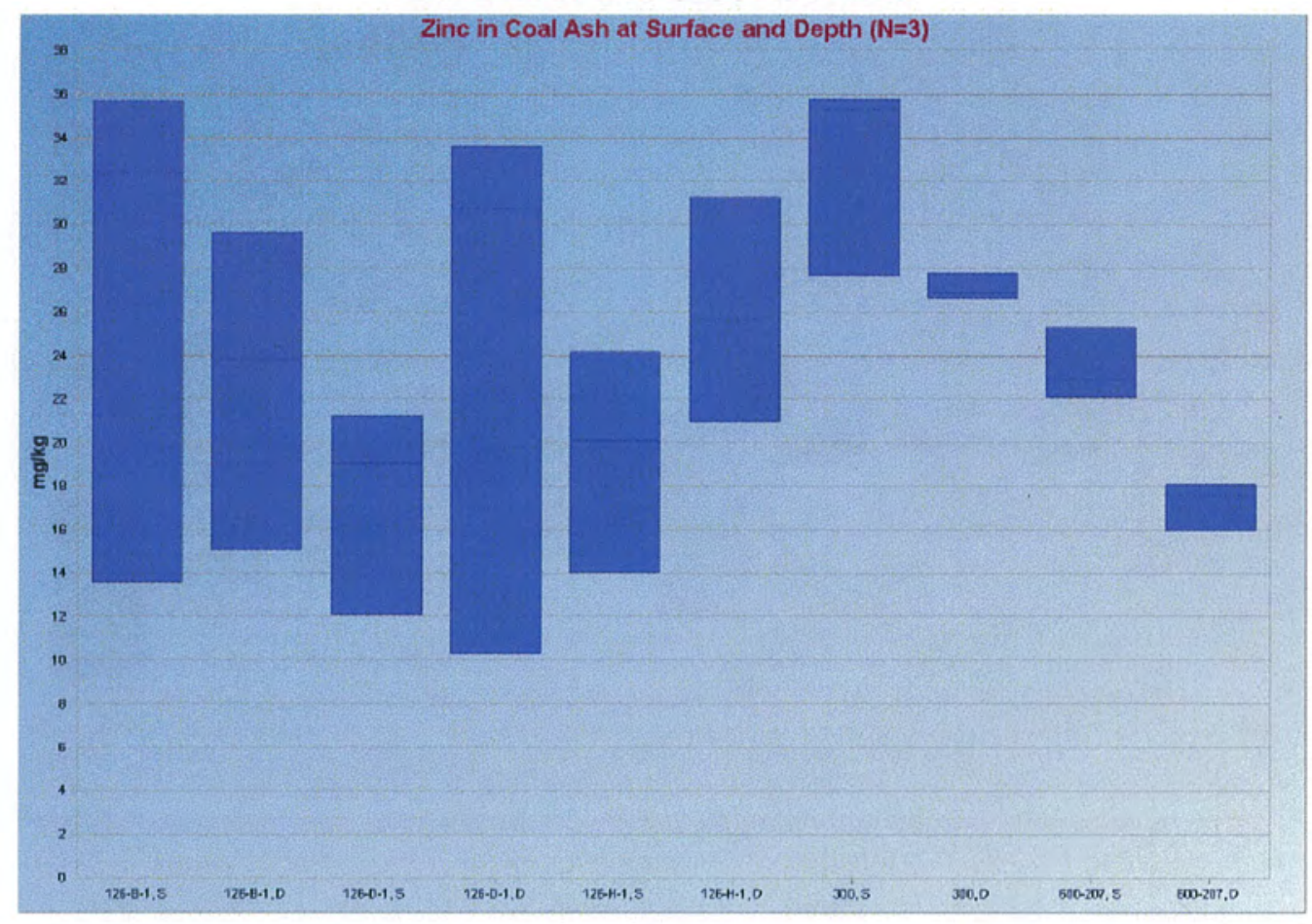


WCH-506

Rev. 0

\section{APPENDIX G}

\section{DATA QUALITY ANALYSIS}


WCH-506

Rev. 0 
WCH-506

Rev. 0

\section{APPENDIX G}

\section{DATA QUALITY ANALYSIS}

A data quality analysis (DQA) was performed to compare the sampling approach and resulting analytical data with the sampling and data requirements specified in the Sampling and Analysis Plan for Characterization of Hanford Site Coal Ash Components (SAP) (DOE-RL 2011a). This DQA was performed in accordance with data quality objectives described in Section 2.5 .2 of the SAP.

A review of the sample designs in the SAP, associated change notices (DOE-RL 2011b, 2011c), field logbook (WCH 2011), and applicable analytical data packages has been performed as part of this DQA. All samples that were required by the SAP were collected.

To ensure quality data, the SAP data assurance requirements and the data validation procedures for chemical and radiochemical analysis (BHI 2000a, 2000b) are used as appropriate. This review involves evaluation of the data to determine if they are of the right type, quality, and quantity to support the intended use (i.e., decision-making purposes). The DQA completes the data life cycle (i.e., planning, implementation, and assessment) that was initiated by the data quality objectives process (EPA 2000).

\section{G.1 COAL ASH ANALYSES}

Samples collected from prescribed locations at each of the five coal ash sample sites were analyzed for inductively coupled plasma (ICP) metals (antimony, arsenic, barium, beryllium, boron, cadmium, chromium [total], cobalt, copper, lead, manganese, molybdenum, nickel, selenium, silver, thallium, vanadium, and zinc) and mercury. A subset of samples were analyzed for total uranium, and polycyclic aromatic hydrocarbons (PAHs) (acenaththene, acenaphthylene, anthracene, benzo(a)anthracene, benzo(a)pyrene, benzo(b)fluoranthene, benzo(ghi)perylene, benzo(k)fluoranthene, chrysene, dibenz[a, $h]$ anthracene, fluoranthene, fluorene, indeno(1,2,3-cd)pyrene, naphthalene, phenanthrene, and pyrene) by U.S. Environmental Protection Agency SW-846 method 8270.

A subset of coal ash samples underwent serial batch leach testing with $\mathrm{pH} 5.2$ water (three dilutions with one replicate) with analysis of the subsequent leachate for ICP metals, mercury, and uranium (total). Each of the coal ash samples (solid) used for batch leaching testing was analyzed in quadruplicate for ICP metals and mercury.

A separate subset of coal ash samples underwent additional serial batch leach testing with non-pH-adjusted water (three dilutions with one replicate) with analysis of the subsequent leachate for ICP metals only. Each of the coal ash samples (solid) used for batch leaching testing was analyzed in quadruplicate for ICP metals.

\section{G.1.1 Major Deficiencies}

Major deficiencies are discussed by the following sample delivery group (SDG). If no comments are made about a specific analysis it should be assumed that no deficiencies in the quality of 
the data were found. Unless otherwise noted, deficiencies listed below are specific to the individual SDG and apply to all samples within that SDG.

\section{G.1.2 Minor Deficiencies}

Minor deficiencies are discussed by SDG as follows. If no comments are made about a specific analysis it should be assumed that no deficiencies in the quality of the data were found. Unless otherwise noted, deficiencies listed below are specific to the individual SDG and apply to all samples within that SDG.

\section{G.2 126-B-1 COAL ASH SAMPLES}

Coal ash samples collected at 126-B-1 were provided by the laboratories in three SDGs: SDG K3346, SDG K3365, and SDG K3599. SDG K3346 was submitted for third-party validation.

\section{G.2.1 SDG K3346}

This SDG comprises 10 field samples (J1HHW9, J1HHX0, J1HHX1, J1HHX4 through J1HHX8, $\mathrm{J} 1 \mathrm{HJ} 08$, and $\mathrm{J} 1 \mathrm{HJ} 09$ ) collected from the 126-B-1 coal ash site.

\section{G.2.2 SDG K3346, Coal Ash Samples}

Minor deficiencies noted in SDG K3346 are as follows:

- In the ICP metals analysis, the matrix spike (MS) recoveries for antimony (31\%) are outside the quality control (QC) limits of $70 \%$ to $130 \%$ (DOE-RL 2011a). Third-party validation qualified all antimony results in SDG K3346 with "J" flags as estimated data. Estimated data are usable for decision-making purposes. Due to method blank contamination, UJ flags were added to antimony results for samples J1HJ08, J1HHW9, J1HHX1, J1HHX4, J1HHX5, and $\mathrm{J} 1 \mathrm{HHX7}$.

- There are no major or minor deficiencies for uranium by kinetic phosphorescence analysis (KPA).

- In the semivolatile organic compound (SVOC) analysis for PAHs, sample J1HHX8 had a single surrogate recovery just below the laboratory QC acceptance criteria. Other acid surrogates were relatively low but were within acceptance criteria. Since only a single surrogate was outside of control limits, no qualifiers were added to the data by third-party validation.

- In the SVOC analysis for PAHs (J1HJ08, J1HJ09, J1HHX0, and J1HHX8), the laboratory control sample (LCS) for naphthalene and matrix spike duplicate (MSD) recoveries for benzo(g,h,i)perylene and indeno(1,2,3-cd)pyrene were within the specified lab control limits but were below the control limits specified in DOE-RL (2011a) (70\% to 130\%) in the range of $56 \%$ to $68 \%$. Results for these PAHs detected above the practical quantitation limit (PQL) were not qualified as estimated in the laboratory or validation report, but may be considered estimated. Estimated data are usable for decision-making purposes. 
The relative percent difference (RPD) values for the laboratory duplicate were evaluated. The laboratory duplicate associated with SDG K3346 calculated for metals and SVOC analysis were all within the acceptance criteria. The data are usable for decision-making purposes.

\section{G.2.3 SDG K3346, Coal Ash Batch Leach Solid and Leachate Samples}

In the ICP metals analysis of the coal ash quadruplicate solid samples (J1HJO8 and J1HJ09, $-1,-2,-3$, and -4 ), the MS recoveries for seven ICP metals (antimony, barium, chromium, copper, lead, manganese, and vanadium) are out of acceptance criteria. An MSD was not performed given these samples were quadruplicate analyses of the primary sample. For barium, chromium, copper, manganese, and vanadium, the spiking concentration was insignificant compared to the native concentration in the sample from which the MS was prepared. For these analytes, the deficiency in the MS is a reflection of the analytical variability of the native concentration rather than a measure of the recovery from the sample. To confirm quantitation, a post digestion spike (PDS) and serial dilution was prepared for all seven analytes with acceptable results. The analytes antimony and lead did not have mismatched spike and native concentrations in the original MS measured $35.7 \%$ and $69.1 \%$, respectively. These data may be considered estimated. Estimated data are usable for decision-making purposes.

There are no major or minor deficiencies for ICP metals or KPA analyses of leachate samples (J1HJ08-A1, -B1, -B2, and -C1 and J1HJ09 -A1, -B1, -C1, and -C2).

\section{G.2.4 SDG K3346, Coal Ash Batch Leach Solid and Leachate Samples - Follow-On Analysis with Non-pH-Adjusted Leachate}

In the ICP metals analysis of the coal ash quadruplicate solid samples (J1HHX8 $-1,-2,-3$, and -4 , and $\mathrm{J} 1 \mathrm{HHX} 8$ and $\mathrm{J} 1 \mathrm{HJ} 08,-5,-6,-7$, and -8 ), the MS recoveries for five ICP metals (antimony, barium, lead, selenium, and thallium) are out of acceptance criteria. An MSD was not performed given these samples were quadruplicate analyses of the primary sample. For barium, the spiking concentration was insignificant compared to the native concentration in the sample from which the MS was prepared and the deficiency in the MS is a reflection of the analytical variability of the native concentration rather than a measure of the recovery from the sample. To confirm quantitation, a PDS and serial dilutions were prepared for all five analytes with acceptable results. The analytes antimony, lead, selenium, and thallium did not have mismatched spike and native concentrations in the original MS. The original MS recoveries measured $27.1 \%, 64.4 \%, 68.4 \%$, and $61.1 \%$, respectively. These data may be considered estimated. Estimated data are usable for decision-making purposes.

ICP metals analysis of leachate samples (J1HHX8-A1, -B1, -C1, -C2, -D1, -E1, -E2, and -F1 and J1HJ08 -D1, -D2, -E1, and -F1) - no major or minor deficiencies.

\section{G.2.5 SDG K3365}

This SDG comprises 18 field samples (J1HJ00, J1HJ03 through J1HJ05, J1HJ10, J1HHV4 through J1HHV9, J1HHW0, J1HHW1, J1HHW3 through J1HHW6, and J1HHW8) collected from the 126-B-1 coal ash site. 


\section{G.2.6 SDG K3365, Coal Ash Samples}

Minor deficiencies noted in SDG K3365 are as follows:

- In the ICP metals analysis, the MS recovery for antimony was outside the QC limits. To confirm quantitation, a PDS and serial dilution was prepared with acceptable recovery for antimony $(98.7 \%)$. The original MS recovery for antimony was $25 \%$. The results for antimony were not qualified as estimated by the laboratory, but may be considered estimated. Estimated data are usable for decision-making purposes.

- There are no major or minor deficiencies for uranium by KPA.

- In the SVOC analysis for PAHs (J1HJ03, J1HJ05, J1HJ10, J1HHW3), the LCS result for naphthalene and MS recoveries for acenaphthalene, acenaphthylene, benzo(a)pyrene, benzo(g,h,i)perylene, dibenz[a,h]anthracene, fluorene, indeno(1,2,3-cd)pyrene, and naphthalene were within the specified lab control limits but were below the control limits specified in DOE-RL 2011 a $(70 \%$ to $130 \%)$ in the range of $62 \%$ to $68 \%$. All MSD results were within QC control limits. No other QC deficiencies were noted in the field samples. The detected results for these PAHs were qualified as estimated in the laboratory report. Estimated data are usable for decision-making purposes.

- The RPD values for the laboratory duplicate were evaluated. The laboratory duplicate associated with SDG K3346 calculated for metals analysis were all within the acceptance criteria. The data are usable for decision-making purposes.

\section{G.2.7 SDG K3599}

This SDG comprises seven field samples (J1HHX9, J1HJ01, J1HJ02, J1HHX2, J1HHX3, $\mathrm{J} 1 \mathrm{HHW} 2$, and J1HHW7) collected from the 126-B-1 coal ash site.

Minor deficiencies noted in SDG K3599 are as follows:

- In the ICP metals analysis, the MS recovery for antimony was outside the QC limits. To confirm quantitation, a PDS and serial dilution was prepared with acceptable recovery for antimony (105.1\%). The original MS recovery for antimony was $19 \%$. The results for antimony were not qualified as estimated by the laboratory, but may be considered estimated. Estimated data are usable for decision-making purposes.

- The laboratory duplicate associated with SDG K3599 indicated elevated RPDs for the analytes beryllium, chromium, cobalt, copper, lead, vanadium and zinc were above the acceptance criteria ranging from $31.7 \%$ to $37.1 \%$. The data are usable for decision-making purposes.

- There are no major or minor deficiencies for uranium by KPA.

\section{G.2.8 126-B-1 Field Quality Assurance/Quality Control}

Relative percent difference evaluations of main sample(s) versus the laboratory duplicate(s) were performed and reported by the laboratory. Any deficiencies in those calculations are reported by SDG in the previous sections. 
Field quality assurance (QA)/QC measures are used to assess potential sources of error and cross contamination of samples that could bias results. Field QAVQC samples from 126-B-1, listed in the field logbook (WCH 2011), are shown in Table G-1. The main and QA/QC sample results are presented in Appendix $\mathrm{C}$.

Table G-1. Field Quality Assurance/Quality Control Samples at 126-B-1.

\begin{tabular}{|c|c|c|}
\hline Sample Area & Main Sample & Duplicate Sample \\
\hline $100-\mathrm{B}-15$ & $\mathrm{~J} 1 \mathrm{HHW} 8$ & $\mathrm{~J} 1 \mathrm{HJ} 04$ \\
\hline $100-\mathrm{B}-25$ & $\mathrm{~J} 1 \mathrm{HHX} 8$ & $\mathrm{~J} 1 \mathrm{HJ} 03$ \\
\hline
\end{tabular}

Field duplicate samples are collected to provide a relative measure of the degree of local heterogeneity in the sampling medium, unlike laboratory duplicates that are used to evaluate precision in the analytical process. The field duplicates are evaluated by computing the RPD of the sample/duplicate pair(s) for each contaminant of potential concern (COPC). Relative percent differences are not calculated for analytes that are not detected in both the main and duplicate sample at more than five times the target detection limit. Relative percent differences of analytes detected at low concentrations (less than five times the detection limit) are not considered to be indicative of the analytical system performance. The calculation brief in Appendix D provides details on duplicate pair evaluation and RPD calculation.

The RPDs for zinc (57.5\%) in the duplicate sample from sample location 100-B-15 and for arsenic $(61.4 \%)$, barium (44.1\%), beryllium (45.4\%), and copper $(33.7 \%)$ in the duplicate from sample location 100-B-25 are above the acceptance criteria of $30 \%$. A secondary check of the data variability is used when one or both of the samples being evaluated (main and duplicate) is less than five times the target detection limit (TDL), including undetected analytes. In these cases, a control limit of \pm 2 times the TDL is used (Appendix D) to indicate that a visual check of the data is required by the reviewer. For the 100-B-25 primary and duplicate (J1HHX8 and $\mathrm{J} 1 \mathrm{HJ} 03$ ), this review was indicated. The results show a difference of more than two times the TDL. The review determined that the primary and duplicate samples had been split into separate analytical batches. Therefore, the difference in the primary and duplicate results between $\mathrm{J} 1 \mathrm{HHX} 8$ and $\mathrm{J} 1 \mathrm{HJO} 3$ are at least partially attributable to the separate batch analyses in addition to the natural heterogeneities inherent to environmental samples. The data are usable for decision-making purposes.

A visual inspection of all of the data was also performed. No additional major or minor deficiencies were noted. The data are usable for decision-making purposes.

\section{G.3 126-D-1 COAL ASH SAMPLES}

Coal ash sample data collected at 126-D-1 were provided by the laboratories in two SDGs: SDG K3572 and SDG K3600. SDG K3572 was submitted for third-party validation. 


\section{G.3.1 SDG K3572}

There are two, separate analytical batches contained in SDG K3572 with a total of 15 field samples collected from the 126-D-1 coal ash site. One batch was analyzed for ICP metals and mercury with select samples analyzed for PAHs and KPA (J1J3W3, J1J3X0 through J1J3X2, $\mathrm{J} 1 \mathrm{~J} 3 \times 4, \mathrm{~J} 1 \mathrm{~J} 3 \times 7, \mathrm{~J} 1 \mathrm{~J} 400, \mathrm{~J} 1 \mathrm{~J} 401, \mathrm{~J} 1 \mathrm{~J} 408, \mathrm{~J} 1 \mathrm{~J} 411)$. The other analytical batch (J1J412, $\mathrm{J} 1 \mathrm{~J} 414$, and $\mathrm{J} 1 \mathrm{~J} 442$ through $\mathrm{J} 1 \mathrm{~J} 444$ ) included these analyses as well as subsequent batch leach testing on appropriate samples.

\section{G.3.2 SDG K3572, Coal Ash and Coal Ash Batch Leach Samples (Pre-Leach Quadruplicate Analysis)}

Minor deficiencies noted in SDG K3572 are as follows:

- In the ICP metals analysis, the MS recoveries for antimony (30.2\%), boron $(64.2 \%)$, lead $(69.1 \%)$, thallium $(66.6 \%)$, and zinc $(23.8 \%)$ associated with $\mathrm{J} 1 \mathrm{~J} 3 \mathrm{~W} 3, \mathrm{~J} 1 \mathrm{~J} 3 \mathrm{X} 0$ through $\mathrm{J} 1 \mathrm{~J} 3 \times 2, \mathrm{~J} 1 \mathrm{~J} 3 \times 4, \mathrm{~J} 1 \mathrm{~J} 3 \times 7, \mathrm{~J} 1 \mathrm{~J} 400, \mathrm{~J} 1 \mathrm{~J} 401, \mathrm{~J} 1 \mathrm{~J} 408$, and $\mathrm{J} 1 \mathrm{~J} 411$ were outside the QC limits. Third-party validation qualified all subject analyte results with "J" flags as estimated data. MS recoveries for barium (-86.6\%) and manganese $(-120 \%)$ are also outside of QC limits. For barium, boron, manganese, and zinc, the spiking concentration was insignificant compared to the native concentration in the sample from which the MS was prepared and the deficiency in the MS is a reflection of the analytical variability of the native concentration rather than a measure of the recovery from the sample. To confirm quantitation, a PDS and serial dilution was prepared for with acceptable recovery for all subject analytes. The data are usable for decision-making purposes.

- The MS recoveries for antimony (21.3\%), boron (65.8\%), lead (57.6\%), manganese (53.1\%), thallium (64\%), and zinc (66.1\%) associated with $\mathrm{J} 1 \mathrm{~J} 412$, J1J414, and $\mathrm{J} 1 \mathrm{~J} 442$ through J1J444 were outside the QC limits. For barium, boron, lead, manganese, and zinc, the spiking concentration was insignificant compared to the native concentration in the sample from which the MS was prepared and the deficiency in the MS is a reflection of the analytical variability of the native concentration rather than a measure of the recovery from the sample. These MS recovery results are also associated with coal ash quadruplicate solid samples $\mathrm{J} 1 \mathrm{~J} 442$ through J1J444, $-1,-2,-3$, and -4 . These samples are part of the batch leaching analysis but were not subject to validation. To confirm quantitation, a PDS and serial dilution was prepared with acceptable recovery for all subject analytes. Third-party validation qualified all subject analyte results for samples J1J412, J1J414, and J1J442 through J1J444 with "J" flags as estimated data. The subject results for samples J1 J442 through $\mathrm{J} 1 \mathrm{~J} 444,-1,-2,-3$, and -4 were not qualified as estimated by the laboratory, but may be considered estimated. The data are usable for decision-making purposes.

- The LCS recovery for antimony (69.9\%) in the LCS associated with J1J412, J1J414, and $\mathrm{J} 1 \mathrm{~J} 442$ through $\mathrm{J} 1 \mathrm{~J} 444$ was outside of control limits and was qualified by third-party validation with a "J" flag as estimated data. Estimated data are usable for decision-making purposes.

- The RPD values for the laboratory duplicate in each analytical batch within SDG K3572 were evaluated. The laboratory duplicate associated with samples J1J3W3, J1J3X0 through J1J3X2, J1J3X4, J1J3X7, J1J400, J1J401, J1J408, and J1J411 calculated for metals showed zinc was outside of $Q C$ limits at $42 \%$, therefore, the associated zinc results 
were qualified by third-party validation with a "J" flag as estimated data. The data are usable for decision-making purposes.

- The laboratory duplicate associated with $\mathrm{J} 1 \mathrm{~J} 3 \mathrm{~W} 3, \mathrm{~J} 1 \mathrm{~J} 3 X 0$ through $\mathrm{J} 1 \mathrm{~J} 3 \times 2, \mathrm{~J} 1 \mathrm{~J} 3 X 4$, $\mathrm{J} 1 \mathrm{~J} 3 \times 7, \mathrm{~J} 1 \mathrm{~J} 400, \mathrm{~J} 1 \mathrm{~J} 401, \mathrm{~J} 1 \mathrm{~J} 408$, and $\mathrm{J} 1 \mathrm{~J} 411$ indicated an elevated RPD for zinc (32\%) slightly above the acceptance criteria. The associated zinc results were qualified by third-party validation with a "J" flag as estimated data and are usable for decision-making purposes. All other laboratory duplicate results were acceptable.

- There are no major or minor deficiencies for uranium by KPA.

- In the SVOC analysis for PAHs (J1J3X0, J1J408, and J1J401), MS recoveries for benzo(a)pyrene, benzo(g,h,i)perylene, indeno(1,2,3-cd)pyrene, and naphthalene were below the control limits specified in DOE-RL 2011 a (70\% to $130 \%)$ in the range of $44 \%$ to $49 \%$. Results for those PAHs detected above the PQL were not qualified as estimated in the laboratory or validation report, but may be considered estimated. Estimated data are usable for decision-making purposes.

- Samples J1J412, J1J414, and J1J442 through J1J444 were originally extracted with the hold time for SVOC analysis but the LCS was inadvertently not spiked. Therefore, all five samples were reextracted within two times the hold time. All results associated with these samples were qualified by third-party validation with a "J" flag as estimated data and are usable for decision-making purposes. Estimated data are usable for decision-making purposes.

- In the SVOC analysis for PAHs, sample J1J444 had a single surrogate recovery below the QC acceptance criteria. Other surrogates were also low but within acceptance criteria. Since only a single surrogate was outside of control limits, no qualifiers were added to the data by third-party validation.

- In the SVOC analysis for PAHs associated with J1J412, J1J414, and J1J442 through $\mathrm{J} 1 \mathrm{~J} 444$, the MS recoveries for acenaphthene, acenaphthylene, anthracene, benzo(a)pyrene, benzo(g,h,i)perylene, benzo(k)fluoranthene, chrysene, dibenz(a,h)anthracene, indeno(1,2,3-cd)pyrene, naphthalene, and pyrene were within the specified lab control limits but were below the control limits specified in DOE-RL (2011a) $(70 \%$ to $130 \%)$, in the range of $55 \%$ to $67 \%$. The MSD recoveries were within control limits with the exception of benzo(g,h,i)perylene $(66 \%)$ and naphthalene $(68 \%)$. Results for those PAHs may be considered estimated. Estimated data are usable for decision-making purposes.

- The laboratory duplicate associated with $\mathrm{J} 1 \mathrm{~J} 412$, J1J414, and J1J442 through J1J444 indicated an elevated RPD for benzo(k)fluoranthene (32\%) slightly above the acceptance criteria. The associated benzo(k)fluoranthene results were qualified by third-party validation with a "J" flag as estimated data. Estimated data are usable for decision-making purposes. All other laboratory duplicate results were acceptable. 


\section{G.3.3 SDG K3572, Coal Ash Batch Leach Leachate Samples}

In the ICP metals analysis of the coal leachate samples (J1J442-A1, -A2, -B1, and -C1, $\mathrm{J} 1 \mathrm{~J} 443-\mathrm{A} 1,-\mathrm{B} 1,-\mathrm{B} 2$, and $-\mathrm{C} 1$, and $\mathrm{J} 1 \mathrm{~J} 444-\mathrm{A} 1,-\mathrm{B} 1,-\mathrm{C} 1$, and $-\mathrm{C} 2$ ), the MS recoveries for barium ( $8.35 \%)$ and silver $(43.2 \%)$ are out of acceptance criteria. An MSD was not performed given these samples were quadruplicate analyses of the primary sample. Results for both barium and silver in the leachate and originating solid sample are consistent with results from other coal ash samples. Therefore, it is possible that the low recoveries are due to analytical variability of the native concentration rather than a measure of the recovery from the sample. These data may be considered estimated. Estimated data are usable for decision-making purposes.

There are no major or minor deficiencies for uranium by KPA.

\section{G.3.4 SDG K3600, Coal Ash Samples}

SDG K3600 comprises 20 field samples (J1J3W4 through J1J3W9, J1J3X3, J1J3X5, J1J3X6, $\mathrm{J} 1 \mathrm{~J} 3 \times 8, \mathrm{~J} 1 \mathrm{~J} 3 \times 9, \mathrm{~J} 1 \mathrm{~J} 402$ through $\mathrm{J} 1 \mathrm{~J} 407, \mathrm{~J} 1 \mathrm{~J} 409, \mathrm{~J} 1 \mathrm{~J} 410$, and $\mathrm{J} 1 \mathrm{~J} 413$ ) collected from the $126-\mathrm{D}-1$ coal ash site.

Minor deficiencies noted in SDG K3600 are as follows:

In the ICP metals analysis, the MS recoveries for antimony (21.1\%), barium $(55.0 \%)$, boron (56.1\%), copper $(60.4 \%)$, mercury $(139 \%)$, and thallium $(69.7 \%)$ were outside the QC limits. For barium, boron, and copper, the spiking concentration was insignificant compared to the native concentration in the sample from which the MS was prepared and the deficiency in the MS is a reflection of the analytical variability of the native concentration rather than a measure of the recovery from the sample. To confirm quantitation, a PDS and serial dilution was prepared for with acceptable recovery for all subject analytes. These data may be considered estimated. Estimated data are usable for decision-making purposes.

\section{G.4 126-D-1 FIELD QUALITY ASSURANCE/QUALITY CONTROL}

Relative percent difference evaluations of main sample(s) versus the laboratory duplicate(s) were performed and reported by the laboratory. Any deficiencies in those calculations are reported by SDG in the previous sections.

Field QAVQC measures are used to assess potential sources of error and cross contamination of samples that could bias results. Field QAVQC samples from 126-D-1, listed in the field logbook (WCH 2011), are shown in Table G-2. The main and QA/QC sample results are presented in Appendix C.

Table G-2. Field Quality Assurance/Quality Control Samples at 126-D-1.

\begin{tabular}{|c|c|c|}
\hline Sample Area & Main Sample & Duplicate Sample \\
\hline 100-D-17 & $\mathrm{J} 1 \mathrm{~J} 3 \mathrm{X} 9$ & $\mathrm{~J} 1 \mathrm{~J} 413$ \\
\hline 100-D-26 & $\mathrm{J} 1 \mathrm{~J} 408$ & $\mathrm{~J} 1 \mathrm{~J} 412$ \\
\hline
\end{tabular}


Field duplicate samples are collected to provide a relative measure of the degree of local heterogeneity in the sampling medium, unlike laboratory duplicates that are used to evaluate precision in the analytical process. The field duplicates are evaluated by computing the RPD of the sample/duplicate pair(s) for each COPC. Relative percent differences are not calculated for analytes that are not detected in both the main and duplicate sample at more than five times the target detection limit. Relative percent differences of analytes detected at low concentrations (less than five times the detection limit) are not considered to be indicative of the analytical system performance. The calculation brief in Appendix $D$ provides details on duplicate pair evaluation and RPD calculation.

The RPDs for boron (30.4\%) in the duplicate sample from sample location 100-D-17 and for zinc $(40.4 \%)$ in the duplicate from sample location 100-D-26 are above the acceptance criteria of $30 \%$. Elevated RPDs in environmental samples are generally attributed to natural heterogeneity in the sample matrix. The data are usable for decision-making purposes.

A secondary check of the data variability is used when one or both of the samples being evaluated (main and duplicate) is less than five times the TDL, including undetected analytes. In these cases, a control limit of \pm 2 times the TDL is used (Appendix D) to indicate that a visual check of the data is required by the reviewer. No data required this check. A visual inspection of all of the data is also performed. No additional major or minor deficiencies are noted. The data are usable for decision-making purposes.

\section{G.5 126-H-1 COAL ASH SAMPLES}

Coal ash sample data collected at $126-\mathrm{H}-1$ were provided by the laboratories in two SDGs: SDG K3632 and SDG K3649. SDG K3632 was submitted for third-party validation.

\section{G.5.1 SDG K3632}

This SDG comprises 16 field samples (J1HJ63 through J1HJ67 and J1HJ72 through J1HJ82) collected from the $126-\mathrm{H}-1$ coal ash site.

\section{G.5.2 SDG K3632, Coal Ash Samples}

Minor deficiencies noted in SDG K3632 are as follows:

- In the ICP metals analysis, the MS recoveries for antimony (32.3\%), barium (354\%), boron $(212 \%)$, lead $(63.5 \%)$, manganese $(51.1 \%)$, mercury $(131 \%)$, nickel $(65.3 \%)$, and thallium $(62.6 \%)$ are outside the QC limits of 70 to $130 \%$ (DOE-RL, 2011a). Third-party validation qualified associated results for antimony, lead, manganese, mercury, nickel, and thallium in SDG K3632 with "J" flags as estimated data. For barium, boron, and manganese, the spiking concentration was insignificant compared to the native concentration in the sample from which the MS was prepared and the deficiency in the MS is a reflection of the analytical variability of the native concentration rather than a measure of the recovery from the sample. To confirm quantitation, a PDS and serial dilution was prepared for all abovementioned analytes with acceptable recovery reported. Associated data not qualified with "J" flags by third-party validation may be considered estimated. Estimated data are usable for decision-making purposes. 
- Due to method blank contamination, UJ flags were added to the zinc result in the equipment blank, J1 JH79 by third-party validation.

- There are no major or minor deficiencies for uranium by KPA.

- In the SVOC analysis for PAH, sample J1HJ72 had a single surrogate recovery just below the QC acceptance criteria. Other acid surrogates were relatively low but were within acceptance criteria. Since only a single surrogate was outside of control limits, no qualifiers were added to the data by third-party validation. Sample J1HJ77 had three surrogate recoveries slightly below the QC acceptance criteria, however, these surrogates are associated with halogenated organics and are not applicable to PAH results. The data are usable for decision-making purposes.

- The RPD values for the laboratory duplicate were evaluated. The laboratory duplicate associated with SDG K3632 calculated for metals and SVOC analysis were all within the acceptance criteria. The data are usable for decision-making purposes.

\section{G.5.3 SDG K3632, Coal Ash Batch Leach Solid and Leachate Samples}

In the ICP metals analysis of the coal ash quadruplicate solid samples (J1HJ80 through J1HJ82, $-1,-2,-3$, and -4 ), the MS recoveries for antimony (29.7\%), barium (164\%), copper $(144 \%)$, and manganese $(68.0 \%)$ are out of acceptance criteria. An MSD was not performed given these samples were quadruplicate analyses of the primary sample. For barium, copper, and manganese, the spiking concentration was insignificant compared to the native concentration in the sample from which the MS was prepared. For these analytes, the deficiency in the MS is a reflection of the analytical variability of the native concentration rather than a measure of the recovery from the sample. To confirm quantitation, a PDS and serial dilution were prepared for all seven analytes with acceptable results. These data may be considered estimated. Estimated data are usable for decision-making purposes.

There are no major or minor deficiencies for ICP metals analysis of leachate samples (J1HJ80-A1, -B1, -B2, and -C1, J1HJ81 -A1, -B1, -C1, and -C2, and J1HJ82 -A1, -A2, -B1, and $-\mathrm{C} 1)$.

There are no major or minor deficiencies for uranium analysis of leachate samples by KPA.

\section{G.5.4 SDG K3632, Coal Ash Batch Leach Solid and Leachate Samples - Follow-On Analysis With Non-pH-Adjusted Leachate (Sample J1HJ80)}

In the ICP metals analysis of the coal ash quadruplicate solid sample J1HJ80 -5, $-6,-7$, and -8 , the MS recoveries for antimony $(25.1 \%)$, barium $(-44.3 \%)$, copper $(194 \%)$, manganese $(60.8 \%)$ silver $(69.3 \%)$, and thallium (66.4\%) were outside the QC limits. For barium, copper, and manganese, the spiking concentration was insignificant compared to the native concentration in the sample from which the MS was prepared and the deficiency in the MS is a reflection of the analytical variability of the native concentration rather than a measure of the recovery from the sample. To confirm quantitation, a PDS and serial dilution was prepared for with acceptable recovery for all subject analytes. These data may be considered estimated and are usable for decision-making purposes. 
ICP metals analysis of leachate samples J1HJ80-D1, -E1, -E2, and -F1 (non-pH-adjusted leachate), no major or minor deficiencies are noted.

\section{G.5.5 SDG K3649}

This SDG comprises 19 field samples (J1HJ48 through J1HJ62 and J1HJ68 through J1HJ71) collected from the $126-\mathrm{H}-1$ coal ash site.

\section{G.5.6 SDG K3649, Coal Ash Samples}

Minor deficiencies noted in SDG K3649 are as follows:

- In the ICP metals analysis, the MS recovery for antimony (28.4\%) is outside the QC limits of $70 \%$ to $130 \%$ (DOE-RL 2011a). To confirm quantitation, a PDS and serial dilution was prepared for antimony with an acceptable result. The antimony data may be considered estimated. Estimated data are usable for decision-making purposes.

- The RPD values for the laboratory duplicate were evaluated. The laboratory duplicate associated with SDG K3649 calculated for metals analysis were all within the acceptance criteria. The data are usable for decision-making purposes.

\section{G.6 126-H-1 FIELD QUALITY ASSURANCE/QUALITY CONTROL}

Relative percent difference evaluations of main sample(s) versus the laboratory duplicate(s) were performed and reported by the laboratory. Any deficiencies in those calculations are reported by SDG in the previous sections.

Field QA/QC measures are used to assess potential sources of error and cross contamination of samples that could bias results. Field QA/QC samples from $126-\mathrm{H}-1$, listed in the field logbook (WCH 2011), are shown in Table G-3. The main and QA/QC sample results are presented in Appendix C.

Table G-3. Field Quality Assurance/Quality Control Samples at 126-H-1.

\begin{tabular}{|c|c|c|}
\hline Sample Area & Main Sample & Duplicate Sample \\
\hline $100-\mathrm{H}-16$ & $\mathrm{~J} 1 \mathrm{HJ} 63$ & $\mathrm{~J} 1 \mathrm{HJ} 78$ \\
\hline $100-\mathrm{H}-26$ & $\mathrm{~J} 1 \mathrm{HJ} 72$ & $\mathrm{~J} 1 \mathrm{HJ} 77$ \\
\hline
\end{tabular}

Field duplicate samples are collected to provide a relative measure of the degree of local heterogeneity in the sampling medium, unlike laboratory duplicates that are used to evaluate precision in the analytical process. The field duplicates are evaluated by computing the RPD of the sample/duplicate pair(s) for each COPC. Relative percent differences are not calculated for analytes that are not detected in both the main and duplicate sample at more than five times the target detection limit. Relative percent differences of analytes detected at low concentrations (less than five times the detection limit) are not considered to be indicative of the analytical 
system performance. The calculation brief in Appendix D provides details on duplicate pair evaluation and RPD calculation.

The RPD for zinc (38.8\%) in the duplicate from sample location $100-\mathrm{H}-25$ is above the acceptance criteria of $30 \%$. Elevated RPDs in environmental samples are generally attributed to natural heterogeneity in the sample matrix. The data are usable for decision-making purposes.

A secondary check of the data variability is used when one or both of the samples being evaluated (main and duplicate) is less than five times the TDL, including undetected analytes. In these cases, a control limit of \pm 2 times the TDL is used (Appendix X) to indicate that a visual check of the data is required by the reviewer. No data required this check. A visual inspection of all of the data is also performed. No additional major or minor deficiencies are noted. The data are usable for decision-making purposes.

\section{G.7 300 AREA COAL ASH SAMPLES}

Coal ash sample data collected at the 300 Area coal ash sampling site were provided by the laboratories in two SDGs: SDG K3648 and SDG K3669. Both SDGs were submitted for third-party validation.

\section{G.7.1 SDG K3648}

This SDG comprises 20 field samples (J1HJP1 through J1HJP9, J1HJR0 through J1HJR9, and $\mathrm{J} 1 \mathrm{HJTO}$ ) collected from the 300 Area coal ash sampling site.

Minor deficiencies noted in SDG K3648 are as follows:

- In the ICP metals analysis, the MS recoveries for antimony (28.0\%), barium (186\%), manganese $(141 \%)$, mercury $(252 \%)$, and zinc $(46.4 \%)$ are outside the QC limits of $70 \%$ to $130 \%$ (DOE-RL 2011a). Third-party validation qualified associated results for antimony, barium, manganese, and zinc with "J" flags as estimated data. For barium, manganese, mercury, and zinc, the spiking concentration was insignificant compared to the native concentration in the sample from which the MS was prepared and the deficiency in the MS is a reflection of the analytical variability of the native concentration rather than a measure of the recovery from the sample. To confirm quantitation, a PDS and serial dilution was prepared for all abovementioned analytes with acceptable recovery reported for all. Associated data not qualified with "J" flags by third-party validation (mercury) may be considered estimated and are usable for decision-making purposes.

- The laboratory duplicate indicated elevated RPDs for nickel (53.9\%) and zinc (33.7\%) above the acceptance criteria. The associated zinc results were both greater than five times the TDL and were qualified by third-party validation with a "J" flag as estimated data. Results for nickel are both less than five times the associated TDL. Elevated RPDs in environmental samples are generally attributed to natural heterogeneity in the sample matrix. These data are usable for decision-making purposes. All other laboratory duplicate results were acceptable. 


\section{G.7.2 SDG K3669, Coal Ash and Coal Ash Batch Leach Samples}

This SDG comprises 15 field samples (J1HJN6 through J1HJN9, J1HJP0, J1HJT1 through J1HJT7, and J1HJV0 through J1HJV2) collected from the 300 Area coal ash sampling site.

Minor deficiencies noted in SDG K3669 are as follows:

- In the ICP metals analysis, the MS recoveries for antimony (27.9\%) and zinc (68.4\%) were outside the QC limits. Third-party validation qualified all subject analyte results with "J" flags as estimated data. For zinc, the spiking concentration was insignificant compared to the native concentration in the sample from which the MS was prepared and the deficiency in the MS is a reflection of the analytical variability of the native concentration rather than a measure of the recovery from the sample. To confirm quantitation, a PDS and serial dilution was prepared for antimony with acceptable recovery. The data are usable for decision-making purposes.

- Due to method blank contamination, UJ flags were added to lead and zinc results in the equipment blank sample J1HJT7.

- Data validation noted the detection of selenium in the laboratory QC blank and, therefore, applied $U J$ flags to selenium data less than five times the blank result (all samples except J1HJT2, J1HJT4, and J1HJV0). However, the preponderance of other coal ash data in this characterization study clearly indicates that low levels of selenium are present in the substrate. Therefore, the project regarded the associated selenium data as detections with a " $\mathrm{J}$ " flag as estimated data and reported these data as detections. The subject selenium data are usable for decision-making purposes.

- There are no major or minor deficiencies for uranium by KPA.

- In the SVOC analysis for PAH, sample J1HJV2 had a single surrogate recovery below the $\mathrm{QC}$ acceptance criteria. Other surrogates were relatively low but were within acceptance criteria. Since only a single surrogate was outside of control limits, no qualifiers were added to the data by third-party validation. The data are usable for decision-making purposes.

\section{G.7.3 SDG K3669, Coal Ash Batch Leach Solid and Leachate Samples}

In the ICP metals analysis of the coal ash quadruplicate solid samples (J1HJV0 through J1HJV2, $-1,-2,-3$, and -4$)$, the MS recoveries for antimony (22.8\%), barium (145\%), mercury (294\%), and thallium (69.5\%) are out of acceptance criteria. An MSD was not performed given these samples were quadruplicate analyses of the primary sample. For barium and mercury, the spiking concentration was insignificant compared to the native concentration in the sample from which the MS was prepared. For these analytes, the deficiency in the MS is a reflection of the analytical variability of the native concentration rather than a measure of the recovery from the sample. To confirm quantitation, a PDS and serial dilution was prepared for all four analytes with acceptable results. These data may be considered estimated. Estimated data are usable for decision-making purposes.

There are no major or minor deficiencies for ICP metals or KPA analyses of leachate samples (J1HJV0 -A1, -A2, -B1, and -C1, J1HJV1 -A1, -B1, -B2, and -C1, and J1HJV2 -A1, -B1, -C1, and $-\mathrm{C} 2)$. 


\section{G.8 300 AREA ASH FIELD QUALITY ASSURANCE/QUALITY CONTROL}

Relative percent difference evaluations of main sample(s) versus the laboratory duplicate(s) were performed and reported by the laboratory. Any deficiencies in those calculations are reported by SDG in the previous sections.

Field QA/QC measures are used to assess potential sources of error and cross contamination of samples that could bias results. Field QA/QC samples from 300 Area ash, listed in the field logbook (WCH 2011), are shown in Table G-4. The main and QA/QC sample results are presented in Appendix C.

Table G-4. Field Quality Assurance/Quality Control Samples at 300 Area Ash.

\begin{tabular}{|c|c|c|}
\hline Sample Area & Main Sample & Duplicate Sample \\
\hline $300-04$ & J1HJN9 & J1HJT5 \\
\hline $300-06$ & J1HJP1 & J1HJT6 \\
\hline
\end{tabular}

Field duplicate samples are collected to provide a relative measure of the degree of local heterogeneity in the sampling medium, unlike laboratory duplicates that are used to evaluate precision in the analytical process. The field duplicates are evaluated by computing the RPD of the sample/duplicate pair(s) for each COPC. Relative percent differences are not calculated for analytes that are not detected in both the main and duplicate sample at more than five times the target detection limit. Relative percent differences of analytes detected at low concentrations (less than five times the detection limit) are not considered to be indicative of the analytical system performance. The calculation brief in Appendix D provides details on duplicate pair evaluation and RPD calculation.

The RPD for zinc (59.4\%) in the duplicate from sample location 300-06 is above the acceptance criteria of $30 \%$. Elevated RPDs in environmental samples are generally attributed to natural heterogeneity in the sample matrix. The data are usable for decision-making purposes.

A secondary check of the data variability is used when one or both of the samples being evaluated (main and duplicate) is less than five times the TDL, including undetected analytes. In these cases, a control limit of \pm 2 times the TDL is used (Appendix D) to indicate that a visual check of the data is required by the reviewer. No data required this check. A visual inspection of all of the data is also performed. No additional major or minor deficiencies are noted. The data are usable for decision-making purposes.

\section{G.9 600-207 COAL ASH SAMPLES}

Coal ash sample data collected at 600-207 were provided by the laboratories in two SDGs: SDG K3702 and SDG K3704. SDG K3702 was submitted for third-party validation. 


\section{G.9.1 SDG K3702}

There are three, separate analytical batches contained in K3702 with a total of 17 field samples collected from the 600-207 coal ash site. One batch with 14 samples was analyzed for ICP metals and mercury with select samples analyzed for PAHs and KPA (samples J1HHM9, J1HHN7, J1HHT7, J1HHP3 through J1HHP9, and J1HHR0 through J1HHR3), a second batch included the same analyses for samples J1HHT8, J1HHT9, and J1HHV0, and the third batch of samples (J1HHT8, J1HHT9, and J1HHV0 -1, -2, -3, and -4) includes batch leach testing on appropriate the subject samples. Additional leach tests were performed on sample J1HHN7 with both $\mathrm{pH}$-adjusted 5.2 water as well unadjusted water.

\section{G.9.2 SDG K3702, Coal Ash Samples}

Minor deficiencies noted in SDG K3702 are as follows:

- In the ICP metals analysis associated with samples J1HHM9, J1HHN7, J1HHT7, J1HHP3 through J1HHP9, and J1HHR0 through J1HHR3, the MS recoveries for antimony $(40.9 \%)$, barium $(-18.8 \%)$, boron $(-0.300 \%)$, chromium $(61.1 \%)$, copper $(28.8 \%)$, manganese ($296 \%$ ), nickel $(66.5 \%)$, thallium (69.8\%), and vanadium $(66.3 \%)$ are outside the QC limits of $70 \%$ to $130 \%$ (DOE-RL 2011a). Third-party validation qualified associated results for antimony, boron, chromium, copper, nickel, thallium, and vanadium in SDG K3702 with "J" flags as estimated data. For barium, boron, chromium, copper, manganese, nickel, and vanadium the spiking concentration was insignificant compared to the native concentration in the sample from which the MS was prepared and the deficiency in the MS is a reflection of the analytical variability of the native concentration rather than a measure of the recovery from the sample. To confirm quantitation, a PDS and serial dilution was prepared for all abovementioned analytes with acceptable recovery reported for all analytes except for barium which was slightly less than QC limits at $68.8 \%$. Associated data not qualified with "J" flags by third-party validation (barium, manganese, and thallium) may be considered estimated and are usable for decision-making purposes.

- The laboratory duplicate associated with J1HHM9, J1HHN7, J1HHT7, J1HHP3 through J1HHP9, and J1HHR0 through J1HHR3 indicated an elevated RPD for barium (35.2\%), boron $(83.7 \%)$, copper $(70.6 \%)$, manganese $(35.4 \%)$, molybdenum $(35.0 \%)$, and nickel $(30.1 \%)$ above the acceptance criteria. The associated barium, boron, copper, and manganese results were qualified by third-party validation with a "J" flag as estimated data and are usable for decision-making purposes. Associated data not qualified with " $\mathrm{J}$ " flags by validation (molybdenum and nickel) may be considered estimated and are usable for decision-making purposes. All other laboratory duplicate results were acceptable.

- In the ICP metals analysis associated with samples J1HHT8, J1HHT9, and J1HHV0, the MS recoveries for antimony $(35.5 \%)$, barium $(-78.4 \%)$, beryllium $(69.5 \%)$, chromium $(66.8 \%)$, copper $(41.1 \%)$, manganese $(-204 \%)$, and vanadium $(49.7 \%)$ are outside the QC limits of $70 \%$ to $130 \%$ (DOE-RL 2011a). Third-party validation qualified associated results for antimony, beryllium, chromium, copper, and vanadium in SDG K3702 with "J" flags as estimated data. For barium, beryllium, chromium, copper, manganese, and vanadium the spiking concentration was insignificant compared to the native concentration in the sample from which the MS was prepared and the deficiency in the MS is a reflection of the analytical variability of the native concentration rather than a measure of the recovery from the sample. To confirm quantitation, a PDS and serial dilution was prepared for all 
abovementioned analytes with acceptable recovery reported for all analytes. Associated data not qualified with "J" flags by third-party validation (manganese) may be considered estimated and are usable for decision-making purposes.

- The laboratory duplicate associated with J1HHT8, J1HHT9, and J1HHV0 indicated an elevated RPD for beryllium (41.7\%) above the acceptance criteria. The associated beryllium results were qualified by third-party validation with a "J" flag as estimated data and are usable for decision-making purposes. All other laboratory duplicate results were acceptable.

- There are no major or minor deficiencies for uranium by KPA.

- In the SVOC analysis for PAH associated with samples J1HHM9, J1HHN7, J1HHT7, $\mathrm{J} 1 \mathrm{HHP} 5$, and J1HHR2, the MS and MSD recoveries for benzo(a)anthracene $(63 \%$ and $65 \%$ ), benzo(a)pyrene ( $47 \%$ and $44 \%$ ), benzo(b)fluoranthene $(62 \%$ and $63 \%)$, benzo(ghi)perylene ( $34 \%$ and $32 \%)$, benzo(k)fluoranthene $(58 \%$ and $65 \%)$, chrysene $(65 \%$ and $68 \%)$, dibenz(a,h)anthracene ( $38 \%$ and $37 \%)$, fluoranthene $(69 \%$ in MS only), and indeno(1,2,3-cd)pyrene (36\% and 34\%), respectively, are outside the QC limits of $70 \%$ to $130 \%$ (DOE-RL 2011a). Third-party validation qualified associated results for benzo(a)pyrene, benzo(ghi)perylene, dibenz(a,h)anthracene, and indeno(1,2,3-cd)pyrene, with "J" flags as estimated data. The sample material from which the MS and MSD were prepared was J1HHM9. Analysis of J1HHM9, as well as both the MS and MSD, required $3 X$ dilutions due to matrix interference from the coal ash. Associated data not qualified with "J" flags by third-party validation (benzo(a)anthracene, benzo(b)fluoranthene, benzo(k)fluoranthene, chrysene, and fluoranthene) may be considered estimated and are usable for decision-making purposes.

- In the SVOC analysis for PAH associated with samples J1HHT8, J1HHT9, and J1HHV0, the MS and MSD recoveries for acenaphthylene (62\% in MSD only), anthracene (63\% in MSD only), benzo(a)anthracene (60\% in MSD only), benzo(a)pyrene (47\% and $36 \%$ ), benzo(b)fluoranthene $(68 \%$ and $57 \%$ ), benzo(ghi)perylene ( $35 \%$ and $25 \%$ ), benzo(k)fluoranthene $(65 \%$ and $55 \%)$, chrysene (63\% in MSD only), dibenz(a,h)anthracene (45\% and $32 \%$ ), fluoranthene (65\% in MSD only), indeno(1,2,3-cd)pyrene (41\% and $29 \%$ ), and phenanthrene (66\% in MSD only) respectively, are outside the QC limits of $70 \%$ to $130 \%$ (DOE-RL 2011a). Third-party validation qualified associated results for benzo(a)pyrene, benzo(ghi)perylene, dibenz(a,h)anthracene, and indeno(.1,2,3-cd)pyrene, with " $\mathrm{J}$ " flags as estimated data. The sample material from which the MS and MSD were prepared was $\mathrm{J} 1 \mathrm{HHT} 8$. Analysis of $\mathrm{J} 1 \mathrm{HHT} 8$, as well as both the MS and MSD, required a $3 X$ dilution in order to compensate for matrix interference from the coal ash. Therefore, the deficiency in the MS and MSD is attributable to the analytical variability from matrix interference rather than a measure of the recovery from the sample. Associated data not qualified with " $\mathrm{J}$ " flags by validation (acenaphthylene, anthracene, benzo(a)anthracene, benzo(b)fluoranthene, benzo(k)fluoranthene, chrysene, and fluoranthene) may be considered estimated and are usable for decision-making purposes.

- The laboratory duplicate for SVOC analysis associated with J1HHT8, J1HHT9, and J1HHV0 indicated an elevated RPD for benzo(ghi)perylene (33\%), dibenz(a,h)anthracene (33\%), and indeno( $1,2,3-\mathrm{cd})$ pyrene $(32 \%)$ above the acceptance criteria. The associated results were qualified by third-party validation with a "J" flag as estimated data and are usable for decision-making purposes. All other laboratory duplicate results were acceptable. 
WCH-506

Rev. 0

\section{G.9.3 SDG K3702, Coal Ash Batch Leach Solid and Leachate Samples}

In the ICP metals analysis of the coal ash quadruplicate solid samples J1HHT8, J1HHT9, and J1HHV0 $-1,-2,-3$, and -4 , MS recoveries associated with antimony $(30.8 \%)$, barium $(168 \%)$, and manganese (238\%) were outside the QC limits. For barium and manganese, the spiking concentration was insignificant compared to the native concentration in the sample from which the MS was prepared and the deficiency in the MS is a reflection of the analytical variability of the native concentration rather than a measure of the recovery from the sample. To confirm quantitation, a PDS and serial dilution was prepared with acceptable recovery for all subject analytes. The subject results were not qualified as estimated by the laboratory, but may be considered estimated. The data are usable for decision-making purposes.

The MS recoveries associated with leachate samples J1HJV0-A1, -A2, -B1, and -C1, J1HJV1 -A1, -B1, -B2, and -C1, and J1HJV2 -A1, -B1, -C1, and -C2 for antimony (59.2\%) and barium $(0.290 \%)$ were outside the QC limits. An MSD was not performed given these samples were quadruplicate analyses of the primary sample. Results for both barium and silver in the leachate and originating solid sample are consistent with results from other coal ash samples. Therefore, it is possible that the low recoveries are due to analytical variability of the native concentration rather than a measure of the recovery from the sample. These data may be considered estimated. Estimated data are usable for decision-making purposes.

There are no major or minor deficiencies for uranium analysis of leachate samples by KPA. .

\section{G.9.4 SDG K3702, Coal Ash Batch Leach Solid and Leachate Samples - Additional Leach Test Follow-On Analysis with pH-Adjusted and Non-pH-Adjusted Leachate for J1HHN7}

There are no major or minor deficiencies noted in the ICP metals analysis of the coal ash quadruplicate solid sample J1HHN7 $-1,-2,-3$, and -4 and J1HHN7 $-5,-6,-7$, and -8 .

There are no major or minor deficiencies noted in the ICP metals analysis of leachate samples J1HHN7-A1, -B1, -B2, -C1, (pH 5.2 leachate) and J1HHN7-D1, -E1, -F1, -F2 (non-pH-adjusted leachate).

\section{G.9.5 SDG K3704}

This SDG comprises 18 field samples (J1HHM3 through J1HHM8, J1HHN0 through J1HHN6, J1HHN8 through J1HHN9 and J1HHP0 through J1HHP2) collected from the 600-207 coal ash site.

Minor deficiencies noted in SDG K3704 are as follows:

In the ICP metals analysis, the MS recoveries for antimony (28.0\%), barium (38.3\%), beryllium $(68.0 \%)$, boron $(56.3 \%)$, chromium $(66.3 \%)$, cobalt $(66.2 \%)$, copper $(60.4 \%)$, lead $(64.9 \%)$, manganese $(-3.29 \%)$, molybdenum $(66.3 \%)$, nickel $(63.9 \%)$, thallium $(61.6 \%)$, and vanadium (58.9\%) are outside the QC limits of 70 to $130 \%$ (DOE-RL 2011a). For barium, beryllium, boron, chromium, copper, manganese, and vanadium, the spiking concentration was insignificant compared to the native concentration in the sample from which the MS was prepared and the deficiency in the MS is a reflection of the analytical variability of the native concentration rather than a measure of the recovery from the sample. To confirm quantitation, 
a PDS and serial dilution was prepared for all abovementioned analytes with acceptable recovery reported for all. Associated data may be considered estimated and are usable for decision-making purposes.

The laboratory duplicate indicated elevated RPDs for cobalt (34.8\%) and nickel (30.1\%) above the acceptance criteria. Results for cobalt and nickel are both less than five times the associated TDL. Elevated RPDs in environmental samples are generally attributed to natural heterogeneity in the sample matrix. These data are usable for decision-making purposes. All other laboratory duplicate results were acceptable.

\section{G.12 600-207 COAL ASH FIELD QUALITY ASSURANCE/QUALITY CONTROL}

Relative percent difference evaluations of main sample(s) versus the laboratory duplicate(s) were performed and reported by the laboratory. Any deficiencies in those calculations are reported by SDG in the previous sections.

Field QA/QC measures are used to assess potential sources of error and cross contamination of samples that could bias results. Field QA/QC samples from 600-207, listed in the field logbook (WCH 2011), are shown in Table G-5. The main and QA/QC sample results are presented in Appendix C.

Table G-5. Field Quality Assurance/Quality Control Samples at 600-207.

\begin{tabular}{|c|c|c|}
\hline Sample Area & Main Sample & Duplicate Sample \\
\hline $600-15$ & J1HHN7 & J1HHR2 \\
\hline $600-24$ & J1HHP6 & J1HHR3 \\
\hline
\end{tabular}

Field duplicate samples are collected to provide a relative measure of the degree of local heterogeneity in the sampling medium, unlike laboratory duplicates that are used to evaluate precision in the analytical process. The field duplicates are evaluated by computing the RPD of the sample/duplicate pair(s) for each COPC. Relative percent differences are not calculated for analytes that are not detected in both the main and duplicate sample at more than five times the target detection limit. Relative percent differences of analytes detected at low concentrations (less than five times the detection limit) are not considered to be indicative of the analytical system performance. The calculation brief in Appendix $D$ provides details on duplicate pair evaluation and RPD calculation.

The RPDs for boron (36.5\%), lead (38.7\%), and vanadium $(59.4 \%)$ in the duplicate from sample location 600-15 are above the acceptance criteria of 30\%. Elevated RPDs in environmental samples are generally attributed to natural heterogeneity in the sample matrix. The data are usable for decision-making purposes.

A secondary check of the data variability is used when one or both of the samples being evaluated (main and duplicate) is less than five times the TDL, including undetected analytes. In these cases, a control limit of \pm 2 times the TDL is used (Appendix D) to indicate that a visual check of the data is required by the reviewer. No data required this check. A visual inspection 
of all of the data is also performed. No additional major or minor deficiencies are noted. The data are usable for decision-making purposes.

\section{G.13 SUMMARY}

Limited, random, or sample matrix-specific influenced batch QC issues such as those discussed above, are a potential for any analysis. The number and types seen in these data sets are within expectations for the matrix types and analyses performed. The DQA review of the coal ash characterization sampling data found that the analytical results are accurate within the standard errors associated with the analytical methods, sampling, and sample handling. This DQA review concludes that the reviewed data are of the right type, quality, and quantity to support the intended use. The analytical data were found acceptable for decision-making purposes. The verification sample analytical data are stored in the Environmental Restoration project-specific database prior to being submitted for inclusion in the Hanford Environmental Information System database. The verification sample analytical data are also summarized in Appendix C.

\section{G.14 REFERENCES}

BHI, 2000a, Data Validation Procedure for Chemical Analysis, BHI-01435, Rev. 0, Bechtel Hanford, Inc., Richland, Washington.

BHI, 2000b, Data Validation Procedure for Radiochemical Analysis, BHI-01433, Rev. 0, Bechtel Hanford, Inc., Richland, Washington.

DOE-RL, 2011a, Sampling and Analysis Plan for Characterization of Hanford Site Coal Ash Components, DOE/RL-2010-113, Rev. 0, U.S. Department of Energy, Richland Operations Office, Richland, Washington.

DOE-RL, 2011b, Tri Party Agreement Change Notice, TPA-CN-431, Modify Sampling and Analysis Plan for Characterization of Hanford Site Coal Ash Components (DOE/RL-2010-113, Rev. 0) to add Additional Analysis for Total Uranium on a Subset of Coal Ash Samples, U.S. Department of Energy, Richland Operations Office, Richland, Washington.

DOE-RL, 2011c, Tri Party Agreement Change Notice, TPA-CN-451, Modify Sampling and Analysis Plan for Characterization of Hanford Site Coal Ash Components (DOE/RL-2010-113, Rev. 0) to 1) Update Sample Area of 126-D-1 to Exclude an Area Containing Minimal Coal Ash and 2) Identify Waste Management Approach for Waste Related to Coal Ash Sampling Activities, U.S. Department of Energy, Richland Operations Office, Richland, Washington.

EPA, 2000, Guidance for Data Quality Assessment, EPA QA/G-9, QA00 Update, U.S. Environmental Protection Agency, Office of Environmental Information, Washington, D.C.

WCH, 2011, Characterization of Hanford Coal Ash Components, Logbook EL-1658, Washington Closure Hanford, Richland, Washington. 
WCH-506

Rev. 0 
WCH-506

Rev. 0

\section{DISTRIBUTION}

U.S. Department of Energy

$\underline{\text { Richland Operations Office }}$
J. A. Hansen
A5-11
J. P. Hanson
A5-11
J. P. Neath
A3-04
J. P. Sands (6)
A5-11
G. L. Sinton
A6-38
M. Thompson
A6-38

Washington Closure Hanford

J. M. Capron

$\mathrm{H} 4-22$

S. W. Clark

$\mathrm{H} 4-23$

J. A. Lerch

$\mathrm{H} 4-22$

R. W. Ovink

$\mathrm{H} 4-23$

H. M. Sulloway

$\mathrm{H} 4-23$

CH2M HILL Plateau Remediation Company

D. E. Dooley

R3-50

B. H. Ford

H8-43

U.S. Environmental Protection Agency

$\begin{array}{ll}\text { L. Buelow B1-46 } & \text { B }\end{array}$

Washington Department of Ecology

$\begin{array}{ll}\text { A. Boyd } \quad \mathrm{HO}-57 & \end{array}$

Document Control H4-11

Administrative Record H6-08 
WCH-506

Rev. 0 